\title{
Pharmaceutical Patents in Europe
}

\author{
Bengt Domeij
}

\section{Contents}

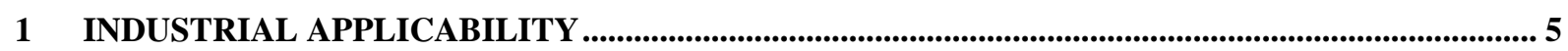

1.1 INDUSTRIAL APPLICABILITY OF PHARMACEUTICAL PRODUCT PATENTS …………………….................. 5

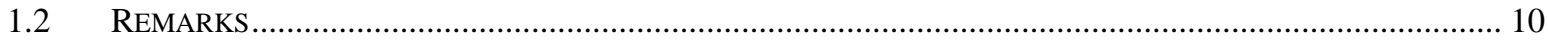

1.3 INDUSTRIAL APPLICABILITY OF MEDICAL PROCESSES........................................................................ 14

1.3.1 Processes applied to the human or animal body.................................................................... 14

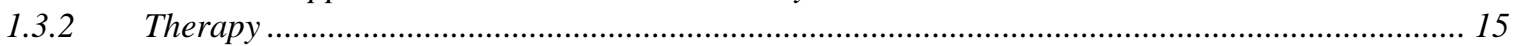

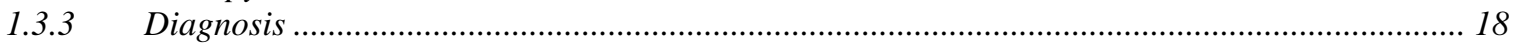

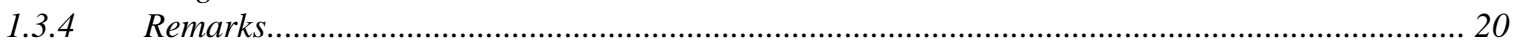

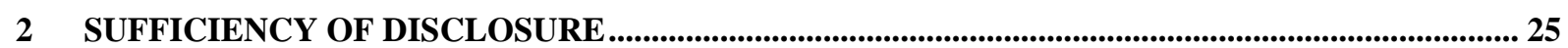

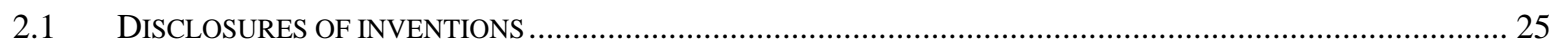

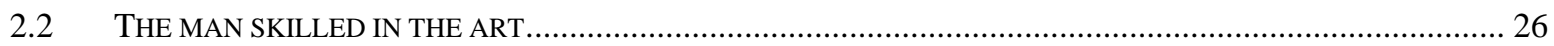

2.2.1 The different aspects of the man skilled in the art....................................................................... 26

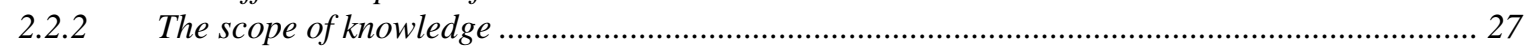

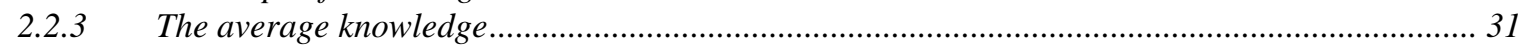

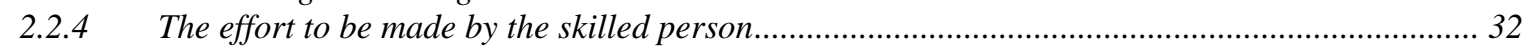

2.2.5 Parts of the patent application that the skilled man may use............................................................... 35

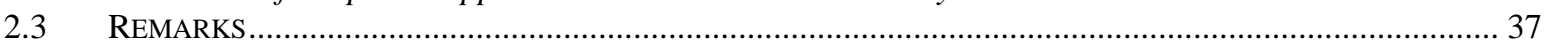

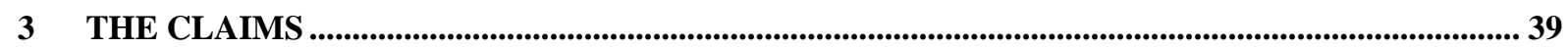

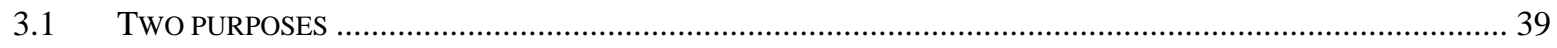

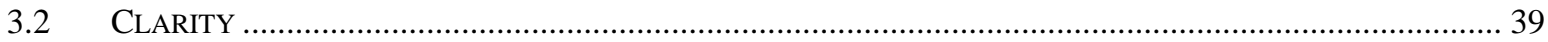

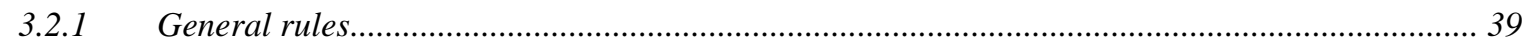

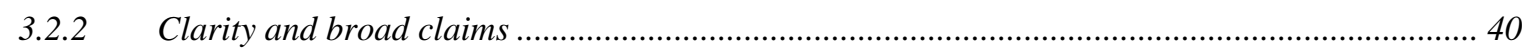

3.2.3 Definitions of chemical compounds …………………............................................................ 42

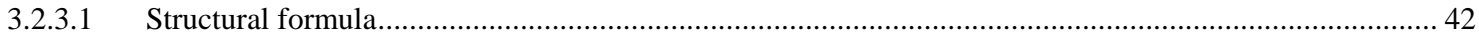

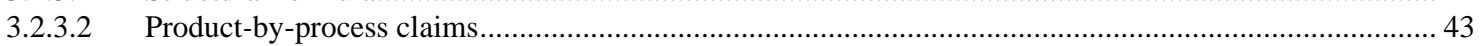

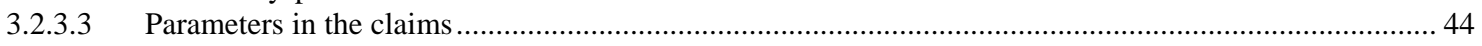

3.3 THE RELATIONSHIP BETWEEN THE INVENTION AND THE SCOPE OF PROTECTION ……............................... 46

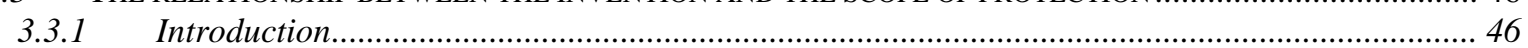

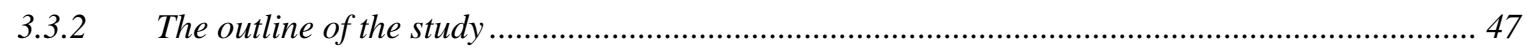

3.3.3 Structural features - Markush claims ........................................................................................ 49

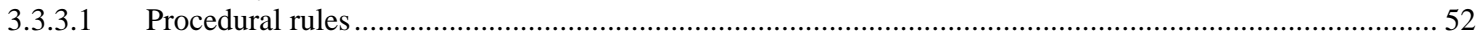

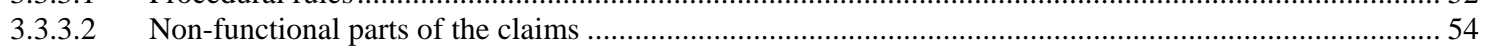

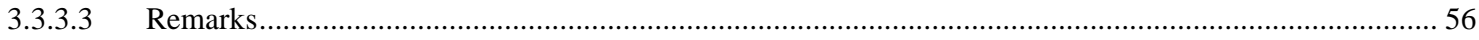

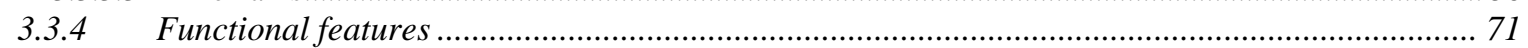

3.3.4.1 Functional features - particularly in biotechnology ........................................................................ 71

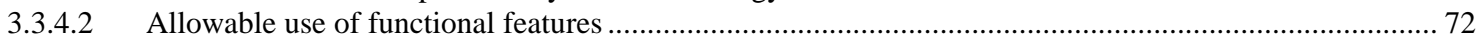

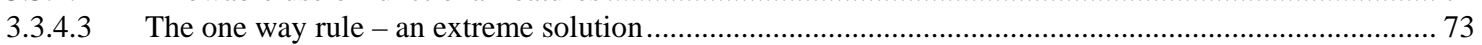

3.3.4.4 Functional claims in cases of deposition ..................................................................................... 76

3.3.4.5 Generalisation from one to more organisms ......................................................................................... 81

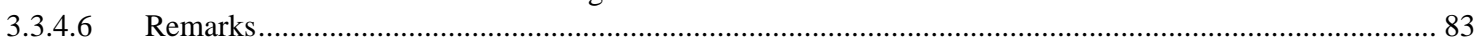

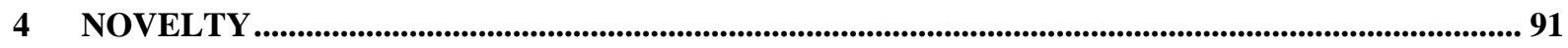

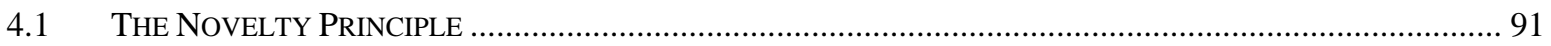

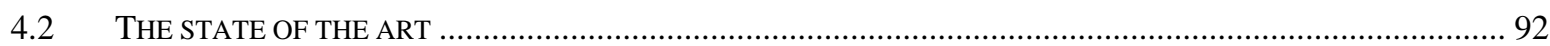

4.2.1 Absolute and global novelty - an extensive definition of the state of the art ................................ 92

4.2.2 The skilled man must be able to use the state of the art................................................................. 94

4.3 NOVEL TECHNICAL FEATURES IN PRODUCT PATENTS FOR ACTIVE SUBSTANCES ..................................... 98 


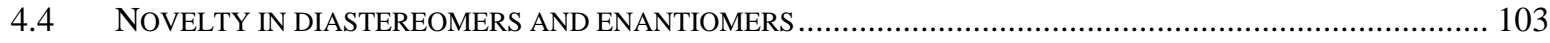

4.5 A STARTING SUBSTANCE AND A PROCESS GIVING THE CLAIMED SUBSTANCE .................................... 107

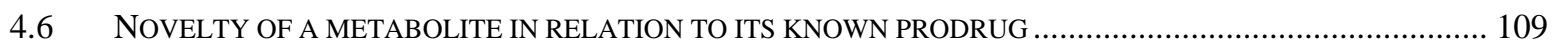

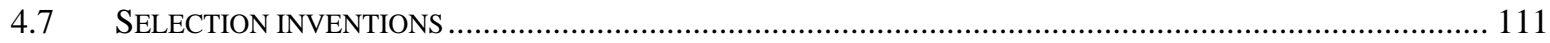

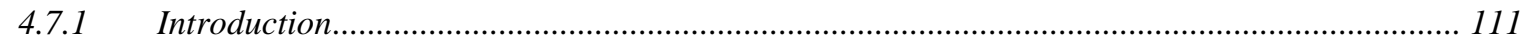

4.7.2 Selection with formulae and with individual substances...................................................... 112

Selection from Markush formulae .............................................................................................. 114

Selection from an unlimited number of possibilities ............................................................... 116

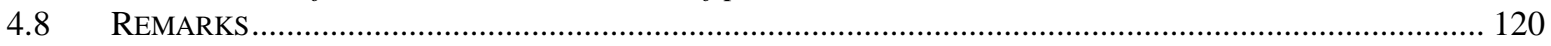

4.8.1 The global and absolute novelty requirement ................................................................. 120

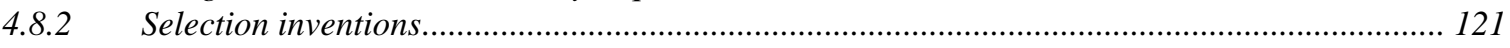

5 NEW MEDICAL USES OF KNOWN COMPOUNDS …............................................................... 127

5.1 NEW MEDICAL INDICATIONS AND THE SYSTEMATISATION OF PATENT LAW ................................... 127

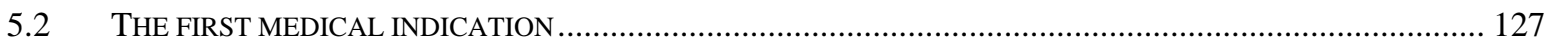

5.2.1 The breadth of patent claims to a first medical indication...................................................... 128

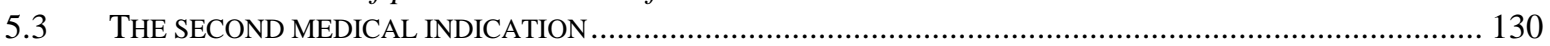

5.3.1 Introduction of patents for a second medical indication........................................................... 130

5.3.2 Breadth of claims for a second medical indication ............................................................. 133

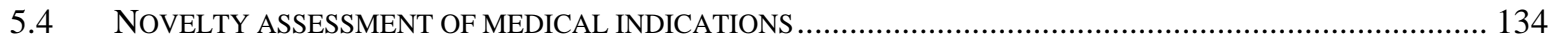

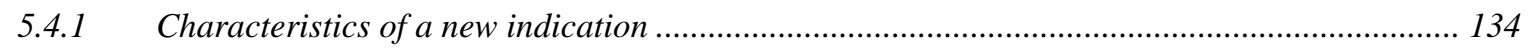

5.4.2 New biological mechanism as a new indication ................................................................ 135

5.4.3 New category of patients as a new indication................................................................. 137

5.4.4 Method of administration as a new indication ....................................................................... 138

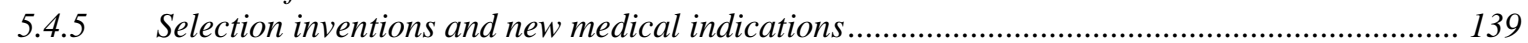

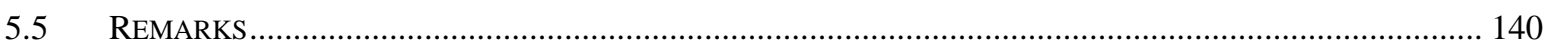

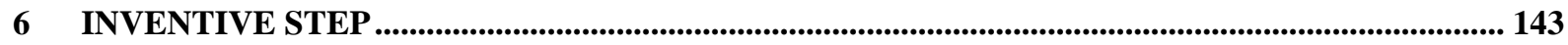

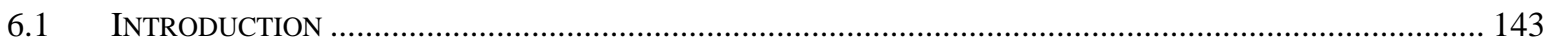

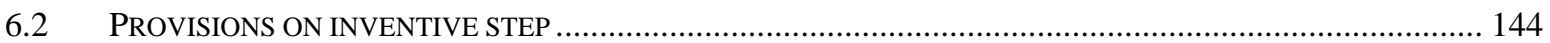

6.3 SPECIAL CONDITIONS APPLYING TO PHARMACEUTICAL INVENTIONS .............................................. 144

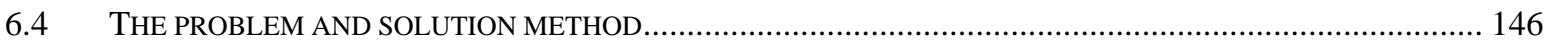

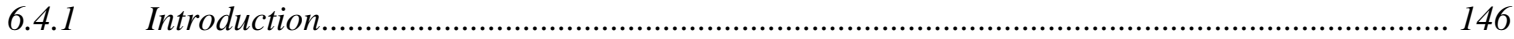

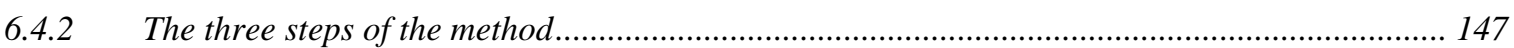

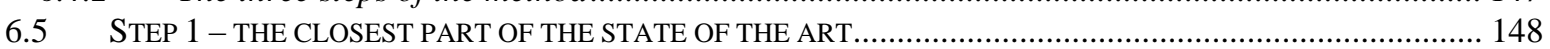

6.5.1 Consideration of technical effect and structure .................................................................. 148

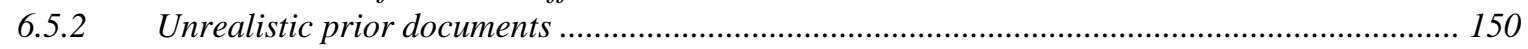

6.5.3 Circumstances disclosed after the priority date ..................................................................... 152

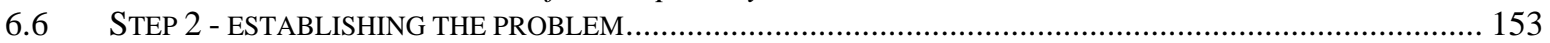

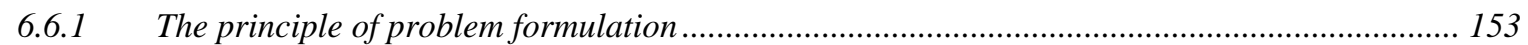

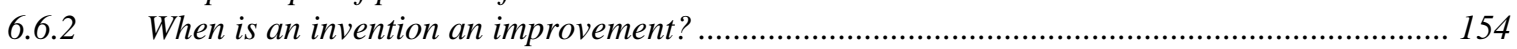

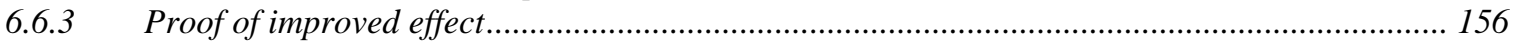

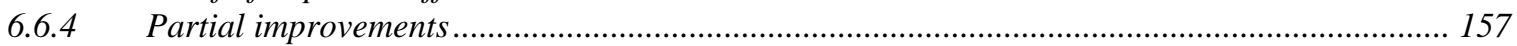

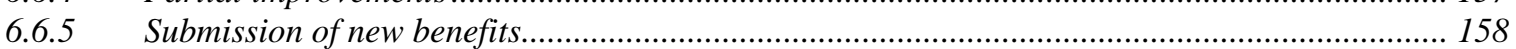

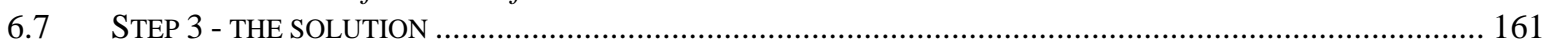

6.7.1 The solution - the features producing the technical effect..................................................... 161

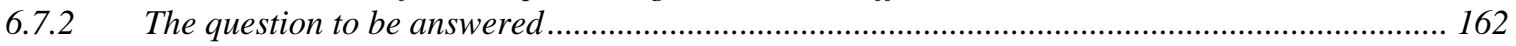

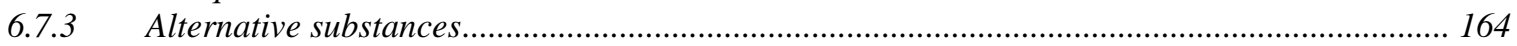

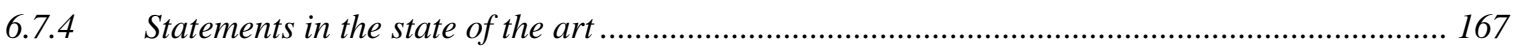

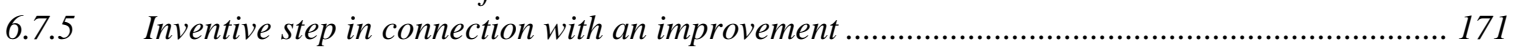

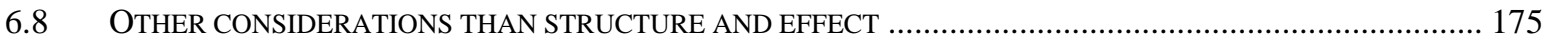

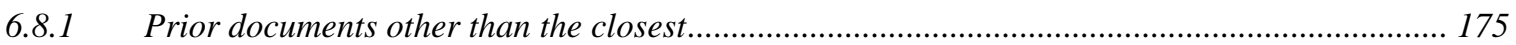

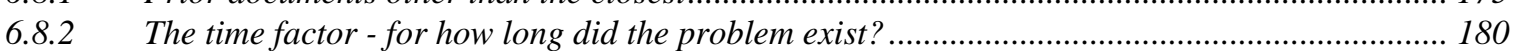

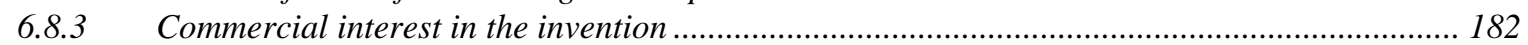

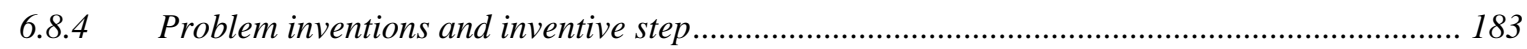

6.8.5 Inventive step in the creation of biotechnology ..................................................................... 186

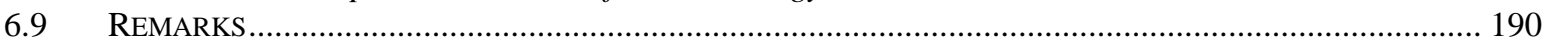

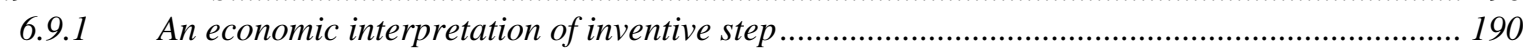

6.9.2 The "market" for the creation of pharmaceutical inventions .................................................... 192 


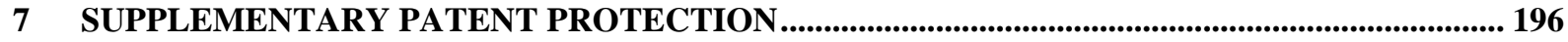

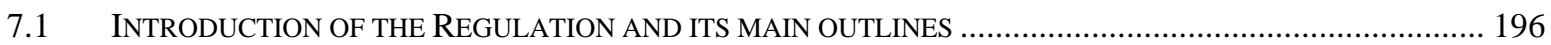

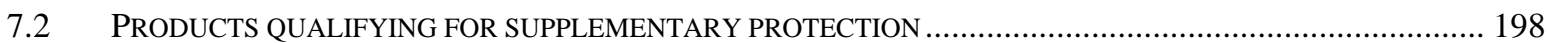

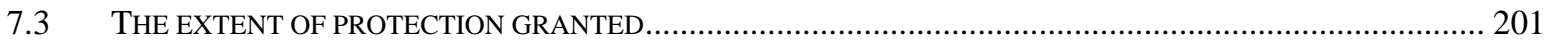

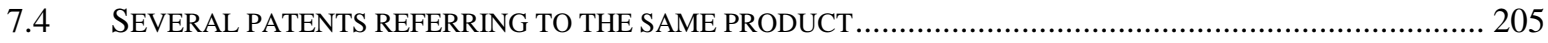

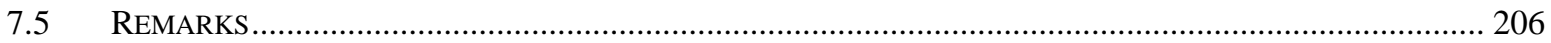

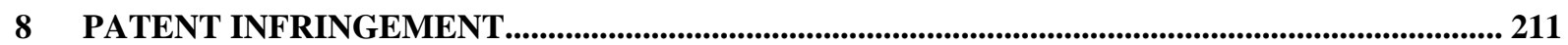

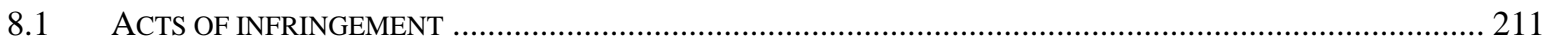

8.1.1 Direct products of a patented process................................................................................. 211

8.1.2 Exceptions from the exclusive right ........................................................................................ 213

8.1.3 Exhaustion of the patent right - parallel imports of medicinal products..................................... 213

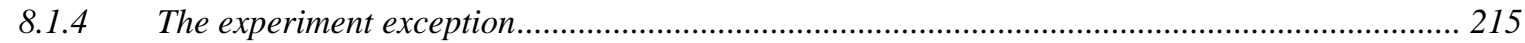

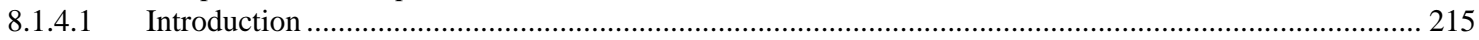

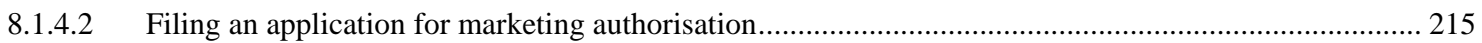

8.1.4.3 Submission of samples of medicinal products to the authorising authority ……………………………. 216

8.1.4.4 Experiments for the collection of data................................................................................................... 217

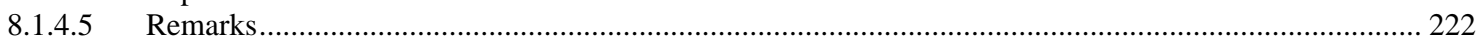

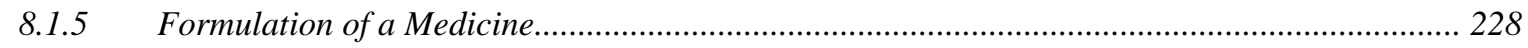

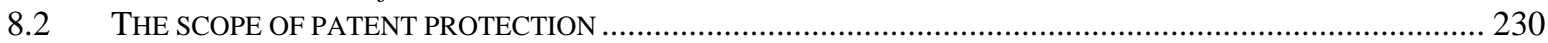

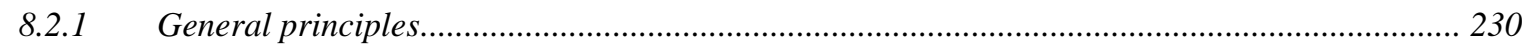

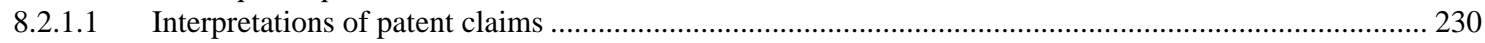

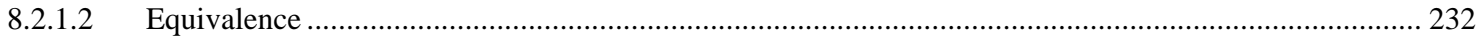

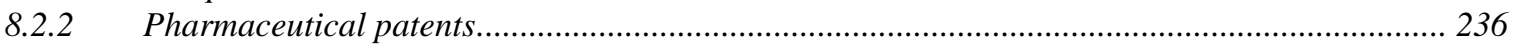

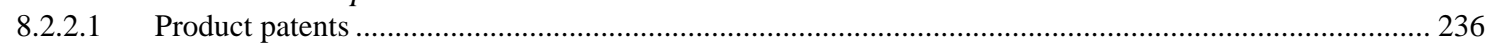

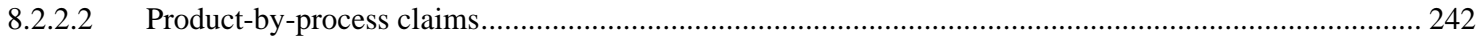

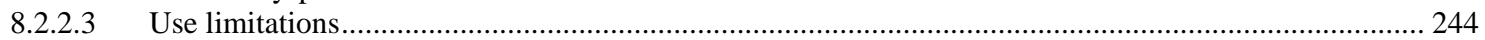

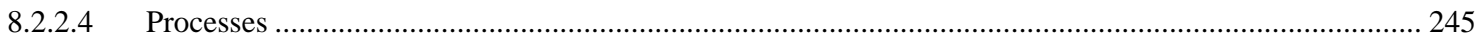

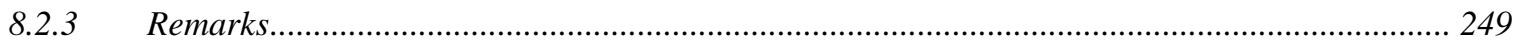

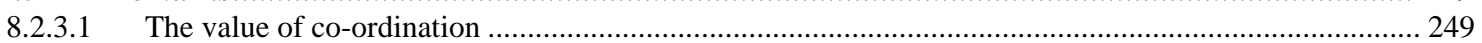

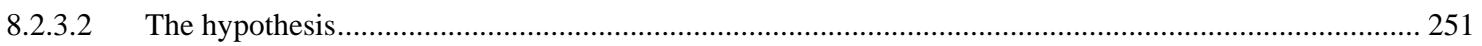

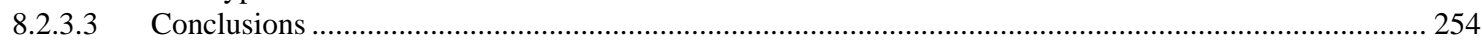

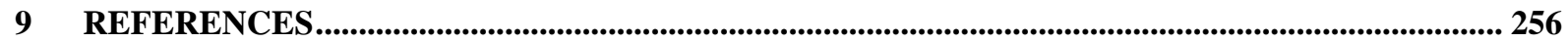




\section{Abbreviations}

$\begin{array}{ll}\text { Art. } & \text { Article } \\ \text { EC } & \text { European Community } \\ \text { ECJ } & \text { European Community Court of Justice } \\ \text { ECR } & \text { European Court Reports } \\ \text { EIPR } & \text { European Intellectual Property Law Review } \\ \text { EPC } & \text { European Patent Convention 1973 } \\ \text { EPO } & \text { European Patent Office } \\ \text { F.S.R. } & \text { Fleet Street Reports } \\ \text { GRUR } & \text { Gewerblicher Rechtschutz und Urheberrecht } \\ \text { GRUR Int. } & \text { Gewerblicher Rechtschutz und Urheberrecht/Internationaler Teil } \\ \text { IIC } & \text { International Review of Industrial Property and Copyright Law } \\ \text { JPTOS } & \text { Journal of Patent and Trademark Office Society } \\ \text { NIR } & \text { Nordiskt Immateriellt Rättsskydd } \\ \text { OJ EPO } & \text { Official Journal of the EPO } \\ \text { R.P.C. } & \text { Report of Patent Cases } \\ \text { SPC } & \text { Supplementary Protection Certificates }\end{array}$




\section{Pharmaceutical patents in Europe}

\section{Industrial applicability}

\subsection{Industrial applicability of pharmaceutical product patents}

One of the most basic exercises for a student of organic chemistry is that of using well-known methods of synthesis to manufacture chemical compounds. With a few reactive starting materials and equipment for utilising different reactions, the student will be able to manufacture an almost unlimited number of compounds. It is probable that, from a patent law perspective, one of the compounds could be new, in the sense of having no previously recorded existence. But no one would seriously think that the student had invented anything, because what chemists, like any other engineers, are after is products with practical uses. Short of being a new element, a new chemical entity is hardly useful or particularly interesting in itself. A new chemical entity is only interesting if it has been demonstrated to have features that are sought-after for some reason. Only then is it relevant to view the compound as an invention and possibly to apply for a patent.

Research leading to new compounds with pharmaceutical uses mainly involves a series of minor changes to the structure of one or more so-called leading compounds. The changes are evaluated by tests done in vitro or in vivo. If the research is successful, one of the compounds will have an interesting biological effect. That effect may be of value to society, so that the compound meets the requirement of industrial applicability in Art. 52 EPC. This test is similar to the American patent law requirement of utility.

A new pharmaceutical needs official regulatory approval before it can be sold, but the patent law requirement of industrial applicability will be fulfilled much earlier. Perhaps only a small percentage of all patents for compounds with a pharmaceutical use mentioned in the application later protects a product that is approved for sale. There are a plethora of possible reasons for a patented compound not being used and sold. Apart from the strictly economic considerations, the clinical trials may prove the biological effect to be less in humans than in the earlier pre-clinical trials. It may also be that the compounds cause unacceptable side effects. In this part we shall be considering what consequences knowledge of these kinds of problems may have in the patent law test for industrial applicability.

Rule 27(1)(f) EPC provides that the patent description should:

"[I]ndicate explicitly, when it is not obvious from the description or nature of the invention, the way in which the invention is capable of exploitation in industry."

If no use for the compounds is mentioned in the description, there is probably reason to conclude that there is no industrial applicability, but this may also be grounds for finding a lack of inventive step. In case T 939/92 the Board of Appeal found that it was questionable whether a chemical compound without any known useful property was an invention at all. However, the application was refused on the ground that there was no inventive step. If a compound had no known use, any compound could do what the invention did, i.e. nothing. Therefore the man skilled in the art would have been able to do what the applicant had done. An arbitrary selection of chemical compounds therefore lacked inventive step. ${ }^{1}$

1 T 939/92, OJ EPO 1996, 309: "Although the board is not convinced that, in the absence of any technically useful properties, the claimed compounds could be regarded as being a technical invention at all ..., the board has nevertheless examined whether the notional person skilled in the art would have considered the claimed compounds as a solution of such a hypothetical 'technical problem'... [I]n the board's judgement the answer to the question as to what a person skilled in the art would have done depends on the result he wished to obtain .... 
Regularly patent applications on pharmaceuticals carry a statement about the use for the compounds and reports of some experiments where this biological effect has been found. This is usually accepted by the EPO. In T 156/95 the technical problem confronted was to provide further chemical compounds useful as analgesic agents. In view of the inhibition of phenylquinone-induced writhing in mice, reported for two representatives of the claimed group of compounds, the Board was satisfied that the subject-matter of the claims solved the said technical problem. ${ }^{2}$ In the EPO Guidelines the general principles for evaluation of such proof are described as follows:

"Facts adduced by a party will ... normally be deemed true, even without supporting evidence, if it is clear that no doubts exist concerning them, if they do not contradict one another or if no objection is raced. In such cases the facts need not be supported by evidence.”3

Only if the examiner has serious doubts about the effect stated in the application, e.g. if they appear contradictory or otherwise unlikely, will supplementary proof be demanded, by means of new experiments. ${ }^{4}$ This means that the test for industrial applicability does not usually play a material role in the examination process.

It is not even necessary in principle to report any experiments. In case T 964/92 an application had been made pertaining to compounds for the treatment of angina pectoris. The applicant stated that the compounds had better pharmaceutical effects than compounds used conventionally for the same indication, but he did not refer to any examples to support this. The Board of Appeal observed that the compounds were closely related structurally to compounds known to have the use that was mentioned in the claims. This made it likely that the compounds in question had the therapeutic effect mentioned in the application. Compounds with similar structures can normally be assumed to have similar characteristics. The most instructive part of the judgement, though, was the Board's conclusion that if a biological effect is so probable, due to structural considerations, that examples are not necessary, then the effect cannot at the same time be used as support for the existence of inventive step. ${ }^{5}$ Thus, a claim by the applicant relating to the industrial applicability of the

If this result is only to be seen in obtaining further chemical compounds, then all known chemical compounds are equally suitable as the starting point for structural modification, and no inventive skill needs to be exercised in selecting, for instance, the compound of formula XIV of D3 for this purpose. Consequently, all structurally similar chemical compounds, irrespective of their number, that a skilled person would expect, in the light of the cited prior art, to be capable of being synthesised, are equally suitable candidates for solving such a hypothetical 'technical problem', and would therefore all be equally 'suggested' to the skilled person. It follows from these considerations that a mere arbitrary choice from this host of possible solutions of such a 'technical problem' cannot involve an inventive step .... In other words, the board holds that ... the selection of such compounds, in order to be patentable, must not be arbitrary but must be justified by a hitherto unknown technical effect which is caused by those structural features which distinguish the claimed compounds from the numerous other compounds."

2 T 156/95 of 30031998.

3 Guidelines E IV 1.2.

4 Guidelines C VI 14.2.

5 T 964/92 of 2308 1994: “Although the application documents do not contain any test result showing that at least one of the group of compounds defined by formula I possesses the desired activity, the Board is satisfied that the above-defined technical problem is credibly solved by the claimed compounds, having regard to the common general knowledge reflected by document (2). This document provides an example for the widely accepted assumption that chemical compounds of similar structure normally have similar properties, including biological activity. ... The question as to what extent the structure of a chemical compound can be modified without causing major changes in its biological activity, is equally relevant in respect of the question as to whether or not it is credible that all members of a certain group of chemical compounds solve a particular technical problem, in the present case whether they can be fairly assumed to be useful in the therapeutical treatment of angina pectoris, and to the question of whether or not such an activity can be expected on the basis 
compounds can be supported by their structural similarity to compounds already known to have the effect in question. It is sometimes feasible to make such an analogy about pharmaceutical effects. Reports from experiments, then, are not necessary. But this will probably be of little use to applicants, since the pharmaceutical effects the compounds are believed to have, then will be "automatically" deemed obvious to the man skilled in the art. This will probably mean that there is no inventive step if there are no examples to support the industrial applicability.

In the far majority of cases, therefore, patent applicants will describe some experiments in order to establish the probability of the therapeutic effect. These experiments will not generally have been performed on humans. Humans are normally involved at a much later stage in the pharmaceutical research process. This may complicate matters, because there is normally considerable diversity between the reactions in humans and in the test models used in the early stages of pharmaceutical research. However, tests on animals or in vitro tests are still normally understood to be sufficient from the patent law perspective, also when they refer to a new therapeutic technique, such as gene therapy. ${ }^{6}$ The necessary confirmation of industrial applicability should not have to be on a high level. ${ }^{7}$

An example of sufficient proof of usefulness is the capability of the compounds to bind to certain biological receptors. Receptors are situated on the cell surfaces and this is where pharmaceuticals usually interact to cause biological effects. In case T 643/96 the Board stated:

"It follows that the technical problem to be solved can be seen in providing further chemical compounds useful in the treatment and/or prophylactic of dementia in mammals. In view of the reported radio ligand binding tests which are indicative for the muscarinic binding activity of the tested

of the state of the art. If the answer to the former question can only be 'yes' with respect to considerations forming part of the common general knowledge, then the question whether or not the solution of the same technical problem by providing the same group of compounds was obvious must be answered on the basis of the same considerations, since in the Board's judgement the same common general knowledge must be taken into account in respect of all aspects of patentability ... It is thus not permissible that an applicant, who, as in the present case, has alleged the solution of the technical problem by a group of chemical compounds comprising individual compounds which have neither been synthesised nor tested for the desired biological activity, wishes, at the same time, to have any kind of structural modification with respect to the state of the art to be considered inventive. In other words, if the solution of the underlying technical problem is only credible because it can be fairly assumed that compounds of similar chemical structure would to a certain extent have similar therapeutical activity, then the skilled person would consider the same kind of structure-activity relationship when looking for further compounds having the same activity."

6 T 856/94 of 0506 1997: "The technical contribution to the state of the art by the disclosure of the application consists of providing a technical solution to the treatment of diseases of the CNS by means of host cells expressing therapeutic molecules. The technical contribution achieved by the application is not a new technique for gene therapy in the CNS, but achievement of the technical result foreshadowed at a theoretical level ... In view of Examples II and III disclosed in the application in suit, the Board is satisfied that the technical problem mentioned above has been solved. Example II shows that donor cells genetically modified to express NGF and implanted into the brains of rats with a surgical lesion of the fimbria-fornix, prevent the degeneration of cholinergic neurons that would die without treatment. Example III shows that cells genetically modified for expressing tyrosine hydroxylase (TH), an enzyme capable of catalyzing the conversion of tyrosine to L-DOPA, once implanted into the rostral-caudate striatum, significantly reduce drug-induced rotations, a rat model for Parkinson's disease.”

7 In regard to EPO-case law, it has been said that: "In the case of inventions relating to new products, for instance new chemical compounds ... at least one process for preparing them and their utility, or, better, 'industrial applicability' in the sense of Article 57 EPC, should be described. A predicted utility is sufficient. It is not necessary to provide absolute proof that the utility indeed exists, e.g., for a pharmaceutically useful compound, it can be sufficient to refer to in vitro data or to data obtained by animal experiments, as long as this reference is credible under the circumstances.” See Jaenichen, H-R., The European Patent Office’s Case Law on the Patentability of Biotechnology Inventions p. 344. 
compounds ... which in turn is indicative of their usefulness as anti-dementia agents ... the Board is satisfied that the subject-matter of Claim 1 solves the said technical problem."

The receptor-binding property of the compounds was sufficient proof of their anti-dementia activity. In decision T 795/94 the applicant claimed that some compounds had an agonistic activity at the muscarinic receptor and therefore could be use in the treatment and/or prophylaxis of dementia. ${ }^{9}$ In support of this the applicant supplied a sheet having the title "Data comparison", with IC50-values (the concentration which inhibits binding of the ligand by $50 \%$ ) for the claimed compounds compared to some known compounds. The Board concluded that this comparison gave an indication of the agonist character of the compounds and that therefore the therapeutic effect was credible. It is a low requirement of proof of industrial applicability to accept inhibition of ligand-binding as indicative of a therapeutic effectiveness. The technical uncertainties that have to be overcome before a pharmaceutical can be sold are considerable.

Another side of the requirement for industrial applicability, however, is that it is unacceptable for the applicant not to have performed certain necessary tests, owing to the expense entailed or other practical problems. In case T 164/83 the applicant did not carry out animal tests with the pharmaceuticals because it would have been very expensive and the tests would allegedly not have been permitted in Germany. The Board of Appeal concluded that these arguments could not be accepted if the tests were necessary in order for the Board to be able to decide the issue of patentability. The lack of universally accepted rules as to animal tests made it impossible for the Board to take into account the situation in the different EPO member states. ${ }^{10}$

The test for industrial applicability tends to be more at work when an opponent questions the reliability of the tests that have been done by the applicant. Then the actual effects of the invention may be judged. Both parties will be in a position to carry out practical experiments and the Office can make a comparison between the proof that is presented to it. In the EPO-Guidelines it is for this situation stated:

"If the Opposition Division has serious doubts as regards the possibility of performing the invention and repeating it as described, the burden of proof as regards this possibility, or at least a demonstration that success is credible, rests with the proprietor of the patent. This may be the case where, for example, experiments carried out by the opponent suggest that the subject-matter of the patent does not achieve the desired technical result."11

The test to be applied was described in T 270/90 as a "balance of probabilities" and was to be contrasted with other possibilities such as "beyond all reasonable doubt" or "absolute conviction". ${ }^{12}$ This meant that an opposition regarding the industrial applicability of the

\footnotetext{
$8 \mathrm{~T} 643 / 96$ of 14101996.

9 T 795/94 of 21081997.

10 T 164/83, OJ EPO 1987, 149: "The argument about the costly character of the comparative tests is not persuasive ... Whether or not in a particular country experiments on animals may also be problematic under national law cannot be taken into consideration either, since this or similar difficulties could arise in connection with the testing or development of any invention or some ground or another in various countries of the world, and no special considerations could apply to applicants of various nationalities on such grounds. The Board has therefore come to the conclusion that the possibility of a prohibition of experiments with animals in one Contracting State of the EPC is not a sufficient reason for declining the submission of test results in comparison with the closest state of the art if the inventive step can only be demonstrated in this manner."

11 Guidelines D-V 4.3.

12 T 270/90, OJ EPO 1993, 725: "When arriving at their decisions, the Boards, in addition to exercising their inquisitorial powers, (should this be necessary), decide the issues before them on the basis of the evidence adduced by the parties. Their decision need not, and in deed in most cases could not, be based on absolute
} 
invention required the opponent to have done more than simply claim that the invention does not work. The opponent can do what the patent office cannot, namely carry out practical experiments. This should be done by the opponent using the practical knowledge of the man skilled in the art. Furthermore, the experiments must be of such a nature as to allow the patentee an opportunity repeating them. ${ }^{13}$

The legal standard that the patent office should adhere to thereafter when "balancing the probabilities" is not whether the invention is economically feasible or if it is a "good" invention. It is a strictly technical question. Does the invention work at all? In case T 923/92 it was stated:

"[T]he Board observes that the later evidence submitted by the appellants [the opponents] does not prove that it was impossible to obtain expression of human t-PA molecule in E.coli, but that expression of active t-PA 'at a significant level' was not shown, ... or that 'expression is poor'. It should be remembered that at issue here is whether the teaching of the patent in suit is sufficient in order to achieve expression of human t-PA in E.coli at any level."14

Another instructive case in this respect is T 449/90. The invention was a process to heat blood plasma and was characterised by the functional feature of any AIDS or Hepatitis virus becoming "substantially inactive". An opponent pointed out that if the AIDS virus were not fully inactivated, patients would get the disease and probably die. The Board commented by making a statement to the effect that the applicant had not claimed that the process was perfect. It was one step towards a method of completely inactivating virus in donated blood, and that was sufficient from a patent law perspective. ${ }^{15}$ Further, in case $\mathrm{T}$ 548/91, an opponent claimed that some structural elements in the compounds automatically gave unwanted side effects. This, however, did not suffice for the Board of Appeal:

"Appellant [the opponent]... finally contested the sufficiency of disclosure by arguing, that some of the claimed compounds possess some structural elements which would automatically confer to the corresponding compounds some undesirable properties. In the present case, this technical argument is

conviction, but has, instead, to be arrived at on the basis of the overall balance of probability, in other words on the footing that on set of facts is more likely to be true than the other".

13 T 122/84, OJ EPO 1987, 177: "[E]ven the presentation of test results will not as a rule suffice as definitive evidence of the validity of the argument against maintaining the patent. This is especially true if, as in the present case, the patent specification cites examples of how the invention can be carried out. With chemical processes in particular, the desired result is often not obtained under all conditions apparently possible in the light of the description. Even a well-intentioned opponent may, when reproducing a chemical process, meet with failures which can only be overcome by additional experiments that he could reasonably be expected to perform. The revocation of a process patent in the field of chemistry solely on the grounds that it cannot be carried out can hardly be justified without the patent proprietor having been given an opportunity to check the opponent's experiments and to confirm by means of new tests of his own that the invention is in fact reproducible."

14 T 923/92, OJ EPO 1996, 564.

15 T 449/90 of 0512 1991: "The Board would like to note here that the argument put forward by the Respondents that the inactivation of the viruses in the case of AIDS has to be complete because otherwise the composition might be fatal, is a question of a certainly highly desired effect; it is, however, not a question of the requirements of Article 83 EPC with regard to the fact that the claim has the wording 'substantially inactivated'. These circumstances are already described in the original disclosure ... where it is made clear that the claimed composition provides a product in which the titer of viruses is reduced so low that infusion of therapeutic quantities of the product into a plurality of normal animal hosts for the virus will fail to produce clinical or serological evidence of infection in a host population, or will delay significantly the onset of infection in such population. There was, therefore, no claim that a hundred per cent, i.e. complete inactivation of viruses can be ensured, whereas the claimed compositions are to be considered as a step further in the direction of the certainly desired situation of compositions being completely free of any infectious viruses which may put life at risk. There is, thus, no requirement as to the mentioned article of the EPC that the composition has to be entirely free of any viruses.” 
not relevant, since it is part of the common general knowledge in this technical field that some compounds with a complex structure exhibit simultaneously different pharmaceutical activities and insofar as Appellant ... failed to provide experimental data showing that the undesirable properties are preponderant over the desired property."16

The Board essentially stated that toxic effects can be accepted, if they do not preponderate over the desired property of the compounds. This means that the known side effects may not be so severe that a practical use of the valuable properties is excluded. Of course, it is enough if the advantages are present for a certain category of patients. Only if one can see no possibility of a practical use for the compounds should it be concluded that they have no industrial applicability. This is a marginal patentability requirement and does not often come into question.

\subsection{Remarks}

The pharmaceutical research process is particularly long and expensive. For that reason it is of considerable importance that patents can be awarded at an early stage in the process. The patentee will then know that only he may take advantage of the investments that are subsequently made in a particular group of compounds. This will increase his incentives. The merit of this has been much emphasised.

"With the selection of the lead compound, the chemist and biologist embark on an extensive program to improve its potency, the specificity of biological effect with concomitant reduction in toxicity, oral absorption, duration of action, metabolic pattern and pharmacokinetic pattern. This will involve extensive structure-activity (SAR) studies. Needless to say, the lead structure series must be patentable."17

From a social point of view it also allows companies to do parallel, i.e. co-ordinated, research efforts, rather than duplicative and therefore, on the whole inefficient research on the same compounds.

"[I]t could be argued that unpatentability of ligands [compounds binding to receptors] will only incite the industry to maintain its secrecy until further development (lead optimisation) yields a patentable compound. In such a case, the only net effect of ligand unpatentability would be that drug candidates are patented later in their development. ... [But], recognition that the optimised ligand could be patented later would thus result in the entire optimisation process occurring without legal protection beyond a possible trade secret. Until the patent is obtained, the developer has no way of knowing whether competitors are developing the same compound in parallel, what the status of such development is, and so forth. Although lead optimisation does not constitute the bulk of drug development costs, it remains significant. 'Later patenting' would possibly make this investment too risky to be worthwhile and could actually deter development."18

Early patenting has the valuable effect of reducing wasteful duplicative research. This suggests that it is essential that patents can be applied for at a time when the applicant has only made in vitro or animal tests. The test for industrial applicability should not include a need to do human tests. Many years of research need to be invested in a group of compounds before clinical trials can commence. Developments in the USA are a good example of the

\footnotetext{
16 T 548/91 of 07021994.

17 See de Stevens, G., 266, in Hansch, C. and Dayton, C. (eds.), Comprehensive Medicinal Chemistry.

18 See Ducor, P., 22 Rutgers computer \& technology law journal 1996, 461.
} 
importance of applicants not having to demonstrate results from clinical trials. The requirements for utility in relation to pharmaceuticals was reduced in 1995 in the USA, mainly in response to complaints from small biotechnology companies that were unable to obtain the resources necessary for carrying out clinical trials without already having a patent. $^{19}$ The importance of a low threshold for the utility of compounds has increased over the last years because pharmaceutical companies try to move away from animal test towards more in vitro test. ${ }^{20}$ In vitro tests are often more cost-effective, reliable and also do not cause the same public protests as animal tests sometimes do. It is obvious that the current acceptance by the EPO of in vitro tests as confirmation of industrial application is important and does not generally result in premature patenting.

But there is probably no dispensing entirely with the requirement that there should be a substantiated practical use mentioned in a patent application for a chemical compound. The present-day low threshold of 'industrial applicability' is helping to create a situation where almost everything can be patented, and this may come expensive to society. It has been said that:

\begin{abstract}
"Today, patents are avidly pursued all along the lengthy road from the most basic science through to the market-place for pharmaceuticals. Because every step along the way draws another patent application, the path towards public possession of real benefits is increasingly observed by dense thickets of intersecting, overlapping, and cross-blocking patents. Those operating at the beginnings of the road are most insistent on their right to nail down leverage that will remain formidable despite marketplace rejection of the uses to which they saw their inventions may be put. The frank aim of these early stage workers is to control ultimate applications discovered by others. The system is abused if those who would benefit in this way from the later labour can posit patents on the most strain utilities imaginable". ${ }^{21}$
\end{abstract}

This development has become particularly pronounced in the biotechnology field. Patents have e.g. been applied for gene fragments, i.e. parts of full-length genes. ${ }^{22}$ The fragments, being only a portion of the full-length gene, cannot be used to manufacture a full protein. When the patent applications are made, it is usually not known what biological function the full-length gene and the corresponding protein have in the human body. This is the most expensive step in the pharmaceutical research process, and it may become unattractive when fragments of the genes are already patented. ${ }^{23}$

"If marginal inventions such as the NIH's [the first and much debated patent applications regarding gene fragments] are patented these will raise the costs of companies attempting to sell new gene-based drugs as they will need to pay royalties to the licensees of these marginal products, for example, for recombinant deoxyribonucleic acid (rDNA) technology (for which Stanford University and the University of California earned \$17 million in royalties in 1991), other 'enabling technologies', and patents for specific genes. Failures to pay these costs results in increased infringement litigation. If the NIH's application succeeds, companies may abandon products development to take up routine genetic sequencing in order to stake a claim to as much of the genome as possible."24

The test for industrial applicability can fill an important function in this situation. Patent offices and courts can use it to only permit patents where the applicant has demonstrated that he can develop the commercial products that fall within the granted protection. Patents should

\footnotetext{
19 The American development is described in Kjeldgaard, R. H., Marsh, D. R., [1997] EIPR 16.

20 See Ducor, P., 22 Rutgers Computer \& Technology Law Journal 1996, 388.

21 See Kiley, T. D., 257 Science 1992, 915.

22 For a technical background see Oser, A., IIC 1999, 3.

23 See Straus, J., GRUR 1998, 317.

24 See Roberts, C., [1994] EIPR 31.
} 
not function only as claims to what others may develop in the future. To avoid this it can be tested by the patent offices whether the applicant has proved that he has the technical capacity that it realistically takes to use the production opportunities within the scope of protection. If the applicant has not demonstrated such a capacity, then there are advantages in maintaining a situation of continuing competition. Co-ordination through the patent system is only advantageous when production opportunities have been identified and the applicant has disclosed a realistic use for them. Then, but only then, can inefficient duplicative research work be avoided.

If only a fragment of a gene has been sequenced and no knowledge exists about the biological function of the full-length gene, no therapeutical or diagnostical uses of the gene can be efficiently exploited. But perhaps the chromosomal localisation of a gene section, or the identification for individual-related, tissue-specific or forensic uses, can be exploited commercially. ${ }^{25}$ Thus, the patentee has not shown that he can use all the production opportunities that would be protected by such a patent, but only some non-therapeutic ones. If later someone else develops the therapeutic uses, infringement would probably occur. The fragments are parts of the full-length genes and therefore probably the genes would infringe a patent for the fragments, of course depending on the actual claim wording. Usually it is patent infringement to incorporate a patented product (the fragment) in a larger commercial product (the full-length gene or protein). The claimed fragment will be a part of the product that may become used therapeutically or diagnostically. ${ }^{26}$ One example of a patent claim that would not, on the normal principles of claim construction, exclude the full length gene is:

“A nucleic sequence comprising a sequence of nucleotides as shown in SEQ ID No X”27

It is probably efficient to allow patents for gene fragments, but it seems to be essential that the scope of protection is more pruned back, namely to the uses of the fragments that the patentee has demonstrated or at least made likely that he can exploit commercially. This means that the claims may, for example, protect the opportunity to use the fragments to search for full-length genes, i.e. as a research tool. That is a practical possibility that the fragments possess and which the patentee will be able to use commercially. But the scope should not encompass the gene-based pharmaceuticals or diagnostics that may be developed in the future with knowledge of biological functions, unless for example the applicant has shown a sequence homology to a gene for which a function has already reliably been assigned. This use of bioinformatics may be a way to assign a putative function or functions to the gene and corresponding protein in question. Once the function of a gene is known it can be a target for the screening of compounds with the aim of finding one which modifies the function of the gene or the corresponding protein and hence has therapeutic potential. However, it is questionable whether it is enough to assign a putative function entirely on the basis of computer analysis, without the use of biochemical studies. It is a technical question. Bioinformatics is reasonably reliable at assigning functional domains to particular proteins, but in a proteins natural environment there are many other things which actually influence the function of the molecule, such as, the presence of particular co-factors or cellular localisation. At present it can only be concluded that the patentability of such sequences will rest on the technical evidence which can be advanced at the time of examination. ${ }^{28}$

\footnotetext{
25 See Oser, A., IIC 1999, 9.

26 See Caskey, T. C., Tribble, J. L., Science and Public Affairs Autumn 1997, 52.

27 See Baldock, C., Patent World March 1999, 18.

28 See Baldock, C., Patent World March 1999, 19.
} 
Besides the difficulties concerning sufficient evidence, the purely legal question of acceptable functions is equally uncertain in Europe, despite the passing in July 1998 of the Directive 98/44/EC on the Patenting of Biotechnological Inventions. ${ }^{29}$ The Directive (Article $5: 1$, Recital 16) lays down that the mere discovery of a sequence or a partial sequence of a human gene is not patentable. Article 5:3 further states that the industrial application of a sequence or a partial sequence of a gene must be disclosed in the application. But no provision sheds light on what kinds of functions are acceptable industrial applications. Is a biological or therapeutical function for the gene and the corresponding protein necessary to disclose in the application or is perhaps the use as a probe or gene marker sufficient? That is not made clear.

\footnotetext{
${ }^{29}$ Directive 98/44/EC published on July 30, 1998 in OJ EC No. L213/13.
} 


\subsection{Industrial applicability of medical processes}

New industrial processes for manufacturing of pharmaceuticals can be patented. That is uncontroversial. But processes that have a direct medical effect (therapeutic, surgical or diagnostic) cannot, due to Art. 52(4) EPC. This limitation on the scope for patentable inventions is usually explained by ethical considerations. It has traditionally been thought in Europe that medical and veterinarian practitioners should not be influenced by the patent system in their professional activities. ${ }^{30}$ It is explicitly stated in the EPC that this prohibition should not affect the patentability of products. ${ }^{31}$ Thus, only new medical uses of known devices cannot be patented. In the pharmaceutical field this will affect new pharmaceutical uses of known compounds. If a compound is known and the inventor has demonstrated a new therapeutic, surgical or diagnostic effectiveness for it, the claims must be given a particular wording, in order to avoid the prohibition of patents for new medical uses. The provision does not render unpatentable any inventions using a pharmaceutical, but particular claim wording becomes necessary. ${ }^{32}$ The claim language is discussed in chapter 5 . The main practical effect on the scope of patentable inventions of the provision is, therefore, that it entails caveats to the patenting of methods of treatment that do not include the use of a pharmaceutical, i.e. new medical techniques that are directly used by doctors or veterinarians.

\subsubsection{Processes applied to the human or animal body}

It is evident from Art. 52(4) EPC that only processes that are applied directly to the human or animal bodies are excluded. This was to limit the application to processes that are used in medical and veterinarian clinics. More "industrial" processes, e.g. for diagnostic analysis in laboratories, should be patentable. ${ }^{33}$ The process must be applied to a living body if the prohibition is to be applied. Problems of interpretation may occur in this respect when the process pertains to the treatment of an implant. T 24/91 was concerned with a process whereby an implanted synthetic eye lens was shaped by a laser. The Board concluded that this was a process applied to the human body. This was because the lens was fixed to the lenticule and thereby permanently integrated into the body. The process would directly affect the patient's eyesight. It would have been different if the process had been applied to an arm or leg prosthesis. Then it would not have been deemed a medical treatment. ${ }^{34}$

\footnotetext{
$30 \mathrm{~T}$ 116/85, OJ EPO 1989, 13: "The exclusion of such methods from patentability is not a new provision under the EPC. Prior to the coming into force of the EPC, such methods were excluded from patentability under the national laws of many European countries. The policy behind the exclusion of such methods is clearly in order to ensure that those who carry out such methods as part of the medical treatment of animals should not be inhibited by patents.".

31 Art. 52(4) 2 sentence EPC: "shall not apply to products, in particular substances or compositions, for use in any of these methods."

32 See Paterson, G., GRUR 1996, 1094.

33 See Doc BR/177/172, quoted in Moufang, R., IIC 1993, 29.

34 T 24/91, OJ EPO 1995, 512: "The appellant further argued that the claimed process did not comprise any surgical step or therapeutic treatment of the human body but, instead, comprised physical treatment of a synthetic lenticule, i.e. a specific type of prosthesis, only. ... [T] he lenticule having been secured to the cornea of the eye is a real implant, in contrast to, for example, an arm or leg prosthesis. .... Both the lens and the lenticule form a unit with the patient's cornea and serve to correct the patient's refractive error. Ablating portions of the lenticule as claimed in claim 1, therefore, directly results in a change of the refractive power of the patient's eye. Because of this direct influence of the claimed process on the refractive power of the patient's eye and his vision, the process is to be considered as treatment of the human body.”
} 
The Board moved in the same direction in T 1077/93. The application was for the use of a compound to prevent skin burns due to too much sun. The applicant's view was that the compounds worked as a filter, i.e. did not interact with the skin. It was no more therapeutic than a parasol. The Board appears to have accepted this line of argument in principle, but decided that the effect of the compound was at least to some part due to interaction with the skin and therefore the use was therapeutic. ${ }^{35}$

Finally, the requirement for a direct treatment of a body has been mentioned in relation to treatments of parasitic attacks. However, it was concluded in $T$ 116/85 that treatments of parasites are treatments of the body. It is not the parasites that are being treated. ${ }^{36}$ Treatments of parasites may therefore not be patented with use claims.

\subsubsection{Therapy}

It is often the case that a new process has both a medical and a non-medical (i.e. patentable) effect. In these situations the process should, according to case law, be viewed as a whole. One may not word the claims to only include the non-medical effect, if the two effects are associated in normal use. The occurrence of a therapeutic effect as a result of the process is sufficient to render use or process claims unallowable.

"[T]he question for the purposes of Article 52(4) EPC is not whether the main or even the only reason for carrying out the whole of the claimed method is non-therapeutic. Rather a method claim falls under the prohibition of Article 52(4) EPC already if the administration of one of the substances is a treatment by therapy, and the administration of this substance is a feature of the claim."37

One example is a process for treatment of hair loss and stimulation of hair growth. This can obviously be used for non-therapeutic reasons. The Board, however, observed that the process was not solely used for non-therapeutic reasons. Therefore, the exclusion of patents on medical processes had to be taken into account. ${ }^{38}$ In assessments of whether the two effects are inseparably linked or correlated it is significant if the times necessary for appreciating the effects are different. ${ }^{39}$

There is a presumption that if for safety reasons a process has to be carried out by persons with medical training, then, due to the exclusion in Art. 52(4) EPC, the process is not patentable. ${ }^{40}$ It can therefore be important to be able to show by experiments that no

\footnotetext{
35 T $1077 / 93$ of 30051996.

36 T 116/85, OJ EPO 1989, 13.

37 T 820/92, OJ EPO 1995, 113.

38 T 143/94, OJ EPO 1996, 430: "The therapeutic character of the treatment of hair loss using trigonelline as an active ingredient was no longer questioned by the parties. Nor can the Board find any justification for viewing the treatment of hair loss as an exclusively non-therapeutic measure. Article 52(4) EPC must therefore also be borne in mind when assessing the patentability of the subject-matter of claim 1 according to the main request." 39 T 469/94 of 0107 1997: "It is the board's view that the two effects of choline are not inseparably linked or correlated but, on the contrary, are readily distinguishable because they involve groups of persons (or patients) undoubtedly distinct. The one consists of patients known to have a muscular disease, muscular injury or epilepsy, whereas the second comprises healthy persons who will receive no therapeutic benefit from the treatment. Moreover, the times necessary for appreciating the different effects (days for the therapeutic effect and minutes or hours for the non-therapeutic effect [reducing fatigue]) would appear to be so different that no unwanted overlap of the treatment could occur."

40 T 24/91, OJ EPO 1995, 512: "The intention underlying this article is to ensure that nobody who wants to use the methods specified in this article as part of the medical treatment of humans or animals should be prevented from this by patents. Such medical treatments need not necessarily be carried out by physicians .... However,
} 
serious risks are involved in practising the claimed method. ${ }^{41}$ However, the fact that a method has to be carried out by medically trained persons or under the supervision of such persons, is not sufficient to conclude that the method is unpatentable. In T 329/94 the method in suit facilitated blood donation by increasing the flow of blood towards the extraction point. The method was to be used by medically trained persons, but since the effect was neither therapeutic nor surgical, it was patentable. There was no functional link between what was done and a bodily therapeutic effect. Changes in the blood flow were not a therapeutic effect. ${ }^{42}$

It has also been decided that a method may be deemed therapeutical even if it is not usually understood to be a treatment of an illness, i.e. having therapeutic effect. Pain, physical discomfort or reduced physical capacity, may be the consequences of illnesses, but may also be the result of physical processes such as ageing. ${ }^{43}$ The symptoms may be the same and therefore no clear patent law distinction can be made between treatments of normal and pathologic bodily changes. This resulted in a process for treatment of menstrual pain being deemed therapeutic in $\mathrm{T}$ 81/84. The Board declared itself unable to make a distinction between treatments that heal and treatments that merely provide relief. Processes are considered therapeutic irrespectively of if they treat the reasons for the pain or only the

where, in view of the health risks connected with such a treatment, a claimed method for treatment has to be performed by a physician or under his supervision, it will normally fall within the exclusion of the first sentence of article 52(4) EPC. ... Since the physician is bound to exercise extreme care both during the medical treatment and during the use of medical-technical apparatus and, moreover, is responsible for the surveillance of his medical and non- medical staff, the board is convinced that the claimed process is and has to be carried out by the ophthalmologist or ophthalmic surgeon himself or at least under his supervision. This fact indicates that the claimed process falls under the exclusion of Article 52(4) EPC.”

41 T 469/94 of 0107 1997: "Moreover, as set out in decision T 655/92 ... a method for treating the human or animal body, though technical, is nevertheless excluded from patentability pursuant to Article 52(4) EPC in view of the high risk for the patient's health involved in one essential part of said method. The reduction in the perception of fatigue does not appear to entail the same considerations since it does not apparently increase the risk of causing an injury in the muscle tissue. In fact, as stressed by the expert accompanying the appellant's representative at the oral proceedings, no evident relationship exists between the increase of endurance in the muscular system of a fit person performing major exercise and the occurrence of a muscle injury. The expert argued that the gap between the situation of reduced perception of fatigue and the situation in which the muscle tissue may become prone to damage as a result of excessive effort remains very broad and controllable, even after administration of choline. As a matter of fact, no evidence of any injuries was detected during the trials on runners, swimmers and basketball players. The board does not see any reason to question the appellant's arguments, which are therefore accepted."

42 T 329/94 of 1106 1997: "La Chambre ... conclut que l'objet de la revendication 12 selon la requête principale vise pour l'essentiel à faciliter un flux soutenu de sang veineux vers un point d'extraction du sang, localisé dans la veine d'un membre supérieur du corps humain, en alertant le donneur de sang au moyen d'un stimulus tactile automatique et sélectif, afin qu'il participe au maintien du flux sanguin désiré. A cet égard, la Chambre estime qu'il importe peu de savoir si la mesure est réalisée par un praticien ou une autre personne possédant des connaissances médicales, ou encore sous la supervision d'une telle personne. ... Ce seul critère n'est pas suffisant pour déterminer si l'étape de la méthode appelle des objections au titre de l'art. 52(4) CBE, même si les compétences médicales du praticien peuvent constituer à première vue une indication utile. L'objectif et l'effet inévitable de la caractéristique examinée sont bien plus importants. ... [I]l n'existe aucun lien fonctionnel, ni aucun lien de causalité physique entre la mesure mise en oeuvre et un quelconque effet thérapeutique sur le corps auquel cette mesure est appliquée. La Chambre peut donc souscrire à l'avis du requérant, selon lequel la revendication 12 ne couvre pas l'extraction du sang proprement dite. ... En effet, le procédé auxiliaire d'extraction sanguine qui est revendiqué n'a pas une visée thérapeutique ou chirurgicale, mais revêt simplement un caractère technique et a pour seul but d'améliorer l'efficacité de la prise de sang chez un donneur. On peut donc clairement distinguer ce procédé d'un effet thérapeutique ou diagnostique”.

43 T 24/91, OJ EPO 1995, 512: “The meaning of the term 'therapy' is not restricted to curing a disease and removing its causes. Rather, the term covers any treatment which is designed to cure, alleviate, remove or lessen the symptoms of, or prevent or reduce the possibility of contracting any disorder or malfunction of the human or animal body." 
symptoms. The biochemical reactions are not different in any relevant respect. ${ }^{44}$ If, however, the treated symptom is only that of fatigue induced by the performance of exercises, then simple use claims are permitted. The Board observed in T 469/94 that fatigue is a transitory physiological condition caused by natural circumstances and removable by simple rest. Pain or serious suffering were not normal manifestations of fatigue, which therefore was not comparable with the pathological state typical of a disease or an injury. The use of choline for reducing the perception of fatigue was thus not considered therapeutic. ${ }^{45}$ Some relief of pain or of discomfort seem to have to be involved in a therapeutic treatment, but if that is the case all processes for maintaining or restoring health, including prophylactic treatment, e.g. vaccination, are excluded from the patentable sphere. ${ }^{46}$

In line with the definition of therapeutic, it was in $\mathrm{T}$ 820/92 concluded that contraception was not a therapeutic effect. All the same, the application was refused. This was because the process did not only have a contraceptive effect. Some side effects usually associated with the contraceptive effect were also avoided, and this was deemed a therapeutic effect. If side effects are avoided, then the process may be therapeutic, even if the main object of adopting the process is not. ${ }^{47}$

Another application for a contraceptive process was tried in $T$ 74/93. The claims were directed to the application to the body of compounds with a contraceptive effect. The Board stated that the prohibition against patents for medical processes was not an obstacle to patenting in this case. But this was not the end of the matter. It was also necessary that the process in itself could be applied industrially. The three categories of medical processes were thus not an exhaustive enumeration of the medical methods excluded from the patentable sphere, due to the necessity for industrial applicability. The justification given was that the physical integrity of humans should be respected. The

44 T 81/84, OJ EPO 1988, 207: "In the view of the Board, the concept of therapy should not be confined narrowly. There are many chemical agents which are used by physicians to relieve pain, discomfort and incapacity. Although at least some such experiences may have been caused by natural circumstances (e.g. menstruation, pregnancy or age etc.) or by a reaction to situations in the human environment (e.g. atmospheric conditions provoking tiredness, headaches, etc.), these overlap with and are often indistinguishable from symptoms of a disease or an injury. The biochemical effects and mechanisms which medicaments generate in order to restore the normal, capable and painless state for the body are often very similar or identical in these instances and in cases of disease, irrespective of the nature of the real cause. It would be impossible and undesirable to distinguish between basic and symptomatic therapy, i.e. healing or cure and mere relief. The use of medicaments may be called for whenever the human body is suffering from a disease, illness, pain or discomfort or incapacity, and the administration thereof could provide or contribute to either full or partial healing, or relief or restoration of fitness. These are part of the everyday therapeutic activities of the medical profession, in addition to surgery and diagnostic methods also mentioned in Article 52(4) EPC. Contrary to other situations where the boundaries with the non-medical handling of the human body are not at all clear in view of the involvement of different specialists (e.g. cosmeticians), the treatment of pain normally is a matter exclusively reserved for the physician. For these reasons it is the view of the Board that irrespective of the origin of pain, discomfort or incapacity, its relief, by the administration of an appropriate agent, is to be construed as 'therapy' or 'therapeutic use' in the sense of Article 52(4) EPC, and must be considered in the light of the decision of the Enlarged Board of Appeal in respect of claims 'directed to the use of a substance or composition for the manufacture of a medicament for a specified, new and inventive therapeutic application.'”

$45 \mathrm{~T}$ 469/94 of 01071997.

46 T 19/86, OJ EPO 1989, 24: "[B]oth prophylactic and curative treatments of disease should be regarded as falling within the meaning of the word 'therapy' in the sense that that word is used in Article 52(4) EPC, since both are directed to the same objective, i.e. the maintenance or restoration of health."

47 T 820/92, OJ EPO 1995, 113: "In the case of a method involving administration of two or more substances, the question for the purpose of article 52(4) EPC is not whether the main or even the only reason for carrying out the whole of the claimed method is non-therapeutic. Rather a method claim falls under the prohibition of Article 52(4) EPC already if the administration of one of the substances is a treatment by therapy, and the administration of this substance is a feature of the claim.” 
patenting of the process for using the contraceptive was deemed an infringement of the physical and personal integrity and therefore unpatentable. ${ }^{48}$

This decision can be criticised for two reasons. Firstly, it is a well-established dogma of legal interpretation that lex specialis legi generali derogat. The three specific prohibitions of different medical process are the interpretation of "industrial applicability" that has been made by the legislator. Further exclusions should not in such a case be added in case law by interpretations of the more general requirement for "industrial applicability". Secondly, in all European countries private acts cannot be patent infringements. Thus, there is no risk of conflict with the personal integrity of patients, and accordingly this need not be an issue at the granting stage.

\subsubsection{Diagnosis}

The second category of direct medical effects that render a process unpatentable is diagnostic. The term refers to processes for identification and differentiation between pathological situations. Other processes for investigations of the body can be patented, e.g. ways of finding out about one person's capacities for sport or for a particular occupation. Furthermore, only those processes are banned from patenting which are carried out directly on a living body. Therapy can of course only be carried out on a living patient, but diagnosis can sometimes also be performed on a dead body, as an autopsy. Diagnosis can also be performed on tissue samples or body fluids that have been permanently removed from the body. If the material is not returned to the same body, the process does not fall under the prohibition against patents on diagnostic processes. Accordingly, a process to diagnose HIV infection in blood is patentable, because the blood sample will not be returned to the body. However, a process for dialysis of blood would not be patentable because the blood is returned to the body. 49

A material caveat is that the prohibition only encompasses processes giving a direct indication of what medical steps should be taken. This considerably limits the scope of the prohibition. In case T 385/86 a process for measuring the body temperature and $\mathrm{pH}$-values was not deemed diagnostic because there was no phase in which the values were compared to average values. Thereby no decision was taken in the process on which disease the patient was suffering from. ${ }^{50}$ The reasoning was that a medically trained doctor did not have to be

\footnotetext{
48 T 74/93, OJ EPO 1995, 712. For a comment, see Pagenberg, J., IIC 1996, 106.

49 Guidelines IV 4.3.

50 T 385/86, OJ EPO 1988, 308: “[T] he only diagnostic methods to be excluded from patent protection are those whose results immediately make it possible to decide on a particular course of medical treatment. This means that to answer the question whether a method is a diagnostic method for the purposes of Article 52(4), first sentence, it is necessary to ascertain whether the method claimed contains all the steps involved in reaching a medical diagnosis. Methods providing only interim results are thus not diagnostic methods in the meaning of Article 52(4), first sentence, even if they can be utilised in making a diagnosis. ... In the present case the result of the measures claimed is a quantitative expression of an isolated physical variable, namely the temperature or $\mathrm{pH}$ value within a given volume of a human or animal body. In the view of the department of first instance the fact that the product of the measures claimed are measurements of the body's own values which thus show up a divergence from the norm that can be used to make a diagnosis was sufficient to constitute a diagnostic method. For the reasons stated above the Board is unable to concur. On the contrary it is still necessary to consider whether the measurements obtained immediately indicate the diagnosis. The measures claimed in Claim 1 result in the obtaining of a specific value for body temperature. While the department of first instance is correct in stating that such a value implicitly contains information which is of use in making a diagnosis, it is not a matter of the possibilities outside the scope of the claim that the value opens up. What is decisive is whether the nature of the disease is already immediately clear from that very value, which in the present case is not so. The presence or absence of a particular disease cannot be determined solely from the value of the body temperature
} 
involved in the process until a complete diagnosis was made. ${ }^{51}$ Therefore a patent on the process would not conflict with the work of doctors. Similarly a process for measuring the blood sugar level has been found patentable since it did not provide any immediate answer to whether the patient was suffering from diabetes. ${ }^{52}$

This interpretation dictates that very few processes are diagnostic. It would be necessary that the process in suit has a phase where data is collected, a phase where data is compared to average values and a phase where the symptoms are related to a particular disease. ${ }^{53}$ Especially, the last phase is unusual in new medical processes. It is difficult to "mechanically" diagnose a disease from values only. It usually requires a doctor's experience of what the symptoms may be for different patients.

However, the quite recent case, $\mathrm{T}$ 655/92, may have changed the interpretation and lead to a more frequent application of the prohibition in the future. It was known on the priority date of the application that a certain magnetic material could be used for treatment of persons suffering from a lack of iron. The applicant had found that the same material could be used as a contrast agent in NMR (Nuclear Magnetic Resonance) X-ray if it was injected intravenously. An X-ray does not result in a direct diagnosis. But the Board pointed out that the process included steps for in vivo injections and thereby non-negligible medical risks. Only medically trained staff could use this process due to the health risks involved. The Board stated that any other conclusion than that the process was excluded from the patentable sphere, would have been in conflict with article 52(4) EPC.54 It is not primarily the nature of

as measured. For this it would first be necessary to establish whether the actual temperature measured differs significantly from a reference temperature value regarded as normal, i.e. non-pathological, and to what clinical picture the quantitative variance can be attributed. Only if these operations of differentiation and comparison were to be incorporated into the claim would the claimed method of measuring a physical variable (temperature) on the human or animal body become a diagnostic method in the meaning of Article 52(4), first sentence, EPC, and would then be so regardless of whether these operations were performed by a doctor or by computer."

51 T 655/92 of 1102 1997: "With regard to methods for obtaining chemical/physical data from inside the living body by means of diagnostic apparatus registering these data or reproducing images, the Boards have ruled that these do not fall within the exclusion of Article 52(4) EPC and that only such methods are excluded which provide results immediately enabling a decision to be taken on a particular line of medical treatment ( $\mathrm{T}$ 385/86 ...). This ruling was based on the consideration that in such methods the step sequence for which protection is sought does not include any stage having the character of medical diagnostic activity or medical treatment or any measure requiring a doctor to carry them out. Rather the method therein claimed could be carried out by a technician in order to provide a basis for the doctor's subsequent activity of diagnosis”.

52 T 83/87 of 1401 1988: “Gegenstand des vorliegenden Anspruchs 1 ist ein Verfahren zur Konzentrationsbestimmung von Zucker in Gegenwart störender Fremdsubstanzen. ... Der Vorinstanz ist zwar darin zuzustimmen, daß der für die Zuckerkonzentration repräsentative Meßwert im Rahmen einer Diagnosestellung verwendet werden kann. Für sich selbst betrachtet, d.h. ohne Bezugnahme auf einen als normal zu betrachtenden Sollwert und auf die Zuordnung einer möglicherweise ermittelten Abweichung vom Sollwert zu einem bestimmten Krankheitsbild, kann dieser Meßwert jedoch nicht unmittelbar eine Diagnose im Sinne der Erkennung eines pathologischen Zustands liefern. Wie die Kammer ... dargelegt hat, sind aber nur solche Verfahren vom Patentschutz auszunehmen, deren Ergebnis unmittelbar gestattet, über eine medizinische Behandlung zu entscheiden. Verfahren, die lediglich Zwischenergebnisse liefern, sind noch keine Diagnostizierverfahren im Sinne des Artikels 52(4) Satz 1 EPÜ, selbst wenn sie beim Stellen einer Diagnose verwendbar sind. Diese Auslegung des Art. 52(4) EPÜ führt dazu, daß auch das vorliegende, im Anspruch 1 definierte Verfahren kein Diagnostizierverfahren im Sinne dieser Vorschrift ist.”

53 See Moufang, R., IIC 1993, 46.

54 T 655/92 of 1102 1997: "The method is an in vivo NMR imaging technique using contrast agents. Unlike the technique envisaged in T 385/86, supra, the method of the present invention is characterised by the parenteral administration (iv) of the contrast agent of claim 1, which transforms the NMR imaging from a non-invasive to an invasive technique. Additionally the intravenous injection of dextran-magnetite, i.e. Imferon ..., is not devoid of any risk of side-effects, some severe, as is well documented by (46) the rare anaphylactic reaction, which may be fatal in spite of treatment. While only a few such deaths have been reported, it remains a deterrent to the use of iron dextran. ... It is indisputable that the task of 'determining whether any signs or symptoms of anaphylaxis 
the process that is decisive, but who uses the process. If a doctor needs to be involved the process may not be patented, even if the process does not result in a complete diagnosis.

\subsubsection{Remarks}

Reasons of an economic or ethical nature? It is possible that the ban on patents for medical processes has negative social consequences. Maybe valuable new medical treatments are not developed because doctors do not have the same research incentives as other professions do. However, the usual legal justification for the prohibition is of an ethical and not economic nature. Ethical and economic considerations, of course, are not directly comparable, and so the exclusion could be justified regardless of its economic consequences. In this part it will, however, be argued that there is no viable ethical elucidation of the way in which the prohibition is interpreted and applied today, and that the present limitations on patentability can best be explained by the economic effects of the patent system.

First, it should be mentioned that it is paradoxical that the work of doctors is excluded from the patentable sphere. Probably no other profession has benefited and still benefits in their professional capabilities to such an extent from the patent system. Today almost all new pharmaceuticals and most other kinds of advanced new medical equipment are patented, and a large portion of these would probably not have existed if they could not have been patented. Second, product patents are issued on medical inventions, including patents on new products that can only be used by doctors, e.g. new surgical instruments. This is not perceived as a moral problem. ${ }^{55}$ That is paradoxical because these patents will create a stronger position for the patentee then a patent for a new process can ever do. Whoever develops the new instrument can block all uses of it. But whoever patents a new use for a known instrument could, if the prohibition was lifted, still only gain a patent on the particular new use that he has found. That would be a weaker position in relation to the medical profession. This is a situation that is difficult to justify from an ethical perspective.

The importance of economic considerations in regard to patents for medical processes can also be viewed in a historical context. Today, at the turn of the century, new pharmaceuticals are no longer being developed by doctors and pharmacists working directly with patients and at the same time doing research. Particular innovative companies have replaced them. This has been due to the dramatic increase in research costs. At present no more than perhaps 100 multinational companies have sufficient resources to cover all stages of pharmaceutical research. As the most efficient innovator has become susceptible to the lure of the patent system, the patent system has gone through a process of change. Product patents for pharmaceuticals have, for example, become the international standard protection for

appear' can only be the responsibility of medical staff who have to recognise the earliest symptoms of anaphylaxis or any other undesired reaction and accordingly either have to adapt the treatment to the specific situation, or interrupt the administration or even undertake without delay all those measures necessary to control and minimise the side-effects already evident. Therefore, unlike the processes of the previous cases, the present diagnostic process, when considered in its totality, comprises at least one step essential for the desired diagnostic result, which cannot fall under the exclusive responsibility of the technician skilled in NMR technology. While for a process whose steps as a whole are non-medical but technical it is legitimate not to derive the in vivo diagnostic character from its final diagnostic purpose, this does not apply to a process for a diagnostic purpose which is to be implemented in its essential steps by medical staff or under the responsibility of a doctor. A different interpretation would be in clear conflict with the spirit of Article 52(4) EPC.”

55 See Thums, D., GRUR Int. 1995, 283. 
pharmaceuticals. ${ }^{56}$ At the beginning of the century this was deemed ethically unacceptable. But it has become ethically acceptable, largely because the patent system nowadays serves an important purpose for researchers in this field. When the patent system works economically it seems to be deemed ethically acceptable to society. Caveats to the patentable sphere are perhaps generally economically instrumental in a similar manner. If patents on medical processes are needed from an economic perspective they may become ethically justified. It will now be considered whether there is such an economic need for lifting the prohibition of patents for medical uses.

Incentives. The incentive function of the patent system is dependent on the existence of innovative work done for economic reasons. Research done by pharmaceutical companies is fully evaluated in economic terms and controlled by this. Every research project is reassessed several times per year and if the value is negative it will be ended. However, it is unusual that pharmaceutical companies develop new medical processes with a direct medical effect. In a majority of cases doctors working in clinics do these important inventions. They have the unique opportunity to "experiment", more or less consciously, on the job. 57 This creates considerable advantages in a competition with other possible innovators. It would be painstakingly difficult for a company to compete in the development of new medical process. The innovative work of doctors has very low costs. It has been said that:

\begin{abstract}
"On average, it may be cheaper to develop new methods of treatment than it is to bring a new drug onto the market. Generally speaking, the development of a new drug may involve the focusing of the resources of a large drug company over a period of several years. After years of screening suitable compounds, promising results may be obtained with a few compounds. Further studies are then focused on these compounds. Many experiments and trials must be conducted to prove the efficacy and safety of the new drug and to eventually gain regulatory approval from government agencies. Finally, after much risk and expense, a new drug makes it onto the market.

In contrast, the development of a new surgical technique may be made by an intellectually curious and creative surgeon who has an idea. The idea might occur quite quickly, and with no expenditure of capital. Regulatory approval may not be required at all before the technique can be widely adopted .... . Peer review serves as the main mechanism for regulating the adoption of medical processes. Therefore, the need to provide an incentive to invest in research via the patent system may be less in relation to methods than it is for drugs and devices."58
\end{abstract}

It is interesting to observe that the same circumstance that makes it impossible to patent a process - no new product - is that which gives doctors a competitive advantage in relation to other possible inventors. Doctors have no or very limited opportunities to make a prototype for a new instrument or pharmaceutical and thereafter invest the marketing resources that are necessary. Production of new physical products demands a considerably broader competence than just the medical. In addition, there will be a considerable need for venture capital, at least at the time the new product is to be introduced on the market. This will make companies the most efficient innovators if a new product has to be produced. Therefore it is evident that an

\footnotetext{
56 "Hier scheint sich überwiegend die Erkenntnis durchzusetzen, daß das Verbot des Schutzes chemischer Stoffe auf wirtschaftspolitische Vorstellungen beruht, die heute keine Gültigheit mehr haben und daß die chemische Industrie ein anzuerkennendes wirtschaftliches Interesse daran hat, für neuentwickelte chemische Stoffe einen angemessenen Patentschutz zu erhalten.” See Beier, K-F, GRUR Int 1960, 106.

57 "[T] he advancement [in medical procedures] occur through gradual, on-the-job improvements and refinements of known methods in operating rooms and physicians offices."; see statement by the witness Dr. Charles Kelman, President of The American Society of Cataract and Refractive Surgery, at a Hearing Before the Subcomm. on Courts and Intellectual Property of the House Comm. on the Judiciary, 104th Cong., 1st Sess (1995), quoted by Reisman, J. M., 10 High Technology Law Journal 1995, 355, footnote 208.

58 See Culbert, P., Patent World May 1997, 38 (footnotes excluded).
} 
opportunity to patent, and corresponding economic incentives, should exist when a new product has to be manufactured. In many cases this will be necessary to motivate the investments. Such a patenting opportunity exists today. The close correspondence between the legal requirement and the economic necessities is evidence for the instrumental economic logic that is at work in the patent system. It leads to an economically efficient legal interpretation, even if the common justification for a certain provision is ethical.

The need to be able to patent new products cannot of course be a reason for making patents for medical processes impossible. But it is questionable whether the incentives could be used efficiently by the main innovators, i.e. the doctors. Probably market demand does not have a very direct influence on the innovative work that is done by doctors. In the "International Code of Medical Ethics" adopted in London in 1949, we read:

"Physicians shall not permit motives of profit to influence the free and independent exercise of professional judgement on behalf of patients.”

Of course, doctors in general do research on the most important medical problems. But their personal profit from this research is seldom the decisive factor in the project. Research is highly valued by the medical profession, but mostly because of the professional status it creates, not due to the immediate personal economic advantages. Therefore an opportunity to patent new medical process would probably not create so much more incentives. Probably even more notable is that new medical treatments can be introduced on the market without much further technical input or marketing investments, at least much less than in the case of a new pharmaceutical. New medical procedures are mainly introduced through a process of peer review and are not particularly dependent on commercial marketing efforts.

Probably other actors would need to become the most efficient developers of new medical processes in order to warrant a change in the legal situation. The inventors need to have a business perspective on the innovative work, in order to be significantly influenced by the incentives in the patent system. It is difficult to see who these new inventors of medical processes would be and how a change could happen today. However, one possible development is than gene therapy will develop into an important medical process used frequently in clinics. It may be that companies are more efficient innovators in this particular field, the reason being that the relationship between the processes used and the therapeutic result is very complex and costly to study. ${ }^{59}$ A multidisciplinary research similar to pharmaceutical research may be needed, mainly because the treatment is done on a molecular level. This may make medical practitioners inefficient innovators. This is a medical area that at present may be going through the same process as the pharmaceutical research process did in the beginning of the century, i.e. the creation of particular research companies and therefore a need may occur for the patent system.

At present it is important to follow closely how the patentable sphere is delineated. If the development of new medical processes becomes so complex and costly that the larger resources of a company are needed, then all processes should be included in the patentable sphere. A prohibition of the patenting of medical processes may not entail any particular economic disadvantages today, but according to this analysis the prohibition may need to be interpreted in a new way in order to avoid possible future negative consequences. It is material for the patentable sphere who the efficient inventors of certain processes normally are. Ethical considerations are subordinate. Inventions that require sizeable investments in order to be achieved, or more importantly, to be exploited commercially,

${ }^{59}$ Cf. Straus, J., GRUR 1996, 10. 
should belong to the patentable field. Others may be excluded without necessarily causing negative consequences.

This conclusion may be related to case T 227/91, which probably should have been decided differently. At the time of the patent application a device for laser surgery was known. The applicant had found a new way to direct and focus the laser beam so as to avoid effects on other biological material than the object in question. A complex control equation achieved this. The claims had been crafted in accordance with the rules for a second medical indication, see part 5.3. But the Board still refused the application. This particular claim wording was only deemed available for new pharmaceuticals. Surgical equipment could not be patented with these types of claims. The Board explained that the reason was that the equipment did not disappear after it had been used, as a pharmaceutical does. ${ }^{60}$

The decision can be questioned from a strictly legal dogmatic perspective. There is no indication in the decision G 1/83 from the Enlarged Board of Appeal, that it is necessary for the product to dissolve when used, in order for the applicant to be able to use claims worded according to the solution available for a second medical indication. More material, though, is that the particular use of the laser that the applicant had discovered was the result of advanced engineering and medical research. It could probably not have been developed by surgeons during their day to day handling of the instrument. The manufacturer of the instrument was probably the most efficient inventor in this situation (in this particular case it was also, as a matter of fact, the manufacturer of the instrument that had filed the patent application, not a doctor or any other user). In situations where the medical users are inefficient inventors of new modes of application due to the necessary research costs, the manufacturers (companies) should be able to take advantage of the incentives that the patent system can create. Such an interpretation would mean that the patentable sphere is dependent on when the most efficient inventors are making hardheaded cost/benefit analysis. If they do, then the inventions should be patentable. This interpretation would create flexibility and be well attuned to future developments in the medical field where research may become increasingly costly.

In the interesting recent case T 317/95 an economic interpretation of Art. 52(4) EPC was suggested, not very unlike the one argued here. The invention related to the prescribing of a specific drug regimen for what was a known medical treatment. Discussing the novelty of such a claim the Board stated:

\footnotetext{
"It was apparently the intention of the Enlarged Board of Appeal [in G 5/83] ... to provide a certain compensation for the restriction on patent rights in the industrial and commercial field resulting from Article 52(4) EPC .... This suggests that ... it appears appropriate to consider the question of whether the sole distinguishing feature ... relates to non-commercial and non-industrial medical activities. The board has no reason to question the appellants' submission that the pharmaceutical industry, too, is engaged in optimizing the use of drugs and medicaments by investigating the optimum regimen for their administration to achieve the maximum possible therapeutic effect. Notwithstanding this, determination of the best individual treatment schedule, in particular the prescribing and modification of drug regimens used for administering a particular medicament, so as to comply with the specific needs of a patient, appear to be in the first place part of the typical activities and duties of the doctor in attendance in exercising his professional skills of curing, preventing or alleviating the symptoms of suffering and illness. These are, however, typical non-commercial and non-industrial medical activities which Article 52(4) EPC intends to free from restraint."61
}

The Board's reasoning is not far removed from the legal position that the patentable sphere should be dependent on whether efficient innovative work in the particular field is based on

60 T 227/91, OJ EPO 1994, 291.

61 T $317 / 95$ of 26021999. 
an economic cost/benefit analysis. Probably, though, it would be even better to avoid difficult assessments of this kind and do in Europe as has been done in the USA. ${ }^{62}$ No prohibition exists there. Instead practising doctors are given immunity against patent infringement actions. That would create an even better "fit" with situations in which the patent system works. The patent applicant will have to decide if the patenting is worthwhile even though he cannot receive any payments from medical practitioners.

The aim of the European solution was to protect medical doctors from the effects of patenting. It appears obvious that the American solution does this in a much simpler and more nuanced manner. The U.S. patent office does not in each case have to try whether a patent for a particular process could become a problem for the medical profession. The profession is simply excluded as such from the effects of patents granted. The European solution creates a considerable waste of resources. Another advantage, if the prohibition of patents for medical processes was abolished, would be that the claims to new uses for known pharmaceuticals could be given the normal use format. The artificial and difficult European rules on this point do not appear to lead to a better result. On the contrary, the best adjustment of the patentable sphere is probably achieved if it is left to the inventors to decide whether the inventions are worth patenting even though they cannot prevent practising doctors from adopting them.

Finally, it is valuable that veterinarian processes can be patented in the USA and that veterinarians also can infringe patents. Much veterinarian research is done for economic reasons, i.e. to increase the output of meat or other farm products. It is research driven primarily by cost/benefit analysis and could therefore be undertaken in response to the lure of a strong patent.

62 See e.g. Miller, S. A., 36:2 IDEA 1996, 255. 


\section{Sufficiency of disclosure}

\subsection{Disclosures of inventions}

The pharmaceutical industry is dependent on efficient ways of accumulating, systematising and communicating chemical and medical information. Over the last 50 years, opportunities in this respect have radically increased, mainly due to the development of computers. Access to the information has become decisive for the competitive edge of pharmaceutical companies. For the patent system this has meant that its information side has gained increasing importance. ${ }^{63}$ The information in the patent registers is of course used by the research and developments divisions, but also by the marketing and sales divisions of pharmaceutical companies.

The nature of the available information in the patent registers will among other things be dependent on Art. 83 EPC, i.e. the requirement that the invention must be disclosed in the application in a manner that enables the man skilled in the art to practise the invention. The application must contain information about what the invention can be used for and what steps should be taken in order to accomplish its effect. ${ }^{64}$ In traditional chemistry, disputes about this have been quite unusual. The substances have had low molecular weight and they have been unambiguously identifiable by structural formulas. The information in the patent application has made it feasible to manufacture the substances with generally available starting materials, and by utilising some more or less standardised reactions. The most valuable information in the application has been what the compounds could be used for, i.e. the industrial applicability, not how they could be manufactured. The growing importance of biotechnology in the pharmaceutical industry has changed this. Larger molecules are being used therapeutically and much more complicated manufacturing processes are being utilised, e.g. human proteins and the metabolism of living organisms. Therefore, the repeatability of what the applicant has achieved has progressively diminished. A majority of the decisions that will be discussed in this chapter therefore belong to the field of biotechnology. ${ }^{65}$

Persons working with patents are often asked if it is necessary to disclose knowhow in patent applications. The answer must be that it depends on whether a man skilled in the art can practise the invention without the knowledge. If the skilled person cannot attain the envisaged effect of the invention without the know-how, it must be disclosed in the application. It is always the practical and functional that is stressed in the patent-granting procedure. Therefore it is not necessary to disclose information about the scientific basis on which the invention and its effects rest. 66 The invention can be practised without such an explanation. This is important for patents on pharmaceuticals. It will usually take some time from filing the patent application until the inventor can explain how the substances lead to the biological effect. The interaction between the active substance and the biological receptor is hard to identify, even with knowledge that permits the effect to be used practically.

Nor does the test for sufficient disclosure require that the applicant discloses information that would make it possible to use the invention in the most competitive manner.

\footnotetext{
63 See Beier, F-K., Strass, J., IIC 1977, 393 and Merges, R. P., 7:1 High Technology Law Journal 1992, 66.

64 G 2/93, OJ EPO 1995, 275: "In order to meet the requirements of Article 83 EPC, a European patent application must therefore contain sufficient information to allow a person skilled in the art, using his common general knowledge, to perceive the technical teaching inherent in the claimed invention and to put it into effect accordingly.”.

65 See Hirsche, F., Hansen, B., Protecting Inventions in Chemistry, 51.

66 See Koktvedgaard, M., Østerborg, L., Patentloven, 65.
} 
European patent law has no equivalent to the American "best mode" requirement. ${ }^{67}$ The material factor is that the technical effect can be attained at all. If, however, a particular embodiment or effect is claimed, that must be available to the man skilled in the art. ${ }^{68}$ The claim defines the invention and effects mentioned in the claims must therefore be available to the man skilled in the art. An effect of the invention that is only mentioned in the description is another matter. It is not necessary that the man skilled in the art can repeat examples in the description. 69

\subsection{The man skilled in the art}

\subsubsection{The different aspects of the man skilled in the art}

In patent law it is often necessary to make qualitative judgements of technical accomplishments. Then the man skilled in the art is invoked. This is, however, a difficult concept. It is highly ambiguous. At times it appears only to be a way to equip a subjective decision with an objective wording. There is a risk that the concept is not related to facts that may be objectively determined. One purpose in this part is to study what characteristics the man skilled in the art has. The findings will be of relevance to the test in Art. 83 EPC of sufficient disclosure, but also in a number of other situations, in particular of course in

\footnotetext{
67 T 431/96 of 2302 1999: "As for the question of the need of a deposit of the particular hybridomas of the examples ..., the prescription of Rule 28(1) EPC cannot be interpreted such that there is an obligation to deposit material to facilitate the reproduction if the invention can be repeated on the basis of the written description, even if this should be a much more cumbersome way than by merely growing the deposited micro-organism (here: the hybridoma). Such is the case here. There was thus no obligation to assist the disclosure by making the hybridomas of the examples available by way of a deposit, because the best mode requirement is not part of the European Patent system.”
}

68 T 281/86, OJ EPO 1989, 202: "It is always the case in chemistry that the outcome of experiments show some fluctuations in yield, quality etc. This is irrelevant for sufficiency unless the invention requires certain characteristics in this respect. It should therefore be even less relevant if only the conditions and the means used to carry out a process show inevitable variations as long as the ultimate result is the same. ... It is therefore the view of the Board that there is no requirement under Article 83 EPC to the effect that a specifically described example of a process must be exactly repeatable. Variations in the constitution of an agent used in a process are immaterial to the sufficiency of the disclosure provided the claimed process reliably leads to the desired product. As long as the description of the process is sufficiently clear and complete, i.e. the claimed process can be put into practise without undue burden by the skilled person taking common general knowledge also into consideration, there is no deficiency in this respect."

69 T 251/95 of 1012 1998: "Article 83 EPC requires that the disclosure of the invention be clear and complete so as to be sufficient to enable a person skilled in the art to carry it out. In the present case, the invention as claimed in present claims 1 to 7 consists of certain products, more specifically: (i) anti-inflammatory pharmaceutical effervescent compositions in solid form, eg granules or tablets. ... However, the respondents' objection of insufficiency goes wider than this and is based on the allegation that the appellants were unable to demonstrate that a person skilled in the art was indeed in a position to produce compositions exhibiting the particular properties mentioned in the description .... In this respect it is to be noted that neither the degree of solubility of the claimed composition of $98 \%$ minimum nor the period of 30 to 90 seconds maximum required to obtain a solution are features which form part of the definition of the claimed invention. Hence, contrary to the respondents' assertion, the question whether or not compositions falling within the scope of the present claims indeed exhibit the above-mentioned particular properties and capabilities is immaterial to the question of sufficiency, as long as the person skilled in the art (ie the person at whom the disclosure of the contested patent is aimed) was able - on the basis of the original disclosure and possibly by using his common general knowledge to supplement the information contained in the application as filed - to carry out the invention, that is to say to prepare effervescent pharmaceutical preparations containing ibuprofen”. 
relation to inventive step. ${ }^{70}$ The study of the man skilled in the art and his common general knowledge has been organised under three headings: ${ }^{71}$

The scope of knowledge, part 2.2.2

The average knowledge, part 2.2.3

The effort expected, part 2.2.4

\title{
2.2.2 The scope of knowledge
}

The concept of "the man skilled in the art" conveys that the knowledge is limited and restricted to a particular technical field. One man skilled in the art is used when the invention is a turbine, another when the invention is a new kind of plastic. However, the way the man skilled in the art is defined should be the same in all cases: it is he who usually confronts the kind of problems that have been solved by the invention. ${ }^{72}$ One example of how this is done can be found in $\mathrm{T} 251 / 95$. The invention was an anti-inflammatory pharmaceutical composition with an ibuprofen base. The board concurred with the appellant's submissions during oral proceedings that the addressee of the patent was the formulator in the pharmaceutical industry who was a specialist or a team of specialists of that skill being familiar, inter alia, with all kinds of materials and methods used for the preparation of effervescent pharmaceutical compositions, the particular chemical and physical properties of ibuprofen and its salts, specifically in context of their solubility in water, and the standard methods used for testing effervescent pharmaceutical preparations. ${ }^{73}$

The particular skilled person who would be asked to confront the problem depends on how the problem was perceived at the time of filing. In T 317/95 it was stated that:

\begin{abstract}
"[T]he board is of the opinion that the skilled person in the present case is a specialist in gastroenterology or a team of specialists of that skill aiming to simplify the known combined therapy and to improve compliance. The appellants' allegation that there was at the priority date a certain concern about possible detrimental interactions between the two particular kinds of drugs used in the present simultaneous treatment requiring the assistance of a clinical pharmacologist does not find any support in the cited state of the art and has likewise not been substantiated in the appellants' submissions."74
\end{abstract}

It is quite unusual, though, for the competence of the man skilled in the art to be crafted as directly and explicitly as in these cases. The skills are often only considered indirectly when it is decided what documents from the state of the art are pertinent and what is to be understood from them. The man skilled in the art is a "background figure" when these questions are decided and seldom personalised or made more concrete. It is the relevance and meaning of

\footnotetext{
70 T 60/89, OJ EPO 1992, 268: "The Board adopts the view that the same level of skill has to be applied when, for the same invention, the two questions of sufficient disclosure and inventive step have to be considered.”

71 "The person skilled in the art is skilled in the art corresponding to the technology with which the invention is concerned. The person skilled in the art is one of average knowledge and average ability; his level will depend on the nature of the technology in question. The person skilled in the art does not have the whole technology at his fingertips; he knows the state of the art which is part of the average knowledge required in his professional work; he knows also the state of the art revealed in the patent.” See AIPPI Yearbook 1978/II, $158 \mathrm{ff}$.

72 See Pagenberg, J., Münchener Gemeinschaftskommentar 56.22.

73 T 251/95 of 10121998.

74 T 317/95 of 26021999.
} 
different documents that are discussed explicitly. But the more documents that are considered relevant, the wider will be the scope of knowledge attributed (indirectly) to the man skilled in the art. This is a practical way to work. In most cases it is unnecessary to discuss the man skilled in the art as a separate question.

It has, however, been decided in case law that the knowledge of the man skilled in the art is that which is stated in basic handbooks and textbooks in the field of the invention and furthermore, it includes the references mentioned in said books. ${ }^{75}$ All that which belongs to the state of the art is not part of his knowledge. ${ }^{76}$ More specialised sources, e.g. Chemical Abstracts, do not belong to the knowledge of the man skilled in the art. ${ }^{77}$ Nor do "standard" journals in the technical field, even if people who work in the field usually read the journals in question. ${ }^{78}$ However, the man skilled in the art may be considered to have knowledge of sales or technical brochures, ${ }^{79}$ but normally not published patents. ${ }^{80}$ An exception in regard to patents may be made, however, if the invention belongs to a new technical field where no general textbooks have yet been written and therefore the only available literature is published patents. ${ }^{81}$ Furthermore, patents are deemed known to the man skilled in the art if they are referred to in the patent application in question. ${ }^{82}$ References may of course also have

75 T 206/83, OJ EPO 1987, 5: "Basically any cure of insufficiency lies with the addressee of the document, i.e. the person skilled in the art who has common general knowledge at his immediate disposal. It would be unfair to the public if more were to be expected of him, i.e. an awareness of the whole state of the art. It is normally accepted that common general knowledge is represented by basic handbooks and textbooks on the subject in question. The skilled person could well be expected to consult these to obtain clear advice as to what to do in the circumstances, since the skills of such persons not only includes knowledge about particular basic prior art but also knowledge as to where to find such information. Such books may indeed refer him to articles describing specifically how to act or at least giving a fairly generally applicable method for the purpose, which can be used without any doubt."

76 Cf Guidelines C IV 9.6: "The person skilled in the art should be presumed to be an ordinary practitioner aware of what was common general knowledge in the art at the relevant date. He should also be presumed to have had access to everything in the 'state of the art', in particular the documents cited in the search report".

77 T 206/83, OJ EPO 1987, 5: “The indexes of Chemical Abstracts cover virtually the whole state of the art, and represent therefore much more than what is assumed to be the common general knowledge of the addressee of the specification. Reliance on the contents of Chemical Abstracts to rectify insufficiency might be tantamount to leave the skilled reader to carry out a search in the whole state of the art, which would be an unacceptable burden on the public."

78 T 475/88 of 1123 1989: "Der Inhalt von Fachzeitschriften, auch solchen, von denen zu unterstellen ist, daß sie der Fachmann regelmäßig liest, so daß sie als 'Standardzeitschriften' bezeichnet werden können, gehört jedoch nach Auffassung der Kammer ebenso wie der Inhalt von Patentschriften in der Regel nicht zum allgemeinen Fachwissen des Durchschnitsfachmanns, weil ihr Inhalt normalerweise nicht zum präsenten Wissen desselben gehört, sondern erst durch eine umfassende Recherche erschlossen werden muß.”

79 T 654/90 of 0805 1991: "In the present circumstances, it can be assumed that the skilled person would be aware of the technical brochures, such as documents I, XIV and XVI, issued by the manufacturers of alkylolated melamines."

80 T 206/83, OJ EPO 1987, 5: “Normally patent specifications are not part of common general knowledge and cannot therefore cure apparent insufficiency".

81 T 51/87, OJ EPO 1991, 177: "In the present case, the C-076 starting compounds are highly elaborated microbial metabolites opening a brand new field of research, so that any technical knowledge acquired in this field at the beginning, through basic pioneering work had not yet been distilled into the form of textbooks. By contrast, in the prior decision T 206/83 the situation was quite a different one, namely that the man skilled in the art was a person working in the field of classical herbicide chemistry, which was not a new developing field like that of the chemistry of C-076 compounds. The man skilled in the art, therefore, cannot be presumed to possess the same common general knowledge in both cases."

82 T 449/90 of 0512 1991: "The Appellants do not contest that at the time of filing of the patent in suit there were no test procedures available to test the presence or absence of the AIDS virus as such or that the available tests for identifying the hepatitis virus were difficult ... There is, however, ... reference .. made to a prior art document PCT International Application WO 82/03871, which document is incorporated into the disclosure by 
been made to other publicly available documents and they then become part of the knowledge of the man skilled in the art. In such a case it is necessary that the referral is unambiguous and that the document referred to is easily retrievable. Common general knowledge is not confined just to material for which the addressee is supposed to have mental recall or can recite more or less verbatim. But documents that can only be found after a considerable search do not belong to the knowledge of the man skilled in the art.

The knowledge of the man skilled in the art may in some cases extend beyond his normal field of work. A Board of Appeal has decided that the knowledge of the man skilled in the art may extend to a larger or otherwise related technical field, but only under the condition that similar technical problems exist in the skilled person's special area of competence and in the other field. The thought is that the man skilled in the art does not normally work in the other field, but that he has some knowledge of developments done there. ${ }^{83}$ One example is $\mathrm{T} 161 / 84$. The invention was an apparatus used for automatic insulin injections. The Board of Appeal stated that the man skilled in the art was an expert in the field of injection devices, but this person was also considered to have knowledge in the narrowly related field of pacemaker technology. The elucidation given was that the two fields had many problems in common. ${ }^{84}$ Another example is $\mathrm{T} 1 / 85$. The Board of Appeal found that the person skilled in processes for making hard dosage forms could be another than the person skilled in relation to processes for making soft dosage forms. However, the Board concluded that the differences in the field of work were not decisive, because in the two fields similar technical measures were applied to similar technical problems. The technical knowledge as such was overlapping, even if the fields of work were perhaps not. A man skilled in one of the fields, therefore, could be expected to follow the developments made in the other field. The conclusion drawn was that technical solutions from the "other" field should be taken into account. 85

reference. This document relates in particular to a method for the inactivation of viruses in compositions containing blood clotting factor enzymes, for example Factor VIII. ... In the Board's opinion this disclosure has to be included into the disclosure of the patent in suit by reference; it provides the information necessary to carry out available model tests with regard to a certain desired degree of inactivation of a virus”.

83 T 176/84, OJ EPO 1986, 50: "While it is indeed perfectly reasonable to expect a person skilled in the art if need be, i.e. in the absence of useful suggestions in the relevant field as to how a given problem might be solved, to look for suitable parallels in neighbouring fields, the question of what is a neighbouring field is one of fact and the solution depends, in the opinion of the Board, on whether the fields are so closely related that the person skilled in the art seeking a solution to a given problem would take into account developments in the neighbouring field. It is furthermore quite reasonable to expect a skilled person to refer to the state of the art in the general field of technology in which the same problems or problems similar to those in the special field of the application extensively arise and of which a person skilled in the art must be expected to be aware.”

84 T 161/84 of 2808 1986: "Zur Frage des zur Beurteilung der erfinderischen Tätigkeit maßgeblichen Fachmannes sei angemerkt, daß im vorliegenden Fall wohl der Fachmann für das Sachgebiet der Infusionsgeräte zuständig ist, von dem jedoch erwartet werden muß, daß er die Technik eines eng benachbarten Gebietes der Medizinaltechnik kennt, auf dem viele gemeinsame Probleme zu lösen sind, nämlich jenes der Elektrostimulation z. B. der Herzschrittmacher, oder sich zumindest dort umsieht. Deswegen konnte der Fachmann, der die in Punkt 3.2 erwähnte Aufgabe zu lösen hatte, auch Dokumente, die sich auf Herzschrittmacher beziehen, heranziehen.”

85 T 1/85 of 2207 1986: "Die Beschwerdeführerin hält dem entgegen, daß das Füllen von Hart- und von Weichkapseln nicht auf dem gleichen oder einem benachbarten Fachgebiet liege und der Hartkapselfachmann daher zur Lösung seiner Probleme nicht auf dem Gebiete der Weichkapselfüllung Ausschau halten werde. Sie will daraus herleiten, daß die Kombination von (A) mit (B) für den maßgebenden Fachmann nicht nahegelegen habe. Die Kammer verkennt nicht, daß Hartkapseln üblicherweise mit Feststoffen, Weichkapseln dagegen mit Flüssigkeiten gefüllt werden, was Unterschiede in der Art und Weise des Abfüllens bedingt. Es mag auch zutreffen, daß das Abfüllen von Hart- und dasjenige von Weichkapseln in der Praxis von verschiedenen Fachleuten ausgeführt werden. Darauf kommt es jedoch nicht an; entscheidend ist vielmehr, ob es sich bei den fraglichen Gebieten um technische Nachbargebiete handelt, von denen erwartet werden kann, daß der Fachmann 
Decision T 69/94 was also related to a pharmaceutical dosage form with a particularly slow dissolution. The dosage form contained a silica material with a particularly high absorption of liquids, which had not previously been used in the pharmaceutical field. But the Board decided that the man skilled in the art could be expected to search for and test materials that were available in the wider area of liquid absorbing substances. The material was known in this technical field and therefore it was deemed to be a part of the knowledge of the man skilled in the art. ${ }^{86}$ A similar extension of the skilled person's knowledge occurred in T 379/96 where the invention related to medicinal aerosol formulations having a propellant system being as little ozone damaging as possible. The Board stated:

"The skilled person in the present case has special knowledge in the field of medicinal aerosol
formulations and is deemed to have access to the whole literature relating to that field. He must
also have knowledge about the basic chemistry necessary to determine on the basis of physico-
chemical properties of the components of a formulation their interactions and what follows from
their use in a formulation. When difficulties resulting from the use of some components of a
formulation also occur in an analogue manner in other fields, such fields can be considered as
neighbouring technical fields. If it is the case, as here with environmental problems, the skilled
person will of course be interested to know the solution proposed in such neighbouring fields"87

As for research on active pharmaceutical substances, it is probable that the field of work is particularly narrow. It is a highly specialised field. Commonly researchers only work within a certain therapeutic field and maybe only on some particular substances. In T 20/83 the Board of Appeal decided that the man skilled in the art in relation to certain anti-allergic substances did not posses knowledge of appetite-suppressing pharmaceuticals. ${ }^{88}$ However, in a biotechnology decision, $\mathrm{T}$ 455/91, the Board decided that the man skilled in the art in relation to proteins expressed by yeast should have knowledge of the technique applied when bacteria was used for the same purpose. ${ }^{89}$

des einen Weiterentwicklungen auf dem anderen im Auge behält. Benachbart sind zwei technische Gebiete insbesondere dann, wenn sie zweckbedingte Berührungspunkte haben, so daß der Fachmann des einen Lösungen eines konkreten Problems auf dem anderen Gebiet vermuten kann. Dies ist hier der Falle .... Somit besteht bei der Herstellung pharmazeutischer Dosiereinheiten in Hartkapseln einerseits und Weichkapseln andererseits eine zweckbedingte Zusammengehörigkeit, die die fachmännische Beobachtung des jeweils anderen technologischen Nachbargebietes erwarten läßt. Es ist daher nur natürlich, daß sich der Hartkapselfachmann angesichts der hier bestehenden Teilaufgabe auf dem Weichkapselgebiet nach einer Lösung umgesehen hätte.”

86 T 69/94 of 1806 1996: "As far as a selection of the silica material is alleged, the Board generally considers it as forming part of the normal activities of the person skilled in the art first of all to have a look at materials available on the market. In view of the Respondent's own argument that there was no high absorption material available in the field of pharmaceuticals, it is all the more likely that the skilled person would have inevitably turned to the best silica material already available in the broader general field of the absorption of liquids”.

87 T 379/96 of 13011999.

88 T 20/83, OJ EPO 1983, 419: "The intention was apparently to show that the appetite-suppressing effect of the phenethylamines does not depend on the presence or absence of the cyano residue. This finding in what is, in terms of structure and effects, a very narrow specialist field would not have been applied by a skilled person to the -in this respect far-removed - field of anti-allergic 4-oxo-benzothiopyrans.”

89 T 455/91, OJ EPO 1995, 684: “[I]t should be borne in mind that the skilled person in the field of expression of polypeptides in yeast had good reasons to move in the direction of the technical teaching of the patent in suit, because the skilled person knew how to adjust the technical teaching in (24') from an adjacent neighbouring field, namely the bacterial art. This was a sufficient incentive for an expert at least to try to transform knowledge from the bacterial art to yeast. It is observed that in this respect the expert in the bacterial art and for yeast is the same." 


\subsubsection{The average knowledge}

When the field of knowledge has been crafted, the next issue at stake is the level of skills in this field. Also in this situation it is the person who usually is entrusted with problems similar to the one solved by the invention, who is the reference point. Depending upon the technical field to which the invention belongs the skilled person may have everything from a research education to only practical experience. The skilled person will have a high level of education in fields of "high technology". 90

It has been decided that the skilled person can on some occasions be a research team. In Guidelines it is stated that this is particularly relevant in relation to inventions in fields of high technology, e.g. the manufacture of complex chemical substances. ${ }^{91}$ In T 60/89 the Board described the skilled person in relation to a biotechnology invention made in 1978. The skilled person was not to be deemed as a Nobel Prize laureate, although a number of practitioners at this time actually received the Nobel Prize. The skilled person was described as a team of graduated scientists who at that time tried to find practical applications for gene technology. ${ }^{92}$ In a junior decision, $\mathrm{T}$ 223/92, the Board understood this to be a too highly qualified skilled person. It should not be a team of graduated scientists, as in $\mathrm{T} 60 / 89$, but a highly qualified technician. ${ }^{93}$ This, however, has been criticised in the literature, because gene technology in 1981 was a relatively new and unexplored field and the question to ask was who usually solved problems in the field at the time of the application. ${ }^{94}$ In T 412/93 the Board also made some comments on how the man skilled in the art had been described in $\mathrm{T}$ 223/92. It was mentioned that the highly qualified technician had a doctor degree. ${ }^{95}$

In $\mathrm{T}$ 412/93 the parties agreed that the man skilled in the art was a team of scientists, composed of one person with a Ph.D.-exam and several years of experience in the field of biotechnology and biochemistry. The two others were laboratory technicians with good knowledge of the processes pertinent for the biotechnology invention. The Board of Appeal observed that this definition was in accordance with the thinking of the Board and

\footnotetext{
90 See Pagenberg, J., Münchener Gemeinschaftskommentar, Europäisches Patentübereinkommen Art. 56.44.

91 Guidelines C IV 9.6: "There may be instances where it is more appropriate to think in terms of a group of persons, e.g. a research or production team, than a single person. This may apply e.g. in certain advanced technologies such as computers or telephone systems and in highly specialised processes such as the commercial production of integrated circuits or of complex chemical substances."

$92 \mathrm{~T} \mathrm{60/89,} \mathrm{OJ} \mathrm{EPO} \mathrm{1992,} \mathrm{268:} \mathrm{"It} \mathrm{is} \mathrm{the} \mathrm{opinion} \mathrm{of} \mathrm{the} \mathrm{Board} \mathrm{that} \mathrm{the} \mathrm{skilled} \mathrm{person} \mathrm{in} \mathrm{the} \mathrm{field} \mathrm{of} \mathrm{genetic}$ engineering in 1978 is not to be defined as a Nobel Prize laureate, even if a number of scientists working in this filed at that time were actually awarded the Nobel Prize. Rather, it is understood that the skilled person was to be seen as a graduate scientist or a team of scientists of that skill, working in laboratories which developed from molecular genetics to genetic engineering techniques, at that time.”

93 T 223/92 of 2007 1993: "[T]he skilled person must be assumed to lack the inventive imagination to solve problems for which there do not exist already routine methods of solution, the appropriate comparison here being not with a team but with a highly skilled technician carrying out a project where the initial instructions received are already adequate to tell the technician how to overcome any problems likely to arise.”

${ }^{94}$ In regard to T 223/92 it has been said that: "There is no good reason to define the level of knowledge of a person skilled in the art lower in molecular biology than in other fields of technology, such as chemistry or engineering. Rather, the opposite would be plausible because molecular biology is a highly complex technical field in which people practising it at their bench and planning new projects must have a complex background of knowledge and training. This is something that a technician certainly does not have.” See Jaenichen, H-R., The European Patent Office's Case Law on the Patentability of Biotechnology Inventions, 47.

95 T 412/93 of 2111 1994: "In decision T 223/92 .. where the priority claimed was for much the same time, 1983, as in this case, the Board considering that case defined the 'skilled person' as a highly skilled technician ..., which in real terms would mean a PhD. researcher for the knowledge. The notional skilled person in terms of patent law can then be treated as comprising this researcher and two laboratory assistants having the necessary manual dexterity and lack of fatigue”.
} 
therefore that skilled person was used in deciding the case. ${ }^{96}$ Thus, once more the skilled person in a biotechnology case was described as a team, but perhaps with a little less competence than in $\mathrm{T} 60 / 89$.

In Bayer plc v. Octapharma Ltd, the English Patents Court, ruled on the qualities of the skilled man in regard to a patent that involved trying to enhance the stability of aqueous immunoglobulin (antibody) preparations. ${ }^{97}$ Save that the parties agreed that the addressee in such a field should be a team and that it should not include a statistician, they were some way apart when it came to identifying the constitution of such a team. Bayer's addressee was modest in size: primarily a synthetic organic chemist and a biochemist who would have access as required to pharmacologists and immunologists. By contrast, Octapharma proposed a larger team, a line-up that appeared to the court to be more like the sort of research team which one would expect to find in a major multinational pharmaceutical company. The court ruled that the notional skilled addressee is the ordinary man who may not have the advantages that some employees of large companies may have. Therefore Bayer's selection was nearer the mark.

In T 142/94 it was decided that the skilled man should have knowledge of and the capacity to apply, the theoretical calculus made on the pharmacokinetic qualities of different dosage forms. ${ }^{98}$ This is advanced technical knowledge usually only applied by researchers.

In $\mathrm{T}$ 383/88 it was tried whether the man skilled in the art could manufacture a particular active substance by traditional chemical synthesis. The applicant had in support of enablement handed in a statement from a professor at a medical faculty. The Board said that the statement had been made by a person that was too highly qualified to have any influence on the outcome. It is not clear from the decision how the statement had been drafted. Had the professor said that he had been able to manufacture the substances at the particular time? In that case it was reasonable to decide that he had been too highly qualified. ${ }^{99}$

\subsubsection{The effort to be made by the skilled person}

\footnotetext{
96 T 412/93 of 2111 1994: "[T] of three composed of one $\mathrm{PhD}$. researcher with several years experience in the aspect of gene technology or biochemistry under consideration, assisted by two laboratory technicians fully acquainted with the known techniques relevant to that aspect. This applied equally whether the question under consideration was obviousness for the purpose of Article 56 EPC or sufficiency for the purpose of Article 83 EPC. For different aspects the composition of the team might vary depending on the knowledge and skills required by that particular aspect. This definition of the skilled person coincides with the view of the Board, and references in this decision to 'skilled person' are to be interpreted as meaning this team. ... The notional skilled person in terms of patent law can then be treated as comprising this researcher and two laboratory assistants having the necessary manual dexterity and lack of fatigue."

97 Patents Court, Bayer plc v. Octapharma Ltd, of 05011999.

$98 \mathrm{~T} 142 / 94$ of 16011997.

99 T 383/88 of 0112 1992: "The affidavit of Professor David H. Coy of the Department of Medicine of Tulane University could, on the face of it, be regarded as irrelevant, on the ground that it was given by a person who was too highly qualified to be regarded as a notional skilled addressee in what is, after all, a well-developed technical field. However, even if the Board could accept that Professor Coy could properly be regarded as the notional, that is to say, average skilled person in the present field of chemistry of pyrimidines, the fact remains that the Board has only a single affidavit from a notional skilled addressee, and it cannot normally be the case that an affidavit by a single person suffices to discharge the burden of proof to the strict standard that is required”.
} 
The third and final aspect of the man skilled in the art is the effort to be expected of him. Under Art. 83 EPC, it is not the technical feasibility of individual steps that is relevant, but the necessary total effort for the man skilled in the art. ${ }^{100}$ Even if each of the different steps involved could be considered as being feasible with a certain amount of trial and error, the total amount of experimental effort necessary to successfully advance step by step may still be regarded as undue. ${ }^{101}$ It is the total effort that must not constitute an "undue burden". This is an important limitation on what may be expected of a man skilled in the art. All that the skilled person has the theoretical knowledge to do cannot be expected of him. The reason may be lack of time, but also his particular working methods or perhaps even his general attitude to his work. In T 500/91 the research team that constituted the skilled person was said to be orientated towards practical results and therefore was not able to solve problems in unexplored areas. ${ }^{102}$ In another biotechnology case, $\mathrm{T}$ 455/91, a similar attitude was described. The Board of Appeal stated that the man skilled in the art had a conservative and cautious approach to his work. He resisted work in a manner that contradicted established opinions. Nor did he take actions that had unforeseeable consequences. He only did modifications that involved limited risks and efforts. ${ }^{103}$

In $\mathrm{T}$ 418/89 the invention was certain monoclonal antibodies. The only information in the description directly related to the antibodies pertained to the antigens that had been used to stimulate production of the antibodies. The Board concluded that this did not suffice, because it meant a considerable effort to create a hybridoma able to express the

100 T 639/95 of 2101 1998: "Although it can be said that at the priority date of the present application, all the techniques referred to were to some extent manageable, it cannot be said that it was a trivial exercise to put them in practice in the sequence outlined by the complex experimental plan given on pages 30 to 32 of the description. Each individual step therein presented the skilled person with some degree of difficulties and uncertainties. What is remarkable is the discrepancy between the lack of technical details in respect of the procedural steps to be taken according to the general plan and the complexity of the plan as a whole. Thus, even if each individual experimental step per se can be considered as being feasible with a certain amount of trial and error, the total amount of experimental effort necessary to successfully advance step by step towards the desired final goal may still be regarded as undue for a skilled person.”

101 T 994/95 of 1802 1999: “The Appellants argued that each of the steps involved in the isolation and therapeutic functionality of antisense oligonucleotides could be made to work to some extent and that this should be enough to consider the whole invention as sufficiently disclosed. The Board, however, cannot agree. ...

[E]ach of the necessary steps needs to be combined with the other steps to get to the therapeutic compound. If all steps can only be carried out with 'some' efficiency, then it is to be expected that the overall efficiency of their combination will be dismally low. Moreover, even if each individual experimental step per se could be considered as being feasible with a certain amount of trial and error, the total amount of experimental effort necessary to successfully advance step by step towards the desired final goal is still to be regarded as undue burden for a skilled person, especially in the absence of any concrete guidance and experimental verification.”

102 T 500/91 of 2110 1992: "[I]n accordance with the established jurisprudence of the Boards of Appeal, the notional skilled person who may be represented by a team of appropriate specialists ... is oriented towards practicalities ... and the development of the art normally expected by him does not include solving technical problems by performing scientific research in areas not yet explored.”

103 T 455/91, OJ EPO 1995, 684: "[T] he said expert would constantly be conditioned by the prior art and, before taking action, would carefully ponder any possible modification, change or adjustment against the background of the existing knowledge. Under these circumstances, in the board's view, the skilled person would adopt a conservative attitude. However, this must not be seen in the sense of being reluctant or opposed to modify or adjust a known product or process, but rather in the sense of being cautious. For example, the skilled person in question would neither go against an established prejudice nor try to enter into 'sacrosanct' or unpredictable areas nor take incalculable risks. However, within the normal design procedures, the said expert would readily seek appropriate, manifest changes, modifications or adjustments which involve little trouble or work and no risks or only calculable risks, especially for the sake of obtaining a more handy or convenient product or of simplifying a procedure. In particular, the skilled person working in one field (e.g. expression in yeast) would regard a means conveniently adopted in a neighbouring field (e.g. the bacterial art) as being readily usable also in that field, if this transfer of technical knowledge involves nothing out of the ordinary." 
antibodies according to the claims, if it was at all feasible. ${ }^{104}$ After the Board had concluded that the written description was insufficient, the Board tried whether a deposition made by the applicant disclosed the invention sufficiently. Normally a deposition of a hybrid cell is enough to disclose an invention. The deposition will make it possible to receive samples from the deposition institute and the sample cells will express the antibodies. In this case, though, it proved to be difficult to produce antibodies with the deposited culture. Only after the opponents had requested two, and at times three, samples, could minute quantities of the antibodies be produced. From this the Board concluded that the skilled person was not able to practise the invention. It was unacceptable that he had to repeatedly request new samples from the depositions institute and only received minute quantities, despite adopting a technique considerably more sophisticated than the one the deposition institute had recommended. 105

Decision T 412/93 is instructive in several ways. The application was on the recombinantly produced and commercially very successful protein erythropoietin (Epo). The most controversial question was whether the skilled person had been able to practice the invention on the priority date, 13 December 1983. The application did not contain a deposition, or a description of the DNA-sequence that coded for the protein. The most valuable information was an oligomer that, according to the application, could be used as a probe to find the Epo-gene in a gene bank. But this meant that the skilled person had to go through many steps in order to practise the invention. ${ }^{106}$

The patent application had been filed at such an early stage because an intense race had preceded the application. The main technical problem had been to find a probe that would render it feasible to locate the Epo-gene. The other necessary steps did not normally cause insurmountable problems. But, nevertheless, once the skilled person had the probe much work remained. During the proceedings in this case the patentee and the opponents had carried out the necessary steps on a number of occasions. It had been found that it would have taken one person 25 weeks to do it, 9 weeks for a team of three persons (together 27 weeks) and 14 weeks for a researcher and his assistant (together 28 weeks). Witnesses in the proceedings stated that a few months was a realistic period for the work. The Board ruled that this was a considerable amount of time, but that it was not an undue burden. Thus, the invention was sufficiently disclosed and a deposition had not been necessary. ${ }^{107}$ It is unusual

104 T 418/89, OJ EPO 1993, 20: "The Board considers that in the circumstances of the present case, where the written description of how to produce a hybridoma is basically the known cumbersome and random general process and a specific technical teaching is provided only by identifying the type of the antigen, being E-rosette positive purified normal human peripheral T-cells, the requirements of Article 83 EPC are not met.”

105 T 418/89, OJ EPO 1993, 20: "A disclosure provided by a deposit according to Rule 28 EPC is not regarded as being sufficient within the meaning of Article 83 EPC, if and when it is only possible to reproduce the invention after repeated requests to the depository institution and by applying techniques considerably more sophisticated than those recommended by the depository institution.”

106 T 412/93 of 2111 1994. The Board of Appeal described the steps in the following manner: “(a) obtaining an available gene bank, for example, the Lawn gene bank ... or producing this [a gene bank] in accordance with published literature, such bank having a high probability of containing a clone including the gene sequence coding for human Epo;

(b) making a DNA oligomer to act as a probe to isolate clones with the Epo gene from the gene bank;

(c) isolating, sequencing and characterising the clone;

(d) obtaining available vectors, and constructing a suitable expression vector;

(e) obtaining host cells in which the DNA could be expressed;

(f) transfection of the host cells, amplification of the cells, and cultivation to produce Epo;

(g) producing standard antibodies for an assay for Epo;

(h) purification of the Epo so produced, and testing that it has the desired properties.”

107 T 412/93 of 2111 1994: "This need for a deposit cannot be introduced by reference to the concept of undue burden. This concept relates more to cases where the route that the reader is to follow is so poorly marked that 
that the public should have to make such a considerable effort in order to practise a patented invention. But maybe it was accepted in this situation due to the considerable importance of the invention and the general research intensity in this field.

The importance of relating the efforts of the skilled person to the resources available in the particular technical field is clear from $\mathrm{T}$ 456/91. The claims pertained to a dosage form that made it possible to release the active substances during a period of one month. Opponents had pointed out that it would cost thousands of dollars to test whether one particular embodiment would have the mentioned effect. Nevertheless, the Board found that the application satisfied the requirements of Art. 83 EPC. The main reason was the huge costs associated with the development of a new pharmaceutical even after the basic discovery had been made. Thus, it was not unreasonable to expect that relatively large sums were spent on testing whether a certain dosage form was suitable. ${ }^{108}$

\subsubsection{Parts of the patent application that the skilled man may use}

According to Arts. 78 and 83 EPC the application in its entirety may be used in order to enable the skilled person to practise the invention. This means that the description, including any drawings, may be used. This has been confirmed in case law. ${ }^{109}$ However, it is made clear in Art. 85 EPC that the abstract may not be used. ${ }^{110}$

The descriptions of pharmaceutical inventions usually start by a statement about the therapeutic field that the inventions belong to, e.g. ulcer treatment, antibiotics or vaccine. Thereafter some to the invention closely related substances in the therapeutic field are mentioned. Further, it is usually stated how a man skilled in the art can manufacture the substances from broadly available starting materials; the technique used to manufacture the dosage forms for the finished pharmaceuticals; and the therapeutic result that can be obtained. In addition, the description normally refers to some particular embodiments of the invention, the working examples. ${ }^{111}$

In some applications tests are described that the applicant has not really carried that out. These are called paper examples or prophetic examples. However, it may sometimes be a contentious issue whether the applicant really has made the experiments or not. But, due

success is not certain .... If the route is certain but long and laborious, the patentee is under no obligation to assist the disclosure by making actual physical samples, that is, the 'factory' available. To come to the opposite conclusion would be effectively to introduce a requirement to make the best mode immediately accessible to the public, and such a requirement is not part of the European patent system.”

108 T 456/91 of 0311 1993: "[I]t is well known that developing and testing a marketable product in the medical field is commonly very expensive and time consuming even once the basic research has already been completed. Development of a product that is to achieve sustained release over one month, can be expected to be more expensive than average. In view of this the Board does not consider that the time scale and costs quoted ... are so different from what could reasonably be expected, as to amount to evidence of the description of the patent being insufficient because an undue burden is imposed on anyone wishing to carry out the teaching of the patent in suit.”

109 T 14/83, OJ EPO 1984, 105: "Pursuant to Article 83 EPC the invention is sufficiently clearly and completely disclosed if it can be carried out by a person skilled in the art. The source of the disclosure of the invention within the European patent application is of no importance”.

110 T 169/83, OJ EPO 1985, 193: "It is worth noting that the only element of the application to which the Convention gives a ranking is the abstract when it states that the latter is to serve merely as technical information and may not be taken into account for any other purpose (Article 85). This makes it logical to assume that the drafters of the Convention would have devoted a similar clause to the rank of drawings had this been their intention.”

111 See Rule 27(1) EPC. 
to the particularly unpredictable nature of the pharmaceutical field, applicants writing paper examples often use especially cautious statements. T 60/89 was related to a patent for a biotechnological process. Expressions such as "presumably", "probably", "will be” and "in principle" were used in the examples. The Board stated that this indicated that the examples had not actually been performed. The description was found insufficient, but this was not solely because the examples had not been carried out, it was a technical assessment of the disclosure. ${ }^{112}$ The pertinent question is always whether the man skilled in the art could have practised the invention with the information in the application; it is not decisive whether the applicant had done this at the time of filing. The test is strictly objective. ${ }^{113}$ If the statements in the description are sufficient there is no basis for complaint. However, there have still been cases where paper examples have raised particular doubt. In T 918/94 the Board observed:

"[W]riting down a mere reaction scheme is not synonymous with sufficient disclosure, if the skilled person is not also taught by way of experimental verification whether the reaction actually works, and if it does not, which measures should be taken to remedy the failure.”114

Although the importance of empirical verification was stressed it was stated that a strictly theoretical description of the invention could be sufficient, even though it was not so in this case. ${ }^{115}$ The lack of practical experience in the patent application can thus be a disadvantage to the applicant. But it is only one circumstance among many others. If the information in the application as a whole enables the man skilled in the art, the lack of working experiments is immaterial. Post-published documents can be used as evidence whether the invention merely disclosed at a general conceptual level was indeed reproducible at the relevant filing date. ${ }^{116}$

112 T 60/89, OJ EPO 1992, 268: “As to the question of 'working example', raised above, the wording of the description in column 8 using words such as 'presumably', 'probably', 'will be' and 'in principle' indicates that the work described has not yet been done. One can, therefore, hardly interpret the information in column 8 as a working example. According to the Board's opinion the arguments put forward by the Respondents that, if the skilled persons had worked exactly according to the rather vague information provided in column 8 in the sense of a working example, within a reasonable amount of time and investigation, they would have failed, are convincing. In particular in 1978, as emphasised by the Respondents, cleaving the penicillinase gene with the Taq restriction enzyme would have produced seven fragments and at that time it would have been extremely difficult to handle this problem. Further, the control over the enzymes nibbling back the DNA was not yet well developed so that it would have been very cumbersome to stop the reaction mixture exactly at that point where it was necessary to form a perfect fusion. Thus, the proposal given in column 8 would not have enabled a skilled person to carry out the process of Claim 2 when working exactly along the wording of this part of the description, without undue burden, if at all.”

113 “[O]bjections to 'paper examples' are only difficult to overcome if these 'paper examples' fail to disclose all essential elements that are necessary to carry them out”. See Jaenichen, H-R., The European Patent Office's Case Law on the Patentability of Biotechnology Inventions, 346.

114 T 918/94 of 06071995.

115 T 918/94 of 0607 1995: "The only evidence submitted is that of Prof. Gilles, based on theory and not experiment, that the reactions should work. In these circumstances the Board can make no assumption in the Appellant's favour that the method would work. If it had been shown that a method exists that does work, the Appellant might have been given the benefit of the doubt, at the application stage at least, on the question of whether the skilled person would have hit on this method. But where, as here, there is no experimental demonstration of success to set against very substantial indications that it does not work, this is not appropriate. The Board has thus to come, through a slightly different line of argument than the Examining Division, to the conclusion that on the evidence presently available neither reaction (a) nor (b) are workable and therefore the requirements of Article 83 EPC are not fulfilled for the subject-matter of the main request."

116 T 994/95 of 1802 1999: "[T] stabilized in any known way and complementary to a portion of an mRNA will have to be tested in respect of its ability to enter a cell and hybridize to said mRNA in such a way as to block translation, methods being available to characterize any mRNA portion and to isolate and stabilize any oligonucleotide. Whenever such an oligonucleotide is found, it is a therapeutic agent according to claim 1. The Appellants themselves defined this 


\title{
2.3 Remarks
}

It is well known that research based pharmaceutical companies closely watch the patents that are applied for in their field, as do most other large manufacturing companies. ${ }^{117} \mathrm{~A}$ study has been made on how much the patent registers are used by oligopolistic companies, which is a proper description of much of the pharmaceutical industry. In industries where less than 20 companies had more than 90 per cent of the market, 97 per cent of the companies regularly followed the patent applications that were published. ${ }^{118}$

The patent registers are of course particularly important for the research departments. In one study, interviews were made to find out more about the research process in one of the world's leading pharmaceutical companies. The following description was given of the policy the company had in relation to publication of research results:

\begin{abstract}
"Beyond a possible brief delay to prepare patent filings, the firm encourages publication of research results. ... We see some danger of losing our competitive advantage by publishing, but a much greater danger if we do anything that deters the best scientists from coming here. Further, we need for our scientists to have great reputations in order to bring others like them to [the firm]. We are the beneficiaries of worldwide scientific research, and thus we also need to contribute to this pool of scientific knowledge, creating a public good". 119
\end{abstract}

It may be important for pharmaceutical companies to permit their researchers to publish in scientific journals, but nothing is published before it has been evaluated for possible patenting. Patents are the company's primary aim, and once the applications have been filed the inventions will become known to the engaged firms if the inventions are of any value. That will be the case, with or without additional publication in a scientific journal. The fact that such a large portion of the privately funded medical research is published is therefore mainly attributable to the existence of the patent system, and not much to a general desire in companies to contribute to a common pool of knowledge. The information that is made public in the scientific journals is there much thanks to the patent system.

The decisive economic advantage of publication of research results after 18 months is that a co-ordination between rivalrous companies is achieved, i.e. a division of labour. ${ }^{120}$ That may be particularly valuable with regard to projects that have been abandoned by one company after a patent application has been published.

"When a decision ... is made to abandon a drug during the long discovery and development
process, the usual outcome is simply greater uncertainty, rather than dramatic evidence of
success or failure. We simply stop producing information upon which to make a definitive
judgement. When a project is dropped by a company or by an academic research director

teaching as conceptual in nature and the Board certainly agrees that it is so. In the absence of any tangible proof in the patent specification that the claimed concept can be put into practice, post-published documents can be used as evidence whether the invention merely disclosed at a general conceptual level was indeed reproducible without undue burden at the relevant filing date.”

117 See Jucker, E. M., American Patent Lawyers Association Quarterly Journal 1982, 85 and Allcock, H. M., Lotz, J. W., 18 Journal of Chemical Information \& Computer Science, 1978, 65 and Koenig, M. E. D., 12 Research Policy 1983, 15.

118 See Hofinger, S., EPI Information 3/1996, 87.

119 See Zucker, G. L., Darby, M. R., 26 Research Policy 1997, 438 (a footnote is excluded from the quotation).

120 This has been described in Eisenberg, R., 97:2 The Yale Law Journal 1987, 219 and Krol, T.F., Coleman, J.

C., Bryant,, J., 30 Drug Information Journal 1996, 251. 
because of lacks of funds, all that remains is the lingering doubt of a few knowledgeable scientists about what might have been."121

It may very well be that another company can use the results if they are publicly available. They may interpret them differently or simply make a different assessment of the possibilities for success. Dimasi has studied the reasons why clinical trials are abandoned. He sent questionnaires to pharmaceutical companies. The answers that the companies gave were grouped into three categories: safety (e.g. toxicity), effectiveness (e.g. too little therapeutic response or none at all) and economic reasons (e.g. the market was too small or the return lower than initially calculated). 27 per cent of the clinical trials were abandoned for safety reasons, 46 per cent due to low efficiency and 23 per cent for economic reasons. ${ }^{122}$ It is not unlikely that these reasons for abandoning certain substance could be re-evaluated in the future by other companies. They may have new information or a different research situation within the company. The publication of patent applications will be of considerable importance for the possibility for such re-evaluation of results that have been attained by others.

121 See Helms, R. B., Preface, xxii, in Helms, R. B., (ed.) Drugs and Health.

122 See Dimasi, J. A., 58:1 Clinical Pharmacological Therapy 1995, table on page 8. 


\section{The claims}

\subsection{Two purposes}

Once the industrial applicability of the invention is known and the ability of the man skilled in the art to practice the applicability has been ascertained, it is possible to start to formulate a definition of the invention. That is done in the patent claims. The claims should as a principle correspond to the field in which the man skilled in the art can take advantage of the industrial applicability of the invention. This, however, is not simple. How patent scope is crafted in more details will be the topic treated in this chapter.

The inventor will want as broad claims as possible, because there is a direct link between the breadth and the economic value of the patent. But the examiner must see to that the claims correspond to the technical contribution that has been made by the inventor, so that competition is not adversely affected. Other patentability decisions are constrained by a number of quite well-defined legal principles, but patent offices have a considerable room for discretion when deciding which claims should be admitted and which ones should be pruned back or rejected due to their scope. There is also another question that must be considered by the examiner when the claims are formulated: as much legal certainty as possible is required. The claims define a monopoly that will be used in relation to competitors. If the scope of the monopoly is ambiguous the parties may need to enter into negotiations and perhaps in the end a court may have to settle the meaning of the claims. The requirements as to the precision of the claims will be the first topic in this chapter. Thereafter will the allowable breadth of the claims be discussed.

\subsection{Clarity}

\subsubsection{General rules}

Art. 84 EPC provides that the claims shall be clear and concise. This dictates that a man skilled in the art should know what subject matter is encompassed by the claims. It is the definition of the monopoly that has to be clear from a practical perspective. There should be no need to carry out undue investigations as to the precise limits of the invention. ${ }^{123} \mathrm{~A}$ further provision of importance in this respect is Rule 29 EPC. It provides that the patent claims shall consist of two parts divided by the words "characterised by". The first part should include the features that belong to the state of the art and the second - "characterising" - part should contain the novel features. According to EPO Guidelines this wording of the claims shall be used "wherever appropriate". One example mentioned in the Guidelines of when it is not appropriate is, however, product patents on chemical compounds. ${ }^{124}$

When chemical formulas are included in the claims it should be possible to determine the scope of protection only by reading the claims. Rule 29(6) EPC provides:

123 T 720/92 of 3105 1994: “Article 84 EPC requires the claims to be clear. It is a necessary but not sufficient condition of clarity that the claims be drafted in comprehensible language. Patent claims are directed to concrete subject-matter. In consequence, it is the practical meaning of the language of the claims which is of importance. The person skilled in the art should thus understand what is meant by the language of a claim without ambiguity and without complicated, time-consuming investigations, i.e. without undue burden.”

124 Guidelines C III 2.3. 
"Claims shall not, except where absolutely necessary, rely, in respect of technical features of the invention, on references to the description or drawings. In particular they shall not rely on such references as: 'as described in part ... of the description', or 'as illustrated in figure ... of the drawings'."

One example of how claims may not be crafted is the following: "Any one of the compounds of Formula I listed in Examples 1 to 39 herein”, i.e. a reference to compounds used in the description as examples. ${ }^{125}$ The possibility for applicants to make references to the description is limited to information that cannot easily be conveyed in words, e.g. graphs or diagrams. However, a Board of Appeal has found that there may be situations in which it is permitted to include references in the claims to chemical formulas in the description. ${ }^{126}$ In Guidelines it is also mentioned that methods for measuring may be stated in the description if the claims otherwise would become difficult to comprehend. ${ }^{127}$ In one application antibodies were defined in the claims by their "specific activity". The Board of Appeal accepted that the method of measuring the activity was defined in the description. ${ }^{128}$ It is for the applicant to demonstrate that the information cannot be easily included in the claims. ${ }^{129}$ In T 269/87 claim 9 read: "Plasmid pCT 70". The terminology was arbitrary and contained in itself no technical information about the plasmid. The Board of Appeal resisted the claim, because the description contained further structural information that could have been incorporated in the claim. ${ }^{130}$ After this decision arbitrary terminology is broadly deemed to be in conflict with Art. 84 EPC. 131

\subsubsection{Clarity and broad claims}

In $\mathrm{T}$ 238/88 the question was whether the mere breadth of the claims constituted a lack of clarity. It was decided that that was not the case. Breadth in itself was not a reason to question the clarity. The feature "alkyl" was unambiguous to the man skilled in the art, even though it covered an unlimited number of chemical structures. The Board also decided that the same applied to a number of other common chemical terms. ${ }^{132}$ Furthermore, it has been decided

125 T 792/90 of 21021991.

126 T 271/88 of 06061989.

127 Guidelines C III 4.10.

128 T 139/85 of 2312 1986: "One way to draft a concise claim is by making use of features which are clearly defined in the description. There are no objections to such a method, unless the clarity of the claim is so affected that a person skilled in the art would have difficulties understanding what is meant by the claim. No such difficulties arise in the present case. The statement in the description that all human interferon titres are quoted in Reference Research Units using the human interferon-alpha reference research standard 69/19 ... thus gives clear guidance to the skilled man as to how the term 'specific activity' in the claims has to be interpreted."

129 Guidelines C-III-4.10.

130 T 269/87 of 2401 1989: "In order to enable the public to ascertain the scope of the protection provided, the claims should be clear in themselves, whenever this is possible, and contain as much structural information as available and necessary in the circumstances ... The specification, in particular Figure 5, contained detailed structural information which could have been included in claim 9 to enable the public to identify and compare plasmids vis-à-vis the claimed subject-matter.”

131 See Jaenichen, H-R., The European Patent Office's Case Law on the Patentability of Biotechnology Inventions, 89.

132 T 238/88, OJ EPO 1992, 709: "The criticised feature 'alkyl' in the definition of the R substituents undoubtedly relates to a well-known technical term of art which is commonly used in the chemical field and which does not imply any lack of clarity or ambiguity as alleged by the Examining Division, i.e. it is clear as it 
that the term "substituted" was clear in a product patent, although it was not defined in the claim how the molecule could be substituted. The meaning was that the molecules could be substituted in any manner and this did not as such render the scope of protection ambiguous. ${ }^{133}$

Clarity is a matter of quality and not quantity. It is not dependent on the scope of protection, but on the precision of the limits of that protection. ${ }^{134}$ This became evident in $\mathrm{T}$ 338/95. The Board decided that the feature "lower alkyl”, which does not cover particularly many possibilities, was unclear from a technical viewpoint. ${ }^{135}$ The important question was how well the feature delineated the outer boundaries of the monopoly, not how much they monopolised. Clarity in Art. 84 EPC is therefore not a caveat to the allowable breadth of the claims. Other legal requirements have that effect.

In Rule 31 EPC it is provided that the applicant shall pay additional fees for applications that contain more than 10 claims. In T 156/82 the Examining Division decided that the applicant had tried to circumvent this by including 13 individual chemical compounds in one claim. The Board of Appeal reversed and found the claims acceptable. It was stated that Rule 31 was designed to compensate for the extra examination that was necessary in applications with many claims, but that the 13 disparate compounds in one claim did not confront the examiner with any additional problems. Nor did the claims become ambiguous. ${ }^{136}$

In T 246/91 it was not the content of one claim that was particularly large, but the number of claims. The application originally had 191 independent and dependent claims.

stands. Unless sound reasons are given, the breadth of the term cannot be a bar to its incorporation into the claim. The Examining Division's criticism in this respect amounts to no more than a mere allegation not supported by any evidence or reference to the prior art. The same considerations apply, mutatis mutandis, to the features 'aryl, cycloalkyl, a heterocycle ... and alkoxycarbonyl' which follow in this listing."

133 T 939/92, OJ EPO 1996, 309. The first instance had: “considered that the term 'substituted' could not be given its ordinary meaning in the context of products which were only claimed because of their biological activity. Since the special meanings ascribed to this term in the description were different in each case it was used, the claims were held to be unclear." The Board, however, decided that: "the present independent claim covers certain chemical compounds per se, and not just those compounds having a particular biological activity. Hence the biological activity of these compounds is not an essential technical feature of the claimed subjectmatter, and thus not part of the definition of the claimed subject-matter, so that there is no reason for the term 'substituted' not to have its ordinary technical meaning, namely 'substituted by absolutely anything' .... This meaning is, moreover, the one that was clearly intended by the appellant. On this basis, claim 1 is, in the board's judgement, clear in the sense of Article 84 EPC.”

134 T 456/91 of 0311 1993: “According to the jurisprudence of the Boards of Appeal, the clarity of a claim is not diminished by the mere breadth of a term of art contained in it, if the meaning of such term is unambiguous for a person skilled in the art, either per See or in the light of the description .... As in the present case it is clear from the claims when read in the light of the description ... which peptides are suitable for the present invention, the present claims cannot be challenged under Article 84 EPC for the sole reason that an extremely large number of compounds can be used for carrying out the invention.”

135 T 338/95 of 3001 1996: “[T]he term 'lower alkyl' on its own, i.e. in the absence of any unambiguous reference point, does not have a 'well-recognised meaning' in the art of organic chemistry. This would have been needed for the use of such a relative term in Claim 1 to be legitimate .... According to the available evidence, there exists no meaning of 'lower alkyl' generally accepted by those skilled in the art. In particular, it is unclear what the upper limit of the carbon atom number is for a (saturated) hydrocarbon radical to be a 'lower alkyl'. This term is therefore not suitable for clearly defining the subject-matter for which protection is sought in Claim 1 which is directed to a group of organic compounds per se.”

136 T 156/82 of 0901 1984: “Art. 84 EPÜ enthält drei deutliche Erfordernisse für Einzelansprüche, die der Deutlichkeit, Knappheit und Stützung durch die Beschreibung. Das letzte Erfordernis ist eindeutig gewährleistet. Die Deutlichkeit ist auch nicht zu beanstanden, da die Namen der Verbindungen nomenklaturgemäß sind. Der Knappheit wegen verstößt der Anspruch 5 auch nicht gegen Art. 84 EPÜ. Dies bedeutet nicht, daß eine Beanstandung aufgrund dieses Erfordernisse, zum Beispiel wenn die Übersichtlichkeit nicht mehr gewährleistet wäre, in ähnlichen Fällen niemals gerechtfertigt wäre.” 
The number had been reduced to 157 at the time of the decision by the Board of Appeal. The Board found that the expression "concise" in Art. 84 EPC and Rule 29(5) did not establish any exact limit as to the number of claims permitted in a patent application, but this did not mean that the requirement was without legal effect. The extra fee for applications with more than 10 claims was not the only limitation. The Board mentioned that the number of claims affected the legal certainty. The work that was necessary to assess the scope of a patent with 157 claims was considerable and totally unnecessary. Therefore the application was in conflict with Art. 84 EPC and Rule 29(5). ${ }^{137}$ A similar analysis was made in T 79/91 where 10 independent claims with partially overlapping scope was found to be an undue burden to competitors that wanted to determine the scope of protection. ${ }^{138}$ In T 552/91 the Board stated that a situation in which two independent claims were overlapping was, as such, sufficient grounds to reject the application. ${ }^{139}$

\subsubsection{Definitions of chemical compounds}

Compounds with therapeutic effects can be defined with sufficient clarity in three different ways or with a combination of the three different ways: structural formula, product-byprocess claims and parameters relating to the compounds. ${ }^{140}$

\subsubsection{Structural formula}

The most precise way to define a chemical compound is by way of a structural formula. That should be used if feasible. A new synthetically manufactured compound is usually delineated in the claims by a generalised structural formula. This is known as a Markush claim. ${ }^{141}$ The scope of such a claim depends on which compounds may be created by combining the different alternatives mentioned for the different positions in the formula. This type of claim makes it possible to define a very large number of compounds concisely. In dependent claims

\footnotetext{
137 T 246/91 of 1409 1993: "What is or what is not reasonable depends on the facts and circumstances of each particular case. In deciding the matter, regard has to be had to the interest of the relevant public since patents should not be allowed to erect a legal maze or smoke screen in front of potential users of the inventions to which they lay claim. Patent claims, taken singly as well as in totality, must be clear and concise (Article 84 EPC) in order to enable such potential users to ascertain, without undue burden, let alone recourse to litigation, whether their planned commercial use is likely to infringe the patent monopoly. Having to construe the 157 claims (let alone 191 claims), and to form a valid and commercially useful opinion on whether or not any one of them could prevent or hinder the commercial activities must in the nature of things impose a severe and totally undue burden on the public."

138 T 79/91 of 1109 1991: "In the present case, what the Applicant considers according to his main request to be the invention has been set out in at least ten independent claims of different, though to a greater or lesser degree overlapping, scope. This presentation makes it difficult, if at all possible, to determine the matter for which protection is sought and places an undue burden on others seeking to establish the extent of the monopoly."

139 T 552/91, OJ EPO 1995, 100: "In actual fact the main request would have to be rejected if only because, as the appellant has itself submitted, claims 10 and 17 relate to the same subject-matter, so that the set of claims as a whole cannot be regarded as concise within the meaning of Article 84 EPC.”

140 Guidelines C III 4.7a.

141 See in general about these kinds of claims Brown, L. J., 31 Journal of Chemical Information and Computer Science 1991, 2.
} 
the most commercially interesting individual compounds are usually singled out. The independent and broadest claim is most efficient when someone tries to "invent-around" the patent. The dependent and narrower claims are "safety measures" if it would turn out that the first claim lacks novelty or inventive step. In addition, the dependent claims are valuable because they may make it impossible for competitors to make selection inventions within the scope of the broader claims, see part 4.7.

\subsubsection{Product-by-process claims}

A product-by-process claim is a definition of a compound by way of how it is manufactured. It may be used if the compound cannot be defined by any other means. In general it is naturally occurring chemical compounds, catalysts (enzymes), macromolecules and products of microbiological process, that are defined by how they are manufactured. These compounds are particularly susceptible to structural variations, which makes it difficult to define them unambiguously by their structure. ${ }^{142}$

It must be borne in mind that product-by-process claims are definitions of new chemical compounds. The compound in itself must fulfil all patentability requirements. It is not sufficient that the manufacturing process is novel. The process only serves as a definition; it is not the invention. ${ }^{143}$

Product-by-process claims will often create more uncertainty than structural definitions. Chemical reactions rarely follow only one path. Normally disparate reactions occur simultaneously. This will lead to by-products and a separation will be needed before the end-product is available. Legal certainty therefore demands that a structural definition is used if possible. ${ }^{144}$ The applicant has not a free choice. However, on one occasion a Board of Appeal has accepted product-by-process claims in a situation where a structural definition in principle would have been better. In T 552/91 the applicant had initially made a mistake in the structural formula that was filed and it would have been in conflict with Art. 123(2) EPC to

142 Even "macro-organisms", i.e. animals, can be patented with product-by-process claims. When the first instance examined the Oncomouse application, in V 4/89, OJ EPO 1989, 451, it was stated that: "Clarity (Article 84 EPC) Claim 17 is drafted as a 'product-by-process' claim. It has been recognised by a Technical Board of Appeal that claims for products defined in terms of a process of manufacture as admissible provided they fulfil the requirements for patentability and there is no other information available in the application which could enable the Applicant to define the product satisfactorily by reference to its composition, structure or some other testable parameter .... Since in the present case the claimed products are not individually definable biological entities, which could be characterised by their physiological or morphological features, there is no way of defining the animals other than by the process of their production."

143 T 124/93 of 1008 1995: “According to established case law of the Boards of Appeal ... product-by-process claims give protection for the products as such, independent from the process by which they were made, and the said products, therefore, likewise have to fulfil the requirements of patentability such as novelty and inventive step independent from the novelty and inventive step of the process." T 495/90 of 3006 1992: “[T]he information given in document (1) provides the skilled person, with all he needs regarding the starting material, being the inhalation drug solution and the spray-drying method, for preparing the claimed substance. It has again to be emphasised that Claim 1 is a product claim. Certain features of the product claim are defined by process elements, so that Claim 1 could be regarded as a product by process claim. But this definition of a product does not change the patent category. Claim 1 still remains a product claim and is only patentable if the product itself is novel. Even if the process were novel, this did not necessarily render the product of this process novel. It is self-evident that by new and patentable processes known products may be produced, but this does not happen to be the case here."

144 T 130/90 of 2808 1991: “According to this case law the conditions to define a certain product by its process are that there are no other parameters available for a further definition of the product.” 
correct this. However, in the application the correct process for manufacturing the compound had been described. The Board decided that in such a situation the applicant was allowed to define the product by the process with which it was manufactured. ${ }^{145}$

\subsubsection{Parameters in the claims}

Parameters pertaining to characteristic features of the compounds are at times used together with product-by-process claims. The combination will normally lead to the most unambiguous scope of protection, when the structure is not available. ${ }^{146}$ By parameters are meant characteristic aspects of a product. It may be things that can easily be measured, e.g. melting point, optic rotation and electric resistance. ${ }^{147}$ Parameters may also be more or less complex mathematical formulas that define a relationship between different values. Parameters are possible definitions because differences in parameters can only be explained by corresponding structural diversity. Parameters are a function of the chemical structure. ${ }^{148}$ But as with product-by-process claims, parameters as the only definition may cause ambiguities. ${ }^{149}$ Their acceptance hinges on that they do not create an undue burden for the man skilled in the art. ${ }^{150}$

145 T 552/91, OJ EPO 1995, 100: "It may be that claim 17 [the structural definition] complies better with the requirement of clarity (Article 84 EPC) than the corresponding 'product-by-process' claim 10. Correcting the incorrect formula by submitting this claim may therefore seem desirable in the interests of legal certainty. As, however, the subsequent amendment of formula I ... cannot be regarded as rectification of an obvious mistake within the meaning of Rule $88 \mathrm{EPC}$, as the appellant conceded in the oral proceedings, it can only be allowed if it does not infringe Article 123(2) EPC, which likewise serves to safeguard legal certainty. ... Apart from the title 'Chromanol or chromene derivatives' - which, although general, is accurate - and the method of production, the application as filed contains no details which would help characterise more clearly and in an admissible manner those substances to be protected by claim $10 \ldots$ Similarly, the indication of the melting point and the appropriate general title in each case, together with the production measures in claims 11 to 16 , ensures the greatest possible clarity within the bounds of what is admissible. The wording of claims 10 to 16 as 'product-byprocess' claims is therefore compatible with the requirement of clarity in Article 84 EPC.”

146 Cf Hirsche, F., Hansen, B., Protecting Inventions in Chemistry, 80.

147 Guidelines C III 4.7a.

148 T 205/83, OJ EPO 1985, 363: "To establish novelty ... [i]t is also sufficient, however, if distinct differences in the products' properties are demonstrated; this is because, according to an empirical principle in chemistry, a products properties are determined by its structure, so that differences in the properties of products indicate a structural modification.”

149 Guidelines C III 4.7a: "Characterisation of a chemical product solely by its parameters should, as a general rule, not be allowed. It may however be allowable in those cases where the invention cannot be adequately defined in any other way, provided that those parameters can be clearly and reliably determined either by indications in the description or by objective procedures which are usual in the art."

150 T 720/92 of 3105 1994: "Article 84 EPC requires the claims to be clear. It is a necessary but not sufficient condition of clarity that the claims be drafted in comprehensible language. Patent claims are directed to concrete subject-matter. In consequence, it is the practical meaning of the language of the claims which is of importance. The person skilled in the art should thus understand what is meant by the language of a claim without ambiguity and without complicated, time-consuming investigations, i.e. without undue burden. This applies also to functional features, i.e. features which become manifest only on exposure to qualifying conditions (here: testing of 'oxidisability' of the 'oxidisable polymer component' by the oxygen permeability measurement of the composition); the acknowledgement of their clarity depends on the amount of verifying input necessary to conclude whether or not a functional parameter is met. In areas where experimentation is required, clarity can for these reasons be recognised only if with usual methods or methods disclosed in the application, possibly together with common general knowledge, the skilled person is in a position to purposefully design embodiments of the invention by routine experimentation. That is, clarity cannot be recognised if verification of a functional feature involves the working out of experimental activities or even strategies which are not 
Ambiguities may particularly arise with unconventional or previously unheard of parameters in the claims. It is stated in the Guidelines that the examiner should be cautious if the parameters are unusual or require measuring instruments that are not generally available. ${ }^{151}$ The applicant may then have chosen the parameters to hide a lack of novelty. ${ }^{152}$ A further reason for caution is that unusual parameters render the patent documents less valuable as sources of technical information. But these considerations do not preclude that sometimes it may be necessary to use previously unknown parameters. There is no complete bar against the use of new measurements or features, but new types of parameters may not be used if there are known means of characterisation.

In some decisions, therapeutically useful proteins or DNA-sequences have been characterised by their biological effect. It has then been questioned if the skilled person was able to determine the biological effect in a way that would make the scope of protection sufficiently clear. In $\mathrm{T}$ 301/87 the application concerned a recombinant DNA-molecule characterised by that it coded for a polypeptide of IFN- $\alpha$ type (alpha-interferon fulfils a function in the human immune defence and is used in cancer treatment). The Board of Appeal accepted the wording of the claims. There would be borderline cases as to whether a polypeptide had alpha-interferon activity, but this did not, according to the Board, mean that the terminology was unclear, presupposing that there were a number of polypeptides that without doubt fulfilled the claim definition. ${ }^{153}$ Also in $\mathrm{T}$ 391/91 the Board accepted a definition of a DNA-sequence by a statement of its function (coded for the protein INA), together with a statement about the size of the sequence $(10 \mathrm{~kb})$ and from where it had been extracted (a one cellular organism). ${ }^{154}$ However, a statement in the claims that the recombinant protein was different from the naturally occurring version with the same biological function, was found to be an unclear definition of a protein in $\mathrm{T}$ 412/93. The Board decided that the feature "recombinant" without any additional structural features meant that the skilled person faced an undue burden. ${ }^{155}$

disclosed in the application and are not within common general knowledge, thus imposing on the expert an undue burden."

151 Guidelines C III 4.7a.

152 Guidelines C IV 7.5.

153 T 301/87, OJ EPO 1990, 335: "There is, of course, the possibility that the skilled person might, on repeating the process of the patent, obtain some precursor candidates, among others, which might show borderline functional characteristics. The fact that in a set of candidates prepared in this manner a few are borderline cases in as much as they show less marked functional characteristics than others becomes irrelevant if indeed there are many others in the relevant core areas which are satisfactory. The problem for the skilled person may be real in some situations and this might be undesirable, but this does not affect the fact that members of the claimed class may be obtained with sufficient certainty and frequency. The Board can therefore see the need for a partial reliance on functional characteristics in such situations, in view of the special circumstances which prevail in this field."

154 T 391/91 of 2211 1993: "[T]he technical features used for its definition, namely its size (10kb), its activity (encodes INA) and the source (derived from a unicellular source having INA), are, in the context of the present application, sufficient to clearly identify the product (an isolated DNA sequence) for which protection is sought. In fact, the skilled person can readily recognize and test whether a specific DNA sequence falls within the terms of the claim.” The decision has , however, been criticised, see Jaenichen, H-R., The European Patent Office's Case Law on the Patentability of Biotechnology Inventions, 96.

155 T 412/93 of 2111 1994: “Claim 19 ... 'and not being identical to erythropoietin isolated from urinary sources' ... does not comprise any indication of the technical feature or the degree of difference on which non identity of r-Epo with u-Epo should be based, but rather leaves this to the reader's imagination. This puts the claim clearly in the category of claims which are not clear. The whole burden of distinguishing over the prior art $\mathrm{u}$-Epo is put on the reader. In any case, if the reader were to start research to get reliable technical data to examine whether this condition is fulfilled, he would of necessity face all the difficulties discussed above ... Claim 19 ... is thus not allowable as not being in accordance with Article 84 EPC ... must therefore be refused.” 
Ambiguities may also occur in definitions of pharmaceutical dosage forms by use of parameters. In T 954/92 the dosage form had been defined by the amount of the active compound that was dissolved in vitro during 12 hours and by the points in time when the highest level of the active compound was attained in blood plasma, in vivo. The Examining Division refused the application because the definition was so unclear that it would be difficult to decide whether the patent covered a certain dosage form. But the Board of Appeal accepted the wording of the claims. As to the in vitro-values the Board observed that there was a reliable method for measurement and that it was described in the claims. As to the in vivo-measurements, the Board found that they would be difficult, but since they were related to the in vitro values it was only an additional feature. ${ }^{156}$

In $\mathrm{T}$ 893/90 the application was for a process for manufacturing pharmaceutical dosage forms. One component had been defined as "present in amounts and proportions just sufficient to arrest bleeding". The Board of Appeal found that this could be bothersome to test, but that it was still possible with routine methods. Therefore the claim was accepted. ${ }^{157} \mathrm{~T}$ 820/92 was another case where a medical effect was used to limit the scope of protection. A contraceptive process had been defined by the feature that one compound should be used in a "contraceptively effective amount" and another compound in a "physiologically effective amount”. Without giving any particular justification, the Board stated that the wording of the claims was in accordance with established case law. ${ }^{158}$ In T 18/91 a chemical compound for dental treatment had been defined as having an acceptable "shelf-life", i.e. when stored it did not go through a process that made it useless for dentists. The Board of Appeal found that this feature was unclear and unacceptable. The man skilled in the art had no opportunity to assess what was acceptable shelf life. ${ }^{159}$

\subsection{The relationship between the invention and the scope of protection}

\subsubsection{Introduction}

\footnotetext{
156 T 954/92 of 2805 1993: "In vitro dissolution rate tests are well known and well accepted methods for evaluating the suitability of dosage forms for therapeutic administration. Methods, apparatus and means for interpreting such tests are available, for example, from the US Pharmacopeia ... Features (b) and (c) provide instructions which are sufficiently clear for the expert to reduce them to practise. In fact, once the in vitro dissolution criteria to be satisfied are set forth, the expert in preparing the dosage form merely needs to carry out the appropriate adjustments of the ingredients as well as the necessary measurements. No undue experimentation is needed therefore. ... Although the testing of said functional feature [in Vivo plasma values] may be prima facie bothersome, nevertheless it constitutes only a further criterion for the verification of the oral dosage form. As there is likely to be a good correlation between in vivo plasma levels and in vitro release characteristics, it would seem relatively easy to adjust the nature of the delivery system so as to satisfy the criterion”.

157 T 893/90 of 2207 1993: “The feature 'being present in amounts and proportions just sufficient to arrest bleeding' is indeed a functional feature which defines a technical result. However, said feature constitutes also a testable criterion which has to be satisfied by the claimed pharmaceutical composition. Its testing might appear prima facie bothersome, but it is nothing out of the ordinary for the field of medicines and involves only routine trials. Thus, the adopted functional language is allowable and in line with the EPO case law .... The claimed subject-matter is clearly enough defined to meet the requirements of Article 84 EPC without a limitation of Claim 1 by introduction of a reference to specific amounts and/or proportions of the components.”

158 T 820/92, OJ EPO 1995, 113.

159 T 18/91 of 2602 1993: "The Board agrees with the objections of the Respondents that the reference to 'acceptable shelf life' contravenes Article 84 EPC .... The expression 'acceptable shelf-life' is clearly a vague concept without any generally recognised technical meaning in the present context. Nor is there any help in the description as to how to interpret the expression which cannot therefore be considered appropriate in defining the matter for which protection is sought. Accordingly the main request must be rejected for lack of clarity”.
} 
It is important that the claims are clear and unambiguous. But once that has been ascertained it is necessary to consider the acceptable breadth of the claims. This is done in European case law under two different provisions: sufficient disclosure as provided in Art. 83 EPC and support for the claims as provided in Art. 84 EPC. Together the two provisions require an appropriate relationship between the inventor's contribution to the state of the art and the monopoly that is granted. Behind the two provisions is the common thought that the scope of protection should cover the contribution made to the state of the art, but not any more. ${ }^{160}$ This relates to the so-called reward theory, which is perhaps the most common justification for the patent system. He who sows shall reap. But behind this simple idea is a number of contentious issues at stake, issues that reach to the basic rationale of the patent system, i.e. under what circumstances private monopolies can be motivated. That will be discussed in the remarks section.

Decisions on the allowable breadth of claims are a part of the daily work with patents. An inventor will usually describe his invention, to e.g. a patent agent, as certain practical possibilities. It may be some compounds that can be used therapeutically or a chemical process that has a better yield if the temperature is held within a certain range. The patent agent will have to try to generalise this knowledge to define some outer boundaries of the invention. In a case where some compounds have been found to have therapeutic effects, the agent may ask the inventor if it can be expected that closely related compounds will also have the same usefulness. The agent may also ask if the effect requires any particular dosage or formulation. Some of these questions may the inventor be able to answer, at least he may hazard a qualified guess. But he may also have to go back to the laboratory in order to do some more tests. One thing is however clear: there is no definite answer to what the invention actually is and where its boundaries are. It will in the end always be a complex assessment built on probabilities.

Of course, the allowable breadth of a claim is mainly dependent on what is new and has an inventive step. But that is not the questions that is studied in this part. Here it is taken as granted that novelty and inventive step are present. The question is what the appropriate relationship then should be between the practical results the applicant has disclosed and the coverage that he is entitled to.

\subsubsection{The outline of the study}

It has already been mentioned that two provisions are pertinent to decisions on the scope of protection. This would probably not have been a problem but for the fact that only one of the provisions is an explicit ground for revocation of a granted patent. If a man skilled in the art is unable to practise the invention, the patent can be revoked (art. 100 and 138 EPC). But if the claims are not supported by the description this cannot happen. The latter requirement is precluded once the patent has been granted. "Support" is not a ground for opposition, nor is it available in national revocation procedure. It is stated in the Guidelines that:

160 T 409/91, OJ EPO 1994, 653: “Although the requirements of Article 83 and Article 84 are directed to different parts of the patent application, since Article 83 relates to the disclosure of the invention, whilst Article 84 deals with the definition of the invention by the claims, the underlying purpose of the requirement of support by the description, insofar as its substantive aspect is concerned, and of the requirement of sufficient disclosure is the same, namely to ensure that the patent monopoly should be justified by the actual technical contribution to the art." Cf Crespi, R. S., IIC 1997, 609. 
"[A]lthough an objection of lack of support is an objection under Art. 84, it can often, as in the above examples, also be considered as an objection of insufficient disclosure of the invention under Art. 83, the objection being that the disclosure is insufficient to enable the skilled person to carry out the 'invention' over the whole of the broad field claimed (although sufficient in respect of a narrow (invention'). Whether the objection is raised as lack of support or as insufficiency is unimportant in examination proceedings; but it is important in opposition proceedings since there only the latter ground is available". ${ }^{161}$

Only if the applicant has modified the wording of the claims in an appeal procedure is lack of support a ground for revocation. ${ }^{162}$ The non-availability of "support for the claims" has been quite severely criticised. ${ }^{163}$ Mistakes may be made when the Examining Division decides on the breadth of the claims and a possibility to correct these should be available.

Some relatively recent cases, however, indicate that the Boards of Appeal have identified and acted to overcome this deficiency in the EPC. ${ }^{164}$ In T 409/91 it was observed that claims that contravene Art. 84 EPC by being too broad, may also be in conflict with Art. 83 and therefore be possible to revoke. ${ }^{165}$ This decision was followed by T 923/92 in which the Board stated that the circumstances that are relevant for Art. 84 EPC may also be relevant for Art. 83 EPC. The grounds for Art. 84 would then still be available after the patent has been granted, although not the provision as such. ${ }^{166}$ This indicates that case law, by a purposive construction of the EPC, has corrected what appears to be an unfortunate mistake in the drafting of the EPC. The House of Lords has also ruled that overly broad patents can be nullified. ${ }^{167}$ Furthermore, the EPO Boards of Appeal have on some occasions revoked previously granted patents with unduly broad claims by applying the requirement of inventive step. ${ }^{168}$ It thus appears as if unduly broad claims are handled under several different provisions. Probably it would be advantageous if the EPC could be revised and lack of

161 Guidelines C III 6.4.

162 T 23/86, OJ EPO 1987, 316.

163 See Brandi-Dohrn, M., IIC 1994, 648 and Wibbelmann, J., [1997] EIPR 518.

164 See Wibbelmann, J., [1997] EIPR 520.

165 T 409/91, OJ EPO 1994, 653: "In the present case ... the reasons why the invention defined in the claims does not meet the requirement of Article 83 EPC are in effect the same as those that lead to their infringing Article 84 EPC as well, namely that the invention extends to technical subject-matter not made available to the person skilled in the art by the application as filed, since it was not contested by the appellant that no information was given to perform the claimed invention successfully without using the structurally defined class of additives. Therefore, the Board does not find that the description discloses the invention defined in the present claims in the manner prescribed by Article 83 EPC."

166 T 923/92, OJ EPO 1996, 564: "In the Board's view, in order to fulfil the requirement of Article 83 EPC, the application as filed must contain sufficient information to allow a person skilled in the art, using common general knowledge, to carry out the invention within the whole area that is claimed. Claims which by omission of an essential feature extend to subject-matter which, after reading of the description, would still not be at the disposal of the person skilled in the art, are objectionable both under Article 83 and 84 EPC. ... Furthermore, it is observed that the feature 'has human tissue plasminogen activator function' is quite vague and ambiguous. Although this feature was not amended during opposition proceedings, its significance and thus its clarity have to be reconsidered within the framework of the amendments to the claims in view of its influence on the decision on the issue of the sufficiency of disclosure".

167 House of Lords, Biogen v. Medeva of 3110 1996, [1997] R.P.C. 1 and IIC 1997, 740.

168 T 939/92, OJ EPO 1996, 309: "[I]t has for long been a generally accepted legal principle that the extent of the patent monopoly should correspond to and be justified by the technical contribution to the art ....[T]his general legal principle was applied in relation to the extent of the patent protection that was justified by reference to the requirements of Articles 83 and 84 EPC, the same legal principle also governs the decision that is required to be made under Article 56 EPC, for everything falling within a valid claim has to be inventive. If this is not the case, the claim must be amended so as to exclude obvious subject-matter in order to justify the monopoly." See further T 131/87 of 0709 1989; T 742/89 of 0211 1992; T 626/90 of 02121993 and T 741/91 of 2209 1992. For a comment on this practice see Wells, N., [1999] EIPR 220. 
support made an explicit ground for revocation. This would improve the comprehensibility of the EPC and legal certainty for applicants. ${ }^{169}$ However, it may be that, at least for the time being, it is more practical to make a purposive construction in case law, taking into account the practical difficulties associated with a revision of the EPC. ${ }^{170}$

The somewhat confusing legal picture on the question of breadth of claims has had consequences for this study. It has not been possible to discuss the breadth of the claims only with regard to one provision, which is what is done in other parts. The topic must be treated without characterising it as only a question of sufficient disclosure or support. ${ }^{171}$ There is no real dividing line between the two provisions. One and the same situation may fall under either provision. ${ }^{172}$ Therefore in this part there will be an analysis of the legal content of both Arts. 83 and 84 EPC. A distinction will instead be made between two different types of features that may be used in the claims: structural and functional features. By structural features are meant information on the physical appearance of the invention, in pharmaceutical patents usually in the form of structural formulas. A functional feature is different. It is a statement of a valuable result. A technical effect serves as a limiting feature, not the means by which the effect is attained. The patent will then cover all products or processes that achieve the effect mentioned in the claims.

The difference between a structural and a functional definition can easily be exemplified. A pharmaceutical is defined structurally if the claims contain a chemical formula. The same invention is defined functionally if the claims have a statement about the biological effect that the pharmaceutical has. Then the scope of protection will cover all means to achieve the effect. Normally a patent claim has a mixture of structural and functional features. Furthermore, many features are in themselves impossible to classify as only one or the other. A word may in its normal use have a mix of functional and structural connotations. Nevertheless, the distinction is made because the case law pertaining to claim breadth can be purposefully related to these two kinds of features.

\subsubsection{Structural features - Markush claims}

The usual structural definition of a pharmaceutical is a Markush claim, see part 3.2.3.1. The more groups that are named as alternatives, the broader the claim becomes. The pertinent question is what alternatives may be named considering the examples that the applicant has provided in the description. The answer depends on what changes that can be made in the structure of the molecule without losing its useful effects. In one survey of 1,282 chemical patents it was found that some of the most common generalising features were: alkyls (in 798 cases), halogens (416 cases), phenyls (336 cases), aryls (225 cases) and lower alkyls (214 cases). ${ }^{173}$

In the Guidelines the following statement is directed to the question of generalisation of structural features in claims:

\footnotetext{
169 See Crespi, R. S., IIC 1997, 622.

170 Cf Roberts, T., [1994] EIPR 373.

171 See Wibbelmann, J., [1997] EIPR 518.

172 It has sometimes been difficult for the Boards of Appeal to decide under which article the case should be decided. T 923/92, OJ EPO 1996, 564: "The Board examines here first the compliance of these requests with the requirements of Article 84 EPC (clarity and support by the description) and 83 EPC (sufficiency of disclosure) ... . The two requests are examined in conjunction as the question at issue is the same for both." In T 583/93 of 0401 1996, the Board tried the broadness of the functional claim under the heading “Article 83/84”.

173 See Dehlinger, P. J., JPTOS July 1992, 464, footnote 5.
} 
"In many cases a single example or single embodiment will suffice, but where the claims cover a broad field the description should not usually be regarded as satisfying the requirements of Art. 83 unless it gives a number of examples or describes alternative embodiments or variations extending over the area protected by the claims. However, regard must be had to the facts of the particular case. There are some instances where even a very broad field is sufficiently exemplified by a limited number of examples or even one example”. ${ }^{174}$

It is made clear that the allowable breadth of the claims depends on the possibility to predict whether the examples are representative for the area that is covered. But beyond that the Guidelines offers little help when it comes to the allowable generalisation of product claims on chemical compounds. A description of one way to practise the invention may be sufficient, but is not always sufficient. ${ }^{175}$ In case law too, claim breadth has been a contentious issue. It is often stated that the protection has to be crafted on a case by case basis. ${ }^{176}$ It is rather unsatisfying that one of the most important and basic questions cannot be answered in a more enlightening and precise manner. The only reliable conclusion seems to be that the allowable breadth of the claims depends on the predictability of the technical field. The possible generalisation is inversely related to the predictability in the technical field. ${ }^{177}$

In the mechanical and electrical field, one single embodiment of the invention is often sufficient to permit predictions of the technical results within broadly crafted claims. Therefore there are good opportunities for extending the claims to all that is novel and has an inventive step. Chemical reactions and particularly biological responses are much more difficult to predict. This is what makes the breadth of the claims such an important question in this field. In case law the predictability in the pharmaceutical field has been described in the following manner:

"It is the prevailing opinion that such properties [biological effects] are essentially determined by the substances structure, which according to current thinking is comprised of characteristic structural elements, the basic structure and the type and position of substituents. Since scientifically attested connections between the structure and properties of chemical substances are the exception rather than the rule, empirical data and analogy have to be relied upon, more often than not, in predicting the properties of such substances having regard to their structure. The legitimacy of conclusions based on analogy depends primarily on how much is known and on the particular specialist's field. As a rule, predictions by persons skilled in the art is no longer possible where the substances whose properties have to be assessed have been theoretically synthesised, by interchanging all the structural elements from compounds forming the state of the art and having the same kind of effect."178

\footnotetext{
174 Guidelines C II 4.9.

175 “The arguments which consider whether a Markush is scientifically reasonable or outside statutory limitations is an open-ended discussion which probably has no exactly correct or exactly incorrect answers.” See Brown, L. J., 31 Journal of Chemical Information and Computer Science 1991, 3.

176 T 133/85, OJ EPO 1988, 441:“[T] he requirement in Article 84 EPC that the claims shall be supported by the description is of importance in ensuring that the monopoly given by a patent granted generally corresponds to the invention which have been described in the application, and that the claims are not drafted so broadly that they dominate activities which are not dependent on the invention which has been described in the application. On the other hand, Article 84 EPC clearly envisages (by use of the word 'supported') that the 'matter for which protection is sought' can be defined in a generalised form, compared to the specific description of the invention. The permissible extent of generalisation from the description to the claims, having regard to the requirement of Article 84 EPC, is a question of degree and has to be determined, having particular regard to the nature of the invention which has been described, in each individual case.”

177 See Brandi-Dohrn, M., IIC 1994, 652.

178 T 20/83, OJ EPO 1983, 419.
} 
Knowledge about variations that can be done in a molecule without affecting the biological effect is of a very limited scope. There are some further statements by the Boards of Appeal on the predictability in the pharmaceutical field. It has been said that it is well known that even minute structural diversity may radically change the biological effect, but that it is also well known that biological effects are dependent on the chemical structure and that therefore there are possibilities, but quite limited, to make predictions about biological effects. ${ }^{179}$ It is difficult to make predictions with the skills that are available to an average practitioner or even theoretical tools more advanced than that. The research strategy in the pharmaceutical field is therefore often to try in assays as many compounds as possible and see whether an interesting effect is discovered.

The prevailing technical uncertainty appears to have blocked the development of reliable legal rules pertaining to the breadth of the claims. There has been no viable starting point for a legal assessment of what can be accepted in respect of non-functional parts inside the claims. This has at times been handled in case law by recourse to an alternative rule: the requirement of unity. ${ }^{180} \mathrm{~A}$ patent application may not contain more than one invention. Thus Art. 82 EPC has been used to limit the breadth of claims to structurally defined pharmaceuticals. As to "unity" and Markush claims it is stated in the Guidelines that:

"Where a single claim defines (chemical or non-chemical) alternatives, i.e. a so-called 'Markush grouping', unity of invention should be considered to be present when the alternatives are of a similar nature ... When the Markush grouping is for alternatives of chemical compounds, they should be regarded as being of a similar nature where:

(i) all alternatives have a common property or activity, and

(ii) a common structure is present, i.e. a significant structural element is shared by all of the alternatives, or all alternatives belong to a recognised class of chemical compounds in the art to which the invention pertains.

A 'significant structural element is shared by all of the alternatives' where the compounds share a common chemical structure which occupies a large portion of their structures, or in case the compounds have in common only a small portion of their structures, the commonly shared structure constitutes a structurally distinctive portion in view of existing prior art. The structural element may be a single component or a combination of individual components linked together. The alternatives belong to a 'recognised class of chemical compounds' if there is an expectation from the knowledge in the art that members of the class will behave in the same way in the context of the claimed invention, i.e. that each member could be substituted one for the other, with the expectation that the same intended result would be achieved.”181

There is unity if the compounds covered by the claim have the same useful effect and an important structural feature in common. ${ }^{182}$ In case W 52/92 the International Search Authority had found that the two medical indications mentioned in the application, viz. portal hypertension and migraine, were not related, but belonged to different fields of medical

179 T 939/92, OJ EPO 1996, 309: “[I]t can therefore be accepted as undisputed common general knowledge that even small structural modifications may cause major differences in biological activity. Nevertheless, it is also well accepted that the properties of chemical compounds do indeed largely depend on their chemical structure, and that a skilled person would therefore normally expect that the properties of two compounds would become the more similar the more similar their chemical structures became ... In view of all the above considerations, the board finds that reasonable predictions of relations between chemical structure and biological activity are in principle possible, but that there is a limit beyond which no such prediction can be validly made.”

180 Art. 82 EPC states that the application shall: "relate to one invention only or to a group of inventions so linked as to form a single inventive concept.”

181 Guidelines C III 7.4a.

182 T 169/96 of 30071996. 
science. Since there was neither relationship nor link between the medical indications there was no unity and an additional search fee had to be paid. However, after protest (Article 154(3) EPC), the Board of Appeal decided that the additional fee should be reimbursed. It was explained that the invention solved the problem of providing a further medical use for certain compounds, the treatment or prophylaxis of portal hypertension and migraine. The two medical indications were considered related in that both resulted from disorders due to excessive vasodilatation, i.e. were dependent on the same biological function. ${ }^{183}$ But application of such a legal rule presupposes that it is possible to judge if the compounds actually have the technical effect that is mentioned in the application; that was not an issue in W 59/92. If that knowledge is lacking it is difficult to apply the concept of unity, according to the explanation offered in case law and in the Guidelines. ${ }^{184}$ It will not be any easier to apply the legal requirement of unity in relation to the allowable breadth of the claims, than it would be to apply the provision regarding support in Art. 84 EPC. Thus "unity" seems to offer no opportunity to avoid the difficulties associated with assessing claim breadth under conditions of low predictability.

\subsubsection{Procedural rules}

The difficulties associated with finding a firm legal basis for assessments of the breadth of the claims have made the procedural rules gain in importance. When neither the applicant nor the examiner know or have any practical opportunity to test for the existence of the technical effect, the burden of proof becomes decisive. In Guidelines it is in this respect provided that:

"As a general rule, a claim should be regarded as supported by the description unless exceptionally there are well-founded reasons for believing that the skilled man would be unable, on the basis of the information given in the application as filed, to extend the particular teaching of the description to the whole of the field claimed by using routine methods of experimentation or analysis. Support must however be of a technical character; vague statements or assertions having no technical content provide no basis.

Since the examiner should raise an objection of lack of support only if he has well-founded reasons, it follows that the applicant should be given the benefit of the doubt. Where objection is raised, the reasons should preferably be supported specifically by a published document.”185

This passage is of considerable significance for the allowable breadth of Markush claims in European patent practice. It is the examiner who has to show why the claims are too broad. The examiner must support his assertion with technical circumstances. ${ }^{186}$ This has lead to a

\footnotetext{
183 W 52/92 of 0204 1993: "As stated in the description, this medical utility is ascribed to the fact that the compounds of structure (I) are agonists at 5-HT2 and/or 5-HT1-like receptors which are believed to be effective in a) portal hypertension through constriction of mesenteric arterioles, and partial constriction of paraesophageal collaterals with consequent reduction of portal flow and portal pressure and b) migraine through constriction of cerebral arteries (5-HT1- like agonists) and constriction of temporal artery (5-HT2 agonists). It is thus clear that contrary to the assertion of the ISA portal hypertension and migraine are two diseases which are closely related in that both result from disorders due to excessive vasodilation, which the present application proposes to treat with specific vessel constricting medicaments, i.e. agonists at 5-HT2 and/or 5- HT1-like receptors”.

184 See Brennan, J., 31 Journal of Chemical Information and Computer Science 1991, 11.

185 Guidelines C III 6.3.

186 T 484/92 of 3012 1993: "If an Examining Division takes the view that despite following all the instructions given in an example the desired results are not attainable, it has the burden of substantiating the point. As an Examining Division has neither the time nor the equipment for performing tests, at least it ought to back an
} 
liberal case law at least in ex parte proceedings, because it is usually very difficult to theoretically show that the effect does not exist for some of the compounds in a claim. ${ }^{187}$

Decision T 939/92 is interesting in this context, because it appears as if the burden of proof at times maybe different from what the wording of the Guidelines suggests. ${ }^{188}$ The application was on a fungicide that had been defined in the claims by a Markush formula with three substituent groups. The group R3 was defined as a substituted phenyl group, i.e. a phenyl group that could be substituted by anything. In the description there was a large number of examples, but R3 had not in any case been anything other than halogen atoms or methyl groups. The applicant argued that it was the examiner who had to show that the effect would be lost if the R3-group was substituted by anything else than halogen atoms or methyl groups. The Board of Appeal explained that this was an inaccurate description of the burden of proof. Someone who supports his case by a certain fact has to prove that fact. Furthermore, the Board observed that in this case it was highly unlikely that the herbicide effect was unaffected by how R3 was substituted. Therefore the application was refused. ${ }^{189}$

There are, however, only isolated instances where the applicant/patentee has had to prove why his generalisation is reasonable. Only in extreme cases have applications been rejected for unreasonable generalisations. In T 1055/97 claim 1 related to therapeutic compositions comprising polynucleotides having at least one nucleotide of the general formula P-B-S-Sig. The length of the polynucleotides was not specified, nor was there any indication regarding the number of altered P-B-S-Sig nucleotides they may contain. B, S and $\mathrm{P}$ were generically defined as belonging to three chemical groups (bases (B), sugars (S) and phosphate (P)). Claim 1 comprised compositions where the therapeutic agent was the claimed polynucleotide, but it needed not necessarily be so. The application disclosed that if the therapeutic agents were vaccines or immuno-competent agents, the polynucleotides enhanced their effects. A specific reference to the stimulation of interferon production and the use of the polypeptides as chemotherapeutic agents was made. The Board ruled:

"[T]hese claims virtually cover an unlimited number of therapeutic compositions. ... If one is to accept as argued by the Appellant that, at the filing date, it was a matter of common general knowledge to isolate the P-B-S chemical backbones, there remains nonetheless that claim 1 of both requests comprises a myriad of P-B-S-Sig moieties differing by the structure, nature and conformation of Sig .... Furthermore, the skilled person is left without guidance how to test the polynucleotides for therapeutical uses (even how to test those cited in the specification). When

objection of the kind which has been made here by some argument as to why it is of the opinion that an example is inherently unlikely to achieve the desired result."

187 T 610/90 of 1501 1992: "In ex-parte pre-grant procedures, it is the practice of the EPO and of the Boards of Appeal to adopt a liberal view concerning the application of Article 84 to the breadth of claims”.

188 Cf. Karet, I., [1996] EIPR 561.

189 T 939/92, OJ EPO 1996, 309: “In the tests which are reported ... a great number of compounds were used. However, in all these compounds ... R3 was always phenyl substituted by halogen atoms or methyl groups. Thus, despite the number of tested compounds, these test results do not support the alleged herbicidal activity of compounds in which e.g. the phenyl ring R3 may be substituted by absolutely everything, having regard to the common general knowledge relied upon by the appellant himself, namely that the influence of structural modifications on the desired herbicidal activity is unpredictable. ... According to the appellant's submission, in a case such as this one, where the credibility of the alleged herbicidal activity is at issue, the burden of proof that the presence of the alleged herbicidal activity is not credible rests on the EPO, here upon this board of appeal. This submission is clearly contrary to the legal principle that any one who alleges a fact has the onus of proving his allegation (in these proceedings to the standard of the balance of probabilities) by appropriate evidence .... Thus, if neither the examining division nor the board of appeal is in the position to discharge this burden to the above or to any other standard, and if it is evident that the number of compounds claimed is such that it is inherently unlikely that all of them, or at least substantially all of them, will possess the promised activity, then the burden of proof of that fact, i.e. the possession of that activity, can indeed rest only upon the shoulders of the person alleging it." 
asked by the Board for the likelyhood that polynucleotides containing, for example, P-B-Sfluorescein would have a therapeutic value, the Appellant's representative indicated that this was not known, but that some compositions had been found to stimulate the induction of interferon from interferon producing cells.... In the present case, wanting to reproduce the invention over the claimed range undoutedly amounts to devising a research program comprising both the setting up of methods for the isolation of the majority of the P-B-S-Sig polynucleotides and the investigation of the therapeutical indications, they might be useful for. Alternatively, for those therapeutic indications mentioned in the description it involves finding out under which conditions they might be useful. This exceeds by far in terms of efforts, time and skills what a skilled person can be expected to investigate in addition to the restricted disclosure of the patent application."190

Normally, however, only very limited requirements are at work in respect of proof of utility, at least in the ex parte patentability examination. The examiner will, on the basis of his skills, make an assessment of the probability for the effect being retained within the scope of protection. If this is improbable, he may ask for more examples. But mostly examiners are unable to present technical reasons why the effect would be lost.

\subsubsection{Non-functional parts of the claims}

It has already been found that there are very limited opportunities to thoroughly assess whether the technical effect is present within the whole scope of protection. An analysis based on the examples in the description and by use of the skilled person's common knowledge will not reach very far. It is the low predictability in the biological field that is the cause for this uncertainty. However, that is not the end of the matter. The low predictability will also mean that a claim to thousands or even many more compounds must in most cases be expected to have non-functional parts. In the Guidelines the following statement describes how nonfunctional parts should be treated in an opposition proceeding:

\footnotetext{
"The fact that only variants of the invention e.g. one of a number of embodiments of the invention are not capable of being performed should not immediately give rise to the conclusion that the subject-matter of the invention as a whole is incapable of being performed, i.e. is incapable of resolving the problem involved and therefore of achieving the desired technical result. Those parts of the description relating to the variants of the invention which are incapable of being performed and the relevant claims must however then be deleted at the request of the Opposition Division if the deficiency is not remedied. The patent specification must then be so worded that the remaining claims are supported by the description and do not relate to embodiments which have proved to be incapable of being performed. Any failure to rectify these deficiencies without good reason will result in the patent being revoked."191
}

The Boards of Appeal have also on some occasions decided that all parts of the claims must be functional. It has been stated that an opponent does not have to question the function of the examples mentioned in the description, he may just as well attack any other part of the scope of protection. This is because the whole area inside the claims should be functional. ${ }^{192}$

$190 \mathrm{~T} 1055 / 97$ of 17121998.

191 Guidelines C V 4.4 and 4.4.1.

192 T 741/91 of 2209 1993: "In the Board's opinion, the relevance of experiments, which aim at establishing that the promised result cannot be obtained over the whole range of a process claim, does not depend on the exactness of repeating an example of the patent in suit. On the contrary, it is quite sufficient that such experiments are carried out according to the process claim. The reason is that the claims define the subjectmatter for which a monopoly right (i.e. the patent) is granted under the EPC, and that, according to Article 52 
Therefore it could appear as if the legal situation was clear on this point: the industrial applicability or utility for the invention has to be present within the entire scope of protection. But the situation is not so clear. Other statements in case law indicate that it is impossible to uphold such an absolute requirement of functionality.

In $\mathrm{T}$ 14/83 the Board concluded that it was not decisive that some points of the claim were impossible to use for a man skilled in the art. But two things were necessary in such a case. Firstly, it should not be possible to formulate the claims in another way that would still create sufficient protection. Secondly, if the man skilled in the art by accident choose unsuitable variants he should, with the help of the description, still be able to take advantage of the industrial applicability. ${ }^{193}$ When case law was summarised by a Board of Appeal it was stated that "substantially" everything within the claims must work. It was acceptable with some points of non-functionality. ${ }^{194}$

This is a vague rule compared to a rule according to which everything inside the claims must function. But it is of considerable practical significance to make this distinction. It can be exemplified by T 311/93. The invention was a chemical manufacturing process. One feature in the claim was a "basic medium". The Board concluded that this feature was to be interpreted as everything with $\mathrm{pH}$ above seven. However, a catalyst that was used in the process would poison some such mediums. The Board did not find that this meant that the invention did not function. The reason was that the man skilled in the art was aware of the problem and could adjust the conditions with only routine experiments. ${ }^{195}$ This more liberal position on non-functional alternatives is broadly based on the approach that it can be expected of the man skilled in the art to make some adjustments inside the claims. ${ }^{196}$ That is also an approach that has been adopted by the German Federal Patent Court. ${ }^{197}$ It is, however,

EPC, it is justified to grant such rights only for new, inventive and industrially applicable subject-matter. Therefore, all embodiments falling within a process claim have to meet the requirements of patentability set out in Articles 52 to 57 EPC.”

193 T 14/83, OJ EPO 1984, 105: “[A] teaching cannot be defined in a claim precisely enough to rule out occasional lack of success, such a claim is not to be objected to provided it is possible to deduce from the description the action to be taken -- which also cannot be precisely defined -- by tuning of the fine variables.”

194 T 939/92, OJ EPO 1996, 309: "It follows directly from these considerations that a technical effect which justifies the selection of the claimed compounds must be one which can be fairly assumed to be produced by substantially all the selected compounds”.

$195 \mathrm{~T} 311 / 93$ of 16011997.

196 T 639/95 of 2101 1998: ”Article 83 EPC requires that the disclosure of a claimed invention must be 'sufficiently clear and complete for it to be carried out by a person skilled in the art' ... . According to the established case law of the boards of appeal, this means that a skilled person, having read the description, should be able at the filing date, also on the basis of common general knowledge, to perform the invention with undue burden and without needing inventive skill within the whole area claimed. Although a reasonable amount of trial and error is permissible when it comes to the sufficiency of disclosure in an unexplored or difficult field, there must then be available adequate instructions in the specification or on the basis of common general knowledge which would lead the skilled person necessarily and directly towards success through the evaluation of initial failures or through an acceptable statistical expectation rate in case of random experiments”.

197 The EPO-patent in suit was for a fungicide that was defined by a chemical formula. The compound could be in the form of two enantiomers - L or D - which was not defined in the claims. In subsequent experiments it had been established that only one of the enantiomers was an efficient fungicide. From this the Bundespatentgericht concluded that the claim could be interpreted in two different ways; it either covered both compounds or only the efficient compound. However, this was not decisive for the validity of the patent: "[I]n diesem Fall bedurfte es angesichts der erfaßten Zahl von zwei Enantiomeren zur herbeiführung der Erkenntnis, welches der beiden Enantiomeren zu dem angestrebten Erfolg führt und damit jedenfalls brauchbar ist, jedoch nur weniger orientierender Versuche, mit deren Hilfe sich der Fachmann ohne eigenes erfinderisches Bemühen alsbald ein zuverlässiges Bild über das geeignete Enantiomer verschaffen und das ungeeignete ausscheiden konnte; damit ist, nachdem das D-Enantiomer unstreitig die behauptete herbizide Wirkung aufweist, Ausfürbarkeit unter dem aspekt der Brauchbarkeit aber bereits zu bejahen ... Versteht man dagegen den verteidigten Patentanspruch 8 als 
in these situations imperative that the skilled man knows what result he should achieve. The Board of Appeal ruled the following in T 656/94:

\begin{abstract}
"According to the Appellant, an unacceptable effort was required for the skilled person to identify, among the huge number of claimed DNA sequences, those DNA sequences encoding polypeptides with hpG-CSF biological properties, having regard that Example 10 of the patent in suit showed that only one of the five polypeptides prepared according to Example 8 exhibited biological activity ... . In the Board's view, however, this objection is beside the point when discussing Article 83 EPC requirements, since the patent teaches how to prepare hpG-CSF analogs and how to check the biological activity thereof by using the $3 \mathrm{H}$ thymidine uptake and the CFU-GM and WEHI-3B D+ assays ... . If a given analog does not fulfil the biological activity requirement recited in the claims, it has to be discarded. In spite of the considerable amount of theoretically possible variation of the DNA and hence amino acid sequence in the present case, there is still likely to be a structural similarity among all the variants covered by the present claims. The situation here, where the claimed DNAs are limited to those having a certain structural relation to one another, and encoding proteins with a testable narrowly defined activity, must be distinguished from situations where either the structure or the activity or both is/are not defined in a disputed claim". 198
\end{abstract}

The view that the man skilled in the art can be expected to make some adjustments to fulfil features in the claim seems reasonable due to the practical problems that exist in an unpredictable technical area such as the pharmaceutical field. The claims will be valid and not too broad if the man skilled in the art can without undue burden travel down a road that permits him to practically take advantage of the technical effect mentioned in the description. This may unfortunately be a rather vague legal rule and patent applicants may try to take undue advantage of it. Therefore one probably should add, as has been done in some EPO cases, that substantially everything inside the claims has to be functional. It must be a strong presumption that the claims define the limits to what can be used to attain the technical effect. If that is not the case the claims are too broad and vulnerable to attack under Arts 83 and 84 EPC. A strict legal solution could cause considerable problems for the use of structural definitions of pharmaceuticals. A large part of all patents would perhaps be formally invalid and necessary to reformulate if they were involved in litigation. The legal situation should take this into account and concentrate on whether the claims are so formulated that they pose a real problem for a man skilled in the art that wants to practise the invention. If that is not the case and the claims have been formulated in good faith, i.e. so as to try to find, as far as possible, a limit for the usefulness of the invention, then the definition should be accepted, although non-functional parts can be found.

\title{
3.3.3.3 Remarks
}

The problem. The allowable breadth of the claims is decisive for the consequences of the patent system. ${ }^{199}$ The broader the claims, the more difficult it becomes for competitors to substitute the invention by other means. It is characteristic for the pharmaceutical industry that the competition to a large extent takes the form of such attempts to product

von vornherein auf das herbizid wirksame der beiden Enantiomere beschränkt, bedurfte es dieser Versuche um herauszufinden, um welches der beiden Enantiomere es sich handelte; diese Betrachtung führte ebenfalls nicht zur Verneinung der Brauchbarkeit.” See Bundespatentgericht 3 Ni 26/94 of 1409 1995, GRUR 1996, 822.

198 T 656/94 of 16071998.

199 An empirical study of the relationship between the breadth of the claims and the value of the patent has been made in the biotechnology field, see Lerner, J., 25:2 RAND Journal of Economics Summer 1994, 319. 
differentiation. Within the therapeutic segment (indication) research-based companies try to differentiate their products from competitors patented products and develop so-called me-too products. One or a few atoms are changed in the structure of the molecule. This process creates new therapeutic alternatives for patients. Normally there will also be some patients that find the new molecule advantageous, e.g. experiences less side effects. When the competition takes the form of attempts to product differentiation this will, however, lead to a simultaneous reduction in price competition. The latter will not be necessary, because the market is segmented. 200

At present there is an on-going debate on whether it is advisable to grant broad patents within the pharmaceutical field. By broad patents are usually meant those that "reach through" to possible second generation uses, i.e. valuable application that cannot be understood only by reading the first patent application. ${ }^{201}$ Above it has been concluded that the legal rules are quite vague on this point. There is room for discretionary decisions by the patent offices and courts. Much of the explanation for this is that the two most straightforward legal alternatives are both quite inadequate. It would be unreasonable if it was only permitted to patent the compounds that have been tested in practical experiments. The scope would become too narrow and the patents easy to escape. Some minor structural change would result in a compound that was free to market. It would also mean that applicants would try to compensate for this by making a large amount of tests before a patent application was filed. The tests would be of little technical value. The other obvious legal solution is equally unreasonable. It would not work to allow applicants to include all chemical compounds in the claims, provided the compounds satisfied the novelty and inventive step requirement. Taken to the extreme, all novel compounds could then be included in the claim and it could be asserted that it is only a routine experiment to test whether a certain compound has the biological effect stated in the description. That would soon render competition through product differentiation impossible. ${ }^{202}$

Clearly, the appropriate legal rule comes somewhere in between these two extremes. But the problem one then confronts is that it is very difficult to find any intermediate situations. How is one to define, in concrete and structural terms, a good balance between functional and non-functional definitions of the invention? It seems to have been difficult for case law to reach much further than to a rather vague statement that "substantially" everything within the coverage must be functional. The most important elucidation of the difficulties in finding an intermediate position is that the rule must be based on two factors with unknown interdependence: chemical structure and therapeutic result. The aim is to make the structural claim correspond to the extent of the therapeutic utility. Although it is in theory clear that the two correspond - a change in structure affect the therapeutic effects - it is at the time of the patent application not clear how this happens in all details. The economic value of the invention is the therapeutic effect, but the value of the patent is to be "calibrated" by structural features. This will always be a contentious issue. It has been described as one of the unresolved problems in patent law. ${ }^{203}$ In the following one solution will be discussed.

\footnotetext{
200 "The impact of the patent system has not been to create monopoly positions ..., but rather it has been to foreclose to a great extent rivalry between identical chemical entities or standardized commodities about which price competition might develop. It has strengthened and encouraged the high degree of chemical product differentiation which is the primary form taken by technical change within the pharmaceutical industry." See Comanor, W. S., 31 Economica 1964, 379. See also Cockburn, I., Henderson, R., 3:3 Journal of Economics and Management Strategy 1994, 494.

201 See Crespi, R. S., IIC 1997, 607.

202 See Roberts, T., [1994] EIPR 372.

203 See Szabo, G., IIC 1995, 484.
} 
Use limitations. All attempts in the patent system to make predictions about the existence of biological effects will by necessity lead to the same difficulties as all research in the pharmaceutical industry faces: a very high degree of unpredictability. Researchers deal with this by doing practical experiments in vitro and in vivo. For obvious reasons this road is not open to patent offices. However, there is no reason why patent offices and patent law should try to repeat the technical achievements of research, because the therapeutic utility has to be known and disclosed when the patent application is filed.

In the following it will therefore be examined what the consequences would be if the pharmaceutical utility that motivates the grant of a patent is used when patent claims are formulated, i.e. if there should be some kind of use limitation in otherwise structural patent claims on pharmaceuticals. It is today built deep into the practice in all major countries to allow absolute product patents for chemicals. ${ }^{204}$ The scope of protection does not have to be pruned back to any particular use of the compounds. The protection will cover all uses of the compounds in the claims, including uses that the compounds may have outside the pharmaceutical field. Although the legal situation is clear, there are reasons for studying the question of use limitations. One such reason is that even with absolute product patents being granted, the aim is to have a use limitation in one aspect, namely the one studied above: the outer limits of the claims shall be determined by the existence of the therapeutic effect. The scope of protection should not extend to compounds that do not have the therapeutic effect mentioned in the description. But at the same time, within the structural boundaries of the claims there is not attempt to limit the scope to the use that the applicant has found.

Thus, the industrial applicability is considered material when the outer limits of the structural definition are crafted, but not for the scope of protection within the structural definition. This appears to be a less-than-optimal legal solution. Either it is suitable to permit protection of uses that are unknown at the time of the patent application, or it is better to try to limit the scope of protection to the uses that the applicant has found. The tension between these two alternatives will be discussed in the following. This means that one has to ask a question of fundamental importance for patent protection: when is it advantageous to issue private property rights to unknown but possible future technical production opportunities? The answer to this question has to guide decisions as to the allowable scope of protection. But the same question is also decisive in a number of other patent law issues, e.g. novelty and inventive step. It is probably fair to say that questions related to the value of private property rights are the most basic within the patent system. All patentability requirements have one thing in common, they are "boundaries" between public and private property. They define when a public ownership (or no ownership) can become private ownership. Therefore the conclusions that are drawn in this part will affect several later parts of the study.

Outline of the analysis and patents as prospects. The term "prospecting" will be used here to describe one part of the scope of protection in a patent granted. It refers to the valuable commercial uses that are protected by a patent, but which a man skilled in the art is unable to use with the information that is available in the patent. It is a "bonus" that a patent applicant sometimes receives, in addition to the scope of protection that is granted to him in respect to production opportunities that he has disclosed in the application. In the case of absolute product patents on chemical compounds, the patentee will receive a scope of protection that

\footnotetext{
204 The decision to require that developing countries grant product patents for pharmaceutical innovations as a condition of membership in the World Trade Organization was very contentious. See generally in this regard Lanjouw, J. O., The Introduction of Pharmaceutical Product Patents in India: "Heartless Exploitation of the Poor and Suffering”?, National Bureau of Economic Research Working Paper No. W6366, issued in January 1998.
} 
includes the new uses of the compounds that are discovered during the lifetime of the patent. This "prospect" part of the patent entitles the patentee to newly discovered production opportunities, independent of who actually makes the discoveries. If someone else finds the new production opportunity he may apply for a use patent. But this will become a dependent patent in relation to the product patent, for the sole reason that the product patent has a "prospect" part. It should also be mentioned that when a use patent is granted the product patentee may not take advantage of the new use without permission from the patentholder with use claims. Thus they may both exclude each other from the new use. ${ }^{205}$ After a licence negotiation, the value of the new use will be divided between the product patentee and the proprietor of the new use patent. The value of the prospect part in a patent is in general dependent on the remaining lifetime of the patent and the likelihood that there will be discovered new production opportunities within the scope of protection.

A discussion of the effects that occur in the pharmaceutical industry due to the prospecting function of the patent system can be found in a decision by the German Federal Supreme Court, the Bundesgerichtshof. 206 There was a product patent for human recombinant immuninterferon. A competitor to the patentee carried out clinical tests in order to search for possible new indications for the interferon, e.g. treatment of cancer, AIDS, allergies, leukaemia, asthma, etc. The court described the effects that this research would have on the patentee in the following manner:

"Die Möglichkeit, daß klinische Versuche zur Erkenntnis neuer Indikationen des geschützten Stoffs und zu einem entsprechendem Verwendungspatent führen können, gefärdet nicht den wirtschaftlichen Wert des Erzeugnispatents. Das Stoffpatent behält vielmehr infolge der Abhängigkeit des Verwendungspatents seinen wirtschaftlichen Wert, weil der Inhaber des jüngeren Patents zu dessen Benutzung die Zustimmung des Inhabers des älteren Patents benötigt und das ältere patent gegenüber Dritten auch bezüglich der durch das jüngere Patente geschützten Verwendung seine volle Feltung behält. ... Die Erschließung neuer Verwendungsmöglichkeiten durch weiterfürenden Versuche Dritter führt grundsätzlich zunächst sogar zur Steigerung des Wertes des Sachpatents und des davon bestehenden ausschließlichen Nutzungsrechts des älteren Erfinders.”

The other type of new production opportunities in the prospect part of the product patent is the possibility that somewhere within the claim is hidden a better effect of a kind that has already been known to exist to a lesser degree. This is the situation of so-called selection inventions. If a choice has been made within a generic field and a particular high efficiency is found then the selection may be patented. This is analysed in detail in part 4.7. A selection invention will, in the same way as a new use patent, become a dependent patent in relation to a patent on the more general field from which the selection was made.

The two types of dependency relationships have the consequence that a product patent with claims to several chemical compounds includes a value comprising possible subsequently discovered new uses and better effects. They are "prospected" to some extent. For reasons of clarity, only prospecting of new uses will be analysed in this part. The necessary considerations are different in the situation of selection inventions. Therefore they will be analysed in a separate part, 4.8.2.

205 Cour de Cassation, Alfuzosin of 2610 1993, OJ EPO 1995, 252, in English translation: "Whereas the proprietor of a product patent can stop exploitation of a new use which is the subject-matter of another patent, he himself is unable to exploit it without the consent of the other patent's proprietor; admittedly a new use of a known patented product is a dependent invention, but the right to this invention belongs to the proprietor of the second patent which is dependent on the first one."

206 Bundesgerichthofs, X ZR 99/92 of 1107 1995, GRUR 1996, 109; [1997] R.P.C., 623. 
It is not new to describe one effect of the patent system as a kind of prospecting. In the American law \& economics literature on patents, the analogy was introduced by Kitch. ${ }^{207}$ He used the term because the patent system has some features in common with real prospecting. Before someone decides to fully explore the value of minerals on a plot of land, he will normally have made some preliminary investigations and found indications of valuable minerals. But the true extent and value of the finding cannot be determined without expensive explorations. In order for there to be incentives for such an expensive undertaking, the prospector becomes the proprietor of whatever he may find. The patent system affects pharmaceutical research in a similar manner. Investments in research and development often only become feasible after the inventor knows that he has an exclusive right to the uses that he may later find and develop as a consequence of the initial discovery.

The new aspect in Kitch's analysis was that granted patents were not only viewed as an economic drawback to society, that had to be accepted in order to create incentives for the next generation of inventors. Kitch pointed to the material functions that patents have after they have been granted. They create incentives for the patentee to explore the known and yet unknown opportunities that may exist within the scope of protection. Possibly the most valuable aspect of the patent system is that it encourages the development of inventions into marketable products. The most painstaking and expensive work often comes after the patent application has been field. In the pharmaceutical field this includes clinical trials, purchase of manufacturing equipment, investments in marketing etc. This work can be facilitated through creation of property rights. It must, however, also be borne in mind that it is during the exploitation phase that patents cause monopoly losses and negative incentives for others who could have searched for new and unknown production opportunities within the granted claims. ${ }^{208}$

The need for incentives. There can only be a need to analyse the consequences of patent prospecting in the pharmaceutical industry if unknown uses within the scope of protection of granted patents, are not discovered "automatically". Only when investments are necessary in order to discover possible new therapeutic uses for known substances does it matter how the patent system does this. Some practical examples may show that there is indeed such a need. It is well known that potential uses of known pharmaceuticals can be hidden for many years. The compound hydroxyurea (Hydrea) was first synthesised and used as a pharmaceutical in 1869. In the 1960s scientists took a new interest in hydroxyurea, but they had no success because they were searching for a use in cancer therapy, e.g. in treatment of leukaemia, and no such use was to be found. In recent years, however, new uses have been found for hydroxyurea: heart attacks can be reduced for patients suffering from sickle-cells anaemia. It has also been reported that hydroxyurea has at least in vitro-efficiency against AIDS.

It is not unusual that new uses are discovered by accident. The use of nitrogen gas as an anaesthetic was discovered when its euphoric effects were displayed publicly and one volunteer did not react to a wound he received on his leg. It is particularly common for new uses to be discovered during a clinical trial phase. The compound may turn out to be

\footnotetext{
207 See Kitch, E. W., 20 Journal of Law \& Economics 1977, 265.

208 "Today, patents are avidly pursued all along the lengthy road from the most basic science through to the market-place for pharmaceuticals. Because every step along the way draws another patent application, the path towards public possession of real benefits is increasingly observed by dense thickets of intersecting, overlapping, and cross-blocking patents. Those operating at the beginnings of the road are most insistent on their right to nail down leverage that will remain formidable despite marketplace rejection of the uses to which they saw their inventions may be put. The frank aim of these early stage workers is to control ultimate applications discovered by others. The system is abused if those who would benefit in this way from the later labour can posit patents on the most strain utilities imaginable”. See Kiley, T. D., 257 Science 1992, 915.
} 
useful for another purpose than that for which it was originally tested. One example is a compound tested for treatment of allergic reactions, but it did not have any such useful properties. One volunteer, though, reported that her illness when travelling by car had ceased, and the compound has been used for this indication ever since. ${ }^{209}$ However, the large majority of new uses are discovered during research in laboratories, where systematic studies are made of pharmacological and physiological effects. The list of new uses that have been found in such trials is very long. The aim, therefore, is not to narrow the research field too much in the early phases of a research project.

One economic study of pharmaceutical research attempted to establish a relationship between the investments in research within a particular field and the number of patents that were granted to the companies in that field. But when the patents were matched to the different research projects it was discovered that some patents were granted in technical fields where the companies reported no research projects. From this the conclusion was drawn that there was a significant factor of research "spill-over" within the pharmaceutical industry, between disparate areas in which companies carry out research efforts and also to some extent a spill-over into fields in which the companies had no established research programs. The latter kind of "spill-over" was, however, rather unusual.210

Even if new medical uses are to some extent discovered by pure chance and without particular research efforts, it must be borne in mind that investments are still necessary in order for the new uses to reach the market. It may be cheaper on average to introduce a new indication for an already existing compound; the safety aspect is probably to some extent already explored. But it does not come free of charge. The conclusion to be drawn from this is that new medical uses for known compounds are not discovered "automatically", but require investments both prior to the patent application and after the patent has been granted. Proper patent incentives for this will therefore generate more available treatments. Thus there are good reasons for trying to analyse how this can best be done within the patent system.

The design of incentives. An analysis of the present legal situation, with prospecting of new therapeutic uses, can be done by looking at how prospects are taken advantage of by the proprietors. In the analysis, which will be attempted here, it is considered efficient if possible production opportunities, in the form of prospects, are made available to whoever can take best advantage of them, i.e. can exploit them most efficiently in research or product development. Such an allocation may in theory be achieved by negotiations, e.g. licence agreements. But in practice this is usually impossible, due to the presence of transaction costs. Therefore, one very material aspect of the patent granting process is that rights to new production opportunities are allocated to efficient users; it cannot be taken for granted that the most efficient user of a prospect is able to reach an agreement with a less efficient user.

It is difficult to assess how high the costs for an agreement concerning the prospect parts of a patent would be, but it is clear that they would be considerable. Patent licences are in general expensive to negotiate. An agreement by which the prospected area in a product patent is licensed would be particularly expensive. Trade in prospect rights is of course legally quite possible, but it is rarely economically feasible. One reason for this is that the value of the prospect is expensive to establish. No one knows what the value is - if one did, it would not be a prospect - and uncertainty increases transaction costs. Potential buyers would have to make extensive technical investigations before they could make a good

209 See Sneader, W., 61, in Hansch, C., Taylor, J. B., (ed.), Comprehensive medicinal chemistry: The rational design, mechanistic study and therapeutic application of chemical compounds.

210 See Cockburn, I., Henderson, R., 3:3 Journal of Economics and Management Strategy 1994, 514. 
estimate of the value. Furthermore, with knowledge about this, the product patentee will be watchful when someone wishes to license or buy the prospect right. If the product patentee is approached this conveys to him that there is some value in his patent that he has previously neglected. This will perhaps make the product patentee decline the offer and try to develop the prospect himself. It is a very difficult negotiating situation and therefore it entails a risk that the production opportunities will not be taken advantage of at all. In situations where negotiations are difficult it is particularly important that the legal system allocates property rights only to the most efficient user.

Where product patents for pharmaceuticals are concerned, it has to be investigated whether it is most efficient to permit one actor - the product patentee - to prospect unknown production opportunities or if it is preferable to have a situation of free competition pertaining to the still unknown production opportunities. If the latter is deemed preferable, then some form of use limitation should be required in pharmaceutical patents. If this is done the uses that are later discovered will not become dependent on the senior product patent. Junior inventors will not have to negotiate with the product patentee. To try to answer this question, a comparison will be made between the incentive structure with or without use limitations.

The analysis will start by looking at a situation in which only patents with use limitations are granted. In this situation both the party which has applied for a patent for the first discovered use and any junior inventor of a new use may be granted independent patents. The second patent will not become dependent on a pre-existing product patent (as there will not be any product patent, but only patents with use limitations). In this situation there is no need for any transactions before production can start. Whoever has found the first use of the compound can market the substance for that use and whoever has found a later use may market the compound for that use. It is notable that the two possible actors may be one and the same. The most common situation is that the same company finds and develops the first and also any subsequent medical uses for a particular substance. Since it is possible for whoever has found the first use to secure a new patent for any subsequent use, it appears not to cause the first inventor any significant loss to have to include a use limitation in his first patent. The difference is the effects on competitors. They will have increased incentives to search for new medical uses for known pharmaceuticals. When the patent on the first discovered indication is use bound to the discovered indication there will be no prospecting of possible later uses.

This situation with use limitations will now be compared to the present situation where absolute product patents are granted. It can be done by a simple example. ${ }^{211}$ A product patent is granted on a compound that has a therapeutic utility with a market value of 100 . If there is no further use for the compound, and all actors are aware of this, the patent will have a market value of 100 . This is because the patent has no prospect part. That is the normal situation within the patent system; a large majority of inventions has no "hidden" uses. The situation will be different if there is a chance of further uses for the compounds and the new uses belong to the scope of protection of the patent granted. It is here assumed that the subsequently discovered medical use also has a value of 100 , i.e. the same value as the first indication (a second medical use is normally as valuable as the first). Further, it is assumed that a patent may be granted for the second use. This patent will, according to the present legal situation, become dependent on the product patent. If it is a competitor to the product patentee that has patented the new use, he must enter into negotiations with the product patentee. A likely outcome of such a negotiation is that the product patentee receives 50 of the value of the new production opportunity that has been patented by the use patent. This will

211 The example comes from Mergers, R. P., Nelson, R. R., 90 Columbia Law Review 1990, 865, footnote 117. 
result in the division of 150 to the product patentee and 50 to the proprietor of the use patent. $^{212}$ In this situation the value of the prospect in the product patent has been 50 . This will of course reduce the incentives to search for new uses for everyone but the product patentee.

One further example will serve to illustrate the effects of the prospect function in the patent system. Let us assume that a product patent covers a compound that may be used to make diagnosis. This use has a market value of 100 . The second medical use is a therapeutic use with a market value of 900 . After negotiations have been concluded the division of profits will be such that the product patentee receives 550 and the use patentee 450. This happens even though the inventor of the second use has made an invention that is of considerably more value and one which was not obvious in relation to the product patent. Had the new use been obvious, the product patentee would have gained the total value of the new use (1000 in total), since no new patent would have been granted and therefore no possibilities for negotiations would have existed.

These two examples show that the prospect function in pharmaceutical product patents creates a situation in which competitors lose up to half of their incentives to search for new uses. How large the prospect value will be in each case turns on a large number of factors, foremost among them how long the remaining lifetime of the product patent is when the new use is discovered, but also the negotiation skills of the parties and other factors. However, the uncertainties about the details of the situation should not obscure the main conclusion: the prospect function of the patent system reduces incentives to search for new uses for all but the product patentee. If, on the other hand, the prospect function is avoided by introduction of compulsory use limitations, the product patentee would still be able to protect additional uses that he discovers later. Thus, a use limitation would not mean a significant loss of incentives for him.

This analysis seems to indicate why the patent system in general is designed to make the scope of the claims correspond to the applicant's contribution to the state of the art, i.e. why prospecting of unknown production opportunities generally is avoided. Prospecting means that the patentee's competitors suffer a loss without an apparent corresponding gain to the patentee. As a preliminary conclusion, therefore, the granting of absolute product patents in the pharmaceutical field can be unfortunate from an economic viewpoint. But before making this our final conclusion, we have to ask whether there may be some other reasons why prospecting of unknown production opportunities should be permitted.

Interdependence of inventions as a reason for prospecting. In Guidelines it is stated that inventions that open up a whole new technical field should be granted wider protection than inventions pertaining to advances in established technical fields, on the principle that whoever creates opportunities for further inventive input should gain some of the value of the later developments. ${ }^{213}$ This is a common thought in many analyses of the patent system. ${ }^{214} \mathrm{~A}$ Board of Appeal (T 292/85) has also voiced it:

\footnotetext{
212 The division may of course be different. Decisive is the negotiating skills of the parties. But the most common outcome of a negotiation is that the parties meet "half-way".

213 Guidelines C III 6.2: "Most claims are generalisations from one or more particular examples. The extent of generalisation permissible is a matter which the examiner must judge in each particular case in the light of the relevant prior art. Thus an invention which opens up a whole new field is entitled to more generality in the claims than one which is concerned with advances in a known technology. A fair statement of claim is one which is not so broad that it goes beyond the invention nor yet so narrow as to deprive the applicant of a just reward for the disclosure of his invention."
} 


\begin{abstract}
"Unless broad, yet proper terminology is allowable, subsequent investigations by third parties might be encouraged to concentrate on finding alternatives outside the claims instead of trying to pursue progress through dependent inventions. The lack of recognition of the full significance and the interdependency of technical contributions could adversely affect progress in the area of microbiology and biochemistry."215
\end{abstract}

The Board motivated broad claims by the stimulating effect that one invention may have on further developments, refinements etc. This may intuitively appear reasonable. If it is clear that one inventor has benefited from an earlier invention some reward to the first inventor appears warranted. But its truthfulness becomes doubtful if one avoids this retrospective approach and instead looks to the future and the incentives available. The decisive question is whether there is any reason to believe that new medical uses within the scope of a product patent will be discovered earlier if the incentives are limited for all but the product patentee. That is the practical effect of prospecting, nothing else. It does not create any extra incentives for the product patentee. He may still patent a subsequent use when his first patent has a use limitation.

With this perspective, the authorship of a previous invention seems to become irrelevant. The material factor is how the incentives for achieving new inventions can be maintained at a high level. The product patentee has had access to the information that may have facilitated subsequent inventions by others. If someone has used that information in a manner that the first inventor did not, then this must mean that the junior inventor used the available research opportunities better and that competition proved advantageous. The - no doubt, very real - interdependence of inventions does not override the conclusion that someone who has made a further inventive input has proved the value of competition in the search for new uses. Therefore, avoiding or limiting the prospect function does not lead to unjustified advantages for competitors to the product patentee, but rather maintains an equal footing and free competition between possible later inventors of new medical uses.

The Board stated in $\mathrm{T}$ 292/85 that it would be a problem if inventors were encouraged to find alternatives outside the claims rather than trying to pursue progress through dependent inventions. But this is hardly a correct description of the problems that occur when broad claims are allowed. The problem is not to prevent research being done outside the scope of patents granted. There are unlimited opportunities for research outside the scope of patents granted and such research will always be carried out. The true problem with broad claims and prospecting is that there may be too little research done within the scope of granted patents. This may be a problem if there are valuable unknown production opportunities within the scope of granted patents. Whether new production opportunities are found inside or outside the scope of granted patents is irrelevant from an economic point of view. It is their discovery that matters. This being so, the real problem clearly concerns the unknown production opportunities existing within the scope of patents already granted, not the possibilities of research being done outside the scope of granted patents. When considering the scope of protection, therefore, care must foremost be taken to ensure that there will not be a lack of incentive to search for yet unknown production opportunities within the scope of already granted patents.

Thus, interdependence of inventions seems not to be a convincing justification for allowing prospecting. The question of creating efficient incentives should be related to the

\footnotetext{
214 Green and Scotchmer have argued that it is an economic advantage for a later invention to become dependent on an earlier invention, if the later invention could not have been done without knowledge of the earlier one. See Green, J. G., Scotchmer, S., 26:1 RAND Journal of Economics Spring 1995, 25.

215 T 292/85, OJ EPO 1989, 275.
} 
future exploitation of production opportunities. What matters then is not who has created certain technical knowledge (an invention), but how the search for new information can be made efficient. In other words, how to avoid an unnecessary reduction of the incentives for further research when a patent is granted. It may be that one invention has been essential for a later one. But the fact remains that in such a case the junior inventor has made better use of the impetus for further research than the senior inventor has. Therefore it appears that prospecting of new medical uses cannot really be justified by the interdependence of technological development. ${ }^{216}$

Possible merits of limitations to the incentives. So far in this analysis it has been found that prospecting of unknown production opportunities within the scope of the patent leads to the reduction of incentives for all but the product patentee. This is not balanced by any apparent benefit to the product patentee, because he can also apply for a use patent if he finds subsequent new production opportunities. This indicates inefficiency and cannot, as it seems, be justified by the technical interdependence of inventions. However, the problem is even more complicated than that. There may be aspects of the research situation that actually make it advantageous to limit the incentives available to the product patentee's competitors. There is the risk of an inefficient racing situation. ${ }^{217}$ Several actors may work in parallel on the same technical problem, which may cause inefficient repetition and over investment. This is a problem that is liable to occur when efforts are rewarded on a first-past-the-post basis, as is the case in the patent system.

In a situation where a number of pharmaceutical companies are researching, say, a treatment for Alzheimer's disease, each company will have made some investments, e.g. they will have built research facilities and perhaps obtained some positive preliminary results. In their ongoing decisions on the further funding of their projects, these companies will presumably be willing to invest until their marginal return is zero, i.e. the return equals the interest they have to pay. Such decisions are optimal for the corporate return. But the problem is that such investment decisions also affect the position of their competitors, if only one company can gain a patent. When one company is making an investment in a situation where several competitors are confronting the same problem, each investment will be a cost item for others. The competitors will be worse off after the investment, in a situation where the winner takes all. However, the company that makes the investment naturally does not consider these costs. Consequently there may be reason to invest more from the perspective of each company than from the viewpoint of society. The external effects of investment decisions are only included in the social calculus. This particularity of the research situation may lead to excessively fierce competition, i.e. more investments than can be justified by the available research opportunities.

If "inefficient racing" is in some instances accepted as an adequate description of the pharmaceutical field, then there might be reason to believe that incentives should at times be limited and the prospect function of the patent system be valued for having this effect. When a product patent is granted, it will reduce the incentives for competitors within the prospected areas. They may stop doing research, thereby ending the race. The product patentee can then co-ordinate the exploitation of the known and as yet unknown production opportunities within the scope of protection. The patentee will invest in due proportion to the value of the possible production opportunities. His competitors will do research outside the scope of protection.

${ }^{216}$ Cf. Scotchmer, S., 5:1 Journal of Economic Perspective 1991 s. 29.

217 The literature on the inefficiency of "racing" is reviewed in Cockburn, I., Henderson, R., 3:3 Journal of Economics And Management Strategy 1994, 485. 
Kitch's conclusion was that prospecting within the patent system was an economically advantageous effect of the patent system. The creation of property rights at an early stage in a technical field, according to Kitch, creates the most efficient exploitation of the rewards technical research may have. The explanation is that inefficient duplicative work is avoided. ${ }^{218}$ There is no doubt that there are considerable risks of inefficient duplicative work in pharmaceutical research, as witness the following: 49 biotechnology companies tried simultaneously to develop the protein t-PA (which serves to dissolve blood clots); 94 companies tried simultaneously to develop interferon (an anti-cancer drug); 60 companies tried to develop interleukin-2; and 38 companies were active in the field of human growth hormones. ${ }^{219}$ Due to the patent system they may have been engaged in an inefficient winnertakes-all race, with excessive commitment of resources. It might have been more efficient if the products had been prospected and the research thereby co-ordinated by the prospect owner.

Despite the apparent risks of inefficient duplicative work, there are also signs of co-operation within the pharmaceutical field between research departments in different pharmaceutical companies. ${ }^{220}$ This is corroborated by an empirical investigation, in which no chronological connection was found in the timing of investments by pharmaceutical companies within a given therapeutic field. This meant that there was no indication of the pharmaceutical companies acting as if they were involved in a race. Unlike the investment decisions, there was interdependence between the research successes of the companies in a certain therapeutic field. Research success was measured by the filing of patent applications. When one company filed a patent application, this increased the likelihood of another company filing an application in the same field. This interdependence was interpreted as an indication of research "spill-over", i.e. a kind of informal research co-ordination. Companies were able to take advantage of each other's advances. Considering the findings of this study, one can hardly describe research in the pharmaceutical sector as broadly leading to identical results and thus being inefficient as postulated in a racing situation. The research has considerable elements of division of labour. The winner does not take all. The results obtained by his competitors will also be of value. ${ }^{221}$

The importance of trying to avoid racing depends greatly on the efficiency by which the prospector, i.e. the product patentee that by his legal position reduces racing, searches for and tries to exploit the unknown production opportunities. Research done by competitors will only be wasteful if it duplicates what is already done by the product patentee. This has been pointed out in the legal debate on the prospect function of the patent system. Merges and Nelson have criticised Kitch's view of prospecting in general as a positive feature of the patent system.222 They have argued that competition is preferable, at least in certain situations, because sometimes the prospector does not take advantage of what he has prospected:

“Once a firm develops and becomes competent in one part of a 'prospect,' it may be very hard for it to give much attention to other parts, even though in the eyes of others, there may be great promise there."223

\footnotetext{
218 See Kitch, E. W., 20 Journal of Law \& Economics 1977, 279.

219 See Werner, R., 27 Advances in Drug Research 1996, 1091.

220 See Penan, H., 25 Research Policy 1996, 354.

221 See Cockburn, I., Henderson, R., 3:3 Journal of Economics And Management Strategy 1994, 508.

222 See Mergers, R. P., Nelson, R. R., 90 Columbia Law Review 1990, 839.

223 See op. cit., 873.
} 
If a technical field has been prospected the result for the prospector is all carrot and no whip (competition). The latter limits the patentee's efforts to exploit potential uses within the prospect before someone else does. New uses may not be taken advantage of as early as would have been the case in a competitive situation. This is particularly likely if the prospector is already selling another product in the market to which the prospected product would belong. If the patentee has already developed a product, a new, similar product would compete with his existing one, and this reduces his incentives for developing a new product.

In support of the value of competition in research, Merges and Nelson describe a number of empirical examples of how companies that have one product have not developed a patented invention that would compete with their existing product. The companies saw no reason to invest in and exploit new technical opportunities once other companies were debarred from using the same technical opportunities. The conclusion reached by Merges and Nelson was that the patent system did not function well if it permitted "hoarding" or speculation in future technical opportunities. The reduction of inefficient duplicative research did not offset the losses caused by the prospector's inefficient exploitation of research opportunities. ${ }^{224}$

The point made by Merges and Nelson may be pertinent to product patents for chemical compounds. The patentee will not have any particular interest in the development of a prospect if the only outcome would be a new pharmaceutical that would compete with his existing products. This, however, is not an accurate description of the situation when a pharmaceutical company has to decide whether to search for new medical uses that may exist for a compound that they have already patented. The possible new uses will not usually belong to the same market as the known use of the pharmaceutical. Therefore the later uses will not reduce the profits that the company derives from the first known use. In respect to prospected new medical uses, a product patentee can therefore be expected to efficiently exploit these. This indicates that it is not so important to maintain a competitive situation. There is not such a great need for a "whip", because the "carrot" will usually suffice. The perceived efficiency of the product patentee in the search for new medical uses will increase the risks that duplicative efforts by others become inefficient.

Selection inventions are a different matter. They will belong to the same market as the use that is already mentioned in the product patent. The product patentee will therefore not have the same strong incentives to search for possible selection inventions that have already been prospected by him. Any new product would compete with his existing products. The consequences of this will be discussed in part 4.8.2.

There is one more material reason why research for new medical indications would be inefficient if done by competitors to the product patentee, i.e. why prospecting of new medical uses may be a positive feature as it reduces the amount of inefficient duplicative work. Competitors probably have technical disadvantages in this kind of research compared to the product patentee. Most new uses are discovered when the product patentee tries to exploit the use that he has shown in his product patent. Therefore new uses are to some extent discovered as a side effect of the research done in relation to the first discovered use. This means that the product patentee will be able to search for new medical uses in a particularly cost effective manner.

A survey has been done of the "internal spill-over of knowledge" in researchbased pharmaceutical companies. The effect was due to the fact that these companies at any one time had on average between 10 and 15 research projects, each with a budget in excess of

224 See op. cit., 875 . 
500,000 US dollars per year. ${ }^{225}$ The number of projects generated scale advantages that probably lead to increased research productivity. It was found that the scale advantages increased with the number of projects existing within a company. The medical research findings were co-ordinated within the company. These are similar kinds of efficiencies to those which the patent system creates, to some extent, between rival companies, when property rights are awarded and trade thereby becomes feasible. The opportunity to trade with patents creates a sort of scale advantages. Research findings can be utilised which would otherwise not have been taken advantage of, due to possibilities created for co-ordination. The company that made the discovery can trade the patent and does not have to be in a position to utilise the invention itself.

The "research spill-over" in larger pharmaceutical companies indicates that in a patent system where the claims always had to be bounded to the identified medical use, i.e. a system without prospecting, it would probably still be the inventor of the first medical use that most often found subsequent uses. The first inventor's technical advantage makes him more efficient in the search for later uses. As a consequence the incentives that others, his competitors, lose due to the prospecting, are normally not so significant. The competitors would in most cases be unable to compete efficiently with the product patentee, even if they had the same patent incentives. Their disadvantages are due to technical factors that cannot easily be influenced by the patent system.

Conclusions. The analysis of use limitation in patents on chemical substances has led to the conclusion that a balance needs to be struck between two effects. Absolute product protection, i.e. no use limitations, reduces, for all parties but the product patentee, the incentives to search for new uses. This is unfortunate, but, on the other hand, to some extent it also reduces the amount of wasteful duplicative effort. A valuable division of labour is achieved when the product patentee searches for new uses and his competitors do research outside the scope of the patents granted. This is a difficult balancing of incentives, but perhaps the reduction of duplicative efforts is more material that the losses occurring due to lack of incentives. This would mean that the prospecting of new uses within the pharmaceutical field is in general a positive feature in the patent system.

This belief is based on an emphasis placed on the two factors discussed above. Firstly, the product patentee will have strong incentives to search for new prospected uses for his patented substances. The possible new uses will belong to new markets and the sale that he may gain will not reduce his sales for the first use. This will make the product patentee an efficient user of the incentives he receives through the prospecting function of the patent. There is an effective carrot and so there is less need for the "whip of competition". Secondly, the product patentee will, for technical reasons not directly related to the incentives that are created by the patent system, normally be the most efficient researcher in the field of new uses for his previously patented products. Even if use limitations to the indications discovered were required in the claims, competitors would find it difficult to take advantage of the new incentives.

The conclusion that no use limitations to specific pharmaceutical uses discovered are needed is, however, not applicable to new uses outside the pharmaceutical field. A pharmaceutical company will not efficiently exploit the possible new uses a patented compound has in other fields of industry, e.g. as a detergent or fungicide. For the same reasons, a company that has patented a compound due to its usefulness as a catalyst in the

225 See Cockburn, I. Henderson, R., 27 Rand Journal of Economy 1996, 34. Another figure that has been mentioned is that between 10 and 25 projects exist in a larger pharmaceutical company, see Wiggins, P. N., The Pharmaceutical Research and Development Decision Process, 63, in Helms, R. B. (red), Drugs and Health. 
chemical processing industry or as a food additive will not efficiently exploit the same compound in the pharmaceutical field. The product patentee will only efficiently search for and exploit the uses that belong to his field of industry and not all uses that are covered by the scope of protection. A limitation in patents for new chemical compound to the industry to which the discovered use belongs would therefore not cause a significant loss to the patentee. But it would increase the incentives for companies working in other industries.

Due to the transaction costs involved in patent licensing, one cannot expect these problems to be solved by licensing. The costs are probably too high for it to be expected that rights to the uses falling outside the field of business of the product patentee could be traded to any significant extent, even though they may be more valuable to competitors than to the patentee. Therefore the conclusion drawn from this analysis is that patent claims with structural definitions of new chemical compounds should preferably be required to contain an additional feature: a limitation of use to the industry to which the disclosed industrial applicability belongs. This could mean, for example, a limitation of use to the pharmaceutical industry, to the chemical process industry, the field of fungicides, or the food industry.

Within the pharmaceutical field there already exists a situation in which claims have to be limited along these lines. When a patent is applied for a first medical use for a known compound, the claims must be bounded to the use in the pharmaceutical field; see part 5.2.1. From the analysis it has been concluded that a similar use limitation should also be required in situations where the compounds are new. A use limitation in regard to the industry to which the discovered use belongs is broadly motivated in the chemical field. With such a requirement the prospecting function of the patent system would be used where it is beneficial to avoid duplicative research, but not limit the incentives beyond those situations. Within the industry to which the discovered use belongs one can expect an efficient search for new uses by the product patentee, but that cannot be expected in regard to possible uses outside that industry.

There are other statements in the patent law literature to the effect that the granting of absolute product patents on chemical compounds seems to create an imbalance. ${ }^{226}$ Koktvedgaard, analysing the desirability of use limitations in chemical compound patents, reached a similar conclusion to the one reached here. He concluded that patents on chemical substances should protect uses in fields where the patentee faces competition from other companies. But outside the situation of competition there was no reason to limit the opportunities of other companies. This assessment is similar to the suggestion made here. ${ }^{227}$

Marquis has undertaken an economic analysis of patents for chemical compounds. He concluded that the availability of use-bounded patents for chemical compounds was essential to the incentives for innovation in the chemical field. But it was doubtful whether, in addition to this, absolute product patents should also be granted. The main reason for Marquis' doubts was that product patents were granted too easily, before the patent applicant had shown a truly valuable use. ${ }^{228}$

226 "[I]f mere making [of a new substance] is not invention, yet discovery of one use allows a patentee to claim the substance itself in all its uses, an evident imbalance results: only if the first identifier of a use can patent for that use alone would it seem reasonable to deny any protection to the first maker of the substance. But both issues for the moment remain unresolved.” See Cornish, W. R., Intellectual property: Patents, Copyright, Trade Marks and Allied Rights, 139.

227 See Koktvegaard, M., Konkurrencepraegede Immaterialretspositioner, 244.

228 "While this analysis tends to support the conclusion that new use protection may be essential to encourage the discovery of new uses, there is considerable doubt about the desirability of also providing compound protection. Some decrease in output is likely to occur when the new use and compound patents are owned by different entities. We do not know to what extent this decreased output may be offset by an increased incentive to discover new uses resulting from the availability of compound protection. The most serious objection to 
Non-prospecting in supplementary protection certificates. The EC regulation concerning supplementary protection certificates (SPC) is analysed in chapter 7. But it is already at this point instructive to note that SPCs have a particularly narrow scope of protection, narrower than the patents on which they are based. Only the compounds that have been approved for sale are protected, and only for the use for which the compounds may be sold. ${ }^{229}$ The supplementary protection is thereby narrowly limited structurally and also use-limited. This means that, 20 years after the filing of the patent application only direct copies can be stopped by the inventor. This scope of protection has no prospect part, and that is plainly justified 20 years after the application was filed. During this time the inventor has had ample time to search for new uses within the prospect in the original patent. If these incentives have still not led the product patentee to any new uses, there is no reason to limit the efforts made by his rivals.

The SPCs indicate that it is advantageous if the prospect parts of patents granted are diminished over time. The more time that has elapsed after the filing of the patent application, the more important it becomes that competitors have strong incentives to search for new uses. Such a gradual reduction happens today, because any patent for a new use will of course only be dependent on the product patent during the product patent's lifetime. As the twentieth anniversary of the filing of the product patent approaches, competitors will have gradually increasing incentives within the scope of the product patent. This is valuable.

compound protection, as it now exists in practice, is that the routine patenting of new chemical compounds is permitted. This rewards the person who conceives the structure of the new compound, which is usually not a very creative step. In most cases, the 'new' compound is a member of a known class of compounds and is closely related in structure to known members. The inventor simply writes a structural formula for the new compound in accordance with well established chemical bonding rules, which is within the capacity of a good chemical student.” See Marquis, H. L., 63:1 JPTOS 1981, 40.

229 Article 4 in the Regulation: "Within the limits of the protection conferred by the basic patent, the protection conferred by a certificate shall extend only to the product covered by the authorization to place the corresponding medicinal product on the market and for any use of the product as a medicinal product that has been authorized before the expiry of the certificate.” 


\subsubsection{Functional features}

\subsubsection{Functional features - particularly in biotechnology}

The study now turns from structural definitions of pharmaceuticals to the use of functional features in the claims. Functional features can be used both when a product and a process are claimed. In other words, both a product and a process may be delineated by a statement that it achieves a certain result. However, functional features of this sort can lead to a very broad scope of protection. It may make it possible to claim not just one, but all solutions to a particular problem. However, a scope of protection that is independent of the actual means by which an effect is achieved, is in many cases legitimate and even necessary. Features such as "fastening means", "tightening material", and "drill" have a number of structural connotations, but are mainly functional. They are regularly used in the mechanical field without the scope of protection becoming unjustifiably broad. In the electronic field it is not unusual to find features such as "amplifier" or "AND gate". "Catalysts" and "reduction agent” are examples of functional features used in chemistry.

There are two main reasons why these features appear to be justified. Firstly, they serve as a definition of a technical result that is not new. Secondly, the technical result mentioned in the claim may be achieved in many possible ways that are difficult to capture with more concrete features. These considerations can be exemplified by case $T$ 139/85. The applicant had developed a dosage form that was to be used together with certain active substances that were defined in the claims only with the feature 'physiologically functional'. The Board explained that it would have been very difficult, maybe impossible, to find one or more chemical terms that would capture now existing and possible future derivatives that would function. Therefore the Board concluded that any other limitation than "physiologically functional" would create a too narrow scope of protection. ${ }^{230}$

Functional features become a problem in particular when they are used at the point of novelty in the claim and if it is impossible to foresee from the patent application what future technical solutions that may achieve the technical effect used as the functional feature. In such situations the functional claims may cover unknown solutions that are difficult to develop and require research incentives. Functional features are particularly questionable if the scope of protection will cover junior solutions that are more valuable than those that have been developed by the patentee.

230 T 139/85 of 2312 1986: "The question then is simply whether it is permissible to define a derivative solely by the term 'physiologically functional'. The term 'physiologically functional' is, of course, itself a functional feature. The Examining Division has - correctly - not objected to this term to the extent it is being applied to salts and esters. This Board has [previously] decided ...: 'Functional features defining a technical result are permissible in a patent claim if, from an objective viewpoint, such features cannot otherwise be defined more precisely without restricting the scope of the invention, and if these features provide instructions which are sufficiently clear for the expert to reduce them to practise without undue burden, if necessary with reasonable experiments' The afore-quoted criteria are met, in the present case, by the term 'physiologically functional', irrespective of whether it is applies to salts, to esters or to 'other' derivatives (or simply to 'derivatives' in general): It is extremely difficult, if not impossible, to find a specific chemical term or plurality of terms defining all those derivatives -existing and yet to be synthesised - of gamma-linolenic and of dihomo-gamma-linolenic acid which are 'physiologically functional', i.e. which - in the context of the invention - have the same physiological function as the free acids, e.g. by being convertible in the body thereto. ... The said feature is also 'sufficiently clear for the expert to reduce (it) to practise without undue burden'; for the expert clearly understands its meaning ..., and if in doubt with respect to a particular derivative whether it is "physiologically functional', he can without undue burden, i.e. by means of reasonably simple experiments, determine this.” 
With functional claims the examiner is required to strike an appropriate balance between two interests. On one hand, the applicant's right to efficiently protect the technical contribution he has made, and on the other hand, the risk that the scope of protection becomes so broad that further solutions to the same problem are not sufficiently encouraged. This balance has been particularly difficult to strike in cases of biotechnological inventions. ${ }^{231}$ Therapeutically useful proteins in general tolerate more structural changes than other active compounds. ${ }^{232}$ They are macromolecules where a change of two similar amino acids or some other change in a non-critical part of the structure can be done without necessarily affecting the biological function. Structural variations in the DNA coding for a protein are called allelic variations and are not uncommon in nature. But exceptionally an allelic variation in a gene causes a genetic disease. That is the case e.g. with cystic fibrosis. The protein that is expressed does not fulfil its proper biological function.

In addition to the tolerance to structural changes, another particularity of DNA makes it difficult to capture its value by the use of structural features in the patent claims. There is a surplus of information in genes coding for proteins. All information that is contained in the nucleic acid sequence in a gene is not retained in the protein that is expressed by the gene. This is called the degeneration of the genetic code. The applicant, of course, does not wish to include such surplus information in the claims. The facts that proteins tolerate structural changes to an unusual degree and that there is surplus structural information in genes have led patent applicants to use functional terminology particularly in biotechnology claims, i.e. use the biological function of the protein or gene as a limiting feature. This will mean that any structure with the biological effect in question falling within the scope of protection. 233

\subsubsection{Allowable use of functional features}

It is clear that there are situations where functional features are permissible. ${ }^{234}$ But it is also well known that functional features may lead to unjustifiably broad scopes of protection. The question is what the limitations are. With reference to functional features it is stated in the Guidelines that:

231 “[T]he mode of definition utilized in most patent thought presumes that inventions are descriptively reducible to their ultimate elements, which in turn are capable of reassembly in accordance with some concise set of instructions. Clearly this style of characterization prescribed by patent law is linked to the mechanistic form of technology and the structure of scientific thought most prevalent during the growth of the patent system. The major difference between the classical and biotechnological invention is that, unlike the former, the latter can not be described in terms of its component parts, nor in terms of the elements of which it is composed. For rather than utilizing a mechanistic mode of definition, biotechnological inventions are necessarily defined in either manipulative or functional terms.” See Sherman, B., 10 Oxford Journal of Legal Studies 1990, 280.

232 See Sheiness, D., JPTOS February 1996, 121.

233 T 694/92 of 0805 1996: "Claim 1 at issue is generally directed to a method for genetically modifying a plant cell by transferring into it a combination T-DNA/plant promoter-plant gene, such that expression of the protein encoded by the said plant structural gene is detectable in said plant cell (feature 'such that...'). No specific details are given in the claim in respect of the structural arrangement of the combination T-DNA/plant promoterplant gene ... Thus, the skilled person is essentially instructed by the claim to transfer a DNA vector such as the one disclosed in document (5a) ... into a plant cell 'such that expression of the protein encoded by the said plant structural gene is detectable in said plant cell'. Failing any limitations in respect of the kind of plant cell and/or plant gene and/or plant promoter, the claim is thus directed to a method whenever it works (see the feature 'such that...') for a whole range of applications."

234 See AIPPI Yearbook 1998/VII, 48. 
"As a general rule, claims which attempt to define the invention, by a result to be achieved should not be allowed, in particular if they only amount to claiming the underlying technical problem. However, they may be allowed if the invention either can only be defined in such terms or cannot otherwise be defined more precisely without unduly restricting the scope of the claims and if the result is one which can be directly and positively verified by tests or procedures adequately specified in the description or known to the person skilled in the art and which do not require undue experimentation."235

It is further stated in the Guidelines that:

"A claim may broadly define a feature in terms of its function, even where only one example of the feature has been given in the description, if the skilled reader would appreciate that other means could be used for the same function”. 236

It is evident from this that caution is necessary when functional features are present in the claim. But it is also stated that such features may be permitted if they are necessary in order for the applicant to receive an adequate protection and that the invention cannot otherwise be defined more precisely. Further guidance can be found in case law.

\subsubsection{The one way rule - an extreme solution}

Decision T 292/85 has formed the basis for one approach to functional claims that has sometimes, foremost in English case law, been referred to as the one-way-rule. ${ }^{237}$ By this is meant that the broadness of the claim is acceptable if the man skilled in the art knows one way to use the effect (the functional feature). The invention in suit was a plasmid, i.e. a naturally occurring DNA-element that may be used to insert foreign DNA into a host organism. Only functional features had been used to define the plasmids. The Examining Division refused the application, finding it inappropriate to use functional features in biotechnology if the scope of protection would include technical solutions (new plasmids) that were unavailable to the man skilled in the art on the application day. However, the Board of Appeal allowed the claim. One circumstance the Board pointed to was that the limits to the use of functional features were dependent on the applicant's possibilities of having a fair scope of protection, but not on the technical field. The scope of protection would become insufficient in this case if it did not include future solutions that gave the same result. It was indicated that one way to attain the result mentioned in the claim justified the functional feature. ${ }^{238}$ Applicants that have used particularly broad claims have often quoted from this

235 Guidelines C III 4.7.

236 Guidelines C III 6.5.

237 See Crespi R. S., Biotechnology, Broad Claims and the EPC, [1995] EIPR 267.

238 T 292/85, OJ EPO 1989, 275: "The suggested features in the claims are essentially functional terms in this particular context, in spite of structural connotations, and may cover an unlimited number of possibilities. It follows that the features may generically embrace the use of unknown or not yet envisaged possibilities, including specific variants which might be provided or invented in the future. This Board concurs with the decision of another Board ... in which the possibility of using functional terminology in claims was approved if 'such features cannot otherwise be defined more precisely without restricting the scope of the invention' and their reduction to practise was not an undue burden. The Board sees no valid reason why this should not be equally true for the field of biotechnology as in other fields of technology. In appropriate cases, such as the present, it is only possible to define the invention (the matter for which protection is sought - Article 84 EPC) in a way which gives a fair protection having regard to the nature of the invention which has been described, by 
decision. A one-way-rule was also applied in two English High Court Cases, but in the second of these (Biogen v. Medeva plc) the House of Lords overruled, see below. ${ }^{239}$

The one-way-rule can be described as an extreme standpoint on the permissibility of functional features. It actually means that there are no particular limits to the use of functional features. If the applicant has shown that there is one way to reach the effect, the claims may be crafted so as to include all solutions that can be used to gain the effect, including yet unknown alternatives. The claims do not have to correspond with the ways in which the man skilled in the art is able to practise the invention. It is, of course, impossible to accept such a solution. It would be run counter to the most basic aspects of the patentability examination. ${ }^{240}$ The-one-way-rule could perhaps be viewed as a minimum requirement, but then it does not mean anything more than the self-evident requirement that the man skilled in the art should at least be able to achieve what is claimed. That is already made explicit in Art. 83 EPC and therefore there is no need to speak of this as a particular new rule.

In has in junior EPO-cases become evident that the-one-way-rule cannot be used to decide on the permissibility of functional features. In T 435/91 the Board of Appeal found that the functional features used did only amount to an invitation to start research projects aiming at alternative ways to reach the effect that was stated in the claims. The claim wording was not permitted because the description did not indicate how such new solutions could be found. The applicant asserted that this was in conflict with the one-way-rule, as formulated in T 292/85. But the Board stated that the applicant had made an incorrect interpretation of the case law when arguing that one way to practise the invention always was sufficient for a functional wording of the claims. The correct interpretation of case law was, according to the Board, that functional terminology was allowed if only in exceptional cases the skilled man was unable to attain the effect within the whole scope of protection. ${ }^{241}$

A somewhat similar case is T 128/92. The application was for DNA-sequences that coded for certain polypeptides. The amino acid sequence of the polypeptide was given in

using functional terminology in the claims. ... Unless variants of components are also embraced in the claims, which are, now or later on, equally suitable to achieve the same effect in a manner which could not have been envisaged without the invention, the protection provided by the patent would be ineffectual. Thus it is the view of the Board that an invention is sufficiently disclosed if at least one way is clearly indicated enabling the skilled person to carry out the invention. Consequently, any non-availability of some particular variants of a functionally defined component feature of the invention is immaterial to sufficiency as long as there are suitable variants known to the skilled person through the disclosure or common general knowledge, which provide the same effect for the invention. The disclosure need not include specific instructions as to how all possible component variants within the functional definition should be obtained."

239 Molnlycke AB v. Proctor \& Gamble Ltd, [1994] R.P.C. 49, IIC 1995, 92 and Biogen v. Medeva plc., Court of Appeal [1995] R.P.C. 25, House of Lords [1997] R.P.C. 1 and IIC 1997, 740.

240 Cf Wibbelmann, J., [1997] EIPR 517.

241 T 435/91, OJ EPO 1995, 188: "[I]t is clear that the above definition of the additive is not more than an invitation to perform a research programme in order to find other 'additives' which meet the functional requirements set out in claim 1 . In other words, through this definition in the present claim the respondent tries to claim not only the solution of the technical problem of providing surfactant compositions in the form of a hexagonal liquid crystal gel phase made available to the person skilled in the art by the disclosure in the patent specification, but, in addition, all other possible solutions of this problem ... without giving any or any useful technical guidance as to how to obtain, with a reasonable expectation of success, further suitable 'additives' which are not 'hydrotropes'. Neither the patent specification nor the relevant common general knowledge provide guidance as to how these further additives may be traced out or according to which criteria they might be selected. Therefore the board holds that the patent does not disclose a self-sufficient technical concept which adequately corresponds to the 'functional' used for the 'additive' (b) in the present claim 1.... [T] dhe disclosure of an invention is only sufficient if the skilled person can reasonably expect that substantially all embodiments of a claimed invention which this skilled person would envisage on the basis of the corresponding disclosure and the relevant common general knowledge can be put into practise. In other words, only exceptional failures can be tolerated. In the present case, however, the possibility of failure is far from being exceptional.” 
the claim (a structural feature), but it was also stated that other sequences belonged to the scope of protection, if they could be used to manufacture proteins with activity similar to interleukin-2 (a functional feature). The patent had been granted by the Examining Division and been upheld in an opposition procedure, but an opponent appealed the decision. He stated that more than 20,133 different amino sequences came within the scope of protection. The Board, nevertheless, upheld the patent because on the priority date the skilled person could vary the amino acid sequence and thereafter test to see if the sequences had the required activity. The possibility of new polypeptides with interleukin-2 activity being developed in future was no grounds for refusing the claim. The Board considered it justified that such later inventions should be dependent on this patent. ${ }^{242}$

An illustrative case is T 1024/96. A pharmaceutical formulation had been claimed mainly by designating that it allowed controlled absorption at a rate sufficient to achieve therapeutically effective blood levels over a period of 12-24 hours following oral administration (in vivo profile) and that the formulation displayed a particular in vitro dissolution rate (in vitro profile). The Examining Division refused the application on the grounds that the claims did not comply with the requirement of Article 84 EPC. The Division maintained that the claims did not contain any technical feature characterising the structural composition of the claimed formulation and therefore did not indicate how the desired effect could be achieved. It also held that the claim contained an almost literal repetition of the underlying technical problem and that the dissolution profile defined in the claim merely represented a method of testing whether a given formulation manifested the desired properties. The Board, however, granted the patent. It explained that a functional characterisation was normally accepted and in line with previous decisions when the claimed subject matter could not be otherwise delineated without unduly restricting the protection sought and if this feature provided instructions which were sufficiently clear for the skilled person to reduce them to practice without undue experimentation. It was found that the two requirements were met in this case:

\footnotetext{
"It is immediately evident to the board that the claimed functional characteristics can be matched by a multiplicity of structurally different formulations obtained by combining in the correct amounts the different polymers and additional components thoroughly disclosed in the description and available to the skilled person. Therefore, in the board's judgement, any structural characterisation of the claimed formulation, as suggested by the examining division, would undoubtedly and unjustifiably limit the scope of the claim to a specific and practical embodiment of the invention."243
}

\footnotetext{
242 T 128/92 of 3011 1994: "[T]he skilled person would at the priority date have been in a position with routine methods to make fragments with sequences coding for polypeptide sequences differing from the one listed in the first part of the claim in the manner stated in one of features (a), (b), (c), (d) or (e), and would have been able to test these for IL-2 activity, and select those which had such activity. This would lead to an indefinite number of fragments, other than the one specifically described, which could be used to make a polypeptide having IL-2 activity. There is no evidence that the skilled person could not find, without undue burden, many embodiments. The Board cannot, in these circumstances, see any grounds for saying that the invention is not disclosed in a manner sufficiently clear and complete for it to be carried out by a person skilled in the art. By providing the information in the patent, the Respondent has enabled the skilled person to make not only the polypeptide with the sequence recited in the claim, but to find many others that will have IL-2 activity. The protection granted should correspond to this disclosure. The fact that others, starting from the information in the patent might by research identify a DNA fragment encoding for a polypeptide with improved IL-2 activity infringing the present patent, is not a ground for refusing Claim 1. Such an improvement may deserve a patent of its own, but the possibility of such an improvement is no ground for limiting the present claim. Thus, even if the Appellant is correct in stating that the claim potentially covers more than 20133 embodiments, this is irrelevant.”

243 T 1024/96 of 16061997.
} 
Regarding the Examining Division's opinion that the claims comprised nothing but the almost literal paraphrasing of the underlying technical problem, the Board observed that once the in vitro dissolution and the in vivo absorption criteria had been set forth, the additional contribution required from the skilled person in manufacturing the formulation was merely that of carrying out the appropriate adjustments of the ingredients as well as the necessary measurements by relying on the exhaustive disclosure of components and procedures taught in the specification. Moreover, it was emphasised that all the necessary components were commercially available and all the necessary procedures were well known per se. Therefore, no undue experimentation or inventive merit was required of the skilled person.

\subsubsection{Functional claims in cases of deposition}

A valid deposition enables the man skilled in the art to practise the invention. But it opens just one such possibility. In T 301/87 the application was on DNA sequences that coded for a polypeptide of the IFN- $\alpha$ type (alpha-interferon fulfils a function in the human immune system and serves in the treatment of cancer). Claim 1 had a reference to certain deposited recombinant micro-organisms that expressed $\alpha$-interferon. But in the claims functional terminology was used that did not limit the scope of protection to the polypeptides that were expressed by the deposited cultures or polypeptides identical with these. The functional terminology meant in practice that all DNA molecules that coded for the human protein $\alpha$ interferon came within the scope of protection. The Examining Division granted the patent in accordance with the patent claims. When the Board of Appeal tried the case they found that the depositions supported the functional claims. However, it was also made clear that a deposition did not automatically make a functional definition justifiable. It was decisive whether the man skilled in the art was able to take advantage of the practical value of the invention. ${ }^{244}$

In $\mathrm{T}$ 740/90 the applicant claimed that the deposition justified a functional wording of the claims. But in this case the Board did not accept this. It was pointed out that there were caveats to the one-way-rule. One way to practise the invention had been sufficient in $\mathrm{T}$ 292/85, according to the Board, because the man skilled in the art had been aware of other opportunities to achieve the same result. ${ }^{245}$

The notable English litigation between Biogen and Medeva led to the first biotechnology decision by the House of Lords. The invention in suit made it possible for the first time to recombinantly manufacture hepatitis B antigen material. Such material may, for example, be used in the manufacturing of a vaccine against hepatitis $B$ and in the

\footnotetext{
244 T 301/87, OJ EPO 1990, 335: “[T]he Board finds no case for insufficiency. On the contrary, there is a detailed description of the actual reduction to practice of the invention, and a great number of depositions which could provide a variety of immediate short cuts for the public to carry out the invention without having to go through the cumbersome route from natural sources. The description establishes a good basis for obtaining other variants, if this is desired. Nothing has been presented so far to cast doubt on the workability of the approach presented in the specification. The requirement for sufficiency is not a matter of satisfying the perfectionist but to enable the skilled person to handle the invention in normal practice.”

245 T 740/90 of 0210 1991: "[T] re reference to this decision is inappropriate. The decision T 292/85 relates to an invention in which an element defined in functional terms was by nature such that certain particular variants were not suitable. The Board of Appeal decided that the disclosure was sufficient since those skilled in the art, through the disclosure of the invention or common general knowledge in their technical field, knew appropriate variants which provide the same effect for the invention."
} 
manufacturing of diagnostic equipment. ${ }^{246}$ The case turned on the right to use functional terminology after a deposition has been made.

The technical background to the invention was a scientific article from 1970 showing that blood from persons with hepatitis B-infection contained a particle that was approximately 42 nanometers in diameter. ${ }^{247}$ After its discoverer it was named the "Dane particle". It was thought to contain at least two antigens, a surface protein and a core protein. With this knowledge there was a possibility of manufacturing a vaccine against hepatitis $\mathrm{B}$. If Dane particles were gathered from infected blood and inactivated, they could thereafter be used as a vaccine. This, however, entailed risks because such material may at times revert to its virulent state. If that happened the patients would be infected, not vaccinated but infected. Another disadvantage would be the limited availability of infected blood. Therefore the process would both be dangerous and expensive.

In the early 1970s recombinant DNA-technology was developed. This made it possible to change the DNA in an organism and thereby make the organism produce a sought after protein (express an exogenous gene). Recombinant technology can, for example, be used to express antigen polypeptides that can be used as vaccines. A recombinant vaccine would be both safe and possible to manufacture in unlimited quantities. A prerequisite was, however, that the DNA coding for a surface or core protein in the hepatitis B virus, could be extracted from the Dane particle and introduced into a host organism that would express the antigen polypeptide.

Today it is routine to sequence DNA, but in the 1970s it was both difficult and time-consuming. Not until June 1979 was the full DNA-sequence of the hepatitis B virus published. $^{248}$ But six months earlier, on 22 December 1978, had a British patent application been filed for a recombinant DNA-molecule that coded for a polypeptide with hepatitis B antigenicity. The applicant was Biogen and one of its founders, Professor Murray. Murray had chosen not to sequence the genome of the virus. He had extracted DNA from the Dane particles and thereafter "cut" it in fragments that, hopefully, would code for a polypeptide with a structure similar to an epitope - a characteristic part - of the hepatitis B virus. The random DNA-fragments of the Dane particle were introduced into E.coli and thereby polypeptides could be produced. Tests showed that some E.coli cultures expressed polypeptides with hepatitis B antigenicity, i.e. polypeptides that induced antibodies that would attack hepatitis B viruses. Murray deposited the cultures and a patent application was filed.

In the application the most important part of the claim was the following: " $\mathrm{A}$ recombinant DNA molecule characterised by a DNA sequence coding for a polypeptide or a fragment thereof displaying HBV [hepatitis B virus] antigen specificity...”. The DNAmolecule was thus characterised partly by the manufacturing process, recombinant, partly with the result that was achieved when the molecule was expressed: a polypeptide that induced antibodies against hepatitis B virus. The latter feature was functional, a statement of the valuable result that was achieved.

In T 296/93 the Board of Appeal tried the EPO-application filed by Biogen, where priority was claimed from the British application. ${ }^{249}$ Biogen needed a valid priority because the invention would be obvious in relation to the full DNA-sequence of the hepatitis $\mathrm{B}$ virus, i.e. the content of the article from June 1979. The Board mentioned that the British

\footnotetext{
246 House of Lords, Biogen v. Medeva of 3110 1996, [1997] R.P.C. 1 and IIC 1997, 740.

247 See Dane, D. S., et al., The Lancet 1970, 695.

248 See Valenzuela, P., et. al., 280 Nature of 197908 30, 815.

249 T 296/93, OJ EPO 1995, 627.
} 
application contained no information on the structure or capabilities of the polypeptides, except their hepatitis B antigenicity. But:

"The evidence on file rather indicates that, by proceeding experimentally as taught in the BI priority document [the British application from 22 December 1978], expression of proteins having the antigen specificity and antigenicity of either HBcAG [core protein] or HBsAG [surface protein] was achieved to some extent. The European patent specification confirms the validity of the approach and demonstrates inter alia that deposits A and B express polypeptides with antigen specificity...”.

The patent was granted because the invention could be practised. The Board decided that the man skilled in the art could by use of the disclosure in the British application manufacture proteins with antigenicity for hepatitis B core and surface proteins.

Parallel to the proceedings in the EPO, a nullity action against the EPO patent was pending in the British courts. This was ended by the judgement of the House of Lords on 31 October 1996. ${ }^{250}$ Biogen had accepted in the British case that the patent lacked inventive step in relation to the article on the DNA-sequence of hepatitis B virus. This made a valid priority a necessity. Thus the situation was the same as in the EPO.

In the judgement by the House of Lords it was initially established that a sufficient disclosure was a central part of the patent system and that a valid priority required the man skilled in the art to be able to practise the invention by use of the priority document. It was further concluded, with reference to EPO case law, that the patent monopoly, as defined by the claims, should correspond to the technical contribution to the art in order for it to be supported, or justified. Thereafter Lord Hoffman accepted the conclusion that the patent had enabled the making of the antigens. However, he continued by stating:

\begin{abstract}
"But the fact that the skilled man following the teaching of Biogen 1 would have been able to make HBcAg [hepatitis B core antigen] and HBsAg [hepatitis B surface antigen] in bacterial cells, or indeed in any cells, does not conclude the matter. I think that in concentrating upon the question of whether Professor Murray's invention could, so to speak, deliver the goods across the full width of the patent or priority document, the courts and the EPO allowed their attention to be diverted from what seems to me in this particular case the critical issue. It is not whether the claimed invention could deliver the goods, but whether the claims cover other ways in which they might be delivered: ways which owe nothing to the teaching of the patent or any principle which it disclosed.
\end{abstract}

It will be remembered that in Genentech I/Polypeptide expression the Technical Board spoke of the need for the patent to give protection against other ways of achieving the same effect 'in a manner which could not have been envisaged without the invention.' This shows that there is more than one way in which the breadth of a claim may exceed the technical contribution to the art embodied in the invention. The patent may claim results which it does not enable, such as making a wide class of products when it enables only one of those products and discloses no principle which would enable others to be made. Or it may claim every way of achieving a result when it enables only one way and it is possible to envisage other ways of achieving that result which make no use of the invention."

Lord Hoffman, further, discussed the technical contribution to the art which Professor Murray made in 1978 and compared it to the scope of protection. The applicant had found one way the first - to manufacture the antigens in a prokaryotic host cell. But that did not justify a monopoly on any recombinant method of making the antigens. It was stated that:

250 House of Lords, Biogen Inc. v. Medeva of 3110 1996, [1997] R.P.C. 1 and IIC 1997, 740. 


\begin{abstract}
"The claimed invention is too broad. Its excessive breadth is due, not to the inability of the teaching to produce all the promised results, but to the fact that the same result could be produced by different means. Professor Murray had won a brilliant Napoleonic victory in cutting through the uncertainties which existed in his day to achieve the desired result. But his success did not in my view establish any new principle which his successors had to follow if they were to achieve the same results. The inventive step, as I have said, was the idea of trying to express unsequenced eukaryotic DNA in a prokaryotic host. Biogen 1 discloses that the way to do it is to choose the restriction enzymes likely to cleave the Dane particle DNA into the largest fragments. This, if anything, was the original element in what Professor Murray did. But once the DNA had been sequenced, no one would choose restriction enzymes on this basis. They would choose those which digested the sites closest to the relevant gene or the part of the gene which expressed an antigenic fragment of the polypeptide. The metaphor used by one of the witnesses was that before the genome had been sequenced everyone was working in the dark. Professor Murray invented a way of working with the genome in the dark. But he did not switch on the light and once the light was on his method was no longer needed. ... The respondents Medeva, who use restriction enzymes based on knowledge of the HBV genome and mammalian host cells, owe nothing to Professor Murray’s invention.”
\end{abstract}

The patent was invalidated, because it lacked priority, which in turn was due to the broadness of the claims in relation to the ability to practise the invention in the British application. The judgement is notable inter alia because it limits the permissible use of functional features in patent claims when a DNA-sequence has been deposited. Lord Hoffman's statement captures very well the problem: it is necessary to look into the future and consider whether new ways to solve the same problem will be developed, ways that do not rely on the teaching in the patent application. The situation in this case was exceptional in this aspect. Biogen had with a combination of luck and skill been the first to recombinantly manufacture hepatitis B antigen material and they thereafter deposited the cultures. Shortly thereafter the DNA-sequence became known and then the technique that had been used in the application was obsolete, i.e. only six months after the application had been filed. Probably this influenced the court. The applicant's achievement was not of such significance that other more advanced and efficient ways to produce hepatitis B antigen material should be protected. In a later English case, the question whether the claims of the patent go beyond the technical contribution to the art made by the inventor, has been coined "the extended form of insufficiency". 251

With hindsight it is obvious that the scope of protection in the EPO patent was very broad in relation to the possibilities the man skilled in the art had to practise the invention. The ways that were enabled were obsolete only six months after the application had been filed, but the scope of protection was not, thanks to the functional wording of the claims. On the contrary, the scope of protection had become much more valuable, owing to the new developments in the art. It is of course very difficult to judge beforehand whether such input will occur. It is not easy to predict what alternative solutions will be developed during the lifetime of the patent. However, it is certainly quite unusual for such a short time to separate the filing of the patent application and the occurrence of a much better solution falling within the scope of a functional claim. Thus, the situation was particular, but the decision at least shows that functional features are not always acceptable when the applicant is the first to solve a particular problem. It is also evident from the decision that the limitations pertaining to the use of functional features are linked to what other means to achieve the effect that may be developed during the lifetime of the patent.

The House of Lords ended their judgement by commenting on the fact that the outcome was different from that in the Board of Appeal. It was explained that EPO decisions are generally deemed to have considerable persuasive authority in British patent law, but that the rules applied by the House of Lords in this case did not deviate from established EPO case

251 See Patents Court, Playhut Inc v. Spring Form Inc. of October 8, 1999. 
law. ${ }^{252}$ Particular attention was drawn to the decisions T 292/85253 and T 409/91. The House of Lords thereby indicated that it considered T 296/93, where Biogen's patent had been granted, to deviate from EPO's own case law. One EPO Board of Appeal has, however, found that it was the position taken by the House of Lords that was at variance with the established practice in the EPO (T 636/97):

\begin{abstract}
"For the board it is a fundamental principle of patent law that a claim can validly cover broad subject matter, even though the description of the relevant patent does not enable every method of arriving at that subject matter to be carried out. Otherwise no dominant patent could exist, and each developer of a new method of arriving at that subject matter would be free of earlier patents. In many cases in the field of biotechnology, patent protection would then become illusory. This is not to say that some claims might not be too broad in scope and not be enabled over their whole scope for the purpose of Article 83 EPC .... The boards have considered this question of allowability of a broad claim versus the requirements of Article 83 EPC, strictly on a case by case basis, influenced by the extent to which the information in the patent could be used to develop further embodiments without a major conceptual leap. The question of the allowable width of claims in relation to sufficiency under Article 83 EPC, may be an important question of law which may at some stage have to be considered by the EBA, in the light of recent national case law which shows that the view of some EPC Contracting States national courts may not be the same as that expressed here (cf. House of Lords in Biogen v. Medeva (1997 RPC 1))."254
\end{abstract}

T 409/91, which the House of Lords referred to as an example of a case to be followed, does not belong to the pharmaceutical field, but has caused considerable interest because the legal problem associated with functional claims was so palpable. The decision will be discussed. Thereafter the presentation will return to the pharmaceutical field.

The invention in $\mathrm{T}$ 409/91 was a solution to the well-known problem that mineral oils, e.g. diesel, lose fluidity at low temperatures. This is due to the crystallisation of wax particles and may, for example, cause clogging of fuel filters. The applicant had discovered that the wax crystals in the oil became small enough to pass through the filter if a certain compound was added to the fuel. The additive was disclosed in the description with a chemical formula, but it was not mentioned in the claims. The applicant had chosen to define his invention in the claims as a fuel with an average particle size below 4,000 nanometers, i.e. by a statement of the result that was achieved when the additive was used. According to the description it had not been possible to achieve any smaller average particle size than 1,000 nanometers. The Board therefore found that this limitation had to be included in the claim, or else the man skilled in the art would be unable to practise the invention within the entire scope of protection.

In the next step of the decision the Board tried the alternative claims that had the additional feature: "above 1,000 nanometers". Now the interesting question occurred: Did the applicant deserve a monopoly on all diesel fuel with wax particles between 1,000 and 4,000 nanometers, when his contribution to the state of the art amounted to the additive that for the first time enabled the man skilled in the art to manufacture a fuel according to the claim?

During the hearing the applicant conceded that he did not know of any other way to manufacture mineral oils with average wax crystals between 1,000 and 4,000 nanometers than by using the additive. From this the Board of Appeal concluded that the application did not meet the requirement of Art. 84 EPC. The claims did not state one of the essential technical features of the invention. The Board further stated that there was a general patent law principle according to which the scope of protection should correspond to the

\footnotetext{
252 Cf Mcinerney, A., [1998] EIPR 20.

253 T 292/85, OJ EPO 1989, 275.

$254 \mathrm{~T}$ 636/97 of 26031998.
} 
contribution to the art made by the inventor. In this case, the scope of protection would not be proportional to the ways in which the man skilled in the art was able to practise the invention. ${ }^{255}$

It had of course been very valuable for the applicant if the functional feature had been accepted. A monopoly on the market for diesel fuel that does not block fuel filters at low temperatures would indeed have been very valuable. However, in this case there was no reason to accept such a functional wording. The applicant had only developed one way of solving the problem and there was no difficulty in stating this way in a concrete manner in the claims. Such a claim could not easily have been circumvented by competitors, but perhaps after further research it could have been invented-around. Therefore the outcome of the case was justified, due to the importance of maintaining incentives for further developments of competing products. All the same, the legal reasoning is none too attractive. The Board did nor really explain how to decide what features are essential in an invention and therefore may not be defined functionally. It only concluded that the essential features had to be included in the claim. Legal analysis, however, has seldom been developed much further than this as regards the use of functional features. ${ }^{256}$

\subsubsection{Generalisation from one to more organisms}

Recombinant biotechnology is possible because all living organisms use the same "language": the DNA-code. A DNA sequence from one organism can in principle be "understood" by another organism. If a human DNA-sequence coding for an interesting protein has been determined, the sequence can in principle be introduced into disparate host organisms, from a bacterium to a sheep, whereupon the recombinant organism starts to manufacture the therapeutic protein. It is not certain, however, that all organisms work or all DNA sequences

255 T 409/91, OJ EPO 1994, 653: "In the Board's judgment, this part of the description of the invention is of a fuel oil composition which must contain, as an essential constituent, 'certain additives'. It is precisely this feature that is missing from the present claims, which, therefore, do not meet the requirement of Article 84, first sentence, EPC, since they do not define the claimed subject-matter by reference to all its essential technical features. Insofar as these claims omit to include this essential feature, they are not definitions of the invention actually described in the body of the description, but mere incomplete descriptions of it. Accordingly, if there be a definition in these claims, it is of some other invention of which the nature of the additives is not an essential part; namely the invention of the principle of smallness, referred to in item II above. ... Consequently, a technical feature which is described and highlighted in the description as being an essential feature of the invention, must also be a part of the independent claim or claims defining this invention .... Since the Board could not find in the rest of the description anything which might be inconsistent with the above clear statement of what the claimed invention is, in particular because all the worked examples describe fuel oils containing additives, and, furthermore, all those examples which do not contain an additive of the group of 'preferred additives' mentioned in the paragraph bridging pages 3 and 4, are marked 'comparative examples' and produce, after cooling, wax crystals which do not meet the requirements set out in Claim 1, the description does not support (or justify) a claim which is directed to a fuel oil without any additives. Therefore, the present claim is not supported by the description and, therefore, offends Article 84 EPC.”

256 The legal situation was summarised in T 694/92 of 0805 1996: "In certain cases a description of one way of performing the claimed invention may be sufficient to support broad claims with functionally defined features, for example where the disclosure of a new technique constitutes the essence of the invention and the description of one way of carrying it out enables the skilled person to obtain without undue burden the same effect of the invention in a broad area by use of suitable variants of the component features .... In other cases, more technical details and more than one example may be necessary in order to support claims of a broad scope, for example where the achievement of a given technical effect by known techniques in different areas of application constitutes the essence of the invention and serious doubts exist as to whether the said effect can readily be obtained for the whole range of applications claimed". 
can be expressed. One question, therefore, is to what extent a discovery that one organism can be used to express a protein may be generalised so as to include the use of other organisms for the same purpose.

In $\mathrm{T}$ 81/87 the claims were to certain recombinant cells that could express the protein rennin. The cells according to the claim were sponges, yeast, bacterial and mammalian cells, thus all cells were included in the scope of protection. The claims also contained the functional limitation that only those cells were covered that actually had the ability to express rennin. The Examining Division refused the application because the description only contained examples with the bacteria Escherichia and Saccharomyces. The Board of Appeal, however, accepted the claims because it considered that it would be unfair to limit the scope to that which was described as examples. At the time of filing it was known that there were other alternative cells that worked and the applicant should therefore be permitted to include future uses of the invention within the scope of protection. It was also observed that there was no need for any particular analysis of whether all other cells actually worked, because the claim had a functional feature that assured that only workable alternatives were protected. ${ }^{257}$ This reasoning, however, is not very useful in relation to the permissibility of functional features. The characteristic aspect of functional claims is that they do not include non-functional parts within the scope of protection, but at the same time do include all functional alternatives. It is the latter consequence that is the problem, not the question of "non-functionality".

T 386/94 was another situation where a Board had to consider what organisms could be used to express a protein. Only examples with the micro-organism E.coli were included in the description. The question was whether it was permissible to expand the scope to all micro-organisms. The Board found this to be the case. There was a detailed description of how to achieve expression, and the protein had been expressible in such disparate organisms as bacteria and calves. This indicated that the skilled person would be able to use also other micro-organisms. Nothing in the state of the art indicated that expression would be impossible in micro-organisms in general. ${ }^{258}$

257 T 81/87, OJ EPO 1990, 250: “As regards the use of cells of various origins according to Claims 1 and 2, the disclosure repeatedly emphasises that these are such as to enable expressions of proteins. It was known at the time of filing that in addition to bacterial cells, various fungi, e.g. yeast cells, were suitable for the purpose. The specification itself refers to various preferred Escherichia and Saccharomyces strains in this respect. Thus, the terms in the claims are not without formal support, since it would be unfair to restrict the claims to the exemplified strains in the disclosure excluding those which may be used in the future. From the disclosure, it is implied for the skilled person that only those cellular organisms are relevant for use are capable of providing expressions of the desired proteins ... Thus, the disclosure in the European application is not insufficient or unsupported in these respects under Articles 83 and 84 EPC.”

258 T 386/94, OJ EPO 1996, 658: "The Board considers that the patent specification discloses the cloning of the cDNA encoding an almost full length preprochymosin gene with the help of standard protocols. No specific experimentation is described, but adequate reference is given to all pertinent techniques. Detailed information on how to construct the vectors necessary to produce chymosin or its precursors in E.coli, starting from the originally cloned cDNA is also provided, alleviating the need for a deposition of the recombinant clones. The protocol for recovery of the recombinant proteins is described. The written specification, thus, teaches that the genes encoding preprochymosin and its maturation forms may successfully be expressed in a biological environment which is phylogenically extremely far apart from the one, they were isolated from (E.coli versus calf). Moreover, it suggests the possibility of expressing said proteins in micro-organisms, in general. The state of the art, on the other hand, contains no evidence that foreign genes cannot be expressed in other organisms than E.coli. To the contrary, both Appellants I and III seem to accept that expression in alternate hosts had already been tried. The Board, thus, believes that one way to carry out the invention is clearly indicated and that there exist no serious doubts that the invention could eventually be carried out with other micro-organisms than E.coli”. 
The generalisation to disparate organisms that has attracted by far the greatest amount of public attention is the Onco-mouse decision, T 19/90. The claims were worded so as to include all non-human mammalians, genetically changed to have an activated onco-gene sequence. This change made the organism particularly sensitive to carcinogenic substances. But the description only contained examples of how this change could be done on mice. The Examining Division was not convinced that all non-human mammalians could be made to have an activated onco-gene sequence. There were material differences as to the number of genes and the immune system. Therefore the application was refused because the claims were too broad. The Examining Division did, however, mention that it would have been willing to grant the patent if the claims had been limited to rodents. The Board of Appeal came to a different conclusion and granted the patent in accordance with the applicant's claims. The Board made a reference to T 292/85 (the one-way-rule) and reasoned that the application could not be refused simply because the claims appeared broad. It was necessary for the patent office to refer to technical circumstances that showed that there was considerable uncertainty as to the possibilities to use the invention. Furthermore the Board stated that it was unclear why the Examining Division had been willing to accept a generalisation to all rodents. It was deemed arbitrary to expect that all rodents would have the same biological characteristics, but at the same time deny that all mammalians would. Thus, claims to all nonhuman mammalians were accepted. ${ }^{259}$

\subsubsection{Remarks}

The legal situation. The use of functional features in claims appears to be growing more and more frequent. This may be because patent applicants are more aware of the opportunities that functional features offer, or it may be because many objects today are normally described by their function rather than by their structural design. Functional features obviously have much practical merit, but it is still quite unclear how they should be treated legally. It is difficult to summarise and draw any conclusions from the EPO case law in this field. ${ }^{260}$ The European patent system does not appear to have yet formed any clear view on when an invention and its scope of protection may be delineated by the technical effect that is achieved. Mainly it is an in casu assessment where the Boards have taken into consideration what uses there are for the invention and how they can be defined in the claims. Particularly functional terminology has been allowed in the field of biotechnology. But this has also meant that claims in biotechnology often have been considered broader than in other technical fields. ${ }^{261}$

The study that has been made above has at least led to the conclusion that the Boards of Appeal have not accepted the one-way-rule. One way to reach a new effect is not

259 T 19/90, OJ EPO 1990, 476: "The Board, in contrast to the Examining Division, considers that the ... invention clearly indicates how the skilled person can achieve chromosomal incorporation of an activated oncogene sequence into the genome of a non-human mammal, disclosing as it does an activated mouse mycogene introduced into a suitable plasmid and then micro-injected into mouse eggs at a given stage of cellular development. Firstly, this ensures that the invention can be reproduced on mice. Secondly, it may be assumed that the skilled person is aware - in the same way as in case T 292/85 - of other suitable mammals on which the invention can likewise be successfully performed. There is thus no reason why the application should be refused on the ground that it involves an extrapolation from mice - as particularly featured in the application - to mammals in general."

260 See Wibbelmann, J., [1997] EIPR 520 and Jaenichen, H-R., The European Patent Office's Case Law on the Patentability of Biotechnology Inventions, 322.

261 See Barton, J. H., IIC 1995, 605. 
sufficient to allow a claim to all possibilities to achieve the result. ${ }^{262}$ If that had been the case, there would no longer be a required relationship between what uses the applicant has found and the scope of protection. Another part of the case law is built on the requirement that all "essential features" of the invention must be mentioned in the claims. The expression "essential features" is to be found in the Guidelines. ${ }^{263}$ But this can hardly be a workable legal rule. There are no parts of an invention that are a priori essential and others non-essential, at least it has yet to be explained in case law how this determination is to be arrived at. "Essential features" therefore become those which the patent office finds necessary to include in the claims in order for the scope not to be too broad in relation to the contribution made by the inventor.

A third possible rule is implicit in the judgement of the House of Lords on 31 October 1996 and was made explicit in T 435/91: functional features may not be used if they only amount to an invitation to search for alternative solutions to those which have been mentioned in the description. The interesting aspect of this rule is that it is related to the future inputs in the technology. There is also support in the literature for the notion that the assessment of functional features must be made by looking at the possible future technical solutions that may have the effect mentioned in the claims. ${ }^{264}$ With such a perspective it may be possible to locate a workable solution, because the problem with functional features is that the scope of protection will include solutions which competitors to the patentee may need incentives to develop. It is the future technical developments that may suffer from functional claims.

An assessment where a relationship is defined between the solutions that are available to the man skilled in the art on the filing date and those that may be developed in the future is valid, because it is normative, i.e. a true rule. That makes it different from only a reference to the need to include all essential features in the claims. Furthermore it is a rule that addresses the problems associated with functional features: the possible hindrance of future technical developments. One problem with this rule, however, is that it will be impossible to assess the claims solely with reference to the state of the art on the filing date. The patent office has to attempt a forecast of future technical developments. Will there be new solutions to the problem that the applicant has solved in one way, and will these junior solutions be so different from the applicant's that it would be improper to include them in the scope of protection? This is not an easy assessment to make, but it is probably the most pertinent one for which case law provides support.

In this assessment there are a number of factors to be considered. Implicitly it is made clear in the rule that some expansion of the effective scope of protection is acceptable during the lifetime of the patent, i.e. after the application day and as new solutions within the

\footnotetext{
262 Cf Jaenichen: "[I]n general, it could be said that the EPO takes a pragmatic position as regards the allowable breadth of claims relating to biotechnology inventions. Functional features are allowable in the claims for characterizing the invention. The functional language may also encompass embodiments which remain to be developed in the future, e.g. the term 'heterologous DNA' would also encompass DNA sequences encoding not yet known polypeptides which have not yet been 'invented'. When a group of compounds is claimed, a single embodiment usually provides sufficient support, i.e. the 'one way-rule' is applied." See Jaenichen, H-R., The European Patent Office's Case Law on the Patentability of Biotechnology Inventions, 143.

263 Guidelines C III 4.4.

264 "Exceptionally ... a patentee who discovers one method of reaching a known desired goal may be entitled to a claim covering all methods if no other form of claim could protect his invention. That could be the case if once one method have been found to open the door to others to find other methods of achieving the same result. In such a situation, unless the patentee were permitted to claim the product defined in terms of the result, the benefit of the invention could be lost to competitors and the objectives of the patent system, of giving a limited monopoly in return for disclosure, would be frustrated.” See Lunzer, R., Singer: The European Patent Convention 56.10A.
} 
functional feature become known. What is required is a certain relationship between the known and as yet unknown solutions; there is no prohibition of a scope of protection that includes solutions developed in the future. Nor is it in conflict with the rule that some of the new solutions are unobvious to the man skilled in the art, i.e. will be patentable as dependent inventions. What is required, however, is an assessment of how many such new uses there may be and how valuable they will be, in relation to the solutions that the applicant has disclosed. It should be difficult to imagine any new, important and principally disparate ways to achieve the effect, if the functional feature is to be allowed.

Perhaps the most material part of this assessment, though, is that it should not be possible to use a more concrete wording of the claims. A structural definition must always be the main rule. There must be reasons particular to the case for functional features to be allowed. A structural definition has to be inadequate for an efficient scope of protection. But if this is the case then it is reasonable to make an assessment of the relationship between the solutions that are available to the man skilled in the art on the filing day and the ones that may be developed in the future. The greater the number of known solutions in relation to those that can be expected to be developed in the future, the more acceptable the functional feature becomes.

Such a limitation to the use of functional features is motivated by the fact that the applicant will only be able to exploit that which is available to the man skilled in the art on the filing date. Important incentives for marketing are created when a patent is granted, but these incentives can of course only be effective in relation to uses that are known to the applicant. It is therefore fair to assume that the only uses of the invention, which will be efficiently exploited by the patentee, are the ones that the man skilled in the art can understand from the application. The incentives for exploitation can justify the granting of a patent, but there is no such justification if a patent protects solutions that the applicant is unable to describe in the application. This will only limit the search by others for such new solutions.

With this view, the rule regarding functional features may become inefficient in two situations. Firstly, it is inefficient if the production opportunities that are available to the man skilled in the art on the application day do not fall within the scope of protection. In such a case the development and marketing incentives available in the patent system are not used. This may happen if functional features are not accepted, although a structural definition will not suffice to protect all that the man skilled in the art will be able to use with the information disclosed in the patent application. The other situation of inefficiency occurs if functional features create marketing incentives that the patentee for technical reasons is unable to use. Then the claims are too broad. Incentives should then instead remain available for later inventors that have proved their capacity to take advantage of those solutions. It is a balance between these two situations that will have to be struck when functional features are evaluated.

In a situation where small pharmaceutical molecules are patented the analysis appears to be adequate. If in such a situation the claims had the following wording: "compounds that inhibit gastric acid secretion to 70 per cent", it would be inefficient, because the applicant will only be able to use the compounds that he has found and close structural analogous to these. There are probably a number of other ways to achieve the effected mentioned in the claim, but the applicant has not shown that he is able to exploit those opportunities. Then he should not be awarded marketing incentives in this respect. The scope of protection should be defined structurally, i.e. by a chemical formula that defines the compounds found to give 70 per cent inhibition and the analogs that due to the discovery also can be used by the man skilled in the art. 
This analysis also corresponds to how assessments are made in case law. In T 409/91 it was not permissible to define the invention by the small size of the wax particles in the mineral oils that the applicant for the first time had been able to produce. The applicant had to mention in the claim the additive that was used. Probably other ways of producing similar oils would be developed in the future, but nothing indicated that the applicant had any such capacity. Perhaps he did not even have any incentive for doing so. The new product would compete on the same market as the additive that he had found to be one solution to the problem. On the other hand, it was very likely that the patent applicant would efficiently exploit the production opportunities that his additive represented. To grant a patent in respect of this use would therefore probably be a powerful marketing incentive for him.

The circumstances in T 409/91 may for the sake of argument be changed somewhat, by assuming that the applicant instead had found a number of effective additives but that they were very difficult to define structurally. This makes the assessment more difficult. One has to take into account that the applicant should be allowed to word the claims so as to gain marketing incentives in respect of all that can be used. If that is not done it is a risk that the new products will not reach the market. It could therefore be motivated to allow a claim to the result, i.e. "fuel with small wax particles".

The same kind of analysis can be performed with respect to patents for therapeutic proteins or DNA sequences. If a structural definition is used in the claims (the nucleotide sequence or the amino acid sequence), then the claims will be of the same kind as traditional claims to compounds with therapeutic effects. There is no reason to believe that such claims are too broad. The DNA sequences and proteins that are covered by such a scope of protection will be possible for the man skilled in the art to exploit. There will remain full incentives in relation to alternative DNA sequences or proteins with similar therapeutic advantages. However, for the patentee such structural claims may be inefficient, because the structure of the protein may be changed quite substantially without any loss of the therapeutic effect. In order for the scope of protection to be efficient, it may be necessary for the applicant to use the therapeutic effect as the limiting feature in the claim. It has been established above that such claims are also usually accepted by the EPO. The judgement by the House of Lords on 31st October 1996 shows, however, that it is not always permissible to use functional features related to the therapeutic result as a definition of a DNA sequence. The British court invalidated the British part of the EPO patent because the scope of protection was too broad in relation to the production opportunity that had been made available to the man skilled in the art. The relatively simple and quickly obsolete process that had been found by the applicant did not justify a scope of protection that included far more efficient means for production that could be used with knowledge of the DNA-sequence. This judgement corresponds well with the analysis of functional features that has been done here. Biogen's patent was revoked because the scope of protection would have meant a greater loss of research incentives for competitors than it would have meant development and marketing incentives for the patentee.

In decision T 128/92 (interleukin-2) the Board of Appeal made a comment that is related to this discussion:

"By providing the information in the patent, the Respondent has enabled the skilled person to make not only the polypeptide with the sequence recited in the claim, but to find many others that will have IL-2 activity. The protection granted should correspond to this disclosure. The fact that others, starting from the information in the patent might by research identify a DNA fragment encoding for a polypeptide with improved IL-2 activity infringing the present patent, is 
not a ground for refusing Claim 1. Such an improvement may deserve a patent of its own, but the possibility of such an improvement is no ground for limiting the present claim."265

It is explained that the patent applicant should be awarded a scope of protection commensurate with the production opportunities he has made available. Further, the Board stated that it is not a problem if new patentable proteins with IL-2 activity become dependent inventions in relation to the functionally worded patent. But it is hardly possible, as the Board did, to take for granted that such a dependency is appropriate. It may be better to allow competitors to search for alternative polypeptides and patent them without the patents becoming dependent. The acceptability of functional features must be determined by considering whether the patentee can be expected to efficiently exploit the known and future production opportunities within the scope of protection. If competition appears to be a more efficient regime, the claims should be narrowed. Such an assessment ultimately turns on technical circumstances. The following has been said in relation to the biotechnology field:

\begin{abstract}
“One very important conclusion, for purposes of assessing ... closely related forms of a protein, is that minor, conservative changes in protein sequences effected through either random mutations or site directed mutagenesis no longer can be treated as an inherently unpredictable feat. The thrust of several recent articles tend to minimise the impact of such conservative changes, concluding that a large number of conservative amino acid changes can be made without significant effects on the overall conformation and activity of the protein, as compared to the naturally occurring or wild type protein."266
\end{abstract}

In most cases the biological effect is not affected by minor structural variations. This resilience to changes conveys that it may be advisable to allow a co-ordination of the exploitation of the known and future production opportunities. It can be expected that the patentee exploits those production opportunities that can be made available through conservative changes in protein sequences. In other words, there are probably particular reasons to allow functional features in claims to DNA-sequences and therapeutic proteins. Much of the research would be routine and its outcome predictable and therefore perhaps not necessary to do in a competitive situation because it would be duplicative. This conclusion, however, still has as a prerequisite that it is difficult to formulate an efficient scope of protection by structural features. This is normally the case with biotechnological inventions, but if it is not, then a functional wording should probably not be accepted.

Furthermore, as the Board pointed out in $\mathrm{T}$ 128/92, competitors will not be completely without incentives. If a new DNA-sequence is found that can be used to manufacture a protein with an improved therapeutic effect, it will probably be inventive. The inventor would normally be able to secure a dependent patent and the better product can then reach the market after a licensing agreement has been reached.

The conclusion that can be drawn from all this is that patentees with claims to therapeutic proteins and DNA sequences usually can be expected to exploit efficiently the production opportunities covered by functionally worded patents. The research that may be necessary is of a relatively simple nature, and co-ordination may therefore be advantageous. It would probably be a greater risk if a structural definition was required. That might lead to a situation where there are not sufficient incentives. Only in exceptional cases - such as the situation tried by the House of Lords - will the production opportunity described in the application be of such limited value that future production opportunities within the scope of

\footnotetext{
265 T 128/92 of 30111994.

266 See Kushan, J. P., 6:1 High Technology Law Journal 1991, 122. See further Bowie, H., et. al., 247 Science 1990, 1306.
} 
protection will be more valuable and also difficult to develop. In such cases, more concrete features should probably be required in order to maintain the research incentives for others.

Particularly valuable dependent inventions. So far it has been concluded that functional features probably are justified when the structure can be modified quite easily and with a reasonably predictable outcome. It may, however, happen that a modification made by a competitor prompts an invention that is considerably more valuable that the invention described in the first patent. The variation may e.g. result in a protein with a considerably improved therapeutic effect. It may also happen that someone finds a particularly efficient manufacturing process for a patented protein. The inventor cannot in this situation exploit his invention until he has a licence from the first inventor. The therapeutically more efficient protein and the more effective manufacturing process will be dependent on the patent with functional claims to the protein.

In this situation it may be justified to apply the legal standard known in American patent law as "the reverse doctrine of equivalents", which has been considered particularly pertinent when the dominant patent has functional claims. ${ }^{267}$ The reverse doctrine of equivalence is most easily understood against the background of the well-known doctrine of equivalence. The "normal" doctrine of equivalence can be applied in an infringement case if the contested embodiment falls outside the literal scope of protection, but still, according to the court, takes advantage of the true value of the patented invention, see part 8.2.1.2. An application of the reverse doctrine of equivalence has the consequence that an embodiment falling squarely inside the literal scope of the claims is propelled outside the scope of protection.

Supreme Court:

The reverse doctrine of equivalence is based upon a statement by the US

\begin{abstract}
"The wholesome realism of [the doctrine of equivalents] is not always applied in favour of a patentee but is sometimes used against him. Thus, where a device is so far changed in principle from a patented article that it performs the same or a similar function in a substantially different way, but nevertheless falls within the literal words of the claims, the doctrine of equivalents may be used [in reverse] to restrict the claim and defeat the patentee’s action of infringement."268
\end{abstract}

The claims in a patent granted may be valid and not necessary to amend, but still an embodiment inside the literal meaning of the claims fall outside the scope of protection. The doctrine is valuable because it maintains a balance in infringement cases. ${ }^{269}$ It has been considered unfair that courts should only be able to reinterpret the literal meaning of the claims to the advantage of the patentee. There may be circumstances that warrant an adjustment to the advantage of the accused infringer.

The reverse doctrine of equivalence fulfils an important function as a remedy in situations where it is difficult for the inventor of a valuable dependent invention to reach an agreement with the owner of the dominant patent. The reverse doctrine of equivalence facilitates the market introduction of particularly valuable dependent inventions, when an agreement with the owner of the dominant patent probably could not be reached. ${ }^{270}$

In American patent law literature it has been said that the reverse doctrine of equivalence would be applicable if a patent has been granted on a DNA-sequence without any

\footnotetext{
267 See Janicke, P. M., 8 Harvard Journal of Law \& Technology 1994, 155, in particular part V C.

268 See Graver Tank \& Mfg Co v. Linde Air Products Co, 339 US 1950, 605.

269 See Merges, R. P., JPTOS November 1991, 880.

270 See Merges, R., 62 Tennessee Law Review 1994, 75, also published in Intellectual Property Law Review 1996, 69.
} 
mention of the use that is made of the DNA by the alleged infringer. ${ }^{271}$ That is a situation in which the reverse doctrine of equivalence appears particularly adequate, because the attacked embodiment is more valuable than the patented invention and quite different from what the skilled person could deduce from the patent. ${ }^{272}$ The doctrine of reverse equivalents could, for example, also have been used in the case Biogen $v$. Medeva in the House of Lords. The manufacturing process that was used by Medeva was considerably more valuable than the one disclosed in the patent. The scope of the claims could have been considered valid, but interpreted not to include hepatitis B antigen material made by a process that built on the knowledge of the DNA-sequence.

Summing up, the reverse doctrine of equivalence is appropriate when a technical development that falls within the literal scope of a previously granted claim has a considerably higher value than the uses that the skilled person can deduce from the patent. If the reverse doctrine of equivalence is not applied in such cases the market introduction of particularly valuable developments may be stifled. Thus, the reverse doctrine of equivalence is useful when the rules on the permissibility of functional features have not functioned well. If in retrospect it is obvious that the first patentee has not properly exploited the production opportunities that apparently existed within the literal scope of his patent, then the actual scope of protection should be narrower than the literal scope. If courts have the opportunity to modify granted claims in this way, the risks with allowing functional features will be less. If junior solutions form a considerable advance over the first invention, the dependency is lifted.

A judgement from the Italian Supreme Court (Corte di Cassazione) in 1990 is probably the only European judgement of any real significance where something similar to the reverse doctrine of equivalence has been applied. The attacked embodiment fell within the literal scope of protection, but had a substantially better therapeutic effect than the examples in the patent application. The claims were of the so-called Markush type and covered, according to the estimate of the court, 10 million compounds. The compound cimetidine, used for ulcer treatment, had not been synthesised when the patent application was filed, but fell within the scope of protection. The court decided that a man skilled in the art had no practical possibility to chose cimetidine with help from the information in the patent. It required a substantial amount of further research to find the particular effects of cimetidine. The court therefore concluded that the patent application had been handed in prematurely and cimetidine did not come within the scope of protection, even though it fell within the claim. ${ }^{273}$

It was probably unfortunate that the court applied something similar to the reverse doctrine of equivalence in this case. It was not a competitor that had found the particularly valuable characteristics of cimetidine. The patentee had discovered them during the lifetime of the patent. The patentee had thus not neglected to exploit any production opportunities. The other party was a generic manufacturer that had copied cimetidine after its valuable properties had been discovered. Thus, there was no need to apply the reverse doctrine of equivalence in order to facilitate market introduction of an improved but neglected solution within the granted claims.

The reverse doctrine of equivalence must be limited to a very small number of cases. It must never become normal infringement practice to narrow the literal wording of the claims. Probably the reverse doctrine of equivalence should neither be as frequently applied as the "normal" doctrine of equivalence. More commonly, the wording of the claims can be avoided but the value of the invention still taken advantage of, than something within the claims being used but the value of the invention is still not taken advantage of. Only if there is

\footnotetext{
271 See Maebius, P. B., JPTOS September 1992, 653.

272 See Merges, R. P., JPTOS November 1991, 888.

273 Corte di Cassazione Cimetidine of 1601 1990, GRUR Int. 1991, 497.
} 
a considerable "added value" in the contested embodiment is the reverse doctrine of equivalence justified. In these special cases the doctrine may, however, be of considerable importance. It may make competitors willing to explore and exploit improved production opportunities within the literal scope of patents granted. Knowledge of the existence of a doctrine of reverse equivalents could also influence the negotiating situations in these special cases and thereby facilitate the market introduction of particularly valuable new pharmaceuticals.

There may, however, be a disadvantage associated with the doctrine of reverse equivalents. The interpretation of patent claims may become more uncertain. The dependency of a selection or new use patent on a dominant patent will with this rule have some exceptions. Someone with a functionally worded claim can not be certain that considerably improved versions of his invention will be covered by his claims. This uncertainty, however, is not any different, as such, from the uncertainty that is caused by the well-known doctrine of equivalence. Thus one should probably not overestimate the problems associated with a reverse doctrine of equivalence. The doctrine has been established for some time in American patent law, however, it has not been applied much. 


\section{Novelty}

\subsection{The Novelty Principle}

So far we have considered how the invention may be formulated in the patent claims in relation to the ways in which the skilled man is capable of using it. Once a definition of the invention has been thus formulated in the claims, the next step is to test its novelty. The novelty requirement, and also the requirement of inventive step, derives its content from the concept of "the state of the art". The relation between the two is a palpable one in European patent law. The novelty requirement is simply a re-wording of the state of the art: that which is not part of the state of the art is new, as is plainly stated in Art. 54(1) EPC:

“An invention shall be considered to be new if it does not form part of the state of the art.”

Novelty requires the patent claims to include at least one essential technical feature not to be found in the state of the art. ${ }^{274}$ The technical features in the patent claim, must not all be contained in one document belonging to the state of the art. If a known document contains an explicit indication of another document, the two are not to be interpreted as separate documents. A separate document, then, need not be a physical document, but it must be a "linked" part of the state of the art. ${ }^{275}$ The limitation of the novelty assessment to just one document at a time implies, for example, that two substances which per se are already known but are indicated together for the first time in the patent claim are new - that is to say, the combination is new. Novelty can also be a combination in the patent claims of a certain known active substance and a certain known formulation, so long as the active substance and the formulation have not previously been used together. ${ }^{276}$

All features are in principle considered equal. No qualitative valuation is made concerning the importance of a feature for the function of the claimed invention. But since the extent of protection is also governed by the claims, the patent will be easily circumvented if the new technical feature is not material for the functioning of the invention. In this sense the assessment of novelty is "self-regulating”. If a technical solution can be put to practical use without any of the features included in the claims being used, a third party can use the valuable part of the invention without being guilty of infringement; in other words, the patent

\footnotetext{
${ }^{274}$ G 2/88, OJ EPO 1990, 93: “A claimed invention lacks novelty unless it includes at least one essential technical feature which distinguishes it from the state of the art.”

${ }^{275}$ Guidelines C IV 7.1: "It should be noted that in considering novelty (as distinct from inventive step), it is not permissible to combine separate items of prior art together .... However, if a document (the 'primary' document) refers explicitly to another document (e.g. as providing more detailed information on certain features), the teaching of the latter may be regarded as incorporated into the document containing the reference. Equally, it is permissible to use a dictionary or similar document of reference in order to interpret a special term used in the primary document." T 153/85, OJ EPO 1988, 1: "When assessing novelty, the disclosure of a particular prior document must always be considered in isolation; in other words it is only the actual content of a document (as understood by a skilled man) which destroys novelty. It is not permissible to 'combine' separate items of prior art together. However, in a case such as the present, where there is a specific reference in one prior document (the 'primary document') to a second prior document, when construing the primary document (i.e. determining its meaning to the skilled man) the presence of such specific reference may necessitate that part or all of the disclosure of the second document be considered as part of the disclosure of the primary document."

${ }^{276}$ T 261/87 of 1612 1988: "The Board has already held allowable ... a product claim for a therapeutic product novel by way of its form of application, in spite of the active ingredient contained therein being known. In applying this principle to the present case, the claimed product as a whole - i.e. peppermint oil in enterically coated capsules - being novel, ... Articles 52 and 54 EPC do not preclude protection by a per-se product claim.”
} 
has no substantial value. In this way, indirectly, the new technical feature is subjected to a qualitative evaluation.

Due to the precision with which a substance can be defined structurally in chemistry, the novelty requirement in connection with product patents tends to become an assessment of the identity of two substances: if there is the slightest difference, the novelty requirement is satisfied. If, for example, someone has manufactured a heterocyclic organic compound which at one point is substituted with a propyl group, novelty is lacking if an identical compound is to be found in the state of the art. It is also the main rule that there must not be any overlap. In other words, there must not be individual substances which belong to the state of the art and at the same time to the patent claims (exceptions can be made for selection inventions; see section 4.7). Novelty exists, however, if the state of the art contains the same heterocyclic basic structure as in the claimed substance, except that one position in the claimed substance has a methyl group instead of the propyl group.

True, the state of the art has to be construed from the point of view of the skilled man, but this does not imply the inclusion of what the skilled man knows to be equivalent alternatives. If the skilled man has an exact notion of the significance of a feature in the state of the art, equivalent alternatives beyond the scope of the definition are to be deemed new. The critical issue in the assessment of patentability will then be inventive step. ${ }^{277}$

\subsection{The state of the art}

\subsubsection{Absolute and global novelty - an extensive definition of the state of the art}

The possibility of patenting a technical solution which was known abroad ceased to exist in the majority of countries at the end of the 19th century. Novelty now began to be assessed, and the state of the art established, at global levels. Certain types of disclosure, however, e.g. public lectures, or the demonstration and exhibition of inventions, were often regarded as not detracting from novelty. Disclosure occurring neither in the form of a printed publication nor through the practical utilisation of the invention did not form a part of the state of the art. Only in recent years has it come to be internationally accepted that all information about an invention is equally detrimental to novelty. The global and absolute novelty requirement applied nowadays dictates that everything which is or has been possible for a skilled man to find out anywhere in the world - whatever the form of disclosure - belongs to the state of the art. Art. 54(2) EPC provides:

"The state of the art shall be held to comprise everything made available to the public by means of a written or oral description, by use or in any other way, before the date of filing of the European patent application.”

Right from the beginning of the EPC negotiations, in 1960, the principle of absolute novelty was made a point of departure. Several countries, France and Italy among them, already had a requirement of absolute novelty in their legislation at the time and were therefore reluctant to

\footnotetext{
${ }^{277}$ Guidelines C IV 7.2: “A document takes away the novelty of any claimed subject-matter derivable directly and unambiguously from that document including any features implicit to a person skilled in the art in what is expressly mentioned in the document, e.g. a disclosure of the use of rubber in circumstances where clearly its elastic properties are used even if this is not explicitly stated takes away the novelty of the use of an elastic material. The limitation to subject-matter 'derivable directly and unambiguously' from the document is important. Thus, when considering novelty, it is not correct to interpret the teaching of a document as embracing well-known equivalents which are not disclosed in the documents; this is a matter of obviousness.”
} 
accept any other solution. Added to this was the necessity of ensuring that a patent granted by the organisation one intended to create would not be declared invalid by national courts. Accordingly, the novelty requirement was adjusted "upwards". 278

The absolute novelty requirement, irrespective of the form of disclosure, is now firmly rooted in EPO case law. Its latest manifestation came in the decision by the Enlarged Board of Appeal in G 1/92. In T 93/89 the Board of Appeal had ruled that a commercially available chemical mixture (an adhesive) did not detract from novelty until the skilled man had special commercial reasons for investigating the chemical composition of the mixture. ${ }^{279}$ That pronouncement prompted the President of the EPO to ask the Enlarged Board of Appeal the following question:

"Is the chemical composition of a product made available to the public by virtue of the availability to the public of that product, irrespective of whether particular reasons can be identified to cause the skilled person to analyse the composition?”

The Enlarged Board of Appeal replied that, in order for the invention to belong to the state of the art, it was quite sufficient for the invention to be available to the skilled person. No further requirements would be made in precedent. The skilled person did not need to have any special reason for arranging for the investigation of a product generally available on the priority date. Thus the Enlarged Board found the ruling in $T$ 93/89 to be erroneous. The novelty requirement was a question of the skilled man's potential access to the invention somewhere, at some time, before the priority date. But the Enlarged Board of Appeal added that a generally available chemical compound did not imply the making available to the public of more than the chemical structure of that compound, i.e. its composition. If a patent application indicates a new use, e.g. a new medical indication, for a known substance, the new use is patentable. Thus a chemical structure in itself does not detract from the novelty of the potential uses of the substance. In the assessment of novelty, a distinction must be made between a product and its possible uses. ${ }^{280}$

\footnotetext{
${ }^{278}$ See Lunzer, R., Singer: the European Patent Convention 54.01.

${ }^{279}$ T 93/89, OJ EPO 1992, 718: “The mere fact that a company launches a new commercially available product is not necessarily sufficient reason for its competitors to test the product to determine its ingredients. There is no evidence to suggest that companies test all their competitors' new products, at least as far as the chemical composition of new commercially available products is concerned. There would need to be a specific reason to justify such an analysis, which after all costs money. In the present case the Board can see no such reason. Of course competitors are interested in comparing new products with their own, but a chemical analysis is not absolutely necessary for this purpose. Initially it is sufficient to establish the properties of the new product, in this case a new adhesive whose properties can be determined without analysis. The appellants did not submit that their new commercially available product 'Ponal express' had such special properties that a competitor would have had reason to carry out a chemical analysis. The Board does not therefore see the mere appearance of a new commercially available product as grounds for a chemical analysis. ... Competitors look at the market from the commercial angle, not with patent specifications clutched in their hands. Only when a product is of interest because of its special properties and is then tested and analysed on those grounds might attention turn to the prior art to discover whether imitation is allowed."

${ }^{280}$ G 1/92, OJ EPO 1993, 277: "Where it is possible for the skilled person to discover the composition or the internal structure of the product and to reproduce it without undue burden, then both the product and its composition or internal structure become state of the art. There is no support in the EPC for the additional requirement referred to by Board 3.3.3 in case T 93/89 ... that the public should have particular reasons for analysing a product put on the market, in order to identify its composition or internal structure. According to Article 54(2) EPC the state of the art shall be held to comprise everything made available to the public. It is the fact that direct and unambiguous access to some particular information is possible, which makes the latter available, whether or not there is any reason for looking for it. ... It may be added that a commercially available product per se does not implicitly disclose anything beyond its composition or internal structure. Extrinsic characteristics, which are only revealed when the product is exposed to interaction with specifically chosen
} 


\subsubsection{The skilled man must be able to use the state of the art.}

Everything, then, that was "generally available" on the priority date is detrimental to novelty. This being said, there are two stages involved in the assessment of novelty and the definition of the state of the art. The first is an assessment of what is meant by "generally". The main rule says that information is general if the group with access to it was not limited in any respect, e.g. by secrecy agreements. Legal issues arising in connection with this assessment are unaffected by the invention being a pharmaceutical, and so the problem will not be dealt with here. ${ }^{281}$ It is worth noting, however, that there is a German case in which the Federal Patent Court found that an assignment to a hospital to carry out clinical trials of a pharmaceutical which was not yet commercially available should be deemed to be based on such a relation of trust between the client and the hospital that the study and its results did not become a part of the state of the art. There was deemed to exist an implicit agreement for secrecy. ${ }^{282}$ Similarly, in an English case, it was found that private conversations about their current work between academics working on the same hospital or academics writing jointly on a publication, had an implicit confidential nature.283 Such discussions might well be regarded by them, or by reasonable people in their position, as "confidential".

Two other cases relating to micro-organisms also deserve to be mentioned. In case $\mathrm{T}$ 128/92 it was decided that if a micro-organism is obtainable from several different institutions, this indicates that it is publicly available, even if the institutions in question are not obliged to maintain the organisms, as are the special deposition institutes. ${ }^{284}$ A related case was T 815/90. The inventors were scientists associated with the National Institute of Health (NIH). They had not made any deposition of the micro-organisms, but claimed that the funding they had received from the NIH obliged them to distribute the material in question to the public if they were asked. According to the applicant this made the material publicly available. However, the Board of Appeal had three reasons to not equated the conditions associated with research funding with a deposition: NIH accepted that researchers could be bound by further obligations if they were being funded by other sources; NIH had no

outside conditions, e.g., reactants or the like, in order to provide a particular effect or result or to discover potential results or capabilities, therefore point beyond the product per se as they are dependent on deliberate choices being made. Typical examples are the application as a pharmaceutical product of a known substance or composition ... and the use of a known compound for a particular purpose, based on a new technical effect .... Thus, such characteristics cannot be considered as already having been made available to the public.”

${ }^{281}$ See instead Straus, J., GRUR Int. 1994, 89 ff.

282 Bundespatentgericht 3 Ni 38/87, of 1703 1987, Mitteilungen des deutschen Patentanwälte 1988, 207.

283 Patents Court, Visx -v- Nidek and Birmingham Optical Group and Optimax Laser Eye Clinics, of 1910 1998.

284 T 128/92 of 3011 1994: “[O]n the evidence, the Jurkat cells were known to be available on request from three different institutions. The Respondent itself acknowledged in the patent ... that cells were obtainable from these institutions, and itself so obtained the cells on which it carried out further research. The fact that there were three different sources from which the cells could be obtained, is a strong indicator for public availability. Even though there is no evidence that any of these institutions were under any duty to maintain these cell lines and make them available to the public, as it would have been required in case of a deposit of the cell line according to Rule 28 EPC, the Board treats the acknowledgement in the patent, uncontradicted by any other evidence, as sufficient proof that the cell line and thus the enriched IL-2 mRNA source of document (19) were publicly available." 
obligation to keep the micro-organisms alive in the future; and NIH had the opportunity to change its policy in the future. ${ }^{285}$

The second question arising in the assessment of state of the art is whether the technical content of the document is "available" in the sense that the skilled man can make practical use of it. The skilled man must have had the technical capacity necessary for using that which is included in the state of the art. If he does not possess such knowledge, the information cannot belong to the state of the art. The state of the art consists solely of practical rules for action. If, for example, it was never possible to procure the necessary input material for manufacturing a product indicated by a chemical formula, that product does not form part of the state of the art. "Paper chemistry" which cannot be realised by a skilled person does not belong to the state of the art.

In the EPO the rule is that if, on the priority date, there was a document indicating certain substances and, moreover, it was possible for the skilled man to manufacture them, those substances are known. ${ }^{286}$ In the Guidelines, this principle is expressed as follows:

"It should be noted that a chemical compound, the name or formula of which was mentioned in a document, is not considered as known unless the information in the document, together, where appropriate, with knowledge generally available on the effective date of the document, enable it to be prepared and separated or, for instance in the case of a product of nature, only to be separated.,287

EPO precedent has established that the requirement for the man skilled in the art to be able to use the content of the state of the art is the same as the requirement made for a patent, under Art. 83 EPC. The same degree of clarity and practical usefulness is required concerning the possibility of using the invention in the application filed and the possibilities of using that which is part of the state of the art. The rule was formulated and established in T 206/83. ${ }^{288}$ The Boards of Appeal have invoked the rule in a large number of cases. The concurrence of the two assessments makes it possible, in an assessment as to whether something belongs to the state of the art, to consult the rules which have already been described in section 2.2, and

285 T 815/90, OJ EPO 1994, 389: “As becomes apparent from the paper 'NIH policy relating to reporting and distribution of unique biological materials produced with NIH funding' ... investigators are reminded that unique or novel biological materials and their products are considered to be inventions and therefore are subject to the various laws and regulations applicable to patents. Accordingly, the NIH requires that grantees and contractors adhere to grant regulations and contract clauses, respectively, pertaining to the reporting of inventions to the NIH. In addition and of equal importance, nowhere is there any obligation on the NIH to ensure that the biological material necessary to carry out the invention in the present case is cultured and kept alive. Finally, the Board notes that NIH policy of releasing biological material developed within NIH research programmes may be changed at any time in such a manner that the release of newly developed biological material could be restricted in any way whatsoever."

${ }^{286}$ T 181/82, OJ EPO 1984, 401: “[T] alkylation for the above-mentioned spiro compounds. This information, which the person skilled in the art can unquestionably put to practical effect, places the designated 8-methyl spiro compounds within the state of the art, irrespective of whether they have been manufactured.”

${ }^{287}$ Guidelines C IV 7.3.

288 T 206/83, OJ EPO 1987, 5: "Claim 1 of the application in suit embraces a number of chemical compounds which were individually identified in Dow's earlier patent application. There is, however, no dispute either that a compound defined by its chemical structure can only be regarded as being disclosed in a particular document if it has been 'made available to the public' in the sense of Art. 54(2) EPC. In the field of chemistry this requirement is, for instance, satisfied if a reproducible method is described in the same document. This need for an enabling disclosure not only applies to documents cited under Art. 54(2) and (3) EPC but is also in conformity with the principle expressed in Art. 83 EPC for patent applications which must, accordingly, 'disclose the invention in a manner sufficiently clear and complete for it to be carried out by a person skilled in the art' .... The requirements as to the sufficiency of disclosure are, therefore, identical in all these instances.” 
so there is no need here for any further rehearsal of the characteristics which the man skilled in the art shall be presumed to possess or of the work input which can be expected of him when assessing his ability to make use of certain information. Instead a few examples will be quoted from case law on the question of whether the man skilled in the art was able to make practical use of a certain prior document, thus making it prior art.

T 301/87 was a case in which the work input was too extensive for the claimed procedure to belong to the state of the art. The patent application referred to certain DNA sequences which coded for polypeptides with IFN alpha activity (these can, for example, be used in the treatment of cancer). The Examining Division refused the patent partly because novelty was lacking. In a DNA library of 240,000 gene fragments, DNA existed which coded for polypeptides of the type referred to in the claim. The Board of Appeal found, however, that it was not possible for the man skilled in the art to locate the gene in question in this large collection. A comparison was made with the assessment of novelty applied to substances occurring naturally: these are not available merely because they exist in nature. Accordingly, a DNA library does not mean that the DNA sequences included in the library are part of the state of the art. In the assessment of novelty, a detailed assessment had to be made of the work input which would have been necessary to enable the man skilled in the art to make practical use of the genetic sequences. ${ }^{289}$

Another example of genes which are accessible in a certain respect but are not to be deemed available in terms of patent law is the human genome. The genes of the genome are not known merely because they occur in human cells. The decisive question is whether the structure and properties of the individual gene or protein are known:

\begin{abstract}
"[E]ven if claims 1-7 are interpreted as including in their scope genomic DNA fragments encoding H2relaxin, there is no question of lack of novelty of these claims. ... It is common ground amongst the parties that until a cDNA encoding human H2-relaxin and its precursors was isolated by the proprietor, the existence of this form of relaxin was unknown. It is established patent practice to recognise novelty for a natural substance which has been isolated for the first time and which had no previously recognised existence. ${ }^{290}$
\end{abstract}

The considerations to be made when assessing whether a man skilled in the art can make practical use of a prior document were elaborated in $\mathrm{T}$ 158/91. The patent application pertained to a biotechnological procedure for producing human growth hormone. An earlier patent indicating a generally serviceable method for manufacturing proteins was invoked as a

\footnotetext{
289 T 301/87 OJ EPO 1990, 335: “[T]he public, represented by a person skilled in the art, does neither get any indication at all that 'Lawn's gene bank' comprises any clone containing DNA sequences which code for a polypeptide of the IFN-alpha type (leukocyte interferon) nor does the public have a reasonable chance to trace out such DNA sequences within 'Lawn's gene bank' by means of their hybridisation properties. ... The assumed presence of some fragments satisfying the criteria of the claim is not like the incidental availability of an unindexed book in a library. The interrogation of a library material is, at least for some members of the public, a direct mental procedure. The collection in the present case must be interrogated by physical interactions, and a consequent biochemical process in each case. Although any vial containing the relevant phage is a separate entity here, it is impossible to get to the vial without working through tens of thousands of samples. The circumstances are such as if the material were under lock and where the key has to be first manufactured and applied. If anything, the situation resembles that prevailing with natural substances, since the availability of phages is not direct, and is rather like the isolation of a component or bacterium from soil where the same exists in admixture with other useless materials. Thus, the idea that the gene bank itself would once for all anticipate an invention relating to a nucleotide sequence which may be contained therein somewhere, cannot be sustained. Accordingly, the mere existence of a DNA sequence coding for a polypeptide of the IFN-alpha type, within the multitude of clones of 'Lawn's gene bank' cannot automatically mean that the chemical compound (polynucleotide) concerned does become part of the state of the art. The latter would only then be the case if the existence of the compound concerned had recognisably been made publicly available."

${ }^{290}$ Opposition Division V 8/94, OJ EPO 1995, 388.
} 
prior document. The earlier patent contained practical examples for the production of proinsulin, but it also stated that other proteins could be manufactured by this method, e.g. human growth hormone, i.e. the same protein as was referred to in the application at issue. The applicant maintained that novelty existed because the earlier document did not contain enough information to enable the man skilled in the art to manufacture human growth hormone by means of the procedure in the senior patent. The Board of Appeal found that biotechnology at the time of the filing of the application (in 1983) was indeed an unusually complex field of technology, but it arrived at the conclusion that, in the present instance, the man skilled in the art could have fabricated human growth hormone with the aid of the earlier patent. The present patent application was best described as a confirmation of the practical feasibility of the procedure indicated in the earlier patent. ${ }^{291}$

It is worth noting that in T 1058/91 the Board of Appeal assessed the possibility of the man skilled in the art putting into practice a teaching which possibly belonged to the state of the art, taking as its starting point the date on which the prior document became generally available. It is well known that the content of technical writing - patents for example - can acquire a new meaning and become easier to put into practice when they are regarded in the light of information appearing subsequently. It is no uncommon situation for a technical solution in a patent to successively acquire new applications. As a consequence, the man skilled in the art will be able to use a known invention in several new ways as time goes on. The EPO, however, has an established rule to the effect that such subsequent "additional information" does not affect the practically useful content of a document invoked as a prior document in novelty assessment. If the prior document dates from the end of the 19th century, the possibility of the man skilled in the art using the document shall also be assessed according to what men skilled in the art were able to do in the 19th century. It shall not be read in the perspective of the man skilled in the art on the day when it is invoked as prior art. $^{292}$ In T 677/91 it was declared:

\footnotetext{
291 T 158/91 of 3007 1991: “The average amount of time and effort to reproduce certain recombinant DNA processes for the expression of protein is usually very high. The Board is well aware of the fact that on the basis of a publication describing a successful expression no reliable predictions are possible as to whether the repetition of this teaching would also lead to successful expression of another gene prepared by recombinant DNA technique. In the present case, however, the DNA sequence of human growth hormone was already known, so that inter alia from the knowledge of certain restriction sites a reasonable extrapolation with respect to a successful application of the recombinant DNA technique described in document (7) was possible. ... Thus, the Board confirms the Examining Division's view that the fact that the mentioned documents provide information that the teaching of document (7) did not work in each and every case does not mean that the disclosure of document (7) did not work in a sufficiently reliable manner in other cases. The Appellants do not deny that at least in some cases the teaching of document (7) works. ... Although the parameters for a successful construction of the respective DNA sequence are complex and may not in each and every case of a protein lead to success, the Board comes to the conclusion that the mentioning of the protein human growth hormone in document (7) apparently was not done merely speculatively, as emphasised by the Appellants, but rather that the selection of proteins in document (7) as being suitable for an analogous process as described in detail for the protein rat preproinsulin was based on such proteins whose DNA sequence was already known or which were otherwise investigated to such an extent that an extrapolation was reasonable from the details given for the preparation of the gene coding for rat preproinsulin to the genes coding for the other proteins mentioned in document (7). Thus, although the description of document (7) does not provide precise technical steps for the preparation of the gene coding for human pregrowth hormone, the Board believes that a skilled person was equipped with the knowledge of the already known DNA sequence for human growth hormone and his common general knowledge in connection with the detailed description in document (7) as far as the other tools were concerned.”

${ }^{292}$ Guidelines C IV 7.3: "In determining novelty a prior document should be read as it would have been read by a person skilled in the art on the effective date of the document. By 'effective' date is meant the publication date in the case of a previously published document and the date of filing (or priority date, where appropriate) in the case of a document according to Art. 54(3).”
} 
"In the Board's judgement, when considering whether a prior document discloses the technical features of a claim, the disclosure of a prior document must be determined at the date of publication of that document. The disclosure of a document does not change from time to time. The technical content of a document is what is disclosed to a skilled person at the time when it was written and published. In contrast, whether the technical features of a claim are obvious in view of a prior document of course has to be determined at the filing date of such claimed subject- matter."293

This rule for the assessment of novelty is open to criticism. ${ }^{294}$ Firstly, it is at times difficult to know when a prior document was first made public. Secondly, the rule could imply that the content of the state of the art changed if the same document was made public more than once, because associations made by the man skilled in the art will be different at different points in time. Thirdly, the rule is theoretical and not closely related to practical conditions: a person intending to use the technical doctrine in a document will not hesitate to do so merely because a man skilled in the art would not have put the same construction on the document at an earlier point in time. The idea behind the assessment of novelty is that things which are known shall not be patentable. All in all, it is difficult to see any tenable grounds for not assessing the novelty-harming content of a document in the same way as when assessing inventive step, i.e. interpreting the document according to what the man skilled in the art could deduce from it on the priority date of the application at issue in the case. There is no practical problem, nor any theoretical one either, entailed by the novelty-detrimental content of a document developing with the passage of time.

\subsection{Novel technical features in product patents for active substances}

Each new inventor builds on what his predecessors have done. Often it happens that one inventor finds a substance with a therapeutic effect, whereupon another varies the structure and finds another substance with an essentially similar therapeutic effect. This is how competition operates between pharmaceutical research enterprises. One problem, however, in the assessment of novelty may be that different characteristics of the same substance become available at different points in the research process. First perhaps the melting point, the molecular weight and biological activity are found, and it is not until some time afterwards that the structural formula becomes known. This means that one and the same substance may come to be known by several different definitions.

The assessment of novelty in these situations is based on it always being physical products, not chemical definitions, that are patented. A new definition of a previously known product does not meet the novelty requirement. A definition in the claims is merely an aid to the assessment of whether the product has previously been available to the man skilled in the art. Demonstration of a new structural characteristic or a certain characteristic property of a product which it was not previously known to possess or which has not previously been used in defining the product, does not mean that the product as such is a new one. ${ }^{295}$ It may very well be that the same product has previously been defined in

\footnotetext{
${ }^{293}$ T 677/91 of 03111992.

${ }^{294}$ See Rogge, R., IIC 1997, 446.

295 T 722/94 of 1612 1997: "[T] 1 he range of percent values of amino acid defined in present claim 1 does not involve any new feature or discriminating technical effect with regard to the teaching in (4). ... In other words, the alleged difference implied by the indicated amino acid amounts lies only in a different wording."
} 
another way. What we have to ask ourselves, then, is whether, in a certain case, it is only the feature, or also the product, that is new in the new definition. ${ }^{296}$ In T 12/81 it was declared:

"[T]he concept of novelty must not be given such a narrow interpretation that only what has already been described in the same terms is prejudicial to it. The purpose of Article 54(1) EPC is to prevent the state of the art being patented again. Article 54(2) EPC defines the state of the art as comprising everything made available to the public before the date of filing in any way, including by written description. There are many ways of describing a substance in chemistry, and this is usually done by giving its precise scientific designation. ... If inventions relating to chemical substances defined by claims of this kind are patented, it necessarily follows that the resulting patent documents, once they enter the state of the art, will be prejudicial to the novelty of applications claiming the same substance although in a different and perhaps more closely defined form." 297

On the basis of the man-skilled-in-the-art criterion, the assessment of novelty must transcend a formal comparison of different definitions and look for the concrete invention. ${ }^{298}$

The rules presented in section 3.2.3, concerning the definition of the invention

in the patent claims, are among other things aimed at making it possible for patents, once filed, to be subsequently used for assessing the novelty of other inventions. Patent applications must be sufficiently clear to serve as prior documents later on. For this it is important that a uniform terminology is employed, as far as possible. The "standard definition" in pharmaceutical patents takes the form of structural formulae. These indicate the possession of the atoms making up the molecules. It is structural differences that establish novelty, not a new definition of a structure otherwise defined previously. Structural formulae, however, are not the only acceptable definition of a chemical substance in the patent claims. Structural formulae are e.g. difficult to use when the invention consists of macro-molecular substances. These may contain tens of thousands of atoms. In these case the applicant can instead indicate how the substance is manufactured (a product-by-process claim) or else indicate typical properties - parameters - of the substance, e.g. UV spectrum and melting point.

When, in case law, definitions other than structural formulae gradually began to be accepted. There were fears that novelty assessment of junior applications would no longer be feasible. The different definitions were in danger of creating a confusion of concepts. But so far, at least - such fears have not proved justified. It has been possible to examine the identity between a claimed substance and the content of the state of the art, in spite of structural formulae for the substances in the state of the art not being available. This has been possible, thanks to the establishment of a rule whereby the applicant has the onus of proving that a claimed substance differs from those in the state of the art. It is for the applicant to show that the technical features which he has indicated in the claims and which distinguish his invention from the state of the art can only be accounted for as reflecting a structural difference between the invention and the state of the art. The Guidelines provide:

"In the case of a prior document, the lack of novelty may be apparent from what is explicitly stated in the document itself. ... Situations ... may also occur when the claims define the invention, or a feature thereof, by parameters.... It may happen that in the relevant prior art a

\footnotetext{
${ }^{296}$ See Rauh, P. A., Jaenichen, H-R., JPTOS May 1988, 318.

${ }^{297}$ T 12/81, OJ EPO 1982, 296.

298 T 184/84 of 0404 1986: "The Examining Division has started from the correct assumption that the inquiry as to identity cannot stop at a formal level and must proceed to find out whether the same invention had in fact been divulged irrespective of formal distinctions in the terms, since these may still cover identical matter. Such principle calls for an investigation as to the essence of the invention in the European patent application in comparison to what was described in both (2) and (3).”
} 
different parameter, or no parameter at all, is mentioned. If the known and the claimed products are identical in all other respects (which is to be expected if, for example, the starting products and the manufacturing processes are identical), then in the first place an objection of lack of novelty arises.",299

The emphasis placed on the location of the burden of proof was made clear in $\mathrm{T}$ 109/91, where a patent had been applied for recombinant plasmids. The Opposition Division found that they were new, in spite of an opponent having shown that there was a plasmid in the state of the art which had 27 restriction enzymes whose restriction map agreed with the claimed plasmids. The Opposition Division ruled that the agreement only showed 5\% of the DNA sequences to be identical, and that this was not sufficient for the opponent to have shown that they were identical. The Board of Appeal, however, arrived at the opposite conclusion. With the evidence presented by the opponent, the burden of proof had been transferred to the patentee. It was for him to show that there were any differences in relation to the known plasmids. ${ }^{300}$

A similar procedure was applied in $\mathrm{T}$ 495/90, where a pharmaceutical formulation had been claimed which made it possible to use the pharmaceutical by inhalation. In the claim assessed it was stated that more than $90 \%$ of the pharmaceutical particle would be less than $10 \mathrm{My}$ in diameter. Formulations of this kind, however, were known from the state of the art. The characterising part of the claim, therefore, also included further technical features: the particles should be spherical, or else have a spherical but collapsed shape, or be ring-shaped and have a certain surface area. The Board of Appeal found, however, that the shape of the particles in these respects was a result of the way in which they were manufactured and that the manufacturing procedure used did not differ from that which had been used in the state of the art. From this the Board concluded that the particle shape could not be a novel technical feature. ${ }^{301}$ It was merely a new definition of a known product. The difficulty of novelty assessment lies in judging whether a feature indicates a structural difference in relation to the state of the art or if it indicates a pre-existing, but unnoticed property of a known substance.

The burden of proving a structural difference rests with the applicant. But if a discrepancy between the claims and the state of the art has been found, an opponent cannot simply state that it is due to an error of determination. The burden of proof has passed to the opponent and he has to substantiate the structural identity between the state of the art and the claims. In T 367/95 the application was to fragments of human Factor VIII:C. The Board made the following novelty assessment:

"If by comparing the amino acid composition of two peptides it is found - like in the present case - that some amino acids are either absent or present in a different molar percentage, it can be concluded that the two peptides, although being possibly similar, are not identical. Of course, the occurrence of errors of determination cannot be excluded. However, in the present case, there is no evidence whatsoever that such errors occurred. Thus, the respondents' allegation that the discrepancies are likely to be due to errors of determination is unsubstantiated. Due to the

\footnotetext{
${ }^{299}$ Guidelines C IV 7.5.

${ }^{300} \mathrm{~T}$ 109/91 of 15011992.

301 T 495/90 of 3006 1992: "The said features are the shape of the drug, being spherical, collapsed spherical or ring doughnut shaped and the surface character being defined by the envelope surface area : total surface area ratio being in the range of 0.5:1.0 and finally the type of the atomiser. As to the shape and surface feature, the facts on file show that they result from the process by which the inhalation drug particles are prepared. Since the spray- drying process described in document (1) is no different from the spray-drying process mentioned in Claim 1, one has to conclude, as did the Opposition Division, that the particles achieved by the spray-drying process described in document (1) must inevitably have the same shape and surface characteristics as the particles obtained by the spray-drying process claimed in Claim 1.”
} 
above mentioned difference in a relevant parameter, the board concludes that the Factor VIII:C complex described in document (5) is not the same as the active Factor VIII:C fragment of claim 1. Novelty of the latter over document (5) can thus be acknowledged.”302

It is normal that a substance can be manufactured in several different ways. Certain manufacturing procedures are perhaps better and more cost-efficient than others, but if they yield identical end products, the demonstration of a new manufacturing procedure is not sufficient to make the products which can be manufactured patentable. The patentability of a claimed product has to be assessed separately from that of a simultaneously claimed method of preparation on the basis of a comparison of its properties and structural features with those known products of the prior art which are structurally close. ${ }^{303}$ Whilst a process may well be novel and inventive, the same may not be true for the product thereby prepared. This principle was made particularly important by the emergence of recombinant biotechnology. A known polypeptide which, for example, can be extracted from natural sources has not be considered patentable, e.g. on the basis of product-by-process claims, when somebody has first succeeded in manufacturing the polypeptide recombinantly. Nor does demonstration of the amino acid sequence of the protein make it new, if the same protein has been previously known by another definition. ${ }^{304}$ Product patents always require structural differences between the claimed product and known products in the state of the art. ${ }^{305}$

A clear example of what constitutes a novelty-founding difference between the state of the art and a claimed biotechnological invention is to be found in $T$ 412/93. The application concerned recombinant erythropoietin, a hormone used in the treatment of anaemia, kidney failure and other pathological states pertaining to the production of red blood cells. Erythropoietin is produced naturally by the body in very small quantities. Before the priority date it was known that erythropoietin could be extracted from urine and then purified, i.e. that it could be produced from a natural source. The definition in the patent claims of recombinant erythropoietin indicated the same properties as in naturally occurring, the only difference being that the claimed erythropoietin was stated to have been produced by recombinant technology. The question before the Board of Appeal was whether the recombinant mode of production was in itself novelty-founding for the product. The Board found that a new production procedure did not necessarily mean that the product manufactured was new. It went on to point out that nothing in the patent application suggested that there was anything about the biological activity of the recombinant protein to distinguish it from protein extracted from natural sources, and moreover that the applicant had stated to the Federal Drug Agency in USA that recombinant erythropoietin had biological effects identical with those of natural occurring erythropoietin. It was concluded that recombinantly produced erythropoietin lacked novelty in relation to the same substance from natural sources. A product manufactured recombinantly for the first time is not new solely by virtue of its mode of production. ${ }^{306}$

\footnotetext{
302 T 367/95 of 27101998.

303 T 223/96 of 29011999.

${ }^{304}$ Cf. Crespi, R. S., IIC 1997, 604.

305 See Jaenichen, H-R., The European Patent Office's Case Law on the Patentability of Biotechnology Inventions, 197.

${ }^{306} \mathrm{~T}$ 412/93 of 2111 1994: "[T]he fact that a product is referred to in a claim as being the result of some process, does not automatically mean that the product is novel even if it is beyond dispute that the process referred to is new. The purpose of the reference to the process was to exclude those products which in the prior art were not obtained by the process. If, on the evidence available, the process appears capable of producing every product meeting the characteristics of the product of the prior art, the reference to the process is not a limitation for the purpose of considering novelty. The process feature in a product claim can only be relied on for establishing novelty over the prior art, where use of that process necessarily means that the product has a
} 
In a set of alternative claims, the applicant tried to overcome the lack of novelty by introducing a further definition: "and not being identical to erythropoietin isolated from urinary sources”. No novelty, however, was found to exist now either, because, with such a claim formulation, the burden of proving any differences between recombinant and natural erythropoietin would devolve entirely on a third party, which was found to make the claims unclear. The claims were contrary to Art. 84 EPC. It was too laborious and difficult for a third party to judge what was protected solely in the light of what was not protected, i.e. what could been isolated from natural sources. ${ }^{307}$

In a further set of alternative claims the applicant had added a definition concerning the recombinant protein which ran: "and having an average carbohydrate composition which differs from that of human erythropoietin isolated from urinary sources." The Board of Appeal ruled that the definition did not afford any guidance to the man skilled in the art, because there were no values given concerning, respectively, the naturally produced and the recombinant erythropoietin. Thus in a practical perspective the definition was devoid of substance. Lastly, the application contained alternative claims in which the applicant had introduced a definition concerning the recombinant protein which read: "and which has higher molecular weight by SDS-PAGE from erythropoietin isolated from urinary sources." This technical feature, which implied a requirement of higher molecular weight than naturally occurring erythropoietin in electrophoretic measurement, was found by the Board to be possible to establish and, therefore, possible to use for the achievement of novelty. This was a measurement indicating the occurrence of a structural feature which distinguished recombinant from naturally occurring erythropoietin. ${ }^{308}$

The EPO, however, does not appear to be altogether consistent in maintaining the line of demarcation between novel structure and novel mode of production. In T 130/90, a patent had been applied for monoclonal antibodies. The method of manufacturing the antibodies was patentable. The question was whether the antibodies as such could also be patented. There were no possibilities of distinguishing them, by their structure, from antibodies occurring naturally. In the claim, therefore, the antibodies had been defined with the feature: “... said antibody is derived from monoclonal source(s).” Thus the difference lay in the way in which they had been manufactured - using a biotechnological hybridoma procedure - and not in their structure. Normally this ought to mean that product claims are excluded. But the Board of Appeal made an exception to the principle of a new manufacturing procedure not making the end product new. The exception was considered possible in this case because the claim read: "produced by [the patentable procedure]" and not "producible by" or some other such expression. The wording meant that the protection was limited to the

particular characteristic and the skilled person following the teaching of the specification would inevitably achieve that characteristic, would be aware of that characteristic and would discard any products not having it."

${ }^{307} \mathrm{~T} 412 / 93$ of 2111 1994: "Claim $19 \ldots$ is different ... by the addition of the words "and not being identical to erythropoietin isolated from urinary sources.' ... The claim does not comprise any indication of the technical feature or the degree of difference on which non identity of r-Epo with u-Epo should be based, but rather leaves this to the reader's imagination. This puts the claim clearly in the category of claims which are not clear. The whole burden on distinguishing over the prior art u-Epo is put on the reader."

${ }^{308}$ T 412/93 of 2111 1994: "Taken by themselves the words 'and having an average carbohydrate composition which differs from that of human erythropoietin isolated from urinary sources' seem to mean no more than 'and not having a carbohydrate composition identical to that of human erythropoietin isolated from urinary sources' as there is no indication of what parameter(s) is (are) to be measured, how it (they) is (are) to be measured and how an average is to be calculated. That a reference to 'average carbohydrate composition' is virtually meaningless, can also be deduced from the fact that it is a feature that the Respondents held not to deserve a place in the recapitulative Table of the distinguishing features between u-Epo and r-Epo... In the Board's opinion, the fact that the Respondents have chosen this 'yardstick' does not lead to a situation of legal uncertainty for third parties, because it is reliably possible to check on a SDS-PAGE gel whether a given r-Epo exhibits a higher m.w than a given u-Epo made available to the public.” 
antibodies actually manufactured by the patented procedure. Depending on the formulation of the claim, the protection was not more extensive than would have followed, without the in question claim, from the protection relating to direct products of a patented process: see section 8.1.1..$^{309}$

\subsection{Novelty in diastereomers and enantiomers}

The study of the three-dimensional (spatial) shape of molecules, i.e. relations between atoms in space, is called stereochemistry. In pharmaceutical research, growing attention has tended to focus on stereochemistry in the past two decades. ${ }^{310}$ Diastereomers are a concept employed in stereochemistry. Diastereomers occur in a situation where a molecule has more than one asymmetric (chiral) atom. A situation of this kind involves several possible three-dimensional structures in molecules having the same number of the same kind of atoms. The structural differences between the diastereomers are usually material for the biological properties of substances and also for other properties.

$\mathrm{T}$ 12/81 is a basic EPO decision which has often been invoked in subsequent chemistry case law. That decision concerned novelty assessment of diastereomers. The claimed compound was chlorophenoxy imidazol dimethyl butanol, but the claims were formulated in such a way that they only included one of the four possible isomers: the threo form. It had a mucolytic effect. The applicant had defined the isomer by indicating, among other things, its melting point (158-159 degrees). The Board of Appeal found that a prior document disclosed the compound chlorophenoxy imidazol dimethyl butanol, i.e. a compound with an identical formula. If the two compounds - the claimed threo form and the one indicated in the prior document - were identical, novelty was lacking. It was not certain, however, that they really were the same compound, because the compounds contain two asymmetric carbon atoms and can therefore appear in four different spatial forms. The assessment of novelty in this case hinged on the chemical compound indicated in the prior patent, according to the examples having a melting point of $145-147^{\circ} \mathrm{C}$, whereas the compound claimed, as we have already seen, had a melting point of $158-159^{\circ} \mathrm{C}$. From this the Board concluded that there were presumably two diastereomers, i.e. two different chemical individuals, involved. The compound indicated in the prior document was no impediment to the novelty of the present patent application: the difference in melting point indicated a structural difference. ${ }^{311}$

\footnotetext{
${ }^{309}$ T 130/90 of 2802 1991: "Consequently, so far as it is not possible to distinguish the antibodies of the patent in suit over the prior art antibodies of document (5) otherwise than by limiting them to the product directly obtained by the specific process of their preparation, such a feature is accepted as a distinguishing parameter for the purpose of novelty. This view is apparently also accepted by the Appellants whose auxiliary request clearly shows that in the present case they accept a significant difference of the meaning of the two expressions 'produced by' and 'obtainable by', for the purpose of defining the antibodies of the patent in suit compared to those described in document (5). The very specific circumstances of the present case, which differ from those having been the basis of the decisions of the Boards of Appeal as mentioned above under paragraph 3.3, which entered into the Guidelines for substantive examination (C-III, 4.7b), make the expression 'produced by' necessary. The respective part in the Guidelines states that: 'A product is not rendered novel merely by the fact that it is produced by means of a new process'. In the present case, however, as reasoned above, it is the process which renders the product novel."

${ }^{310}$ See Witte, D., Ensing, K., Franke, J-P., De Zeeuw, R., 15 Pharmaceutical World Science 1993, 10. See also Daniels, J. M., Nestmann, E. R., Kerr, A., 31 Drug information journal 1997, 639 and cf. Hansen, B., GRUR 1996, 944.

${ }^{311}$ T 12/81, OJ EPO 1982, 296: "The compound mentioned has two asymmetric carbon atoms and can thus occur in four different spatial configurations, i.e. in two pairs of enantiomers having two diastereomeric
} 
T 990/96 is an notable development of $\mathrm{T} 12 / 81$. In a citation was disclosed a compound that was useful in the treatment of atherosclerosis. The same compound and the same indication were mentioned in the application in suit. The only feature of the subjectmatter of the claim which was not literally disclosed in the prior document was the particular diastereomeric purity, erythro : threo ratio of $99.5: 0.5$ or higher. No new uses were described for the high purity product claimed, compared to uses indicated for the product of the prior document, nor was it argued that the threo impurity had any known detrimental effect. It was merely argued that health authorities would prefer substances to be as pure as possible. The Board of Appeal made the following assessment:

\begin{abstract}
"It is ... common practice for a person skilled in the art of preparative organic chemistry to (further) purify a compound obtained in a particular chemical manufacturing process according to the prevailing needs and requirements, e.g. in samples for analytical purposes. Conventional methods for the purification of low molecular organic reaction products such as recrystallisation, distillation, chromatography, etc., which normally can be successfully applied in purification steps, are within the common general knowledge of those skilled in the art. It follows that, in general, a document disclosing a low molecular chemical compound and its manufacture makes available this compound to the public in the sense of Article 54 EPC in all grades of purity as desired by a person skilled in the art.

The Appellant alleged that this general rule would not be applicable in the present case. The Board accepts that there may exist exceptional situations, which could justify a different conclusion. One such exceptional situation could be ... a situation where it was proved on the balance of probability that all prior attempts to achieve a particular degree of purity by conventional purification processes had failed. However, the burden of proving the existence of such an extraordinary situation lies with the party alleging its existence.”312
\end{abstract}

The Board was not satisfied that the Appellant had discharged his burden of proof. A purity of 99.5\% or higher of the erythro compounds could not directly be found in the state of the art. But that did not prove that it was impossible to achieve such a diastereomeric purity. The Board found that the prior document made available the compound in any desired purity and, thus, also in a diastereomeric purity of at least 99.5 percent. Consequently, the degree of diastereomeric purity given in the claim could not be accepted as a new element distinguishing the claimed subject-matter from the state of the art.

If two molecules containing the same number of atoms of the same kind are also each other's mirror images, they are called enantiomers (the $\mathrm{R}$ and $\mathrm{S}$ configuration, sometimes they are also called + or - or else dextro or levo $(\mathrm{D} / \mathrm{L}))$. They contain an asymmetric carbon atom which forms a chiral centre. A mixture in which the two enantiomers are included in equal proportions - which is the normal result of a chemical manufacturing process - is called a racemic mixture or a racemate. Separating the R and S form is not altogether simple because, unlike diastereomers, they have almost identical chemical/physical properties. One difference between the two inverted molecules, however, is that they rotate polarised light in different directions. For this reason, enantiomers are sometimes also called optical isomers. A racemic mixture, however, does not rotate polarised light, because the two enantiomers extinguish each other's effects. ${ }^{313}$

configurations, viz. the so-called erythro and threo forms .... This steric characteristic would seem to support the view that the compound according to Example 3 of the cited document having a melting point of $145^{\circ}-147^{\circ} \mathrm{C}$ is not identical with the compound claimed, which has a melting point of $158^{\circ}-159^{\circ} \mathrm{C}$.”

312 T 990/96 of 12021998.

313 See Spangenberg, R., IIC 1997, 816. 
Using racemic solutions in pharmaceuticals without any further reflection means using a mixture of an active substance and a substance which is often inactive or in some cases even toxic. The presence of the differences in biological effect is due to pharmaceutical receptors as a rule also being asymmetric. The principle of the significance of enantiomers in pharmaceutical research is as simple as the fact of a left-hand glove (the active substance) not fitting the right-hand (the receptor). Nowadays authorities approving new pharmaceutical products for sale are tending more and more often to stipulate that the applicant shall have investigated whether a racemic mixture of a pharmaceutical product can be separated and only the most active isomer used. ${ }^{314}$

One strategy which has grown popular in recent years among researching pharmaceutical companies is the use of chiral chemistry to develop replacements for the pharmaceuticals which are about to lose their patent protection. ${ }^{315}$ Many older patented pharmaceuticals contain a mixture of the $\mathrm{R}$ and $\mathrm{S}$ configuration, but usually only one of the two is active. If the two enantiomers can be separated, a patent application is filed for the active one. As has already been pointed out, the structural differences between them are smaller than those between diastereomers.

In T 296/87, the question arose of the novelty of enantiomers. A prior document indicated a chemical substance which had a asymmetric carbon atom, but it did not specify which of the two conceivable enantiomers was referred to. The patent application in question referred to a mixture containing $80 \%$ D-form, one of the two enantiomers. The claimed mixture had a higher activity than the racemate. The Board of Appeal ruled that a prior document in which it is not made clear whether the $\mathrm{L}$ or the D-form is being referred to means that novelty is lacking in a racemic mixture, i.e. a mixture containing equal proportions of both enantiomers. Failing indication to the contrary, the man skilled in the art takes it to be a racemic mixture. The point at issue, however, was whether novelty existed when the application referred to a different mixture of enantiomers than a racemic mixture. The Board found that novelty existed when a specification of this kind had been made. The choice of an enantiomer or a certain mixture of enantiomers was sufficient to establish novelty. ${ }^{316}$

The principle of an enantiomer being new in relation to a racemic mixture was confirmed and developed in T 1048/92. A prior document contained an example in which the patented substance was referred to. Furthermore, the prior document contained a Markush formula which included the substance from the example. In connection with the Markush formula it was stated that it included "various optically active isomers" and that the "invention embraces such optically active isomers". In the Examining Division the application had been refused on the grounds that the references in the prior document made all optical isomers within the formula known. The Board of Appeal, however, found that novelty existed in this case. The applicant had chosen one of the two conceivable configurations of the substance in the example. The statement concerning the occurrence of optical isomers referred to the whole of the general formula, which was too general to detract

\footnotetext{
${ }^{314}$ See Daniels, J. M., Nestmann, E. R., Kerr, A., 31 Drug information journal 1997, 639.

315 Ibidem.

${ }^{316}$ T 296/87, OJ EPO 1990, 195: “The first requirement regarding novelty is to establish whether a known chemical formula evidently containing a (single) asymmetrical carbon atom destroys the novelty not only of the compound in the form of its racemate, but also of its enantiomers (d-and l-form or D- and L-form). This applies in particular to documents (1) to (3), which indisputably disclose structures overlapping those described in the contested patent, the only difference being that the latter claims D-enantiomers whereas the former makes no mention of them at all ... Documents (1) to (3) say nothing about enantiomers, nor is there any suggestion that they could occur during the manufacturing process described - say through the use of optically active starting materials. The Board concludes that this prior art is concerned only with racemates which do not affect the novelty of the D-forms claimed in the contested patent. This assessment must also apply to products according to the contested patent which - as finally claimed -have 'a content of at least $80 \%$ D-form'.”
} 
from novelty. The statement did not refer to the individual substance where the applicant had made a distinction concerning the steric form. ${ }^{317}$ This decision shows the special character of the assessment of novelty where product patents in chemistry are concerned. Novelty was already found when a choice was made between two possibilities concerning a substance in an example.

That which in $\mathrm{T}$ 1048/92 was lacking in the prior document - an exact indication of the occurrence of isomers in a specified substance - was present in T 658/91. The indication of steric differences was narrowed down to the very point (substance) where the applicant in this case had made a distinction between possible enantiomers. The prior document stated:

"[C]es composés comportant un carbone asymétrique peuvent exister sous la forme de 2 énantiomères. L'invention concerne aussi bien chacun des énantiomères que leur mélange.”318

As a consequence of the precision of this statement in the prior art, a patent application for one of the enantiomers mentioned was not new. This is just about as close as one can get to a direct indication of the enantiomers. The enantiomers were deemed to be available to the man skilled in the art as concrete possibilities of use. ${ }^{319}$ A similar situation arose in $\mathrm{T}$ 600/95,

${ }^{317}$ T 1048/92 of 0512 1994: "Claim 6 of document (1), taken in isolation, does not unambiguously disclose any one of the above two absolute configurations, although it conceptually comprises both of them. In the Board's judgment the above ambiguity is not removed by the disclosure contained in the paragraph on page 4 of document (1) relied upon in the decision under appeal, since this paragraph refers to optical active isomers in general, including a great number of possible diastereomeric and enantiomeric forms, but not to any specific configuration even at the three asymmetric carbon atoms contained in the penem ring system, for which the specific configuration at these three carbon atoms disclosed in Claim 6 is an example. Moreover, there is no indication that this paragraph contains any technical teaching relevant to asymmetric carbon or even sulphur atoms which may be contained in some of the substituents $\mathrm{R}$ comprised by formula Ia, corresponding to formula I in document (1). Rather, the skilled reader would consider this paragraph solely in respect of the essential structural elements of the disclosed class of chemical compounds, i.e. the three asymmetric carbon atoms of the penem ring system. Nevertheless, the Board observes that it does not agree with the Appellant's submission that the above paragraph relating to optically active isomers would not add anything to what the skilled chemist already knew, i.e. that various optical isomers were theoretically possible, since the express statement that such optically active isomers are embraced by the invention goes beyond the conceptual information that such isomers are theoretically possible ... and is therefore a relevant part of the disclosure of document (1). In these circumstances the fact that the disclosure of Claim 6 of document (1) does not embrace more than two possible steric configurations does not take away the novelty of the specific one which is claimed in the present application, because there is no unambiguous technical teaching directed to that configuration in the parts of document (1) relied upon by the Examining Division.”

${ }^{318} \mathrm{~T}$ 658/91 of 1405 1993. The Board described the document in the following way: "Les parties pertinentes dudit document sont l'exemple ... ou il est constaté que l'invention décrite dans ce document est relative à de nouvelles thiéno(3,2-c)pyridines répondant à une formule générale qui comprend le composé de l'exemple 1 mentionné ci-dessus en précisant que 'ces composés comportant un carbone asymétrique peuvent exister sous la forme de 2 énantiomères. L'invention concerne aussi bien chacun des énantiomères que leur mélange’ ”

${ }^{319}$ T 658/91 of 1405 1993: "D'autre part, il n'est pas conforme ... la jurisprudence de la Chambre qu'une divulgation spécifique d'un composé chimique ne soit reconnue que dans le cas où ce composé est nommément indiqué ou même décrit dans un exemple. Il suffit, par contre, que le composé puisse être identifié sans ambiguëté comme étant envisagé individuellement dans le document en cause, car l'article 54(2) de la CBE a pour but d'exclure l'état de la technique du domaine de la brevetabilité. Selon cet article, l'état de la technique est constitué par tout ce qui a été rendu accessible au public avant la date de dépôt et ce, de quelque façon que ce soit .... Cependant, l'enseignement qu'apporte une antériorité n'est pas seulement constitué par les détails fournis par les exemples de réalisation mais par toute information tirée du contenu de cette antériorité .... Dans le cas présent, la mention d'énantiomères dans la description du document (1) n'est pas limitée à l'information conceptuelle sur l'existence de tels énantiomères, information qui peut être tirée de la première phrase citée cidessus, mais cette mention se réfère, dans la deuxième phrase, expressément au fait que ces énantiomères sont considérés comme une partie intégrale de l'invention décrite. De plus, cette dernière phrase citée ci-dessus ne se 
where one of two possible enantiomers (the (-) isomer) of a known pharmaceutical had been claimed. The known pharmaceuticals were referred to in a prior document, and it was stated that:

"[T]he compounds can exist as two diastereoisomers, their cis or trans isomers, and each of which can exist as the racemic mixture of such isomers or each individual optical isomer. Accordingly, the compounds of the present invention will include not only the (+)-racemates, but also their respective optically active (+) and (-)-isomers.”320

The last (-) isomer mentioned in the quotation was the enantiomers which had subsequently been claimed. The Board of Appeal found that novelty was lacking, because the man skilled in the art could produce the claimed substance with the aid of the prior document. The outcome of the case hinged on the indication in the prior document that the known diastereomers had two different enantiomers. The precision of that statement meant that novelty was lacking when one of the enantiomers in the racemic mixture was claimed. The Board of Appeal commented as follows on the above quoted decision in T 1048/92:

"By contrast, in decision T 1048/92 the statements in the description, although quite similarly worded, were not construed by the Board as relating to an optical isomer of one particular example, because it held that in the circumstances of that case the disclosure of steric configurations in the relevant prior art document related ... to all compounds described in that document.",321

It is clear from this that if the claimed enantiomer has been mentioned in a prior document as a specific possibility regarding an individual substance, novelty is lacking. But in order for such precision to exist in the prior document, the reference must not be more general than perhaps concerning two or, at most, four possible steric forms. Precision of this kind, precluding further patenting of isomers, does not exist if the prior document only contains a general statement to the effect that all substances within the claims can appear in a variety of steric forms.

\subsection{A starting substance and a process giving the claimed substance}

So far we have been grappling with situations in which known substances may detract from the novelty of claimed substances. That is the usual situation. Under certain conditions, though, a starting material referred to in a prior document together with certain specified reactions can also detract from the novelty of the end product obtainable. This situation is an important example of the possibility of the same technical content being differently indicated in the prior art and in the patent application. The Guidelines provide that:

“[T]he lack of novelty may be ... implicit in the sense that, in carrying out the teaching of the prior document, the skilled person would inevitably arrive at a result falling within the terms of the claim. An

réfère pas exclusivement à la formule générale, mais à tous éléments de l'invention décrite, y compris les exemples individuels. Elle rend donc accessible à l'homme du métier de façon non- équivoque tous les énantiomères des composés racémiques individuellement décrits dans les exemples, donc l'énantiomère revendiqué dans la présente demande de brevet, qui, comme admis par la requérante, peut être préparé par l'homme du métier en utilisant ses connaissances de base sur les méthodes de la résolution des composés racémiques, à savoir par un procédé d'analogie. L'homme du métier n'a donc pas besoin d'informations supplémentaires pour obtenir cet énantiomère individuel.”

${ }^{320} \mathrm{~T}$ 600/95 of 28111996.

${ }^{321}$ Ibidem. 
objection of lack of novelty of this kind should be raised by the examiner only where there can be no reasonable doubt as to the practical effect of the prior teaching.",322

In $\mathrm{T}$ 181/82, an important decision, a document referring to a group of starting compounds and, moreover, a manufacturing process, was invoked as a prior document. If the process was applied to one of the starting reactants indicated, the compound now claimed was obtained. If, as a starting reactant, $\mathrm{C}_{1}$ alkyl bromide was selected from the group of $\mathrm{C}_{1}-\mathrm{C}_{4}$ alkyl bromide compounds the claimed substance was obtained. From the formula $\mathrm{C}_{1}-\mathrm{C}_{4}$ alkyl bromide, the man skilled in the art understands eight individual compounds of alkyl bromide: methyl bromide, ethyl bromide, 2 isomers of propyl bromide and 4 isomers of butyl bromide. The eight compounds can be expressed in several ways, e.g. "alkyl bromide with not more than 4 $\mathrm{C}$ atoms", "alkyl bromide with fewer than $5 \mathrm{C}$ atoms" or "alkyl bromide with more than 0 and fewer than 5 C atoms". Despite these differences, to the man skilled in the art these expressions have one and the same information content: the eight individual compounds. The question was whether all eight were to be deemed known starting materials for the reaction referred to. The Board of Appeal found that $\mathrm{C}_{1}$ alkyl bromide being expressly mentioned, made the end product part of the state of the art. Only this substance, however, should be deemed to be known as starting material, because it was the only one expressly mentioned in the prior document. With the formulation " $\mathrm{C}_{1}-\mathrm{C}_{4}$ alkyl bromide" the Board of Appeal found that, only the use of methyl bromide $\left(\mathrm{C}_{1}\right)$ and butyl bromide $\left(\mathrm{C}_{4}\right)$ as a family (four individual compounds), had been referred to as a specific technical teaching. This argument suggests that if the definition "not more than four C atoms" had been used, methyl bromide would not have been individualised, in which case the end product resulting from the use of methyl bromide would not be deemed to be known. The other two conceivable definitions mentioned above, thus viewed, individualised neither methyl bromide nor the $\mathrm{C}_{4}$ family. ${ }^{323}$

A novelty assessment of this kind is hard to accept. The eight compounds can be easily purchased or synthesised. This being so, a distinction based on the form of indication in the prior document seems irrelevant. The Board of Appeal put a formalist construction on the prior document. ${ }^{324}$ This can perhaps be put down to the Board having difficulty in finding any other grounds for its assessment than the mention or otherwise of the starting compounds. All communication in patent law, however, must be addressed to the man skilled in the art and read through his eyes. To the man skilled in the art it is obvious that the definition includes eight individual compounds. ${ }^{325}$ A man skilled in the art is hardly likely to take the view that $\mathrm{C}_{1}$ alkyl bromide is more clearly referred to than any other of the eight compounds. The only

\footnotetext{
${ }^{322}$ Guidelines C IV 7.5.

${ }^{323}$ T 181/82, OJ EPO 1984, 401: “The appellant stated further that the range of 'C1-C4 alkyl bromides' could also be defined differently, for example as 'alkyl bromides with a maximum of $4 \mathrm{C}$ atoms', 'alkyl bromides with fewer than $5 \mathrm{C}$ atoms', or 'alkyl bromides with more than 0 and fewer than 5C atoms'. Despite the different formulation, such ranges had an identical information content and were understood by the skilled person as a disclosure of all 8 possible alkyl bromides without their being individualised. It cannot be denied that the eight conceivable alkyl bromides are covered by all these definitions; a strict distinction must be drawn between this purely intellectual content of the definitions and their information content in the sense of a specific teaching with regard to technical action. While the 'C1-C4' definition specifically designates methyl bromide as an individual compound and the butyl bromides as a family (4 possibilities), the 'maximum C4' definition does not individualise methyl bromide because this substance is not designated or otherwise more closely described. The other two definitions skilfully avoid any specific statement about methyl bromide or indeed about the C4 family. When the teaching from a citation is interpreted, special attention must be paid to the material actually disclosed in the sense of a complete, specific technical rule. A group of compounds in which the substituent is characterised by a range teaches the skilled person only about the individuals specifically designated from the group."

324 See Fürniss, P., Chemiepatent Entscheidungen, 45.

${ }^{325}$ See Hirsche, F., Hansen, B., Protecting Inventions in Chemistry, 142.
} 
reason for the substance being expressly mentioned was that it constituted a limit value. In an infringement case, a court would undoubtedly find that protection defined as " $\mathrm{C}_{1}-\mathrm{C}_{4}$ alkyl bromide” protected all eight compounds, and not just those expressly mentioned. Presumably a better basis for the assessment of novelty would be to consider the likelihood of a man skilled in the art having, on the basis of the reference " $\mathrm{C}_{1}-\mathrm{C}_{4}$ alkyl bromide" chosen methyl bromide as the starting material for the reaction. Guidance for such an assessment can be obtained from the rules on selection inventions: see section 4.7.

\subsection{Novelty of a metabolite in relation to its known prodrug}

In the pharmaceutical industry, a special situation arises resembling that which occurs when a starting material and a manufacturing process are indicated in a prior document. A pharmaceutical may be known and frequently used, at the same time as it is not known that the pharmaceutical functions as a "prodrug", meaning that in the body it is turned into a different substance and it is this latter substance that interacts with a receptor or another target molecule. In a situation of this kind, the pharmaceutical is a kind of starting material which, together with the body's metabolism, gives the active metabolite. The metabolism functions in a similar fashion as a reference in a prior document to a manufacturing process. The question is whether the active metabolite is known when the prodrug and its therapeutic use are known.

This question is important because the biological mechanism is seldom known when a patent application is filed for a pharmaceutical substance. A year or so later, when the biological mechanism and the metabolite are known, the pharmaceutical company not infrequently files a patent application for the metabolite, i.e. for what is the true active substance. This situation has been adjudicated in an English case. Merrell Dow was the proprietor of a patent for the pharmaceutical Terfenadine, a non-sedating anti-histamine. When the patent expired in 1992, several generics manufacturers had prepared an introduction of copies. During the term of the patent, however, Merrell Dow had carried on research which showed that Terfenadine in the liver underwent a 99.5\% metabolism. The anti-histamine effect was almost exclusively due to the occurrence of a specific metabolite. Merrell Dow determined the structure of the metabolite and found it to be a previously unknown substance. A patent application for it was filed in $1980 .{ }^{326}$

When the patent expired in 1992, Merrell Dow sued a generics manufacturer, $\mathrm{N}$ Norton \& Co., for infringing the patent for the metabolite, which did not expire until 2000. Merrell Dow's action pertained to indirect patent infringement. It was alleged that, by selling Terfenadine, the generics manufacturer supplied the essential means of practising the protected metabolite invention. Norton filed a counter-action, claiming invalidity of the patent invoked. It lacked novelty because the metabolite had already been used when the first patent was practiced. Norton further submitted that they were only doing what was expressly apparent from the earlier, now expired patent.

The House of Lords found that there are various ways in which a substance can become a part of the state of the art. The fact of the structure of a metabolite not being known when a patent application is filed does not necessarily mean that it is new. The House of Lords found that the metabolite had been known in that the earlier patent contained sufficient information for practical use to be made of the medical possibilities which the metabolite implied. The earlier patent showed that the taking of Terfenadine produced an anti-histamine

\footnotetext{
${ }^{326}$ House of Lords, Merrell Dow Pharmaceuticals Inc v. H N Norton \& Co Ltd, [1996] R.P.C. 76. For a comment see Karet, I., A Question of Epistemology, [1996] EIPR 97.
} 
effect, and this was a kind of indirect definition of the metabolite. True, when the patent application for the metabolite was filed, it was not possible for a man skilled in the art to indicate in chemical terms the metabolite and the reactive process undergone by Terfenadine. But the crucial point, the Lords found, was what could be put to practical use. As an example of this principle, they noted that the same invention was used by the Indians of the Amazonas when referring to the healing properties of the Tree God and using quinine bark for the treatment of malaria, and by a western scientist who knew the chemical formula for quinine and its therapeutic properties but, when stranded in the Amazonas, used quinine in the form of bark. For purposes of patent law, what mattered was not the scientific elucidation but the capacity for making practical use of a technical effect.

The same invention and dispute were also adjudicated in Germany, where the case reached the Appeal Court, the Oberlandesgericht in Munich. ${ }^{327}$ The proceedings in the German court were somewhat different. The action there concerned infringement only, not invalidity. In Germany these two questions are not adjudicated by one and the same court. In the infringement case it was established that there was no infringement, one reason being that what belonged to the state of the art on the priority date for the patent (the prodrug Terfenadine) could not constitute patent infringement; see section 8.2.1.2. Given this outcome of the infringement suit, the patent for the metabolite would probably have been declared invalid if an action had been filed on the subject. ${ }^{328}$

The English and German decisions concerning the novelty of a metabolite are in the ultimate analysis concerned with what justifies the granting of patents. If we assume that all patients who could be treated with the metabolite could previously be treated just as well with its known prodrug, knowledge of the metabolite did not add anything of practical value. The metabolite was really no more than an explanation of what had previously been used unawares. It is, however, a well-established rule that the assessment of novelty in connection with product patents shall refer to the means (the substance) used in order to achieve the effect justifying the patent, not the effect as such. The fact of a treatment result being attainable with one substance does not, therefore, mean that other substances with the same effect are deemed to be known.

The House of Lords deviated from this principle. Clearly, a different outcome in the case was considered unsatisfactory, because the protection would then have been prolonged for more than 20 years. But the principle that the assessment of novelty shall refer to what is indicated in the claims could perhaps be amalgamated with the House of Lords decision in the following way: an invention is a rule of action, i.e. not only the means or effect but both. The invention is the means (a substance in the claims) together with a biological effect. Everything that follows, consciously or unconsciously, when a known substance serves to obtain a known therapeutic effect is to be deemed known. If a process is knowingly applied to a known substance that which follows from this is considered known, although, as a matter of fact, the particular results are not known in all the details. In this way attention is paid to whether the claimed invention contains any new, practically useful mode of action or whether it is just a new definition of a previously known possibility of action.

This approach can account for the decision by the House of Lords, but it does not seem to be applied generally in the patentability assessment of pharmaceuticals. All that is investigated is whether the content of the claims is new; the technical effect is left out of the assessment. Product patents for metabolites are granted when the structure is not known. No attempt is made to investigate whether the claimed structure has previously been produced as

\footnotetext{
${ }^{327}$ Oberlandesgericht München, 6 U 5155/92 of 0606 1993, quoted in Vossius, C., et. al., GRUR 1994, 472 and Tauchner, P., Patent World April 1999, 19.

${ }^{328}$ Cf. Jacob, R., IIC 1997, 880.
} 
a metabolite, i.e. produced through the body's metabolism when a known prodrug is used. The prodrug combined with the metabolism is deemed not to be a relevant part of the state of the art. The explanation is that in practical terms it would be very hard to make the kind of novelty assessment made by the House of Lords, if it already had to be made at the patenting stage. All the same, it is probably necessary that the practical usefulness of an invention ultimately decides whether it is to be deemed known. An alternative therapeutic substance is useful, but a metabolite that does not imply any other usefulness than its prodrug does not appear to add anything of value. With this kind of novelty assessment protection can be prevented from extending beyond 20 years when new metabolites are identified which have previously been used unawares.

\subsection{Selection inventions}

\subsubsection{Introduction}

Selection inventions are probably the most complicated of novelty assessment issues in the chemical sphere. Internationally there is no real uniformity of approach. The patentability of all selection inventions, however, is a modification of the traditional novelty concept. In the normal instance, whatever comes within the scope of a technical disclosure in a known document is to be deemed known. Something which borders on what was known previously is new, but not something that overlaps it.

Where selection inventions are concerned, the legal position is modified through the principle that a generic reference in the state of the art does not necessarily make all individual points within the disclosure known. In the reverse situation - a known smaller area within a claimed larger one - on the other hand, the larger area always lacks novelty. This principle is stated in the Guidelines:

"In considering novelty it should be borne in mind that a generic disclosure does not usually take away the novelty of any specific example falling within the terms of that disclosure, but that a specific disclosure does take away the novelty of a generic claim embracing that disclosure, e.g. a disclosure of copper takes away the novelty of metal as a generic concept, but not the novelty of any metal other than copper, and one of rivets takes away the novelty of fastening means as a generic concept, but not the novelty of any fastening other than rivets.”329

In $T$ 374/94, a Board of Appeal of the EPO expounded, for the first time, the underlying principles of selection inventions:

"Die Frage des Schutzbereiches der Ansprüche unterscheidet sich von der Frage des Offenbarungsgehalts dieser Ansprüche. ... Der Schutzbereich steht im Zusammenhang mit der 'Extension’ (Begriffsumfang) des bzw. der im Anspruch definierten Begriffe, d. h. mit der Gesamtheit aller individuellen Gegenstände, die sämtliche Merkmale dieses bzw. dieser Begriffe aufweisen. Demgegenüber steht der Offenbarungsgehalt im Zusammenhang mit der 'Intension' dieses bzw. dieser Begriffe (Begriffsinhalt), d. h. mit der Gesamtheit der Merkmale, die die gedankliche Zusammenfassung von individuellen Gegenständen ermöglichen .... Wenn ein Anspruch sich mit allgemeinen Begriffen befaßt, dann offenbart er nur diese allgemeinen Begriffe und nicht alle unter diesen allgemeinen Begriffen fallenden speziellen Beispiele.”330

\footnotetext{
${ }^{329}$ Guidelines C IV 7.4.

${ }^{330} \mathrm{~T}$ 374/94 of 19031996.
} 
The Board distinguished between the extent of the technical concepts and their innate intention. The latter is what is placed at the disposal of the person skilled in the art and extends from the individual practical examples towards the extent of the generic terms used in the document. Thus the "minimum value" as regards content detracting from novelty is that which is expressly indicated by individual practical examples. The "maximum value" is the full extent of the generic terms, i.e. that which would imply infringement of the generic disclosure. Somewhere between these two extremes lies the information content which is to be deemed detrimental to novelty for a later selection within the generic terms. ${ }^{331}$

Quite early on the EPO established a liberal precedent for the novelty assessment of selection inventions. The EPO took English case law as its model, avoiding the German interpretation, which is most immediately concerned with being strictly logical and makes it difficult if not impossible to achieve novelty through selection. It has been said that not even the most harmonising construction can reconcile German precedent with that applied by the EPO. ${ }^{332}$ Germany has what is known as "the Bruchhausen doctrine". ${ }^{333}$ The indication of an interval - say 1-100 ppm - is to be understood as an abbreviated enumeration of all conceivable values within that interval. Accordingly, everything within that interval is known, so long as there is a theoretical possibility of the man skilled in the art being able to use it. The interval 5-10 ppm is deemed known in relation to 1-100 ppm. If anyone has shown the interval 5-10 ppm to have a different effect, use claims can be employed. But selection inventions - a better but similar effect - will not be protected. ${ }^{334}$ EPO assessment concentrates more on the broadness of the generic disclosure and what could probably have been put to practical use by a man skilled in the art.

\subsubsection{Selection with formulae and with individual substances}

The most important principle for the novelty assessment of selection inventions is that, the narrower the selection is in relation to the generic term, the more likelihood there is of the selection being deemed new. A selection in the form of one or two individual substances is more likely to be new than a selection of a wider group of compounds. EPO precedent, however, indicates that it is not only the quantitative relation between the generic and the selected field that matters. Emphasis is apparently placed on the way in which the selection has been formulated in the patent claims - generically or as a number of individual substances.

In T 12/90, a patent application had been filed for a large number of chemical compounds using a Markush formula. The compounds functioned as pesticides. In the state of the art there was a patent which also contained a disclosure of a large number of compounds by a Markush formula. The two formulae overlapped: a large number of compounds were

\footnotetext{
${ }^{331}$ T 167/93 of 0305 1996: “[C]onsideration has not only to be given to the examples of a prior art document but also to whether the disclosure of such a document as a whole is such as to make available to the skilled person the subject-matter for which protection is sought. This means, that the technical teaching of examples of a patent document may be combined with technical information disclosed in its description, provided that the examples concerned are representative for the general technical teaching of this document”.

332 "In the recent past, German and European Case Law have provided extreme interpretations with widely differing viewpoints, so that even for the most optimistic onlooker, harmonisation of the GPO and EPO views of disclosure still seems some distance away.” See Hirsche, F., Hansen, B., Protecting Inventions in Chemistry, 127. Footnotes have been excluded. See also Rogge, R., IIC 1997, 464.

333 See Lunzer, R., Singer: The European Patent Convention, 54.15B. Bruchhaus was a prominent German patent judge.

${ }^{334}$ See Rogge, R., IIC 1997, 799 and Lunzer, R., Singer: The European Patent Convention, 54.15B.
} 
common to both. The applicant submitted that he had made a selection invention in relation to the known field. The Board of Appeal pointed out, however, that there was a difference between two types of selection:

\begin{abstract}
“In der von der Beschwerdeführerin ferner angezogenen Entscheidung 'Xanthine' ging es um die Neuheit einer formelmäßig definierten einzelnen Verbindung gegenüber einer durch eine allgemeine Formel umschriebene Stoffgruppe. Dieser Fall ist mit dem vorliegenden, in dem über die Neuheit einer durch eine allgemeine Formel beschriebenen Stoffgruppe gegenüber einer diese teilweise umfassenden anderen, durch eine andere allgemeine Formel beschriebenen Stoffgruppe zu entscheiden ist, nicht vergleichbar, denn das Konzept der 'Individualisierung' paßt begrifflich nur zur strukturellen Definition einer Einzelverbindung, nicht aber eines Kollektivs; ein solches durch eine allgemeine Formel festgelegtes Kollektiv offenbart nach der Rechtsprechung der Kammer nicht die davon umfaßten Einzelindividuen.”,335
\end{abstract}

Further, $\mathrm{T}$ 133/92 suggests that the novelty assessment differs according as to whether the selection is formulated in a general formula or refers to individual substances. The part of the state of the art nearest at hand was a product patent in which part of the structural formula for the molecules was an alkyl with between 1 and 20 carbon atoms. Alkyls with between 6 and 15 carbon atoms were indicated as a preferred form. In the application in suit, alkyl groups with between 6 and 10 carbon atoms were claimed in the independent claim. To distinguish the invention from what had been expressly indicated as a preferred form in the prior document, the claims also stated that the alkyl with 6 carbon atoms should be n-hexyl. This limitation meant that the new invention fell entirely within the scope of the earlier patent, but no part of the compounds now claimed had been explicitly indicated in the earlier patent. The selection comprised barely half the generic disclosure. The Board of Appeal found that an improved effect existed within the selected area, but that this in itself was not sufficient for the novelty requirement to be satisfied. The Board found that the selection comprised a relatively large part of the previously known field and that the man skilled in the art must therefore be deemed to have contemplated using the area now claimed. Accordingly, the claim was disallowed for lack of novelty. ${ }^{336}$

In a first alternative claim, the applicant had made a narrower selection: C6 to C8 (C6 was still to be n-hexyl) but here again the Board of Appeal found that the selection lacked novelty, because, in this situation too the man skilled in the art would have seriously contemplated using the claimed area. In a second alternative claim, the selection had been further narrowed down, this time to the five specific substances: n-hexyl, n-octyl, 2-ethyl hexyl, 3, 5, 5-trimethyl hexyl and n-decyl. The Division found that the selection was now sufficiently limited to be new in relation to the earlier document. It was particularly observed that:

"According to the established case law of the Boards of Appeal a distinction must be drawn between the novelty of a group of compounds defined by a general formula, and the novelty of particular individual compounds, because of the concept of individualisation which only applies to the structural definition of a single compound.”

\footnotetext{
${ }^{335} \mathrm{~T} 12 / 90$ of 23041990.

${ }^{336} \mathrm{~T}$ 133/92 of 1810 1994: "In the present case, the group of compounds as defined in Claim 1, i.e. containing a C6-C10 alkyl group with the proviso that C6 is n-hexyl, forms a relatively large part of the preferred group of compounds having 6 to 15 carbon atoms in the alkyl moiety disclosed in document (1) and, therefore, represents a mere partial copy of the known group of compounds without adding a novel element. Moreover, in the Board's judgment, a person skilled in the art would, in the light of all the technical facts at his disposal, seriously contemplate applying the technical teaching of this prior art document in the range of overlap. Thus, having regard to these considerations, the Board concludes that the 'selected' group of compounds as defined in Claim 1 of the main request lacks novelty."
} 
But the five compounds could have been stated in a Markush formula. Would novelty then have been lacking? That would hardly have been reasonable. The outcome of the novelty assessment could scarcely be made to hinge on the mode of writing. The distinction between generic formulae and individual substances appears, however, to have taken root somewhat in EPO case law. The idea seems to be that it is specific teachings - meaning individual substances - in a document that detracts from novelty, whereas a general formula requires a certain amount of additional work in order to be practically applied; the man skilled in the art has a choice to make.

In $\mathrm{T} 3 / 89$, the patent application pertained to a diagnostic procedure based on antibodies. In order for a reading to be obtained, a marker had to be connected to the antibodies which were capable of detecting substances connected with a pathological state. There was a prior art document stating that enzymes could be used as a marker for antibodies, but also that the use of "other markers" was possible. The earlier patent instanced such "other markers” with fluorescent and radioactive markers. In the present patent application, four more markers were indicated: red blood cells, spheres of polystyrene, particles of dye and metal particles in solution. The Board of Appeal found that the generic term "markers" in the known document, combined with the explicitly indicated alternative "enzyme", did not retract from the novelty of the four new markers. It further ruled that equivalents in relation to the explicitly indicated markers were not to be taken into account in the assessment of novelty. ${ }^{337}$

\title{
4.7.3 Selection from Markush formulae
}

Selection from Markush formulae is a particularly important situation where pharmaceutical patents are concerned. ${ }^{338}$ The Guidelines give an example of a situation when a selection from a Markush formula is not patentable:

\begin{abstract}
"The prior art includes disclosure of a chemical compound characterised by a specified structure including a substituent group designated ' $R$ '. This substituent ' $R$ ' is defined so as to embrace entire ranges of broadly-defined radical groups such as all alkyl or aryl radicals either unsubstituted or substituted by halogen and/or hydroxy, although for practical reasons only a very small number of specific examples are given. The invention consists in the selection of a particular radical or particular group of radicals from amongst those referred to, as the substituent ' $R$ ' .... The resulting compounds
\end{abstract}

(a) are not described as having, nor shown to possess, any advantageous properties not possessed by the prior art examples; or

(b) are described as possessing advantageous properties compared with the compounds specifically referred to in the prior art but these properties are ones which the person skilled in the art would expect such compounds to possess, so that he is likely to be led to make this selection.",339

\footnotetext{
${ }^{337}$ T $3 / 89$ of 1402 1990: "It is not the case here, that by mentioning the general term 'markers' and three examples out of the indefinite number of possible markers the claimed ones are inevitably disclosed. ... Whether or not the four markers mentioned in Claim 5 are equivalent to those described in document (1) in their effect and function, namely to detect a certain antigen in a sample by the use of labelled monoclonal antibodies, does not play a decisive role in the present decision because the Board follows the general approach developed in the case law cited above, namely that equivalents are not to be taken into account in the examining of novelty."

${ }^{338}$ See Spangenberg, R., IIC 1997, 812.

${ }^{339}$ Guidelines C IV 9.8 (C1) iv.
} 
This passage makes it clear that the patentability of a selection turns on the selected substances having a better effect than those generally occurring in the Markush formula and also on the man skilled in the art not being led by the content of the prior generic art to specifically select the claimed and selected substances with the improved effect. On this latter point, it was stated in T 941/98 that the novelty of an individual chemical compound falling under the scope of a general formula can only be denied if there is an unambiguous pointer to its individual configuration in the form of a technical teaching in the prior generic art. ${ }^{340}$ That makes the number of potential variations in the known Markush formula decisive.

In claim 2 of the patent application in suit in T 7/86, the following

individualised substance was indicated:

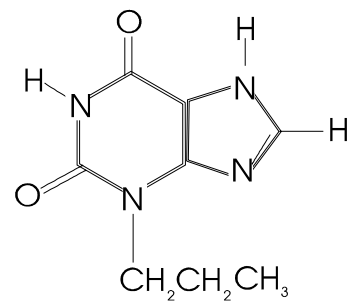

A prior document disclosed the following general formula:<smiles>[R]NC1NC([R])NC1C(N)=O</smiles>

The available choices had for this formulae been specified as $\mathrm{R}_{1}=\mathrm{Me}, \mathrm{Et}, \mathrm{Pr}, \mathrm{Bu}$ and lower alkyls; $\mathrm{R}_{2}=\mathrm{H}$ and lower alkyls. The substances were diuretic. The claimed substance in the case was obtained from the Markush formula if $\mathrm{R}_{1}=\mathrm{Pr}$, i.e. propyl, and $\mathrm{R}_{2}=\mathrm{H}$, i.e. hydrogen. The Board of Appeal found that such a selection was new. The Board ruled that if not all the substances coming within the disclosure $\mathrm{C}_{1}-\mathrm{C}_{4}$ alkyl bromide belonged to the state of the art (the Board referred to $\mathrm{T} 181 / 82$ ), then in this case not all substances coming within a field defined by two groups $\left(\mathrm{R}_{1}\right.$ and $\left.\mathrm{R}_{2}\right)$ could be deemed known. ${ }^{341} \mathrm{~T} 7 / 86$ can be construed in such a way that if there are two variable positions in a general formula, novelty exists when a

\footnotetext{
340 T 941/98 of 30031999.

${ }^{341}$ T 7/86, OJ EPO 1988, 381: “[I]n a previous case, the Board has refused to regard those compounds, which result from the reaction of one compound arbitrarily selected from a group of generically defined reactants ... C1-C4-alkyl bromides (cf. T 181/82 OJ EPO 1984, 401, 410). But if a mere precisely structurally defined (described by a chemical reaction) class of chemical compounds with only one generically defined substituent does not represent a prior disclosure of all the theoretical compounds encompassed by an arbitrary choice of a substituent definition, it must be clearly valid for a group of chemical substances, the general formula of which has two variable groups. Therefore, in the present case, a class of chemical compounds, defined only by a general structural formula having at least two variable groups does not specifically disclose each of the individual compounds which would result from the combination of all possible variants within such groups."
} 
selection is made of one individual substance within the formula. In the case law, a construction of this kind has been described as a general rule. ${ }^{342}$

In the case law, then, emphasis has been placed on the existence of opportunities of choice at two points in a known Markush formula. In T 258/91 an opponent submitted that a selection from the known Markush formula had only been made with respect to one position and that novelty was therefore lacking. The opponent pointed first to a substance indicated in an example in a prior art document and then to the fact that, if a substituent in it was substituted in accordance with the possibilities indicated for the general formula, the claimed substance was obtained. The Board of Appeal found, however, that the opponent's argument was faulty, because it overlooked the fact of the choice to change a certain example being a choice in itself. Novelty existed because two choices had been made: first the example and then a new substituent, which was indicated as an alternative for the general formula. This decision shows that the rule of selection on two points is only to be taken as alluding to a selection made from a general (Markush) formula. If the point of departure is something else in the known document, this in itself is to be construed as a selection. ${ }^{343}$

In the Guidelines paragraph quoted above, it is pointed out that, in order for a selection to be new, the choices made must have a decisive bearing on the technical effect. In $\mathrm{T}$ 163/93, two selections had been made, but one choice did not, in principle, affect the end result. The choice made by the patent applicant was virtually of no consequence: no choice really needed to be made at all. It was only the other parameter which constituted a real choice, affecting the result. In such a situation there is no novelty. ${ }^{344}$

\subsubsection{Selection from an unlimited number of possibilities}

So far we have been grappling with situations in which the selection consisted of chemical compounds. Chemical compounds are individual units and there is a limited number of such units, for example, within a Markush formula, because it is not possible for any structures whatsoever to exist. A reference to "halogens" is interpreted by a chemist, for example, as: fluorine, chlorine, bromine, iodine (there is perhaps some uncertainty as to whether astate is a substance belonging to this group). A different situation arises if the disclosure in the state of the art is by a numeric interval, e.g. "between 5 and 25\%". If so we have a continuum, not a

\footnotetext{
${ }^{342}$ T 169/94 of 3107 1996: "In accordance with previous decisions of the Boards of Appeal concerning the disclosure of general structural formulae (for instance T 7/86, OJ EPO 1988, 381) the Board regards a disclosure of a class of chemical compounds defined only by a general complete formula having at least two variable groups of elements (in the present case $\mathrm{Az}$ and $\mathrm{Bx}$ ) as not being equivalent to a specific disclose of each of the individual compounds which would result from the combination of all possible variants within such groups."

${ }^{343}$ T 258/91 of 1902 1993: "Die Beschwerdeführerin übersieht bei ihrer Betrachtungsweise, daß bei von der Kenntnis der Erfindung unbeeinflußter Interpretation des Inhalts der Druckschrift (1) ein erster Auswahlschritt schon in der Wahl des Beispiels 2 als Ausgangspunkt besteht. Eine Lehre, gerade diese nur beispielhaft erwähnte Verbindung zu modifizieren, ist in Druckschrift (1) jedoch nicht enthalten. Betrachtet man die Angaben über bestimmte Substituenten und das Herstellungsverfahren jedoch im Kontext mit der allgemeinen Formel, auf die sie sich beziehen, so wird unmittelbar deutlich, daß damit nur die Herstellung einer Verbindungsklasse, nicht aber einer bestimmten Einzelverbindung gelehrt wird.”

${ }^{344}$ T 167/93 of 0305 1996: "The Appellant's submission that there would be no difference between the present case and that of the decision T 7/86 cannot be accepted by the Board. In the present case, in which document (3) ... clearly teaches that all the activators have substantially the same properties, the situation is such that each of the specified activators can be equally combined with variants of one variable range, i.e. the range of ratios of available oxygen to activator.”
} 
limited number of choices. Indications of temperature and concentration are typical numeric intervals.

The EPO soon established a special rule for assessing the novelty of a selection when there is a continuous, numerically stated interval in the state of the art. The three requirements which have come to be applied were expressed for the first time in T 198/84. ${ }^{345}$ These have been continuously invoked in subsequent case law. A summary of precedent was made in T 279/89. It was declared that a selection from a numeric interval is new if: ${ }^{346}$

1. The interval selected is narrow;

2. The interval selected is sufficiently far removed from the preferred alternatives in the state of the art, e.g. from examples given;

3. The interval selected should not be arbitrarily chosen but should result in a better technical effect ("purposive selection”).

An example of such a new selection is mentioned in the Guidelines:

\begin{abstract}
"In a process where substance A and substance B are transformed at high temperature into substance $\mathrm{C}$, it was known that there is in general a constantly increased yield of substance $\mathrm{C}$ as the temperature increases in the range between 50 and $130 \mathrm{C}$. It is now found that in the temperature range from 63 to $65 \mathrm{C}$, which previously had not been explored, the yield of substance C was considerably higher than expected.”347
\end{abstract}

This is a narrow selection. A wider numeric selection may, however, lack novelty. In T $247 / 91$ there was a prior document indicating that a temperature interval of 80 to $170^{\circ} \mathrm{C}$ should be used in the production of cyclohexanol. In the patent application in suit, the chosen temperature interval was between 85 and $115^{\circ} \mathrm{C}$. The applicant had shown that within the temperature interval a higher yield was obtained in relation to the energy supplied for the process. The Board of Appeal found that from the prior document a man skilled in the art gained the impression of $135^{\circ} \mathrm{C}$ being the optimum temperature, but that the man skilled in the art would not have taken the interval between 85 and $115^{\circ} \mathrm{C}$ to be excluded. Given this background, the whole range within the prior patent was deemed to be known and the improved effect within the range selected did not make the selection new. ${ }^{348}$

\footnotetext{
345 T 198/84, OJ EPO 1985, 209.

${ }^{346}$ T 279/89 of 0107 1991: "In that case [T198/84] where, like in the present one, the issue of novelty is raised in terms of selection invention, the Board had considered that a selection of a sub-range of numerical values from a broader range is possible when each of the following criteria is satisfied: (i) the selected sub-range should be narrow; (ii) the selected sub-range should be sufficiently far removed from the known range illustrated by means of examples; (iii) the selected area should not provide an arbitrary specimen from the prior art, i.e. not a mere embodiment of the prior description, but another invention (purposive selection).”

${ }^{347}$ Guidelines C IV 9.8 (C2).

${ }^{348}$ T 247/91 of 3002 1992: "[O]n the basis of the examples and the teaching that a lower temperature is associated with a somewhat longer residence time ..., a preference might be construed for the use of a temperature of about $135{ }^{\circ} \mathrm{C}$. However, it was accepted by the Appellant that a skilled reader of document (1) had no reason to exclude the present range of 85 to $115^{\circ} \mathrm{C}$. In these circumstances the teaching of document (1) is clearly not limited to the use the exemplified temperatures but extends to the whole described temperature range. Thus, the complete temperature range of 80 to $170{ }^{\circ} \mathrm{C}$ has been made available to the skilled person as a technical teaching. The Appellant's submission that the temperature range had not been arbitrarily selected, but was based on the surprising effect that in the particular range a higher yield per time unit at a lower energy input is obtained, is not relevant in the present case because the sub-range selected from a larger range cannot be rendered new by virtue of a newly discovered effect, but must be new per se .... Therefore, in the Board's judgment, it follows that the temperature range of 85 to $115{ }^{\circ} \mathrm{C}$ forms part of the state of the art, and that the subject-matter of present Claim 1 lacks novelty.”
} 
One important factor when judging whether the selection is sufficiently narrow is whether the applicant made selections from several intervals at once. In T 245/91 the selection mainly consisted in the applicant having reduced the extent of a number of intervals in a document belonging to the state of the art by between 25 and $80 \%$ and retaining the central parts. These, then, taken separately were not very narrow numeric selections. The Board of Appeal, however, found that novelty existed. It was the large number of selection intervals, more than ten of them, which, taken together, made the selection new in relation to the prior document. ${ }^{349}$

In numeric selections, the area chosen has to be narrow in relation to the known area and, moreover, must be sufficiently far removed from preferred examples within the wider range. In T 198/84 the latter criterion was found to be satisfied, because the distance between the selection ( 0.02 to $0.2 \mathrm{~mol}$ per cent) and what in the state of the art had been indicated as a preferred form (between 2 and 13 mol per cent) was at least ten times (0.2 in relation to 2). ${ }^{350}$ The opposite outcome resulted in $\mathrm{T} 17 / 85$. The selection consisted of a parameter which the applicant had given as 4 to 8 . This was found to lack novelty in relation to a known preferred interval of between 2.6 and 13 . The selection came too close to what had been used previously. ${ }^{351}$

In T 366/90 a time interval for a production process had been disclosed in the state of the art in quite enigmatic terms: "less than between 20 and 72 hours". The Board of Appeal found that the disclosure should be construed as at least some hours. Using a time interval of between 1 and 30 minutes in a similar process was therefore found to be new. It was sufficiently far removed from what the man skilled in the art would have deduced from the prior document. ${ }^{352}$

A narrow selection which is also sufficiently far removed from expressly indicated parts of the state of the art is still not sufficient for a selection to be new. Novelty also requires that some special effect should be associated with the area chosen. Otherwise the selection is arbitrary and of no practical importance. The technical effect must be better within the selected area than that occurring generally within the known, wider range. The improved effect is usually called a requirement of "purposive selection" on the applicant's part. ${ }^{353}$ The crucial question is whether the man skilled in the art would have seriously

\footnotetext{
${ }^{349}$ T 245/91 of 2106 1994: "Even if most of the ranges for these parameters correspond to a more or less central portion of the range limiting the corresponding parameter in the composition according to D8, because of the number of parameters involved, which exceeds 10, the scope of the claimed blends is in reality quite narrow with regard to the breath of the definition of the known composition. For this reason ... the argument of an implicit description in D8 of this narrow selection cannot be accepted.”

${ }^{350}$ T 198/84, OJ EPO 1985, 209.

${ }^{351} \mathrm{~T}$ 17/85, OJ EPO 1986, 406: "The claimed sub-range of $\mathrm{R}=4$ - 8 is not a narrow section of the known subrange of 6.67 - 31.6 nor is it sufficiently far removed from the known range cited as an example, which lies between 2.6 and 13. In fact, it falls in the middle of it. The skilled person does not see these examples as separate, isolated embodiments in connection with the preferred range indicated but as representatives of a range extending at least from the lower particle distribution value $(\mathrm{R}=2.6)$ to the upper $(\mathrm{R}=13)$ and thus covering all the values in between, i.e. including the range claimed here."

${ }^{352}$ T 366/90 of 1706 1992: "In the Board's judgment, the passage quoted from document (2) points ... to MRTs which are, while being 'less than 20 to 72 hours', still in the order of magnitude of some, perhaps some few, hours. Even the values of MRTs derivable from the Examples 2(a) and 2(b) are in the order of magnitude of 1 hour. Thus, the Board concludes that in the absence of additional information to that end, the skilled person would not have seriously contemplated further reducing the MRTs and, consequently, that the feature of the presently claimed process 'a mean residence time of 1 - 30 min. to effect interesterification' had not been made available to the public. It follows that the subject matter of present Claim 1 is novel."

${ }^{353}$ T 198/84, OJ EPO 1985, 209: "It remains to be established whether this view of novelty really entails more than just a formal delimitation of the process concerned vis-à-vis the state of the art. It would be delimited only in respect of the wording of the definition of the invention, but not in respect of its content, if the selection were arbitrary, i.e. if the selected range only had the same properties and capabilities as the whole range, so that what
} 
contemplated using the selected range and thus been able to benefit from the improved effect. $^{354}$

Having thus proceeded from considering different definitions of physical phenomena in the patent claims and the prior documents (e.g. intervals or groups of substances) to considering whether it was likely that the man skilled in the art could have used a demonstrated improved technical effect, we approach what is normally considered to be an assessment of inventive step. The line of demarcation between novelty and inventive step is usually drawn by the man skilled in the art being regarded, in the assessment of novelty, mainly as a passive observer, while in the assessment of inventive step he is active and prepared to make changes to what exists in the state of the art. This, however, is a very hard distinction to maintain where selection inventions are concerned, because the subject of the claim falls within the terms of the prior document. The man skilled in the art does not make any changes which propels him outside the previous disclosure. Instead the question now is how the man skilled in the art understands a document and its possibilities for technical use. The assessment of novelty then becomes very similar to the assessment of inventive step.

The distinction between novelty and inventive step in connection with selection inventions was discussed in T 133/92. The applicant had shown that certain substances produced a better bleaching result than was known from a wider group of substances. With reference to this improvement, the patent applicant claimed that there was an inventive step and that, this being the case, the selection must also be new. This, however, was not accepted by the Board of Appeal. The Board ruled that a selection which has an inventive step need not be new. It shall not be possible to evade the assessment of novelty by demonstrating an inventive step. ${ }^{355}$ Despite the ruling by the Board of Appeal, it is hard to see how it will be possible to maintain any demarcation between the two requisites. Happily, no such distinction

had been selected was only an arbitrary specimen from the prior art. This is not the case, since the effect of the substantial improvement in yield may be believed to occur only within the selected range, but not over the whole known range ('purposive selection')." Cf., however, T 666/89, OJ EPO 1993, 495: "In the Board's view, there is no fundamental difference between examining novelty in situations of so-called 'overlap' or 'selection', and in doing so in other situations, although it may be helpful, in order to verify a preliminary conclusion of a novelty examination in cases of overlap, to investigate whether or not a particular technical effect is associated with the narrow range in question. It needs to be stressed, however, that such a particular effect is neither a prerequisite for novelty nor can it as such confer novelty: its existence can merely serve to confirm a finding of novelty already achieved”.

354 T 666/89, OJ EPO 1993, 495: "In the case of overlapping ranges of physical parameters between a claim and a prior art disclosure, what will often help to determine what is 'hidden' as opposed to what has been made available, is whether or not a skilled person would find it difficult to carry out the prior art teaching in the range of overlap .... A similar approach ... for assessing the novelty of a claim in a case where overlapping numerical ranges of certain parameters exist between a claim and a prior art document, is to consider whether a person skilled in the art would, in the light of all the technical facts at his disposal, seriously contemplate applying the technical teaching of the prior art document in the range of overlap. Provided the information in the prior art document, in combination with the skilled person's common general knowledge, is sufficient to enable him to practise the technical teaching, and if it can reasonably be assumed that he would do so, then the claim in question will lack novelty.”

${ }^{355}$ T 133/92 of 1810 1994: "[T] for deciding selection novelty was identical or closely similar to that employed in determining obviousness. In particular, he put forward the proposition that in cases of overlapping ranges of compounds, a claim to a narrower range as compared with a broader prior art range was always selectively novel if it could be demonstrated that the narrow range was inventive over the broader range. ... Whereas it is undoubtedly true that there can be no selection novelty in a range of overlap where the choice of moving into that overlapping range from the prior art one is obvious, it doesn't either as a matter of law or as a matter of logic follow that the converse is true, namely that if a choice of a narrower range is inventive, then there must of necessity be selective novelty in it. For the above reasons, the Respondent's argument in this respect cannot be accepted.” 
is necessary in the majority of cases. ${ }^{356}$ Probably there are no great practical problems involved in assessment of novelty and assessment of inventive step coinciding to some extent where selection inventions are concerned. Thus, within the novelty assessment of selection inventions, one can and should address the question of whether the man skilled in the art would have contemplated using the selected range.

Since the assessment hinges on the likelihood that the man skilled in the art would have chosen the claimed range, it is important if in the state of the art there are special pronouncements arguing for or against selecting the claimed range. In T 26/85 there was a prior document in which it was stated that a coating should be $3 \mu \mathrm{m}$ or less. A preferred form was given as between 0.1 and $3 \mu \mathrm{m}$. The Board of Appeal found that in relation to this a selection was new in which the layer had been defined as between 0.05 and $0.1 \mu \mathrm{m}$. This conclusion was due to the known document containing a statement which advised the man skilled in the art against using layers less than $0.1 \mu \mathrm{m}$ thick: "if the thickness of the recording layer is too small ... a low or insufficient reproduced output is obtained .... Therefore ... the minimum thickness of the recording layer is at least $0.1 \mu \mathrm{m}$ and preferably at least $0.5 \mu \mathrm{m}$." The applicant's selection fell within the scope of the claim of the previous patent (less than 3 $\mu \mathrm{m})$ and, moreover, directly adjoined a preferred form. In such a situation one may presume that the novelty requirement is only satisfied if the state of the art clearly advises against the range selected. ${ }^{357}$

\subsection{Remarks}

\subsubsection{The global and absolute novelty requirement}

It is commonly said that the task of the patent system is to encourage the development of as much new technical knowledge as possible as quickly as possible. Novelty assessment is thus viewed as an essential part of patent law. But there can be no once-and-for-all definition of what is new. It must be related to its practical consequences. One field in which problems may occur is known medicinal plants. There is a risk of the global and absolute requirement of novelty in patent law causing pharmaceutical research to focus excessively on new, synthetic pharmaceuticals, to the detriment of the development of therapy methods based on traditional medicinal knowledge, which perhaps offer more valuable therapeutic possibilities but at the same time entail greater risks of protection being difficult to obtain or less certain. $^{358}$

If a pharmaceutical company decides to investigate the possibilities of using components of traditional medicinal plants therapeutically, there is a palpable risk of a prior document detrimental to novelty being found somewhere in the world. The Indian Council of Scientific and Industrial Research (CSIRO), for example, has fought a successful opposition action in the USA concerning a patent for the therapeutic use of the medicinal plant turmeric (Curcuma longa). ${ }^{359}$ Two American university researchers had found that this plant could be taken perorally and also applied to the skin to speed up the healing of wounds. A patent was obtained in the USA in 1995 for the use of the plant in the connection with the healing of surgical wounds and gastric ulcers, but in 1997 the US Patent Office revoked it. The use of turmeric in the treatment of wounds was a part of traditional Indian medical knowledge. One

\footnotetext{
356 There is an exception in art. 54(3) EPC.

${ }^{357} \mathrm{~T}$ 26/85, OJ EPO 1990, 22.

${ }^{358}$ Cf. Blakeney, M., [1997] EIPR 298.

359 See Jayaraman, K. S., 389 Nature 0409 1997, 6.
} 
of the prior documents invoked by the CSIRO was an Indian national epic, an Ayurvedic text describing the traditional Hindu science of medicine. For the purpose of defending "national honour" the CSIRO is running a project in which knowledge of 490 traditional Indian medicinal plants is being collected on a compact disk which will be supplied to the EPO and USPTO as a work of reference for the assessment of novelty.

If and when the national patent offices gain access to the compact disk, however, it seems difficult or impossible to give an economic justification for patent applications being refused on the strength of this information. If the traditional Indian medicinal plants have not been previously used in the countries where patents are applied for - the compact disk is the only relevant prior art - there is reason to believe that there has been insufficient incentive for introducing the medicinal plants on the market which the patent is to protect. The technical knowledge is there, but without protection the available incentive in these situations appears to be too limited to lead to production. In such a situation, the absolute and global novelty requirement appears to be inefficient. Thus one risk entailed by the novelty concept in use today is that it only takes into account the existence of technical knowledge, not the need for market incentives, i.e. a sufficient consumer demand as a prerequisite for production. The essential purpose of the patent system is to encourage production, and for this both technical knowledge and sufficient market demand are necessary and, in principle, equally decisive factors.

The accumulation of market demand in favour of one player (the patentee) and against the interests of other players seems justified if a situation of free competition does not lead to production. Technical knowledge has then existed for a considerable time but has not been made use of. The grant of patents in such situations does not give rise to any costs in the form of production constraints on competitors. The willingness of the party applying for the patent to pay the cost of patenting shows that he at least considers the market to be sufficient to justify production, so long as he is able to avail himself of the entire market, i.e. obtain protection. In these situations it is of benefit to consumers for patents to be granted and for the patentee to have the opportunity of testing whether there is a market for the product. If a market is lacking after all, the applicant will stop paying the annual fees and the protection will lapse. Thus whether or not the invention was technically known need not of itself be decisive in the assessment of novelty.

There are, however, administrative considerations which, in the assessment of novelty and patentability, make it difficult to go by actual production instead of technical knowledge. All the same, it would presumably be useful, when establishing the state of the art, to give more conscious consideration to the amount of time that has passed since a prior document became generally available, because in this way the demand side, which is no less important than technical knowledge, can also be taken into account in the assessment of novelty. If a production possibility has been known but a considerable length of time, say 15 or 20 years, has passed without it being put into production, there can hardly be any economical reason for refusing a patent application for the solution. If no production is started during this time, then probably another 20 years will pass without any production beginning. In such a situation there is no cost associated with the grant of a patent. But instead one may reach the critical point where both technical knowledge and demand are sufficient for production. There are examples in EPO case law where Boards of Appeal have considered whether an unusually old document really was a realistic prior art document, see part 6.5.2.

\subsubsection{Selection inventions}


Introduction. The novelty requirement is a necessary but not a sufficient condition of patentability. Normally the assessment of novelty is overshadowed by the requirement of inventive step. This complicates an analysis of the novelty requirement, because the relevant economic question is whether or not the invention is patentable. This is the case with novelty assessment of selection inventions, but in few other situations within the novelty requirement. Inventions of doubtful novelty usually lack inventive step. In selection inventions, though, the two assessments tend to merge, which makes it particularly interesting to analyse the rules surrounding selection inventions.

Selection inventions are quite important for the competitive situation in the pharmaceutical sector. A typical product patent for a pharmaceutical takes the form of a Markush claim. If the Markush claim has eight substituents, the number of individual substances included in the claim can be obtained by multiplying the substituents in each of the eight positions of the molecule. Assume the following numbers of alternative substituents to have been indicated in the eight positions: $8 \times 7 \times 7 \times 3 \times 8 \times 8 \times 17 x 8$. The Markush claim then comprises 10,235,904 individual substances. How are the production possibilities within such a claim to be utilised efficiently? The regulations surrounding selection invention will be decisive, because no-one but the first patentee will carry on research within the claim concerning production possibilities which cannot be patented as selection inventions.

The rules concerning selection inventions create incentives for such research because an innovator who believes that he can patent a new selection within a generic patent claim experiences that there is a consumer demand within the claim. He can count on obtaining a share of the market for such new production possibilities as can find. If a selection patent is granted, this means that the proprietor of the first - the generic - patent will not be able to manufacture and sell the products which the subsequent patentee has shown to have a better technical effect. This situation normally gives rise to a licensing agreement, with the proprietor of the selection patent obtaining a licence for the generic patent and perhaps also the proprietor of the generic patent a licence for the selection patent. The value of the improved possibility of production within the selected range is divided between the two patentees. The actual apportionment of the value turns on the bargaining leverage of the parties.

A situation resembling that connected with selection inventions has been analysed already. In section 3.3.3.3 we studied the incentives in connection with absolute product patents, comparing them with use-related patents for pharmaceuticals. The analysis already undertaken therefore will provide the point of departure for our analysis of selection inventions, because there are points of similarity between the two situations. In both cases there is a prospecting of production possibilities. There are, however, two potentially important differences. The first of these is connected with the extent of the incentives prospected by the first patentee. The second difference concerns the market to which the subsequent dependent invention will belong.

Incentives to do selection inventions. With new uses (indications) for a known pharmaceutical, either half or all of the value accrues to the finder of the new indication. If the first patent is an absolute product patent, the competitor receives half. If instead the first patent is limited to the use for the known indication, the finder of a subsequent indication acquires all rights in it (no dependence). Thus the question as regards new indications was whether the patent system should encourage the search for new indications with all rights in the new indication or only a part, i.e. a dependent patent. It was observed that a dependent patent was probably sufficient within the pharmaceutical sector, i.e. patents should be allowed to be formulated in such a way as to include all medical indications. But on the other hand they should not include the possible non-medical uses of the substance; see section 3.3.3.3. 
In the case of selection inventions, the incentives are different. Depending on whether a selection invention is patentable or not, the party who has made the selection receives either nothing at all or part of the value of the improved effect. Conversely, the proprietor of the patent with generic claims receives half the value of the improved effect if the selection is patentable in relation to the patent with Markush claims. If the selection is deemed to lack novelty, the first patentee receives the full value of the improved effect. Thus the party who has demonstrated the improvement receives nothing if he cannot obtain a selection patent. Within a generic range, there will be no reason for parties other than the patentee to try to optimise production by investigating conceivable improved possibilities which are not admissible as novelties.

The underlying supposition in this section is that incentives within patents previously granted have a diminishing marginal benefit. That is to say, certain incentives experienced by business enterprises with regard to new production possibilities are more important than subsequent, additional incentives to make new inventions within the claims to competitors' patents. The reason is that a not insignificant proportion of all inventions come about as the result of random factors, e.g. in connection with clinical work or when a business enterprise checks the uses of another firm's patented invention. If in situations of this kind the competitor has no incentives, i.e. no possibilities of gaining a share of the market for the improvement, then probably the improvement that has been found will not be put to practical use. The person finding the improvement has no cause to draw the attention of the proprietor of the generic patent to the new opportunity. The limited amount of incentives given by the possibility of dependent selection patents is perhaps insufficient to encourage significant amounts of directed research efforts within a patented field of technology, but the possibility of receiving dependent patents makes it at least probable that inventions occurring by chance will be converted into products on the market, since an agreement can be reached between the proprietor of a generic patent and a proprietor of a dependent patent.

If, on the other hand, someone has found a better effect within a patent previously granted, without being able to obtain a patent of his own, a situation arises which is sometimes termed Arrow's paradox. ${ }^{360}$ If the competitor contacts the proprietor of the generic patent and gives an account of the improvement he has found, then as a rule he no longer has anything to sell, because the patentee will then know about the improvement. At the same time, the party demonstrating the improvement must explain to the proprietor of the generic patent what the improvement implies, otherwise the latter will not know what he is being invited to purchase. In these cases a transaction is unlikely to materialise. Arrow's paradox flags a situation when improved possibilities of production are liable not to benefit the consumers, due to the situation in which the parties finding themselves being conducive to a breakdown of negotiations. ${ }^{361}$ The need to facilitate transactions indicates that it is efficient to open up opportunities for competitors to make selection inventions whenever there is improved possibilities of production within product patents already granted. These limited incentives in the form of dependent patents are probably, in comparison, more valuable than further additional incentives, because they can make transactions possible, which is valuable. ${ }^{362}$

\footnotetext{
360 See Arrow, K. J., in The Rate and Direction of Inventive Activity: Economic and Social Factors, 615.

361 See in general on the negotiations regarding improvement inventions: Merges, R., 62 Tennessee Law Review 1994, 75, also published in Intellectual Property Law Review 1996, 69.

362 "Our general conclusion is that multiple and competitive sources of invention are socially preferable to a structure where there is only one or a few sources. Public policy, including patent law, ought to encourage inventive rivalry, and not hinder it. As the 'race to invent' model show, a rivalourous structure surely has its inefficiencies [the writers refer primarily to unnecessary duplication of research]. But such structures does tend to generate rapid technical progress and seems a much better social bet than a regime where only one or a few
} 
The importance of the market to which the selection invention belongs. Apart from the extent of incentives, there is another difference between selection inventions and inventions of new medical indications, namely in the market to which the new dependent inventions belong. The more efficient new possibilities found in a selection invention will belong to the same market as the possibilities of production indicated in the generic patent. When another medical indication is found, a different situation arises: the new indication will belong to another market, because it is a treatment for another disease. This difference makes selection inventions a category of inventions which will compete with products in which the proprietor of the first generic patent already has an exclusive right. The improved products which can be manufactured with a knowledge of the selection invention will therefore take market shares away from the products which it has been possible to produce with the aid of the content of the generic patent, i.e. products which the first patentee has already spent money on. The risk of a possible new invention preying on an existing invention with the same enterprise is that a proprietor of a first generic patent has little interest in investing in research for new and possibly better substances within the generic claim. ${ }^{363} \mathrm{~A}$ competitor of the proprietor of the generic patent, on the other hand, would disregard in his calculation the losses resulting from a decline in sales of pre-existing pharmaceuticals in the market. The competitor, therefore, tends to be a more efficient user of the incentives which the possibility of a patent for selection inventions implies.

One must bear in mind, however, that a patent in the pharmaceutical industry seldom confers protection comprising a complete or almost a complete market. There are practically always alternative forms of treatment to patented pharmaceuticals. A patentee usually obtains at most about $10-20 \%$ of the market. If so it is not only his sales that will be affected by a new, improved product. Most of the market share for the improved products will instead be taken from the competitors, and in a situation of this kind there is still a relatively effective stimulus for continuing research within a generic patent. ${ }^{364}$ In addition, the proprietor of a generic patent may have a strong interest in making selection inventions, because the protection may then be extended beyond twenty years. First the substance is protected for twenty years by the generic patent, and then protection is conferred for an additional number of years by the selection patent.

Conclusions. The EPO's novelty assessment of selection inventions has already been described as liberal. In practice an assessment is made of the likelihood that a skilled man wishing to exercise the invention in the earlier generic patent would have chosen the field later claimed. It seems that the selection must appear to the skilled man as a suitable alternative in the generic range, in order for novelty to be lacking. If the selection has been made from a Markush formula, it seems to be sufficient for the selection to have been made with disclosure of individual substances, if the Markush formula from which the selection was made had two variable positions. This is a liberal practice. But it should be borne in mind that achievement of a superior technical effect within the selected range is always required as well. This seems to be an appropriate state of the law.

organizations control the development of any given technology.” See Merges, P., Nelson, R., 90 Columbia Law Review 1990, 908.

363 “The firm presently realizing monopoly profits may be less motivated to seek additional profits than one earning only normal profits. It may, in other words, be less hungry for additional profits than the firm without a monopoly position. Several reasons for this is possible. ... [I]t may become more concerned with protecting its current monopoly position than in acquiring a new one.” See Kamien, M., and Schwartz, N., Market Structure and Innovation, 29.

364 See Cockburn, I., Henderson, R., 3:3 Journal of Economics \& Management Strategy Fall 1994, 508. 
When selection inventions are made possible to a great extent, this means that incentives concerning substances with a better effect within a generic patent can, to a considerable extent at least, accrue to the party who makes the selection. The proprietor of a generic patent does, it is true, have an advantage over the competitors in this research. This may be justified, because in this way certain losses in the form of unnecessary duplication are avoided. The competitors concentrate their research on substances outside the protective scope of the patent, while the patentee investigates the occurrence of possible better substances within the protection. At the same time it is important that, within a generic patent, there remain incentives in situations where new possibilities of production can be found. If rivals of the proprietor of the generic patent were to be entirely deprived of the possibility of dependent selection inventions, the co-ordination of research which occurs when competitors can make an agreement with the proprietor of the generic patent would not be achieved. Often, no doubt, it is in any case impossible for agreements to be reached between the proprietor of a generic patent and the party disclosing an improvement. ${ }^{365}$ But with liberal case law, at least the possibilities are improved. It would be especially deplorable if agreements of this kind were prevented in the pharmaceutical sector, where it is common for improved therapeutic results to be achieved by coincidence. The strict German novelty assessment of selection inventions, therefore, probably yields an inferior result to that of the more liberal attitude of the EPO. In situations where improvements can be found, a certain measure of competition is better than no competition at all.

It is especially important to preserve the possibility of selection inventions when the generic patent is so widely formulated as to give the proprietor a large share of the market. The larger the market share obtained by the proprietor of the generic patent by virtue of his exclusive right, the less receptive he will be to incentives concerning new possibilities of production in the same market. He will not willingly compete with himself. Accordingly there may be reason to consider the competitive situation in the market for the products which have been patented with the generic patent. The less competition the generic patent encounters in the therapy segmented concerned (the medical indication), the easier it should be to make selections within the protective scope of the patent. In the assessment of novelty, one should perhaps not only consider the "technical" size of the protection within the generic patent, e.g. the number of individual substances included, and then compare that scope with the scope of the selection. The "economic scope" of the generic patent is also important. This entity can be judged according to whether there are limited possibilities for competitors, outside the generic patent, to develop new products which will compete in the same market as the generic patent. If this is the case, the economic scope is great and the possibilities of selection inventions should also be great. If, on the other hand, the competitors have good possibilities of research outside the generic patent, co-ordination and avoidance of research duplication are more important, i.e. the proprietor of the generic patent conducting research within the patent and his competitors outside the claim.

Given the value of competition within the framework of broad generic patents, it can even be justifiable for selection inventions not to be made at all dependent on the generic patent. A solution of this kind seems suitable if the economic value of the selection invention substantially exceeds the value of the generic invention. There is then reason to allow competitors to have their incentives intact. Otherwise, when the improvement in the technical

\footnotetext{
365 " [I]t seems whimsical to assume that all improvers and potential improvers will be able to bargain with the holders of pioneering patents. Imagine the magnitude of theses costs: identifying all the prospective improvers; agreeing on the value of the pioneering invention and the expected value of the improvement and finding an acceptable division of profits from the 'surplus' created when the improvement is combined with the pioneer invention.” See Merges, P., Nelson, R., 90 Columbia Law Review 1990, 877, footnote 160.
} 
effect is substantial, there are special risks of a breakdown in the event of licensing negotiations. It has been said that:

\begin{abstract}
"Where the original invention contributes most of the value [e.g. to the combination of generic patent and selection patent], or where the original and improvement patent contribute roughly equal value, issuing an improvement patent may be a reasonable solution. But where the original patent contributes very little value compared to the improvement, the hold-up problem may be significant. That is, the holder of the original patent may extract much of the value of the 'original plus improvement' combination from the improver. The reverse doctrine of equivalents solves the problem by, in effect, excusing the improver from infringement liability - and therefore removing the original patentee's hold-up right." 366
\end{abstract}

The reverse doctrine of equivalents referred to in this quotation and applied in American patent law has been described in section 3.3.4.6, where the conclusion was reached that this principle of construction was justified in certain situations with functional claims, more exactly when a subsequent improvement was of substantially greater value than the technical effect indicated in the functionally formulated patent. There should also be a justification for a reverse doctrine of equivalents in certain selection situations. The economic conditions are similar. Suitable incentives are probably achieved if competitors to the proprietors of generic patents have unlimited incentives with regard to new effects whose value significantly exceeds the value of the effect disclosed in the generic patent. The proprietor of a particularly valuable selection patent should have the possibility of petitioning a court, by virtue of the doctrine of reverse equivalents, for confirmation that there is no relation of dependency between the selection invention and the generic patent. Even though actions of this kind may perhaps not be filed in very great numbers, the mere possibility is likely to have a positive impact on the incentives in the research situation and the outcome of licensing negotiations.

\footnotetext{
366 See Merges, P., Nelson, R., 90 Columbia Law Review 1990, 865. Footnotes have been excluded from the quotation. See further Merges, R., 62 Tennessee Law Review 1994, 75, also published in Intellectual Property Law Review 1996, 69.
} 


\section{New medical uses of known compounds}

\subsection{New medical indications and the systematisation of patent law}

A medical indication is a pathological state which can be treated with a pharmaceutical. Reading a pharmaceutical packaging or a medical work of reference, however, one finds that an indication can be many different things: an illness, certain specific symptoms and even preventive effects. Often a pharmaceutical has many different indications. Quinine, for example, can be used for malaria prevention, for the treatment of pneumonia, as an antipyretic and also as an analgesic. It is commoner, however, for different indications of a substance to be more closely related. If so it can be hard to draw a clear line of demarcation between different indications. Not infrequently, new indications for a pharmaceutical are discovered by chance. When a patient used the pharmaceutical Amantadine for a viral infection, an unexpected improvement took place in his Parkinson-related symptoms. Another patient used Cimetidine for treating a gastric ulcer and at the same time experienced an improvement in his psoriasis. The diuretic effects of sulphonamides were discovered when the substances were being used for their antibiotic properties. ${ }^{367}$

If a previously unknown substance has proved to have a new therapeutic effect of this kind or a diagnostic effect, the party disclosing the effect can obtain an exclusive right to all use of the substance, i.e. a product patent conferring absolute protection. If the substance is known, a patent of this kind is ruled out, due to lack of novelty in the chemical structure. Nor can a new therapeutic or diagnostic effect be patented with ordinary use claims, because that would be contrary to the ban on patents for medical procedures (Art. 52(4) EPC, see section 1.3). There is no real difference between patent claims relating to a use of a substance and those relating to a therapeutic procedure: in both cases a new medical activity is patented, i.e. a new way of using one or more known products. Thus the difficulties in European patent law of protecting a new medical indication for a known substance are due to the combination of the novelty requirement (which impedes product claims) and the ban on patents for medical procedures (which impedes use claims).

\subsection{The first medical indication}

In 1971 the International Working Group tasked with drafting the EPC put forward a proposal laying down that medical procedures could not be patented. A French amendment proposal, dated 28 February 1972, stated, however, that the proposal could come to be construed in such a way that certain pharmaceuticals and pharmacological products were excluded from the patentable sphere. The French Delegation argued that this had never been the intention in the negotiations. To ensure that the first medical indication of a pharmaceutical could be patented, but not methods of medical treatment, the French Delegation proposed the following addition to the ban on patents for medical procedures:

"[T]his provision shall not apply to inventions of substances or compounds, whether or not known, presented for the first time as being intended for the purposes stated above."368

\footnotetext{
367 See Sneader, W., 60, in Hansch, C., Taylor, J. B., (red.), Comprehensive medicinal chemistry: The rational design, mechanistic study and therapeutic application of chemical compounds.

368 For the preparatory works see Törnroth, L., NIR 1987, 161.
} 
The French amendment was carried. The Working Group stated that the new wording afforded a use limited product protection for the first medical indication of a known substance. After certain, mainly editorial, alterations, the rule regarding the first medical indication became Art. 54(5) EPC, which came to be worded as follows:

\footnotetext{
"The provisions of paragraphs 1 to 4 [the rules on novelty] shall not exclude the patentability of any substance or composition, comprised in the state of the art, for use in a method referred to in Article 52, paragraph 4 [therapeutic, diagnostic and surgical], provided that its use for any method referred to in that paragraph is not comprised in the state of the art.”
}

In this way a legal situation had been established whereby a first medical indication for a previously known substance was patentable. The patent was regarded as a product patent and, therefore, was not contrary to the ban on patents for medical procedures. But in the novelty assessment of the product patent, the disclosure of the medical use in the claim would mean that the content of the state of the art was limited to the medical sphere. Knowledge of the structure of the substance in a different technical field was not deemed to make it known in the medical field. This innovation of patent law - and breach with the systematisation of patent law - has not created any palpable problems in case law. It is distinctly supported by the EPC. The Guidelines also provide that a first medical indication for a composition can be patented. ${ }^{369}$

\subsubsection{The breadth of patent claims to a first medical indication}

The structure of the substance is already known when a first medical indication is disclosed. A use limited protection therefore has to be resorted to. The question remains, however, how the area of use is to be indicated in the patent claim, i.e. which medical uses a patent for a first medical use may include. Is a patent for the first medical indication to afford protection for all medical use of the chemical compound or only for the specified possibility of medical use disclosed by the inventor?

In T 128/82, chemical compounds had been claimed with the broad disclosure in the claim that they were "therapeutically active". The Examining Division refused the application on the grounds that the specific therapeutic use (improved the function of the brain and the intellectual capacity) needed to be disclosed in the claim. But after the decision had been appealed, the Board of Appeal came to a different conclusion. Noting that absolute product patents were granted for pharmaceuticals, it ruled that broad patents should therefore also be permitted for a first medical indication. Equal cases should be treated equally. All therapeutic use of the compounds, therefore, could be included in the claim. ${ }^{370}$

\footnotetext{
369 Guidelines C IV 4.2.

370 T 128/82, OJ EPO 1984, 164: “[T]he practice of the European Patent Office hitherto has shown that substance and medical preparation claims for therapeutically active compounds not limited to specific indications are allowed, even though as a rule only certain specific activities are stipulated. This practice usually always concerns new compounds. It does not derive from the Convention that compounds which although previously known are still patentable under Article 54(5) are in principle to be treated differently. If an inventor is granted absolute protection in respect of a new chemical compound for use in therapy, the principle of equal treatment would require that an inventor who for the first time makes a known compound available for therapy should be correspondingly rewarded for his service with a purpose-limited substance claim under Article 54(5) EPC to cover the whole field of therapy. Any other treatment would only be justified were Article 54(5) EPC absolutely to forbid a broad protective scope. The fact that Article 54(5) EPC does not contain any requirement that protection should be broad is of itself no reason for refusing to grant such protection. As a general rule the usual practice as it relates to new compounds should be followed."
} 
The principle of $\mathrm{T}$ 128/82 has now become established practice. The patent claim for a first medical indication may be formulated as: "Compound(s) $\mathrm{X}$ for use as a pharmaceutical". But the applicant for a patent for a first medical indication is under no obligation to extend the protection to the whole of the medical field. It was established in $\mathrm{T}$ 143/94 that the applicant may choose to limit his protection to the specific indication or indications he has found and in doing so use the wording developed for a second medical indication. ${ }^{371}$

The Guidelines give examples of patent claims for a first medical indication with successively narrower protection:

"A claim to a known substance or composition for the first use in surgical, therapeutic and/or diagnostic methods should be in a form such as: 'substance or composition $\mathrm{X}$ ' followed by the indication of the use, for instance '... for use as a medicament', '... as an antibacterian' or '... for curing disease $Y^{\prime}$, , 372

Limitation of the scope of protection may be necessary because of the inventive step requirement: a certain medical use - other than that found by the applicant - may have been obvious for the person skilled in the art, in the light of existing knowledge about the compound. If so it may not be protected through the inclusion in the claim of all therapeutic use. ${ }^{373}$

If the new use is mentioned in the claim it must be mentioned as such. It is not allowed to formulate the claims to a product, which is described in a way that shows that it is capable of the new medical use. In T 574/93 the subject-matter of the main claim was "a substantially water-insoluble drug substance in liquid form and a phospholipid monolayer surrounding the anaesthetic". The first auxiliary request had a similar wording: "anaesthetic in liquid form and a phospholipid monolayer surrounding the anaesthetic". The Board of Appeal refused the claims because it was not clear whether protection was sought for a product without any restrictions as to its use, or the protection would relate to a pharmaceutical preparation for use in a method mentioned under Articles 52(4) and 54(5) EPC. Only the latter was, however, allowed. The second auxiliary request was allowed: ”a substantially water-insoluble general or inhalation anaesthetic in liquid form and a phospholipid monolayer surrounding the anaesthetic ... for use in a method of inducing anaesthesia."374

371 T 143/94, OJ EPO 1996, 430: "Headnote: A claim directed to the use of a substance or composition for the production of a medicament for a therapeutic application does not conflict with Articles 52(4) or 57 EPC ..., irrespective of the purpose (protection of a first medical use of a substance or composition, or protection of a further medical use) served by that claim .... Accordingly, prior evidence of a further medical use is not required for this form of claim to be included in a patent application”.

372 Guidelines C IV 4.2.

373 T 128/82, OJ EPO 1984, 164: “[I]n the present case Article 54(5) EPC permits a purpose-limited substance claim stating a general therapeutic purpose. This does not rule out the conceivability of other cases in which because, for example, of insufficient inventive step, the state of the art could make it necessary to limit the scope of application.”

374 T 574/93 of 1902 1998: "The Appellant has based his argumentation on the assumption that the subject matter of claim 1 of the main request and that of claim 1 of the first auxiliary request would relate to a product within the meaning of a so-called first medical indication. However, having regard to the wording of those claims: '1. An aqueous suspension of microdroplets of from $20 \mathrm{~nm}$ to $10 \mu$ in diameter consisting essentially of a sphere of: (i) a substantially water-insoluble drug substance in liquid form (main request); [anaesthetic in liquid form (first auxiliary request)]; and (ii) a phospholipid monolayer surrounding the anaesthetic; the suspension being free of a fat or oil of vegetable or animal origin (emphasis added)', it is not clear whether protection is sought for an aqueous suspension of microdroplets including beside other components a drug (anaesthetic) component and thus the subject-matter of the claim would relate to a product without any restrictions as to its 


\subsection{The second medical indication}

\subsubsection{Introduction of patents for a second medical indication}

The term "a second medical indication" refers to the situation where it has been found that a known pharmaceutical can be used for the treatment of a new disease, i.e. when the pharmaceutical has proved to have a previously unknown indication or use. The term can, however, be misleading, because it refers not only to the numerically second indication but also to every new possibility of treatment with a known pharmaceutical - the third, fourth and so on.

One of the most debated issues of patent law in Europe during the 1980s was whether a second medical indication could be patented. There were three main reasons for this question acquiring such prominence. Firstly, during the $20^{\text {th }}$ century the number of known substances with medical effects had gradually increased, making it increasingly likely that a new medical effect would be found for a previously known pharmaceutical. Secondly, during the 1970s and 1980s there was a shift of pharmaceutical research towards the life sciences. The role of pharmacologists in pharmaceutical research outdistanced that of the chemists. Synthesising thousands of randomly chosen new chemical compounds and then testing their effects gradually became a less efficient research strategy. Instead research began to be based more on biological studies and on known connections between chemical structural elements and biological effects. Research took as its starting point the knowledge already available concerning known pharmaceuticals, which increased the likelihood of finding a new therapeutic effect for a known substance. The third reason was that, from the 1960s onwards in the wake of the Thalidomide disaster - increasingly strict demands began to be made for controlled studies of pharmaceuticals before they could be sold. More toxicological tests were performed and clinical trials were made more meticulous. In such investigations of adverse effects, it is not uncommon for new positive and therapeutically useful effects to be found at the same time.

The EPC does not contain any express provisions concerning a second medical indication, unlike the first. Legal development has proceeded through case law, subsequent to the entry into force of the EPC. The turning point in these developments was the seven parallel cases in which the Enlarged Board of Appeal of the EPO decided that patents were to be granted for a second medical indication for a known pharmaceutical. ${ }^{375}$ The claims, however, were not to be formulated as a new use of the known pharmaceutical, because that would be contrary to the ban on patents for medical procedures. The Enlarged Board of Appeal ruled that there was no material difference between use claims and method claims: a use could always be formulated as a method and vice versa. A use, admittedly, is an activity consisting of one step, while a method contains several steps. But the Enlarged Board pointed out that a use can always be re-worded as several sub-stages. A meaningful patent law distinction could only be maintained between a product and a method, i.e. between a physical phenomenon and an activity. It was therefore concluded that a new medical use of a known

use, or would relate to an aqueous suspension representing as such a pharmaceutical preparation for use in a method mentioned under Articles 52(4) and 54(5) EPC. These articles only permit a purpose-limited substance claim clearly stating a specific or general therapeutic purpose. Since claim 1 of the main request and claim 1 of the first auxiliary request do not clearly and unambiguously define the matter for which protection is sought, the main request and first auxiliary request do not fulfil the requirements of Article 84 EPC.”

375 G 1-6/83, OJ EPO 1985, 64. 
product must be a medical method, i.e. at variance with the ban on patents for medical processes. $^{376}$ Once this had been established, the Enlarged Board of Appeal decided, that instead of use claims, to approve patent claims for a second medical indication, in accordance with the so-called Swiss model, i.e. claims directed to the use of a substance or composition for the manufacture of a medicament for a specified new and inventive therapeutic application, even in a case in which the process of manufacture as such does not differ from known processes using the same active ingredient. ${ }^{377}$

The claim formulation is worded as a manufacturing process and, therefore, was not considered to be in conflict with the ban on patents for methods of medical treatment. Problems arose in another quarter, however. The Enlarged Board of Appeal was aware that the formulation of claims was doubtful from a novelty view point: the manufacturing process and the pharmaceutical resulting from the process did not differ in any way from the pharmaceutical and the manufacturing process used when the pharmaceutical was manufactured for its first indication. On this point, however, the Enlarged Board of Appeal had little to say, except that the manufacturing process could be deemed new when the end product (the pharmaceutical) could be used in a new way. ${ }^{378}$

The basis of this special formulation of claims can only be understood if, like the Enlarged Board of Appeal, we accept that in a method claim novelty and technical effect are assessed with reference to different technical features. The novelty requirement is met with aid of the disclosure of a new indication, while the technical effect requirement is met, and the medical procedure ban avoided, by the feature "production of a pharmaceutical". Only with such a construction of the claims is it possible simultaneously to meet the novelty requirement and avoid the ban on patents for medical procedures. This solution, however, is contrary to an established principle of patent law. The new technical features in the claims are deemed to be those which are to be taken into consideration when assessing whether the invention constitutes a medical method. The argument presented by the Enlarged Board of Appeal in favour of this innovation in patent law was that there was no reason why a second medical indication should be treated differently from a first indication. The novelty of the manufacturing process, therefore, could by analogy be deduced from the new use of the product manufactured. ${ }^{379}$

376 G 1/83, OJ EPO 1985, 64: "The European Patent Convention, in general, allows both method claims and use claims but whether any activity is claimed as a method of carrying out the activity (setting out a sequence of steps) or as the use of a thing for a stated purpose (the sequence of steps being implied), is, in the opinion of the Enlarged Board, a matter of preference. For the European Patent Office there is no difference of substance. In the context of the present case, this means that any artificial distinction according to which, when the invention concerns the employment of a substance or composition for therapy, a method claim excludes and a use claim includes at least the preparation of a pharmaceutical product, with instructions for use in the treatment of illness (which has been called in German the 'augenfällige Herrichtung'), cannot be accepted, because in both cases the active substance or composition for therapy must be in a state capable of exerting its therapeutic activity and this necessarily means that the active material has been formulated and made up into doses.”

377 See Legal Advice from the Swiss Federal Intellectual Property Office, OJ EPO 1985, 91.

378 G 1/83, OJ EPO 1985, 64: "[T] [Te Enlarged Board considers that it is legitimate in principle to allow claims directed to the use of a substance or composition for the manufacture of a medicament for a specified new and inventive therapeutic application, even in a case in which the process of manufacture as such does not differ from known processes using the same active ingredient."

379 G 1/83, OJ EPO 1985, 64: “Article 54(5) EPC provides an exception ... so far as the first use of medicaments is concerned, in respect of which the normal type of use claim is prohibited by Article 52(4) EPC. In effect, in this case the required novelty for the medicament which forms the subject-matter of the claim is derived from the new pharmaceutical use. It seems justifiable by analogy to derive the novelty for the process which forms the subject-matter of the type of use claim now being considered from the new therapeutic use of the medicament and this irrespective of the fact whether a pharmaceutical use of the medicament was already known or not.” 
The Enlarged Board of Appeal pointed out, however, that this formulation of claims was not to be put to general use in method claims. It was only intended for methods with a technical effect of a therapeutic, diagnostic or surgical nature. Where any other kind of technical effect was concerned, it would not be possible for the novelty of the manufacturing method to be deduced from the new use of the product manufactured. Here, then, we come up against the problem dealt with in section 1.3, namely delimitation of the ban on patents for therapeutic and diagnostic methods. Only if the new effect disclosed is therapeutic or diagnostic may the special claim formulation approved in $G$ 1/83 be used. In other cases, where somebody has found a new use for a known product, novelty is lacking in a known method for manufacturing the known product.

The interpretation introduced by the Enlarged Board of Appeal has been confirmed several times by subsequent EPO decisions. In T 570/92, the claim had been formulated in accordance with the rules for a second medical indication, but the claim had also disclosed the formulation and dosage to be used in order to achieve the new therapeutic effect:

\footnotetext{
"Verwendung von Nifedipinkristallen mit einer spezifischen Oberfläche von 1 - 4 m2/g zur Herstellung von festen Arzneizubereitungen zur Erreichung von lang andauernden Blutspiegeln zur oralen Behandlung von Hypertonie durch 1 bis 2 Mal tägliche Applikation.”
}

The Board of Appeal ruled that the claim, in spite of the disclosure of how the substances were to be administered to patients, was still to be regarded as a method for manufacturing a pharmaceutical and not as a method of therapeutic treatment. ${ }^{380}$ Another decision (T 958/94) has laid down that patent claims for a second medical indication need not conform verbatim to the formulation accepted by the Enlarged Board of Appeal. In cases where a patent may be granted with claims directed to the use of a known compound for a specified new and inventive therapeutic application, such a new use may be claimed in the form (i) either of the use of the compound for the manufacture of a medicament for the said therapeutic application, (ii) or of a process for the manufacture of a medicament for the said therapeutic application characterised in the use of the said compound. ${ }^{381}$ Both types of claim include an express reference to the specific second medical use. The Board concluded further in the above-mentioned decision that there is no difference in substance whether the subject-matter of the claimed invention is defined in accordance with the form and wording mentioned under (i) above, or in accordance with the wording and form mentioned under (ii) above. Moreover, the Board accepted in the said decision that one and the same application may contain one set of claims drafted in the form mentioned under (i) above and a second set of claims drafted in the form mentioned under (ii) above for contracting states which have entered reservations in accordance with Art. 167(2)(a) EPC. This ruling was followed in T 853/94. ${ }^{382}$

On the whole, the national patent offices of the EPC countries have also accepted and complied with the Enlarged Board of Appeal's interpretation. Patents for a second medical indication are obtainable from the national patent offices of Italy, Switzerland (which also means Liechtenstein), Austria, the UK, France and Sweden. Patents are also obtainable in Germany, though with a different claim formulation. ${ }^{383}$ Dutch national case law, however, forbids patents for second medical indications. A court in The Hague has also found that an EPO patent formulated in accordance with the rules for a second medical indication

\footnotetext{
380 T 570/92, of 22061995.

381 T 958/94, OJ EPO 1997, 241.

$382 \mathrm{~T} 853 / 94$ of 02111998.

383 See Hirsche, F., Hansen, B., Protecting Inventions in Chemistry, 256.
} 
could not be used to obtain a ban on sales in The Netherlands, the reason being that the formulation of the patent claim was probably invalid. The EPO patent invoked in the Dutch court had been granted in July 1993, with a claim reading as follows:

“[U]se of Taxol and sufficient medications to prevent severe anaphylactic reactions, for manufacturing a medication for simultaneous, separate or sequential application for the administration of from $135 \mathrm{mg} / \mathrm{m}^{2}$ up to $175 \mathrm{mg} / \mathrm{m}^{2}$ Taxol over a period of about three hours or less as a means for treating cancer and simultaneously reducing neutropenia.”384

The court ruled that the claims did not really refer to a method of manufacturing a pharmaceutical, but only to a method of administering the active substance over three-hour periods in doses of between 135 and $175 \mathrm{mg} / \mathrm{m}^{2}$. In particular the court observed that the invention, as disclosed in the claim, was dependent on the area of the patient's skin and, therefore, could hardly be a question of manufacturing method. It made no difference that the use was new and had an inventive step. The court ruled that there was a palpable risk of it declaring the patent invalid, and accordingly the injunction requested by the patentee could not be issued. It is questionable whether the inclusion of a dosage instruction to a doctor makes the claim unpatentable. It can be noted that the EPO Board of Appeal in T 570/92 allowed a claim with the feature "to be administered once or twice daily".

The patentee lodged an appeal before the Court of Appeals of The Hague, which rendered judgement on June 25, 1998.385 The Court held that there was a serious chance that the patent would be revoked for inter alia lack of novelty and sufficiency, and effectively held that the patent did not relate to a second medical use, but to the first (known) use of Taxol together with the special effect that the neutropenia side-effects were actually reduced.

A similar conclusion regarding the same patent was drawn in a revocation action in the UK Patents Court. ${ }^{386}$ Justice Jacob revoked the patent inter alia for want of novelty and inventive step, but accepted that the claim amounted to more than merely a method of treatment. It was to the manufacture of a medicine to be used in a treatment. Exceptions to patentability should be construed narrowly. However, the claims lacked a novel feature, because they essentially only contained more information about the old (first) use. The special effect of the administration of Taxol over a period of three hours or less, i.e. reduced neutropenia side-effects, could not constitute a second medical use when compared to the administration of Taxol over a period of 24 hours.

\subsubsection{Breadth of claims for a second medical indication}

A patent for a second indication, unlike that for a first indication, must be limited to the new indication which the applicant has disclosed. Further new indications - a third and so on will not come within the scope of the protection. Even if it is necessary to disclose the specific therapeutic effects in the claims, several different treatment results can be indicated in the same patent claim, if there is a practical connection between them, i.e. if they meet the requirement of unity in Art. 82 EPC. The Guidelines provide:

384 Court of First Instance in The Hague The Netherlands v. Yew Tree Pharmaceuticals BV et al. of 24071997 , docket no. KG 97/579, ref. by Oosting, B., Patent World, March 1999, 24.

385 Bristol-Myers Squibb v. Yew Tree, The Court of Appeals of The Hague, ref. by Oosting, B., Patent World, March 1999, 24.

386 Patents Court, Bristol-Myers Squibb -v-Baker Norton Pharmaceuticals and Napro Biotherapeutics, of 2008 1998. 
"A claim in the form 'Use of a substance or composition X for the manufacture of a medicament for therapeutic application Z' is allowable for either a first or 'subsequent' (second or further) such application, if this application is new and inventive.... In cases where an applicant simultaneously discloses more than one 'subsequent' therapeutic use, claims of the above type directed to these different uses are allowable in the one application, but only if they form a single general inventive concept (Art. 82).”387

An example of the possibility of a long line of different but closely related therapeutic indications being disclosed together in one claim occurs in T 199/94. The claims read: use of vitamin A acid for manufacturing a mixture which would be applied to the skin for maintaining, deferring or reversing loss of collagen fibres in the skin, abnormal changes in elastic fibres, deterioration of blood vessels and for treatment of abnormal epithelium growth in sun-damaged skin. 388

\subsection{Novelty assessment of medical indications}

\subsubsection{Characteristics of a new indication}

After the Enlarged Board of Appeal had opened up possibilities of patenting new medical indications through decision $G 1 / 83$, it became necessary for the case law to indicate more exactly what was a new patentable medical indication. Lack of novelty in a patent application formulated in accordance with the rules for a second medical indication presupposes that a document belonging to the state of the art contains a disclosure of the substance and a disclosure that the substance can be used for the indication disclosed in the claim. One example of the interaction between novelty assessment with regard to the substance and the indication comes in $\mathrm{T}$ 541/89. The patent application pertained to the use of a substance for manufacturing a pharmaceutical which could be used in the treatment of intermittent claudication. That illness is caused by insufficient blood supply to the ischemic muscles. The application was examined in relation to two documents. A known document stated that:

"[C]ertain, hitherto unknown, 7-(oxoalkyl)-1,3-dialkyl xanthines are very effective in increasing the blood flow through skeletal muscle..."

Ischemic muscles were found to be a new indication in relation to this disclosure that the substance could be used for improving blood flow through skeletal muscles. In another prior document the indication disclosure was identical with the claimed one (ischemic muscles) but, on the other hand, the structure of the disclosed substance was not identical but analogous to that now claimed, i.e. there was a slight variation in a non-critical part of the structure. Novelty, therefore, also existed in relation to the other document. In relation to the two documents combined, however, the patent application was found to lack inventive step. ${ }^{389}$

Basically, then, if substance $\mathrm{X}$ was known for the treatment of illness $\mathrm{Y}$, a use of substance $\mathrm{X}$ for the treatment of illness $\mathrm{Z}$ is new. The important thing is that novelty also exists if substance $\mathrm{X}$ as used previously actually implied a treatment of illness $\mathrm{Z}$, e.g. prophylactic treatment. The decisive thing is that no one was previously aware of this effect occurring and, consequently, it could not have been put to intentional use. It is the possibility

\footnotetext{
387 Guidelines C IV 4.2.

388 T 199/94 of 23031995.

389 T 541/89 of 21031991.
} 
of the skilled man using a technical effect which decides the content of the state of the art, not the actual occurrence or otherwise of the technical effect that is later claimed; see section 4.2.2. 390

\subsubsection{New biological mechanism as a new indication}

Behind every indication of a pharmaceutical there is a biological causal relationship, an active mechanism. It is possible for two pharmaceuticals to have the same therapeutic effect, i.e. the same indication, but to be based on different active mechanisms. Pharmaceuticals can, for example, interact with different receptors. One perhaps relieves pain, while the other perhaps attacks the cause of the pain. From the patient's point of view, the indication for which it is used is the same. Case law, however, has established a rule that the use of a new biological active mechanism is a new indication, even if the result of the treatment is known.

When the patent application in T 290/86 was filed, it was known that if the substance concerned was applied to teeth, the enamel became more resistant to the degradation caused by organic acids. The applicant had now found that when the substance was applied to teeth, it also had the effect of removing plaque. Plaque causes degradation of tooth enamel. The same end result - no degradation of the enamel - was achieved in two different ways. The Board of Appeal found, however, that the removal of plaque was a new technical effect (indication) in relation to the effect known previously. The result of treatment was admittedly the same, but it was achieved by two different modes of action. It was sufficient, the Board ruled, for there to be a new patentable medical indication. The active mechanism was new: a new treatment result was not necessary. ${ }^{391}$

In British patent law, Justice Jacob has critizised this ruling. ${ }^{392}$ He has explained that it led to the repatenting of the old toothpaste when it was discovered that it improved plaque removal. In so holding Justice Jacob made a distinction between true new uses and mere unpatentable new discoveries about old uses. In T 290/86 the additional information contained in the patent in suit did not teach the person skilled in the art to do something which would not have been done without knowing the content of the patent in suit. The remaining possible justification for the ruling could be that there is a value in new knowledge about biological mechanisms as such, even if it does not affect the practical working of the methods of treatment. But it may well be that it is not for patent law to be distorted with such concerns about "pure" knowledge, unrelated to useful applications, since it might greatly complicate an infringement assessment. Furthermore, it can be hard to distinguish a situation with a new active mechanism from a situation where the active mechanism is the same but has been described in a new way. A medical treatment can often be described in a variety of ways. One

\footnotetext{
390 See Paterson, G., GRUR 1996, 1096.

391 T 290/86, OJ EPO 1992, 414: "In the Board's view, even though both document (1) and the claimed invention are concerned with compositions for treating human teeth so as to inhibit tooth decay, the claimed invention represents a further and different therapeutic use from that disclosed in document (1), within the meaning of Decision G 5/83 ..., because the claimed invention is based upon a different technical effect from that which is disclosed in document (1). Document (1) discloses the use of lanthanum salts in dental compositions for the purpose of depressing the solubility of tooth enamel in organic acids such as develop in saliva, thus strengthening the enamel so as to inhibit tooth decay. This is in clear contrast to the technical effect of the claimed invention, namely the improved removal of plaque from teeth by use of compositions including lanthanum salts, so as to inhibit tooth decay which would otherwise result from the presence of the plaque."

392 Baker Norton Pharmaceuticals Ltd and Napro Biotherapeutics Inc v. BMS, August 1998 CH 1997N no.2698/CH 1997 B no. 6446.
} 
can concentrate on the symptom picture or on more fundamental biological causal relationships, such as the ability of a substance to interact with receptors or enzymes. In a situation like this it is important that the applicant should incur the burden of proving that the active mechanism is not the same as that which has been used in the known treatments. It is essential that the active mechanism should be new and not just the description of it.

Even in the EPO, however, T 290/86 may be an exception. The mere discovery of a new effect seems insufficient to satisfy the novelty requirement. The discovery of the new effect must at least to some extent affect how the known pharmaceutical is used. This was decided in $\mathrm{T}$ 254/93. The subject-matter of the claim related to the use of a retinoid in the preparation of a topically administrable medicament for use in the prevention of corticosteroid-induced skin atrophy. Retinoid had for some time been used together with corticosteroid in the prevention of skin atrophy. But none of those numerous documents which related to the combined use of a corticosteroid and a retinoid, told the reader that skin atrophy caused by the topical application of corticosteroids was actually prevented or reduced by the topical use of a retinoid. The applicant referred to the decision $G$ 2/88 by the Enlarged Board of Appeal where it was recognised that the discovery of a previously unknown property of a compound, such property providing a new technical effect, could involve a new and inventive contribution to the art. The Board accepted this, but pointed out that the Enlarged Board of Appeal started from the assumption that the technical effect disclosed for the first time implied a new use for the known substance. In the situations which the Enlarged Board had had in mind such additional new uses were actually possible. There were two different applications for the product which could be separated from each other. In the present case, it was uncontested that skin atrophy was a well-recognised and fully documented clinical condition occurring after prolonged use of corticosteroids. Therefore, it was and had always been an essential aspect in the application of glucocorticosteroids in the treatment of dermatoses to avoid skin atrophy in order not to make the main effect of the glucocorticosteroid meaningless for the patient. The prevention of skin atrophy was an effect inseparably tied to the use of the known pharmaceuticals and therefore there was no novelty.

\footnotetext{
"The Board considers that the mere explanation of an effect obtained when using a compound in a known composition, even if the explanation relates to a pharmaceutical effect which was not known to be due to that compound in the known composition, cannot confer novelty on a known process if the skilled person was already aware of the occurrence of the desired effect when applying the known process.” 393
}

The discovery of the previously "hidden" effect had to have at least some minimal consequences for how the pharmaceutical was used clinically. If not, the new feature did not represent a technical effect within the meaning of decisions $G$ 2/88 and G 6/88.

Similarly, in T 958/90 the Board held that a hitherto unknown increased activity is not a second medical use and added that a known effect cannot become novel for the sole reason that it is present to a hitherto unknown (greater) extent. ${ }^{394}$ Likewise in T 279/93 the Board of

\footnotetext{
393 T 254/93, OJ EPO 1998, 285.

394 T 958/90 of 0412 1992: "Since the information to be considered, namely that the presence of IDA enhances the sequestering activity of NTA in a mixture of NTA and IDA which has already been proposed for use as a sequestering agent, provides no more than an additional reason to use this known mixture in the known way for the known purpose, no contribution to the state of the art in the above sense can be recognised. In other words, what the disputed patent in fact teaches, regardless of how the claims are worded, is to solve a known technical problem by known means, namely to sequester calcium and magnesium ions by NTA in mixture with the specified amounts of IDA, as already proposed by document (1). However, such an additional reason to do what has already been proposed earlier as a solution to the same technical problem cannot be regarded as a new functional technical feature in the sense of Decisions G 2/88 and G 6/88 of the Enlarged Board of Appeal.”
} 
Appeal held that a further reduction in formation of impurities does not constitute a second medical use. The explanation given was that the finding did not affect the practical use. ${ }^{395}$ If the effect does not affect the practical use then the finding is appropriately termed an explanation. In T 863/96 the Board of Appeal stated:

\footnotetext{
"The same reasoning [lack of novelty] applies to claim 1 of the auxiliary request since the insertion for inducing a long lasting and constant inhibition of monoamine oxidase activity represents only an explanation of what already happened when using selegiline alone on a plaster for the treatment of Parkinson's disease according to document (1).” [Emphasis in the decision]. ${ }^{396}$
}

A new effect is not enough. The new effect must entail new practical applications or uses. It is possible that an effect already have inherently taken place, but nevertheless its ex post facto discovery might lead to a new application that can be clearly distinguished. 397

\subsubsection{New category of patients as a new indication}

Many pharmaceuticals are only effective for certain patients, or else certain patients suffer from unacceptable side effects. Situations can therefore arise in which somebody shows a certain category of patient, which it was not previously believed could be treated with a pharmaceutical, to be actually receptive to such treatment. The question is whether a pharmaceutical known for a certain indication shall be patentable for the same indication but for use with the new category of patient. This has been accepted in case law. The situation is probably most common in veterinary medicine.

In $\mathrm{T}$ 19/86 it was considered whether a vaccination of a certain category of piglets could be patented when the same vaccine had been used previously on another category of piglets. The result of treatment was the same. Before the patent application in suit was filed, it was known that the vaccine could be used to immunise piglets that were not resistant to Aujeszky virus. But the piglets which had obtained a certain amount of protection, due to the sow being immune, were not, according to the state of the art, considered receptive to vaccination. The new thing about the invention was the applicant's disclosure that the latter category could also be treated with the vaccine: it was "the patients", not the disease, that separated the patent claims from the state of the art. The Board of Appeal found that practical use of a pharmaceutical (a vaccine in this case) required more than knowledge of the illness to be treated. It was also necessary for the subjects to be treated to have been identified. Since the patient category was a necessary characteristic, it could also, when disclosed in the claim, result in the medical treatment being new. 398

\footnotetext{
395 T 279/93 of 1212 1996: "However, the facts of the present case differ significantly from those underlying decisions G 2/88 and T 59/87. The Board is unable here to find that the words 'in order to reduce the formation of isomelamine impurities' require any new physical activity not already required by the old use of using the alkanolamine with the melamine compound to make the desired end product. There is thus no new technical effect in the sense required by decision $\mathrm{G} 2 / 88$.”

396 T 863/96 of 04021999.

397 T 892/94 of 1901 1999: “[N]ovelty within the meaning of Article 54(1) can only be acknowledged in cases where a newly discovered technical effect of a known substance leads to an invention which is defined in the claim in terms of the use of that substance for a hitherto unknown, new non-medical purpose reflecting said effect.” [Emphasis in the decision]

398 T 19/86, OJ EPO 1989, 24: “[A] new use is not only valuable in cases where a novel area of therapeutic use, i.e. a novel medical indication, is provided but also in those cases where a novel class of animals, which previously did not respond to a medicament, is cured or protected against a disease. The question whether a new
} 
However, in $\mathrm{T}$ 1058/93 the mere reference in the claim to the use of the medicament for critically ill patients did not imply a novel feature. It was not possible to derive any difference between the patients according to the claim in suit over patients usually envisaged for the treatment. 399

\subsubsection{Method of administration as a new indication}

In addition to a new illness and a new category of patient, a new patentable indication for a pharmaceutical can also be the way in which it is administered to patients. In T 289/84 it was known that the pharmaceutical in question could be administered perorally or by injection. The Board of Appeal found that novelty existed when the patent applicant had shown that the same substance could also be applied topically (locally, e.g. on the skin or as eye drops). ${ }^{400}$

The principle of a new mode of administering a pharmaceutical being a new indication was confirmed in $\mathrm{T}$ 51/93. When the patent application was filed, it was known that human choriongonadotropion could be used for treating male infertility. But the substance had only been administered intramuscularly. What the applicant had shown was that a better treatment result could be obtained if the same substance was administered subcutaneously. The Board of Appeal accepted that the new method of administration was a new indication which could be patented with claims formulated in accordance with the special rules for a second medical indication. ${ }^{401}$

However, in T 317/95 some doubt was cast on the thinking that a mere difference in the course of the administration of two drugs confer novelty. The sole difference between the prior art and the subject-matter of the claim resided in the prescribed treatment regimen used for administering a bismuth-containing agent on the one hand, and a H2 receptor blocking anti-secretory agent, on the other, to an individual patient in need of them.

therapeutic use is in accordance with the decision Gr 05/83 should not be answered exclusively on the basis of the ailment to be cured but also on the basis of the subject (in the present case the new group of pigs) to be treated. A medical indication is incomplete if the subject to be treated is not identified; only a disclosure from which both the disease and the subject to be treated are clear represent a complete technical teaching. As already pointed out above, the Applicant has shown that sero-positive piglets could hitherto not be protected against Aujeszky disease. The proposal according to the application to protect such piglets against this disease by intranasally administering a known serum to this particular group of animals was not disclosed in the prior art and, therefore, constitutes a novel therapeutic application in accordance with the above-mentioned decision.”

399 T 1058/93 of 2103 1996: "It remains therefore to be considered whether the mere reference to the use for critically ill patients ... could imply an additional characteristic of the preparation in such a way as to make the claimed preparation distinguishable and non obvious over preparations as envisaged by document (1). ... Taking into account ... the common general knowledge about the metabolism of patients after a trauma or surgical treatment ... it is not possible to derive any quantitative difference from the values specifying the patients according to the patent in suit over patients usually envisaged for parenteral nutrition.”

400 T 289/84 of 1011 1986: "The fact that a chemical compound and pharmaceutical formulations containing the same as an active ingredient are known does not rule out a claim directed to a specific mode of formulation not disclosed by the prior art (in the present case: to a formulation adapted for - only - topical administration), as opposed to only a use claim”.

401 T 51/93 of 0806 1994: "[T]he only difference between the invention as claimed and the disclosure of document (4) is that the claim is directed to an intended method of subcutaneous administration. The claim is drafted in the form approved in decision G 5/83 ... for claims where the novelty is solely that of the intended therapeutic use, so the only question is whether a difference in the mode of administration of a medicament can be treated as a new therapeutic use. ... The mode of administration may be a critical factor in a medical treatment, and no reason can be seen for any a priori bar to relying on this difference when distinguishing over the prior art. Rather patentability must be treated as depending only on whether this modification is in fact novel and inventive. Thus novelty over document (4) can be acknowledged.” 
The known regimen comprised administering said bismuth-containing agent half an hour before the last meal of the day and said H2-receptor blocking anti-secretory agent at bedtime, whereas according to the claim in suit these two active agents were administered within 5 minutes of each other, e.g. concurrently. The Board stated that:

\begin{abstract}
"The invention ... involves the treatment of exactly the same category of patients by separately administering to them exactly the same two commercial drugs in the same concentration, dosage and formulation ... for the treatment of entirely the same illness or disease, with the sole exception that the prescribed regimen for this treatment is slightly modified (BNS and cimetidine are administered to the patient within five minutes of each other). It appears therefore difficult to recognise in the present invention a new field of therapeutic application or any further medical indication in general associated with the claimed combined use of the bismuth-containing agent and an H2-receptor blocking antisecretory agent.” [Emphasis in the decision]. ${ }^{402}$
\end{abstract}

The Board refrained from taking a final standpoint on this issue of novelty and nonpatentability under the terms of Art. 52(4) EPC, since the requests in any case had to be dismissed on the ground of lack of inventive step. As a result, there is some lingering doubts when it comes to the assessment of the possible limits of what can indeed be recognised to be a further medical indication (new therapeutic application) within the meaning of decision $\mathrm{G}$ 5/83, particularly in the field of drug regimens.

\title{
5.4.5 Selection inventions and new medical indications
}

In the description of selection inventions in section 4.7, we saw that the choice of a smaller range within a known wider range could be new if the selection gave rise to a better effect than was known from the wider range. The same principle has proved to be applicable to new indications. If the known indication is formulated in general terms, disclosure of a more exactly defined treatment result can be new. A disclosure in the state of the art that substance $\mathrm{X}$ can be used for the treatment of pain is not necessarily detrimental to novelty in a patent application for the use of the substance for treating headache; a substance known for treating inflammations can be new for the treatment of sinusitis; a substance known for the treatment of diseases of the immune defence can be new when somebody shows that it can be used for the treatment of AIDS. The decisive factor in these situations is whether, on the priority date, a man skilled in the art would have known that the substance could be used for the more closely defined treatment situations.

In T 297/88 there was a disclosure in the state of the art that a substance had a vascular spasmolitic effect in all vascular systems and the same effect in limited vascular systems, e.g. in the central nervous system. A vascular spasmolitic effect indicates that the pharmaceutical causes increased blood flow. The reference in the state of the art, however, was found not to be detrimental to the novelty of an indication consisting of the discovery that the substance could be used for improving the flow of blood through the brain. ${ }^{403}$

\footnotetext{
402 T 317/95 of 26021999.

403 T 297/88 of 0512 1989: "Die allgemeine Aussage über die Wirkung solcher Verbindungen auf p. 11 von D1 wäre dann nicht so zu verstehen, daß jede einzelne der in D1 offenbarten Verbindungen gefäßspasmolytisch und zugleich auch im Zentralnervensystem wirksam ist, sondern so, daß unter den erfaßten Verbindungen auch Vertreter zu finden sind, die im Zentralnervensystem wirken. Damit wäre aber in D1 weder offenbart, daß speziell das Nimodipin zu den im Zentralnervensystem wirksamen Verbindungen gehörte, noch wäre eine Auswahlregel für im Zentralnervensystem wirksame Verbindungen angegeben, die den Fachmann zwangsläufig auf das Nimodipin hingeführt hätte. Es kann daher keine Rede davon sein, daß aufgrund der allgemeinen
} 
In T 879/94 a prior document pointed to the effect of human G-CSF (the commonly used abbreviation for granulocyte colony stimulating factor) on the proliferation and differentiation of bone marrow cells to neutrophiles (this being the characteristic biological activity of this factor). The document suggested a "potential" clinical use in the treatment of leukopenia. But it contained only some in vitro data on bone marrow cells from healthy donors. The data demonstrated the ability of human G-CSF to promote the differentiation of precursor stem cells to granulocytes. Later, the clinical use for treatment of neutropenia was claimed as a new second medical use. The Board of Appeal, for the following reasons, found this use to be new in relation to the disclosure in the prior document:

\begin{abstract}
"(a) firstly, the clinical use of human G-CSF for the treatment of leukopenia is qualified [in the prior document] only as 'potential', ie as a possibility worth investigating; (b) secondly, the said prudent suggestion is made in respect of leukopenia, not of neutropenia. ... [L]eukopenia is a term used in the art in respect of a medical condition in which a generalized reduction in the number of leukocytes, ie of the white blood cells (eg neutrophiles, basophiles, eosinophiles, monocytes), is observed. Leukopenia, although encompassing inter alia neutropenia, cannot be equated thereto. The term neutropenia refers to a particular leukopenic condition in which a decrease in the number of neutrophilic leukocytes is observed. In this respect, it is a distinct medical condition which can be recognised by a doctor on the basis of diagnostic tests.

Thus, while document (1) refers to a potential use of G-CSF in a general therapeutic application (leukopenia), present claim 1 refers - in the terms of a second medical use claim - to a specific therapeutic application (neutropenia). The fact that ... on a clinical basis most cases of leukopenia are due to a decrease in the number of neutrophiles, does not allow the conclusion that the novelty of the specific clinical application is affected by the disclosure in document (1) of the suggestion for a more general clinical application. Consequently, since in assessing novelty any inventive-step elements have to be left out of consideration, document (1) does not take away the novelty of the present claims. ${ }^{404}$
\end{abstract}

Two factors were important, firstly, the more general indication, leukopenia, was only described as "potential" in the prior art document, secondly, a doctor would have differentiated between the more general and the more specific diagnosis.

\title{
5.5 Remarks
}

A steadily increasing number of substances with known therapeutic effects has caused research for new indications to become more and more important in recent years. Altogether 388 new substances were licensed for sale in the USA (a first indication) between 1963 and 1987. Between 6 and 8\% of these were being researched between 1981 and 1987 by American pharmaceutical corporations with a view to finding new indications. $50 \%$ of the projects where new indications were searched for, the clinical trials were successful, i.e. resulted in the pharmaceutical also being registered for sale for the new indication. This is a much higher figure than when permission is obtained for clinical trials of a substance not previously used therapeutically. The greater success rate is mainly due to there being less risk of unexpected side effects when the substance has already been used before as a

Angaben in D1 auch nur die Wirkung einer darin als solcher offenbarten Verbindung auf das Zentralnervensystem zum Stande der Technik gehört hätte. Noch weniger könnte eine spezielle Wirkung einer solchen Verbindung auf das Gehirn, das nur einer von mehreren Bestandteilen des Zentralnervensystems ist, in D1 offenbart sein.”

404 T 879/94 of 19051998. 
pharmaceutical. Thus we may assume that the search for new indications is a particularly cost-efficient form of pharmaceutical research. ${ }^{405}$

A traditional legal interpretation at the beginning of the 1980s, however, could hardly have led to any conclusion but that a second medical indication was impossible to patent. ${ }^{406}$ The fact that patents nonetheless came to be granted for a second medical indication, without the EPC being revised, indicates the great importance of economic considerations in patent law. When the need to be able to patent new medical indications became clear, legal technicalities carried little weight. It was now the Enlarged Board of Appeal that adapted the legal situation to the needs which had not been foreseen when the EPC was written. These developments testify to the flexibility of the structure of patent law and also to the value of construing the law with reference to economic purpose, in order to understand the appearance and development of patent law.

Considering, however, the important part played by economic circumstances in shaping developments, it would have been more satisfactory if, in the making of what can only be termed a change, economic considerations had been explicitly invoked in case law. A statement to the effect that the law as it then stood did not seem to agree with the over arching purpose of patent law was warranted. When case law deviates from what is an established state of law, this should preferably be justified by teleological considerations and not by making as much use as possible of the great flexibility afforded by a more formalistic legal reasoning.

It should be pointed out, however, that there is a certain element of teleological interpretation in G 1/83. For example, the Enlarged Board of Appeal ruled that:

"The intention of Article 52(4) EPC ... is only to free from restrain non-commercial and nonindustrial medical and veterinary activities. To prevent the exclusion from going beyond its proper limits, it seems appropriate to take a special view of the concept of the 'the state of the art'."407

Thus reference was made to the scope of patent law comprising commercial and industrial activity. This can be implicitly interpreted as a reference to economic consequences. The notion glimpsed in this passage is that when the patent system serves an economic purpose, it shall be used. Otherwise the patentable area is interpreted too narrowly. When the incentives available within the patent system can be used to encourage research, this should be done. The incentives can affect innovative pharmaceutical companies, but not medical practitioners to any great extent. Previously, on the basis of this observation, it has been concluded that it is reasonable to consider who is the most cost-efficient innovator for different types of invention when the patentable area is being defined; see section 1.3.4. It is obvious that pharmaceutical companies are efficient users of incentives for new medical indications. Thus it would be unfortunate policy to exclude them.

Only a very few percent of all patent applications for chemical compounds with therapeutic effects are developed into pharmaceuticals that are marketed. Research into the remaining substances is discontinued for technical and/or economic reasons. Often, though, the patent application is made public before the project is discontinued. In this way a considerable proportion of interesting compounds will come to be known as having therapeutic effects. Were it not for the possibility of patenting new medical indications for them, they would subsequently fall outside the patentable field. The substances will belong

405 See DiMasi, J. A., Lasagna, L., 5 Journal of Clincial and Pharmacoepidemiology 1991, 19.

406 A dozen patent law experts stated in 1982 that a new use for a known pharmaceutical was unpatentable. See Gaumont, R., Patentability and Patent Scope of Pharmaeutical Inventions, IIC 1982, 443.

407 G 1/83, OJ EPO 1985, 64 and parallel cases. 
not only to the great category of known substances, but also to the category of known substances with a known medical effect, which would make them unpatentable for all time in the medical field. A legal situation of this kind would be liable to cause widespread losses of incentive. ${ }^{408}$ The incentives created through the possibility of patenting new indications should exist, because pharmaceutical corporations are receptive to this stimulus and because access to the incentive does not have negative effects on any other party. If new medical indications cannot be patented, they cannot be patented by anybody.

Thus, where patents for new medical indications are concerned, we do not have a situation where advantages have to be balanced between different players. The decision that a new indication can be patented for substances known on account of having a medical indication already does not adversely affect any other research enterprise. The new medical indications would come outside the interests of all researching enterprises if they could not be patented. This would have negative consequences for research incentives. The question in this situation is not for whom one is to create incentives but whether one is to create them at all within the patent system. One assumption made here is that they are to be made if they can be taken advantage of. If it is not believed suitable for the patent system to operate in such a way, then surely the target of criticism should be the existence of the whole of the patent system, not the creation, in connection with new medical indications, of incentives for players who can derive benefit from them.

We may add that studies have shown that it is common for new indications for previously known pharmaceuticals to be no less economically important, and not infrequently more so, than the first indication. ${ }^{409}$ This being so, it would be unwise to make the economic outcome of research projects dependent on a circumstance immaterial to the value of the result, namely whether the substance was new or not in the medical sphere. Given that there are tens of millions of known chemical compounds, it is obviously very much a matter of chance whether a project results in the sought-after effect being found in a new chemical substance or in one that is already known. The legal position must take into account that the value created consists in the new possibility of treatment and not in a new chemical compound being used for the first time.

408 Cf. Suchy, H., IIC 1982, 472.

${ }^{409}$ See DiMasi, J. A., Lasagna, L., 5 Journal of Clinical and Pharmacoepidemiology 1991, 19. 


\section{Inventive step}

\subsection{Introduction}

An invention which lacks novelty is directly accessible in the state of the art. But in no modern patent system is novelty the only necessary distinction between the invention and the state of the art. The additional requirement used has been given several names: "inventive step”, “obviousness” and also, in the Nordic countries, "essential difference”. The content, however, is always explained in the same way: the claimed invention must not have been potentially accessible to an average man skilled in the art. ${ }^{410}$ Inventive step exists if those working in the technical field on the priority date would not have been able to make or derive practical benefit from the claimed invention. Often this situation is illustrated metaphorically. Technical knowledge is described as consisting of three layers. The first layer is the state of the art, i.e. all publicly accessible technical knowledge. The second layer comprises certain new technical knowledge but only that which the average man skilled in the art had been able to create and use. Reference is commonly made to the patent-free area in which applications are refused for lack of inventive step. The third, outermost and patentable layer of technical knowledge contains what is not within reach of the average man skilled in the art.

The "gate-keeper function" performed by inventive step in patent law is important. The requirement creates a free zone within which business enterprises can carry on development work without "interference" from competitors. In addition, corporate preferences with regard to technical development are shifted from more predictable development to research with a more uncertain outcome. This may be presumed to be a general purpose of the patent system. The importance of inventive step for the practical effects of the patent system prompts a continuous discussion of the way in which the requirement is to be interpreted. There are, however, great difficulties involved even in analysing this question meaningfully. The stipulation of inventive step is commonly regarded as the most difficult to apply in patent law and also the most difficult to analyse in theory. ${ }^{411}$ The requirement has been dubbed the sphinx of patent law. 412 The difficulties are very much due to the requirement pre-supposing that technical difficulties can be measured in an objective perspective. But technical difficulties can be said to have no such objective side. All problem-solving is by nature subjective. Creative and innovative work overcoming technical difficulties is done by and "within" individual people, never in a quantifiable sphere.

Within the framework of the inventive step requirement, an attempt is, however, made to bridge the gap between the necessarily subjective business of technical problemsolving and the objective assessment aimed for, by making reference to an average man skilled in the art. The man skilled in the art becomes the subject who is always needed for an assessment of degrees of difficulty. But the transition from the subjective to the objective is not painless, because that which makes the assessment objective does not exist in reality. Objectivity is only achieved at the price of introducing a legal fiction. As a result it is the patent examiner's or the patent judge's subjective view of the objective, but non-existent average man skilled in the art which decides the outcome. One may query how much is

\footnotetext{
410 T 513/90, OJ EPO 1994, 154: “Obviousness ... can be explained as an indirect potential availability of the totality of features of a subject-matter through a mental recognition of the consequences of disclosures in the art”.

411 "The evaluative issue that this [inventive step] introduces is the largest single cause of uncertainty about the validity of patents and hence a frequent inflator of the scale and length of patent disputes.” See Cornish, W.R., Intellectual Property: Patents, Copyright, Trade Marks and Allied Rights, 127.

412 See Moufang, R., Genetische Erfindungen im gewerblichen Rechtsschutz, 271.
} 
gained by this: cf. section 2.2, where the content of the "man skilled in the art" yardstick has been investigated.

\title{
6.2 Provisions on inventive step
}

One of the first actions of the EPO was to convene, in 1978, an international conference to discuss how high the inventive step requirement should be pitched. The delegates succeeded, against all the odds, in reaching a consensus. They agreed that the requirement defined in British patent law was too low, while that applied in Dutch precedent was too high. The German assessment of inventive step was considered "just right". ${ }^{413}$ The EPO then became the first, and is perhaps still the only, patent office to issue guidelines on the implementation of inventive step. ${ }^{414}$ They provide partly as follows:

\begin{abstract}
"Thus the question to consider, in relation to any claim defining the invention, is whether at the priority date of that claim, having regard to the art known at the time, it would have been obvious to the person skilled in the art to arrive at something falling within the terms of the claim. If so, the claim is bad for lack of inventive step. The term 'obvious' means that which does not go beyond the normal progress of technology but merely follows plainly or logically from the prior art, i.e. something which does not involve the exercise of any skill or ability beyond that to be expected of the person skilled in the art." 415
\end{abstract}

In these cases the "person skilled in the art" is the same as is referred to in questions of whether the invention is sufficiently disclosed. ${ }^{416}$ The most interesting thing about this quotation from the Guidelines is that "obvious" is confined to that which follows "plainly" or "logically" from the state of the art. One has the impression that that which is obvious must be immediately apparent to a person skilled in the art. This has the effect of minimising the effort expected from that person.

\subsection{Special conditions applying to pharmaceutical inventions}

The requirement of inventive step is of course identically worded in all technical fields, but its practical content is greatly affected by conditions in different technical fields. In the case of pharmaceutical patents, it is the unforeseeable nature of biological reactions which gives the assessment its special character. Two substances which are very similar in structure can produce quite different biological effects, and so it is difficult for a man skilled in the art to modify known technical solutions in a predictable manner so as to obtain a valuable new technical solution. The level of unpredictability is high, with the result that the "patent-free" field of development is limited.

“[T]he principle legal criteria used to determine non-obviousness ... are little more than a legal translation of the notion of serendipity. As a result, drugs yielded by the traditional methods are usually easy to patent because ... these drugs are obtained more by chance and intuition than by reasoned skill, therefore, they are not obvious, even to a person skilled in the field.”417

\footnotetext{
413 See Pagenberg, J., IIC 1978, 351.

414 See Crespi, R. S., JPTOS May 1991, 365.

415 Guidelines C IV 9.3.

416 Guidelines C II 4.1.

417 See Ducor, P., 22 Rutgers computer \& technology law journal 1996, 371. Ducor's main thesis in the article
} 
The assumption of an equivalent biological effect in similar molecules cannot be taken very far in the pharmaceutical industry. Foreseeable connections between structure and effect are more the exception than the rule. If the modification of the state of the art is of any extent, a skilled man in the pharmaceutical sector presumes that the properties of the substances will be affected. In T 548/98 it was said:

\begin{abstract}
"It is to be stressed that in the specific field of 'drug design' any structural modification (even a minor modification in the stereochemical configuration of a compound) is 'a priori', in the absence of any identified strong relationship between a specific structural element and pharmacological activity or in the absence of any established 'bioisosterism', expected to disturb the pharmaceutical activity of the initial structure, particularly when the pharmaceutical activity involves an action on specific receptors.”418
\end{abstract}

Consequences of structural changes are very hard to foresee, at least if one has no knowledge of the structure of the target molecules and the interaction with it of the pharmaceuticals (the ligands). The person skilled in the art must assume that changes of any extent will lead to a loss of the therapeutic effect. From this there follows the rule that inventive step does not presuppose that the claimed substance is having a better effect compared with the structurally most closely related substances having a similar effect in the state of the art. If the claimed substances have a therapeutic effect, i.e. constitute an alternative therapeutic solution, this may be sufficient to establish inventive step. Quantitative particulars of the therapeutic effect, therefore, are not necessary in patent applications: it may be sufficient to establish that the claimed substances have an effect and that there is a structural difference compared with the closest prior art. ${ }^{419}$

Thus no improvement is needed in order to meet the requirement of inventive step. Nor, on the other hand, is the existence of an improvement the same thing as inventive step. Both an alternative and a better solution can be obvious to a man skilled in the art, but neither need be so. An improvement in the technical effect is an indication of inventive step, but never a sufficient condition. ${ }^{420}$ In mechanics and electronics, it is common for minor and relatively simple adjustments or modifications to result in some better technical effect. As we shall see, however, it is unusual in the pharmaceutical sector for an improvement to be deemed obvious. An improvement, therefore, carries a great deal of weight as an indication of inventive step in the pharmaceutical sector. ${ }^{421}$

is, however, that new methods of pharmaceutical research may make new pharmaceuticals obvious and therefore unpatentable.

$418 \mathrm{~T}$ 548/91 of 07021994.

419 T 20/83, OJ EPO 1983, 419: “The Examining Division adjudged the subject-matter of the claim, having regard to the state of the art as contained in citations 1 and 2, to be obvious and made patentability dependent on substantiation of a surprising anti-allergic effect, which it deemed not to be present. The Board, however, views the subject-matter of Claim 1 as qualitatively surprising, so that the question of surprising quantitative superiority can be left aside.”

420 T 65/82, OJ EPO 1983, 327: "It is very relevant in this respect that, whenever a known compound possesses an already recognised capability, the patentability of new close structural analogues which take advantage of that capability must be established by comparison. This should show that the structural modifications represented by the new variants had a technical relevance by bringing about a surprising difference in the effect. This is in contrast to the case where a new type of use is discovered, i.e. new properties for the new variants, which use has been completely unforeseeable beforehand. No comparison with the prior art is therefore necessary."

421 T 181/82, OJ EPO 1984, 401: "In submitting this report on the test the applicant wanted to take account of the fact that in the present case the state of the art approaches the subject-matter of the application so closely that it is necessary to submit an indication of inventive step which is often used in chemistry and frequently carries conviction, i.e. evidence of superior properties." 
The importance of improved effects in the pharmaceutical industry and the chemical sector generally is due to the faint connection between structure and effect, and to this giving the field of technology an empirical character. ${ }^{422}$ That which can be solved theoretically or by means of small, predictable structural modification has usually been done already. Situations are unusual in which a man skilled in the art reads a patent and can see immediately how the properties - toxicity, for example - can be improved in a manner which, the author of the earlier patent application did not see. Technical progress is very much dependent on empirical tests, e.g. in vitro experiments or animal models.

In the pharmaceutical sector, then, the assessment of inventive step involves a bringing together of two types of differences between the invention and the state of the art; those relating to structure and those relating to the technical effect. Technically speaking, these two factors are interdependent, because structure decides the properties of the substance. But in the assessment of inventive step they are regarded as virtually independent of one another, since both a difference of structure and a difference in properties can be sufficient to establish inventive step. The greater the structural distance and the better the technical effect of the invention are compared with the state of the art, the greater the likelihood of inventive step becomes. ${ }^{423}$ This is the vital foundation for the assessment of inventive step.

\subsection{The problem and solution method}

\subsubsection{Introduction}

In order to test the question of inventive step, the EPO has evolved a special method, known as the problem and solution method. In its developed form, this method is unique to the EPO.

\footnotetext{
422 "In general, the German and European Patent Office regard the existence in a new chemical substance of surprising properties superior to known substances with comparable constitution and activity and which a person skilled in the art could not have foreseen, to be evidence of inventive step. One such surprising property might be, for instance, if the protein has increased activity, fewer side effects, better resorption or greater stability. While in the case where a protein of a type not yet known in the state of the art is provided for the first time no specific problems arise in connection with inventive step, the examination for inventive step is particularly critical in those cases where the subject of the invention is a modified protein whose amino acid sequence has been more or less dramatically altered in comparison to a known protein. If, to take the extreme case, a protein is claimed whose amino acid sequence only differs from a known protein by one position, in deciding whether there has been an inventive step crucial significance will then be attached to the proof that the new protein exhibits a surprising effect that is due to said difference. This also applies to the provision of a non-glycosylated protein or a protein having a glycosylation pattern that varies from the known pattern. For in point of fact, the person skilled in the art is already in a position to manufacture a protein modified thus, for example using genetic engineering. Therefore it is the practice at the German and European Patent Offices, in analogy with the examination for patentability of chemico-pharmaceutical inventions, normally only to regard such a modification as involving an inventive step if it also entails a surprising alteration of the properties of the protein.” See Rauh, P. A., Jaenichen, H-R., JPTOS May 1988, 323.

423 "Die Frage des Nichtnaheliegens in der Chemie geht daher dahin, ob es für den durchschnittlichen Chemiker aufgrund des Standes der Technik einen Anreiz oder einen Hinweis gab, in eine bestimmte Richtung zu forschen. Ein solcher Hinweis liegt gewöhnlich bei struktureller Ähnlichkeit der chemischen Substanz vor; der Fachmann muß dann ähnliche Eigenschaften vermuten. Nicht naheliegend sind deshalb abweichende und überraschende Eigenschaften trotz struktureller Ähnlichkeit, aber auch neue und nützliche Eigenschaften neuer Stoffe, von denen strukturell ähnliche Substanzen bisher nicht bekannt waren.” See Pagenberg, J., Münchener Gemeinschaftskommentar art. 56.148.
} 
It was introduced by the newly formed patent office with a view to attaining the greatest possible uniformity of precedent. ${ }^{424}$

The EPC does not expressly provide that the problem and solution method is to be applied, but some support is afforded by Rule 27:

"The description shall ... disclose the invention, as claimed, in such terms that the technical problem (even if not expressly stated as such) and its solution can be understood, and state any advantageous effects of the invention with reference to the background art.”

It is the Boards of Appeal which have established that the problem and solution method is to be used. If the Examining Division has not used it in the assessment, this is sufficient grounds for referring the case back. ${ }^{425}$ In one case, however, a Board of Appeal has ruled that this method is not always necessary, e.g. when the existence of an inventive step is clear. ${ }^{426}$ That ruling, however, has been criticised in subsequent decisions, and so it would appear safe to assume that the EPO's examiners are under an obligation to use the problem and solution method. ${ }^{427}$

\subsubsection{The three steps of the method}

The problem and solution method is based on the genesis of an invention always being amenable to description as a step - of variable difficulty - from the closest part of the state of the art to the technical solution claimed. The problem confronting the person skilled in the art on the application date was that of bridging the distance between the two. From this viewpoint one can assess the level of difficulty in the problem concerned by carrying out a three-stage assessment. ${ }^{428}$

1. In stage one an assessment is made of which individual document - or other disclosure was the nearest state of the art on the priority date, i.e. which "unit of knowledge" the man skilled in the art trying to achieve the invention would have found most helpful.

2. Stage two contains a comparison between the practical usefulness of the technical solution identified as the nearest under point one and, on the other hand, the practical usefulness of the claimed invention. The difference is the useful result of the applicant's innovative effort and, accordingly, that which can justify his being awarded protection. In making the comparison one at the same time formulates the problem by which the average

\footnotetext{
424 See Hirsche, F., Hansen, B., Protecting Inventions in Chemistry, 194 and Szabo, G. P. A., IIC 1995, 457.

425 T 248/85, OJ EPO 1986, 261: "[I]t is observed that a full first instance examination on the basis of the principles developed by this Board, i.e. on the basis of the problem and solution method, has not yet taken place. This alone must have the consequence that the decision under appeal is to be set aside and the case remitted to the first instance, without decision on the merits of the case. In reconsidering the case, the Examining Division must: (1) positively and objectively determine the most relevant piece of prior art, that is, it is necessary both to identify it and to check its teaching; (2) objectively define the problem underlying the alleged invention by comparison of the technical results achieved by the claimed invention with those achieved by the here above designated closest state of the art; (3) satisfy itself that the so-defined problem is solved by the claimed solution; (4) assess the novelty of the subject matter of the claims; (5) assess whether such solutions involves an inventive step, thereby taking account of the prior art."

426 T 465/92, OJ EPO 1996, 32.

427 T 939/92, OJ EPO 1996, 309.

428 See Szabo, G. P. A., IIC 1995, 460.
} 
practitioner was confronted on the priority date: he had to try to achieve the effects which the invention has compared with the state of the art.

3. The final step contains an assessment as to whether the solution to the problem as defined in point two was obvious to the man skilled in the art on the priority date. The characteristic of the problem and solution method now becomes apparent: instead of primarily studying technical details of an invention and comparing them with a prior document in the state of the art, one looks to the practical possibilities of use which the differences of technical detail confers.

With the problem and solution method one tries, firstly, to recreate the situation which existed on the priority date - to abstract the subsequent invention away - by making an individual document in the state of the art the point of departure for the assessment instead of the claimed invention. The purpose here is to avoid as far as possible what everyone considers to be a mistaken retrospective view of technological development; most things appear obvious when they have been done. Secondly, the method means paying special attention to the new, practically useful effects which the invention has in relation to known solutions, i.e. the social value created by the inventor. The applicant's possibilities of obtaining protection are directly linked to the practical value of the invention. A third property of the problem and solution method is that the assessment is lucid. The internal structure - that of the three stages - makes it easier to see what was decisive in the individual case and to discuss where different assessments part company. In short, the problem and solution method is a useful aid to the assessment of inventive step. The present chapter on inventive step has therefore been based on this method.

\subsection{Step 1 - the closest part of the state of the art}

\subsubsection{Consideration of technical effect and structure}

As has already been stated, the problem and solution method is typified by the special importance attached to one prior document, the prior document deemed to be the closest. The need to localise such a prior document makes it possible to draw the conclusion that an invention has inventive step if no reasonable point of departure can be found for further modifications by the man skilled in the art. ${ }^{429}$

The description in the patent application filed shall include a disclosure of what the applicant considers the closest state of the art. ${ }^{430}$ The statement, however, is only subjective and preliminary. If in the course of the examination new, more closely related prior documents are found or a different assessment of relevance is made, other documents shall be made the point of departure for examining the application. ${ }^{431}$ The technical contribution made by the invention may need to be considered from a different perspective than is indicated by the description. Perhaps the invention solves a different problem from that disclosed in the description and perhaps it is just an alternative to known solutions and no longer an

\footnotetext{
429 See Szabo, G. P. A., IIC 1995, 461.

430 Rule 27 (1)(b) EPC.

431 T 24/81, OJ EPO 1983, 133: “Objectivity in the assessment of inventive step is achieved by starting out from the objectively prevailing state of the art, in the light of which the problem is determined which the invention addresses and solves from an objective point of view ... Consideration is given to the question of the obviousness of the disclosed solution to this problem as seen by the man skilled in the Art.”
} 
improvement. Situations of this kind occur commonly. ${ }^{432}$

The most closely related document is established by comparing various prior documents with the invention, i.e. with the technical features of the claims. An attempt is made to ascertain which document would have been most useful to a man skilled in the art trying on the priority date to achieve the invention. ${ }^{433}$ Consideration is paid to the level of agreement between the technical features of the prior document and those of the invention. But before differences in the technical features are considered, the technical effect has to be checked first. The closest prior document must result in the same type of technical effect as the invention. There must be a question of two substitutable technical solutions. This requirement is of little practical importance, for example, in mechanical engineering. There the essential technical features define the possible uses almost completely. But in the chemical sphere, and in the pharmaceutical industry especially, one commonly encounters known substances closely resembling the claimed ones in structure but having known uses which do not agree with those disclosed in the patent application. It is therefore an important rule that only prior documents with the same medical indication can constitute the closest prior document. In T 116/90, the Board of Appeal found that the three medical indications dilation of coronary vessels, dilation of the peripheral vascular system and treatment of the peripheral vascular system - were to be deemed different medical indications. Since no reliable predictions could be made between the occurrence of the indications, a substance with one of the three activities was not relevant to an assessment of inventive step for a substance having another of the indications mentioned. ${ }^{434}$ In T 643/96, the Board of Appeal made the following pronouncement:

"The compounds of document (2) are useful as anti-histamines, neurosedatives, tranquillisers, and for the treatment of Parkinson's Disease.... Thus, their field of application, designated by the Examining Division too as only 'related usefulness', differs from that of the compounds of the present application, namely treatment and prophylaxis of dementia. The Examining Division gave no arguments why a skilled person would have ignored such a difference when looking for a solution to the existing technical problem. The Board, accepting the Appellant's argument that document (2) is not relevant in the present context."435

In the assessment of what is the closest prior document, the relevant technical effect seems to

432 T 13/84, OJ EPO 1986, 253: "The Board is of course aware that when the problem to be solved and its solution are formulated in relation to the prior art as indicated [in an earlier part of the judgement], the invention will appear in a perspective which is somewhat different from that presented in the application as filed. Such a situation is, however, inherent to a patent granting procedure in which a search made after filing may reveal prior art which is nearer to the invention. ... It has consequently been the constant jurisprudence of the Boards of Appeal that the nature of the problem has to be determined on the basis of objective facts, in particular as appearing in the prior art revealed in the course of the proceedings, which may be different from the prior art of which the inventor was actually aware at the time the application was filed."

433 "The reason for making a start in a hypothetical route-tracing exercise from a single starting point is strong. This source is not considered to have been found by the skilled person searching in view of a preconceived purpose, e.g. a problem to be solved, since that would already involve selections and criticism depending on his subjective state of mind and the actual conditions of his environment. It must be assumed that the primary source could have accidentally become available to a skilled person anywhere in the world, in any language, in any situation, e.g. through a non-confidential communication, just by chance. This could be taken as the second maxim of the 'approach'.” See Szabo, G. P. A., IIC 1995, 461.

434 T 116/90 of 1812 1991: "[T] he Appellant conceded in the oral proceedings that coronary dilation, peripheral vasodilation, and curing of pvd are distinct, separate pharmacological activities. Therefore, because a particular xanthine derivative possesses one of these activities, it is not possible to predict that it would also have either one or both of the other activities. By the same token, the Board is not convinced that reliable predictions are possible as regards the influence of the substitution pattern of the xanthines on their pharmacological profile.” 435 T 643/96 of 14101996. 
be narrowly determined. Only very limited variations are permitted in the way in which the technical effect has been expressed in the prior document and in the patent application.

\subsubsection{Unrealistic prior documents}

During the early years of the EPO, structural similarities and concurrent therapeutic applications alone decided the determination of the closest prior art for pharmaceutical patent applications. It was considered possible for the most relevant prior document to be defined almost mechanically, with certainty and objectivity. Later precedent, however, shows that additional nuances have now been introduced, as well as a clear element of assessment. It is explicitly considered how likely the man skilled in the art would really have been to make a certain prior document his starting point, in a situation where his intention was to make the claimed invention. This means that structure and effect are not the only things taken into consideration. ${ }^{436}$

In T 334/92, substances had been claimed which could be used for the treatment of angina pectoris. Nitro-glycerine had been used for this indication for over a hundred years. There were also two other substances on the market for treating the cardiac disorders in question. Comparative experiments performed by the applicant with the three substances showed the claimed substance to have a better effect, both higher activity and lower toxicity. The Examining Division, however, refused the application with reference to a further document containing structurally still more similar substances which were disclosed as being effective in the treatment of angina pectoris. The applicant had not disclosed experiments with these. The decision was appealed. The Board of Appeal found that the first instance had been mistaken in its definition of the closest state of the art. Structural similarities within the framework of the same indication were not the only relevant criteria. It was also necessary to investigate whether the most obvious document was a realistic starting point. In order for it to be so, the man skilled in the art must have interpreted the document on the priority date as a suitable starting point for further technical development. This was not the case. The Board of Appeal found several reasons why it was not. The prior document was a twenty-year-old patent which during this time had not been a subject of any further research or development. At least, there was no standard work of pharmacology which mentioned the substances and their usefulness in connection with treatment of angina pectoris. Furthermore, the prior document did not contain any more detailed information about the level of the therapeutic results obtained in treatment with the substances. This meant that the man skilled in the art would not have been able to evaluate the substances in relation to other pharmaceuticals for angina pectoris without conducting experiments. The Board added, however, that the absence of test results in the prior document did not in itself disqualify that document as "the closest". The Board went on to say that the principle of the closest state of the art being the structurally most similar substance with the same effect should only be departed from in exceptional cases. ${ }^{437}$ For all the prudence of the Board of Appeal's pronouncements, this decision shows

\footnotetext{
436 Cf. Lunzer, R., Singer: The European Patent Convention 56.05.

437 T 334/92 of 2303 1994: “The question of inventive step can only be objectively answered if an artificial, mechanistic and therefore unrealistic approach is avoided. This implies that it is not appropriate to formulate an artificial and unrealistic technical problem which a skilled person, in practice, would not have considered ... It is thus necessary, after having determined the relevant state of the art in respect of chemical structure and technically useful properties, to consider carefully whether or not in the specific circumstances of the case, taking into account all available information about the technical context of the claimed invention, a person skilled in the art would in fact have had any, let alone any good reason to select this piece of prior art as the
} 
that the age of the prior document and the occurrence of examples showing the extent of the technical effect are factors to which importance is attached in the assessment of whether a document is the closest, i.e. whether on the priority date it was a realistic starting point for further technical development.

In $\mathrm{T}$ 69/94, the patent application pertained to a formulation for a pharmaceutical. Here again the most obvious prior document was twenty years old. The applicant objected to this document being invoked as the closest, but the Board of Appeal ruled that:

"Document (6) neither relates to an antiquated technology no more used in industry, nor does it comprise a teaching which at the filing date of the patent in suit was disproved by those skilled in the art. Accordingly, the Board cannot accept the Respondents argumentation that a person skilled in the art would disregard document (6) only because of its publication date about 20 years before the filing date of the application documents forming the basis of the patent in suit."438

It is at least clear that a document from 1921 which has no disclosures of the results obtained shall not be the closest state of the art. ${ }^{439}$ Otherwise, however, it appears to be necessary in the individual case to assess the likelihood that on the priority date the document would have interested a man skilled in the art who intended to make the invention in suit.

Another aspect which perhaps could be taken into account when establishing the closest prior art is whether a certain solution is on the market and, accordingly, may be presumed to have been specially interesting as a point of departure for further development. There are, however, decisions in which the EPO's Boards of Appeal have emphasised that this is of no importance for establishing the closest prior document. ${ }^{440}$ One such case is T

basis for further development. ... In the present case, the Appellant has strongly emphasised that document (1), which was published in 1964, has never been and would never have been considered, let alone seriously considered, by those skilled in the art. This submission is in agreement with the fact that a number of textbooks concerning pharmaceutical chemistry ... Thus the Board accepts the Appellant's assertion that document (1) was disregarded by those skilled in the art for more than 20 years, and, in particular, that no attempt has been made during this long period to take these compounds as a basis for further development. ... It is also significant that this document is completely silent about the extent of the indicated therapeutic activity. Nor does it mention, let alone discuss, the state of the art relevant at the time, which comprised NG as a medicament of recognised value, so that the person skilled in the art would not have been in the position to recognise any technical advantage of these compounds in respect of the relevant state of the art at the time. ... In contrast to the Appellant's submission, however, the Board observes that the absence of quantitative test results alone would not have been sufficient to disqualify document (1) in this respect. ... In conclusion, the Board wishes to observe that the circumstances which had to be taken into account in the present case, in particular the age and the insufficient content of document (1), were rather exceptional and that the direct comparison of a claimed compound with the structurally most closely related compound of the state of the art remains normally the most straightforward, and very often the sole, method of deciding the issue of inventive step."

438 "Document (6) neither relates to an antiquated technology no more used in industry, nor does it comprise a teaching which at the filing date of the patent in suit was disproved by those skilled in the art. Accordingly, the Board cannot accept the Respondents argumentation that a person skilled in the art would disregard document (6) only because of its publication date about 20 years before the filing date of the application documents forming the basis of the patent in suit.

439 T 741/91 of 2209 1993: "[T] 2 he Board refuses to take as its starting point this old document (1), firstly, because it is silent as to yields and secondly, and even more importantly, as it seems to be highly artificial and unrealistic to assume that the technical problem which objectively existed at the priority date of the patent in suit (28 May 1981) should be seen to lie in the improvement of the yields of this process (published in 1925), having regard to the comprehensive and more up-to date state of the art that was cited during the opposition proceedings. Therefore, the Board sees the technical problem underlying the patent in suit as being the increase in the yield of the process as set out in document (5)."

440 T 254/86, OJ EPO 1989, 115: “The fact that the cited closest state of the art is not commercially exploited 
164/83, where substances had been claimed with reference to their anti-histamine and vasodilatory properties. In the state of the art there was structurally similar substances which were known for these properties. The applicant had shown that the substances claimed by him had better properties (higher activity, less toxicity and better vasodilatory effect) than substances marketed for anti-histamine treatment. The applicant had not made experimental comparisons with the substances which were not commercially available but which were structurally most similar. The applicant argued that this was not necessary, partly because there were no known structure/effect relationships in the therapeutic field concerned. The Board of Appeal, however, found that:

\footnotetext{
"It is irrelevant if his claimed solution of the problem is unforeseeable on the basis of less close or structurally remote prior art, as long as it is derivable, together with the required function, from some other, more relevant known compound, which is, for this very reason, termed as the 'closest' state of the art. A compound lacking inventive step over certain disclosures in the state of the art cannot be rendered patentable in view of non-obviousness over other disclosures. This is why the unexpected results of the submitted comparison with structurally unrelated drugs were irrelevant in the present case." 441
}

There have also been EPO cases where the applicant has been forced to give an account of comparative experiments with substances not belonging to the state of the art. A situation of this kind can arise when the invention differs from the state of the art in two respects, but only one of them has been used in the patent claims to define the invention. It is then uncertain whether the difference in technical effect is due to the technical features delimiting the extent of protection. If there is a suspicion of such a situation prevailing, the applicant must compare the claimed substances with substances which differ only with respect to the technical feature used in the claims. This is the case, even though the objects for comparison may not belong to the state of the art. 442

\subsubsection{Circumstances disclosed after the priority date}

The assessment of inventive step has to take as its starting point the situation of the skilled man on the priority date. The assessment hinges on the knowledge which the skilled man then possessed and the information he was able to obtain from different documents. This applies even if, at a later stage of things, that information proves to be incorrect. In T 268/89 the

could be due to various unknown and irrelevant circumstances, and cannot therefore cast shadow on its information content as a disclosure available to the skilled person, let alone lead to the exclusion of the same from consideration. On the contrary, any non-obviousness vis-à-vis such art, if not closest to the invention, would be irrelevant and inconclusive to validity without the assessment of the inventive step in respect of the objectively closest state, i.e. the most promising springboard towards the invention which was, available to the skilled person”.

441 T 164/83, OJ EPO 1987, 149.

442 T 197/86, OJ EPO 1989, 371: "In the present case the Board has concluded that in the case where comparative tests are chosen to demonstrate an inventive step with an improved effect over a claimed area, the nature of the comparison with the closest state of the art must be such that the effect is convincingly shown to have its origin in the distinguishing feature of the invention. For this purpose it may be necessary to modify the elements of comparison so that they differ only by such a distinguishing feature .... This was achieved by preparing unclaimed variants of the state of the art carrying no para-cyano substitution anywhere, as exact counterparts of three exemplified compounds in the claimed area, and comparing their differences in effects attributable solely to the cyano group. The Appellant's criticism that the comparison compound cannot be traced in the state of the art is therefore inappropriate since the evidence showed exactly the unexpected improvements which occur on a direct route towards the invention.” 
patent application concerned magnetised plaster with therapeutic effects on the circulation of the blood. There was an earlier patent for a similar plaster in which the magnets were somewhat differently designed. The applicant declared that it was a part of the state of the art on the priority date that a magnetic plaster designed in accordance with the earlier patent was devoid of therapeutic effect. Therefore, the applicant argued, the earlier document could not be invoked as a prior document against his application. The Board of Appeal, however, ruled that, on the priority date at least, it had not been made clear that the plaster as referred to in the earlier patent application would not work. The fact of it having emerged after the priority date that this was really the case should not be taken into account when establishing what was the closest state of the art. ${ }^{443}$

Accordingly, when assessing inventive step, one should disregard knowledge concerning the usefulness of a prior document if the knowledge only became available after the priority date for the application in suit. The same appears to be the case with regard to knowledge concerning the usefulness of the claimed invention. In T 548/91 the applicant had disclosed comparative experiments in which it emerged that in animal experiments the claimed substances left the body in a more advantageous manner than the substances in the closest state of the art. After the priority date of the application, however, it emerged that the alleged benefits of the claimed substances did not exist in the treatment of human beings. The Board of Appeal found, however, that an improvement in the treatment of human beings had been probable on the priority date and that circumstances discovered later did not change this. ${ }^{444}$ An assessment of this kind is doubtful, however. As regards the claimed invention, one ought to consider its true technical effects, not what was believed about them on the priority date. If the invention falls short in this aspect, national courts can declare the patent invalid.

\subsection{Step 2 - establishing the problem}

\subsubsection{The principle of problem formulation}

The problem which the claimed invention has solved is established by comparing the technical effect of the closest state of the art, identified as shown in the previous section, and the technical effect of the claimed invention. The yardstick used for measuring the shift in the state of the art would then be the practically useful effect of the claimed invention. It may be presumed that the skilled man's aim was to bridge the gap thus formulated, between the state of the art and the claimed invention. ${ }^{445}$

\footnotetext{
$443 \mathrm{~T}$ 268/89, OJ EPO 1994, 50: "Where the inefficacy of a device or process forming part of the prior art is only recognised or alleged after the date of priority or filing, this cannot be cited in formulating the problem.”

444 T 548/91 of 0702 1994: "[T] [Those comparative data reported by the Respondent [applicant] ... established an unexpected preferential elimination pathway of some of the compounds according to the patent in suit when compared with compounds of document (1). Documents (15) and (16), both published after the filing date of the disputed patent, challenge the reliability of the latter comparative data in so far as they show, to some extent, that advantageous pharmacological activities established by means of data derived from experiments with animals, do not automatically occur in humans. The Board is, however, of the view that inventive step is reasonably established by using experimental tools already available at the priority date of the disputed patent and generally considered at this time as reliable as far as the drug design is involved."

445 "[T] invention, i.e. as defined in the claim and by its effects, and the specific disclosures of individual entities in the cited art together with their function. The achievements of the invention structurally or functionally vis-à-vis the prior art are taken as the 'solution' in the broadest sense and this can then be converted into specific 'must-have-
} 
If the claimed invention consists of a new manufacturing method, the technical effect can for example, consist of a better yield or a higher reactive speed in the process. If instead the invention is a new product - a chemical compound, for example - the problem consists in placing a chemical compound with certain properties at the disposal of the general public. The properties of the claimed products are compared with those of the products in the closest state of the art, e.g. their therapeutic activity, the extent of side effects, the capacity of the body to absorb the substances or the stability of the substances when stored in a moist environment. It seems, however, as though the Boards of Appeals of the EPO then generalise in the actual formulation of the problem. At least, the judgement does not show in detail the differences in technical effect between the invention and the state of the art. ${ }^{446}$ The formulation of the problem is limited to one of two alternatives. Whether the claimed invention is an improvement in any practical respect or if it is only a new alternative to the previously known solutions.

\subsubsection{When is an invention an improvement?}

Therapeutic advances are of great importance for competition in the pharmaceutical industry. These advances may consist of new possibilities of saving life, prolonging life or improving the quality of life, e.g. reduced side effects or greater simplicity of use for the patient. They may also consist in a reduction of treatment costs or in a better safety profile for the treatment, e.g. is a simplification of the patient's regimen an improvement. ${ }^{447}$ In addition, a new pharmaceutical has a value if it can be used in situations where existing pharmaceuticals for the same indication ought not to be used (known as counter-indication). ${ }^{448}$ Factors of this kind are taken into account in patentability assessment and in the formulation of the problem which the invention has solved. "Improvement" is given an extensive and benevolent interpretation from the applicant's point of view. Even a relatively marginal improvement has to be taken into account. In T 334/92, substances which could be used for the treatment of angina pectoris had been claimed. The Board of Appeal ruled:

\footnotetext{
"[I]t is clear from the test results included in the application as filed that the technical problem solved by the present application was to provide novel compounds not having just some activity, but compounds having an activity higher than that of NG or ISDN [substances known previously for the treatment of angina pectoris]. In the Board's judgement, it is not permissible to ignore, for the purpose of defining the technical problem, any technical evidence establishing technically useful properties of the claimed compounds, including the obtained level of
}

\footnotetext{
been-the-problem’ definitions”. See Szabo, G. P. A., IIC 1995, 465.

446 For example T 334/92 of 2303 1994: "For these reasons the Board holds that in the present case the closest state of the art for the assessment of inventive step is represented by NG and ISDN, the standard medicaments for treating angina pectoris at the priority date of the present patent application, and that the technical problem underlying this patent application should be seen in providing further chemical compounds which have an improved activity in the treatment of angina pectoris when compared with these known compounds, and are at the same time less toxic.”

447 T 317/95 of 2602 1999: "Given ... the closest state of the art the technical problem the invention sets out to solve may therefore be seen as ... to improve compliance without impairing the beneficial therapeutic effect achieved by the combined therapy. Simplification is in any case a reasonable and desirable objective which the skilled person would obviously seek to achieve. In the particular field of therapeutic treatment simplification of the patient's regimen affords, inter alia, the advantage of minimising the permanent risk of deviation from the prescribed drug treatment which may cause total or partial failure of the therapy".

448 See Drews, J., Ryser, S., 30 Drug Information Journal 1996, 97.
} 
activity."449

According to this decision, all useful therapeutically properties - e.g. anti-allergic, antibacterial and anti-hypertensive - are to be taken into account. Other properties which can facilitate attainment of the therapeutic result are also relevant, e.g. the capacity of the body to absorb the substances in question or the duration of the effect. ${ }^{450}$ Side effects of the active substance, of course, also have a practical bearing on the usefulness of a pharmaceutical. Furthermore, improvements with a bearing on the industrial handling of the pharmaceutical, e.g. its durability or the ease with which the substances can be manufactured, are also taken into account. In case law, account has also been taken of improvements to properties which are not directly - but perhaps indirectly - of practical and economic importance for the user of the invention. Ecological advantages of a chemical manufacturing method ${ }^{451}$ and a medicinal aerosol formulation 452 have been found to be relevant to an assessment of inventive step. However, it has to be proven that an advantage over the state of the art, on balance, does not entail more severe drawbacks. It must be difficult for the patent office, and also for the applicant, to make such an assessment, but it was nevertheless ruled in T 133/94 with regard to the prior art document 3 that:

\footnotetext{
"Den Vortrag der Beschwerdeführerin, daß die Mittel auf der Grundlage der in (3) als geeignet angesehenen Strukturen unter bestimmten Umständen für eine Psoriasisbehandlung nachteilig sein könnten und sogar zu toxischem PAF führen könnten ... hat die Kammer bei der Frage, welche Aufgabe tatsächlich gegenüber dem Stand der Technik nach der Entgegenhaltung (3) gelöst werden könnte, nicht außer Acht gelassen. In Abwesenheit einer direkten Vergleichsmöglichkeit, die gestattet bei der Psoriasisbehandlung, Vor- und Nachteile des in Anspruch 1 verwendeten Mittels gegenüber der Vielzahl unterschiedlicher aus der Entgegenhaltung (3) bekannten Stoffe abzuwägen, also eine Balance der Eigenschaften gestattet, konnte die bestehende Aufgabe aber weder generell auf eine Verbesserung der Psoriasisbehandlung noch auf Vorteile bei der Behandlung spezieller Psoriasisformen gestützt werden." 453
}

The new compounds were only considered to be alternative means for treatment, because there could be disadvantages as well as the proven advantages over the state of the art.

\footnotetext{
449 T 334/92 of 23031994.

450 T 964/95 of 0505 1999: "[T] he technical problem underlying the invention is that of providing compositions having improved properties over the composition of (5), which comprises a beta-blocking agent (timolol). The tests are accordingly so devised as to substantiate such improvement over the beta-blocker activity, as they do. This improvement, however, need not necessarily be an improvement in intensity over the effect of the prostaglandin taken alone, since the intensity of the effect caused by the mixture of medicaments is only one of the properties to be considered when assessing whether the technical problem has actually been solved; the duration of the effect is a further property, which may play an important role when the intensity of the effects of the compared medicaments prove similar."

451 T 91/92 of 0607 1994: "In view of the yields given in the experimental part of the patent in suit (81 to $90 \%)$ and the yield data of the comparative test (89\%), the Board considers the technical problem underlying the disputed patent vis-à- vis the closest prior art to lie in proposing a process of the same type which is environmentally less harmful and provides improved yields.”

452 T 379/96 of 1301 1999: “Accordingly, starting from document (50) and taking into account legal requirements relating to and increasing public interest in environmental protection, the problem underlying the patent in suit may thus be seen in providing medicinal aerosol formulations having acceptable therapeutical effectiveness but being less destructive to ozone.

453 T 133/94 of 01071998.
} 


\subsubsection{Proof of improved effect}

Now that every practically useful improvement in relation to the closest state of the art can be invoked in support of inventive step, an important question is what proof should be required concerning the effects. The effects of the invention are usually indicated in the description and instanced with several practical examples. Biological conditions, however, are by nature hard to quantify and often the results vary considerably when the experiments are repeated. In order, therefore, for comparative experiments in support of the existence of an improvement to be admissible, they must be at least statistically reliable, i.e. the experiments must have been repeated and deviating values not taken into account. ${ }^{454}$ Effects which come within a margin of error are to be disregarded. ${ }^{455}$

Further, causality is required. That is to say, the evidence (the examples) should be of such a kind that they can be unambiguously related to the invention. Other factors shall be discountable as causes of the experimental results. This entails the maximum possible adherence to the instructions, parameters and conditions used in the closest state of the art to make sure that the tests provide a true and reliable account of the results achieved when carrying out the teaching of the state of the art and that the claimed effect has indeed its origin in the distinguishing feature of the invention over what is disclosed in the closest state of the art. The requirements set forth are e.g. not met if there is no reasonable explanation provided for why in comparative tests one drug was administered to the test animals 4 hours after another was given, whilst in the prior art the drugs were administered in reverse order. 456

The causuality also proved to be a problem in $\mathrm{T} 683 / 90$. The examples showed that a better possibility for diagnosing tumours was achieved by using certain fragments of monoclonal antibodies, compared with the previously known technique for tumour diagnosis. The experiments submitted by the applicant showed that with the invention metastases as small as between 0.5 and $1 \mathrm{~cm}$ could be diagnosed, whereas previous technology only gave a positive reading with metastases measuring $2 \mathrm{~cm}$. The problem, however, from the viewpoint of evidence, was that the good results were obtained with measuring equipment (XCL tomography and CAT scans) which was not available and had not been used in the experiments described in the state of the art document. The Board of Appeal concluded that:

\footnotetext{
"The striking discrepancy could well be due to improved instrumentation or computer processing or to other technical progress in that technology achieved in the past decade. Therefore, in the absence of clear evidence that the result submitted to the Board are exclusively due to the use of monoclonal antibody fragments as claimed, they cannot be accepted as a convincing piece of evidence demonstrating an effect going beyond the expectation of the man skilled in the art."
}

In $\mathrm{T}$ 164/83 the applicant tried to avoid the requirement of examples by submitting that new

\footnotetext{
454 T 495/92 of 1909 1995: "It is to be expected that independent experimental measurements of biological activity lead to different results. Biological assays are intrinsically variable because they involve live materials, the behaviour of which is hardly exactly reproducible. This inherent characteristic of biological assays can, however, be taken care of in a standard manner in order to produce significant biological data, which is to repeat the assays and to discard any 'stray value' which may occur. ... In the Board's view, if stray values are not eliminated in the calculation of averages, these averages are objectionable and their comparison meaningless.” 455 T 155/85, OJ EPO 1988, 87: "It is correct to say that even small improvements in yield or other industrial characteristics could mean a very relevant improvement in large scale production ... but the improvement must be significant and therefore above margins of error and normal fluctuations in the field in consequence of other parameters.”

456 T 317/95 of 26021999.

457 T 683/90 of 05051992.
} 
comparative experiments would be unjustifiably expensive and would force him to carry out what was forbidden experiments on animals. The Board of Appeal, however, ruled that conditions of this kind could not be allowed for, because this would lead to different requirements being defined for patent applicants, depending on where they came from. ${ }^{458}$ Thus there do not appear to be any significant exceptions to the rule that examples are needed in order to corroborate an assertion of improved technical effect.

\subsubsection{Partial improvements}

If an applicant maintains that his invention is an improvement in relation to the closest state of the art, a special instance arises of the problems presented in section 3.3.3.2 with regard to technical effect (must the invention work within the entire scope of the patent claims?). The question here is whether the claimed invention needs to constitute an improvement in relation to the closest document throughout the entire scope of the patent claims. This is a slightly different question. But the technical foundation which makes it difficult to answer is the same: biological effects are unpredictable and often need to have been empirically tested before it can be judged whether they exist and, if so, how extensive they are.

In several statements Boards of Appeal have ruled that the applicant must establish the likelihood of the improved effect existing within the whole of the patent claim. ${ }^{459}$ In $\mathrm{T}$ 626/90, for example, a patent had been requested for a procedure for manufacturing nutrient substances which gave particularly low cholesterol values in the blood. With this method, nutrients could be manufactured which gave a blood cholesterol content of $95 \mathrm{mg} / \mathrm{dl}$. Under certain conditions - still within the method claimed - however, a blood cholesterol content of $116-118 \mathrm{mg} / \mathrm{dl}$ was obtained. This corresponded to the values obtained when using known methods and, accordingly, was no improvement. The Board of Appeal ruled:

\footnotetext{
"When defining the technical problem an effect cannot be retained if it is not credible that the promised result is attainable throughout the entire range covered by a claim."460
}

Not until the claim had been limited could the patent be granted. In T 668/94 the claimed compounds achieved an antifungal activity. One opponent objected to the breadth of the claims of the patent in suit based on the feature "optional substituted" defining the substituents in the claims which represented an "open-ended" definition. In the specification of the patent in suit it was stated that some claimed compounds exhibited a plant growth regulating activity, which signified that only a limited, but indeterminate part of all claimed compounds showed this activity. Hence, the Appellant conceded that not substantially all claimed compounds exhibited a plant growth regulating activity. The Board ruled that when

\footnotetext{
458 T 164/83, OJ EPO 1987, 149: “The argument about the costly character of the comparative tests is not persuasive either. Whether or not the structural modifications of the state of the art are associated with an improvement at all is a fundamental aspect of the inventive step. Whether or not in a particular country experiments on animals may also be problematic under national law cannot be taken into consideration either, since this or similar difficulties could arise in connection with the testing or development of any invention or some ground or another in various countries of the world, and no special considerations could apply to applicants of various nationalities on such grounds."

459 T 742/89 of 0211 1992: "Bei der Formulierung der Aufgabe sind nur solche Effekte zu berücksichtigen, deren Auftreten in der beanspruchten Breite glaubhaft erscheint.” See also T 311/93 of 16011997 and T 131/87 of 07091989.

$460 \mathrm{~T}$ 626/90 of 02121993.
} 
only some and not substantially all claimed compounds exhibit a particular technical effect, then the conclusion must be drawn that the invention as broadly defined is not a solution to the technical problem of achieving the particular technical effect, with the consequence that the alleged technical effect of some of the claimed compounds is to be disregarded in the determination of the objective problem underlying the invention, and thus in the assessment of inventive step. This meant that the compounds were only further antifungal compounds and not improved. ${ }^{461}$

One consequence of everything within the claim having to be an improvement is that an opponent can overturn an assertion of better effect by experiments showing that the improved effect is lacking in some part of the claims. The opponent need not elect to test the very examples invoked by the applicant: he is at liberty to refer to anything within the claims. ${ }^{462}$ Normally, though it is not sufficient for the opponent merely to submit an unsubstantiated allegation that the effect invoked by the applicant does not exist in a certain part of the claim. ${ }^{463}$ There are situations where it is hard to judge if the outer fringes of claims confer the improved technical effect referred to in the application. If so, it may seem reasonable to disregard occasional failures to achieve the improved effect if these are not crucial to the practical usefulness of the invention. In T 548/91 the Board of Appeal also ruled that:

\footnotetext{
"The secondary problem of a safer excretion pathway appears to be solved for some of the claimed compounds as established by the experimental data submitted but not solved by other examples of the claimed compounds.... However, the fact ... that the claimed compounds provide only a partial solution to the secondary problem does not prejudice the patentability of the claimed subject-matter as far as it is credible that substantially all of the claimed compounds represent an appropriate solution to the main problem underlying the patent in suit."464
}

Thus a pragmatic view is adopted, so that the applicant will not incur an unduly onerous burden of proof. If the invention implies an improvement in normal practical procedure, it is accepted.

\subsubsection{Submission of new benefits}

An applicant who originally stated that the invention was an alternative to known solutions the technical effect is not better - can in the course of the proceedings change his standpoint and describe the invention as an improvement. ${ }^{465}$ Things become more complicated when, during the proceedings, the patent applicant wishes to invoke new benefits of his invention

$461 \mathrm{~T} 668 / 94$ of 20101998.

462 T 741/91 of 2209 1993: "In the Board's opinion, the relevance of experiments, which aim at establishing that the promised result cannot be obtained over the whole range of a process claim, does not depend on the exactness of repeating an example of the patent in suit. On the contrary, it is quite sufficient that such experiments are carried out according to the process claim. ... Therefore, all embodiments falling within a process claim have to meet the requirements of patentability set out in Articles 52 to 57 EPC."

463 T 281/85 of 1405 1987: "Nach Auffassung der Kammer erscheint es glaubhaft, daß die für diese Verbindung gefundene geringe Mutagenität auch für diejenigen gemäß dem angegriffenen Patent als Kupplungskomponenten zu verwendenden Verbindungen zutrifft, in denen der Rest R eine Alkoxygruppe mit 1 bis 4 Kohlenstoffatomen bedeutet. Diese Glaubhaftigkeit kann durch die unsubstantiierte und unbelegte Behauptung der Beschwerdeführerin nicht erschüttert werden.”

$464 \mathrm{~T} 548 / 91$ of 07021994.

465 T 1/80, OJ EPO 1981, 206. 
which are not mentioned at all in the application originally filed. The question of doing so may arise, for example, when new prior documents emerge during the examination. Or again, the applicant may have developed the invention further during the patentability assessment and in doing so found new benefits which were not known when the patent application was written.

Art. 123(2) of EPC provides that matters not mentioned in the originally filed documents may not be introduced into the patent application. These rules are applicable to assertions of new technical benefits of the invention. But it is important to bear in mind that the assessment of inventive step can be based on effects which may not be included in the patent application. The patent examiner shall take into account more than what may be included in the patent application. The new information is added to the dossier at the patent office and in this way becomes accessible to outsiders. ${ }^{466}$ The difference between what may be introduced in the patent application and what is to be taken into account in the assessment of patentability was expressed in T 564/89. The Board of Appeal ruled that Art. 123(2) of EPC was not to be applied to the formulation of the invention as a problem and the solution. That rule is only relevant if the applicant wishes to re-word the invention as described in the patent application. ${ }^{467}$

When circumstances not indicated in the patent application originally filed are taken into account, the risk is that applications will be filed at too earlier a stage, before the applicant has even had time to evaluate the invention. Especially interesting technical possibilities (e.g. substances) are claimed, and assertions regarding the properties of the substances are then adjusted as and when the applicant has time to investigate their possible usefulness. Realisation of the risk of such developments has prompted certain restrictions on the possibilities of taking assertions of new benefits into account to an extent which favours the applicant.

According to the Guidelines, new technical effects invoked in support of the occurrence of inventive step must either be implicit in the application filed or otherwise relatable to the technical effect indicated in the filed documents:

"Care must be taken, however, whenever new effects in support of inventive step are referred to.
Such new effects can only be taken into account if they are implied by or at least related to the
technical problem initially suggested in the originally filed application ... The invention as filed
relates to a pharmaceutical composition having a specific activity. At first sight, having regard
to the relevant prior art, it would appear that there is a lack of inventive step. Subsequently the
applicant submits new evidence which shows that the claimed composition exhibits an
unexpected advantage in terms of low toxicity. In this case it is allowable to reformulate the
technical problem by including the aspect of toxicity, since pharmaceutical activity and toxicity
are related in the sense that the skilled person would always contemplate the two aspects
together." 468

A lower toxicity, then, is taken into account in the formulation of the problem which the invention has solved even though it, under Art. 123(2) EPC, is not allowed to become part of

\footnotetext{
466 Guidelines C VI 5.7a and b.

467 T 564/89 of 1002 1993: "The Appellant has submitted that any amendment of the technical problem has to be in line with Article 123(2) EPC. This Article, however, governs amendments of a European patent application or - as in the present case - of a European patent. It is not concerned with the issue, whether or not an objectively reformulated technical problem may be used in the course of the so-called 'problem-solution approach' which was developed by the Boards as a tool for achieving objectivity and to avoid ex post facto analysis in the assessment of inventive step. Therefore, Article 123(2) would only come into play if an amended technical problem was incorporated into the description itself, which is not the case here. Thus, the Appellant's objection fails on this ground."

468 Guidelines C IV 9.10.
} 
the description. In T 155/85, the Board of Appeal expounded the implication of the skilled man being able to deduce the occurrence of the new effect from the original patent application. The Board of Appeal ruled that a re-wording of the problem of the invention:

"should not contradict earlier statements in the application about the general purpose and character of the invention.”

The Board further ruled that it was:

"not acceptable to rely on an effect which has previously been described as undesirable ... to present the same suddenly as possibly representing an advantage from another point of view, and thereby to imply that the technical problem ... should take this reversal into account."469

The first point - the general character of the invention may not be altered - probably means, for example, that a substance claimed with reference to an effect as a weedkiller cannot be described later on as therapeutically useful. In T 440/91 the Board of Appeal found, after reciting the rule stated above, that the problem indicated in the patent application filed was that of making available a salt of a certain substance with therapeutic uses (mucolytic and useable as an antidote for paracetamol poisoning). The salt improved the water solubility of the substance compared with previously used formulations containing the active substance, but it was doubtful whether this was sufficient for inventive step to exist. To establish inventive step, the applicant, during the proceedings in the Board of Appeal, invoked two new benefits in relation to known formulations: improved mucolytic effect and reduced side effects in the form of risk of bronchial spasms. The Board took account of these benefits in the assessment of inventive step and declared the new effects to be accessory to that originally stated (improved water solubility). A person skilled in the art would have taken them into account, and so they should also be allowed for in the assessment of inventive step. In this case, then, the new benefits did not alter the general character of the invention. ${ }^{470}$

In $\mathrm{T}$ 1062/ 93 the applicant had originally mentioned the treatment of hypertension. The Opposition Division rejected the application taking the view that the invention did not involve an inventive step with respect to the prior art. During the appeal procedure the applicant submitted a new and additional medical use for the medicament, treatment of kidney problems. The Board of Appeal made the following comments:

”La requérante a contesté que l'effet rénal additionnel puisse être pris en considération pour la formulation du problème technique, car ... cet effet ne serait pas dérivable de la demande telle que déposée ... Des avantages additionnels non indiqués dans la demande déposée mais

469 T 155/85, OJ EPO 1988, 87.

470 T 440/91 of 2203 1994: “[L]'Intimée a ajouté quelques effets techniques additionnels, à savoir l'absence de bronchospasme et une plus forte activité mucolytique, grâce auxquels, selon elle, les sels du brevet attaqué se distinguent de façon non-évidente de la N-acétyl-l- cystéine. La chambre estime que le caracère de l'objet de la présente revendication 1, à savoir un groupe de sels de la N-acétyl-1-cystéine pour l'utilisation comme mucolytique ou comme antidote lors d'intoxication aigu au paracétamol, n'est pas changé par l'indication ultérieure de quelques propriétés thérapeutiques plus spécifiques de ces composés, puisque la présence ou l'absence d'effets accessoires de composés thérapeutiquement actifs, tels que les sels selon la présente revendication 1, est en tout état de cause envisagée par l’homme métier.... Il résulte de ce qui précède que le problème technique relatif à l'appréciation de l'activité inventive de l'objet de la présente revendication 1 ne consiste pas seulement dans la mise en oeuvre des compositions contenant la N-acétyl-l-cystéine qui ont une plus forte solubilité dans l'eau par rapport à la N-acétyl-l-cystéine et évitent en même temps les inconvénients apportés par la présence d'ions de métaux alcalins. Il consiste en outre à l'amélioration des propriétés thérapeutiques, en l'espèce la réduction de la provocation de bronchospasme et l'amélioration de l'activité mucolytique.” 
concernant le domaine d'utilisation cité dans celle-ci peuvent être pris en considération pour la formulation du problème technique et pour l'appréciation de l'activité inventive dès lors que ces avantages ne changent pas le caractère de l'invention. Le fait de compléter le problème technique indiqué dans la demande d'origine par de tels avantages ne change pas le caractère de l'invention lorsqu'il existe une relation technique étroite entre ces avantages et le problème d'origine ... Il était connu de l'homme du métier, comme confirmé par l'intimée à la procédure orale, qu'une altération de la fonction rénale, telle qu'une augmentation de la résistance vasculaire rénale, est une des raisons fréquentes de l'hypertension, elle-même due à la libération excessive de rénine et à l'activation d'angiotensine II qui en résulte. A long terme, une altération de la fonction rénale peut même être la conséquence de l'état d'hypertension. Donc pour l'homme du métier, il existait une relation technique étroite entre l'effet anti-hypertenseur décrit dans la demande d'origine et une éventuelle amélioration de certains paramètres concernant la fonction rénale. Pour cette raison, les effets secondaires sur la fonction rénale peuvent être pris en considération dans l'énoncé du problème technique à résoudre, s'ils sont valablement prouvés." 471

Both the anti-hypertension and kidney effects should be taken into consideration, because medically they were linked. That which must not be taken into account when formulating the problem which the invention has solved is, if anything, the positive - and, to the man skilled in the art, surprising - effects which may exist in a different therapy segment from that indicated originally. However, there is one case where a Board of Appeal has been stricter and refused to take into account new medical benefits of the invention, merely because they were not known to the man skilled in the art on the priority date. ${ }^{472}$

\subsection{Step 3 - the solution}

\subsubsection{The solution - the features producing the technical effect}

So far in our consideration of the problem and solution method we have been studying how the problem solved by the claimed invention is formulated: first the closest part of the state of the art is identified, then the practically useful effects in the identified prior document are compared with those of the claimed invention, and this results in the invention being described either as an alternative or as an improvement. The problem becomes a consequence of the solution, but is still nothing but an aid for re-creating the situation existing on the priority date. The assessment of inventive step hinges exclusively on the difficulties which the skilled man would have in finding the solution, except in the special cases where there exists what is termed a problem invention; see section 6.8.4.

"Solution" is a concise designation for the technical features which are included in the patent claims and which are needed in order to achieve the technical effect. If the patent claims contain technical features which do not contribute to the effect, these are not to be taken into account in the assessment of inventive step. Indications of unnecessary features shall not be deemed to make it more difficult for the man skilled in the art to solve the problem. ${ }^{473}$ Sometimes, though, evidential requirements concerning the importance of

$471 \mathrm{~T}$ 1062/93 of 18061996.

$472 \mathrm{~T}$ 213/93 of 2304 1997: "In identifying the problem, it is not permissible to draw on knowledge acquired only after the priority date ... . In the present case, the Appellant has relied on post facto evidence which turned up later when more thorough investigations on human thrombomodulin were carried out. ... This is not permissible since before the priority date of the patent in suit, the skilled person could not know in advance that human thrombomodulin exhibited an unexpected advantageous effect over rabbit thrombomodulin in view of the fact that human thrombomodulin still awaited to be isolated."

473 T 37/82, OJ EPO 1984, 71: "In assessing the inventive step of a combination of features, consideration must 
technical features for the usefulness of the invention are pitched low. The absence of evidence against the new technical feature influencing the effect can suffice. ${ }^{474}$ Furthermore, no contribution to inventive step is seen in features such as "a therapeutically effective amount" or "in admixture with a pharmaceutically acceptable carrier", as they are measures that a skilled person would routinely adopt when making a pharmaceutical composition. ${ }^{475}$

\subsubsection{The question to be answered}

The crucial question in the problem and solution method is: would a person skilled in the art who, on the priority date, was confronted by the problem formulated, have created a technical solution within the patent claims? Two details of this question merit special attention. Firstly, it is not enough that the person skilled in the art could have solved the problem somehow: he must have reached a solution which comes within the patent claims. There may be many solutions to the same problem: an improved therapeutic effect can usually be achieved with several different known and as yet unknown substances. But it is only solutions within the claims that are relevant to an assessment of the application in question. The more narrowly the patent claims are formulated, therefore, the less likely it is that the man skilled in the art would have found the claimed solution. The other point to be noted is that the question is whether the person skilled in the art would have made the invention, not whether he could have made it. Technical capacity is not a sufficient - though of course it is a necessary condition for an invention to be obvious. This distinction is usually termed the "could/would" distinction. It tells us that inventive step is a practical question to be assessed with reference to the technical and economic limitations to which innovative work is subject. And it is an attempt to avoid ex post facto analysis of inventive step. After the event, and given a knowledge of the invention, it may seem technically possible for a man skilled in the art to combine different prior documents. But this kind of theoretical possibility of success is not sufficient. The probability of success must have been great enough for the person skilled in the art to find it worth while to solve the problem concerned. These two considerations are set out in the Guidelines:

"In the third stage the question to be answered is whether there is any teaching in the prior art as a whole that would (not simply could, but would) prompt the skilled person, faced with the technical problem, to modify or adapt the closest prior art while taking account of that teaching, thus arriving at something falling within the terms of the claims, and thus achieving what the invention achieves." 476

In T 683/90, the "could/would" distinction was used in a case about use of monoclonal antibodies for tumour diagnosis. In the state of the art a procedure was described in which antibodies were successfully used for diagnosing tumours in mice. The applicant, however, submitted that this procedure would not induce the person skilled in the art to use the

be given to a feature only if the applicant has provided evidence that it contributes, either independently or in conjunction with one or more of the other features, to the solution of the problem set in the description."

474 T 816/90 of 0709 1993: "It is true that the existence of a cause/effect relationship between the modification in the RNA isolation step and the successful cloning and expression of CBH II has not been proven. However, at the present stage, neither concrete evidence nor well-founded reasons are available to state that the said modification was unessential and that the skilled person would have achieved the cloning of CBH II merely by using the experimental approach of (1) and (2) in an analogous manner.”

475 T 223/96 of 29011999.

476 Guidelines C IV 9.5. 
antibodies for human diagnosis. The Board of Appeal ruled:

"[T] he relevant question in the present case is whether a skilled person would be encouraged to try a composition in humans with a reasonable expectation of success when advised that a first trial in animals provided a positive result. It may very well turn out that in the human system one encounters pitfalls or difficulties which could not be foreseen from the animal model. However, the Board is not convinced that the man skilled in the art would automatically disregard animal tests as a preliminary step before deciding whether or not it would be worthwhile to carry out the more complex clinical trials needed on humans. One might conclude that, if a test carried out in an animal provides negative results and somebody nevertheless against any reasonable expectation of success tries this method in human beings with success, this could be considered as being surprising, which is clearly not the case here. Consequently the Board cannot see any sound reason why a skilled person, faced with the underlying technical problem would not have arrived at the solution of Claim 7.”477

If the animal experiments had yielded negative results, it is unlikely that a man skilled in the art would have believed that the solution worked on human beings: "he could, but would not". In the present case, however, the animal experiments had had a positive outcome, and so presumably the man skilled in the art "would have" tested the diagnostic equipment on human beings. A successful experiment on only one patient is something which the man skilled in the art interprets as an incentive for going further. True, biological reactions are hard to anticipate in medical research, but a successful result in animal trials is not normally disregarded. ${ }^{478}$ On the other hand, a statement in a prior art document that a certain experiment will be carried out and the biological activity reported elsewhere, is not sufficient grounds to conclude that the skilled person actually would carry out such an experiment. He could technically, but if there is no indication in the document about biological activity he would not necessarily do so. ${ }^{479}$

In a number of decisions the Boards of Appeal of the EPO have chosen to formulate the question in such a way that inventive step was lacking if the man skilled in the art would have had "a reasonable expectation of success" as distinct from a situation where he

$477 \mathrm{~T}$ 683/90 of 05051992.

478 T 915/93 of 2204 1994: "The Board agrees with the view of the Examining Division that both the fact that the conclusions and suggestions in document (1) were based on a unique patient .. would not have deterred the skilled person from trying the suggested approach. [I]n biomedical research data obtained from unique or unusual cases often provide - through the formulation and testing of working hypotheses and suggestions - an important insight into unsolved problems and the line so suggested would be adopted by the skilled person with a reasonable expectation of success."

479 T 549/96 of 0903 1999: "Document (1) discloses ... a process for the preparation of 22,23-dihydro-1á,25dihydroxyvitamin D2, i.e. of 1á,25-dihydroxyvitamin D4, in order to study the effect of unsaturation at the 22,23-position. However, while indicating that the biological activity of this compound will be reported elsewhere, it clearly does not provide any information about its biological activity, let alone about its toxicity. Therefore, in the Board's judgment, document (1) does not give any pointer to the skilled person how the technical problem underlying the present patent application as defined above could be solved. The Board notes in this respect that in view of the teaching of document (1) a skilled person indeed could have tested 1á,25dihydroxyvitamin D4 on its activity. However, according to the consistent case law of the Boards of Appeal for determining lack of inventive step, it is necessary to show that considering the teaching of the relevant prior art as a whole, without using hindsight based on the knowledge of the claimed invention, the skilled person would have arrived at the claimed solution of the technical problem to be solved. However, as indicated above, a skilled person, when trying to solve the technical problem underlying the patent in suit, would not have found any reason in the state of the art to replace vitamin D2 or its active derivatives by the vitamin D4 derivatives as defined in the objected claims. ... [T] he fact that testing of said compound was announced in document (1) cannot be considered as a hint that the preparation of said compound was done in the expectation of any useful pharmaceutical effect.” 
would only have had "a hope to succeed". ${ }^{480}$ In T 202/95 the Board of Appeal described what it meant in practical terms to have a reasonable expectation of success:

"The construction of such fused protein and of the corresponding gene implied merely the association of known elements, all working in a normal way, according to a known scheme with a reasonable expectation of success. For these reasons, an inventive step is to be ruled out". 481

A reasonable expectation of success and, accordingly, the absence of inventive step, has also been contrasted to a situation where:

“[S]omeone ... would only have attempted it despite success being very uncertain, for example because he trusted in his own luck, skill and inventive ingenuity to overcome the known and the as yet unknown problems involved."482

If someone had made the invention by chance or by dint of special skill, this is no reason for supposing that the average man skilled in the art would also have made the invention. On the contrary, it is this very occurrence of chance or special skill in the solution of the problem which is characteristic of situations where an inventive step exists.

\subsubsection{Alternative substances}

When the problem and solution method is used, the technical effect provides the point of departure for an assessment of inventive step. The invention is described either as an alternative or as an improvement in relation to the state of the art. The account which will be given here of the assessment in step three has been constructed according to the results of stage two, i.e. according to whether the invention has been found to be an alternative or an improvement. Within the two categories, we will now consider which structural differences between the invention and the state of the art can be considered sufficiently important for an inventive step to exist. We will start with differences in situations where the invention is a new alternative to state of the art solutions, before going on to consider differences when the invention is an improvement (section 6.7.5).

Where the patent application for substances which are alternatives to substances already known of having the same biological effect is refused for lack of inventive step, this means that the examiner has found that the man skilled in the art could presume on the priority date that the known substances and the new ones were therapeutically substitutable, in spite of the structural difference between them. ${ }^{483}$ What makes the inventive step requirement difficult to apply is the existence of different opinions concerning the likelihood of the biological effect being affected by a structural modification.

\footnotetext{
480 See e.g. T 296/93, OJ EPO 1995, 627; T 386/94, OJ EPO 1996, 658 and T 923/92, OJ EPO 1996, 564.

481 T 202/95 of 21081998.

482 T 223/92 of 20071993.

483 T 852/91 of 0606 1994: “To deny inventive step for novel chemical compounds because of their 'structural similarity' to known chemical compounds amounts to an allegation that a skilled person would have reasonably expected the same or similar usefulness of both the known and the novel compounds as the means for solving the technical problem underlying the application in question. Such an expectation would be justified, if the skilled person knew, be it from common general knowledge or from some specific disclosure, that the existing structural differences of the chemical compounds concerned were so small that they would have no essential bearing on those properties, which are important for solving the said technical problem and could be disregarded."
} 
In $\mathrm{T}$ 116/90, the application pertained to certain substances which were analogous alternatives to known substances for the treatment of vascular diseases. An opponent submitted that if a chemist were to solve such a problem, he would automatically have chosen to modify the functional group. This was one of the simplest structural modifications and therefore, according to the opponent, lacked inventive step. The Board of Appeal, however, ruled as follows:

"The Board doubts the existence of such a general principle that would induce the skilled person to always modify in the first instance functional groups of known pharmacologically active compounds when searching for alternatives; the preparation of e.g. homologues or isomers seems to be equally plausible for such purpose." ${ }^{\prime 84}$

More general rules can seldom be stated concerning the structural modifications which the person skilled in the art can conceivably make. The case law shows a casuistic assessment. One particularly important factor, though, is the number of positions in the claimed molecules distinguishing them from the substances in the closest state of the art.

In T 20/83, certain benzothiopyran derivatives had been claimed. The applicant could not show that they had better anti-allergic properties than the closest prior art in which there were substances having a similar basic structure. To change the known substances so as to obtain the claimed ones, three steps were needed: an amino group needed to be oxidised, after which it would be shifted to another part of the molecule, and finally a cyano or carboxamin group needed to be excluded from the known molecule. The Board of Appeal found the differences in structure to be so significant that the new solution had inventive step, even though no superior effect had been demonstrated. ${ }^{485}$

In $\mathrm{T}$ 164/83 there were structural differences on two points in the claimed antiallergic substance, compared with previously known anti-allergic molecules having the same basic structure. In the first place, the substituents in positions one and seven had changed places. Secondly, there was a hydroxy group in the claimed molecule which had no counterpart in the structurally most similar molecule. The Board of Appeal found that, in view of the close structural connection, the therapeutic effect could be presumed to remain to the same or at a slightly lesser extent. In this case, accordingly, inventive step was found to exist only if the applicant could show an improved anti-allergic effect. ${ }^{486}$

484 T 116/90 of 18121991.

485 T 20/83, OJ EPO 1983, 419: “The applicant set himself the task of preparing additional anti-allergic compounds, a task accomplished by the preparation of the compounds whose structure is defined in detail in Claim 1. These compounds are essentially arrived at mentally in 3 steps by selecting from the thiophenoxamine acid derivatives according to Citation 2 one of the substituents, namely the oxamino acid substituent (Step 1), and combining this with the 4-oxo-benzothiopyran basic structure of the anti-allergic compounds according to Citation 1 (Step 2) and especially attaching this to the fused benzene ring (Step 3). Even the chain of thoughts leading to the first two steps was in itself not obvious from the viewpoint of a person skilled in the art, for both citations show that the anti allergic properties of the substances require the combined action of all their structural components, i.e. either the combination of the 4-oxo-benzothiopyran basic structure with the amino residue and the cyano or carbamido substituents or the combination of the thiophene basic structure with the amino acid group and the cyano residue. The idea of exchanging the basic structure in the two combinations may be inventive per se, because it is contingent on the knowledge that the amino and oxamino acid group have the same effect. ... Finally, the ideas required for step 3, i.e. of shifting the oxamino acid residue from the heterocyclic to the carbocyclic part of the basic structure, were likewise not obvious, because it can be seen from both citations that the amino or oxamino acid residue has to be in the 2-position with respect to the heterocyclic sulphur atom."

486 T 164/83, OJ EPO 1987: "The claimed subject-matter in the present application basically differs from the closest specific compound disclosed in Example 38.2 of (A) by having the substitutions of theophylline at positions 1 and 7 reversed. The nearest compound to this art in the present application is additionally distinguished by having no hydroxy group in the propyl chain linking the homopiperazino group to the aromatic 
In $\mathrm{T}$ 309/91 it was known on the priority date that certain substances which in one position had an amino group or a $\mathrm{CON}=$ group had anti-hypertensive effects. The applicant had found that if a possibly substituted (thio) ureid group, an alkoxy (thio) carbonylamid group or a possibly substituted phenoxy (thio) carbonylamino group were placed in this position instead, the biological effect could be retained, i.e. the antihypertensive effect would not be lost. These substances acted as prodrugs, i.e. were converted in the body into the previously known ones. The Board of Appeal found that there was no concrete indication in the state of the art that the claimed substances could be used as prodrugs, if the structural modification of the previously known substances were made. Thus, there was an inventive step. ${ }^{487}$

In T 334/92, substances had been claimed which could be used in the treatment of angina pectoris. The applicant had not carried out comparative experiments with the substances in the closest part of the state of the art. The Board of Appeal found that this was not necessary either, given the extent of the structural difference compared with the closest prior document. The claimed substances had at least one aromatic nitro group and also a further substituent - mostly lower alkyls - both of which were lacking in the substances previously known for the treatment of angina pectoris. 488

In T 548/91, carboxyalkyl dipeptides were claimed which were said to inhibit acetyl cholinesterasis (ACE) and in this way had an anti-hypertensive effect. There were previously known carboxyalkyl dipeptides with the same effect. The known ones had a Cterminal proline group. In the claimed substances the proline group had been replaced with a large cyclic group. This could in itself have been sufficient for inventive step, but in the present case there were other documents showing that substances with a large cyclic group in the position concerned had an anti-hypertensive effect. Consequently the structural difference was not sufficient. A better effect had to be demonstrated in order for an inventive step to

ring system (cf. p.3, lines 19-20). The question arises whether the antihistaminic or vasodilating activity of such compounds was foreseeable in view of the cited art. In view of the closeness of at least some of the compounds in the application under appeal to those in (A), there must be an expectation of the qualitative retention of the same activities at least to the same or to a somewhat lesser degree. ... Unless evidence refutes this assumption by showing that the small shift in structure to the claimed area was unexpectedly associated with a significant improvement in the property relevant to the solution of the stated problem, the presumption prevails that the compounds represent only predictable effects and are therefore obvious."

487 T 309/91 of 2801 1993: "[D]ocuments (2), (4), (5) and (6) teach that benzopyrans having an optionally substituted amino group or a $\mathrm{CON}=$ as part of a ring possess antihypertensive activity. However, there is no indication in these documents which would lead the skilled person to expect that the solution to the present technical problem would lie in the provision of compounds in which the substituent at the 4-position is an optionally substituted (thio) ureido group, an alkoxy(thio)carbonylamino group or an optionally substituted phenoxy(thio)carbonylamino group. In other words, changing from compounds which may be classed as amines or lactams to ureas, thioureas, carbamates and thiocarbamates. Document (8) discloses in very general terms the prodrug approach to the design of pharmaceutical compounds but is silent on the basic structure or even the activity to which this principle can be applied. This document contains a list of reversible functional groups suitable for the preparation of prodrugs. However, in the absence of any indication that the lactams of document (2) are prodrugs which develop their antihypertensive activity by the hydrolysis of the cyclic amide group, document (8) would not provide with any incentive to investigate the possibility of preparing prodrugs for the optionally substituted amines of documents (4), (5) and (6). Although document (4) refers to prodrugs ..., the only suitable prodrugs mentioned therein are esters of the hydroxy group at the 3-position ... Therefore, in the Board's judgement, the subject-matter ... involves an inventive step.”

488 T 334/92 of 2303 1994: “According to the present amended Claim 1 the claimed compounds must contain at least one aromatic nitro group and an OR group in position 8 of the benzodioxane ring system, structural elements which are not comprised by the general formula of document (1) (see point II above). Thus this document could not suggest these structural modifications as a solution to the above technical problem. It is therefore not necessary in the present case to rely on a direct comparison with a compound described in document (1)." 
exist. ${ }^{489}$

The case law shows that when the substances in the closest prior document and the invention are alternatives, the number of positions in the molecules distinguishing the claimed ones from the state of the art ones are important for the assessment of inventive step. Furthermore, the character of the differences matters, e.g. whether they can be presumed to have a substantial effect on the hydrophobicity, electronic properties and three-dimensional structure of the molecules. The more differences there are and the more important each individual difference can normally be presumed to be, the more likelihood there is of an inventive step existing. Otherwise the assessment is very casuistic. But an inventive step can be said to exist if it is not possible for the differences to be handled predictably by a man skilled in the art. As yet, the pharmaceutical field is so unpredictable that a man skilled in the art seldom has cause to believe that he would succeed in this.

\title{
6.7.4 Statements in the state of the art
}

Sometimes there are particular statements in the state of the art to the effect that certain structural variations do not affect the biological properties. But it may also be that it is only the experience of the man skilled in the art which leads him to believe that the biological effect will presumably be unaffected. One questions which has arisen in the case law is whether assessments of this kind are within the capacity of the man skilled in the art or whether specific statements are needed in the state of the art. In T 116/90, new substances had been claimed which could be used for vasodilatory treatment. The Board of Appeal ruled:

\begin{abstract}
"There is no doubt that ketals [the claimed substances] are structurally closely related to the parent ketones and that, normally, there are no great difficulties to overcome in their preparation. Thus, the skilled person could have considered them as possible and perhaps easily obtainable derivatives of the said parent ketones. This, however, is not the proper question to be asked. According to the established jurisprudence of the Boards it has to be investigated, when it comes to the issue of inventive step, whether a skilled person would have prepared the compounds in question with a reasonable expectation that they would successfully solve the technical problem under consideration. In the absence of any useful information to that end ... the Board cannot see why the skilled person would have suggested the claimed ketals with the expectation that they would be useful in the treatment of pvd [pvd = peripheral vascular disease]."490
\end{abstract}

An inventive step was thus found to exist, because there was no explicit statement in the state of the art to the effect that the modification - from keton to ketal - could be made without the

\footnotetext{
489 T 548/91 of 0702 1994: "Document (4) stresses that any bulky replacement of the C-terminal proline group should be avoided as unfavourable for maximal inhibitory activity. However, to some extent this 'leading away' teaching of document (4) is rebutted by the disclosure of document (1) and also that of document (8), both published after the document (4) but before the priority date of the disputed patent. Documents (1) and (8) which in fact represent the most recent prior art before the priority date, disclose compounds with various degrees of structural similarity but all bearing a bulky substituent on the C-terminal proline group and exhibiting similar ACE inhibitory activity. Therefore, at the priority date, the person skilled in the art would no longer have considered document (4) as representing a particular prejudice against the provision of a bulky C-terminal group on the proline structure when searching for new carboxyalkyl dipeptides. Consequently, the person skilled in the art could have reasonably expected from documents (1) and (8), that the compounds according to claim 1 of the patent in suit would show the same kind and degree of activity. Comparative data were, accordingly, an appropriate means to establish inventive step based on some unexpected property or some unexpected degree of activity.”

$490 \mathrm{~T}$ 116/90 of 18121991.
} 
biological effect being influenced. That conclusion was drawn in spite of the Board of Appeal pointing out that the structures were closely related. Further EPO decisions also indicate that an application is not to be refused for lack of inventive step if the examiner can only refer to the skilled man's “own” knowledge and there are no statements in the state of the art to the effect that the difference does not influence the biological effect. T 852/91 concerned a patent application for substances with leukotriene-antagonistic properties (used in the treatment of allergic and inflammatory conditions, e.g. asthma). The first instance had refused the application on the grounds that there were known substances having the same type and degree of biological effect which, moreover, were structurally similar to the claimed ones. The structural difference was in a substituent. The known substance contained an arylsulphonamide group (-CONHSO${ }_{2} \mathrm{Rg}$ ). The claimed one contained a ketosulphonyl group (-COCH(M)SO $\left.\mathrm{S}_{2}-\mathrm{C}_{6} \mathrm{H}_{4}-\mathrm{Rc}\right)$. The Board of Appeal's ruling on the difference is important from the viewpoint of principle:

"The Examining Division did not cite any particular document in support of their argument that the - $\mathrm{COCH}(\mathrm{M}) \mathrm{SO} 2-\mathrm{C} 6 \mathrm{H} 4-\mathrm{Rc}$ group of the application in suit would act as an equivalent to the CONHSOnRg group of document (A) in respect to the leukotriene antagonising properties of the respective compounds, but obviously relied on the supposed existence of some common general knowledge. This approach is contrary to the principle that in proceedings before the EPO objections against patentability have to be based on verifiable facts. ... [T]aking into account that, according to the Board's own knowledge, even minute structural differences may have a strong impact on the biological or pharmacological properties of chemical compounds, the Board finds that inventive step cannot be denied for the chemical compounds of present Claim 1 merely on the basis of an alleged structural similarity with the chemical compounds known from document (A)."491

The best example of the rule that there has to be explicit statements in the prior art is probably $\mathrm{T}$ 156/95. The benzoxazepine derivatives in the application in suit differed structurally from those disclosed in prior documents by the replacement of two carbon atoms by two nitrogen atoms. The Examining Division found the concept of "bioisosterism" useful for predicting the pharmacological properties of novel chemical compounds and refused the application. On appeal, however, the Board stated:

\footnotetext{
"In the decision under appeal, the Examining Division argued that the replacement of two Catoms of the compounds of document (1) by two N-atoms, resulting in the compounds of the present Claim 1, was obvious in view of the concept of bioisosterism .... This concept belongs to the common general knowledge of those skilled in the art and may provide some general guidance for rationally developing a research program in a particular pharmacological field (as already mentioned by the Appellant to the Examining Division). However, it has to be applied with caution as soon as it comes to reliably predicting the pharmacological properties of novel chemical compounds, since it is not a law of nature of general validity but rather an empirical rule, which in each particular case needs to be experimentally verified in order to establish whether or not it fits. It could only provide a pointer to the claimed solution of the existing technical problem if there was an established case of bioisosterism for the particular class of chemical compounds concerned. The Board accepts that $=\mathrm{N}$ - and $=\mathrm{C}$ - are well known isosteric groups. However, when deciding on inventive step in relation to pharmacologically active compounds, what is essential is not whether a particular sub-structure of a chemical compound is replaced by another known isosteric one, but whether information was available on the impact of such a replacement on the pharmacological activity profile of the (group of) specific compound(s) concerned .... Under the prevailing circumstances of this case, the Examining Division's recourse to the concept of bioisosterism was not justified, since no information (apart
}

$491 \mathrm{~T}$ 852/91 of 06061994. 
from that of the application in suit) was available to them for assessing whether or not this concept could be verified in the field of benzoxazepine analgesics." 492

The decision shows that a patent examiner cannot refer solely to his own assessment of the general knowledge of the man skilled in the art when coming to the conclusion that two groups are interchangeable. A conclusion of this kind always requires some form of support in the state of the art. A statement in this direction also comes in T 964/92, though that case ended in the application being refused, the reason being that there were statements in the state of the art to the effect that the difference between the closest document and the invention did not influence the therapeutic effect. The claimed substances (2-nitrate methyl-benzodioxane derivative) were said to be active in the treatment of angina pectoris, but no improved effect had been demonstrated in relation to the state of the art. The benzene ring of the claimed substances could be substituted with cyano, carboxy or -OR groups. The structurally most related substances had the same basic structure, but instead the substituents were chlorine, bromine or trifluorine. The Board of Appeal ruled:

\begin{abstract}
"Although the Board would concur with the Appellant's submission that a skilled person would not consider substituents having chemical reactivities and electron donor or acceptor properties different from those proposed in the state of the art in cases where no specific information or common general knowledge about the structure-activity relationship exists, the Board is satisfied in the present case, that the skilled person would have expected, in the light of the common general knowledge discussed above [a standard work on pharmacology, in which it was stated that practically all esters of nitrate acid could be expected to be active in the treatment of angina pectoris], that the type of substitution in the benzene ring of the 2nitratoalkyl-benzodioxanes is not important for the activity against angina pectoris and would therefore have been encouraged to replace the substituents mentioned in document (1) by any other pharmaceutically acceptable substituent, in order to solve the present technical problem."493
\end{abstract}

The principle which was applied in T 852/91 and T 964/92 - that there must be statements in the prior art in order for structural modifications to be obvious - was commented on in $\mathrm{T}$ 643/96. That case pertained to a patent application for new alternative substances for the treatment of dementia. The Board of Appeal found that the assumptions concerning bioisosterism must be applied with great caution in the assessment of inventive step, because there is a strong presumption that the therapeutic effect is lost when a biologically active substance is structurally modified. Bioisosterism can at most offer general guidance to those working with medical research, but it can never be seen as a direct indication of the way in which a problem is to be solved. ${ }^{494}$ This argument led to the following conclusion:

\footnotetext{
492 T 156/95 of 30031998

$493 \mathrm{~T}$ 964/92 of 23081994.

494 T 643/96 of 1410 1996: "The ... argument of the Examining Division amounts in fact to an allegation that the existing structural differences between the compounds known from document (1) and those now claimed are so small that a skilled person would have known that such differences have no essential bearing on the properties important for solving the technical problem .... The validity of this argument hinges on the applicability of the concept of bioisosterism from which the skilled person would have drawn this knowledge. The Board agrees that this concept belongs to the common general knowledge of those skilled in the art but, in the Board's judgement, it has to be applied with caution when deciding upon inventive step. In the field of drug design any structural modification of a pharmacologically active compound is, in the absence of an established correlation between structural features and activity, a priori expected to disturb the pharmacological activity profile of the initial structure. This holds true also for an alleged case of bioisosterism, which is one option of a structure-activity relationship, as long as it is not an established case of bioisosterism .... A careful evaluation of all relevant circumstances is therefore required as to whether or not that a priori assumption can indeed be overcome with the aid of the concept of bioisosterism (which in essence is not a law of nature of general validity
} 
"[W]hen deciding on inventive step in relation to pharmacologically active compounds, what is essential is not whether a particular sub-structure of a chemical compound is replaced by another known isosteric one, but whether information was available on the impact of such a replacement on the pharmacological activity profile of the (group of) specific compound(s) concerned.”

Thus the legal position can be deemed such that it is not obvious to a man skilled in the art to make any variation in a structure in the expectation of preserved biological activity if statements are lacking in the state of the art to the effect that the new structural part is bioisosteric with the known one. The same approach also seems to be applied to chemical production methods. ${ }^{495}$ Statements concerning bioisosterism, moreover, are required to be specific in character, i.e. to refer to the types of substances at issue and to the medical indication involved. Only when these two conditions are satisfied and the therapeutic effect is unchanged do new substances with therapeutic effect appear to lack inventive step.

A legal position of this kind has far-reaching consequences. Taken to extremes, it would mean that in the assessment of inventive step, the man skilled in the art only has a kind of "combination function", i.e. is only capable of more or less mechanically adding together disclosures in two or perhaps three prior documents. Inventive step then takes on the character of a wider novelty assessment: several prior documents may be combined, but the man skilled in the art may not make any creative contribution towards solving the problem. He must always have the support of written prior document when modifying a known solution.

A view of inventive step whereby the man skilled in the art is not permitted to "fill in" gaps in the state of the art with his own knowledge or theoretical assumptions is hardly a traditional view and is not common in other technical fields. It does not seem to be applied, for example, to patent applications for new pharmaceutical formulations. ${ }^{496}$ Normally the foremost characteristic of the man skilled in the art is taken to be his capacity for making simple changes to known technical solutions. A requirement that the examiner refer to statements concerning bioisosterism in the state of the art when rejecting an application on grounds of lack of inventive step transforms those situations and is liable to give rise to a formalistic assessment. In technical fields with a limited amount of published material, an approach of this kind can have manifestly unsuitable consequences, because it can be difficult to prove that the man skilled in the art knew the alternatives to be interchangeable. That which in the pharmaceutical sphere might justify the man skilled in the art not being deemed capable on his own initiative with investigating and judging whether two substituents are

but rather an empirical rule, which in each particular case needs to be experimentally verified in order to establish whether or not it fits) ... [T] [he concept of bioisosterism - at least in the circumstances of this case - is to be considered at most as providing some general guidance to the skilled person developing a research program in the particular pharmacological field, but certainly not as a pointer to the solution to the existing technical problem as now claimed”.

495 T 311/93 of 1601 1997: "The Appellant further argued that even in the absence of a direct suggestion in the relevant state of the art it was 'obvious to try' a basic reaction medium, since appropriate tests could easily be performed. However, in the Board's judgment, a chemical process is not 'obvious to try' simply because it can be performed without difficulty, but only if it can be shown by reference to the state of the art that the process concerned could be fairly expected to produce the desired result, or, in other words, to solve the existing technical problem.”

496 T 142/94 of 1601 1997: "The Board agrees with the Appellant's view that it must be expected from the person skilled in the art faced with the problem of prolonging the analgesic activity of hydromorphone not only that he takes account of parameters relating to controlled release formulations known from the prior art and already put into practice, but also that he makes use of theoretical calculations known in the field of pharmacokinetics for the design of drug formulations.” 
bioisosteric might be the low predictability, the large number of options nearly always available and the unusually large quantity of knowledge concerning bioisosterism which has been put down in writing. Even so, a solution of this kind seems dubious and probably unnecessary.

\subsubsection{Inventive step in connection with an improvement}

The Guidelines point out that in the assessment of inventive step great importance is to be attached to the question of whether the claimed invention is a valuable technical advance. ${ }^{497}$ In addition, an example is given of a surprisingly better effect of a pharmaceutical meaning that there is an inventive step. ${ }^{498}$ By better effect is, of course, also meant the achievement of an effect that was previously unattainable. If the invention in question is so good that it has affected the general development of the state of the art - the technical trend - then this is a strong indication of inventive step, or even a sign of a pioneer invention. ${ }^{499}$ Pioneer inventions have a particularly high inventive step and affect generally the technical field.

In spite of the importance to be attached to the occurrence of improvements in technical effect, there are instances in case law where it has been deemed obvious to combine two known pharmaceuticals in such a way that a synergistic and better effect is obtained. In T 379/94 it was known on the priority date that a combination of the substances interferongamma and interferon-alpha was an interesting possibility of treating cancer diseases. ${ }^{500}$ It had been reported that the combined treatment had a therapeutic effect on four different malignant cell strains which among other things caused leukaemia in mice. The patent application in question concerned the use of an interferon combination for treatment of certain human cells with leukaemia. The Board of Appeal found this to be something which the man skilled in the art would have expected to be possible, in the light of what was known on the priority date. It reasoned as follows:

"The Board concedes that any particular tumour cell's reaction to a combination treatment of
various interferons, here interferon-alpha and interferon-gamma, cannot be predicted with
certainty and that the effect of any cytokine on a particular cell depends not only on the presence
or absence of receptors for that cytokine on the cell surface, but also on the type of signal
generated intracellularly if the receptor for that cytokine is present. However, it is not the certain
predictability that leads the skilled person to try to apply a teaching of the prior art to something

497 "If ... an invention is shown to be of considerable technical value, and particularly if it provides a technical advantage which is new and surprising, and this can convincingly be related to one or more of the features included in the claim defining the invention, the examiner should be hesitant in pursuing an objection that such a claim lacks inventive step.” See Guidelines C IV 9.9.

498 "A mixture of medicines consists of a painkiller (analgesic) and a tranquiliser (sedative). It was found that through the addition of the tranquiliser, which intrinsically appeared to have no pain-killing effect, the analgesic effect of the pain-killer was intensified in a way which could not have been predicted from the known properties of the active substances.” See Guidelines C IV $9.8 \mathrm{~B}(2)$.

499 T 292/85, OJ EPO 1989, 275: "There have been many articles in the literature before the priority date involving the insertion of DNA into a plasmid, yet none of them obtained results which would have showed that bacteria can be made to manufacture what the programme prescribes, which is exactly the polypeptide corresponding to an insert. .... In contrast to this, there are more than one hundred publications and dozens of patent applications, which make use of the invention claimed, after it became public ... This sudden cascade of applications after a period when everybody must have strongly desired the breakthrough, should be taken as a confirmation of inventive step, and even as a sign of pioneering significance.”

500 A prior art document stated: “...there is now established a rational optimism for the use of combination IFN [interferon] preparations in antiviral and antitumour therapy.” 
This case is rather unusual, in that a pharmaceutical producing an improved therapeutic reaction in the treatment of human beings was found to lack inventive step. We have already seen that the unpredictable nature of biological reactions means that even an alternative substance, i.e. one without better practical properties, has inventive step in most cases. If the claimed substances have better effects, this of course argues even more strongly for inventive step. In view of the character of an improved technical effect as a positive indication within the framework of the inventive step requirement, the present account of case law will be confined to situations where an application has been refused for lack of inventive step.

The occurrence of an improved effect did not mean that an inventive step existed in T 296/87. The closest prior art consisted of a racemic mixture, i.e. a mixture of two enantiomers in equal proportions (see section 4.4). The applicant had found that if a mixture containing at least $80 \%$ of one enantiomer was used, the effect achieved was the same as when using four times as much of the racemic mixture. The Board of Appeal, however, ruled:

\begin{abstract}
"Under established Board case law, an enhanced effect cannot be adduced as evidence of inventive step if it emerges from obvious tests. Since, in the present case, tests with the enantiomers were obvious in view of the task at hand, discovery of the claimed effect of the Denantiomers compared with corresponding racemates does not involve an inventive step. It should be noted that this conclusion can be generalised only to a limited extent. Thus the outcome might very well differ with compounds having more than one asymmetrical carbon atom, the number of possible isomers multiplying exponentially. Moreover if the basic racemate were indeed known but not in line with the general technical trend, the proposal that enantiomers be produced by splitting the racemate could be inventive. Other cases are also conceivable in which a different result would be achieved - e.g. the isolation of active enantiomers, or ones with a qualitatively different activity, from essentially inactive racemates or ones acting differently. In the present case, however, no such special aspects were put forward by the parties involved, nor are any evident to the Board. ... The conclusion reached above is not affected even by the circumstance that the D-enantiomers in question exhibit not merely double but approximately four times the effectiveness of the relevant racemates - a fact adduced by the appellants and accepted by the Board. After all, if tests with enantiomers suggested themselves to a skilled person as an obvious way of arriving at a solution offering increased activity, the extent of that increase could not as a rule be taken as an indication that the tests - obvious as they were - involved an inventive step. For present purposes there is no need to try and establish whether a different view might be taken in extreme cases where the efficiency factor is so high that the process virtually involves obtaining an active enantiomer from an inactive racemate. With a factor of only 4, that would certainly appear inappropriate." 502
\end{abstract}

The underlying idea in this decision was that if something was obvious to the man skilled in the art, it was obvious regardless of how good the result turned out to be. Similar conclusions have been drawn in the EPO case law on other occasions. ${ }^{503}$ This situation has been termed " $\mathrm{a}$

\footnotetext{
501 T 379/94 of 21051996.

502 T 296/87, OJ EPO 1990, 195.

503 T 507/93 of 1902 1997: "It is clear from the preceding paragraphs that, in the light of the problem stated above, the choice of acetylated lanolin must be regarded as obvious anyhow. Therefore, the fact that the eye ointment according to claim 1 is superior to that of the closest prior art, is irrelevant for the outcome of the present decision. The answer to the question whether 'an invention shall be considered as involving an inventive step' within the meaning of Article 56 EPC depends in each case on the answer to the question whether 'having regard to the state of the art, it is not obvious to a person skilled in the art'. Therefore, in the present case, the fact that a further quantitative improvement could be achieved by the obvious choice of acetylated lanolin could only be considered to be a bonus effect which would have inevitably resulted from the skilled person's noninventive activity.” T 1062/93 of 3004 1997: ”'intimée a aussi invoqué que l'art antérieur décrivant des
} 
one-way street" 504 , a "bonus effect" 505 or "a straightforward situation". ${ }^{506}$ It is mostly in situations of this kind that inventive step has been lacking even though there was a better effect. From the closest prior art, there has been only one reasonable line of development for innovative activity. The man skilled in the art would have chosen it and then "automatically" found the better effect. A bonus effect of this kind does not mean that something which on other grounds is obvious to the man skilled in the art shall be deemed to have an inventive step. An inventive step requires the man skilled in the art to have been able to choose between several possible ways when tackling the problem concerned. ${ }^{507}$ The scope for applying the "one-way street" rule, however, is severely limited. There are situations where the EPO Boards of Appeal have gone so far as to find that the accounts of two or a few possible lines of development have been sufficient for inventive step, so long as a better effect has been found when one of the alternatives has been chosen. 508

associations entre un iACE et un antagoniste du calcium ne pouvait suggérer les effets synergiques, diurétique et natriurétique, des compositions revendiquées. Cependant, comme démontré précédemment l'homme du métier, confronté au problème technique de procurer des compositions pharmaceutiques ayant des effets thérapeutiques sur l'hypertension et sur la fonction rénale, aurait suivi la direction clairement indiquée par le document (23), qui l'aurait conduit de manière évidente au remplacement du vérapamil par le diltiazem dans les associations de (3) et donc aux effets recherchés. Etant donné que la synergie invoquée, bien qu'imprévisible, se produit inévitablement en ayant suivi une voie découlant à l'évidence pour l'homme du métier de l'art antérieur, elle ne saurait rendre cette voie inventive. En fait, pour atteindre ces effets synergiques l'homme du métier n'avait pas dans le présent cas à s'écarter de la voie évidente suggérée par l'art antérieur pour résoudre le problème technique.”

504 T 21/81, OJ EPO 1983, 15: “The Board considers ... that if having regard to the state of the art it would already have been obvious for a person skilled in the art to arrive at something falling within the terms of a claim, because an advantageous effect could be expected to result from the combination of the teachings of the prior art documents, such claim lacks inventive step, irrespective of the circumstance that an extra effect (possibly unforeseen) is obtained."

505 T 673/94 of 0705 1998: "From the preceding paragraphs it follows that on the priority date of the patent in suit a person skilled in the art ... would have tried to use lipid emulsions having a weight ratio of emulsifier to glyceride oil equal to 0.04 ... in the manufacture of a medicament for intravenous infusion to avoid hyperlipidaemia in premature infants and would thus have tried to further reduce the risk of hyperlipidaemia. ... Since the prior art clearly shows a pointer towards using the low PL/TG ratios according to the claimed solution, and since the prior art also shows at least the trend that the triglyceride level decreases when lowering the said $\mathrm{PL} / \mathrm{TG}$ ratio, the Appellant's finding that a reduction in phospholipid levels from a 0.07 ratio to a 0.04 ratio provides much more than the expected proportional effect on triglyceride metabolism as compared with a reduction from 0.12 to 0.07 , and thus, even by regarding the said effect as a surprising one, can only be regarded as a quantitative bonus effect, which itself cannot establish inventiveness of an obvious solution to the problem defined above.”

506 T 830/96 of 1006 1999: "The Board takes the position that the teaching of document (15) creates a 'straightforward' situation for the skilled person .... Accordingly, the improved antimicrobial effect cannot substantiate an inventive step as it has been achieved inevitably on the basis of an obvious measure that the skilled person would have taken in any case since it leads to other predictable advantages ie improved bleaching at low temperature, the more so because both effects (bleaching and microbicide) are in fact achieved simultaneously by the same agent ie active oxygen."

507 T 192/82, OJ EPO 1984, 415: "The skilled man must be free to employ the best means already available for his purposes, although the use of means leading to some expected improvements may well be patentable if relying on an additional effect, provided this involves a choice from a multiplicity of possibilities. The lack of alternatives in this respect may, therefore, create a 'one-way-street' situation leading to predictable advantages which remain obvious in spite of the existence of some unexpected 'bonus' effect."

508 "In general, the current tendency of the Boards of Appeal is to look at the options which present themselves to the skilled worker when faced with a given problem. If the solution adopted involves the use of the only way, or the usual way of solving the problem, the 'one-way-street' metaphor coined in T 192/82 ... may be applied and inventiveness denied. In contrast, where there is more than one way of solving the problem and the one selected affords some new and unexpected effect, inventiveness may be established.” See Lunzer, R., Singer: The European Patent Convention 56.13. 
Another pharmaceutical with an improved therapeutic effect was found to lack inventive step in T 269/93. The invention as claimed was the use of a certain compound (3, 7 , 11, 15-tetramethyl-2, 4, 6, 10, 14-hexadecapentaenoic) for enhancing the effect of anti-tumour agents. The Board defined the problem to be solved as the provision of means to obtain improved anticancer pharmaceuticals. The compound was a known antitumour agent. But the applicant was the first to use it in combination with other anti-tumour agents, so as to achieve the additivity of activity of anticancer agents. The Board found:

\begin{abstract}
"[I]f confronted with the problem as stated above, the skilled person inevitably would turn to other prior art relating to anti-tumour agents and first of all take into account documents containing technical information more generally with respect to the effectiveness of anti-tumour agents. ... [D]ocument (4) contains such information. This prior art clearly proposed the combined use of two or more anticancer agents and clearly draws the skilled person's attention to the fact that additivity of activity of anticancer agents may be achieved by the combined use of at least two anticancer agents and thus also gives an incentive to try to improve in the same way the activity of the anticancer agent 3, 7, 11, 15-tetramethyl-2, 4, 6, 10, 14-hexadecapentaenoic acid or a salt thereof known from document (3)... [T]he Board can only conclude that the subject-matter of claim 1 relates to an obvious use of pharmaceuticals well known in the field of cancer therapy. Accordingly, claim 1 lacks the required inventive step." ${ }^{509}$
\end{abstract}

The enhanced effect was not enough, because the advantages of combining different antitumour agents were well known in the prior art, although no one had previously combined this particular compound with other anti-tumour agents.

There are also examples where the applicant has found a new medical use, but still the application has been refused. In T 913/94 geranylgeranylacetone was in the prior art known for the treatment of experimentally induced ulcer. Its use in the preparation of a medicament against gastritis had been claimed in the application in suit. The Board of Appeal explained that the fact that two distinct diseases have the same origin or are elicited by the same causative factors, is not in itself a reason to deny the inventive merit of the second therapeutic application of a known substance. But if the manifestations of the second more serious disease are known to run through the manifestations of the first disease, and this assumption reliably substantiated was not confuted, then the activity of a medicament against the more serious disease would already strongly suggest an effect also against the less serious one. That was the present situation and therefore the Board concluded that:

\footnotetext{
"[O]n the priority date of the application at issue, it was known that ulcer and gastritis, though distinct diseases, had common causative factors, developed through common, degenerative states and could be treated with the same medicaments. The skilled person, faced with the technical problem underlying the present invention and assisted by this knowledge, would have regarded gastritis as the most obvious and most promising direction for extending the field of application of the compound disclosed in the closest prior art, document (6). This choice would have been further motivated by the knowledge of the mechanism of action of GGA which suggested a protective activity not limited to the pathology typical of ulcer but directed, in general, against the action of aggressive factors including those causing gastritis. For all these reasons, in the board's judgement, the subject-matter of the claim lacks inventive step.”510
}

The fact that the two diseases were dependent on the same biological mechanisms suggested to the skilled person, that the known activity of the medicament against the more serious disease (ulcer) could also be used against the less serious one (gastritis).

509 T 269/93 of 11121997.

$510 \mathrm{~T}$ 913/94 of 27021998. 


\subsection{Other considerations than structure and effect}

An assessment of inventive step means a search for circumstances arguing for or against the supposition that a skilled man faced with the problem concerned on the priority date would have reached a solution within the patent claims. The two most important factors of assessment have been dealt with already, namely the practical usefulness of the invention and its structural similarity, both as compared with the closest part of the state of the art. Case law also includes certain other considerations. One particularly important circumstance is the content of other prior documents than the closest one. The claimed invention may be found to be obvious because a "mosaic" of different prior documents would lead the skilled man to the invention.

The usefulness of the invention and its structural similarity to the closest prior document and other prior documents constitute the concrete or technical part of the assessment of inventive step. Case law also makes use of certain additional circumstances, termed secondary factors, and sometimes known as circumstantial evidence or objective circumstances. They include, for example, the length of time for which the problem existed before it was solved, whether there was a prejudice against acting in accordance with the claimed solution and whether the invention has turned out to be a commercial success. It has, however, been established in EPO case law that circumstantial evidence of this kind cannot replace the technical assessment, nor need it be taken into account if the result of the technical assessment is unambiguous. 511 The secondary factors can be viewed as a complement to the technical assessment. We will here begin by considering the importance of additional prior documents apart from the closest one, and then go on to consider the importance of different secondary factors.

\subsubsection{Prior documents other than the closest}

As we saw in section 6.7.4 above, Boards of Appeals have declared that "verifiable facts" are needed in the state of the art in order for the skilled man to be able to bridge the gap between the closest part of the state of the art and the claimed solution. This means that there have to be particular prior documents. A mere general view among men skilled in the art does not appear to be sufficient. Most rejections by the EPO Boards of Appeal of patent applications for lack of inventive step have been prompted by the man skilled in the art being able to combine the most closest document with other prior documents in such a way as to arrive to the claimed invention. ${ }^{512}$ The importance to be attached to prior documents other than the closest one in questions of inventive step can, in accordance with the Guidelines, be assessed in three steps:

\footnotetext{
511 T 1072/92 of 2806 1994: “Zu diesem Vorbringen ist zu bemerken, daß Beweisanzeichen wie die Befriedigung eines langen bestehenden Bedürfnisses, die Überwindung eines Vorurteils der Fachwelt oder ein großer wirtschaftlicher Erfolg lediglich Hilfserwägungen für die Beurteilung der erfinderischen Tätigkeit bilden. Solche Hilfserwägungen können Voraussetzung für eine positive Beurteilung der erfinderischen Tätigkeit sein, sind dies jedoch nicht zwangsläufig. Ihr Vorhandensein braucht jedenfalls dann nicht festgestellt zu werden, wenn, wie in dem zu entscheidenden Fall, die Prüfung des beanspruchten Gegenstandes nach dem AufgabeLösungs-Prinzip ... bereits zu dem Ergebnis führt, daß das Vorliegen von erfinderischer Tätigkeit zu bejahen ist."

512 See Paterson, G., The European Patent System 10-15.
} 
"In determining whether it would be obvious to combine two or more distinct documents, the examiner should have regard to the following:

(i) Whether the content of the documents is such as to make it likely or unlikely that the person skilled in the art, when concerned with the problem solved by the invention, would combine them - for example, if two disclosures considered as a whole could not in practice be readily combined because of inherent incompatibility in disclosed features essential to the invention, the combining of these disclosures should not normally be regarded as obvious.

(ii) Whether the documents come from similar, neighbouring or remote technical fields.

(iii) The number of documents which need to be combined."513

Thus, unlike the situation in the assessment of novelty, it is possible, within the framework of the inventive step requirement, for several prior documents to be considered together. Some technical features of the invention may be taken from one prior document, others from another. This is commonly referred to as making a mosaic of prior documents. By this method, however, practically any invention could be traced back to the state of the art. Only the present combination makes the invention new. The limit to the permissible mosaic of prior documents is drawn in the assessment of inventive step by not allowing the combination to become an artificial ex post facto analysis bearing no relation to the way in which a practically active skilled man would have gone about things. 514

One requirement for a prior document other than the closest to be taken into consideration is that it must be reliable and complementary to the closest one. ${ }^{515}$ Two or more prior documents must be meaningfully combinable and not be mutually contradictory. It is not only what is said in the form of detailed disclosures in the prior documents that is decisive. More generally phrased statements concerning the suitable direction for further development of the art may be just as important. Thus a prior document can be deemed to make it harder for the man skilled in the art to solve the problem than it would have been if the prior document had not existed. The prior document may contain ideas expressly pointing in a different direction from that of the claimed invention, i.e. leading the skilled man away from the solution within the patent claims.

If there are several clear statements to the effect that further development of the art in the direction of the invention is fruitless or impossible, the applicant is deemed to have overcome a prejudice. This is a well-established indication of inventive step. On the priority date, the prejudice constituted a special problem which the skilled man was forced to overcome on his way towards the invention. Typical prejudices are erroneous statements in the state of the art concerning causal relations or erroneous statements concerning the technical effect obtained under certain conditions. One possible example in the pharmaceutical sphere is the view that a certain part of the molecule exerts an influence on the pharmaceutical receptor, when in actual fact it is another part that does so. Another typical prejudice is the existence of experiments in which a group of substances are wrongfully

513 Guidelines C-IV 9.7.

514 See Pagenberg, J., Münchener Gemeinschaftskommentar 56.21.

515 T 469/94 of 0107 1997: "Since the clinical pictures disclosed in documents (1) [teaches the oral administration of massive amounts of choline during a period of 4 months on medically intractable human complex partial seizures] and (2) [teaches the healing activity of choline or methionine on different muscular diseases or injuries resulting from a multiplicity of situations all apparently characterised by a deficit of adenosine-triphosphate (ATP) in the muscular tissue] are dramatically different the one from the other, no combination of their teaching could have been envisaged by the skilled person in the attempt to provide a solution to the above defined technical problem [to provide new applications for choline or choline-derivatives which release choline upon administration for increasing the acetylcholine level in brain and tissue and thereby reducing the perception of fatigue in a person about to participate in major exercise or having completed major exercise].” 
indicated as being inactive in connection with a certain treatment. 516

In T 236/96 Interleukin 1 (IL-1) and its derivative had been claimed. One known document disclosed the cloning of murine IL-1 cDNA. According to an opponent, the availability of the cloned murine cDNA as a probe would give the skilled person a reasonable expectation of success that human IL-1 cDNA could be isolated. However, the Board found that two other documents emphasized the differences between human and murine IL- 1 . In one document it was stated that: "In this sense (the existence of more than one species of human IL-1), human IL-1 differs from that of the mouse..." and in another: "There is evidence that human IL-1 differs significantly from murine IL-1 with respect to its biochemical and antigenic properties". In the Board's judgement, the skilled person aware of these two documents would not have had a reasonable expectation that the DNAs encoding murine and human IL-1 would be so homologous that the earlier could successfully serve as a probe to isolate the latter. The cloning of IL-1 DNAs and production of variants thereof was, thus, considered inventive. 517

In order for a prejudice to be relevant, it must have existed on the priority date of the application. ${ }^{518}$ No importance is to be ascribed to prejudices existing previously and overcome on the priority date or to prejudices arising after the priority date. ${ }^{519}$ It is further required that importance was attached to the prejudice by men skilled in the art, i.e. that it was so well known as to influence the conduct of practical research on the priority date. The burden of proving that prejudice was that widespread rests with the applicant. ${ }^{20}$ An individual person's opinion is not sufficient proof of the existence of a prejudice, even if the person in question is well-informed of research in the art concerned. ${ }^{521}$ The usual requirement is for the prejudice to have been expressed in works of reference or other standard literature in order for importance to be attached to it. ${ }^{222}$ A prejudice may not be created by arbitrarily selecting isolated disclosures from the state of the art. ${ }^{523}$ The fact of someone having elected

\footnotetext{
516 See Paterson, G., The European Patent System 10-33 and Hirsche, F., Hansen, B., Protecting Inventions in Chemistry, 218.

517 T 236/96 of 13041999.

518 T 341/94 of 1307 1995: “[A] prejudice in any particular field relates to an opinion or preconceived idea widely or universally held by experts in that particular field. The existence of such prejudice is normally demonstrated by reference to the literature or to encyclopaedias published before the priority date. The prejudice must have existed at the priority date; any prejudice which might have developed later is of no concern in the judgement of inventive step.”

519 T 507/93 of 1902 1997: "The Appellant has sought to demonstrate that there was a prejudice against a combination of the teachings in documents relating on the one hand to ointments for the treatment of the skin and on the other hand to ointments for the treatment of the eye. However, each of the four documents filed as evidence in favour of this submission comprises scientific studies published at least two years after the priority date of the patent in suit. Accordingly, the results of these studies could not influence the way in which a person skilled in the art would understand the disclosure of documents (2), (3), (4), (7) and (11) at the priority date of the patent in suit. In other words, the skilled person faced with solving the stated problem would read the prior art as it stands."

520 T 119/82, OJ EPO 1984, 217.

521 T 19/81, OJ EPO 1982, 51.

$522 \mathrm{~T} 341 / 94$ of 13071995.

523 T 317/95 of 2602 1999: "The burden is on the appellants to demonstrate that the alleged prejudice really existed. The existence of a prejudice should normally be demonstrated by reference to handbooks representing the general, commonly accepted specialist knowledge and encyclopaedias published before the priority date. Any prejudice which might have developed after the priority date is of no concern in the judgment of inventive step. Contrary to the requirements set forth above, there was no evidence provided to adequately support the appellants' contention that in the present case at the priority date of the claimed invention a prejudice existed in the state of the art which would have diverted the skilled person away from simultaneous or common administration of the two active agents. It rather appears that, in the absence of a real prejudice in the sense outlined above, the appellants made in their submissions an ex post facto attempt to create a prejudice, which in
} 
not to publish research findings or not to maintain protection of a technical solution closely related to the invention is not convincing evidence of men skilled in the art having considered inventions of the type in question to be useless. There are far too many conceivable reasons for refraining from patent protection for this to be attributed to the existence of a prejudice.524

Large proportions of all substances are toxic to human beings. This, however, does not mean to say that there is a prejudice against using chemical substances for therapeutic purposes. In T 341/94 the Board of Appeal pointed out that:

"A prejudice in the field of oral compositions [a toothpaste with an anti-plaque effect] must not be confused with a reasonable fear regarding the safety of a product which has not yet been clinically tested.”525

Typical risks of failure or other negative consequences existing with regard to all products of the claimed type shall not be regarded as prejudices. A prejudice must be specifically attached to the design of the claimed invention or the smaller group of technical solutions to which that invention belongs. A prejudice presupposes that the man skilled in the art believed alternative lines of development to be more promising for research into the problem concerned.

The opposite of a prejudice is statements in prior documents encouraging the man skilled in the art to choose a direction of research leading to the claimed invention. An encouragement to which men skilled in the pharmaceutical art attach special importance is that of a substance having proved to have interesting effects in animal experiments. In T 199/94 the patent application pertained to substances which could be used in the treatment of human skin damaged by UV radiation. The treatment was intended to limit or prevent the occurrence of wrinkles. As prior document an article was invoked which showed that hairless albino mice exposed to harmful UV radiation could be successfully treated with the substances. The applicant commented concerning the prior document that there were significant differences between the skin of mice and that of human beings and that, accordingly, the animal experiments did not amount to any special encouragement to test the substances on human beings. The Board of Appeal, however, ruled as follows:

"[I]t seems to the Board that the first if not the sole purpose of experiments involving the exposure of mice to UV-light and subsequently applying therapy must be to act as a model for possible application to humans. Whilst it may well be true that successful experiments with mice do not invariably lead to effective therapy for humans, such successful experiments would nevertheless provide a strong incentive at least to try if the results are transferable. In accordance with the case law of the Boards of Appeal, a course of action can be considered

fact did not exist, by more or less arbitrarily selecting certain isolated disclosures from the state of the art and purposively combining them with the benefit of hindsight."

524 T 261/89 of 0903 1990: "The Board cannot agree to the Appellant's argument that the fact that the inventors of document (1) did not publish their results in an acknowledged scientific journal necessarily leads to the conclusion that the skilled person would stay away from further working according to the disclosure of document (1). Whether or not scientific results are published may depend on so many parameters that nonpublishing is no certain indication that any results may be wrong. Also the fact that the inventors in later filed patent applications or later published documents did not refer to their own prior work, in this case to document (1), cannot give rise to the clear conclusion that the inventors themselves thought the disclosure of document (1) to be wrong. For similar reasons, the Board further cannot agree to the Appellant's argument that non-payment of the annuities for document (1) leads to the definite conclusion that document (1) was spurious and thus it was not worthwhile to further prosecute this patent application. Again, there may be so many reasons to abandon a patent application that it is not possible to assume a causality between abandoning the patent application and wrong experimental results. The Board, therefore, cannot see that the mere fact that the patent application had been abandoned would have restrained the skilled circles from further working on the basis of the disclosure of document (1).”

525 T 341/94 of 13071995. 
obvious within the meaning of Article 56 EPC, if the skilled person would have carried it out in expectation of some improvement or advantage .... In other words, obviousness is not only at hand when the results are clearly predictable but also when there is a reasonable expectation of success." 526

Animal experiments are especially important in the prognostication of therapeutic reactions in humans. In vitro experiments are less important, but the skilled man is aware that these too have a connection with effects on humans.

Importance attaches also to the number of prior documents needing to be combined in order to arrive at the claimed invention. One rule to which reference is sometimes made, but which seldom seems to be expressly used by the EPO Boards of Appeal, is the so-called three-document rule, which says that if the man skilled in the art has to use more than two prior documents (the closest and two or more additional ones) in order to find all the technical features of the invention, there is a presumption of inventive step. ${ }^{527}$ If two prior documents (the most obvious and one more) are sufficient, there is instead a presumption that inventive step is lacking. One author who apparently attaches particular importance to the three-document rule is Szabo, a prominent judge in the Board of Appeals and a member of the Enlarged Board of Appeal. Szabo writes:

\begin{abstract}
"The whole hypothetical exercise [the problem and solution method] boils down to the question whether or not the modifying features found in a secondary source by a conscious but limited search for means to obtain the desired effect, are immediately recognised by the skilled person as suitable for modification of the primary source [the most obvious prior document], or at least as clearly worthwhile for a try in view of a promising outcome. Normally a single other specific source must contain what is missing since to collect the features of the invention from three or more sources, with all the question marks about mutual incompatibilities of their background, is considered beyond the 'practical' capability of the ordinary skilled person and beyond obviousness in view of the emerging uncertainties. Only in special circumstances would other publications come into the picture for instance when cross references or other reasons link them to each other. Thus it is the fifth maxim of the 'approach' that the skilled person must find the modifying features in a single secondary source of information, taking his common general knowledge additionally into account as a possible source for shaping the claimed subjectmatter."528
\end{abstract}

The Guidelines are more prudently formulated on this point. They state that a document can be combined with a standard work, and also that one document can be combined with another, so long as the first contains a reference to the second.529 Nothing is said, however, concerning the possibilities of the man skilled in the art combining two completely separate documents which Szabo apparently assumes he is capable of doing. Nor is it said that normally the man skilled in the art shall not be able to combine three documents.

The three-document rule, as formulated by Szabo, probably reflects EPO precedent. On average, an inventive step exists if a combination of more than two documents

\footnotetext{
526 T 199/94 of 23031995.

527 See Grubb, P. W., Patents in Chemistry and Biotechnology, 228.

528 See Szabo, G. P. A., IIC 1995, 473 (footnotes excluded).

529 Guidelines C IV 9.7: "The combining of two or more parts of the same document would be obvious if it would be natural for the skilled person to associate these parts with one another. It would normally be obvious to combine with other prior documents a well-known textbook or standard dictionary; this is only a special case of the general proposition that it is obvious to combine the teaching of one or more documents with the common general knowledge in the art. It would, generally speaking, also be obvious to combine two documents one of which contains a clear and unmistakable reference to the other. In determining whether it is permissible to combine a document with an item of prior art made public in some other way, e.g. by use, similar considerations apply.”
} 
is needed in order for the man skilled in the art to gain access to all technical features of the claimed invention. One might say that the novelty requirement means that the essential technical features of the claimed invention may not occur in one prior document, and that the requirement of inventive step means that not all technical features may occur in two documents. As regards the inventive step requirement, however, this statement greatly simplifies the issue. Probably the three-document rule should be regarded as descriptive, not normative. On average, inventive step exists if three documents have to be combined, but it is hardly possible to draw any conclusions from this in the individual case. The presumption created by the rule is a brittle one, especially if, like Szabo, one considers it permissible to add the general knowledge of the man skilled in the art to the two prior documents which the skilled man shall be able to combine. If the skilled man's knowledge is allowed to become a further point of consideration, the advantages of the three-document rule - clarity and predictability - will be lost. The justifiability of this rule seems to require that the skilled man's own knowledge may not be invoked.

\subsubsection{The time factor - for how long did the problem exist?}

Apart from the technical assessment of the relation between different prior documents and the claimed invention, importance can also be ascribed to other circumstances. If, for example, it can be shown that the problem which the invention has solved existed for a long time, this shall be taken as an indication of the existence of inventive step. The Guidelines provide:

“... [T]he examiner should be hesitant in pursuing an objection that ... a claim lacks inventive step ... [i]f the invention solves a technical problem which workers in the art have been attempting to solve for a long time, or otherwise fulfils a long-felt need.”530

What is taken into account in these cases is the interval of time between the occurrence of the problem and its solution in the form of the claimed invention. What happens is that the interval of time is determined between disclosure of the closest prior document, or sometimes the appearance on the market of a solution according to the closest prior document ${ }^{531}$, (the occurrence of the problem) and the priority date of the application (the solution of the problem). If the problem involved is significant, even a couple of years may indicate inventive step, especially in a field of intense development. If, on the other hand, the occurrence of a solution is caused by new generally applicable technique having appeared just before the priority date and the technique was applicable to the problem concerned (the closest prior document), the interval of time loses values as indication of inventive step. In such a case the interval of time shall instead be calculated from the appearance of the new technique and not from the appearance of the closest prior document.

When the time factor is invoked in support of inventive step, it shall be assessed how important the problem was considered among men skilled in the art and what resources had been invested within the industry in efforts to solve it. Research intensity in particular has

\footnotetext{
530 Guidelines C IV 9.9.

531 T 830/96 of 1006 1999: "The appellant pointed out that the long period of time that elapsed between the first publication relating to the use of organic peroxyacid bleach precursors for laundry compositions (see document (15) referring back to a congress held in 1964) and the present patent (priority date of 3 December 1987) was that TAED was not available on the market before 1980. The respondent did not contest this information and the Board can accept this as a plausible explanation of the delay in using organic peroxyacid bleach precursors for denture cleansing compositions.”
} 
proved to be very important. In $\mathrm{T}$ 22/82 the Board of Appeal found that a problem which it had taken nine years to solve in the research-intensive field of "flavour substances for food" was an indication of inventive step. ${ }^{532}$ In the case of a manufacturing method for therapeutically active substances, an indication of inventive step was found in the fact that the method in an acid environment had been known for six years before the patent application was filed in which the method proved possible to use in a basic environment. ${ }^{533}$ In T 605/91, on the other hand, it was found that a sixteen-year period could not betoken inventive step, because during the interval of time there had not been "general, repeated and unsuccessful attempts at solving the problem". 534 Decision T 386/94 concerned biotechnology, and the applicant submitted that this field was research-intensive, because four parallel research groups had been working to produce recombinant cymosine (a protein used in cheesemaking). It was argued that in such a situation a period of one and a half months for another research group to achieve the results which the applicant had obtained was an indication of inventive step. The Board of Appeal found, however, that this argument could not be admitted, due partly to the shortness of the period of time and also to the difference in time between the respective patent applications by the two research groups being above all connected with their strategy for the timing of patent applications. 535

In $\mathrm{T}$ 116/90, certain substances were claimed with reference to their vasodilatory effects. Structurally similar substances were known to have the indication. No better effect was found in the claimed ones. The Board of Appeal found, however, that inventive step nevertheless existed, partly because it had been known for twenty years that the claimed substances could be produced, without their therapeutic effects having been tested. The Board of Appeal ruled:

\footnotetext{
"In this connection the Board also notes that document ... already published in 1956 and mentioning as intermediates ketals of xanthine derivatives ... evidently did not provide any incentive to the authors of document (2) [the closest] with a priority of 1976 . This indicates that experts ignored the ketals as possible pharmacological alternatives to the parent ketones in the field of peripheral vasodilating xanthines."536
}

One special way of showing the problem to have existed for a long time is by proving that the known technique had drawbacks on the priority date but was nonetheless used. Proof of such a character is presumably more convincing than the mere fact of the closest prior document having been known for a considerable length of time. T 271/84 pertained to a chemical method, and the Board of Appeal ruled that:

"If a process has been performed successfully on a commercial scale for more than 20 years in
spite of economic disadvantages associated with it, and the claimed invention provides a
solution to the technical problem of avoiding such economic disadvantages, this supports a

532 22/82, OJ EPO 1982, 341: “The lengthy nine-year period elapsing in the much researched area of the flavour substances here under consideration ... between the time when hexane-4,5-diol-3,4-dione was first proposed as a suitable intermediate for these flavour substances ... and the time when the present application was filed can be seen as a further indication that document $2 \ldots$ was unable to provide any suggestion for the separate stage as in the application".

533 T 311/93 of 1601 1997: "Documents (3) and (6) had been published some six years before the priority date, and yet no-one had tried the effect of a basic medium. This speaks against obviousness.”

$534 \mathrm{~T} 605 / 91$ of 20071993.

535 T 386/94, OJ EPO 1996, 658: "The board does not find the argument ... relevant because of the time scale involved (one and a half months for Collaborative Res.). It would rather seem that all groups performed the invention in parallel and that the narrow time differences observed in filing the invention are more representative of a filing strategy than of a level of inventive step."

$536 \mathrm{~T}$ 116/90 of 18121991. 
finding of inventive step." 537

If disadvantages have been accepted in practical use that probably should be an indication of the significance of the problem solved that far outweigh that of only one prior document having been published a long time previously. ${ }^{538}$ The incentives for development are much stronger related to the former.

\subsubsection{Commercial interest in the invention}

Neither true inventors (most of them profit maximising enterprises) nor the man skilled in the art are impelled by idle curiosity to achieve inventions. ${ }^{539}$ Their endeavour is to achieve commercial success by deriving benefit from new technical solutions in the market. Given this background, the commercial success of a new technical solution indicates that it was not previously available to competitors or to the man skilled in the art. If a rival of the applicant was capable of using the commercially valuable invention, why did he not do so?

One problem about commercial success as an indication of inventive step, however, is that of causality. The commercial success has to be due to the technical qualities of the invention as indicated in the patent claims. Causality of this kind can be hard to distinguish from other factors irrelevant to patent law, such as marketing or technical solutions used by the applicant even though they come outside the patent claims. ${ }^{540}$ The difficulty of causal assessment has generally resulted in the EPO Boards of Appeal disregarding assertions of commercial success as an indication of inventive step. The applicant has not been able to show to a sufficient extent which commercial successes have been due to the invention and which ones have been due to efficient production and good salesmanship. One instance where commercial success was accepted as an indication, however, occurred when the firm applying for the patent had unusually small financial resources. This made it unlikely that the commercial success had been due to marketing measures. ${ }^{541}$

537 T 271/84, OJ EPO 1987, 405, See "Headnote".

538 T 665/97 of 1310 1998: "The first argument was the time factor which was used to support the existence of a long felt need. In the Board's view, however, the period of 60 years wherein distillation was not used to obtain highly purified lactides cannot amount to an indication of a long felt need on which an inventive step could be based. During that period of time nobody thought to use distillation since there was no need to produce highly purified lactides economically. As specified on page 4, first full paragraph, of the present patent application, producers of medical-related biodegradable products were not concerned about low yields inevitable when using recrystallization; the same applied to extraction, because of the high margin generally expected for sales of such products. The problem of an economic production arose only in connection with large scale production of cheap biodegradable polymers. When a large scale economic production became necessary, there was a need for a continuous purification process hardly possible with purification by recrystallization. It was thus obvious to purify by distillation instead of recrystallization known to cause high losses in the product to be recrystallized."

539 T 939/92, OJ EPO 1996, 309: "[I]n the board's judgment, it follows from this same legal principle that the answer to the question what a skilled person would have done in the light of the state of the art depends in large measure on the technical result he had set out to achieve. In other words, the notional 'person skilled in the art' is not to be assumed to seek to perform a particular act without some concrete technical reason: he must, rather, be assumed to act not out of idle curiosity but with some specific technical purpose in mind.”

540 T 478/91 of 0206 1993: "Toutefois, il est bien connu que la réussite commerciale d'un produit peut aussi s'expliquer par des raisons tout à fait étrangères à ses qualités, notamment par des mesures de rationalisation dans sa fabrication et par des actions de publicité.”

$541 \mathrm{~T} \mathrm{106/84}$, OJ EPO 1985, 132: "The appellant's assertion that the commercial success his device enjoys is merely based on the superior performance of the product related to the features claimed rather than extraordinary 
One special type of "commercial success" occurs when a competitor of the applicant uses the invention at the time of the inventive step being assessed by the patent office. If competitors of the applicant have taken out licences for the invention, this has been regarded as an indication of inventive step. ${ }^{542}$ An alternative to licensing is for use to have been made unlawfully (assuming there is an inventive step). If the competitors have started using the invention, with or without permission, after the application has been made public, this may be an indication of inventive step. ${ }^{543}$

\subsubsection{Problem inventions and inventive step}

In the great majority of cases, the formulation of the problem is only a technique for achieving as objective and sound an examination as possible of the patentability of the solution. It is the solution that justifies the grant of a patent and the formulation of the problem is aimed only at clarifying the practical importance of the solution, measured in terms of useful effects. A different situation applies when a problem invention has been claimed. In cases of this kind the applicant has disclosed a problem previously unknown to the man skilled in the art, i.e. an unknown need for improving the state of the art. Under such conditions there may be an inventive step, even though the solution to the problem is obvious to the skilled man. It should be pointed out, however, that with problem solutions too, there has to be a solution to the problem; the only difference is that the solution is allowed to be obvious to the skilled man. In EPO decision T 2/83 it was ruled:

"The discovery of an unrecognised problem may in certain circumstances give rise to patentable subject-matter in spite of the fact that the claimed solution is retrospectively trivial and in itself obvious." 544

The consistent use of the problem and solution method by the EPO has caused special attention to focus on problem inventions, because they are an anomaly. Problem inventions

sales promotion efforts must be considered in the light of the fact that the appellant's company is rather small, for which reason it is conceivable that it cannot afford to indulge in major sales campaigns and sophisticated marketing techniques. Thus, the Board is prepared to accept in this case that the commercial success stems from the technical advantages related to the features claimed."

$542 \mathrm{~T}$ 351/93 of 0103 1995: "Beweisanzeichen, wie die Befriedigung eines seit langem bestehenden Bedürfnisses, die Überwindung eines Vorurteils der Fachwelt oder ein großer wirtschaftlicher Erfolg, stellen Hilfserwägungen für die Beurteilung der erfinderischen Tätigkeit dar. Die Feststellung des Vorhandenseins solcher Beweisanzeichen, zu denen auch das Bemühen der Marktkonkurrenten um Mitbenutzungsrechte gezählt werden mag, kann zu einer positiven Beurteilung der Frage der erfinderischen Tätigkeit führen, muß dies jedoch nicht quasi zwangsläufig.”

543 T 92/86 of 0511 1987: "In response to the Appellant's assertion that the Respondents copied the subjectmatter of the patent and filed opposition only after negotiations with a view to entering a license agreement failed, the Respondents admitted that, for a limited period of time, they used the drawing of the patent in suit in their own commercial pamphlets. It is not to be denied that in certain circumstances the actions of competitors after the publication of a patent application - in this case: the imitation of the published invention and the unauthorised use of the drawings of the patent specification by the Respondents - can serve as evidence of a general recognition in the art of the merits of its subject-matter and may lead to the conclusion that the claimed invention involves an inventive step even though it appears prima facie to be obvious. In the present case, however, the Appellant's reference to the acts of a single competitor does not establish that there was such a general recognition and is not sufficient to persuade the Board that its conclusion of lack of inventive step based on the above arguments is not well founded."

544 T 2/83, OJ EPO 1984, 265. 
cannot be accurately valued by the problem and solution method. In cases of this kind, the distance between the state of the art and the solution is not the only contribution made by the inventor. The disclosure of the problem also has to be taken into account. In T 225/84 it was ruled:

"[T]he perception of the problem has to be considered as being the main contribution to the inventive merits..."545

In decision $\mathrm{T}$ 109/87, a comment was made concerning the circumstances in which the formulation of the problem would be deemed to contribute to the inventive step:

"The posing of a new problem does not represent a contribution to the inventive merits of the solution if it could have been posed by the average person skilled in the art. Such is the case where a problem consists solely of eliminating deficiencies in an object which comes to light when it is in use." 546

The same thought was applied in T 223/96:

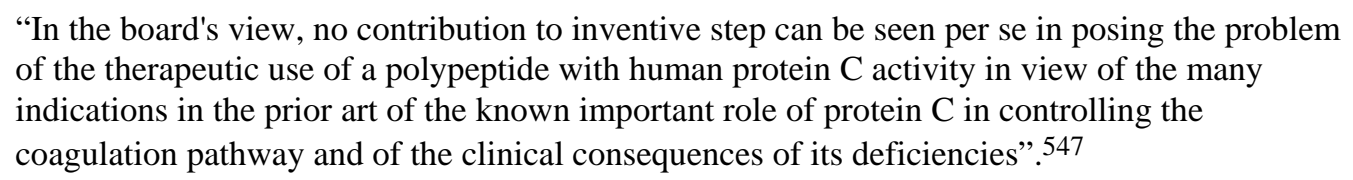

If the man skilled in the art was aware on the priority date, of the problem arising in the use of the then known technique, the problem did not make any contribution towards the inventive step. If e.g. there was poor patient compliance using a formulation as a result of the unpleasant taste, to come up with the idea that something should be done about this, does not add to the inventive merits of the solution to the problem. ${ }^{548}$ On the other hand, if the man skilled in the art was unaware of the drawbacks of the known technology or, if you will, its further possibilities, both the problem and its solution can contribute to the inventive step.

It is characteristic of problem inventions that they occur in situations where known technology has hidden weaknesses. The solution which overcomes the previously hidden weaknesses may not in itself amount to more than skilled adjustments to the state of the art, but the reason for making the changes was previously lacking. There are situations in the pharmaceutical sphere where disclosure of a problem with known solutions from the state of the art has contributed to the inventive step. In $\mathrm{T}$ 2/83 the patent application concerned a formulation consisting of a tablet containing two substances which were separated by a thin layer. The formulation was intended for a medicament for gastric ulcers. The layer prevented the two components from reacting with each other in storage. The Board of Appeal found that it could have been obvious to a man skilled in the art to place a barrier between the two substances for the purpose of preventing a reaction. But the man skilled in the art would not

\footnotetext{
545 T 225/84 of 16071986.

546 T 109/87, OJ EPO 1984, 473.

547 т 223/96 of 29011999.

548 T 566/91 of 1805 1994: "In the Board's view, this approach is not realistic because the mere posing of a problem, even if it were new, does not represent a contribution to the inventive merits of a solution to that problem if it could have been posed by the average person skilled in the art. Such is the case where, as here a problem necessarily comes to light when an object or product is used. Consequently, a problem which amounts to no more than noticing an obvious non-compliance with an obvious desiderata in a given situation, namely poor patient compliance using nystatin formulations as a result of the unpleasant taste of the active substance, cannot be retained as the actual problem to be solved”.
} 
have done so, because he was not aware of the existence of the problem. In the closest state of the art, a third substance had been added for the purpose of preventing a reaction between the other two. This was generally considered to be an adequate measure. But the applicant had shown the known solution to be unsatisfactory. The Board of Appeal ruled:

"Since the [known] tablet was ... a satisfactory answer to the problem of undesirable migration,
the addition of a barrier would have appeared superfluous, wasteful and devoid of any technical
effect. In view of the recognition that a barrier has, after all, a substantial effect, the outcome
was not predictable, and the claimed modification involves an inventive step."549

The relevant question was not whether the man skilled in the art could have inserted a barrier to solve the problem (prevent a reaction), but whether he was aware of the reason why this ought to be done. The man skilled in the art could have solved the problem but would not have done so (see section 6.7.2 for the distinction between "could" and "would").

T 630/92 concerned a patent application for a method for producing living but weakened micro-organisms. They could be used as a vaccine for salmonella. Because the micro-organisms had been weakened, the immunological defence could cope with them easily and would then be capable of recognising and effectively attacking "real" salmonellainducing micro-organisms. The disadvantage of using weakened micro-organisms as a vaccine, however, is that the weakened organisms can sometimes resume their pathogenic character, with the result that the patients are infected instead of being vaccinated. In the closest prior art there was a statement that the micro-organisms used were safe as vaccine for humans, i.e. did not revert to their pathogenic form. The applicant, however, had found a way of producing micro-organisms which gave rise to an even lower frequency of pathogenic properties. Referring to the statement in the state of the art, the applicant maintained that he had achieved a problem invention: not only did the production method (the solution) contribute to the inventive step, but so too did the disclosure that there was a need for further reducing the pathogenic properties of the micro-organisms belonging to the state of the art. The Board of Appeal, however, refused this argument on the following grounds:

"The fact that the auxotrophic mutant strains of (2) were described as 'virtually non-virulent', i.e. safe and effective for use as vaccine in humans, cannot necessarily lead to the conclusion that the skilled person did not perceive potential problems connected with their administration. In the absence of strong indications to the contrary in the state of the art, it is not credible that a skilled person working in the field of vaccines would have regarded the live vaccine of (2) as the ultimate solution to the problem of a possible reversion of attenuated strains to virulent wild strains. Consequently, in spite of the good results reported in (2) (detected reversion frequency $<10-11$ ), the skilled person would not have excluded a priori the possibility of further reducing the reversion frequency of the mutants strains to even lower levels."550

Problem inventions are very similar to the situation where there is a prejudice. In both cases the man skilled in the art must overcome a particular difficulty before he begins to design the solution to the problem in question. Just as with prejudices, however, the existence of occasional statements in the state of the art about the known solution being adequate are not sufficient to establish a problem invention. In problem inventions, the opinion that further development of the art was unnecessary must have been shared by practically everyone active in the field.

549 T 2/83, OJ EPO 1984, 265.

$550 \mathrm{~T} 630 / 92$ of 22021994. 


\subsubsection{Inventive step in the creation of biotechnology}

In 1975 Milstein and Köhler published an article showing that cells producing antibodies, known as lymphocytes, could be amalgamated with tumour cells (myeloma cells). ${ }^{551}$ The hybrid cell quickly begins to divide. Some of the daughter cells acquire a DNA content which causes them to produce a monoclonal antibody and at the same time to multiply without restraint as long as nourishment is available. This latter property, so dangerous in the natural state, is useful in a laboratory environment, because in this way one can quickly obtain a culture of cells which do not die out and simultaneously produce identical antibodies (monoclonal antibodies, all of which bond with the same structure, the antigen). This provides access to an efficient biological "workshop" producing a specific product. The antibodies can be put to practical use in several medical fields.

Milstein and Köhler never patented their invention, but on the other hand they received the Nobel Prize in 1984 for hybridoma technology. ${ }^{552}$ The technology immediately acquired a number of commercial applications. 553 One important application was cancer diagnosis. If radioactive isotopes are bonded with tumour-cells-specific monoclonal antibodies and the antibodies then injected into the body, they will bond with any tumours and can then be located as radioactive accumulations. In 1981 a patent application was filed for an assay for cancer diagnosis based on Milstein and Köhler's technique and consisting mainly of monoclonal antibodies. The application was refused in case $\mathrm{T}$ 683/90 because it was found to be obvious to the man skilled in the art to combine a previously known method of cancer diagnosis, in which polyclonal antibodies were used, with an article in which the advantages of monoclonal antibodies were disclosed. The improved diagnostic result of the claimed equipment was not sufficient for inventive step. ${ }^{554}$ A further example of the same situation occurs in T 906/91. The application pertained to monoclonal antibodies which could be used for measuring the concurrence of theophylline in the blood. Theophylline is an antihistamine medicament with a particularly narrow dosage spectrum, and accordingly the amount of theophylline in the patient's blood has to be closely monitored. This is complicated, however, because coffee, tea and soft drinks contain substances which are similar in structure to theophylline. In the state of the art, polyclonal antibodies had been used for measuring the theophylline content of the blood. With monoclonal antibodies, the accuracy of measurement was improved by a factor of between five and eight. Since, however, on the priority date of the application it was known that polyclonal antibodies could be used for similar measurements, the Board of Appeal found that inventive step was lacking. ${ }^{555}$

The decisions in which monoclonal antibodies have replaced polyclonal antibodies exemplify a relatively common situation in the patent system. A new and better alternative is developed to the previous art and the new method can be applied in several different connections. The inventive step requirement and the ability of the man skilled in the art to combine two or more prior documents then prevents all new possible uses being

\footnotetext{
551 See Köhler, G., Milstein, C., 256 Nature 1975, 495.

552 See Wade, F., 208 Science 1980, 693.

553 See Mackenzie, M., Cambrioso, A., Keating, P., 17 Research Policy 1988, 155.

554 T 683/90 of 0505 1992: “[I]n the Board's judgment ... document (73) provides a strong incentive to solve the problem arising from the short-comings of the polyclonal technique described in document (8), by making use of the technically superior monoclonal antibodies. He would, therefore, certainly have tried to replace the polyclonal antibody fragments by monoclonal ones. Under these conditions the Board considers the improvement to correspond to what was to be expected by the skilled person. Consequently the claimed solution cannot be regarded as surprising."

555 T 906/91 of 25041995.
} 
patented (by other parties than those who devised the generic technology). If the new solution is known to be functionally equivalent to the previously used - a direct substitute - the incentives for the man skilled in the art to replace the earlier solution and thus obtain a better result are so strong that this becomes obvious, unless particular difficulties have been overcome. ${ }^{556}$

Monoclonal antibodies are one part of the new biotechnology. The other, no less important part is recombinant technology. This too is an example of a new technology with generic applications. At the beginning of the 1970s Cohen and Boyer created the tools which made it possible to "cut" the DNA molecule into smaller fragments and introduce these into another DNA molecule. Recombinant biotechnology made it possible to replicating the genetic code and produce therapeutically sought-after proteins. Many patent applications have been filed for the production of proteins using Cohen and Boyer's technique. ${ }^{557}$

These two methods forming the basis of modern biotechnology contain processes for the transfer of information between two naturally occurring substances. Antigen/antibody detection contains an information transfer in that the structure of the antibody is determined by the antigen. A similar information transfer takes place between a DNA sequence and a protein. It is not difficult nowadays for a man skilled in the art to indicate, from a certain amino acid sequence in a known protein, a DNA sequence in which the amino acid sequence is coded; but the man skilled in the art is aware that the degeneration of the genetic code means that, for each given amino acid sequence, there are several conceivable DNA sequences. Conversely, it is not difficult for a man skilled in the art to indicate the amino acid sequence matched by a certain DNA sequence. This process of information transfer is very important in the assessment of inventive step. If there is no starting point in the state of the art for a transfer of information, then an inventive step may be presumed. If the DNA sequence does not belong to the state of the art, a new protein will have inventive step. In T 412/93 the Board of Appeal found that on the priority date there was no reliable source for erythropoietin, i.e. no material to be sequenced, and that the recombinant production of the protein erythropoietin therefore had inventive step. ${ }^{558}$ If, on the other hand, one part of the information transfer belongs to the state of the art, there is a good chance of the man skilled in the art producing the other part. ${ }^{559}$ If only a foreseeable such use

556 “[A] replacement of polyclonal by monoclonal antibodies would be patentable if one or more of the following requirements would be fulfilled: - the selection of the antigen used for immunization was inventive; the degree of improvement of cross-reactivity or affinity to the antigen achieved by the invention was surprising; - it was surprising that the antigen used for the immunization of the host animal is a sufficiently strong antigen; procedural difficulties had to be overcome in order to isolate the claimed monoclonal antibodies; - the prior art had unsuccessfully tried to provide monoclonal antibodies engineered to solve the technical problem underlying the alleged invention; - the person skilled in the art would have tried to solve the technical problem by another route; and - the prior art antisera had been shown to be useless clinically, and the monoclonal antibody fulfilled a long-felt need." See Jaenichen, H-R., The European Patent Office's Case Law on the Patentability of Biotechnology Inventions, $290 \mathrm{f}$.

557 US pat 4,740,470, “Biologically Functional Molecular Chimeras”.

$558 \mathrm{~T} 412 / 93$ of 21111994.

559 " $[\mathrm{T}]$ he relationship between the proteins and the DNA sequences coding for them is of crucial importance for deciding whether DNA sequences have novelty and inventive step. If the protein is to be considered patentable, then random DNA sequences coding for the protein are novel and also involve an inventive step since from the state of the art it could not have been obvious to the person ordinarily skilled in the art how to prepare them; broad claims are then patentable. If the protein is known or obvious, on the other hand, then only a DNA sequence can be patented that is distinguished from the group of synonymous DNA sequences by advantageous properties. The reason for this has to do with the expertise of the person skilled in the art: as a rule that person will be able to indicate and even prepare a DNA sequence coding for a known or obvious protein without inventive effort. ... If the antigen is new (for example a newly discovered virus or some other immunogenic substances as yet unreported) and by virtue of surprising advantageous properties (e.g. suitability 
has occurred, inventive step is lacking. If on the other hand the applicant has overcome some particular difficulties, inventive step may exist. One or more steps may be so complicated that the production of a protein is not obvious to a man skilled in the art. If the DNA sequence does belong to the state of the art it is necessary to consider whether a skilled person could have expressed it on the priority date. The Board of Appeal made such an assessment in T 207/94:

\begin{abstract}
"The point which remains to be decided is therefore whether the skilled person would have reasonably expected the beta-IFN cDNA to be expressed in the recombinant host as an active protein, in the light of the known properties of the human beta-IFN. ... In the case of gene expression, this evaluation necessitates that the properties of the 'expression partners' (the gene to be expressed and its protein product on the one hand, and the recombinant host on the other) be compared. If any one of them has properties which common general knowledge at the priority date would have suggested might be unfavourable to their relationship, it is justified to conclude that the person skilled in the art would have had no reasonable expectation of success. The situation often occurs, however, that no meaningful comparison can be carried out simply because there is not enough available knowledge on both partners. Such a situation must, thus, be assessed in the light of the prior art, as the average person skilled in the art would have done at the priority date. ... The respondent has pointed to a number of properties of beta-IFN and beta-IFN cDNA as potential sources of difficulties for expression: the high hydrophobicity of beta-IFN, the presence of an uneven number of cysteine residues in its amino-acid sequence, the existence of two narrowly spaced ATGs at the 5' end of the beta-IFN cDNA, the presence of the rare Ile AUA codon. All of these properties will be considered in turn. ... In summary, the respondent's submission that the known features of beta-IFN would necessarily have been regarded as insurmountable obstacles for its expression in recombinant form, even in the absence of any suggestions in the art that such kind of features was likely to cause problems for expression cannot convince the board. ... In view of the above findings ... the main request is rejected for lack of inventive step." 560
\end{abstract}

In another decision, $\mathrm{T}$ 923/92, the opposite conclusion was drawn. The Board of Appeal found that it was not obvious to a man skilled in the art in 1982 to produce recombinant tissue plasminogen activator (t-PA, used for the prevention and treatment of embolisms). For one thing, the protein was unusually large and therefore difficult to work with. ${ }^{561}$ The production of recombinant interferon-gamma (used in cancer therapy; patent applied for in 1981) was also found in T 223/92 to have inventive step, because:

"[I]t was common general knowledge at the time of priority that the cloning of each and every gene coding for a certain protein depends on many technical details which are particular to each single gene, for example the size, the existence or non-existence of introns or pre- or pro-

for active immunization and/or diagnosis) also involves an inventive step, therefore in other words is patentable, then as a rule the provision of a monoclonal antibody - likewise new - directed to the patentable antigen will also involve an inventive step, since the person skilled in the art could find no suggestion of it in the state of the art. While the person skilled in the art is basically familiar with the methodology of manufacturing monoclonal antibodies, providing a monoclonal antibody directed to an antigen unknown in the state of the art cannot, however, be taken as obvious from the state of the art .... This means that where an antigen is patentable, the manufacture of any monoclonal antibody, i.e. any random monoclonal antibody directed to the antigen, involves an inventive step.... However, if the antigen is known, then as a rule the technical expertise of a person skilled in the art will enable manufacture of any monoclonal antibody to the antigen without inventive effort.... As with a DNA sequence coding for a known or obvious protein, however, where an antigen is known the provision of a particular monoclonal antibody may involve an inventive step, provided that said antibody proves to be superior to other antibodies directed to the same antigen. Its superiority may, for example, be reflected in the fact that the monoclonal antibody possesses the structure complementary to an essential epitope of the antigen and hence excellent specific binding properties to the antigen.” See Rauh, P. A., Jaenichen, H-R., JPTOS May 1988, 328.

560 T 207/94 of 08041997.

561 т 923/92, OJ EPO 1996, 564. 
sequences and the existence and location of necessary restriction enzyme sites. A reasonable extrapolation from the successful cloning of one gene to another was, therefore, only in rare cases possible at that time."562

If on the other hand the technology in a particular connection does not run into any such special problems, inventive step is lacking. This was found to be the case with an invention claimed in 1981. In T 386/94 it was ruled, concerning production of the protein chymosine (used in the food industry):

\begin{abstract}
"Thus, the teachings of document (87) lead to the conclusion that none of the difficulties expected from the prevailing knowledge on cDNA cloning would be encountered. Accordingly, the person skilled in the art would be fairly confident at the onset of the project that the combination of these teachings and such standard knowledge on biotechnological protocols as gathered in documents (15) or (17) would lead to the successful cloning of the genes encoding preprochymosin and its maturation forms. ... It would, thus, appear that, at the date of priority, the cloning and expression of the chymosin DNA would have been perceived as an endeavour likely to succeed and that achieving this cloning did not pose such problems as to prove that this assumption was wrong. Therefore the board must already conclude on this basis that the main request lacks inventive step.”563
\end{abstract}

Case T 495/92 concerned a patent application filed in 1983 for a DNA sequence which coded for the protein interferon-gamma which can be used in cancer therapy. The difference in relation to a known DNA sequence coding for the same protein consisted solely of one codon in position 420. That difference resulted in a protein which differed from the known one solely with regard to one amino acid. The applicant could not show in a statistically reliable manner that the new protein had a better therapeutic effect. This being so, the Board of Appeal found that inventive step was lacking. The structural variation amounted only to a routine application of recombinant genetic engineering by varying the structure in a predictable manner. ${ }^{564}$ Similarly, in T 886/91, the Board of Appeal found that a new variant of a previously known DNA sequence which coded for a polypeptide with Hepatitis B antigenicity (usable in Hepatitis vaccine and for diagnosis) lacked inventive step. ${ }^{565}$

The borderline between routine application and research concerning new technical possibilities in biotechnology was distinctly expressed in $\mathrm{T}$ 441/93. At the time of the application being filed it was known that the Saccharomyces yeast strain could be used as a host organism for replicating proteins. The applicant had found that the Kluyveromyces strain could also be used. On the priority date Kluyveromyces was already being used in other commercial biotechnology connections. Therefore, the Board of Appeal stated, that in theory it was obvious to a skilled man to investigate whether Kluyveromyces could also be used in the same way as Saccharomyces had been used previously. The Board pointed out, however, that there were a number of problems which made it uncertain whether this would succeed; for one thing, there were problems concerning the availability of suitable vectors. The Board therefore came to the conclusion that inventive step existed, because the use of Kluyveromyces:

"required a substantial amount of work which was not of a routine nature but rather required scientific research, the outcome of which remained uncertain."566

\footnotetext{
562 T 223/92 of 20071993.

563 T 386/94, OJ EPO 1996, 658.

564 T 495/92 of 19091995.

$565 \mathrm{~T}$ 886/91 of 16061994.

566 T 441/93 of 27031996.
} 
The uncertainty regarding the result of the measures is decisive in the assessment of whether or not an invention is obvious to a man skilled in the art. When a generally applicable technology is introduced, there is always some uncertainty to begin with regarding its possible uses. That uncertainty is then successively reduced as the technology becomes commonly known and used.567

\subsection{Remarks}

\subsubsection{An economic interpretation of inventive step}

In the majority of cases there is presumably a rational economic explanation for the way in which a patent law requirement has developed over the years and in case law. It is worthwhile trying to find and describe such motives, because this makes the legal rules easier to understand and apply. The thesis which will be propounded in this section is that the inventive step requirement exists for the purpose of efficiently co-ordinating research efforts in an industry by avoiding, through suitable application of the requirement, the kind of unnecessary duplication of effort which would otherwise be liable to occur when somebody solves a technical problem and several players have been looking for a solution to the same problem. The background is as follows.

There can be two reasons why the patent applicant and nobody else made the invention which is a subject of assessment in a particular case. One explanation is that the applicant has carried out particularly competent technical research, i.e. accomplished something which his rivals were not capable of. The other conceivable reason why an invention was achieved by the applicant and nobody else is that on the priority date there were not enough researching enterprises to be able to use all the possibilities of technical development open to them. In a situation of this kind the applicant was the first to take an interest in the new possibility, but the solution was potentially available to those who had been trying. In these situations of poor competition in the development of the art, average skilled measures can resolve in a valuable new technical solution which then prompts rejection by the patent authorities on grounds of lack of inventive step. It may perhaps sound unlikely that men skilled in the art should not make use of technical possibilities of production which are available to them and have an economic value, but this is often the case. The number of conceivable technical modifications to existing products is so immense that attention cannot be paid to all of them. It is mainly those already manufacturing a product who take an interest in its further development. But it is often also in situations of this kind that patent applications are refused for lack of inventive step.

According to this argument, the reason why the applicant and nobody else became the inventor lies in one or the other situation: above-average technical achievement by the applicant or lack of interest (competition) regarding the further of existing products. The inventive step requirement can be described as a means in patent law of distinguishing between these two possible situations. The inventive step requirement means that a stand is taken on the question of whether the applicant has displayed special competence (there is inventive step) or whether he has devoted himself to a problem which no-one else has paid attention to, i.e. that lack of research competition was the reason for the solution being a new one (there is no inventive step).

567 See Merges, R. P., 7:1 High Technology Law Journal 1992, 1 ff and Ducor, P., 22 Rutgers Computer \& Technology Law Journal 1996, 448. 
If on the priority date competition was lacking as regards efforts to develop the new possibility of production, then at that time there was no need to prevent unnecessary duplication by granting a patent and letting a patentee co-ordinate the further exploitation of the production opportunity. But when there are several enterprises simultaneously and separately investing in research in a field of technology, there is reason, at some stage of the innovation process, to grant an exclusive right and to let it be known that the new possibility of production now exists. The inventive step requirement then serves to limit the extent of duplicated effort in situations where it would otherwise mean a waste of resources. This is achieved by inventive step setting a minimum standard for competition in research into the problem which the invention has solved. What the applicant has achieved must have been beyond the capabilities of others because if this was not the case then there could not have existed any useless duplication of effort after the problem was solved and the patent application filed. It is only in a situation with several firms competing to develop a new solution that one can imagine an agreement whereby firms wish to discontinue research when somebody has solved the problem in hand, i.e. a possibility for increased efficiency. No such agreement is conceivable with regard to variations within the capabilities of a man skilled in the art. The thought is simply that the co-ordination function of the patent system, through the creation of property rights, must be justified by a need for co-ordination.

The two situations - the existence or lack of competition - are concerned with what the applicant's competitors were doing on the priority date, because their activity decides whether efficiency gains can be achieved. But the two situations can also indirectly be distinguished by investigating what the applicant has done. It is suggested here that that is what is primarily done when applying the inventive step requirement. Looking at the accomplishment of the inventor is a way to investigate what his competitors may have been doing on the priority date. As has already been shown, the following factors are taken into account within the framework of the inventive step requirement:

1. Structural differences between the invention and the closest prior document.

2. The practical usefulness of the invention (its technical effect) compared with the closest part of the state of the art.

3. The time factor, i.e. the period elapsed between the occurrence of the problem and the priority date of the application.

4. Commercial success, i.e. whether the invention was of such a kind as to result in a commercial success.

These four points can quite easily be described as different aspects of the question of whether competition existed with regard to efforts to develop the claimed solution. There is, however, a difference between point 1 (structural differences) and the other three points. Point 1 refers to technical conditions and the structural modifications made by the applicant to the state of the art. But after establishing what the applicant has done, conclusions are drawn in this situation as to whether the average man skilled in the art, i.e. the competitors, could have made the invention. If the man skilled in the art could not have done so, there were possibilities of competition in the particular research task and, accordingly, there could be economic reasons for halting the competition when someone had found a solution. Point 1 can be seen as related to the existence of competition, because without some special technical achievement by the applicant, there could not have been competition in the particular research. In point 1 , then, the prime topic of investigation is what the applicant has done, but the important thing about this investigation is that it provides an indication as to whether there were any competitors who could be presumed to have worked on the same problem, i.e. whether there were risks of unnecessary duplication of effort occurring after someone had 
found a solution to the problem.

Points 2, 3 and 4 are different. They are more direct investigations to see whether somebody other than the applicant devoted themselves to the problem, i.e. whether several people were working parallel to the applicant on the priority date. Point 1 builds on a connection between the practical significance of the problem and the number of players who attempt to solve it. The more important the problem, the more competitors there will probably be and the more important it then becomes to grant a patent. In point 3 it is assumed that, the longer the problem has been known, the more people have had time to take an interest in it. The problem must have been known for a time in order for a duplication of effort to have been at all possible. If the closest prior document has been assiduously used in spite of known disadvantages, there is special reason to believe that on the priority date several parallel attempts were being made to solve the problem. Point 4 assumes that there is a connection between the commercial value of the invention and the number of players who have tried to achieve the invention. All in all, the three considerations can be described as investigations regarding the market demand for the invention: its practical value, the time the problem had existed before it was solved and the inventions commercial value. The underlying rationale for these factors can be that market demand functions as a substitute for a direct investigation of whether the competitors were actually working to solve the problem on the priority date. The right to keep $\mathrm{R} \& \mathrm{D}$ secret within a corporation and the natural reluctance of competitors to help a competitor to obtain a patent by disclosing their failures, make it virtually impossible within the patent system to produce direct evidence of what the competitors were actually doing on the priority date. If unsuccessful attempts can be proven these become important indicia for the presence of inventive step. ${ }^{568}$ But usually demand for the invention has to be used as an "approximate value" for the way in which the competitors were conducting their research.

So far it has been argued that the inventive step requirement is a means of distinguishing and making possible the patenting of solutions to problems concerning which, on the priority date, a competitive search was in progress for a solution. This is not the case when an average man skilled in the art could have solved the problem on the priority date. On the basis of this assessment, patent law creates a minimum standard for when it is justifiable to proceed from a competitive situation in research to a situation where there is co-ordinated exploitation of the possibilities of production, the purpose being to avoid wastage of resources on unnecessary duplication of effort. This argument will now be elaborated with reference to the situation applying to pharmaceutical patents.

\subsubsection{The "market" for the creation of pharmaceutical inventions}

Lack of inventive step, as has previously been argued, is equivalent to the establishment of a lack of competition concerning the problem which the claimed invention has solved. In basic economic theory, lack of competition usually is thought to indicate an imperfection of the market, i.e. the absence of players rushing to take benefit from existing possibilities. Viewed in this light, the purpose of the inventive step requirement could be said to correct for the

568 T 73/95 of 1803 1999: "The numerous attempts to deal with this deficiency reported in the patent in suit and in the prior art relied upon by the Appellant show that many solutions involving phosphites had been envisaged ... but none of them could be regarded as really satisfactory. Given the many applications and the industrial importance of the polymers, it is thus legitimate to regard the question of the stabilization of phosphites against hydrolysis as a long-felt need”. 
occurrence of less efficient "markets" for technical development, e.g. situations where a patent application is filed for an improvement to an existing product and only the party who manufactured the previously known product was trying to develop it further. Only in a wellfunctioning market for technological development is there a risk of such unnecessary duplication of effort as can justify the grant of a patent.

It is now necessary to discuss in closer detail the market for inventions to which reference has been made. Inventions are not altogether traditional products. But, like all other markets, the market for inventions can be described as consisting of a supply and a demand side. 569 The supply consists of the state of the art and also of what patent law describes as the capacity of the man skilled in the art for making modifications to the art. The demand side consists of potential buyers of the new invention. They are perhaps unaware of what kind of new product they are demanding, but the important thing is that potential inventors can forecast the demand they are likely to meet if a certain new possibility of production can be developed.

One observation is that in recent years more and more emphasis seems to have come to be placed on the demand side in the assessment of inventive step. It has grown increasingly important to produce evidence of the interest aroused by the problem in the industry before or on the priority date of the application. This emphasis on demand for the invention appears to have been at the expense of the supply side, i.e. assessment of the technical differences between the prior documents and the patent application. This shift towards greater consideration for the demand side seems to be quite clear e.g. from the consistent use made by the EPO of the problem and solution method in its assessment of inventive step, the very starting point for which is the practical usefulness of the invention, i.e. how it is valued. Nowadays consideration of the demand side tends to go so far that every invention with a commercial value - i.e. an invention for which there is a market demand - is deemed entitled to protection. Intellectual property protection is granted for practically all new achievements of commercial value. Even if the technical modification of the state of the art appears to be simple, the conclusion is drawn that inventive step exists if there is a market demand for the change. This development can be seen in all sectors, and not least in the pharmaceutical industry. Quite simply, technical difficulties are perhaps no longer such important considerations. It seems that instead a direct study is made, through the demand for the claimed solution, to see whether there was competition in the search for the solution.

The above review of case law from the EPO Boards of Appeal has revealed that there are only a few cases in which a patent application for a pharmaceutical was refused for lack of inventive step. Any refusals by the Examining Division have probably been appealed and so patent applications of this kind ought to have been identifiable if they occurred to any more palpable extent. In T 296/87 a patent application was refused for a substance with biological effect (a pesticide), on grounds of lack of inventive step. ${ }^{570}$ In this case the most obvious part of the state of the art was a racemic mixture, i.e. a mixture of two enantiomers comprising $50 \%$ of each. The applicant had found that the biological effect was improved with a mixture containing at least $80 \%$ of one of the enantiomers. The Board of Appeal found that inventive step was lacking, because testing different mixtures to ascertain their effect was a routine procedure. Situations like the one in this case are unusual in research-intensive sectors, a category which should be taken to include the pesticide industry. It is still less common for simple actions by a man skilled in the art to lead to a valuable effect in the pharmaceutical industry. The skilled man's variations or investigations have usually preceded an initial patent application, or else the variations are included within the claims, even without

569 Cf. Scherer, F. M., Innovation and Growth, 120.

570 T 296/87, OJ EPO 1990, 195. 
their effects having been practically tested. Research intensity is so high that modifications by the skilled man have already been made before a prior document is disclosed.

Research intensity and thereby the existence of competition means that only achievements surpassing the actions of a man skilled in the art can result in inventions with a market value. The description of the skilled man commonly employed in case law very seldom credits him with the possibilities of making technical changes of a kind which satisfy a demand within the pharmaceutical sector. ${ }^{571}$

Thus, probably unlike many other technological sectors, the market for the further development of existing pharmaceuticals can be deemed efficient, because a skilled man is very seldom likely to achieve new technical possibilities of economic importance. The technical possibilities of value at the disposal of an average man skilled in the art are almost without exception put to practical use. At the same time, however, the intensively competitive efforts made to solve medical problems imply great risks for the occurrence of unnecessary duplication.

As a result of this high research intensity, there is very little need within the patent system to correct for imperfections of the market, by using the inventive step requirement for the rejection of patent applications. Before the average skilled man has the opportunity of making a technical innovation, it will have been made by a team of aboveaverage skilled men. There is nearly always competition in pharmaceutical research and, accordingly, nearly always reason for breaking off competition and the unnecessary duplication of effort which would result if the existing competition were not broken off by granting of a property right.

Thus a situation seems to have arisen within the pharmaceutical industry where research findings with an economic value, i.e. those which are in demand and which somebody wishes to patent, almost without exception have an inventive step. The intensity of competition makes it possible in practice to conclude immediately from the commercial value of an invention, the demand side, that the invention was not obvious to an average man skilled in the art. A conclusion of this kind is possible in efficient markets for inventions. The more intensive the research and development competition existing in a technical field, the more patent law appears to assume the character of protection for the creation of economic values, i.e. protection for a demand. In patentability assessment, then, the prime topic of investigation will be not the technical achievement but the interest of the market in that achievement.

The connection which is being described between the intensity of competition surrounding new inventions in an industry and low probability of claimed inventions being refused for lack of inventive step is perhaps especially noticeable in the pharmaceutical industry. But nearly all enterprises nowadays have to be continuously engaged in product development. New and innovative products have acquired critical importance in all commodity-producing sectors. ${ }^{572}$ This change has occurred within the space of ten years or a bit more. As a consequence, the patent system has more generally assumed the character of protection for economic values; if there is demand for the product, a patent will be available

\footnotetext{
571 T 455/91, OJ EPO 1995, 684: “[T] taking action, would carefully ponder any possible modification, change or adjustment against the background of the existing knowledge. Under these circumstances, in the board's view, the skilled person would adopt a conservative attitude. However, this must not be seen in the sense of being reluctant or opposed to modify or adjust a known product or process, but rather in the sense of being cautious. For example, the skilled person in question would neither go against an established prejudice nor try to enter into 'sacrosanct' or unpredictable areas nor take incalculable risks.”

572 See Edge, G., 25:2 R \& D Management April 1995, 128.
} 
to the creator of the new product. ${ }^{573}$

The aim of this analysis has been to show new and insufficiently observed aspects of the inventive step requirement, and also to some extent to alter the way in which the inventive step requirement is interpreted and discussed. It has often been regarded as a purely technical assessment. But it is suggested here that such a description agrees poorly with present-day reality. The requirement is just as much, if not more so, an appraisal of demand for the invention on the priority date. This is justifiable, because if there was demand for the claimed solution then there was probably competition in the search for the solution and, accordingly, there is a need to move from a competitive situation to a co-ordination of development when further research is not needed, the solution having been found. Coordination of research efforts seems to be the most likely justification for the inventive step requirement, and that purpose is probably more closely connected with demand for the usefulness of the invention than with the technical difficulties in relation to the closest prior art which the applicant has overcome. One can, it is true, study the likelihood of competition solely in terms of the technical difficulties existing on the priority date in relation to the state of the art. If there were no particular technical difficulties, there cannot have been any competition. Beyond this point, however, a purely technical assessment probably bears relatively little relation to the aim of the inventive step requirement, mainly the prevention of unnecessary duplication and effort. Probably, therefore, it is more fruitful to try to establish more directly whether others besides the applicant were actually trying to solve the problem on the application priority date. There are many different ways of investigating this question, but probably the simplest and most effective is by testing to see whether demand existed for the technical effect disclosed in the application.

Finally it should be emphasised that the co-ordination achieved by patents being granted in the great majority of cases at an early stage of pharmaceutical research is very important. Competitors can then access alternative research opportunities outside the patent claims. Inefficient duplicative work is avoided. The appropriate scope of patent claims is of course a further question, but this is dealt with elsewhere; see section 3.3.3.3 and 3.3.4.6.

573 See Levin, M., NIR 1996, 409. 


\section{Supplementary patent protection}

\subsection{Introduction of the Regulation and its main outlines}

There are several sources indicating the average length of time for which a pharmaceutical patent confers protection on the proprietor. A factoring together of various data shows that on average between nine and eleven years elapse between the patent application date and the day when sales can begin. This leaves an effective patent term of between nine and eleven years. ${ }^{574}$

The relatively short effective patent term for pharmaceuticals began to be noticed during the 1980s, i.e. about ten years after the authorities granting permits for the sale of pharmaceuticals began to make more and more exacting demands for clinical trials, which led to a reduction of the effective patent term. In 1984 the USA introduced the possibility of extending the patent term for pharmaceuticals. ${ }^{575}$ Japan introduced a similar possibility in 1987.576 Corresponding legislation was passed in Europe during the 1990s. In 1991 France introduced supplementary protection for pharmaceuticals in the form of a special new intellectual property right giving the proprietor the same rights as a patent but only covering the pharmaceutical substance which the applicant had obtained permission to sell. ${ }^{577}$ Soon afterwards Italy introduced supplementary protection for pharmaceuticals. ${ }^{578}$ By this time it was clear that if the Member States of the EU were allowed to act independently in this matter, the results would vary and one and the same pharmaceutical would come to be protected for different lengths of time in different EU countries. This was a development which the Commission could not accept. It would be contrary to the basic principle of a common market.

The Commission proposed a solution whereby supplementary protection would be obtainable for up to ten years and would confer maximum effective protection for sixteen years. ${ }^{579}$ Following political processing of the proposal, a regulation was adopted which was slightly less favourable to the research-based pharmaceutical companies. The supplementary protection (Supplementary Protection Certificate, SPC) could at most be extended to five years and confer a total effective protection (patent + supplementary protection) of fifteen years. ${ }^{580}$ On this basis of calculation, three different situations can arise:

1. The first authorisation for sale in the EU has been granted within five years of the patent application date. If so, no supplementary protection is obtained.

2. The first sale authorisation has been granted between five and ten years after the patent application date. In a situation of this kind, supplementary protection is obtained for

\footnotetext{
574 See Kolker, P. L., 2 Intellectual Property Quarterly 1997, 249; Grabowski, H., Vernon, J., 10 (Supp. 2) PharmacoEconomics 1996, 110.

575 Drug Price Competition and Patent Term Restoration Act of 1984.

576 The Japanese statute no. 27 from 1987, in force 01011988.

577 The French statute no. 91-1180 of 19111991.

578 The Italian statute no. 349/1991.

579 Proposal for a Council Regulation, EEC, concerning the creation of a supplementary protection certificate for medicinal products, COM (90) 101.

580 EC Regulation 1768/92 art. 13: “1. The certificate shall take effect at the end of the lawful term of the basic patent for a period equal to the period which elapsed between the date on which the application for a basic patent was lodged and the date of the first authorization to place the product on the market in the Community reduced by a period of five years. 2 . Notwithstanding paragraph 1 , the duration of the certificate may not exceed five years from the date on which it takes effect."
} 
between zero and five years.

3. The first sales authorisation was granted between ten and twenty years from the patent application date. In a situation of this kind, supplementary protection is obtained for five years.

Regulation 1768/92 came into force on 2 January 1993.581 A survey of six EU countries has revealed that the average supplementary protection granted under the Regulation has been of just over three years' duration. ${ }^{582}$ More than $95 \%$ of the patents invoked in an application for supplementary protection have been EPO, not national, patents. ${ }^{583}$ Even so, it is the national patent offices, not the EPO, that grant supplementary protection.

The preamble to the Regulation states that its purpose was to encourage research in the pharmaceutical industry, but at the same time attention was drawn to the importance of considering all interests in this unusually complex and socially important industry. The political difficulties involved in reconciling the various interests caused the Regulation to include many points of uncertainty from the beginning. There was, quite simply, no real consensus among the Member States when the Regulation was ready. ${ }^{584}$ As a consequence of these vaguenesses, divergent interpretations have developed in the national patent offices charged with giving effect to the provisions. ${ }^{585}$ The content of the Regulation has been, and will be, successively refined through ECJ case law.

The first question which the ECJ had to consider after the Regulation came into force was whether the EU was competent to issue it. The existence of such competence was affirmed by the ECJ, which at the same time sanctioned further intervention by the EU in European patent law. The ECJ declared:

\footnotetext{
"Neither Article 222 nor Article 36 of the Treaty reserves a power to regulate substantive patent law to the national legislature, to the exclusion of any Community action in the matter. ... It follows that the Community had power to adopt Regulation No 1768/92 concerning the creation of a supplementary protection certificate for medicinal products and extending the duration of the protection conferred by a patent in certain cases. ${ }^{586}$
}

In order for the effects of supplementary protection not to be delayed by many years, it was judged necessary, at the time of drafting the Regulation, for supplementary protection to be obtainable for pharmaceuticals which were already on the market when the Regulation entered into force. Art. 19 therefore provided that supplementary protection would be obtainable for pharmaceuticals, which on the day of the Regulation entering into force (2 January 1993), were protected by a valid patent and were the subject of a marketing authorisation issued within the EU after 1 January 1985. Medicinal products for which marketing authorisation had been granted before that date, on the other hand, were not eligible for supplementary protection. National departures from this rule were possible, however. Germany, Denmark and Finland chose to make the applicable time period run from 1 January 1988, which meant more limited scope for supplementary protection. Belgium,

\footnotetext{
581 EC Regulation 1768/92 of 18061992 published in the Official Journal of the European Communities of $2^{\text {nd }}$ July 1992, No. L 182/1-5.

582 The Netherlands 3.13 years; Belgium 3.11 years; Great Britain, 3.23 years; Luxembourg 2.86 years; France 2.89 years and Germany 3.98 years. See de Pastors, A., 17:3 World Patent Information 1995, 191.

583 Ibidem.

584 See Kolker, P. L., Intellectual Property Quarterly 1997, 250.

585 "[A] piece of legislation which has become notorious for its lack of clarity". See Faircliffe, S., 11 World Intellectual Property Report 1997, 84.

586 Case C-350/92, ECR 1995, I-1985.
} 
Italy and Austria on the other hand applied a more generous policy, granting supplementary protection for medicinal products for which marketing authorisation had been granted after 1 January 1982.587

In Greece, Portugal and Spain, medicinal products were not patentable at all when the EU Regulation was drafted, and a possibility of this kind was only introduced in 1992. These three countries also worked out special rules for the entry into force of the Regulation. Art. 21 of the Regulation provides:

\footnotetext{
"In those Member States whose national law did not on 1 January 1990 provide for the patentability of pharmaceutical products, this Regulation shall apply five years after the entry into force of this Regulation.

Article 19 shall not apply in those Member States.”
}

Since the Regulation entered into force on 2 January 1993, in Greece, Spain and Portugal it began to apply on 2nd January 1998. The interim provisions referred to above, in Art. 19 concerning pharmaceuticals already on the market, were not, however, to apply. Only the main rule of Art. 7 became applicable, and under this an application for supplementary protection must have been filed within six months of authorisation to place the product on the market. As a result of the three countries not applying the interim provisions, it will still be some years - until about 2010 - before all pharmaceuticals in the EU countries are protected for the same length of time.

The deferment by Greece, Spain and Portugal of implementation of the Regulation proved especially important - in a negative sense from the viewpoint of researchbased pharmaceutical companies - when the ECJ decided the united cases C-267/95 and C268/95.588 The ECJ found that weaknesses in an EU country's patent protection or a party feeling himself to have an ethical duty of selling medicinal products in an EU country, are not acceptable grounds for stopping parallel imports to other EU countries. Only if the patentee has a concrete and specific legal duty to sell the product in the country from which further exports then take place, can parallel imports be prevented by the patentee.

The unwillingness of the ECJ to depart from the rule of free movements of goods, except in connection with an express legal duty of sale, e.g. a compulsory licence, can probably be attributed to the difficult problems of demarcation which would arise if any different basis were chosen. ${ }^{589}$ If the court had accepted, as grounds for the prevention of parallel imports, that the research-based pharmaceutical company was under a moral obligation to sell its medicinal products in countries where it could not obtain as a high price as it would have wanted to, a considerable part of ECJ case law on the free movement of goods might be called into question. As a result of the judgement, the absence of supplementary protection in Greece, Spain and Portugal for a number of years to come has implications not only in those countries but everywhere in the EU. If the proprietors of supplementary protection sell the protected medicinal product in the three above mentioned countries, they cannot then prevent parallel imports to countries with supplementary protection.

\subsection{Products qualifying for supplementary protection}

\footnotetext{
587 See Art. 19 in Regulation 1768/92 and Kolker, P. L., Intellectual Property Quarterly 1997, 250; de Pastors, A., 17:3 World Patent Information 1995, 189.

588 The united cases C-267/95 and C-268/95, ECR 1996, I-6285.

589 See Multimear, J., [1996] EIPR 103.
} 
Art. 2(a) of the EC Regulation gives the following definition of the "medicinal products" for which supplementary protection is obtainable:

“(a) 'medicinal product' means any substance or combination of substances presented for treating or preventing disease in human beings or animals and any substance or combination of substances which may be administered to human beings or animals with a view to making a medical diagnosis or to restoring, correcting or modifying physiological functions in humans or in animals;

(b) 'product' means the active ingredient or combination of active ingredients of a medicinal product.”

In this provision a distinction is made between "medicinal product" and "product". The latter term refers to the active substance of the pharmaceutical to which the first term refers. Medicinal products are the category of products eligible for supplementary protection. But the protection as such - the exclusive right - applies only to the product, i.e. to the active substance.

The category of medicinal products and those which can be considered for supplementary protection includes those which are used therapeutically or prophylactically in animals or human beings. In the case of diagnostic products, however, the article requires that they be "administered" to animals or human beings. This indicates that diagnostic equipment used in vitro, e.g. tests used for blood sampling, are not eligible for supplementary protection, while on the other hand, for example, contrasting agents injected and used in X-ray examination are eligible. ${ }^{590}$

Medicinal and veterinary products are equated in the Regulation. As a result, if a substance has first been authorised for sale for the treatment of animals and its sale is later authorised for the treatment of humans, the veterinary application shall count as the first marketing authorisation and shall provide the starting point for computing the duration of the supplementary protection. ${ }^{591}$

Art. 2 of the Regulation provides that every patented product - i.e. active substance - which has to undergo a special authorisation procedure as provided in Directive 65/65 (proprietary medicinal products intended for human use) or 81/852 (proprietary medicinal products intended for animal use) before they can be sold as part of a medicinal product can obtain supplementary protection. ${ }^{592}$ An additive to livestock feed is one example of a substance which does not have to undergo an authorisation procedure as laid down in the Directives. Marketing authorisation for livestock feed additives forms the subject of another Directive. ${ }^{593}$ Accordingly, livestock feed additives are not eligible for supplementary protection. Should it happen, however, that a therapeutic application of a livestock feed additive is approved during the patent term as provided in Regulation 65/65 or 81/851, there are possibilities of supplementary protection being obtained for that use. ${ }^{594}$ Plus there is nothing to prevent supplementary protection being granted for a substance which has been available on the market as a non-medicinal product before being authorised for sale as a

\footnotetext{
590 See von Morzé, H., Hanna, P., JPTOS June 1995, 481.

591 Decision of the General Comptroller of Patents, Designs and Trade Marks in Farmitalia Carlo Erba SRL's SPC Application of 0703 1996, [1996] R.P.C. 111.

592 Art. 2: “Any product protected by a patent in the territory of a Member State and subject, prior to being placed on the market as a medicinal product, to an administrative authorization procedure as laid down in Council Directive 65/65/EEC or Directive 81/851/EEC may, under the terms and conditions provided for in this Regulation, be the subject of a certificate.”

593 Directive 70/524.

594 See von Morzé, H., Hanna, P., JPTOS June 1995, 487.
} 
medicinal product. EPO patent 39981 pertained to a substance which it had been found could be used as a sweetener. Later the substance was also found to have a laxative effect, and authorisation was obtained for the sale of the substance as a medicinal product. Supplementary protection could have been obtained for this latter indication, because the Regulation does not stipulate that the therapeutic indication must have been disclosed in the patent. 595

The marketing authorisation which makes the product eligible for supplementary protection and is based on one of the two Regulations must have been granted at the time of supplementary protection being applied for. This is clear from Art. 3(b) of the Regulation. In English law it has been established that permission of the kind granted by pharmaceutical boards for the conduct of clinical experiments (clinical trials) cannot be equated with the marketing authorisation required by the Regulation. ${ }^{596}$ Supplementary protection is only intended for products actually on the market when the patent expires, not for products which will perhaps be introduced during the term for which a possible supplementary protection could apply. ${ }^{597}$ If, however, an application for supplementary protection is refused, the same applicant could subsequently file a new application for the same medicinal product after obtaining marketing authorisation. A refusal, in other words, has no res judicata effect. 598

A related situation arose in a case tried by the ECJ, C-110/95. When the application for supplementary protection was filed in the UK, marketing authorisation had been granted in France, but not in the UK. The UK Patent Office refused the application with reference to Art. 3 of the Regulation, which reads:

"A certificate shall be granted if, in the Member State in which the application referred to in
Article 7 is submitted and at the date of that application ... a valid authorization to place the
product on the market as a medicinal product has been granted in accordance with Directive
65/65/EEC or Directive 81/851/EEC."

The applicant maintained that marketing authorisation in the country where the application was filed was not necessary. All that was required, according to the applicant, was for marketing of the product to be authorised somewhere, understood within the EU. An interpretation of this kind would create uniformity with regard to the granting of supplementary protection within the EU. The ECJ, however, found that marketing authorisation must have been granted in the country where supplementary protection had been applied for and must exist not later than the supplementary protection application date. ${ }^{599}$ This requirement (Art. 3) has had the consequence that the German Bundespatentgericht refused an application where the marketing authorisation was only granted after the supplementary certificate had been applied for. ${ }^{600}$

The conclusion drawn by the ECJ and the Bundespatentgericht follows from the wording of the Regulation. All the same, the legal position can be questioned with reference to the underlying purposes of supplementary protection. That protection is justified for the very products for which the authorisation procedure, perhaps in a particular country, has been especially protracted. Supplementary protection is intended as compensation for such delays.

\footnotetext{
595 See Hirsche, F., Hansen, B., Protecting Inventions in Chemistry, 301.

596 Decision of the General Comptroller of Patents, Designs and Trade Marks, British Technology Group Ltd. 's SPC Application, 1997 R.P.C. 50.

597 See Kolker, P. L., 2 Intellectual Property Quarterly 1997, 252.

598 British Technology Group Ltd.'s SPC Application, [1997] R.P.C. 50.

599 C-110/95, ECR 1997, I-3251.

600 Bundespatentgericht 15 W (pat) 71/97 of 2106 1999, quoted in Kellner, H., GRUR 1999, 808.
} 
There is no apparent reason why medicinal products affected by very great delays in one or more countries should not be compensated at all. At all events, proprietors of supplementary protection will not be able to benefit from supplementary protection until marketing authorisation has actually been obtained for the product in the country where the supplementary protection has been applied for. But once this has happened, there seems to be special cause for supplementary protection to be granted in these cases. An amendment of the Regulation on this point would be warranted. ${ }^{601}$

\subsection{The extent of protection granted}

As has already been pointed out, supplementary protection is intended for the active substance of a medicinal product. The question, however, is how extensive that protection will be. When the Regulation was being drafted, it was at first proposed that the supplementary protection should have the same extent as a patent for a first medical indication, i.e. include all medicinal use of the active substance. The Commission, however, opposed this, and the result was the narrower protection restricted to the uses for which marketing authorisation has been granted. Art. 4 of the Regulation reads:

\footnotetext{
"Within the limits of the protection conferred by the basic patent, the protection conferred by a certificate shall extend only to the product covered by the authorisation to place the corresponding medicinal product on the market and for any use of the product as a medicinal product that has been authorised before the expiry of the certificate.”
}

A marketing authorisation contains a disclosure of the active substance which may be sold, for which indications it may be sold and, in addition, disclosures of the formulation, i.e. the other substances included in the medicinal product and the proportions of the different substances. It is also indicated how the medicinal product may be presented in different respects, e.g. which recommended doses may be stated on the packaging. Although it does not say so in as many words, the provision should presumably be construed in such a way that protection only includes the medical uses for which the proprietor of the supplementary protection has obtained marketing authorisation.

A different question - the so called "salt-problem"602 - is if the certificate can only be granted for the specific chemical form mentioned in the marketing authorisation for the product or if the protection may be broader. Medicinal products are often administered in combination with different salts, but the choice of salts seldom affects their therapeutic properties. If the certificate could protect only e.g. the particular salt form of the active ingredient mentioned as the active constituent in the marketing authorisation, whereas the basic patent protects the active ingredient as such as well as salts thereof, including the one which is the subject-matter of the marketing authorisation, any competitor would be able, after the basic patent had expired, to apply for and, in some circumstances, obtain marketing authorisation for a different salt of the same active ingredient, formerly protected by that patent. Once the basic patent expired, generic manufacturers would then be free to obtain marketing authorisations for medicinal products using other salts of the same free base, with equivalent therapeutic or diagnostic effects, simply by conducting a small number of bioequivalence tests, which could be carried out in advance outside the Community, in the light of the known literature. It would be possible for medicinal products which were, in principle,

601 Cf. Kellner, H., GRUR 1999, 808.

602 See Adams, J. N., [1995] EIPR 277. 
therapeutically equivalent to that protected by the certificate to compete with the latter. Such an interpretation would greatly belittle the value of supplementary protection.

As regards the breadth of protection in this particular respect, the European national patent offices began to evolve divergent precedent. Precedent in Britain and Italy was generous: supplementary protection was formulated in such a way as to include every therapeutically acceptable salt of the active substance. In Germany, on the other hand, precedent was restrictive, in that only the substance for which the patentee had marketing authorisation was included in his supplementary protection, e.g. the active substance in combination with a certain salt or a certain ester. ${ }^{603}$ This line was, however, challenged before the ECJ. ${ }^{604}$ At issue was not whether a certificate should be granted or not, but its terms.

Farmitalia held a German patent notified on 9 June 1975 for alpha-anomer of 4Demethoxydaunomycin, its manufacturing process and the medicament containing that substance. The short designation for chemical compositions so structured was idarubicin. The patent claims mentioned the salt idarubicin hydrochloride as an embodiment of the invention. Farmitalia subsequently obtained an authorisation to market the products 'Zavedos 5 mg' and 'Zavedos $10 \mathrm{mg}$ ' in Germany as medicinal products for treatment of acute myelitic leukaemias. These products contain the salt idarubicin hydrochloride and, as an ancillary ingredient, dehydrated lactose.

The original patent expired and Farmitalia applied for a SPC for the free base 'idarubicin and salts thereof including idarubicin hydrochloride'. However, the German Patent Office granted a German certificate only in respect of 'the medicament Zavedos containing as its active ingredient idarubicin hydrochloride‘. Farmitalia commenced complaint proceedings before the Federal Patents Court seeking a certificate in the terms initially requested or, in the alternative, for 'idarubicin and idarubicin hydrochloride'. That court rejected both the main and the subsidiary applications. The Bundespatentgericht took the view that neither the main nor the subsidiary application satisfied Article 3(b) of the SPC Regulation, as an SPC could only be granted to a product stated to be an active ingredient of a medicinal product in the relevant marketing authorisation. In the present case, idarubicin hydrochloride was the named active ingredient of the two authorised Zavedos products, so that an SPC could not be granted in wider terms. Furthermore, in the view of the Bundespatentgericht, the main application did not satisfy Article 3(a) of the SPC Regulation, because not all the salts of idarubicin were protected by the basic patent. In addition to the free base idarubicin itself, only one salt, idarubicin hydrochloride, was mentioned in the patent. The Bundespatentgericht considered that the protection by a basic patent required by Article 3(a) did not refer to the effective scope of patent protection in any notional infringement proceedings, but, rather, to the technical doctrine protected by the basic patent, that is, in addition to the matters mentioned expressly in the patent, such other matters which, in the view of a person skilled in the art, are self-explanatory or all but indispensable in regard to the patented discovery without the need for special mention to be made of them, or which the person skilled in the art on an attentive reading of the patent papers can recognise and follow in his own thought processes. This was not the case regarding idarubicin salts as, owing to their different chemical composition in comparison with idarubicin and idarubicin hydrochloride, the expert could at least consider it possible that there might be differences in their therapeutic effectiveness.

On appeal to the Bundesgerichtshof (Federal Court of Justice, BGH), Farmitalia argued, in respect of Article 3(b) of the SPC Regulation, that the term 'active ingredient" should be understood as designating the pharmacologically active base including its

603 See Kolker, P. L., 2 Intellectual Property Quarterly 1997, 251; Hirsche, F., Hansen, B., Protecting Inventions in Chemistry, 300, Kellner, H., GRUR 1999, 807 and Adams, J. N., [1995] EIPR 277.

604 C-392/97 of 16 September 1999. 
derivatives (salts and esters). Article 3(b) did not, therefore, require a marketing authorisation in respect of every possible variant of the active ingredient, provided it had been authorised in one of its possible forms. Regarding Article 3(a) of the Regulation, Farmitalia submitted that the Bundespatentgericht had erred, as a matter of German law, concerning the scope of the protection conferred by the basic German patent, as a person skilled in the art would have known that other pharmaceutically consistent salts of idarubicin would have been equally as suitable as idarubicin hydrochloride as a means of dispensing the active ingredient idarubicin. In the light of the dispute regarding the proper interpretation of the SPC Regulation, the court referred the following questions to the ECJ:

"1. Does Article 3(b) presuppose that the product in respect of which the grant of a protection certificate is sought is described as an "active constituent" in the authorisation for marketing as a medicinal product?

Is, then, Article 3(b) not complied with where a single individual salt of an active ingredient is stated in the notice of authorisation to be an "active constituent", but the issue of a protection certificate is sought for the free base and/or for other salts of the active ingredient?

\section{If the questions at 1 . are answered in the negative:}

According to which criteria is it to be determined whether the product, as referred to in Article 3(a), is protected by a basic patent where the issue of a protection certificate is sought for the free base of an active ingredient including any of its salts, but the basic patent in its patent claims mentions only the free base of that ingredient and, moreover, mentions in an embodiment a single salt of this free base? Is the wording of the claim for the basic patent or the latter's scope of protection the determining criterion?”

Thus, in the first question, the BGH essentially asked whether the product could be understood in wider terms than those used to describe the medicinal product in the relevant marketing authorisation. The issue, in the second question, was whether the protection of a product by a basic patent is determined in accordance with the patent claims or on the basis of the effective scope of protection of the patent. It only arises if it is at least possible to conceive of the product in terms wider than those used in the claims.

The ECJ made a purposive construction. It would not accept an interpretation that would frustrate the purpose of Regulation No. 1768/92: to remedy the insufficient incentives for investments into pharmaceutical research. If the certificate did not cover the actual medicinal product, as protected by the basic patent and one of the possible forms of which is the subject-matter of a marketing authorisation, the objective would not be attained. Moreover, the court added that four years after the introduction of supplementary protection for medicinal products, a similar supplementary protection was being worked out within the EU for plant protection products and the preamble of this latter Regulation was made to include rules on the interpretation of supplementary protection for medicinal products. The Regulation on supplementary protection for plant protection products provided:

\footnotetext{
"13. Whereas the certificate confers the same rights as those conferred by the basic patent; whereas, consequently, where the basic patent covers an active substance and its various derivatives (salts and esters), the certificate confers the same protection;

$\cdots$

17. Whereas the detailed rules in recitals 12, 13 and 14 and in Articles 3 (2), 4, 8 (1) (c) and 17

(2) of this Regulation are also valid, mutatis mutandis, for the interpretation in particular of recital 9 and Articles 3, 4, 8 (1) (c) and 17 of Council Regulation (EEC) No 1768/92.”605
}

${ }^{605}$ Regulation 1610/96, points 13, 14 and 17 in the preamble. 
The clarification in recital 13 and 17 means that supplementary protection for medicinal products can include the active substance and its derivates (salts and esters). The ECJ did not discuss - as Advocat General Fenelly did in this case - the more general question whether the Community legislature was entitled to seek to influence the judicial interpretation of a legislative measure through the inclusion of interpretative "rules" in later legislation which does not purport to amend the earlier measure. The ECJ seems to have accepted this ex post facto measure. Consequently, the ruling on the first question was that where a product in the form referred to in the marketing authorisation is protected by a basic patent in force, the certificate is capable of covering that product, as a medicinal product, in any of the forms enjoying the protection of the basic patent.

The second question was in regard to the criteria for establishing whether or not a product is protected by a basic patent. This is one of the conditions for obtaining a certificate. The ECJ noted that as Community law now stands, the provisions concerning patents have not yet been made the subject of harmonisation at Community level or of an approximation of laws. Therefore, in order to determine, in connection with the application of Regulation No 1768/92 and, in particular, Article 3(a) thereof, whether a product is protected by a basic patent, reference must be made to the national rules which govern that patent.

In addition to certificates that cover all compounds protected by a basic patent, new supplementary protection can be granted if special patents have been granted for new formulations. Recital 14 in the Regulation on supplementary protection for plant protection products provides:

“14. Whereas the issue of a certificate for a product consisting of an active substance does not prejudice the issue of other certificates for derivatives (salts and esters) of the substance, provided that the derivatives are the subject of patents specifically covering them;"606

However, the protection is still limited to the use for which marketing authorisation has been obtained. Given this linkage to use, there should probably not be anything to prevent competitors to the proprietor of the supplementary protection from manufacturing the active substance, e.g. in bulk form, i.e. without it being packaged or otherwise designed for use for the medical indication to which the supplementary protection refers.

Further problems concerning the agreement between patent and marketing authorisation occur when new formulations are developed and given marketing authorisation, but no new patent is obtained. In an English case the substance budesonide had been claimed in 1973. In 1981 the patentee obtained permission to sell budesonide as an anti-allergic agent in the form of an "aerosol, solid suspended in propellants", "with or without tube extension". The patentee developed and later patented a new way of administering the substance, called a turbohaler. In order for the more efficient turbohaler to work, however, budesonide had to be used in a new formulation: "additive-free budesonide in the form of agglomerated micronised particles". In 1990 the patentee obtained permission to sell the new formulation of budesonide. Later supplementary protection was applied for with reference to the 1973 patent and to marketing authorisation having been obtained in 1990, but the application was refused. The Patents Court ruled that the first marketing authorisation had been obtained in 1981, i.e. before the date (1985) determining the applicability of the EC Regulation. A new approved formulation developed later for the patented substance would, the Court found, not obtain supplementary protection unless the new form of the active substance had in itself been patented. Only one supplementary protection could be granted per patent, for one and the same legal subject. The court found the legal position so clear that the question was not to be

606 Regulation 1610/96, points 13, 14 and 17 in the preamble. 
referred to the ECJ. ${ }^{607}$

The decision from the Patents Court in England indicates that the development by a patentee of new formulations within the scope of patent protection cannot form the basis of new supplementary protection unless the new formulation has been patented in itself. It is thus not possible to obtain more and longer supplementary protection as the medicinal product begins to be sold in different formulations. The new forms will admittedly fall within the protection which follows from the supplementary protection based possibly on a first marketing authorisation - this is made possible by the above ECJ decision C-392/97 - but new supplementary protection with longer terms cannot, it seems, be obtained with reference to later, new marketing authorisations. As has already been pointed out, a different situation arises if the new formulation or the new indication is patentable in itself. When a new patent has been granted, new supplementary protection will also be obtainable.

A completely different aspect of the scope of protection concerns which actions are to constitute infringement and what restrictions are made concerning acts of infringement. Art. 5 of the Regulation provides:

"[T]he certificate shall confer the same rights as conferred by the basic patent and shall be subject to the same limitations and the same obligations."

National rules of patent law decide which actions constitute infringements, the possibilities of granting interlocutory prohibitions and the sanctions available to the patentee. One English decision has established that national authorities are able to issue compulsory licences for the supplementary protection applying within the country concerned. ${ }^{608}$

\subsection{Several patents referring to the same product}

We have already seen that under Art. 3(a) of the Regulation there has to be a valid patent when supplementary protection is applied for. But it may also be that several valid patents protect a product. A number of different situations are conceivable where this is the case, e.g. a product patent and a patent for a new indication or a wider patent and a narrower selection patent or a product patent and a patent for a certain formulation containing the substance protected by the product patent. If the same legal subject holds several patents for the product for which there is marketing authorisation, the patentee can choose between his patents. This is not self-evident, but it seems fair to assume that the patentee can choose the patent he applied for first. This is an advantage to him, because the earlier the patent has been applied for, the longer will be the time elapsing until marketing authorisation was obtained and the longer will be the duration of the supplementary protection. According to Art. 3 paragraph 2 of Regulation No. 1610/96 no more than one certificate may be granted for the same plant protection product. Recital 17 of the Regulation makes this rule applicable also to medicinal products. However, this rule is perhaps still disputable. On some occasions, before Regulation 1610/96 was issued, the German Patent Office granted more than one certificate when the medicinal product was protected by more than one patent belonging to the same proprietor. ${ }^{609}$

\footnotetext{
607 Patents Court DRACO A.B.'s SPC Application, [1996] R.P.C. 417. The cases is commented on in Raff, K., [1996] EIPR 508 ff and in Jacob, R., IIC 1997, 883.

608 Patents Court, Research Corporations Supplementary Protection Certificate, of 0610 1994, [1994] R.PC. 667.

${ }^{609}$ See Kellner, H., GRUR 1999, 806 f.
} 
If on the other hand a product is protected by several patents held by different legal subjects, the ECJ has established, in case C-181/95, that each of the patentees shall be able to obtain supplementary protection of his own for the product which may be sold. Several supplementary protections may be granted for one and the same product if the supplementary protections will belong to different legal subjects. 610

It is not uncommon in the pharmaceutical industry for the patentee to be a different party from the holder of marketing authorisation for products coming within the scope of the patent. Many companies, biotechnology companies especially, research and patent substances with therapeutic effects but subsequently license the patents exclusively to large pharmaceutical companies which develop the invention into a finished medicinal product and in this way become the party obtaining marketing authorisation. The patents and marketing authorisation are then in different hands. In such a situation it can be difficult for the patentee to gain access to the marketing authorisation which has to be appended to an application for supplementary protection. In the above mentioned ECJ decision, it was laid down that a patentee shall not be impeded from obtaining supplementary protection merely because he does not have access to a copy of the marketing authorisation issued for his patented product. In purely practical terms, the ECJ found that, in a situation of confidential marketing authorisations for medicinal products to which a patentee cannot gain access, the sanctioning authority shall confidentially transmit a copy of the authorisation to the patent authority, or else a copy of the marketing authorisation, with all quantitative information concealed, shall be surrendered to the patentee. In this way the results of the clinical tests can remain confidential, at the same time as supplementary protection can be granted to the patentee. ${ }^{611}$

If the holder of a marketing authorisation tries to impede a patentee from obtaining supplementary protection, his intention is probably to try to avoid the payment of licensing fees for the length of time during which a supplementary protection would be valid. If the medicinal product is protected by several patents, perhaps the holder of the marketing authorisation tries to arrange things in such a way that supplementary protection is granted solely for the patent for which he pays the lowest licensing charge. Methods of this kind for excluding patentees from further licensing charges have, however, as has already been shown, been thwarted by the ECJ decision.

In earlier licensing agreements it was not possible to foresee the advent of the rules on supplementary protection for medicinal products. Unless otherwise indicated by the licensing agreement, however, it should probably be assumed that a licensing charge is also to be paid during the term of the supplementary protection and at the same rate as during the term of the patent. ${ }^{612}$ One argument in favour of this is that the effects of the two protections are essentially the same. One may therefore assume that if, at the time of their agreement, the parties had been able to foresee the introduction of the rules on supplementary protection, they would probably have agreed on a licensing fee for the term of the supplementary protection. A solution of this kind has, moreover, been codified in German law. ${ }^{613}$

\subsection{Remarks}

\footnotetext{
610 C-181/95, ECR 1997, I-0357.

611 C-181/95, ECR 1997, I-0357.

612 See Kolker, P. L., 2 Intellectual Property Quarterly 1997, 252.

613 The German Patent Act 16a § 3 paragraph: "Lizenzen ..., die für ein Patent wirksam sind, gelten auch für den ergänzenden Schutz.”
} 
The present-day legal position means that all medicinal products which have to undergo a marketing authorisation procedure before they can be sold, i.e. which are sold in finished packagings to consumers, are eligible for supplementary protection. Neutrality prevails here between different therapeutic fields. Indirectly, though, through the way in which the duration of the supplementary protection is computed, certain therapeutic fields may be disfavoured by the rules. In the present section we shall consider how the manner of computing the duration of supplementary protection can influence the prioritisation of different research tasks.

If no sale can take place during only the five first years from the date of a patent application being filed, the patentee is not entitled to supplementary protection. This is because in many technical fields it takes five years from the patent application date for the invention to go on sale. It is only the particularly long development periods in the pharmaceutical industry - those exceeding five years - which are to be compensated for by means of supplementary protection.

Since the first five years do not qualify for compensation through supplementary protection where medicinal products are concerned, there are strong incentives for patentees to try to get the product on to the market within those five years. If successful in this, the patentee obtains a protection of more than fifteen years' duration. It is unusual, however, for the market launch of a medicinal product to come so soon after the patent application. The average time is something like ten years. Probably there is no type of medical indication for which, under normal conditions, a new medicinal product can be launched during the first five years. For practical reasons, therefore, the upper limit for the duration of effective protection (patent + supplementary protection) is neutral as between research in different therapeutic fields.

If marketing authorisation is obtained sometime between five and ten years after the patent application date, supplementary protection is obtainable. The supplementary protection will have a duration equalling the period between five years after the patent application and the day on which marketing authorisation was obtained. Because the period between five and ten years from a patent application is compensated on a one-to-one basis by supplementary protection, the total duration of protection is not affected by the point during the period at which the inventor launches the medicinal product. A regulation of this kind entails a risk, because it may be that the patentee will devote fewer resources to a rapid market launch than he would have done if he had not obtained compensation in the form of supplementary protection. But this risk is presumably limited, the reason being that a new patented medicinal product seldom confers anything resembling a true monopoly, i.e. a situation in which patients do not have acceptable alternative treatment possibilities to choose from. The products may perhaps have somewhat different properties, but they are interchangeable in many treatment situations. In a market of this kind, it is crucially important to be first or at least among the first in the market, because one can then establish a trademark and build up a market position which latecomers may have difficulty in breaking. Another important reason which research-based pharmaceutical companies probably do not reduce their efforts to launch new medicinal products quickly is that future earnings have to be discounted; that is, the further ahead in time they come, the less they will be worth. From this we may infer that the extension is unlikely to result, to any considerable extent in new medicinal products being launched later than they would have been otherwise.

The maximum effective protection (fifteen years) is constant from five years until ten years have passed from the patent application to the commencement of sales. The time after ten years from the patent application date and until the patentee obtains marketing authorisation is not compensated for by the rules of supplementary protection. If it takes twelve years from the patent application for the marketing authorisation to be obtained, the effective patent terms falls from fifteen to thirteen years (eight years' patent + five years' 
supplementary protection). Thus development time over and above ten years from the patent application is not compensated for by supplementary protection. This is liable to entail a negative incentive for research in therapeutic fields requiring particularly long development times. The average time lag for a research project in the pharmaceutical field from patent application to sale is often ten years. The patent term will be less than ten years and the effect of protection less than fifteen years in those therapeutic fields where the development time above all the time taken by the clinical trials - is longer than average. Long development times are especially common in research projects relating to chronic diseases. In such cases, long-term toxicity tests are needed, as well as tests of the carcinogenic properties of the substances and sometimes also long-term efficacy tests, i.e. comparisons between the therapeutic effects of the new medicinal products and those of existing products against the same illness. Where certain diseases are concerned, it takes a very long time before any treatment result can be demonstrated at all, as for example with the treatment of osteoporosis. 614

The choice of research field in the pharmaceutical industry is strongly influenced by the return which can be expected in different research fields. The researchbased pharmaceutical company inaugurating projects in therapeutic fields where there are very long development times involved, can perhaps only count on an effective patent term of between five and ten years. In other therapeutic fields the researching enterprise can count on between ten and fifteen years' protection. In consequence, pharmaceutical companies will devote fewer resources to research in the above mentioned fields and more to research relating to diseases where development times do not normally exceed ten years.

From the consumer's points of view, demand does not automatically decline when 25 years $(20+5)$ have passed from the day of the patent application, i.e. when the development time up to the market launch exceeds ten years. But from the viewpoint of the research-based pharmaceutical company, demand is reduced in this way, the reason being that generic competition then takes market shares. In this way there comes to be a lack of agreement between actual demand and the demand which research-based pharmaceutical companies calculate with. Therefore, it might be negative to limit the duration of maximum supplementary protection to five years, because this is discriminatory against research projects in therapeutic fields where development periods commonly exceed ten years.

How, then, should the rules on the duration of supplementary protection be framed? Neutrality between medicinal products in different therapeutic fields would require effective protection to be of the same duration regardless of the length of the development period, so long as development has been expeditiously prosecuted by the patentee. Under the existing rules, effective protection is obtained for fifteen years, but only if no more than ten years have passed between the patent application date and the market launch. In the interests of neutrality it is proposed that new medicinal products, once they have been patented, should qualify for fifteen years' protection with effect from the day when marketing authorisation was obtained for them. In such cases the supplementary protection could last for anything up to fifteen years. The maximum of fifteen years, however, would only be obtained in the unusual situation where the patent had already expired when marketing authorisation was granted. In other cases the patent-protected term would be subtracted from the maximum fifteen-year supplementary protection.

With an arrangement of this kind, a new medicinal product which was once patentable and which gets onto the market will be guaranteed fifteen years' protection from the day of its first marketing authorisation. As an adjunct, however, it should be possible for patent offices and competitors to demand an account of the way in which development work

614 See von Morzé, H., Hanna, P., JPTOS July 1995, 518. 
under the project has been prosecuted. If the patentee has not prosecuted development with sufficient dispatch, the term of supplementary protection should be reducible, following an assessment of the conditions in the individual case. Development periods resulting from necessary safety and efficacy controls should be accepted, but not delays due, for example, to the patentee not having had the economic resources to conduct the project at a normal speed. This control is intended to counteract a situation where companies acquire more exclusive rights than they can effectively exploit the therapeutic potential of.

The proposal roughly outlined here is aimed at achieving better agreement between the commercial and social valuations of research in different therapeutic fields. A situation of efficiency exists when market demands for medicinal products is allowed to govern the research strategy of pharmaceutical companies, rather than that strategy being governed by the length of time which clinical trials can be expected to occupy in different therapeutic fields. The proposal, which is intended to establish greater neutrality, derives a measure of support from other studies. ${ }^{615}$

A protection whose duration is computed from the day when sales may begin and whose extent is confined to the therapeutic indications for which marketing authorisation has been obtained resembles, in its practical consequences, the right to "regulatory data protection" obtainable under EC Directive 87/21. ${ }^{616}$ Under that Directive, an application for marketing authorisation for a generic medicinal product may not contain a reference to the pre-clinical and clinical trials conducted by an innovative enterprise less than six or - if the Member State so chooses - ten years from the date on which marketing authorisation was granted for an innovative medicinal product. Most of the EU countries have opted for ten years. In principle these rules do not confer protection, because generics manufacturers are always in a position to carry out the necessary experiments for themselves. In practice, however, cost is an effective barrier to such experiments, except in special cases. ${ }^{617}$ Thus the introduction of a supplementary protection which can be extended for up to fifteen years from the marketing authorisation date is not quite as radical as it might appear at first sight. Tenyear protection is already obtainable in most cases, regardless of the effective patent term.

One possible objection to the permission of more than five years' supplementary protection for particularly long development periods has already been dealt with and for the most part found to be unjustified: there is hardly any reason to believe that the possibility of longer supplementary protection would mean pharmaceutical enterprises not launching their new medicinal products as quickly as possible. Competition and the necessity of discounting future earnings are probably sufficient motive forces for projects in the great majority of cases to get onto the market quickly. Another conceivable objection to more than five years' supplementary protection if the development time has been very long might be that the situation prior to the introduction of supplementary protection entailed still greater discrimination against research into diseases requiring especially protracted clinical trials. The negative selection which now takes place as regards projects requiring more than ten years' development used to occur immediately, i.e. on a sliding scale (the longer the

\footnotetext{
615 The following has been said in regard to the American situation: "If policymakers are to consider changes in the 1984 Act, we think the first priority should be for products that have little or no effective patent life. In particular, attention should be focused on new drug candidates that must rely on the 5-year data exclusivity for their basic protection. We don't know how many potentially useful compounds have not reached the market because of too little patent protection. But we do know that five years is a very short term to recover the hundreds of millions of dollars of R\&D investment, required by the typical new drug introduction”. See Grabowski, H., Vernon, J., 10:2 PharmacoEconomics 1996, 110.

616 Directive 87/21/EEC.

617 See Grabowski, H., Vernon, J., 10 (Supp. 2) PharmacoEconomics 1996, 110 ff and Cook, T. M., Patent World, March 1998, 32.
} 
development time, the shorter the effective patent term). But this is not really a sustainable objection. One cannot assume that the historical design of patent law has in every respect agreed with its purpose. The character of patent right as an exclusive right of limited duration has probably, historically speaking, had a distorting and therefore negative effect on research in certain therapeutic fields. Research has come to be concentrated excessively on fields where new medicinal products could be introduced very quickly onto the market. The character of patent right as a relatively short exclusive right has been detrimental to patients suffering from chronic, unusual diseases. A situation of inefficiency has probably existed, because the priorities of research-based pharmaceutical companies have not agreed with the aggregate value to consumers of various new medicinal products. 


\section{Patent infringement}

\subsection{Acts of infringement}

\subsubsection{Direct products of a patented process}

One of the first steps to be taken when assessing whether a patent has been infringed is to decide whether the actions taken by a competitor are of a kind reserved for patentees in general. Concerning products, patentees have an exclusive right to making, offering for sale, putting on the market or using the subject-matter of the patent, or importing or stocking the product for these purposes. In the case of process patents, there is an exclusive right to use the process and to offer it for use. There is also an exclusive right to products made by the patented process. This is especially important where pharmaceutical patents are concerned.

Art. 64(2) of EPC, the only EPC provision, apart from Art. 69, grappling with matters of infringement, was introduced at the 1973 Munich conference following a proposal by the Swiss delegation, which had made itself the spokesman for the chemical and pharmaceutical industry. ${ }^{618}$ Art. 64(2) reads:

"If the subject-matter of a European patent is a process, the protection conferred by the patent shall extend to products directly obtained by such process."

Pharmaceutical companies have an extensive knowledge of ways in which new and more efficient synthesisation methods can be developed. Often new processes are claimed and come to be of great value in the production of a pharmaceutical. It is not practically possible, however, to patent the new manufacturing process in every country of the world. If the product is not protected, the process patent would be of little practical value, because the end product could be imported by competitors from countries where use of the process was unrestricted. In a case concerning imports of medicinal products manufactured abroad by a process protected in Portugal, the Portuguese Supreme Court found that the extended protection for process patents is to be interpreted against the background of this being a mean of maintaining the value of the process patent. 619

The importance of the provision hinges on the meaning of "direct result" where patents for pharmaceutical production process are concerned. It is not uncommon for a medicinal product to have been manufactured by a patented process but then to have passed through an additional reactive step before being imported to the country where the production process is patented. Basically, one can imagine such changes as being of three kinds: 620

1. A "trivial" further step has been taken, e.g. grinding, addition of an inactive component or formulation of the substance in dosages.

2. A further chemical process is performed which is of a particularly simple kind, e.g. esterification, a salt formation or suchlike.

3. One or more additional chemical reactions is performed which leads to a significant structural change in the active molecule, e.g. changes in the positioning of the substituents, the addition of further substituents or some other such change.

As regards the first point, the German Federal Patent Court has found that an active substance

618 See Beier, F-K., Ohly, A., GRUR Int. 1996, 975.

619 The Portuguese Supreme Court Trimethroprim of 1403 1979, GRUR Int. 1979, 482.

620 See Wibbelmann, J., [1996] EIPR 175. 
made by a patented process remains the direct result of that method after it has become part of a formulation. Additions of, say, thinning agents and carrier compounds which did not affect the therapeutic properties did not make the active substances possible to import to a country where the process for manufacturing the substances was patented.621 Consultation among professional practitioners in the patent system has yielded the same result. Situation 1 is generally taken to constitute an infringement. ${ }^{622}$ Finished medicinal products contain more constituents than just the active substances, and genuine extended process protection would be lacking if imports of the end product could not be prevented in cases of this kind. Simple production of a formulation, then, does not prevent the medicinal product from being regarded as the direct result of the production process used for making the active substance. ${ }^{623}$

Presumably situation 2 would also as a rule be regarded as an infringement of the to products extended process protection. It is well known in pharmacology how an active substance can be esterified or given a salt form. The therapeutic properties remain completely or almost completely unchanged, but practical handling and storage of the medicinal product are perhaps made easier. If on the other hand a simple reaction of this kind has led to a not insignificant change in the biological properties of the substance, the end product ought hardly to be regarded any longer as the direct result of the manufacturing process. What constitutes the direct result of a patented production process can be judged from whether there has been any significant effect on the usefulness of the end product.

One or more "normal" structural changes in end products mean that they are no longer to be regarded as the "direct" result of the production process. An interpretation of this kind is supported by the decision by the Dutch Supreme Court in 1983. The appellant was the proprietor of three patents for methods of manufacturing the substance Methacyclin. The substance Doxycyclin had been imported to The Netherlands. It was obtained after Methacyclin had undergone a further reactive stage which, according to the court, could be termed neither simple nor obvious. Without further explaining how the requirement of "direct result" was to be interpreted, the court found that the importation of Doxycyclin did not constitute an infringement. ${ }^{624}$ One reason given for a "normal" structural change not meaning that the end product is the direct result of the patented production process is that the patentee should take responsibility for not having included the final reactive stage in his patent claim and, accordingly, not having made his exclusive rights sufficiently clear to a third party. ${ }^{625}$

It is of course unclear what constitutes a "normal" structural change to a product. How many and what kinds of structural changes can occur? In this respect a technical assessment cannot be wholly decisive. Instead, as hinted above, one should consider the practical importance of the structural change which the end product has undergone. What is decisive as regards extended process protection is whether the action taken by the infringer, apart from the use of the patented process, has conferred some significant additional value on the end product. It is the relation of the patented production process to the additional production stage that matters. In German doctrine this interpretation of the to products extended process protection has been termed "Eigenschaftstheorie", as distinct to an interpretation where the "direct result" requirement has been taken to mean the requirement of chronological connection between use of the patented production process and the further changes made subsequently to the end product. ${ }^{626}$

\footnotetext{
621 Bundespatentgericht of 0710 1976, Blatt für Patent-, Muster-, und Zeichenwesen 1977, 274.

622 See Wibbelmann, J., [1996] EIPR 175.

623 See Beier, F-K., Ohly, A., GRUR Int. 1996, 981.

624 Hoge Raad Doxycyclin of 1006 1983, GRUR Int. 1984, 705.

625 See Beier, F-K., Ohly, A., GRUR Int. 1996, 980.

626 See op. cit., 973 and 983.
} 
The reason why the extended process protection is interpreted according to whether the properties are preserved in the imported product is that those properties govern its commercial value and the very purpose of Art. 64 EPC is to give the patentee an exclusive right to use of commercial value. It is an accepted general principle that acts of infringement shall be interpreted to confer an exclusive right to the entire commercial value of the invention. ${ }^{627}$ It should therefore be possible to assess the to products extended process protection according to whether the cause of the import of the to some extent modified end product was the value generated by the patented production process in relation to alternative production possibilities existing on the application date, or whether it was the value of the additional modification made by the importer to the end product. An attempt must be made to differentiate between the additional value contributed by the patentee and the importer to the end product, according to the situation on the market to which the product has been imported.

\subsubsection{Exceptions from the exclusive right}

Usually, national European patent legislation makes exceptions from the patentee's exclusive right in four cases:

1. non-commercial use;

2. consumption of the patent right (parallel imports of medicinal products);

3. experiments with the inventions;

4. formulation of medicinal products in dispensaries as prescribed by a physician.

As regards point 1 , non-commercial use of the invention, the restriction is probably of no practical importance to proprietors of pharmaceutical patents. Producing by himself the medicinal products needed by the family or his immediate circle of friends is hardly a realistic alternative for anyone. The non-commercial use exception is normally not interpreted to allow any further use of the invention than that. The other exceptions from the exclusive right will now be dealt with one by one.

\subsubsection{Exhaustion of the patent right - parallel imports of medicinal products}

The regional European exhaustion of patent rights is of great importance to the pharmaceutical industry, because in this way the patentee is not given an exclusive right to patented commodities which he or someone acting with his consent has put into circulation within the European Economic Area. The exclusive right is exhausted once the medicinal products have been placed on the European market. This means, for example, that if a Swedish patentee or someone acting with his permission (a licensee) has sold a medicinal product in Italy, the Swedish patentee cannot afterwards prevent a third party buying the medicinal products in Italy and then importing and selling them in Sweden or some other EEA country.

627 "Es ist also ... festzuhalten, daß sie dem Patentinhaber primär die (zeitlich begrenzte) Anwartschaft auf die wirtschaftliche Ausbeute der patentierte Erfindung sicherstellen soll. Jede Benützung, die eine solchen Erfolg nach sich ziehen kann, fällt unter sein ausschlißches Recht, auch wenn sie nicht zum Kern desselben ... gehört.” See Pedrazzini, M. M., GRUR Int. 1996, 375. 
The rule opens up possibilities of parallel imports, i.e. sales of pharmaceuticals to one side of the channels controlled by the patentee. Parallel imports limit the patentee's possibilities of differentiating prices in the EEA countries. It will be difficult for him to differentiate according to demand in different European national markets. He will be unable to charge an optimum high price in countries where there is a high willingness to pay if at the same time he wants to sell in countries where that willingness is a good deal lower. An excessive difference in prices will lead to parallel imports.

The EU rules on a single market and the rules dealing more specifically with parallel imports of pharmaceutical products are complicated, very extensive and subject to rapid and ceaseless development through the medium of ECJ case law. These rules generate serious legal problems, as regards both practical implementation and theoretical analysis. One significant factor, for example, is that pharmaceutical markets are regulated and consumers' willingness to pay government-subsidised in different ways in different countries. Such is the range of this question that, in a study like the present, focusing on central patent law, it cannot be dealt with in any great detail. On the other hand, the rules on parallel imports are so important that they cannot be completely disregarded either. The main points will now be passed in review.

Article 30 of the Treaty of Rome lays down that "quantitative restrictions on imports and all measures having equivalent effect" are "prohibited between Member States". The ECJ has continuously affirmed that every measure which, directly or indirectly, actually or potentially, impedes exchange between the Member States is equivalent to quantitative restriction on imports and is therefore prohibited. ${ }^{628}$ Art. 36 of the Treaty of Rome provides that the provision of Art. 30 shall not preclude a prohibition of imports through national legislation if necessary for the protection of industrial and commercial property. In this way Art. 36 opens up a possibility for the EU countries of having national rules which enable patentees to prevent imports from other EU countries of products infringing a patent applying in the country concerned. The ECJ has established, however, that the protection of industrial and commercial property shall not be taken to such lengths that patents can be used for obstructing imports to a country of products whose sale the patentee himself has permitted in another EEA country.

The ECJ has developed and articulated the assessment of when the patentee shall be deemed to have consented to the sale of certain products and in doing so made it possible for those products to circulate freely within the Common Market. If the products medicaments, for example - have come on the European market by virtue of a compulsory licence, the patentee shall not be deemed to have consented thereto in which case further exports of the medicaments to other EEA countries can be prevented by the patents in the other European countries. ${ }^{629}$ In the event of compulsory licence, the patentee has not himself been in a position to judge whether he wishes to sell in a country where the price has to be pitched particularly low. Therefore he has not been able to take into account the risk of parallel imports to countries which are ready to pay more.

The ECJ has established that a binding legal obligation to place the medicament on the market - a compulsory licence, for example - is needed in order for the patentee to be able to prevent parallel imports. It is not enough for the patentee to feel that he is under an ethical obligation to sell his patented medicament in a certain country. ${ }^{630}$ Nor is it sufficient, for the prevention of parallel imports, that a certain country applies price controls to pharmaceutical products in such a way that the patentee cannot independently determine the

\footnotetext{
628 See e.g. the EC Court C-8/74, ECR 1974, 837 and C-412/93, ECR 1995, 179.

629 C-19/84, ECR 1985, 228.

630 United cases C-267/95 and C-268/95, ECR 1996, I-6285.
} 
price in the country from which the medicament is perhaps exported subsequently. A patentee who cannot accept the price set by the authorities has the possibility of refraining altogether from selling in that country. Lastly, the absence of a patent in the country from which the medicament is exported does not affect the assessment of parallel imports to a country where there are patents. What matters is whether the medicament has come on the market with the consent of the patentee in the importing country, regardless of whether a patent has existed in the exporting country. In a word, the rule is that if the patentee has opted to sell his product in a certain EEA country, that decision is deemed to have been prompted by commercial considerations and in such cases the patentee is deemed to have factored the consequences of any parallel imports into his decision.

\subsubsection{The experiment exception}

\subsubsection{Introduction}

It is a well-established and almost universally accepted rule of patent law that experiments with a patented invention cannot be prevented by the patentee. The patent system exists for the promotion of technical progress, and this being so, the obstruction of experiments would be counter-productive. This rule exists in all European countries. ${ }^{631}$ In the pharmaceutical industry, however, the experiment exception has recently given rise to many problems of interpretation and several legal disputes. The growing importance of the experiment exception is due partly to the progressively more exacting demands which pharmaceutical products have had to meet in recent decades before marketing authorisation can be granted. This has led to a growing interest on the part of generics manufactures in being able to carry out necessary experiments at a time when a rival - a research-based pharmaceutical company - has patent protection in force. If such experiments may be performed, a generic version of the patented medicament can be introduced on the market immediately after the patent expires. This is of great economic value. Given the keen competition existing between different generics manufacturers, it is also important to be first in the market with a cheaper generic alternative when the patent has expired. ${ }^{632}$ The experiment exception has also come to have a more and more interesting bearing on competition between researching pharmaceutical companies. Pharmaceutical research intensity has grown in recent years. Several pharmaceutical corporations experiment with and develop alternatives to their rivals' successful and patented products. An attempt is made to differentiate the patented product within the same therapeutic segment by varying the chemical structure and in this way acquiring a share of the market. Experiments with competitors’ products are an important portion of this research.

\subsubsection{Filing an application for marketing authorisation}

Pharmaceutical products may not be sold until they have been shown, in various experiments, to be safe and effective. Marketing authorisation can be obtained from national pharmaceutical authorities in Europe or from the European Agency for the Valuation of

\footnotetext{
631 See AIPPI Yearbook 1991/V Q105: Experimental use as a defence to a claim of patent infringement. See also Benyamini, A., Patent Infringement in the European Community, 266.

632 See Grabowski, H., Vernon, J. M., 35 Journal of Law and Economics 1992, 346.
} 
Medical Products. It is an established rule that the "paper actions" implied by an application for marketing authorisation, e.g. the disclosure to a public authority of the substances which it is intending to use in the medicinal product, shall not be deemed to constitute patent infringement, e.g. offering for sale. This rule has been established by the Stockholm Court of First Instance. ${ }^{633}$ The same conclusion has been reached in English law. ${ }^{634}$ In no European country have "paper acts" been deemed to constitute infringement of a pharmaceutical patent. 635 An application cannot be a use of the invention, because the application does not contain the essential technical features of the invention as disclosed in the patent claims. Disclosing the essential technical features of the invention is not the same thing as manufacturing an infringing product. A parallel situation occurs when patent applications are filed for dependent inventions. In these cases the filing of a patent application is not to be deemed an infringement of the dominant patent. ${ }^{636}$ The distinction between real use of the invention and "paper acts" have been explained as follows by the German Supreme Court in an infringement case concerning a patent for a plant protection product:

"[A]nders als bei der Stellung eines Zulassungsantrags, der als solcher noch keinen Eingriff in der geschützte Erfindung darstellt, können die Feldversuche ... nicht durchgeführt, ohne daß dadurch der Gegenstand der geschützten Erfindung unmittelbar gebraucht wird."637

A marketing authorisation application cannot be an offer for sale either, since there is no intention of inducing the authority which grants the authorisations to acquire physical copies of the medicinal product. The Dutch Superior Court has laid down that the documents transmitted to pharmaceutical authorities do not constitute patent infringement. In particular the Court pointed out that this measure was not to be deemed as offering the product for sale. The requirement of marketing authorisation was statutory not voluntary, and so the transmission of the product was not to be interpreted as an offering of the product for sale to the pharmaceutical authority. Nor were the application papers to be deemed an essential means of exercising the invention, because such means needed to be of a technical character. Accordingly there was no indirect infringement either. 638

\subsubsection{Submission of samples of medicinal products to the authorising authority}

Together with the application for marketing authorisation, the applicant is required in several - though not all - European countries to enclose a physical specimen of the medicinal product to which the application refers. A submission of specimens of the medicinal product is not deemed to come within the scope of the exception made for experiments, nor to be permissible to competitors on any other grounds without the patentee's consent. The consensus view is that submission of specimens of the medicinal product is the critical factor

633 Stockholm City Court T 7-536-93 and T 7-737-94 of 15061995.

634 Patents Court The Upjohn Company v. T. Kerfoot \& Co. Ltd., [1988] F.S.R. 1.

635 "In no case has it been held that the mere submission of an application for a marketing authorisation without more, and in particular without the submission of a sample, infringes.” See Cook, T. M., Patent World February 1997, 38.

636 See Pedrazzini, M. M., GRUR Int. 1996, 375.

637 Bundesgerichthof X ZR 53/89 of 2102 1989, GRUR 1990, 997.

638 The Appellate Court in the Hague, 96/761, Glaxo-Wellcome v. Stada-Centrafarm, quoted in Ebbink, R., Patent World February 1998, 33. 
which creates patent infringement when the normal preparations are being made for the market launch of a generic product. ${ }^{639}$ The competitor must therefore put off enclosing specimens until the patent has expired. This rule has been established in The Netherlands. ${ }^{640}$ In Sweden the Stockholm Court of First Instance has arrived at the same conclusion. ${ }^{641}$ The question has been touched on, but not directly adjudicated, by the ECJ.

The question which the ECJ addressed was whether it was compatible with the EC rule concerning a single market for the national patent law of an EU country to prohibit the submission of samples and the procurement of marketing authorisation while a patent was in force. The ECJ did not make a direct pronouncement as to whether the submission of specimens constituted a patent infringement, because this was a question for national law. But EC law cannot be used as a means of expanding the rights conferred on patentees by national law. The EC rules on the free movement of goods or competition can only be used to restrict a patentee's possibilities of asserting an exclusive right under national law. In the case referred to, however, the ECJ found that it was not contrary to Community law for rules of national patent law to forbid other parties than the patentee, during the term of a patent, to submit specimens of a patented medicinal product to a marketing authorisation authority. A possibility of this kind under national law should not be denied the patentee with reference to Community law. That judgement enables, but does not oblige, EU Member States to allow patentees to prevent competitors from submitting specimens of a patented medicinal product while the patent is in force. A further observation made by the court concerned the sanctions available when there had been premature submission of specimens and, by the same token, patent infringement. The ECJ found that a national court could ban sales of the generic medicinal product, also with reference to the period following the expiry of the protection (patent or supplementary protection), if the purpose of the measure was to compensate a patentee for the loss of protection entailed by the premature application for marketing authorisation. ${ }^{642}$

\subsubsection{Experiments for the collection of data}

Verification of data in a patent. The description and claims in a pharmaceutical patent contain a large number of particulars of a technical nature, e.g. how the claimed substances can be manufactured and what biological reactions are obtained. For practical reasons, patent offices have no real possibility of checking these particulars. Only in cases where the particulars appear downright improbable do the patent offices require the applicant to furnish additional evidence. A system of this kind works if the necessary control is instead exercised

639 See Cook, T. M., Patent World February 1997, 38.

640 The Supreme Court in the Netherlands (Hoge Raad) has in German translation stated that: "Soweit bei der Herstellung von Arzneimitteln mit Atenolol gemäß dem patentierten Verfahren und dadurch, daß dem Gremium zur Prüfung von Arzneimitteln Muster davon zur Verfügung gestellt werden, überhaupt von einer 'Untersuchung des Gegenstands des Patents' die Rede sein kann, erfolgte die Untersuchung weder zu einem rein wissenschaftlichen Zweck noch mit einem den Zweck des Patentgesetzes verwirklichenden Ziel; Medicopharma und Pharbita bezwecken mit dieser Forschung nicht die Weiterentwicklung der Technik, sondern wollen lediglich bereits während der Dauer des Patents das gemäß dem WGV Erforderliche tun, um ihrerseits das bereits bekannte, vom Patent geschützte Arzneimittel unmittelbar nach Ablauf des Patents auf den Markt bringen zu können.” See Hoge Raad Atenolol of 1812 1992, GRUR Int. 1993, 887, OJ EPO 1994, 220.

641 Stockholm City Court T 7-536-93 and T 7-737-94 of 15061995.

642 EC Court C-316/95, ECR 1997, I-3929. 
by the applicant's competitors, who, if the particulars are incorrect, can lodge oppositions with the patent offices or file invalidation proceedings with a court.

One finds here a foundation for the accepted interpretation of the experiment exception: competitors may conduct experiments referring to the patented invention, but not experiments in which the invention is used for investigating something else. ${ }^{643}$ The invention may not be an aid, e.g. a measuring instrument, used in the conduct of an experiment but shall be the object of the experiment. ${ }^{644}$ This interpretation makes it permissible for competitors to investigate the particulars furnished in the patent application. It becomes permissible to check whether a copy of the invention, e.g. a medicinal product, can be manufactured by the method disclosed in the description, and it also becomes permissible to verify other particulars, e.g. disclosures of therapeutic effects. The German Supreme Court has ruled:

"Versuche an dem Gegenstand dem patentierten Erfindung und - damit notwendigerweise auch - die Herstellung einer Verkörperung dieses Gegenstands für solche Versuche patentfrei zu lassen, sei ... Zweck des § 11 Nr. 2 PatG [the experiment exception].»645

Presumably one consequence of the permissible experiments having to refer to the practical usefulness of that invention will be that, if a patentee holds more than one patent, it is not permissible to use one invention as a means of investigating another invention belonging to the same patentee. The situation of several patents belonging to the same legal subject has been discussed in an English case. The plaintiff held two patents for a substance used in the gastric ulcer medicine Cimetidine and a third patent for a certain polymorphous form of Cimetidine. The defendant used the invention, according to the first two patents, to investigate and file an opposition to the third patent. The defendant argued that, by virtue of the experiment exception, he was entitled to use the plaintiff's other two, technically very similar, patents when checking the invention disclosed in the third patent. He maintained that there was implicit consent to this when someone had filed a patent application. But the English Patents Court found that:

"[F]or there to be consent ... there must be some act or perhaps omission of SK\&F [the patentee] over and above that of applying for and being granted a patent.”

Further, as regards the rule that the experiments shall concern the patented invention, the Court ruled:

"[I]f an act is to fall within [the experiment exception] it must be done for purposes relating to the subject matter of the invention found in the claims of the patent alleged to be infringed."

The Court elucidated the expression "relating to" by stating that the connection between the invention and the experiments must be "real and direct" in order for the experiment exception to be applicable. No such direct connection was found to exist with regard to one of the plaintiff's patents which had been used by the defendant in connection with a dispute concerning another patent. The Court attached importance to the purpose of the experiments (invalidating another patent) not amounting to an investigation of the invention protected by the patent. The fact of a patent being used in an experimental context was not enough: the experiments had to refer to the invention in the patent concerned. Nor was it sufficient for the

\footnotetext{
643 See Gilat, D., Experimental Use and Patents, 22 and Pedrazzini, M. M., GRUR Int. 1996, 375.

644 See Straus, J., in European Research Structures - Changes and Challenges, 18.

645 Bundesgerichthof X ZR 99/92 of 1107 1995, GRUR 1996, 109, IIC 1997, 103; [1997] R.P.C., 623.
} 
patents to be technically very similar. 646

The English Court's interpretation of the experiment exception is probably representative of the legal position in Europe, but the policy behind this interpretation is open to question. ${ }^{647}$ With this interpretation, for example, it could become difficult for competitors to carry out comparative experiments aimed at checking whether a certain claimed medicinal product produces a better therapeutic result than certain other patented medicaments. If the known medicinal products cannot be procured in an anonymous market and the two patents have the same proprietor, that proprietor is unlikely to grant a licence for using a patented invention for the purpose of investigating the reliability of the particulars in another of his patents. It would be unsuitable if such experiments could be prevented. But at the same time there is a need for the issuing of effective patents for inventions which could be used for verifying particulars in patent applications. It would not be a good thing, therefore, if all verifications of particulars in patent applications were permitted with reference to the experiment exception. One can imagine a situation involving a patent for a commonly occurring chemical reagent. If someone wishes to use it for verifying particulars furnished in a patent application for a medicinal product, there is no reason why the proprietor of the patent for the reagent should be deprived of the right to a license fee, e.g. by permitting production of the reagent for the experiments. A solution of this kind would generally reduce the value of patents used mainly as research tools. ${ }^{648}$ This question is analysed more closely below, in section 8.1.4.5.

Clinical trials. There may be various reasons for someone other than the product patentee wishing to carry out clinical trials with a patented medicinal product while the patent is still in force. Some of the motives are at direct variance with the patentee's interests, as for example in the case of a generics manufacturer wishing to carry out the trials needed in order to obtain marketing authorisation for the product. After carrying out the experiments, the generics manufacturer may inaugurate commercial sales as soon as the patent expires. Another motive for carrying out clinical trials is that of trying to find new indications for the substance patented with product claims. Such motives are entertained by researching pharmaceutical companies competing with the product patentee. The competitors wish to patent new indications for the substance and to share the value of them with the product patentee. Here we can see a difference between two types of experiment: market-oriented and researchoriented. ${ }^{649}$ Generics manufacturers wish to conduct experiments with a view to marketing a pharmaceutical for a known indication, while research-based companies wish to carry out experiments aimed at obtaining a new patent. This distinction has become very important in recent European case law.

Clinical trials conducted solely with a view to marketing authorisation do not as a rule come under the experiment exception and can therefore be prevented by the patentee.650 These investigations imply a reiteration of tests already performed by the researching pharmaceutical company and, therefore, are not deemed to be genuine research activity but rather a commercial use of an existing invention. In order to be possible without the patentee's permission, the experiments must be capable of resulting in a new technical

\footnotetext{
646 The outcome of this case, however, was that the experiments were allowed, the reason being that the English Court decided that the experiments had not been performed for commercial reasons. Patents Court SK\&F Laboratories Ltd. v. Evans Medical Ltd., [1989] F.S.R. 513.

647 See Gilat, D., Experimental Use and Patents, 24.

648 See Eisenberg, R., 56 The University of Chicago Law Review 1989, 1035.

649 See Gilat, D., Experimental Use and Patents, 4.

650 See Cornish, W. R., 29 IIC 1998, 753.
} 
possibility which was not available when the experiments began. The mere verification of a certain company's ability to manufacture a medicinal product of good quality and safety is not sufficient as a research purpose. ${ }^{651}$ In an English case it was considered whether field experiments with a plant protection product amounted to patent infringement; the field experiments were allegedly necessary in order for marketing authorisation to be obtainable for the plant protection product. The Court of Appeal ruled that:

\begin{abstract}
"Trials carried out in order to discover something unknown or to test a hypothesis or even in order to find out whether something which is known to work in specific conditions, e.g. soil or weather, will work in different conditions can fairly, in my judgement, be regarded as experiments. But trials carried out in order to demonstrate to a third party that a product works or, in order to amass information to satisfy a third party, whether customer or a [regulatory body], that a product works as its maker claims are not, in my judgement, to be regarded as acts done for 'experimental purposes'.”652
\end{abstract}

The Court refused to adopt a narrow construction which would preclude any experiments which was directed to commercial exploitation. But it limited the permissible test to those were the data yielded was not intended for contacts with a third party. The same result was reached when the dispute was litigated in other countries. ${ }^{653}$

In The Netherlands the Supreme Court found in 1992 that the experiment exception only applied to scientific experiments, i.e. those aimed at verifying whether the invention could be used for the purpose indicated in the patent application or whether the invention could be developed further. ${ }^{654}$ Therefore it was not allowed to make a generic version and submit samples to the Medical Supervisory Authority in The Netherlands during the patent term. In a further Dutch decision, confirmed by the Supreme Court, it was considered whether there was patent infringement when clinical trials regarding a folliclestimulating hormone had been conducted in ten European countries and at thirty or more different research centres. The defendant maintained that the research was conducted in order to investigate whether the invention worked and whether it could possibly be developed further. The plaintiff in the infringement dispute argued, however, that the investigation had been conducted with the primary purpose of obtaining data for an international registration of the medicinal product for sale. In summary proceedings for a cross-border injunction the Court applied the test developed by the Dutch Supreme Court in the previously mentioned case and found that the defendant had not established the probability of the scientific purpose adduced by him necessitating experiments on such a large scale. The main purpose of the experiments, therefore, was presumed to be that of obtaining marketing authorisation. Given this background, the Court prohibited any further clinical trials. ${ }^{655}$

A common method of approach appears to be evolving in European cases of this kind. If the experimenter acts independently, i.e. does not intend to use the data in dealings with other parties, that may give some basis for suggesting that the testing in dispute is a genuine attempt to find out more about the product. Nevertheless the courts will require convincing that the trials are for this purpose and are not merely to persuade licensing authorities or customers of the virtues of the products. ${ }^{656}$

\footnotetext{
651 See AIPPI Yearbook 1992/III, Resolution 4.

652 Patents Appeal Tribunal Monsanto v. Stauffer, [1985] R.P.C 515; IIC 1986, 115; GRUR Int. $1987,108$.

653 See Cornish, W. R., IIC 1998, 745 and Cook, T. M., Patent World February 1997, 37.

654 Hoge Raad Atenolol of 1812 1992, GRUR Int. 1993, 887, OJ EPO 1994, 22.

655 The decision is that of the Court of Appeal of The Hague, Follikestimulationshormon II of 23061995 , GRUR Int. 1997, 838; IIC 1998, 702. The Supreme Court (Hoge Raad) only confirmed its correctness.

656 See Cornish, W. R., IIC 1998, 746.
} 
The legal position was articulated in favour of a similar interpretation of the experiment exception by the German Supreme Court in 1995, when it distinguished between different types of clinical trial depending, not so much on how the resulting data was used, but on the purpose for which the trials were conducted. In German law, as a result of this and a later judgement by the same court, the crucial consideration is not whether the experiments are performed commercially or with a view to profit but whether they amounted, at least in part, to a search for new knowledge. ${ }^{657}$ The first decision pertained to the medicinal product Gamma-Interferon, which consisted of a protein containing 146 amino acids. The German patent had claims in which the amino acid sequence was disclosed, and it was evident from the claim that alleles of the sequence came within the protection. The patentee had authorisation to sell the protein in Germany for the treatment of rheumatic diseases, but it was conceivable that the protein could also be used for the treatment of other indications. The Court found that the experiment exception made it permissible for the patentee's rivals to carry out clinical trials aimed at investigating the existence of new indications. The Court pointed out that new indications could only be developed if known medicinal products were allowed to be tested clinically, and further that:

\begin{abstract}
"Da das Patentrecht - im nationalen Bereich ebenso wie prinzipiell auch im ausländischen und supranationalen Recht - daruauf zielt, den technichen Fortschritt zu fördern und den Erfindergeist für das Gewerbe in nutzbringender Weise anzuregen ..., nicht aber den Technik ungebürlich in ihrer Entwicklung zu hemmen, wäre es mit diesem Zweck nicht zu vereinbaren, wenn Versuchshandlungen ausgeschlossen würden, die der Forschung und Fortentwicklung der Technik dienen." 658
\end{abstract}

The defendant had carried out clinical trials concerning twenty new conceivable indications for Gamma-Interferon, e.g. treatment of cancer, Aids, allergies, leukaemia, asthma and chronic hepatitis. The aim was to apply for a patent for possible new indications. If the research was permissible, the product patentee might find himself having to compete with others within the scope of his patent. The Court found this suitable and in agreement with the purpose of the patent system. It pointed out that any patents for the new indications would come to be dependent on the product patent and therefore, in principle, be of benefit to the product patentee, even if he, in order himself to sell the protein for the new indication, would have to reach an agreement with the party who had found that indication.

The German Supreme Court took account of the English decision related above. ${ }^{659}$ It found, however, that the two cases involved different situations. In the English decision the sole purpose of the experiments had been to meet the requirements of the authority granting marketing authorisation. In the case tried by the German Court, the clinical trials were found to have the dual purpose of investigating whether Gamma-Interferon could be used for new indications and of collecting sufficient data to be able, subsequently, to obtain marketing authorisation for any new indications which might be demonstrated by the experiments. The Court ruled:

"Die Zulässigkeit klinischer Versuche steht schließlich nicht entgegen, daß die Bekl. diese mit dem weiteren Ziel einer arzneimittelrechtlichen Zulassung durchführen bzw. unterstützen. Klinische Versuche an Menschen zur Erprobung eines Wirkstoffs zielen zwangsläufig auf eine Zulassung als Arzneimittel.”

\footnotetext{
657 See Hieber, T., GRUR 1996, 447.

658 Bundesgerichthof X ZR 99/92, GRUR 1996, 109; [1997] R.P.C., 623; IIC 1997, 103 :

659 Patents Appeal Tribunal Monsanto v. Stauffer of 1106 1985, R.P.C. 1985, 515; IIC 1986, 410; GRUR Int. 1987, 108
} 
The expression "weiteren Ziel" is critical to the interpretation of this case. The purpose of obtaining marketing authorisation does not prevent the experiments, regarded as a whole, having an experimental purpose. If the research purpose exists, the commercial purpose may also exist while a patent is in force. During the term of the patent, research-based pharmaceutical companies are permitted to conduct clinical experiments for the purpose of finding, and subsequently obtaining marketing authorisation for, new medicinal products or new indications for known products. But generics manufacturers will probably not be permitted to carry out experiments with a view, after the patent expires, to selling a medicinal product whose therapeutic properties are the same or almost the same as those of the existing and patented product. If the experiments can result in medicinal products which, for some patients at least, have clinical advantages over the patented product, then probably the experiment exception can be successfully invoked. ${ }^{660}$ This means that both experiments aimed at devastating a new indication and experiments to devastate an improved therapeutic effect (a selection invention) of a substance within a product patent already granted should be permitted during the term of the patent. 661 If, on the other hand, the intention is to produce a similar medicinal product which, after the expiry of the patent, can compete only in price with the patented product, the clinical experiments will presumably not come within the scope of the experiment exception.

In a decision from 1997, the German Supreme Court confirmed its interpretation in the 1995 decision. ${ }^{662}$ The trials in this case were designed to obtain knowledge about the properties of the active ingredient within a known indication; the data sought would remove uncertainties regarding the effects and the tolerability of the pharmaceutical composition in which the patented substance was placed. The Court held that equally such trials fell within the permitted experimental use. Clinical research with a patented medicinal product was permissible so long as it took place on a planned basis and for the purpose of gaining insights serving to dispel uncertainties regarding the therapeutic effects or toxicity of substances. The fact of the experiments also being intended to serve as the basis for the grant of marketing authorisation for a medicinal product did not prevent them from being permissible with reference to the experiment exception. Experiments with a research purpose, however, should not be conducted in such a way or on such a scale that the patentee suffers a loss of demand regarding known possible uses of the patented medicinal product. Given the authority of the German Supreme Court, these two judgements are likely to exert a great deal of influence on other national European jurisdictions. 663 The Hague Court of Appeal decided already in 1994 similarly that it was permissible to do experiments designed to discover a further indication of the blood coagulant erythropoietin, as it was furthering the development of technology. ${ }^{664}$

\subsubsection{Remarks}

Transaction costs connected with licences to experiment. A few decades ago, it was common for research tools and information about research methods to be shared freely between different laboratories, without any particular consideration of rights and commercial

\footnotetext{
660 See von Meibom, W., Pitz, J., Patent World June/July 1997, 29.

661 See Straus, J., GRUR 1996, 16.

662 Bundesgerichtshof X ZR 68/94 of 1704 1997, [1998] R.P.C., 423.

663 See Cornish, W., IIC 1998, 753.

664 See The Hague Court of Appeal, Kirin Amgen v. Boehringer Mannheim of 0302 1994, reported in Cornish, W. R., 29 IIC 1998, 735.
} 
possibilities. It was the end products of research that were considered commercially interesting. But the development of biotechnology since the mid-1970s has led to the starting up of many new enterprises which have only a research function and at the same time are in great need of venture capital from financiers. Their situation has stimulated interest in the commercial value of patenting things which create possibilities of research. As a result, it has become increasingly common for licensing agreements or agreements for the transfer of research tools to be concluded, specifying that a certain proportion of the value of the products developed with the research tools shall accrue to the proprietor of the rights to the research tools. "Reach through" clauses of this kind are not necessarily negative, because they can lead to the rapid dissemination of a new research tool through the proprietor's marketing. But they can also lead to long, complicated and expensive negotiations before the preconditions of efficient research are put in place. In recent years there seems to have been growing irritation among researchers over their failure, due to patent rights, to gain access to the equipment they need for their research.

When sizing up the situation which has arisen, one can begin by noting that if a patented product - e.g. an enzyme, reagent or medicament - is available in an anonymous market at a realistic price, the experiment exception, giving everyone the chance of manufacturing and using copies of the invention for experiments relating to that invention, will not be of any real practical significance. Those wishing to carry out the experiments will be able to acquire the product in the open market, directly from the patentee or from someone with his permission to sell the product, and then use it as they please, the protection to the particular product having been exhausted; see section 8.1.2.

If the patentee should put a particularly high price on the product, this may limit the interest of competitors in carrying out the experiments, but it will also affect other buyers of the invention in the anonymous market. ${ }^{665}$ The important point is that, in an anonymous market, the patentee cannot differentiate between ordinary consumers of the invention and those wishing to investigate it, experiment with it and develop it further. From this we may conclude that the experiment exception does not serve any decisive purpose in situations where the invention can be procured in an anonymous market. Consumption of the protection is probably sufficient for further technical development to be possible.

Not all patented inventions, however, are obtainable in an anonymous market. Certain inventions are only obtainable by "face to face" contract with the patentee. Patented methods are one such example. Where patented medicinal products are concerned, there is no anonymous market for the products which have not yet come onto the market, nor for substances which never will come on the market but are protected all the same. The first mentioned are pharmaceuticals which the patentee is in the process of developing and clinically testing. The second may be a substance which comes within the scope of a product patent but which the patentee has not chosen to launch. In both cases there may be development possibilities of which the patentee is not making optimum use. And, in the absence of an anonymous licensing market, there is a risk that competitors of the patentee will not be able to utilise the possibilities of production. With these situations in mind, it is essential that the experiment exception be suitably formulated.

The licences which confer the right to carry out experiments, but which the patentee normally objects to, should he be asked, have a special character: they improve the licensee's possibilities of becoming a producer in the market to which the patented invention belongs. In this situation the patentee regards his opposite number in the licensing negotiations as a competitor whose intention is to erode the value of the patent and prevent future profits from it. The opposite number is not regarded as an ordinary consumer of the

665 See Eisenberg, R., 56 The University of Chicago Law Review 1989, 1072. 
invention, i.e. as somebody contributing to the patentee's monopoly profits.

The licences which can make it easier for the licensee to become a producer can be of several different kinds. A possibility of carrying out certain experiments with a patented invention can, for example, result in the patent later being declared invalid, should the patent application be found to contain particulars which are incorrect or insufficient. When the patent is declared invalid, the licensee has the possibility of becoming a producer in the market to which the invention belongs. It is that consequence which can motivate competitors for checking up on previously granted patents. A licence to carry out experiments can also result in the party conducting the experiments succeeding in developing a new, patentable and rival product in the same market as the earlier invention. This may be, for example, a more effective substance which comes within the patent claims of the invention to which the experiments referred (a new selection invention). It may also be a new indication for a substance within the product patent claims (a new use invention). In both cases possibilities are opened up for the competitor to become a producer. Lastly, the experiments can also result in the licensee being able, very quickly after the expiry of the patent, to become the producer of a generic copy of the patented medicinal product. These situations are to be compared with the situation where the user of the patented medicinal product is a patient and has no prospects of becoming a producer.

Justification for the existence and structure of the experiment exception in patent law can be sought in the negotiating situation which arises when a use of the invention improves the user's possibilities of becoming a producer in the market to which the patent applies. In these situations, negotiations for patent licences take on a special character. The situation is different from where the licensee is an ordinary consumer of the invention. In this analysis we shall investigate when the experiment exception is suitable, according to the character of the negotiating situation.

Negotiations conferring a right of carrying out experiments which can make the licensee a producer within the scope of protection are difficult or unlikely for a number of reasons. The parties to the agreement will probably have different opinions concerning the value of the patented invention, the validity of the patent and its scope of protection. In addition, their opinions will differ concerning the value of carrying out further experiments, i.e. the likelihood of the experiments yielding a new product and, in such an eventuality, what the properties of the new product would be. Certain problems of this kind can perhaps be solved by gearing the license fee to the experimental outcome. Even so, the transaction costs would be considerable, especially if the experiments require access to several inventions belonging to different patentees. In addition there is a major problem which cannot be solved by suitable wording of the licensing agreement: the party with an interest in carrying out experiments must be extremely chary of informing the patentee of his plans for developing the invention further. The danger is that the patentee will refuse to grant a licence and carry out the experiments himself. The fact that research plans cannot be protected with intellectual properties - only as trade secrets - generates high and in many cases insuperable transaction costs in connection with agreements for the conduct of new research. To solve this problem of high transaction costs obstructing the conduct of experiments, a rule of patent law seems to have been established whereby no transaction is required between the patentee and the party wishing to carry out the experiments. At least, this appears to be a likely explanation. ${ }^{666}$

\footnotetext{
666 A similar explanation for the fair use doctrine in copyright has been spelled out in an influential article by Gorden. Gordon described the fair use doctrine in terms of "market failure”. Market failure occurs when the transaction costs of a voluntary transfer are so high that a consensual transfer is unlikely to take place spontanously. Gordon's key insight was that fair use makes sense where no functioning market for copyrighted works exists. See Gordon, W., 82 Columbia Law Review 1982, 1600 and further Merges, R. P., 12:1 Berkely Technology Law Journal 1997.
} 
Investigations of the usefulness of the invention. When experiments are conducted for the purpose of checking the reliability of particulars furnished in a patent, this amounts to a supplementation of the patentability examination conducted by the patent offices. The controlling activities of competitors mean that there will be fewer formally valid but really invalid patents. In such cases, those performing the experiments have an important function. The person wishing to check the validity of a patent by means of experiments would, however, be regarded by the patentee as a potential producer in the market to which the invention belongs and not as a consumer of the invention. The consequence would be high, probably prohibitive, transaction costs. But if checks cannot be freely made, the competitors will not be able to produce the invention in situations where the patent is invalid. Such a situation is clearly unfortunate. If the patentability requirements are seen to be justified, they must also be sustained, especially as proprietors of materially valid patents are not adversely affected when checks are made by competitors.

However, the current legal position also appears to be that a patentee can prevent someone from using his invention to verify particulars which he himself has furnished in a patent application for another invention. The rule that experiments shall have the invention as an object and not as a tool has been prompted by a bid to preserve the effects of the patent system concerning inventions used mainly in research. ${ }^{667}$ Otherwise research tools would not be given any meaningful patent protection, because the normal users of the invention would not then be infringing the patent. Inventions used very commonly in pharmaceutical research include, for example, chemical reagents and instruments for the study of molecular structures. The development of the well-known onco-mouse would also have lacked incentives if all pharmaceutical research could be conducted by virtue of the experiment exception, i.e. regardless of whether an invention was the object of the experiments or a tool for them. The onco-mouse is a "tool" in cancer research. ${ }^{668}$

When a patented invention serves as a tool in pharmaceutical research, a viable market for licences normally arises, because the transaction costs are limited. The patentee regards the licensees as ordinary consumers of the possibilities of use. There are, however, certain types of patented tools for pharmaceutical research where the patentee regards licensees mainly as potential producers in the market for his invention. This is the case where the research use of the patented invention is highly specific, i.e. a licensee's measures will compete with the patentee's own use of the specific research possibility which the invention affords. The situation of this kind arises, for example, when a patented invention can only be used for verifying and investigating a certain other patented product. If the two patented inventions belong to different legal subjects, the transaction costs need not be prohibitive. But if they belong to the same legal subject, it would probably be impossible for competitors to carry out the experiments. The patentee does not want competitors using one invention for the purpose of checking his other patents.

One way of avoiding this problem is by interpreting the experiment exception in such a way that all patents belonging to a certain patentee can be freely used for the purpose of investigating particulars in another patent belonging to the patentee. The range of the experiment exception is then limited according to the individual patentee, not according to the individual invention. In this way it can also be made possible, by virtue of the experiment exception, to make free use of patents belonging to enterprises with a common interest, for checking patents belonging to other enterprises within that same community of interest. This

667 See Eisenberg, R., 56 The University of Chicago Law Review 1989, 1074.

668 The Onco-mouse case, T 19/90, OJ EPO 1990, 476. For an economic analysis of the experiment exception in relation to the onco-mouse, see Eisenberg, R., 56 The University of Chicago Law Review 1989, 1084. 
would probably be a suitable solution, because in such cases the party outside the community of interest will be viewed by the patentee primarily as a rival producer, not as a consumer.

The proposed interpretation would mean that, generally speaking, a patentee could not stop anyone using his technique to check his patent claims. The possibility of control will then be independent of whether or not the patentee's technology is "divided up" into several closely related patents. In the case of "true" research tools, those with a more general use in pharmaceutical research, this interpretation would make no difference on the whole, because if the research tool is generally useful, the market for it will be much greater than just for checking up on the patentee’s own patents. 669

Further development of a patented medicinal product. There are two basic ways in which therapeutically useful substances protected by patents can be further developed by rival research enterprises. Firstly, a new therapeutic effect of a different kind can be disclosed, i.e. a new medical indication. Secondly, somebody may achieve a selection invention, i.e. disclose, within the claims of the patent, one or a small number of substances with a better therapeutic effect of the same kind as that previously known within the wider scope. In both cases the person who has carried out the experiments can obtain a patent, but this will be dependent on the first patent. The question is whether experiments for the purpose of achieving such new inventions should be permitted and, if so, how extensively they can be accepted within the framework of the experiment exception, e.g. as regards clinical trials.

If experiments are permitted during the term of the patent without the patentee's approval being required, a cost arises, consisting of a risk of unnecessary duplication of effort. There is a risk of the patentee and one or more other research-based pharmaceutical companies simultaneously carrying out one and the same experiment. In this way the coordinated exploitation of research opportunities which is a decisive benefit of the patent system will be lost. This, however, is not to say that rival experiments are inappropriate, because they have to be balanced against the positive effects which may stem from research competition within the scope of the patent granted. Production possibilities are usually located more rapidly if there is competition. Both the benefits of co-ordination - less duplication - and those of competition - more intensive research - can predominate.

In section 3.3.3.3, however, we came to the conclusion that patents for chemical compounds should be confined to use in the sector to which the technical effect disclosed in the patent application belongs, which in the present case means the pharmaceutical industry. The proposed use limitation to the pharmaceutical industry, with its implied reduction of incentives for competition by other pharmaceutical enterprises, as compared with a situation where the protection is limited to the particular new indication disclosed, may perhaps be wrongly interpreted as meaning that competition in the pharmaceutical industry during the term of a patent should not be aimed for, i.e. that the decisions by the German Supreme Court are inappropriate. ${ }^{670}$ This is not the case. The situation of use limitation is different from that governed by the experiment exception.

When a product patent is framed in such a way as to include all therapeutic indications for the substances concerned, there will still remain incentives for competitors. This is shown, for example, by the German decisions. True, the competitors' patents will acquire a relation of dependency to the first patent, but they can be used in negotiations with the holder of the first patent. In order for rivals to the product patentee to experience any incentives at all within the framework of a patent granted, however, there must always be an experiment exception. If the experiment exception cannot be invoked, there is no possibility

669 For a similar conclusion see Gilat, D., Experimental Use and Patents, 22.

670 Bundesgerichthof X ZR 99/92 of 1107 1995, GRUR 1996, 109, [1997] R.P.C., 623. 
of anyone but the first patentee searching for new indications. As has already been shown, it is unlikely that a licence can be negotiated for these experiments, because the patentee regards the licensee as a possible future producer. In reality, therefore, competitors of the product patentee experience no incentives within the patent granted, unless the experiment exception can be invoked. If on the other hand experiments are permitted, but the patents obtainable assume a relation of dependency, this results in the very structure of incentives proposed in section 3.3.3.3. Thus the experiment exception is needed so that competition will not be impeded within the scope of patents already granted.

One important factor in this connection is that the experiment exception is nationally limited. In countries where patents for the substances concerned are entirely lacking or the experiment exception can be invoked, patentees' competitors will be able to search for new indications and carry out clinical trials without any regard for impediments to research which a patent may imply. When the experiments, any clinical trials included, have been carried out, the competitors can then make use of the results, e.g. inventions, even in countries where the conduct of the experiments would have been an infringement. The mere filing of a patent application containing an account of research which would have amounted to patent infringement is not a patent infringement in itself. A restricted construction of the experiment exception in a certain country - which prevents certain research - therefore does not prevent research from being carried out, because there will always be countries where patents have not been applied for or where the experiment exception is more extensively construed. Thus a restrictive construction of the experiment exception would probably only result in research being conducted in a different country. ${ }^{671}$

The German Supreme Court's interpretation thus appears well-founded. It leads to a certain amount of competition, i.e. opportunities for competitors to demonstrate and clinically evaluate new indications within somebody else's product patent. At the same time any new patents will be dependent on the product patent and, accordingly, there will for the most part be a co-ordination of research. Co-ordination of research through the patentee will doubtless be the dominant feature of research, even if experiments are permitted. Companies will in all essential respects carry out research on such inventions which will not be dependent. But there seems not to be a forceful argument against competitors being able to carry out research on patented substances. If competitors are induced to carry out this research, in spite of the limited economic incentives, this suggests that the product patentee does not make efficient use of the possibilities of production existing within the scope of his patent, in which case a co-ordination of research through his agency appears less suitable. Accordingly, it is reasonable that the experiment exception can be invoked by competitors of a patentee who are conducting research aimed at demonstrating a new indication or a better therapeutic effect for a substance within the scope of a product patent already granted. If research can result in a product with added value, the experiment exception should probably be applicable. It is not probable that this kind of research would happen without a research exception. The transaction costs would prevent competitors from negotiating a license.

671 "The only viable option left to Israel's generic chemical industry was to move their research and development departments outside Israel to countries which permitted trials ... during the patent term. Since such an act would cause production lines to eventually be moved outside Israel, the State of Israel was therefore faced with a potentially severe drain on its brain power and/or ten-to-fifteen thousand families added to the unemployment rolls and a severe cutback of export income from the generic chemical industry." Israel accordingly introduced a possibility for generics manufacturers to carry out necessary clinical trials during the patent term. See Cohen, A. D., Patent World June 1998, 25. See also Eisenberg, R., 56 The University of Chicago Law Review 1989, 1078. 


\subsubsection{Formulation of a Medicine}

Extemporaneous formulation of medicinal products as prescribed by a physician in the individual case or a measure using a medicinal product thus prepared is excluded from the patentee's exclusive right under the majority of European patent laws. Usually the provision was introduced in response to the new situation arising when product patents began to be granted for medicinal products. So long as only the production methods could be patented, there was little risk of dispensary staff using patented methods. When medicinal products could be patented as products, this increased the risk of pharmacists' personnel incurring liability for patent infringement. This was deemed inappropriate.

As a result of this provision, medicaments on prescription will always be supplied by pharmacists' staff without any regard to the patent situation. But the exception to the patentee's exclusive right only refers to formulation in individual cases. The pharmacy is not allowed to prepare medicinal products for stock without taking the patent situation into account. It also follows from the wording of the provision that veterinary products may not be prepared in the individual case without regard to the patent situation.

This provision is of little practical importance today, since only a few medicinal products are now manufactured in pharmacies. Those which may still come into question are for the most part skin lotions. One form of pharmacy production which might be of commercial interest is that occurring in pharmacies organisationally integrated with large hospitals. In such cases, hundreds of prescriptions from physicians can be made up every day. But so long as these activities are not being conducted by a competitor, the majority of pharmaceutical enterprises probably prefer not to disrupt the customer-pharmacy relationship. Besides, the exception probably only matters in situations where there is medical justification for the pharmacy making up the medicament on the premises. In other situations pharmacy staff doubtless prefer to sell an existing, ready-packaged medicament. Economic considerations are usually of minor importance in these situations. Should it ever happen that hospital pharmacies systematically, but still for individual patients, and for economic - that is not medical - reasons choose to manufacture patented medicinal products under their own auspices, a teleological interpretation of the provision suggests that such action must be regarded as patent infringement, because the provision implies only sporadic, improvised and medically prompted use of patented medicinal products. ${ }^{672}$

The restriction concerning dispensary production has the same basic motive as the ban on patents for medical methods. The idea is that certain professional categories should be excluded from the effects of the patent system. The legal structure which has been introduced to "protect" pharmacists, however, is quite different in character from that intended to serve the corresponding purpose for physicians. Pharmacists as a professional category, are given immunity from patent infringement proceedings. For historical reasons, physicians have instead come to be protected by the exclusion, under Article 52(4) EPC, of the possibility of patents being granted for therapeutic, surgical and diagnostic methods. These differences in legislative technique have resulted in pharmacists being protected in a more appropriate, less expensive and at the same time more balanced way that is the case with physicians. In the case of pharmacists, it is left to the inventors to judge whether the invention is worth patenting, even if the exclusive right cannot be invoked in relation to pharmacists. As regards protection for physicians, the patent examiners have to judge which inventions will be excluded from patenting, on the grounds that patents can have an unsuitable impact on the working situation of the medical profession. Earlier, in section 1.3.4, it was proposed that, just as has happened already in the USA, Europe should introduce legislation for physicians

672 See Benyamini, A., Patent Infringement in the European Community, 282. 
matching those already existing for pharmacists, i.e. should abolish the ban on patents for medical methods but equip medical practitioners with immunity from patent infringement actions. 


\subsection{The scope of patent protection}

\subsubsection{General principles}

\subsubsection{Interpretations of patent claims}

While decisions regarding patentability are constrained by a number of legal requirements in both the EPC and national patent law, the legal basis for decisions on scope of protection solely resides in Art. 69, which provides that patent claims define the extent of patent protection, but that the description may be used to interpret the claims. However, in practical litigation no such abstract "extent of patent protection" is ever delineated. A concrete comparison is made between the invention, primarily the patent claims, and the alleged object of infringement. The assessment of the coverage involves two principal steps: an interpretation of the features of the patent claims, and secondly an assessment of whether and if so how far the protection should extend beyond what follows from the technical meaning of the features, i.e. a possible equivalence interpretation. It can at times be hard to maintain a distinction between the interpretation of patent claims and application of the doctrine of equivalence. But the distinction is established in the patent law tradition and usually it facilitates a discussion as to the nature of the differences between the patent claims and the object of infringement and as to whether or not the differences are significant.

Infringement pre-supposes that the object of infringement has all the technical features of the claims, at least in an equivalent manner. This applies regardless of whether the technical features are to be found in the preamble or in the characterising portion of the claims. This "all elements rule" is fundamental in the adjudication of both infringement and patentability. If the object of infringement has further characteristics over and above those to be found in the patent claims, the object of infringement can be patentable, but there is still infringement in the senior patent. If the object of infringement is patentable, there exists a dependent invention.

The skilled person may not fall back on the abstract when deciding on the extent of patent protection. ${ }^{673}$ On the other hand, as mentioned, the description may be used in assessing how the man skilled in the art would have interpreted the terms used in the claims. Often the description turns out to be very important. An example is a decision from the German Supreme Court. One feature in the claims had, according to the standard work: "Ullmann, Enzyklopädie der technischen Chemie", a special and carefully defined meaning, but another definition had been given in the patent description. The Court ruled that in a situation of this kind, with contrary definitions involved, the definition in the description should form the basis for determining the extent of protection. ${ }^{674}$

In 1999 the English Patents Court interpreted a claim in a patent for aqueous immune serum globulin (IgG, antibodies) preparations. ${ }^{675}$ One of the construction issues was whether the claims of the patent covered 'reconstituted IgG', ie IgG solutions ready for intravenous use which had been made up from freeze-dried solid. They were found not to. This conclusion was reached by the judge reading the specification and thereby establishing what the patentee intended by the introductory words of the claim "An immune serum globulin preparation in the form of an aqueous solution which is stabilised ...”. Literally taken the claim did not exclude IgG made up from freeze-dried solid. But the availability of

\footnotetext{
673 See art. 85 EPC.

674 Bundesgerichthof X ZR 7/82 of 3101 1984, GRUR 1984, 425.

675 Patents Court, Bayer plc v. Octapharma Ltd, of 05011999.
} 
freeze-dried IgG products at the priority date of the Patent was a matter of common general knowledge. On the other hand, from start to finish, the aqueous solution with which the description was concerned was a solution whose practical virtue was that it could be stored as a solution from the very time of its manufacture, for use as and when required. There was no mention in the patent of made-up solutions of the freeze-dried product which were well known at the priority date. Indeed, with freeze-dried IgG products, some terms used in the specification would have been quite meaningless. Therefore, the patent was not accorded the more comprehensive disclosure and broader scope of claim.

Support for the importance of the description when interpreting the claims is also to be found in a protocol from the conference in Munich in 1973. Representatives of several of the States Parties were of the opinion that Art. 69 EPC did not sufficiently regulate the important question of the scope of patent protection. ${ }^{676}$ There were divergent national traditions in this field which needed to be reconciled. A compromise between the traditions was worked out and, in accordance with the rules of Art. 164(1) EPC, made an integral part of the convention. The Protocol provides:

\begin{abstract}
"Article 69 should not be interpreted in the sense that the extent of the protection conferred by a European patent is to be understood as that defined by the strict, literal meaning of the wording used in the claims, the description and drawings being employed only for the purpose of resolving an ambiguity found in the claims. Neither should it be interpreted in the sense that the claims serve only as a guideline and that the actual protection conferred may extend to what, from a consideration of the description and drawings by a person skilled in the art, the patentee has contemplated. On the contrary, it is to be interpreted as defining a position between these extremes which combines a fair protection for the patentee with a reasonable degree of certainty for third parties."
\end{abstract}

The description, then, can be used for more than just eliminating points of uncertainty in the claims. Like the German Supreme Court in the decision mentioned above, it is in particular circumstances possible to change the skilled man's normal interpretation of the claims on the basis of the description. The Protocol also conveys that one should avoid two extreme solutions, namely the one in which narrowly formulated claims are broadly interpreted with reference to the existence of a general inventive concept, and the one in which the claims are narrowly and literally construed, thereby giving competitors the chance of evading the protection by means of cosmetic changes to what falls squarely within the claims. The interpretation should be a compromise between the two approaches described (the central and the peripheral tradition of interpretation). ${ }^{677}$

The central tradition of interpretation dictates that the court tries above all to decide what protection an inventor deserves, in view of what he has actually invented. The peripheral tradition of interpretation dictates that the court mainly tries to decide what protection the inventor has claimed and informed the general public of. ${ }^{678}$ In the central tradition of interpretation, patent claims serve as an indication of what is central and vital about the invention, i.e. the inventive idea or inventive concept, meaning above all a technical problem and its solution. The essential technical features in patent claims are interpreted with reference to an over arching functional purpose. References in the interpretation to the inventive idea render the claims possible to stretch. This also shifted the centre of gravity for deciding the extent of protection from the patent offices to the courts. Germany was the country where the central tradition of interpretation achieved its most prominent position. In

\footnotetext{
676 See Lunzer, R., Singer: The European Patent Convention 69.01.

677 See Takenaka, T., Interpreting patent claims: The United States, Germany and Japan, 3.

678 Cf. Paterson, G., The European Patentsystem 11-11.
} 
countries with the contrary attitude, the peripheral tradition of interpretation, the claims were seen as the outer limits of the protection, regardless of whether the claims constituted a decisive limit to the effectiveness or patentability of the invention. This tradition of interpretation was most firmly rooted in Britain. The handling of infringement cases by the courts was characterised by deference to legal safeguards.

The successive internationalisation of patent law, especially in Europe, has made it increasingly difficult for the two approaches to exist side by side. ${ }^{679}$ It will be manifestly inappropriate if EPO patents were to be interpreted from essentially deviating points of view. Here the Protocol has served an important purpose in European infringement precedent and there has been a successive convergence, at least on the level of principle. In 1986 the German Supreme Court, referring to the Protocol, ruled that patent claims should not only be the point of departure for defining the extent of patent protection but should constitute a decisive limit on the extent of protection. ${ }^{680}$ A House of Lords decision established that patents are to be construed according to their purpose and no longer strictly according to the letter.681 The Protocol has also come to play a very material function in the evolution of precedent in other EPO countries. ${ }^{682}$

\title{
8.2.1.2 Equivalence
}

Courts may stretch the scope of protection beyond the literal meaning of the claims and include matters that they consider equivalent to certain features in the claims. This so-called doctrine of equivalence has historically been a much-debated feature of the patent system. Some uncertainty is undoubtedly created. But, the doctrine must probably be accepted as a necessary component of a fair and well-functioning patent system, though it has indisputable drawbacks. ${ }^{683}$ In a notable decision from 1986, the German Federal Supreme Court resisted the argument that the doctrine of equivalence was in conflict with the via media for claim interpretation prescribed in the Protocol associated to Art. 69 EPC:

\begin{abstract}
"In accordance with this Protocol the extent of the protection conferred by a patent is not confined to what is defined by the strict literal meaning of the wording used in the claims. This opens the way for extending the scope of protection beyond the wording of the claim to encompass modifications of the invention as claimed. The extension of the scope of protection beyond the wording of the claim to include equivalent embodiments, corresponds in the opinion of this Court, to the intention of the Member States of the EPC, although significant differences regarding both the approach of determining the extent of protection of patents and the scope of the protection granted, still exist." 684
\end{abstract}

When it comes to the detailed application of the doctrine of equivalence, the German Supreme Court observed that significant differences exist between Member States of the EPC.

\footnotetext{
679 See Takenaka, T., Interpreting patent claims: the United States, Germany and Japan, 3.

680 Bundesgerichthof X ZR 28/85 of 2904 1986, IIC 1987, 795, OJ EPO 1987, 551.

681 The House of Lords suggested "purposive construction" in Catnic v. Hill and Smith, R.P.C. 1982, 183, GRUR Int. 1982, 136 and IIC 1981, 699.

682 See Austrian Oberster Patent- und Markensenat Op 5/83 of 2711 1985, IIC 1988, 676 and the Swiss Handelsgericht des Kantons Zürich in Tool Retainer Spindles of 0512 1988, IIC 1991, 398. See also the Dutch Supreme Court (Hoge Raad) Contact Lens Liquid of 1301 1995, IIC 1997, 748.

${ }^{683}$ See for a discussion concerning the room for a free-standing or independent doctrine of equivalence: Jacob, R., NIR 1999, 475.

${ }^{684}$ Bundesgerichthofs X ZR 28/85 of 2904 1986, IIC 1987, 795 and OJ EPO 1987, 551.
} 
However, it seems that courts at least have in common two important aspects in their equivalence analyses: if there is a functional similarity between the invention and the contested embodiment and if a person skilled in the art was able to replace the features in the claims not used with some corresponding features in the contested embodiment. The House of Lords has referred to these factors in its leading judgement Catnic v. Hill and Smith from 1982:

\begin{abstract}
"The question in each case is: whether persons with practical knowledge and experience of the kind of work in which the invention was intended to be used, would understand that strict compliance with a particular descriptive word or phrase appearing in a claim was intended by the patentee to be an essential requirement of the invention so that any variant would fall outside the monopoly claimed, even though it could have no material effect upon the way the invention worked.
\end{abstract}

The question, of course, does not arise where the variant in fact has a material effect upon the way the invention worked. Nor does it arise unless at the date of publication of the specification it would be obvious to the informed reader that this was so. Where it is not obvious, in the light of the then existing knowledge, the reader is entitled to assume that the patentee thought at the time of the specification that he had good reason for limiting his monopoly so strictly and had intended to do so, even though subsequent work by him or others in the field of the invention might show the limitation to have been unnecessary." 685

Functional similarity and the knowledge of the man skilled in the art are consistently considered when English courts try infringement actions. ${ }^{686}$ There is no equivalence if the difference between the defendant's product and the claims have a material effect upon how the technical solutions work or if the attacked embodiment was not obvious for the man skilled in the art starting from the litigated patent. British courts have formalised the ruling of the House of Lords in the following test:

\begin{abstract}
"Does the variant have a material effect on the way the invention works? If yes the variant is outside the claim. If no:

Would this (i.e. that the variant had no material effect) have been obvious at the date of publication of the patent to a reader skilled in the art? If no, the variant is outside the claim. If yes:

Would the reader skilled in the art nevertheless have understood from the language of the claim that the patentee intended that strict compliance with the primary meaning was an essential requirement of the invention? If yes, the variant is outside the claim.

On the other hand, a negative answer to the last question would lead to the conclusion that the patentee was intending the word or phrase to have not a literal but a figurative meaning (the figure being a form of synecdoche [a figure by which a more comprehensive term is used for a less comprehensive or vice versa; as whole for part or part for whole, genus for species or species for genus, etc.] or methonomy [a figure of speech which consists in substituting for the name of a thing the name of an attribute of it or of something closely related.]) denoting a part of things which include the variant and the literal meaning, the latter being perhaps the most perfect, best known or striking example of the class.”687
\end{abstract}

In the above-mentioned German Supreme Court ruling it was also decided that functional similarity and the ability of the man skilled in the art, are decisive considerations:

\footnotetext{
685 House of Lords Catnic v. Hill and Smith of 2711 1980, [1982] R.P.C. 183, GRUR Int. 1982, 136 and IIC $1981,699$.

686 See e.g. Court of Appeal Kastner v. Rizla Limited of 1606 1995, IIC 1997, 114.

${ }^{687}$ Court of Appeal Improver Corporation v. Remington Consumer Products, [1990] F.S.R. 181, IIC 1990, 860.
} 


\begin{abstract}
"What must be considered is the scope of the invention as it may be recognised by a person skilled in the art. It has to be examined whether a person skilled in the art, based on the invention as claimed, is able to solve the problem solved by the invention as claimed with equivalent means, i.e. to achieve the desired result with different means also leading to that result. Means which the average person skilled in the art, due to his knowledge and skill and based on considerations oriented on the invention as claimed, can identify as being equivalent are generally covered by the extent of the protection conferred by the patent. This is required by the goal of fair remuneration for the inventor under consideration of the aspect of legal certainty." ${ }^{688}$
\end{abstract}

Given this similarity between English and German case law, we may conclude that equivalence in Europe is, at least in large parts, ${ }^{689}$ an investigation into whether the skilled man knew that the infringing product was a functional alternative to the solution according to the patent claims. These principles are also helping Swedish courts in decisions on equivalence. ${ }^{690}$ The apparent similarity in approach, at least in theory, to equivalence can probably be explained by the difficulties in finding any alternative test. How else is one to make a broadly applicable qualitative comparison between two substitutable technical solutions?691 The difficulties in finding an alternative legal teaching are evident from the close resemblance between the test for equivalence and the other qualitative test that is applied to differences between two closely related technical teachings: inventive step. Inventive step is basically also a question of whether a man skilled in the art was able to modify a known technical solution but still use its function.

The close resemblance between the tests of inventive step and equivalence must have been evident in a plethora of cases on infringement. Still it is unusual that courts discuss if and how they distinguish between the legal scenarios of inventive step and equivalence. 692 One explanation for the silence could be that it is difficult to explain so-called dependent inventions if that which is patentable cannot at the same time be equivalent to a known and patented invention. If the two tests actually are the same qualitative yardstick, then it would appear as if there could be no dependent inventions. Modifications that were not available to the man skilled in the art starting from a certain patent have inventive step but, at the same time and for the same reason, are not equivalent modification of the earlier patent. ${ }^{693}$

In certain cases of dependent inventions, this conclusion is plainly untrue and there is therefore room for dependent inventions - for example, when a new use (indication) is found for an already patented substance or when a better effect of the same kind is found in one or a few substances belonging to a larger group of substances known to have this effect (selection inventions). In these situations the junior inventions can have inventive step, but still come within the scope of protection of the senior invention (be subservient). This is because the protection flowing from the first patent is not intended to correspond to the feasible uses that the man skilled in the art can derive from the disclosure of the invention. The scope of protection is larger and consequently there is "room" for dependent inventions, i.e. new inventions within the scope of the first patent. The claims have not been crafted with the intention that all production opportunities inside the claims should be available to the man skilled in the art through the disclosure. They can therefore be patented subsequently.

These, however, are special situations. Usually an attempt is made to align the protection as closely as possible with the uses that are made available to the man skilled in the

\footnotetext{
688 BGH X ZR 28/85 of 2904 1986, IIC 1987, 795 and OJ EPO 1987, 551.

689 Cf. Grabinski, K., GRUR 1998, 861 and Scharen, U., GRUR 1999, 285.

690 See Westlander, O., Törnroth, L., Patent - Allmänna domstolarnas praxis åren 1983-1991, 284.

691 Pagenberg, J., IIC 1995, 231.

692 Takenaka, T., 22 Rutgers Computer \& Technology Law Journal 1996, 492.

693 See Scharen, U., GRUR 1999, 285 and cf. Strauss, J., NIR 1999, 470 ff.
} 
art. So if the invention and the possibly infringing device are two products or two processes, an assessment of whether a man skilled in the art knew of the functional similarity is a feasible test for equivalence. The patentee can be granted coverage corresponding to what the man skilled in the art knows of as alternatives to the features in the claims. Competitors are free to use solutions that are not obvious to the man skilled in the art, i.e. non-equivalent solutions. The test is possible as long as the scope of protection corresponds to what the man skilled in the art learned from the patent application. New production opportunities, similar to the contested embodiment, are not expected within the scope of protection. Whoever tries to design-around or develop a patent further must then make a contribution beyond that which can be expected of the man skilled in the art. This appears to be a reasonable and practical interpretation of the doctrine of equivalence. ${ }^{694}$ Someone wishing to avoid the risk of committing patent infringement must interpret the claims in good faith, i.e. accept as protected such modifications to the claims that are available to the man skilled in the art. On the other hand, someone who does not draw the usual conclusions from the patent has reasons to believe that his interpretation does not correspond to the scope of protection. This could be someone who makes a further inventive input, but it could also be someone who in bad faith makes an unduly literal interpretation of the claims in order to easily escape them.

When applying the skills of an average practitioner in deciding equivalence, one has to decide on the pertinent time. Is it the ability of the skilled man on the priority date of the litigated patent? Or is it the date when the patent was published or perhaps the date of the infringement? The scope of equivalence will differ accordingly, because as time elapses the man skilled in the art will become aware of new equivalent alternatives to the features in the patent claims.

The point in time from which to evaluate equivalence has, however, been difficult to decide. There is a risk involved in allowing the time of infringement to be decisive. This would mean that the scope of protection is expanding over time and perhaps will come to include too many equivalents. On the other hand, decisions on equivalence based on the state of knowledge on the priority or publication date could mean the patent for an important invention losing its value, due to unrelated developments of new technology allowing circumvention of the claims. If the true value of the invention has still been used it appears reasonable to use the time of infringement when deciding what the man skilled in the art was aware of. 695

A compromise is applied, at least in some European countries. ${ }^{696}$ This test for equivalence takes as its starting point the knowledge possessed by the man skilled in the art on the day when the patent was applied for or published. But the range of equivalents should not necessarily be bounded to these. If the knowledge possessed by the skilled man on the day previously mentioned would have been sufficient for him to realise that some junior solutions were functional alternatives, these would also be equivalents. This position is somewhat obscure, but it has e.g. been advocated in Scandinavian patent law. ${ }^{697}$

There is one additional way of using the knowledge of the man skilled in the art in questions related to infringement, namely by looking at what was obvious to the man

\footnotetext{
694 Takenaka, T., Interpreting patent claims: The United States, Germany and Japan, 298.

695 This is the situation in the US. The US Supreme Court stated on the 3 March 1997 in the case WarnerJenkinson Company Inc. v. Hilton Davis Chemical Co.: "Insofar as the question under the doctrine is whether an accused element is equivalent to a claimed element, the proper time for evaluating equivalency-and knowledge of interchangeability between elements--is at the time of infringement, not at the time the patent was issued."

696 For English law see, Patents Appeal Tribunal Improver Corporation v. Remington Consumer Products, [1990] F.S.R. 181. For German law, Benkard, G., Patentgesetz Gebrauchmustergesetz § 14 Rdn 172 and Beton, J., [1994] EIPR 281.

697 Godenhielm, B., Patentskyddets omfattning, 302 (1994).
} 
skilled in the art, starting from the state of the art on the priority date of the litigated patent. In this test, unlike the previous one, it is not tried whether the man skilled in the art could overcome the differences between the claims and the infringing device. Instead one looks to the relationship between the state of the art and the infringing device. This is a defence built on the principle that a patent should not be permitted to encompass subject matter that was possible for the man skilled in the art to use on the priority date of the patent. That which was once freely available should not be recaptured through an application of the doctrine of equivalence. In Germany it has been ruled that all technical solutions available on the priority date of a litigated patent fall outside its coverage.698 The same line of defence has been known in British patent law for a long time ${ }^{699}$ and is also established in Swedish patent law. ${ }^{700}$ The argument that there is no mentioning of the state of the art in the Protocoll to Art. 69 EPC has not prevented the establishment of this defence. ${ }^{701}$

Summing up, in an infringement suit based on the doctrine of equivalence the defendant has two lines of defence. He may argue that his product was not obvious to the man skilled in the art in relation to the claims of the litigated patent. That would mean that there is no equivalence. The defendant may also argue that his device was obvious in relation to the state of the art on the date the patent application was filed. If one of these two arguments is valid there can be no infringement. By this reasoning the coverage, beyond the literal meaning of the claims, is established through an assessment of what was obvious functional alternatives for a man skilled in the art. A different technical approach appears hard to work out.

\subsubsection{Pharmaceutical patents}

\subsubsection{Product patents}

In a product patent for a pharmaceutical the claims define a chemical structure, usually by way of a structural formula. There is no statement in the claims about a use for the pharmaceutical. A structural formula will define the literal scope of protection with good accuracy. A common remark is that this clarity should be preserved and the murky waters of equivalence strictly confined, although they may not be completely absent. The salt of an active substance could probably be deemed equivalent with the active substance. Equivalence may also be present when two functional groups are swapped or a polar group is inserted in the molecule in order to increase the water-solubility. But mindful of the practical ins and outs of making further assumptions than that about functional similarity most commentators

\footnotetext{
698 Bundesgerichtshof X ZR 28/85 of 2904 1986, IIC 1987, 795 and OJ EPO 1987, 551: "Bei der Bestimmung des Schutzbereichs nach 14 PatG 1981 ist der Einwand zugelassen, die angegriffene und als angeblich äquivalente Benutzung in Anspruch genommene Ausführungsform stelle mit Rücksicht auf den Stand der Technik keine patentfähige Erfindung dar. Der Bekl. kann sich im Patentverletzungsprozeß daher nicht nur damit verteidigen, die als patentverletzend beanstandete Ausführungsform sei durch den Stand der Technik bekannt, sondern auch damit, mit Rücksicht auf diesen stelle sie keine Erfindung dar." See for a comment Strauss, J., NIR 1999, 468.

699 Gillette Co. v. Anglo Co. of 3006 1913, [1913] R.P.C 465.

700 Westlander, O., Törnroth, L., Patent - Allmänna domstolarnas praxis åren 1983-1991, 282.

701 Cf. Scharen, U., GRUR 1999, 287.
} 
are inclined to largely close the door on the doctrine of equivalence in the pharmaceutical field. ${ }^{702}$

The need for the doctrine of equivalence is probably not so great in these cases either. The literal scope of protection is usually generously allowed by the patent offices. Another reason is the very large investments that are necessary in order to bring a new compound to the market. Bearing them in mind, the patent-holder will have strong incentives to choose to market a substance that is indisputably protected, thereby preventing generic companies from introducing an approved compound that may or may not be an equivalent to those literally covered by the patent. Furthermore, due to the high regulatory costs research engaged competitors are well-advised to select compounds that are clearly not related to already granted patents, in order to avoid a possible finding of equivalence. These considerations have made case law on the doctrine of equivalence very limited.

A premium placed on the grant of claims with literal coverage entails that the scope of protection is finally crafted by the patent offices. Compounds falling within e.g. a Markush-claim are protected, but not those falling outside. In one significant European case the first portion of this teaching was, however, not followed. The Supreme Italian Court (Corte di Cassazione) tried an infringement case relating to a patent for the ulcer drug cimetidine. The claims were of the Markush type and the court estimated that they included some 10 million compounds. The compound manufactured by the patentee, i.e. the active substance in cimetidine, not having been synthesised at the time of filing, was not explicitly mentioned in the patent. The patent only disclosed a small number of examples with compounds inside the claims. The court ruled that there was no practical opportunity for a man skilled in the art to choose, from the disclosure in the patent, the active substance in cimetidine. A considerable amount of further research was needed to find that this compound was particularly valuable. On this theme the court concluded that the patent application had been filed too early, and as a consequence, that the active substance in cimetidine was not part of the coverage, in spite of the substance falling within the literal or technical meaning of the claim. ${ }^{703}$ It should, however, be added that this decision is unique. There is no other example where a court has strayed from the principle that what the skilled man identifies as coming within the literal meaning of the claims is protected. This is also true when something within the claims is novel and inventive in relation to the patent, i.e. becomes a dependent invention.

As was mentioned in part 8.2.1.2, a functional similarity between the invention and the infringing product is a necessary condition of a finding of equivalence. But of course a functional similarity - use for the same medical indication - is not sufficient grounds for extending the coverage beyond the literal meaning of the claims. The definitional function of claims must be upheld. The argument can, however, be reversed. If the defendant's substance is structurally closely related to those in the claims, but has not been used to attain an effect mentioned in the patent, then there is probably no infringement. The doctrine of equivalence requires the infringing product to have the functional implications mentioned in the patent. True, a product patent contains no use-limitation and thus the scope of protection is not bounded to certain pharmaceutical uses, but this only applies to the literal scope of protection. Outside the literal scope the patentee must rely on the doctrine of equivalence, and this requires the invention and the infringing product to solve the same problem, i.e. share a function. The protection that the doctrine of equivalence may offer is therefore limited to the

\footnotetext{
702 See Wegner, H. C., Patent Law in Biotechnology, Chemicals \& Pharmaceuticals, § 302; Benkard, Patentgesetz Mustergesetz, § 14 Rdn 53 and Hirsche, F., Hansen B., Protecting Inventions in Chemistry, 330. However, the opinion that the scope for equivalence in chemical product patents should be no less than for other patents is argued in Lederer, F., IIC 1999, 275.

703 Corte di Cassazione Cimetidine of 1601 1990, GRUR Int. 1991, 497.
} 
uses that the man skilled in the art learns from the patent. ${ }^{704}$ This is unaffected by the infringing product in fact having a potential for a use mentioned in the patent. The important factor is for what the possible infringer has used or sold the products. It can be taken from this that if substances just outside a Markush claim have more than one indication and the defendant has manufactured and used the substances for one that is not mentioned in the patent, infringement is at least unlikely. ${ }^{705}$

The typical situation for equivalence is, however, the opposite. There is a functional similarity, but also a structural difference between the claims and the contested embodiment. The active substance $\mathrm{X}$ is patented as a product and the description states that $\mathrm{X}$ may be used for treatment of $\mathrm{Y}$. Some years later the question arises whether another substance that also can be used for treatment of $\mathrm{Y}$ falls within the protective scope of the patent for $\mathrm{X}$. On the whole, as we have already seen, equivalence must remain an exception in these cases. It is necessary for equivalence that the man skilled in the art would know that the structural difference is unimportant for the utility of the pharmaceutical - an unlikely situation, in view of the general difficulties involved in predictions of therapeutic effects.

Infringement litigation in the pharmaceutical field seems to be concentrated to the field of macromolecules, such as proteins. Structural definitions, like Markush-claims, are often difficult to use in these cases. Instead product-by-process claims and parameters are often employed, but they will cause more ambiguity concerning the literal scope of protection and perhaps litigation. One example is a German biotechnology case from the appellate court, Oberlandesgericht, in Düsseldorf. The patent was on the protein erythropoietin. In the claims erythropoietin had been defined as a "polypeptide" and characterised as the product of prokaryotic or eukaryotic expression from an exogenous DNA sequence. The possibly infringing erythropoietin was glycosylated, i.e. contained a sugar residue, and was therefore a glycoprotein. The court ruled that the man skilled in the art had included sugar residues in the term "polypeptide", if the product was the result of the particular expression that was described in the patent. ${ }^{706}$

The same appellate court in Germany has also tried a case regarding equivalent infringement of a patent for the trombolytic - blood-clot-dissolving - enzyme Pro-Urokinase. In the claims the enzyme had been defined, not by its amino-acid sequence, but with a number of physico-chemical parameters, e.g. its molecular weight and the enzyme being isolated from a "biological source". ${ }^{707}$ Thus the patent claim at issue used the accepted possibility of describing the teaching in a manner other than by means of a chemical structural formula. The possibly infringing embodiment was a recombinant single-chain Pro-Urokinase enzyme, expressed by E.coli. Furthermore, it was a structurally deviating embodiment from the enzyme obtained from the biological source. It was distinguished from the claimed compound in having a different molecular weight (the attacked product lacked sugar residues that made up around 3 per cent of the total weight of the molecule). An expert witness stated in the proceedings that a man skilled in the art would, on the priority date of the patent, have

\footnotetext{
704 See Lederer, F., IIC 1999, 283.

705 See Benkard, Patentgesetz Mustergesetz $§ 14$ Rdn 53.

706 Oberlandesgericht in Düsseldorf 2 U 128/92, 2804 1994, as quoted in Hirsche, F., Hansen, B., Protecting Inventions in Chemistry, 328.

707 Thrombolytic with plasminogen activator isolated from a biological source such as urine or tissue culture and consisting essentially of urokinase, characterised by a) a molecular weight of approximately 56,000 Dalton, b) a specific activity of approximately 40,000 to 50,000 CTA units/mg when tested on a fibrin plate, c) the appearance of a single-chain structure corresponding to a molecular weight of 56,000 Dalton as shown by a single protein band on $7.5 \%$ polyacrylamide gel with sodium lauryl sulfate gel electrophoresis on unreduced urokinase, d) the appearance of a single-chain structure as shown by a single protein band on $7.5 \%$ polyacrylamide gel with sodium lauryl sulfate gel electrophoresis on urokinase, that has been reduced by $0.1 \mathrm{~m}$ dithiothereitol, a significant binding affinity for fibrin/celite.
} 
recognised that the differences between the attacked embodiment and the claims - the way of manufacturing and the molecular weight - did not essentially influence the therapeutic uses for the enzyme. Based on the opinion of the expert patent infringement by equivalent means was found. 708

This decision on equivalence is far-reaching. From a practical and economic perspective, it is important whether an enzyme is manufactured recombinantly or extracted from natural sources. It does not necessarily influence the therapeutic use, but by virtue of the recombinant technique the product is cheaper to manufacture and the risks for unwanted clinical side-effects are smaller. To a man skilled in the art, the statement that the enzyme was extracted from natural sources would have been a material part of the patented invention, and therefore it is surprising that this feature was not decisive for the coverage. The line taken in most cases is that a practically significant claim feature is decisive for the scope of protection. The possibilities for a patentee to fall back on the doctrine of equivalence are limited to differences of no particular practical or economic significance.

There are numerous examples of such possible minor modifications of patented polypeptides. It is regularly immaterial if one amino-acid is replaced by another with the same physico/chemical qualities, e.g. "X-Gly-Ser-Glu-Y" is replaced by "X-Gly-Ser-Asp-Y". If one single amino-acid has been modified merely in order to circumvent a patent, it appears reasonable to apply the doctrine of equivalence and find an infringement. By the mid-1980s, DNA-technology had reached a point where it was possible to produce a series of polypeptides with structures that were varied systematically at individual points. The doctrine of equivalence, however, probably ought not to be applied in these situations if the contested polypeptide has obtained new valuable qualities as a result of the minor modification. That could not have been predicted by the man skilled in the art. 709

One modification that is usually of limited practical importance, and therefore could warrant application of the doctrine of equivalence, occurs when a possibly infringing product is a prodrug to the patented pharmaceutical. This means that the attacked compound is metabolised in the body into the patented active substance. The supreme British court, the House of Lords, has tried one such case. In British patent law a particular expression is at times used to describe the doctrine of equivalence: "the pith and marrow doctrine". ${ }^{710}$ The idea is that if someone uses the important parts of an invention then it is an infringement, even if there are minor differences between the patent claims and the infringing product. The patent in this infringement case was for the antibiotic ampicillin, a semi-synthetic penicillin, and a major commercial success. The first claim had the following wording:

“(1) Penicillin derivatives of the general formula:

\footnotetext{
708 Oberlandesgericht in Düsseldorf 2 U 52/89 of 3112 1992, as quoted in Hirsche, F., Hansen, B., Protecting Inventions in Chemistry, 331 and Lederer, F., IIC 1999, 280.

709 "Application of the doctrine of equivalence to actions for infringement of protein patent claims must be approached carefully by the courts when it weighs the equities of the case before it. For example, few would hesitate to label a 'conservative' amino-acid substitution in a polypeptide sequence of a complex, patented protein, which was implemented for the sole purpose of escaping the literal scope of a patent claim, as anything but an 'unimportant and insubstantial' modification. Allowing the patentee to expand the enforceable scope of protection of a patent claim without restriction, however, seems inequitable to later parties who do not derive their work from that of the patentee, especially where a modified protein is superior to the earlier, patented protein.” See Kushan, J. P., 6:1 High Technology Law Journal 1991, 113 (footnotes excluded).

710 See for a background Jacob, R., NIR 1999, 476 f.
} 


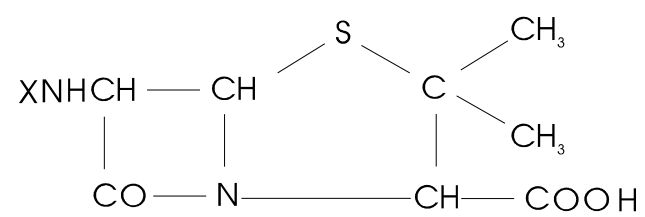

where $\mathrm{X}$ is an amino-substituted acyl group containing up to 20 carbon atoms having the formula:<smiles>[R]C(N)C(=O)OC</smiles>

where $\mathrm{R}$ is hydrogen or an amino, carboxyl or substituted or unsubstituted alkyl, aralkyl or heterocyclic group and $\mathrm{n}$ is zero or an integer, and non-toxic salts thereof.”

$\mathrm{X}$ was according to the claims a free amino group $\left(\mathrm{NH}_{2}\right)$ in the alpha position. All claims had this feature. But the contested embodiment, the antibiotic hetacillin, did not have such a group. Its formula was the following:

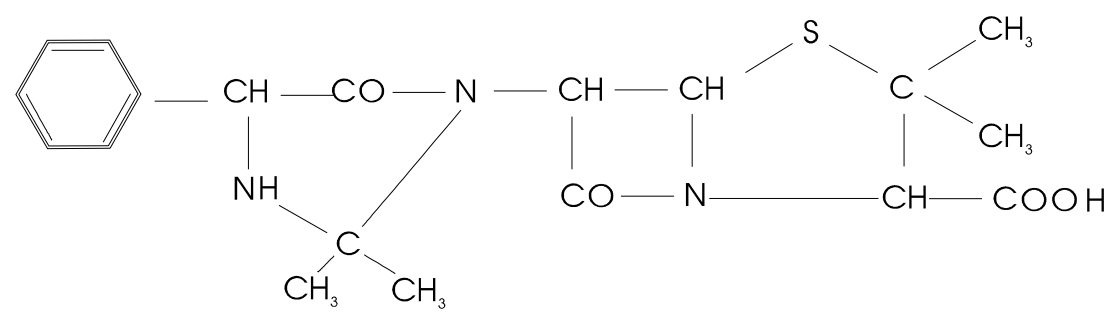

Thus there was a structural difference between the claims and the contested embodiment. But the above compound, hetacillin, could be manufactured from the patented ampicillin and acetone. The reaction resulted in hetacillin and water. Importantly, the reaction was also reversible: in the presence of water, hetacillin hydrolysed to ampicillin and water. It had been found that the attacked embodiment, hetacillin, did not in itself have an antibiotic effect. Its therapeutic utility was entirely due to its ability to work as a prodrug to ampicillin: when hetacillin came in contact with water in the gullet and stomach it converted to the patented antibacterial substance ampicillin. One witness described hetacillin as: "ampicillin with a hat on”. Lord Diplock, who wrote the judgement of the House of Lords, stated something similar as he concluded that there was infringement:

"I have already expressed my opinion that the pith and marrow doctrine is applicable to claims for new products as well as to new processes; and I agree with the Court of Appeal that the relationship of hetacillin to ampicillin provides a clear case for its application. It was argued that what is claimed in the patent as an essential feature of the class of products to which ampicillin belongs is the presence of an amino group in the alpha position, and that this feature is absent in hetacillin. This is literally true at the time of importation and sale but it ceases to be true as soon as hetacillin is put to use for the only purpose for which it is intended. The substitution for the postulated amino group of the variant incorporated in hetacillin is evanescent and reversible and for all practical purposes of use can be regarded as the equivalent of the amino group in ampicillin. In the apt phrase used by the Court of Appeal, it is the reproduction of the substance ampicillin, albeit temporarily masked.”711

\footnotetext{
${ }^{711}$ House of Lords Beecham Group v. Bristol Laboratories of 3003 1978, [1978] R.P.C 153.
} 
It can be taken from Lord Diplock's statement that the absence of any "practical purposes of use" for the structural difference is crucial if the protective scope of a patent is to be stretched beyond the claims.

In Germany an appellate court has also tried a prodrug-situation. The litigation was in relation to the anti-histamine Terfenadine, with the following structural formula: ${ }^{712}$<smiles>CCCCCC(O)c1ccc(C(C)(C)C)cc1</smiles>

The pharmaceutical Terfenadine was converted in the body into the active metabolite MDL 16,455:<smiles>CCCCC(O)c1ccc(C(C)(O)C(=O)O)cc1</smiles>

The difference between the prodrug and the metabolite was merely that a carboxy group in the latter (to the far right in the structural formulas) had replaced a methyl group in the prodrug. Terfenadine had been patented, but the patent had lapsed when the infringement suit was commenced. In the litigation a "follow-up" product patent was invoked, which protected the metabolite MDL 16455. This patent had been applied for when it was unknown that Terfenadine in the body became the active metabolite. But at the time of the litigation this was known. After the priority date of the metabolite patent tests initiated by the US Federal Health Authorities revealed that the substance MDL 16,455 was a metabolite (degradation product) of Terfenadine. In the years between 1980 and 1982, several test reports were published addressing the relationship between Terfenadine and the metabolite. It became a well known fact in the art that MDL 16,455 was an active metabolite of Terfenadine. Therefore, as in the British case, the man skilled in the art knew that using Terfenadine (the prodrug) meant that the active metabolite was used. Nevertheless, the Appellate Court in Munich found that the use of Terfenadine was not an infringement of the metabolite patent. The court reasoned that the senior patent had been possible to use without knowing that the compound was metabolised to the active metabolite. Furthermore, the court observed that:

\footnotetext{
"Spätere Erkentnisse und Mitteilungen haben mit der Bekanntheit und Ausführung der Lehre gemäß dem Patent 2303306 [the senior patent] nichts zu tun. Bessere Erkentnisse der Wirkungszusammenhänge begrunden keine schützenswerte Erfindung, wenn weiterhin nach den 'alt hergebrachten Methoden', die patentfrei geworden sind, verfahren wird. Die neue isolierte Herstellung und direkte Anwendung von MDL 16455 mag nach dem Klagepatent geschützt sein, jedoch kann nicht über diesen 'Umweg' der Schutz für ein patentfrei gewordenes Verhalten verlängert werden." 713
}

Claims should not be stretched so as to include subject-matter that was available to the man skilled in the art on the priority date of the litigated metabolite patent. Terfenadine was known

712 Oberlandesgericht in München 6 U 5155/92 of 0306 1993, ref. by Vossius, C., et al., GRUR 1994, 472 and Tauchner, P., Patent World April 1999, 19.

713 Oberlandesgericht in München 6 U 5155/92 of 0306 1993, ref. by Vossius, C., et. al., GRUR 1994, 472. 
on the priority date of the patent for the metabolite. Therefore the sale of Terfenadine could not be an equivalent infringement of the patent for the metabolite MDL 16455.

The British and German cases on infringement by a prodrug in a patent for a metabolite had different outcomes. In the British case the court found that the prodrug was an infringement, while in the German case there was no finding of infringement. However, there is no real conflict between the two decisions. The material difference is the time relation. ${ }^{714}$ In the British case the metabolite was the first patented and practically used substance. At a later time a competitor started to manufacture a prodrug that was found to be an equivalent to the metabolite. In the German case the time relationship was the opposite. The prodrug was the first invention and it belonged to the state of the art when the patent application for the metabolite was filed. Then the patent for the metabolite should not be allowed to monopolies uses that had been available to the man skilled in the art on the priority date of the metabolite patent. Subsequent knowledge about Terfenadine's metabolism did not make Terfenadine part of the scope of protection of the metabolite patent. The man skilled in the art knew when the infringement suit was filed that Terfenadine in practical terms was a use of the metabolite. But such new knowledge of functional relationships did not extend the coverage to something that had been available as a practical teaching on the date the litigated patent was applied for.

If the active substances are not manufactured by human metabolism, but by an industrial process, the starting material can be called intermediates. Intermediates may be patented by product claims. In these cases, as in any other, the principle of compound protection applies, i.e. the scope of protection is independent of the use. ${ }^{715}$ For practical reasons there may, however, be exceptions to this rule. There may sometimes be traces of the intermediate in the end-product. If the intermediate, but not the end-product, is patented or if the patent for the intermediate and the end-product belong to different patentees, traces of the intermediate in the end-product can give rise to patent disputes. There is a German decision from Landesgericht in Düsseldorf where the end-product contained small traces (between 0.1 and 0.4 per cent) of the intermediate. The intermediate had no practical bearing on the utility of the end-product. The court concluded that there was no infringement of the literal wording of the patent or a use by equivalent means, the rationale being that infringement required a professional use of the patented invention and that the small amount of the intermediate was not considered as such, because it had no function for the utility of the end-product. ${ }^{716}$ This reasoning - non-professional use - may be surprising, but the outcome was clearly warranted.

\subsubsection{Product-by-process claims}

A product that fulfils the patentability requirements but cannot be defined in the claims by its structure may be defined as the end-product of a particular manufacturing process, i.e. by using product-by-process claims (chapter 3.2.3.2). However, if the product is new there is some doubt about the resulting scope of protection. Will it be the same as in a product claim? Is the manufacturing process a limiting feature of the claim or merely mentioned because the substance's structure was unavailable?

One opinion is that the answer turns on the particular wording of the claim. If the applicant desires absolute compound protection, claims not having been pruned back to how the product was manufactured, the claim must read: "Product $\mathrm{X}$ obtainable by process

\footnotetext{
714 See Tauchner, P., Patent World April 1999, 21.

715 Bundesgerichthof X ZB 1/71 of 2504 1972, GRUR 1972, 642.

${ }^{716}$ Landesgericht Düsseldorf 4 O 310/84 of 0608 1985, GRUR 1987, 896.
} 
Y" or something similar, e.g. "producible”. It must be indisputable that the process is merely one of many possible ways to manufacture the substance. ${ }^{717}$ The process should not be crafted as a limitation. Only then will the coverage be equal to that of an absolute product patent. ${ }^{718}$ The difference can be seen in the following two claims:

1. "Compound, obtained by ... [process].

2. "Compound, obtainable by... [process].

Claim alternative 1 only protects products manufactured with the process that is described in the claim. ${ }^{719}$ If instead claim alternative 2 has been used the novel product is protected, irrespective of the manufacturing process used. ${ }^{720}$ Alternative 2 therefore has a wider scope. It is stated in the Guidelines that a product-by-process claim normally should have the form "obtainable" and that the form "obtained" should be avoided. ${ }^{721}$

This interpretation of product-by-process claims is faithful to the linguistic content of the claims. But there are examples of other interpretations in case law. The Supreme Federal Court in Germany has on one occasion concluded that claims with the format: "Produkt X, erhalten durch verfahren Y" was not bounded to the products manufactured by the process in the claims. The process was only a way to avoid the practical problem associated with a definition of the structure of the patented product. Such problems should not affect the scope of protection. The court "translated" the manufacturing process into structural features and all products with said features were protected, irrespective of how they had actually been manufactured. ${ }^{722}$ A parallel decision worthy of note has as a matter of fact been handed down in the EPO, T 223/96. The question was whether certain amendments offended against Article 123(3) EPC, i.e. broadened the scope of protection, which is inadmissible after grant. The granted claims had the form "produced by", but nevertheless the protection conferred was found to be absolute, i.e. encompassed any product the features of which were identical with those of the product resulting from the disclosed process. The Board of Appeal ruled:

\footnotetext{
"It is true that a 'product-by-process' claim confers absolute protection to the product as such, ie to any product - however made - the features of which are identical with those of the product resulting from the process to which reference is made. The difficulty in such a situation, especially when dealing with complex molecules such as proteins, lies in defining the precise contribution of the process to the structure and properties of the product and thus in establishing the identity of all the features which characterise it.”723
}

\footnotetext{
717 See Jaenichen, H-R., The European Patent Office's Case-law on the Patentability of Biotechnology Inventions, 93.

718 See Boeters, H. D., Handbuch Chemiepatent B I 13.

719 For German law see Bundesgerichthof X ZB 9/70 of 0607 1971, GRUR 1972, 80.

720 See T 130/90 of 2802 1991. For a comment see Jaenichen, H-R., The European Patent Office's Case-law on the Patentability of Biotechnology Inventions, 93.

721 Guidelines C III 4.7b.

722 "Denn Gegenstand des Patents ist trotz der Beschreibung durch das Herstellungsverfahren das Erzeugnis als solches, das unabhängig von seinem Herstellungsweg die Voraussetzungen für die Patentierbarkeit erfüllen muß. In dieser Art der Beschreibung liegt auch keine Beschränkung des Schutzes für das Erzeugnis auf den zu seiner Kennzeichnung angegebenen Verfahrensweg. Die Beschreibung des Züchtungswegs dient nur der eindeutigen Kennzeichnung des Erzeugnisses”. See Bundesgerichthof X ZB 13/90 of 3003 1993, GRUR 1993, 655.

723 T 223/96 of 29011999.
} 


\subsubsection{Use limitations}

If a use for the patented compounds is mentioned in the claims, infringement demands that the attacked compounds have been used for the stated purpose. That follows from the allelements-dogma in interpretation of patent claims. A use limitation can, however, be of varying broadness. If a first medical indication has been found for a known chemical entity, the patent claims may be worded so as to encompass all medical uses of the compound. But if a second medical use is found that particular use must be recited in the claims.

In German case law it has been established that claims to the medical use of a pharmaceutical protect the manufacturing and formulation of the patented pharmaceutical (“augenfällige Herrichtung”, i.e. roughly the "manifest preparation"), if these acts are done in order to take advantage of the particular medical use that is stated in the claims. ${ }^{724}$ The therapeutic use in the claims is reinterpreted to protect the manufacturing of a pharmaceutical suitable for the use mentioned in the claim. ${ }^{725}$ Also the inclusion of instructions for the patented use in the patient information leaflet, which is added when the substance is marketed, is protected, as well as other commercial preparatory steps taken before the medical use. The EPO Guidelines too state that claims to a medical use of a chemical substance give coverage that includes substances and mixtures presented or formulated for the medical use stated in the claim. ${ }^{726}$ Patents related to new medical uses - a first or a second - can thus be used to prevent competitors from manufacturing the substances in a formulation - package and dose - intended for the use that is stated in the claims. On the other hand, if the claims have been limited to a particular medical use, the patentee cannot prevent engaged firms from using the substances for another indication. In an infringement case tried by the Federal German Supreme Court there was only one claim and it had the following wording:

“Antivirusmittel, dadurch gekennzeichnet, daß es 1-Aminoadamantan oder 1Aminoadamantanhydrochlorid enthält.”727

The substances had been known when the patent was applied for, but their use as an antiviral drug was novel and made them patentable for a first medical indication. When the new pharmaceutical reached the market a doctor on one occasion used it to treat, for influenza, a patient who was also suffering from Parkinson's disease. The doctor unexpectedly discovered an improvement in the Parkinson-related symptoms. The discovery later led a competitor to start manufacturing the substances. When his pharmaceuticals were sold, only the indication "Parkinsonismus" was recited on the package. The court concluded that infringement in this situation had required a product patent, i.e. claims that only contained a structural definition of the substances, or as an alternative, claims where the substances were described together with a statement that they could be used as pharmaceuticals. The latter kind of claims had been possible in this case, because the substances had merely been known outside the pharmaceutical field. But now when the claims specifically mentioned the antiviral indication, there was no infringement.

The explanation for the unnecessarily pruned back claims was that the patentee had relied on older German law according to which a use stated in the claims was not a limitation, if the claims had the so called "Mittelanspruch" (“Antivirusmittel”) form. It was only a limiting feature if it had the use format ("pharmaceuticals for treatment of virus

\footnotetext{
${ }^{724}$ Bundesgerichthof X ZB 4783 of 2009 1983, GRUR 1983, 729.

725 See the comment by Klöpsch, F. to the case BGH X ZB 4783 of 2009 1983, GRUR 1983, 734.

726 Guidelines C IV 4.2.

${ }^{727}$ BGH X ZR 51/86 of 1606 1987, GRUR 1987, 794.
} 
infections”). However, the Federal German Supreme Court concluded that this distinction was no longer valid. The critical issue was whether the defendant had used the claimed indication when he designed the doses and stated the indication on the package. The court reversed the lower court's ruling, according to which the claim in question protected all compounds with a potential for antiviral use. The antiviral indication was a significant component of the claim and could not be ignored when the scope of protection was crafted. Infringement required that the indication mentioned in the claim had been used commercially.

\subsubsection{Processes}

Introduction. There can be two kinds of differences between a patented manufacturing process and a contested process. The starting material in the patent claims and in the contested process may be different. There may also be differences as to the reaction steps that are used, e.g. the number, the type and the order. These diversities may, however, be of minor practical importance and possible to bridge by the knowledge of the man skilled in the art, i.e. through an application of the doctrine of equivalence. Probably the scope for the doctrine of equivalence is larger in regard to process claims than in regard to product claims. This is because the literal scope of a process claim often has more ambiguities than a product claim.

Analogy processes. Analogy processes are inventions where the patent claims define processes for manufacturing a new, valuable and previously unknown substance. The valuable invention that motivates the grant of a patent is the end-product of the process, but the granted scope of protection is all the same bounded to certain ways of manufacturing that product. Analogy process claims have been the usual form of protection for new pharmaceuticals in countries where product patents have not been permitted. But sometimes this protection has been brittle. For the protection to be effective, it is essential that all economically feasible processes for the production of the new substance are included in the claims. An end-product that has been manufactured by a process not mentioned in the claims would be freely available. This has meant that patent applicants have been spending time and money on otherwise non-essential work. It has been necessary to search at an early stage for all processes for manufacturing the new substance that have valuable properties. This is a difficult task, and so not infrequently competitors have subsequently been able to find a manufacturing process that has not been mentioned in the claims. This has at times led to litigation and the question has often been whether a certain process, differing somewhat from the patented ones, infringes under the doctrine of equivalence.

In Denmark the Supreme Court decided in 1993 an infringement case related to an analogy process for manufacturing the commercially successful ulcer drug rantidine (Zantac). Its chemical formula was the following: ${ }^{728}$

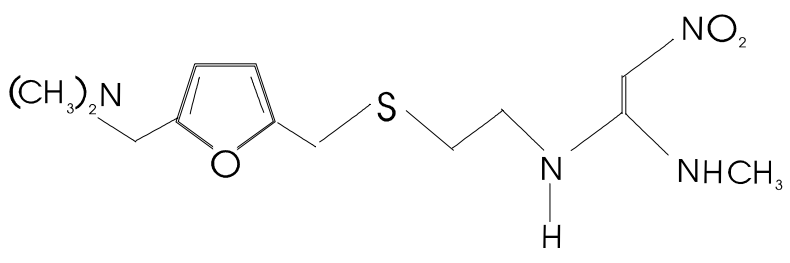

\footnotetext{
728 Højesteret Allen Hanburys Ltd v. A/S GEA Farmaceutisk Fabrik of 2708 1993, Ugeskrift for Retsvæsen 1993, 859.
} 
The defendant had used largely the same synthesis steps as in the patent claims. But due to somewhat different starting material, the result of the process was not the same as in the claims, i.e. not rantidine, but a substance with the following structure:

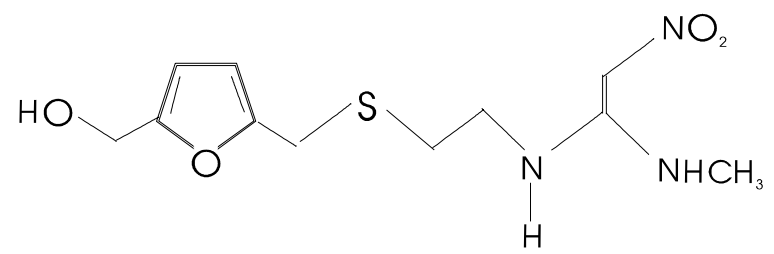

That substance was an intermediate. The defendant used an additional reaction step with the following substances:

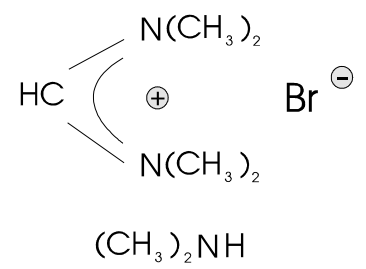

Now the end-product was rantidine. The final reaction step was not mentioned in the patent on the analogy process. Furthermore, the last step was so unpredictable that it was unobvious to the man skilled in the art. It had been patented, on its own merits, by the defendant in the infringement process. The Danish Supreme Court ruled, taking these facts into consideration, that the contested process was not an equivalent to the patented analogy process. It was pointed out that the claimed analogy process as such did not have the character of an invention. The unexpected and valuable features of the end-product motivated the analogy patent, not the process in the claims. Therefore the claims should be particularly narrowly interpreted. Furthermore, the defendant had used an additional step in his process, which had no counterpart in the claims and which was not obvious to the man skilled in the art. The Court concluded that the differences were so material that a broad interpretation of the analogy process patent should not be done.

The Danish Supreme Court's reasoning is not altogether convincing. The Court found that a patent for an analogy process did not justify the same broad interpretation of the claims as an "ordinary" process patent, i.e. a process that in itself has inventive step. The doctrine of equivalence was considered more appropriate for inventions where a significant technical advance had been defined in the claims. Usually the novel features of an invention are stated in the claims and then this principle is correct and important. There should e.g. be more equivalents in relation to pioneer inventions, simply because the doctrine is designed to prevent theft of valuable technical advances through immaterial cosmetic changes. But patents for analogy processes are special in that the valuable technical achievement is not to be found in the claims; the claims only state traditional and usually well-known processes of manufacturing. This means that the justification for applying the doctrine of equivalence cannot really be evaluated from the viewpoint of the features in the claims. However, the Supreme Danish Court did just that.

When the claims do not disclose the true value of the invention, special opportunities for applying the doctrine of equivalence seems to be present. The literal meaning of the claims do not have the same significance. A liberal use of equivalence in relation to analogy processes has been argued in Scandinavian $\operatorname{law}^{729}$ and has been

\footnotetext{
${ }^{729}$ See Godenhielm, B., Om ekvivalens och annat gott, 114.
} 
established in German law. ${ }^{730}$ Thereby inventors can profit from the true value of their inventions. An infringer who has manufactured the end-product has taken advantage of the actual merit of the invention, irrespective of whether he has used a process mentioned in the claims or not. A generous use of the doctrine of equivalence in these cases means that the protection is interpreted more like one of a product patent.

It is, however, probably unfair to fully equate analogy process patents with product patents. Therefore, the Danish case was most likely correctly decided - there was no infringement - although the reasoning in the decision is none too convincing. An application of the doctrine of equivalence should stop short of modifications that are unavailable to the man skilled in the art. Differences between the claims in the analogy process patent and the infringing process must be obvious to the man skilled in the art. Only then is it possible to put on an equal footing analogy process patents and product patents. In the Danish case the differences between the analogy processes and the process that had been used by the defendant were such as to render the defendant's process patentable. In such a situation the doctrine of equivalence should probably not be applied. In the case in question there was a further reason not to apply the doctrine of equivalence: the defendant's process had better yield, i.e. the new process added some extra value to the processes mentioned in the litigated patent.

The Court of First Instance in Stockholm has delivered a judgement in a patent infringement case relating to an analogy process for manufacturing the ulcer drug cimetidine. ${ }^{731}$ In the patent description the following substances, named A, was mentioned as starting material:<smiles>CC1=C(C)NCN1</smiles>

It was also stated in the description that A reacted with another substance, called B:<smiles>NCCS</smiles>

Thereafter in the main claim it was explained that the substance resulting from the process in the description, i.e. $\mathrm{AB}$, should react with the following substance, called $\mathrm{C}$ :

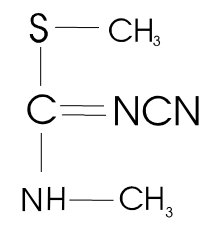

By this process cimetidine was manufactured, i.e. the following compound, where the three different starting materials can be identified:

\footnotetext{
730 See Hirsche, F., Hansen, B., Protecting Inventions in Chemistry, 338.

731 Stockholm Court of First Instance T 7-213-83 of 20061985.
} 


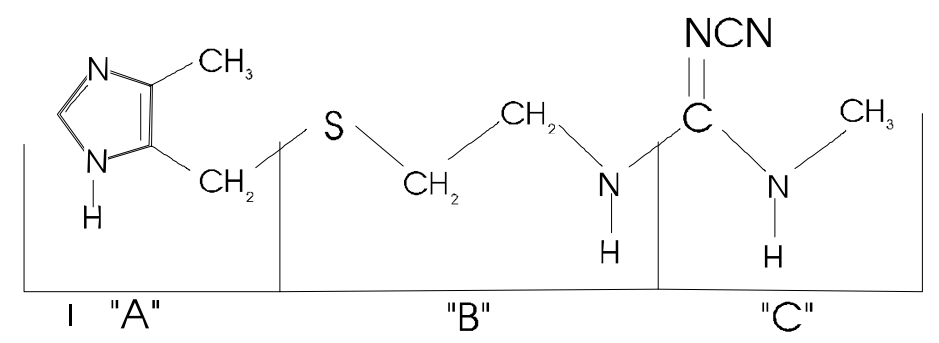

The same three substances had been used as starting materials in the attacked process for manufacturing cimetidine. A difference was, however, the order with which the synthesis was carried out. In the possibly infringing process the first step was substance B being made to react with $\mathrm{C}$. Thereafter $\mathrm{BC}$ was made to react with $\mathrm{A}$. This meant that the patent claims described the reaction: $\mathrm{AB}+\mathrm{C} \rightarrow \mathrm{ABC}$, while the possibly infringing process used the reaction: $\mathrm{B}+\mathrm{C} \rightarrow \mathrm{BC}+\mathrm{A} \rightarrow \mathrm{ABC}$. The Stockholm Court of First Instance remarked that the claims only mentioned $\mathrm{AB}$ and $\mathrm{C}$ as starting materials, but taking into consideration the description, where it was described how $\mathrm{AB}$ could be manufactured, the reaction $\mathrm{A}+\mathrm{B}$ should also be considered in an interpretation of the claims. Furthermore, the Court found that the same chemical groups were used in the same manner in the two processes. Thus, they were chemically similar. The defendant had pointed to the yields of the two processes, which were not the same, and to the fact that different reaction conditions were used. That, however, was not decisive for the Court. These were modifications that a man skilled in art was expected to make, and even if the modifications were not available to the man skilled in the art, the infringing process could be a dependent invention. The Court concluded that this was a case of infringement by equivalents.

This is quite an extensive use of the doctrine of equivalence. It had been demonstrated that the reaction $\mathrm{BC}+\mathrm{A}$ in the infringing process required a basic environment, while the reaction $\mathrm{A}+\mathrm{B}$ according to the patent description only took place in an acid environment. Another notable difference was that the reaction $A B+C$ according to the claim required 24 hours to be completed, while the reaction $\mathrm{B}+\mathrm{C}$ in the infringing process occurred practically momentous. These differences are significant. But an even more material difference was that the infringing process had a better yield. It produced 70 per cent more cimetidine than the process in keeping with the claims. It also produced pure cimetidine, whereas the patented process required a further purification step. These differences are important from a practical perspective and it is questionable whether it was correct to consider that the attacked process represented equivalent infringement.

The Danish and the Swedish cases relating to patents for analogy processes have some similarities. In both cases the contested processes yielded more than the patented processes and the contested processes were inventive in relation to the patented processes. However, the outcomes were different. In the Danish case there was no finding of equivalence, but in the Swedish case there was. However, one of the judges in the Swedish case, Westlander, has since stated that he questions the correctness of the judgement. ${ }^{732}$ More importance should, therefore, perhaps be attached to the Danish decision, particularly as it was handed down by the Supreme Court. From it one can infer that the doctrine of equivalence is applied to claims to analogy processes and probably more often so than in relation to most other claims, but the infringing process must still be an interchangeable and obvious functional alternative for a man skilled in the art, starting from the technical content of the litigated patent.

\footnotetext{
732 See Westlander, O., Törnroth, L., Patent - Allmänna domstolarnas praxis åren 1983-1991, 213.
} 
Chemical manufacturing processes. Today very few patents are granted on analogy processes. However, the above decisions are more than of historical interest only. At least in part they represent and exemplify important principles of assessment that will be of continued value when seen and adapted to patents for chemical processes where the process features are inventions worthy of protection. Sometimes research on chemical manufacturing processes prompts the development of a whole new way of synthesis with perhaps 10 individual steps. More often, however, a small modification is made to a well-known manufacturing process, e.g. another $\mathrm{pH}$ is used. This may lead to a higher yield or greater purity of the end-product.

There is one case from the Finnish Supreme Court relating to the interpretation of claims to new processes. The invention was a process for production of tetracycline. This antibiotic was known on the day of the patent application and had been produced by other means. The new manufacturing process was microbiologic. Some particular micro-organisms of the species Streptomyces aureofaciens had been used to produce large quantities of tetracycline in a certain fermentation process, where the conditions were controlled in a particular manner. The level of chloride ions was controlled so as to make the main result of the process tetracycline. The defendant in the infringement case had imported to Finland tetracycline from Poland, manufactured under conditions according to the claims, with one exception: the chloride ion level was not within the limits specified in the claims. This difference was possible because a micro-organism with slightly different features had been used in the attacked process. ${ }^{733}$

The Finnish Supreme Court found that the way the chloride ions were controlled was an important part of the invention. Therefore it was not an infringement to manufacture without using the particular chloride ion level. The fact of the same end-product - tetracycline - resulting from the processes was not enough for the doctrine of equivalence to apply. The pharmaceutical as such was known and the defendant had not used a material feature of the patented process. Coverage reaching beyond the literal wording would not be reasonable.

Summing up, under the doctrine of equivalence a comparison is made between the claims and the contested process regarding the starting material and the reactions used. The end-product is also taken into consideration, e.g. the yield and purity. It is decisive that a man skilled in the art knew of the interchangeability between the claims and the corresponding features of the infringing process. The scope for the doctrine of equivalence in patents for chemical production processes seems to lie somewhere in between, on the one hand, the particularly limited scope in regard to product patents and, on the other, the particularly large number of equivalents existing for analogy process patents.

\subsubsection{Remarks}

\subsubsection{The value of co-ordination}

It has throughout this work been argued that the patent system can generally be seen as a way to co-ordinate research and exploitation done in secret by competing firms. It will here be endeavoured to show that the rules for interpretation of patent claims can and do fulfil an important function in this governmental co-ordination effort. Courts engaged in claim interpretation fine-tune the incentives available for research into production opportunities located close to the boundaries of claims already granted.

\footnotetext{
733 The Finnish Supreme Court of 0509 1973, NIR 1974, 185.
} 
It is indisputable that there will always be competitors who find new production opportunities on or in close approximation to the boundaries of granted patent claims. This may be because the engaged firms have not noticed that a patent has been granted. It may also be that a company by pure coincidence, i.e. without doing systematic research, finds a new production opportunity close to a granted patent. In addition, there will be competitors who do research simply in a bid to escape granted patents (known as "inventing around").

A competitor, who has made a new development close to the boundaries of an already granted patent, has a choice between four main alternatives. He can end his research project and abandon the finding; he may change the direction of the project in order for the result to be propelled clearly outside the scope of the already granted patent; he may seek a licence from the proprietor of the patent or, finally; he may carry on with the project and argue, perhaps in court, that the new product does not infringe. In order to make a rational choice between the alternatives, someone will have to decide how the claims in the competitor's patent are to be interpreted. It is in this situation that the legal rules on equivalence play an important role for the incentives created by the patent system and thereby for the total research efficiency in the industry. Efficiency through co-ordination can generally be equated to a situation in which projects that add value to already known production opportunities are carried on, but not projects without such a positive value to society. An efficient result of that kind would occur if the different projects belonged to the same company, i.e. there was a co-ordination through a common research leadership, one that would continue all projects with a positive present value.

However, the prevailing competition may lead to inefficient situations when independently acting firms find new and valuable knowledge about production opportunities within or in close approximation to a valid patent. It is unfortunate if such knowledge is simply abandoned and no information about the new production opportunity is given to the patentee. One example would be if a research-based company in in vitro or in animal tests finds a substance that may become an innovative drug, with better therapeutic qualities than the known compounds, but the company concludes that the compound possibly infringes a competitor's patent and decides not to do any further research on that compound or inform the patentee of the finding. If the two parties could expect to come up against the same situation many times in the future, one would probably see the occurrence of a pattern of co-operation between them. They would be well-advised to "invest in co-operation", i.e. send a signal of co-operation to the other party and expect him to do the same in a similar situation in the future. That would create a bigger "pie" for them to share. However, the number of multinational research-based pharmaceutical companies is probably too large for this cooperation mechanism to occur spontaneously. Instead a legal structure will have to be relied on in the hope of avoiding losses of valuable information due to the competitive situation.

The significance of new discoveries close to the boundaries of granted patents has been indicated in an American study of the patents that biotechnology companies applied for in the pharmaceutical field. ${ }^{734}$ A significant relationship was found between the classes in which the companies patented and the level of their expenditure on possible patent infringement litigation. Companies with high litigation costs (which were approximated as companies with limited financial resources and no previous experience of patent infringement disputes) on average patented in classes where the previous patent had been granted 303 days earlier. Other companies, with lower expected expenditure on infringement disputes, patented in classes where the previous patent had on average been granted 208 days earlier. The difference could not be explained by any other circumstances than the risk that the companies associated with ending up in a patent dispute. The study indicates that small companies find it

\footnotetext{
734 See Lerner, J., 38:2 The Journal of Law \& Economics 1995, 463.
} 
painstaking to reach agreements that would permit them to make full use of all new production opportunities that they discover. They choose to avoid "crowded" fields, for fear of infringement actions. Any legal teaching facilitating the exploitation of new production opportunities of disputable ownership seem to be of considerable value to society.

Poor co-ordination can in particular be anticipated if a competitor who has found a new production opportunity cannot obtain a dependent patent. It is then almost impossible for him to enter into a negotiation. The opportunities for filing dependent patents can thus be seen as one way to confront the problems that arise when a competitor finds a new production opportunity within or possibly within a granted patent. Another partial solution is the rules on compulsory licences. If they are applied it can be said that a court simulates the elusive negotiations, i.e. the judge decides on a licence fee according to what the situation would have been if there had been a smoothly running market for the licence in question. Although compulsory licences are unusual, the mere possibility is probably important because it facilitates efficient market transactions. Parties know that the other party has an alternative to a plainly unreasonable offer.

So far in this analysis, the focus of attention has been on a significant problem in the patent system. The transition from a situation of free competition in research to an exploitation phase where the patentee orchestrates the use of the protected production opportunities is not always without expense. For a number of reasons there will be situations where a patentee's competitors have gained technical knowledge that would be of value to the patentee. But this knowledge may be lost due to transacting difficulties. Attention has also been drawn to a number of mechanisms whereby the patent system is designed to minimise these costs, i.e. permit the exploitation of new production opportunities found by engaged competitors within or on the boundaries of granted claims. One more example of this can probably be seen in the interpretative rules and doctrines that serve to craft the scope of protection in an infringement dispute.

\subsubsection{The hypothesis}

The hypothesis is that the scope of protection and particularly an application of the doctrine of equivalence hinge on the relationship between the commercial value of the attacked product and the value of the patented invention. In situations where there is some added value in the attacked product, courts interpret the patent claims narrowly so that the attacked product does not infringe. If courts indulge in such assessments it can create value because when a patent is published and this interpretation is known, there will be special incentives for competitors to carry on with precisely those projects that society values. If on the other hand there is no added value in the new product, courts make a broad interpretation of the claims in order to force such competitors to reach an agreement with the patentee. Thereby courts do not create the same incentives for projects that add no extra value to consumers. These projects will either be abandoned when the patent is published or the competitor may seek a licence if he e.g. can produce and market the product more efficiently than the patentee. By using the available discretionary scope between a narrow and a broad interpretation of the claims, courts can guide the research efforts of an industry to create extra value for society, i.e. a favourable co-ordination through the incentives created by the patent system.

A need for this kind of co-ordination by interpretations of claims can be explained by looking more at the details of the negotiating situation. If the possibly infringing product has no extra value in relation to the products according to the patent, there are no 
specific transacting problems. There is a functioning market, mainly because the patentee does not have to reach an agreement with the particular infringer, nor must the possible infringer necessarily reach an agreement with the patentee. They can just as well reach agreements with other parties. The presence of many possible parties to an agreement generally reduces transaction costs. Particular transacting difficulties are present only when a competitor has some valuable knowledge that is unknown to the patentee. Then the two must reach an agreement with each other, if the value of the newly discovered production opportunity is not to be lost. The possibly infringing product may be patentable and the two patentees may perhaps then be able to reach an agreement, even though they must reach an agreement with each other and have no alternative parties. The problems are mitigated by the above mentioned rules regarding compulsory licences. The most problematic situation occurs, however, when the new production opportunity is not patentable, but at the same time valuable. It will then be very difficult to negotiate. There will be a considerable risk of the competitor neither using the new production opportunity nor informing the patentee of its existence. That was the situation within the pharmaceutical area before new uses for known pharmaceuticals could be patented. The problems still exist to a lesser extent in regard to production opportunities on the boundaries of granted patents, but can be mitigated by proper claim interpretation.

Summing up, the hypothesis is that the added value to consumers of the possibly infringing product, in relation to the value of the patented invention, is a good indication of whether a court finds an infringement. ${ }^{735}$ The larger the added value, the greater the likelihood of no infringement being found. In situations when the technical meaning of the claims is doubtful or where the doctrine of equivalence comes into question, the commercially added value is presumed to be the most important factor for decisions on the scope of protection. This will now be tested.

The Oberlandesgericht in Düsseldorf ruled that a recombinant protein was equivalent to a protein that was defined by being extracted from biological sources. One expert witness stated in the proceedings that a man skilled in the art would not have considered the differences between the claims and the contested product significant for the therapeutic use and the court therefore concluded that it was an infringement by equivalent means. Although the therapeutic use of the products did not differ as such in this case, it is difficult to reconcile the decision with the hypothesis. When a protein for the first time can be manufactured recombinantly, it will be available at lower cost, in larger quantities and create less health risks for the patients. This probably amounts to a significant added value for consumers of the invention (the patients) and thereby also for the manufacturers. ${ }^{736}$

One must therefore conclude that either the hypothesis regarding the importance of the added value is incorrect or the judgement from the German court is an exception. It appears as if the latter could be the case. The German court probably decided the case with too narrow a perspective. It only considered the type of the therapeutic effect and found no difference between the two proteins. But if the court had taken into account all factors of significance for the use of the infringing product, it would have been obvious that the added value was considerable. Therefore, strong incentives for development of this research project would have been motivated, even after the patent application on the protein derived from biological sources was published. One cannot be certain that the inventor of the recombinant protein could have got a licence from the patentee, in any case it would have been costly and

\footnotetext{
${ }^{735}$ Further support for this justification for the doctrine of equivalence can be found in the seminal article Merges, R. P., Nelson, R. R., Columbia Law Review 1990, 867.

736 Oberlandesgericht Düsseldorf 2 U 52/89 of 3112 1992, ref. by Hirsche, F., Hansen, B., Protecting Inventions in Chemistry, 329 and Lederer, F., IIC 1999, 280.
} 
the negotiations would had created uncertainty in the recombinant research project. If this judgement is followed in similar situations, the danger is that new inventions of this kind, which consumers particularly value, will not be made or developed to marketable products.

The House of Lords has ruled that a prodrug to ampicillin was equivalent to the patent for ampicillin. When the prodrug came into contact with water in the gullet it underwent a chemical reaction and became the active substance ampicillin. There was no therapeutic or other added value associated with the use of the prodrug. The court decided that this was an infringement by the use of equivalent means, despite the existence of not insignificant structural diversity between the claims and the infringing product. In this case it was plainly important that the prodrug did not add any value to the known invention. The judgement can very well be explained by the hypothesis that courts do not want to create incentives for competitors who do not add any commercial value to a patented product. They should be forced to reach an agreement with the patentee. ${ }^{737}$

The Danish Supreme Court decided that there was no infringement in a patent for an analogy process for manufacturing of an ulcer drug. ${ }^{738}$ The reasoning in the case has been somewhat criticised above, but the conclusion found to be correct. That the defendant had improved upon the patented process in a material and non-obvious manner was the elucidation furnished for why the outcome was probably warranted. The defendant in this case had established a new way of synthesis with advantages as to both yield and the environment. Hydrochloride acid did not have to be used, which meant that no special equipment was needed. In addition, the purification process was less complicated. Together these differences added considerable value to the patented manufacturing process. The decision therefore accords well with the hypothesis that attacked embodiments representing an added value are non-infringing.

In part 8.2.2.4 it was also concluded that patents for analogy processes are interpreted in a particularly broad manner. The reason given was that the value of the invention was to be found in the end-product and not in the processes in the claims. Since the claims do not reflect the true value of the invention, a competitor who has found a new process has not necessarily added any value. To these claims the doctrine of equivalence can therefore be applied quite extensively. This is in accordance with the hypothesis pertaining to the importance of "added value" in claim interpretation.

The Court of First Instance in Stockholm has also tried a case relating to an analogy process. The court ruled that it was an infringement by equivalent means, because the modifications of the process were within the knowledge of the man skilled in the art. In this case that was a relatively far-reaching application of the doctrine of equivalence, because the practical effects of the differences were considerable. The synthesis according to the claims took 24 hours, while the attacked process was almost instantaneous. Furthermore, the attacked process yielded 70 per cent more of the end-product than the patented processes. Finally, the infringing process produced pure cimetidine, while the patented process required a final purification step. These differences are of practical importance within the pharmaceutical business and of economic significance. One must therefore conclude that this judgement does not accord well with the hypothesis. Some support for the hypothesis can, however, be found in the fact that one of the judges has since stated that he questions the outcome of the case. The reason for his hesitation was the very fact of the differences between the invention and the infringing process being significant from a practical perspective. ${ }^{739}$

\footnotetext{
${ }^{737}$ House of Lords Beecham Group v. Bristol Laboratories of 3003 1978, [1978] R.P.C 153.

${ }^{738}$ Højesteret Allen Hanburys Ltd v. A/S GEA Farmaceutisk Fabrik of 2708 1993, Ugeskrift for Retsvæsen 1993, 859.

739 Stockholm Court of First Instance T 7-213-83 of 20061985 and Westlander, O., Törnroth, L., Patent Allmänna domstolarnas praxis åren 1983-1991, 213.
} 


\subsubsection{Conclusions}

The comparison between the hypothesis and available case law indicates that courts attach importance to whether the contested embodiment adds any value to the production opportunities that are disclosed in the patent. Courts limit the reach of patents in the face of substantial improvements. No definite conclusions can be drawn, however. It is difficult to determine whether the value added by the possibly infringing object is decisive for the scope of protection, because many judgements lack an explicit analysis of the economic value of the attacked product in relation to the invention. This could of course be taken to indicate that the courts do not consider this relevant. However, it is evident that courts attach some significance to the question.

One may perhaps go so far as to conclude that the added value of the infringing product is one of the most important circumstances, if not the most important, when the scope of equivalence is crafted. It can, however, never be the only consideration. There must always be opportunities for competitors to design-around patent claims, without simultaneously improving upon the function of the patented invention. Considerations about the added value can therefore primarily be used as a defence against an application of the doctrine of equivalence. A presence of economically significant differences between the contested embodiment and the claims should block a finding of equivalence, even if the technical differences, taken as such, are marginal in relation to the claims. This would make it easier for competitors to carry on research projects leading to valuable further developments of already patented inventions. Legal considerations of "added value" create incentives for those technical developments that are especially valued by customers. Socially less important, but perhaps technically more remote, developments of a patented invention do not receive the same incentives. In the latter cases an agreement with the patentee will be needed, not in the former. But, as has already been pointed out, these considerations are primarily a defence against the application of the doctrine of equivalence.

Legal certainty would perhaps benefit if courts more systematically discussed the added value of the attacked product. This is a factor that can be studied quite objectively by looking at the market for the invention and the attacked product. Today the explicit analysis of equivalence is mainly technical in character. It is tested whether a man skilled in the art knew of the interchangeability between features in the claims and the contested embodiment. It is thereby considered to some extent, but indirectly and implicitly, whether the contested embodiment has added some value to the invention. A connection exists between the technical difficulties that have been overcome and the added value of the contested embodiment. If the contested embodiment was obvious to the man skilled in the art, this probably conveys a lack of economic value. But this grappling with the doctrine of equivalence has led to the conclusion that courts would be well-advised to investigate the added value of the attacked embodiment in a more direct manner. The important problem, seemingly inescapable in the patent system, that some valuable research results of questionable ownership may not reach the marketplace is primarily related to the added value of the research results, not to the technical difficulties that have been overcome.

If increased importance is attached to the added value of the attacked embodiment in determining the scope of protection, granted claims will have an area of genuine uncertainty from a technical viewpoint. The scope can never be fully determined in technical terms in the claims, because it is also dependent on the value of the attacked product. The patent office would make a decision on the protection by establishing the 
technical coverage. But thereafter the courts would make the final decision, and they can apply the doctrine of equivalence because they know the value of the contested product. This division of labour between patent offices and courts exists today and is probably unavoidable. All claims and doctrines for interpretation will have some discretionary scope. The proposal made here is only that the portion of the scope of protection that is decided by the courts should mainly be thought of as an ex post facto adjustment taking into account the value of the attacked embodiment. The doctrine of equivalence could primarily be seen as an economic test and not a technical one. The patent claims define the invention, but does not conclusively define the scope of protection. This is because it is impossible at the granting stage to foresee possibly infringing products that add something of importance and therefore should be encouraged by courts letting inventors know that they need not in these situations reach agreements with competitors.

In part 3.3.4.6 it was argued that there should be a place in Europe for applying the reverse doctrine of equivalence, as it is in the USA. The rationale for the reverse doctrine of equivalence is the same as the rationale that has been advanced here for the "normal" doctrine of equivalence. ${ }^{740}$ Both are ways of facilitating the market introduction of new products of particularly high value to consumers, in situations where they would otherwise encroach in the scope of a patent granted. The reverse doctrine of equivalence excludes from the literal scope of protection an embodiment with particular value. The interpretation of the "normal" doctrine of equivalence, that has been presented here, limits the application of the doctrine of equivalence to situations where no value or almost none has been added by the competitor. In both cases additional incentives are created for the market introduction of products that are particularly highly valued by consumers.

740 See Merges, R. P., Nelson, R. R., Columbia Law Review 1990, 867. 


\section{References}

\section{Table of Cases}

\section{European}

The EC Court of Justice

C-8/74, ECR 1974, 837:

C-19/84, ECR 1985, 228:

C-350/92, ECR 1995, I-1985:

C-412/93, ECR 1995, 179:

C-110/95, ECR 1997, I-3251:

C-181/95, ECR 1997, I-0357:

C-267/95 and C-268/95, ECR 1996, I-6285:

C-316/95, ECR 1997, I-3929:

C-392/97 of 16 September 1999:

\section{EPO}

The Enlarged Board of Appeal

G 1/83, OJ EPO 1985, 64:

G 2/88, OJ EPO 1990, 93:

G 1/92, OJ EPO 1993, 277 :

G 2/93, OJ EPO 1995, 275 :

The Boards of Appeal

T 1/80, OJ EPO 1981, 206:

T 12/81, OJ EPO 1982, 296:

T 19/81, OJ EPO 1982, 51:

T 21/81, OJ EPO 1983, 15:

T 24/81, OJ EPO 1983, 133 :

T 22/82, OJ EPO 1982, 341:

T 37/82, OJ EPO 1984, 71:

T 65/82, OJ EPO 1983, 327:

T 119/82, OJ EPO 1984, 217:

T 128/82, OJ EPO 1984, 164 :

T 156/82, of 0901 1984:

T 181/82, OJ EPO 1984, 401:

T 192/82, OJ EPO 1984, 415:

T 2/83, OJ EPO 1984, 265:

T 14/83, OJ EPO 1984, 105 :

T 20/83, OJ EPO 1983, 419:

T 164/83, OJ EPO 1987, 149 :

T 169/83, OJ EPO 1985, 193 :

T 205/83, OJ EPO 1985, 363 :

T 206/83, OJ EPO 1987, 5:

T 13/84, OJ EPO 1986, 253:

T 81/84, OJ EPO 1988, 207 :

T 106/84, OJ EPO 1985, 132:

T 122/84, OJ EPO 1987, 177 :

T 161/84 of 2808 1986: 
T 176/84, OJ EPO 1986, 50:

T 184/84 of 0404 1986:

T 198/84, OJ EPO 1985, 209:

T 225/84 of 1607 1986:

T 271/84, OJ EPO 1987, 405:

T 289/84 of 1011 1986:

T 1/85 of 2207 1986:

T 17/85, OJ EPO 1986, 406:

T 26/85, OJ EPO 1990, 22:

T 116/85, OJ EPO 1989, 13:

T 133/85, OJ EPO 1988, 441:

T 139/85 of 2312 1986:

T 153/85, OJ EPO 1988, 1:

T 155/85, OJ EPO 1988, 87:

T 248/85, OJ EPO 1986, 261:

T 281/85 of 1405 1987:

T 292/85, OJ EPO 1989, 275:

T 7/86, OJ EPO 1988, 381:

T 19/86, OJ EPO 1989, 24 :

T 23/86, OJ EPO 1987, 316:

T 92/86 of 0511 1987:

T 197/86, OJ EPO 1989, 371:

T 254/86, OJ EPO 1989, 115:

T 281/86, OJ EPO 1989, 202:

T 290/86, OJ EPO 1992, 414:

T 385/86, OJ EPO 1988, 308:

T 51/87, OJ EPO 1991, 177:

T 81/87, OJ EPO 1990, 250:

T 83/87 of 1401 1988:

T 109/87, OJ EPO 1984, 473:

T 131/87 of 0709 1989:

T 261/87 of 1612 1988:

T 269/87 of 2401 1989:

T 296/87, OJ EPO 1990, 195:

T 301/87, OJ EPO 1990, 335:

T 238/88, OJ EPO 1992, 709:

T 271/88 of 0606 1989:

T 297/88 of 0512 1989:

T 383/88 of 0112 1992:

T 475/88 of 1123 1989:

T 3/89 of 1402 1990:

T 60/89, OJ EPO 1992, 268:

T 93/89, OJ EPO 1992, 718:

T 261/89 of 0903 1990:

T 268/89, OJ EPO 1994, 50:

T 279/89 of 0107 1991:

T 418/89, OJ EPO 1993, 20:

T 541/89 of 2103 1991:

T 564/89 of 1002 1993:

T 666/89, OJ EPO 1993, 495:

T 742/89 of 0211 1992:

T 12/90 of 2304 1990:

T 19/90, OJ EPO 1990, 476:

T 116/90 of 1812 1991:

T 130/90 of 2808 1991:

T 270/90, OJ EPO 1993, 725 :

T 366/90 of 1706 1992:

T 449/90 of 0512 1991:

T 495/90 of 3006 1992: 
T 513/90, OJ EPO 1994, 154 :

T 610/90 of 1501 1992:

T 626/90 of 0212 1993:

T 654/90 of 0805 1991:

T 683/90 of 0505 1992:

T 740/90 of 0210 1991:

T 792/90 of 2102 1991:

T 815/90, OJ EPO 1994, 389:

T 816/90 of 0709 1993:

T 893/90 of 2207 1993:

T 958/90 of 0412 1992:

T 18/91 of 2602 1993:

T 24/91, OJ EPO 1995, 512:

T 79/91 of 1109 1991:

T 109/91 of 1501 1992:

T 158/91 of 3007 1991:

T 227/91, OJ EPO 1994, 291:

T 245/91 of 2106 1994:

T 246/91 of 1409 1993:

T 247/91 of 3002 1992:

T 258/91 of 1902 1993:

T 309/91 of 2801 1993:

T 391/91 of 2211 1993:

T 409/91, OJ EPO 1994, 653:

T 435/91, OJ EPO 1995, 188:

T 440/91 of 2203 1994:

T 455/91, OJ EPO 1995, 684:

T 456/91 of 0311 1993:

T 478/91 of 0206 1993:

T 500/91 of 2110 1992:

T 548/91 of 0702 1994:

T 552/91, OJ EPO 1995, 100 :

T 566/91 of 1805 1994:

T 605/91 of 2007 1993:

T 658/91 of 1405 1993:

T 677/91 of 0311 1992:

T 741/91 of 2209 1992:

T 852/91 of 0606 1994:

T 886/91 of 1606 1994:

T 906/91 of 2504 1995:

T 91/92 of 0607 1994:

T 128/92 of 3011 1994:

T 133/92 of 1810 1994:

T 223/92 of 2007 1993:

T 334/92 of 2303 1994:

T 465/92, OJ EPO 1996, 32:

T 484/92 of 3012 1993:

T 495/92 of 1909 1995:

T 570/92, of 2206 1995:

T 630/92 of 2202 1994:

T 655/92 of 1102 1997:

T 694/92 of 0805 1996:

T 720/92 of 3105 1994:

T 820/92, OJ EPO 1995, 113:

T 923/92, OJ EPO 1996, 564:

T 939/92, OJ EPO 1996, 309:

T 954/92 of 2805 1993:

T 964/92 of 2308 1994:

T 1048/92 of 0512 1994: 
T 1072/92 of 2806 1994:

T 51/93 of 0806 1994:

T 74/93, OJ EPO 1995, 712:

T 124/93 of 1008 1995:

T 167/93 of 0305 1996:

T 213/93 of 2304 1997:

T 254/93, OJ EPO 1998, 285:

T 269/93 of 1112 1997:

T 279/93 of 1212 1996:

T 296/93, OJ EPO 1995, 627:

T 311/93 of 1601 1997:

T 351/93 of 0103 1995:

T 412/93 of 2111 1994:

T 441/93 of 2703 1996:

T 507/93 of 1902 1997:

T 574/93 of 1902 1998:

T 583/93 of 0401 1996:

T 915/93 of 2204 1994:

T 1058/93 of 2103 1996:

T 1062/93 of 1806 1996:

T 1077/93 of 3005 1996:

T 69/94 of 1806 1996:

T 133/94 of 0107 1998:

T 142/94 of 1601 1997:

T 143/94, OJ EPO 1996, 430:

T 169/94 of 3107 1996:

T 199/94 of 2303 1995:

T 207/94 of 0804 1997:

T 329/94 of 1106 1997:

T 341/94 of 1307 1995:

T 374/94 of 1903 1996:

T 379/94 of 2105 1996:

T 386/94, OJ EPO 1996, 658:

T 469/94 of 0107 1997:

T 656/94 of 1607 1998:

T 668/94 of 2010 1998:

T 673/94 of 0705 1998:

T 722/94 of 1612 1997:

T 795/94 of 2108 1997:

T 853/94 of 0211 1998:

T 856/94 of 0506 1997:

T 879/94 of 1905 1998:

T 913/94 of 2702 1998:

T 918/94 of 0607 1995:

T 958/94, OJ EPO 1997, 241:

T 73/95 of 1803 1999:

T 156/95 of 3003 1998:

T 202/95 of 2108 1998:

T 251/95 of 10121998.

T 317/95 of 2602 1999:

T 338/95 of 3001 1996:

T 367/95 of 2710 1998:

T 453/95 of 1603 1999:

T 600/95 of 2811 1996:

T 639/95 of 2101 1998:

T 964/95 of 0505 1999:

T 994/95 of 1802 1999:

T 169/96 of 3007 1996:

T 223/96 of 29011999. 
T 236/96 of 1304 1999:

T 379/96 of 1301 1999:

T 431/96 of 2302 1999:

T 643/96 of 1410 1996:

T 830/96 of 1006 1999:

T 863/96 of 0402 1999:

T 990/96 of 1202 1998:

T 1024/96 of 1606 1997:

T 450/97, OJ EPO 1999, 67:

T 636/97 of 2603 1998:

T 665/97 of 1310 1998:

T 1055/97 of 1712 1998:

T 941/98 of 3003 1999:

W 52/92 of 0204 1993:

Opposition Division

V 4/89, OJ EPO 1989, 451:

V 8/94, OJ EPO 1995, 388.

\section{National Cases}

Austria

Oberster Patent- und Markensenat Op 5/83 of 2711 1985, IIC 1988, 676:

Denmark

Højesteret Allen Hanburys Ltd v. A/S GEA Farmaceutisk Fabrik of 2708 1993, Ugeskrift for Retsvæsen 1993, 859:

England

House of Lords

Gillette Co. v. Anglo Co., [1913] R.P.C 465:

Beecham Group v. Bristol Laboratories, [1978] R.P.C 153:

Catnic v. Hill and Smith, [1982] R.P.C. 183, GRUR Int. 1982, 136; IIC 1981, 699:

Merrell Dow Pharmaceuticals Inc v. H N Norton \& Co Ltd, [1996] R.P.C. 76:

Biogen v. Medeva plc., [1997] R.P.C. 1; IIC 1997, 740:

Court of Appeal

Monsanto v. Stauffer, [1985] R.P.C. 515; IIC 1986, 115; GRUR Int. 1987, 108:

Molnlycke AB v. Proctor \& Gamble Ltd, [1994] R.P.C. 49; IIC 1995, 92:

Biogen v. Medeva plc., [1995] R.P.C. 25:

Kastner v. Rizla Limited, [1995] R.P.C. 585; IIC 1997, 114:

Improver Corporation v. Remington Consumer Products, [1990] F.S.R. 181; IIC 1990, 860:

Patents Court 
The Upjohn Company v. T. Kerfoot \& Co. Ltd., [1988] F.S.R. 1:

SK\&F Laboratories Ltd. v. Evans Medical Ltd., [1989] F.S.R. 513:

Research Corporations Supplementary Protection Certificate, [1994] R.P.C. 667:

DRACO A.B. 's SPC Application, [1996] R.P.C. 417:

Bristol-Myers Squibb -v- Baker Norton Pharmaceuticals and Napro Biotherapeutics, 20081998 :

Visx -v- Nidek and Birmingham Optical Group and Optimax Laser Eye Clinics, of 1910 1998:

Bayer plc v. Octapharma Ltd, of 0501 1999:

Playhut Inc v. Spring Form Inc. of 0810 1999:

Decision of the General Comptroller of Patents, Designs and Trade Marks

Farmitalia Carlo Erba SRL's SPC Application, [1996] R.P.C. 111:

British Technology Group Ltd.'s SPC Application, [1997] R.P.C. 50:

Finland

Finnish Supreme Court of 0509 1973, NIR 1974, 185:

France

Cour de Cassation, Alfuzosin of 2610 1993, OJ EPO 1995, 252:

Germany

Bundesgerichtshof (The Federal Supreme Court)

X ZB 9/70 of 0607 1971, GRUR 1972, 80:

X ZB 1/71 of 2504 1972, GRUR 1972, 642:

X ZB 4783 of 2009 1983, GRUR 1983, 729:

X ZR 7/82 of 3101 1984, GRUR 1984, 425:

X ZR 28/85 of 2904 1986, IIC 1987, 795; OJ EPO 1987, 551:

X ZR 51/86 of 1606 1987, GRUR 1987, 794:

X ZR 53/89 of 2102 1989, GRUR 1990, 997:

X ZB 13/90 of 3003 1993, GRUR 1993, 655:

X ZR 99/92 of 1107 1995, GRUR 1996, 109; [1997] R.P.C. 623:

X ZR 68/94 of 1704 1997, [1998] R.P.C. 423:

Bundespatentgericht (The Federal Patent Court)

Judgement of 0710 1976, Blatt für Patent-, Muster-, und Zeichenwesen 1977, 274:

3 Ni 38/87, of 1703 1987, Mitteilungen des deutschen Patentanwälte 1988, 207:

3 Ni 26/94 of 1409 1995, GRUR 1996, 822:

$15 \mathrm{~W}$ (pat) 71/97 of 2106 1999:

Oberlandesgerichts (Appelate Courts)

Oberlandesgericht München, 6 U 5155/92 of 0606 1993, ref. by Vossius, C., et. al., GRUR 1994, 472:

Oberlandesgericht in Düsseldorf 2 U 128/92, 2804 1994, ref. by Hirsche, F., Hansen, B., Protecting Inventions in Chemistry, 328:

Oberlandesgericht in Düsseldorf 2 U 52/89 of 3112 1992, ref by. Hirsche, F., Hansen, B., Protecting Inventions in Chemistry, $331 \mathrm{f}$ :

Landesgericht (First Instance Courts) 
Landesgericht Düsseldorf 4 O 310/84 of 0608 1985, GRUR 1987, 896:

Italy

Corte di Cassazione, Cimetidine of 1601 1990, GRUR Int. 1991, 497:

The Netherlands

Hoge Raad

Doxycyclin of 1006 1983, GRUR Int. 1984, 705:

Atenolol of 1812 1992, GRUR Int. 1993, 887; OJ EPO 1994, 220:

Contact Lens Liquid of 1301 1995; IIC 1997, 748:

Court of Appeal of The Hague

Glaxo-Wellcome v. Stada-Centrafarm, quoted in Ebbink, R., Patent World February 1998, 33:

Follikestimulationshormon II of 2306 1995, GRUR Int. 1997, 838; IIC 1998, 702:

Kirin Amgen v. Boehringer Mannheim of 0302 1994, quoted in Cornish, W. R., IIC 1998, 735:

Bristol-Myers Squibb v. Yew Tree Pharmaceuticals BV et al., of 2506 1998, ref. by Oosting, B., Patent World, March 1999, 24.

Court of First Instance in The Hague

The Netherlands v. Yew Tree Pharmaceuticals BV et al. of 2407 1997, ref. by Oosting, B., Patent World, March 1999, 24:

\section{Portugal}

Supreme Court Trimethroprim, GRUR Int. 1979, 482:

Sweden

Stockholm City Court (Stockholms tingsrätt)

T 7-213-83 of 2006 1985:

T 7-536-93 and T 7-737-94 of 1506 1995:

\section{Switzerland}

Handelsgericht des Kantons Zürich Tool Retainer Spindles of 0512 1988, IIC 1991, 398:

USA

Supreme Court

Graver Tank \& Mfg Co v. Linde Air Products Co, 339 US 1950, 605:

Warner-Jenkinson Company Inc. v. Hilton Davis Chemical Co, 117 S Ct. 1040 (1997): 


\section{Bibliography}

\section{Books}

AIPPI Yearbook 1978/II (Munich 15-19 May 1978).

AIPPI Yearbook 1991/V (Tokyo 5-11 April 1992).

AIPPI Yearbook 1992/III (Tokyo 5-11 April 1992).

AIPPI Yearbook 1998/VII, (Rio de Janeiro 24-29 May 1998).

Benkard, G., Patentgesetz Gebrauchmustergesetz, München 1993.

Benyamini, A., Patent Infringement in the European Community, Weinheim 1993.

Boeters, H. D., Handbuch Chemiepatent, Heidelberg 1989.

Cornish, W.R., Intellectual Property: Patents, Copyright, Trade Marks and Allied Rights, London 1989.

Fürniss, P., Chemiepatent Entscheidungen, Heidelberg 1986.

Gilat, D., Experimental Use and Patents, Weinheim 1995.

Godenhielm, B., Patentskyddets omfattning i europeisk och nordisk rätt, Helsingfors 1994.

-, Om ekvivalens och annat gott, Ekenäs 1990.

Grubb, P. W., Patents in Chemistry and Biotechnology, Oxford 1986.

Hirsche, F., Hansen, B., Protecting Inventions in Chemistry, Weinheim 1997.

Jaenichen, H-R., The European Patent Office's Case Law on the Patentability of Biotechnology Inventions,

München 1997.

Lanjouw, J. O., The Introduction of Pharmaceutical Product Patents in India: "Heartless Exploitation of the Poor and Suffering”?, National Bureau of Economic Research Working Paper No. W6366, issued in January 1998.

Lunzer, R., Singer: The European Patent Convention, London 1995.

Kamien, M. I., Schwartz, N. L., Market Structure and Innovation, Cambridge 1982.

Koktvedgaard, M., Konkurrencepraegede Immaterialretspositioner, København 1965.

Koktvedgaard, M., Østerborg, L., Patentloven, Køpenhavn 1979.

Moufang, R., Genetische Erfindungen im gewerblichen Rechtsschutz, Köln; Berlin; Bonn; München 1988.

Paterson, G., The European Patent System, London 1992. First Supplement, London 1994.

-, Second Supplement, London 1997.

Scherer, F. M., Innovation and Growth, London 1984.

Takenaka, T., Interpreting Patent Claims: The United States, Germany and Japan, Weinheim 1995.

Wegner, H. C., Patent Law in Biotechnology, Chemicals \& Pharmaceuticals, New York 1992.

Westlander, O., Törnroth, L., Patent - Allmänna domstolarnas praxis åren 1983-1991, Stockholm 1995.

\section{Articles}

Adams, J. N., Supplementary Protection Certificates: The 'Salt’ Problem, [1995] EIPR 277.

Allcock, H. M., Lotz, J. W., Patent Intelligence and Technology - Gleaning Pseudoproprietary Information from Publicly Available Data, 18 Journal of Chemical Information \& Computer Science 1978, 65.

Arrow, K. J., Economic Welfare and the Allocation of Resources for Invention, 609, in The Rate and Direction of Inventive Activity: Economic and Social Factors, National Bureau of Economic Research 1962.

Baldock, C., Patenting of ESTs, Patent World, March 1999, 18.

Barton, J. H, Patent Scope in Biotechnology, IIC 1995, 605.

Beier, K-F, Die vom Patentschutz ausgeschlossenen Erfindungen in rechtsvergleichener Sicht, GRUR Int 1960, 105.

Beier, F-K., Ohly, A., Was heißt ”unmittelbares Verfarenserzeugnis”?, GRUR Int. 1996, 973.

Beier, F-K., Strass, J., The Patent System and Its Informational Function - Yesterday and Today, IIC 1977, 387.

Beton, J., Are International Standards for Patent Claim Interpretation Possible?, [1994] EIPR 276.

Blakeney, M., Bioprospecting and the Protection of Traditional Medical Knowledge of Indigenous Peoples: An Australian Perspective, [1997] EIPR 298.

Bowie, H., et. al., Deciphering the Message in Protein Sequences: Tolerance to Amino Acid Substitutions, 247 Science 1990, 1306.

Brandi-Dohrn, M., The Unduly Broad Claim, IIC 1994, 648.

Brennan, J., Very Broad Markush Claims; A Solution or a Problem, Proceedings of a Round Table Discussion, 31 Journal of Chemical Information and Computer Science 1991, 9.

Brown, L. J., The Markush Challenge, 31 Journal of Chemical Information and Computer Science 1991, 2.

Caskey, T. C., Tribble, J. L., Who owns the code?, Science and Public Affairs, Autumn 1997, 52. 
Cockburn, I., Henderson, R., Racing to invest? The dynamics of competition in ethical drug discovery, 3:3 Journal of Economics \& Management Strategy Fall 1994, 481.

- Scala, scope, and spillovers: the determinants of research productivity in drug discovery, 27:1 Rand Journal of Economics Spring 1996, 32.

Cohen, A. D., Exceptions to Experimental Use and Limited Patent Term Extension in Israel, Patent World June 1998, 24.

Cook, T. M., Pharmaceutical Patents and the Generics Sector in Europe, Patent World February 1997, 36.

-, Regulatory Exclusivity, Patent World March 1998, 31.

Comanor, W. S., Research and Competetive Product Differentiation in the Pharmaceutical Industry in the United States, 31 Economica 1964, 372.

Cornish, W. R., Experimental Use of Patented Inventions in European Community States, 29 IIC 1998, 735.

Crespi, R. S., Inventivness in Biological Chemistry, JPTOS May 1991, 351.

-, Biotechnology, Broad Claims and the EPC, [1995] EIPR 267.

-, Recombinant DNA Patents in Litigation - A Comparative Study of Some EPO and UK National Court Decisions, IIC 1997, 603.

Culbert, P., Patent Law Reform in New Zealand: Should Methods of Medical Treatment be Patentable?, Patent World May 1997, 32.

Dane, D. S., et al., Virus-like particles in serum of patients with Australian-antigen-associated hepatitis, The Lancet 1970, 695.

Daniels, J. M., Nestmann, E. R., Kerr, A., Development of stereoisomeric (chiral) drugs: a brief review of scientific and regulatory considerations, 31 Drug Information Journal 1997, 639.

Dehlinger, P. J., A Not-so-Radical Proposal for Selecting Radical Substitutions in Markush-Type Claims, JPTOS July 1992, 463.

Dimasi, J. A., Success rates for new drugs entering clinical testing in the United States, 58:1 Clinical Pharmacological Therapy 1995, 1.

DiMasi, J. A., Lasagna, L., Development of Supplemental Indications for Already-Approved Drugs by the United States Pharmaceutical Industry, 5 Journal of Clinical and Pharmacoepidemiology 1991, 19.

Drews, J., Ryser, S., Innovation deficit in the pharmaceutical industry, 30 Drug Information Journal 1996, 97.

Ducor, P., New drug discovery technologies and patents, 22 Rutgers computer \& technology law journal 1996, 369.

Ebbink, R., Overview of Patent Litigation in Europe 1997, Patent World February 1998, 33.

Edge, G., Thinking about the technology future, 25:2 R \& D Management April 1995, 128.

Eisenberg, R. S., Proprietary Rights and the Norms of Science in Biotechnology Research, 97 The Yale Law Journal 1987, 177.

-, Patents and the Progress of Science: Exclusive Rights and Experimental Use, 56 The

University of Chicago Law Review 1989, 1017.

Faircliffe, S., Court of Justice Ruling Clarifies Regulation on SPCs for Medicines, 11 World Intellectual Property Report 1997, 82.

Gaumont, R., Patentability and Patent Scope of Pharmaceutical Inventions, IIC 1982, 431.

Gordon, W., Fair Use as a Market Failure: A Structural and Economic Analysis of the Betamax Case and its Predecessors, 82 Columbia Law Review 1982, 1600.

Grabinski, K., Kann und darf die Bestimmung des Schutzbereichs eines europäischen Patents in verschiedenen Ländern zu unterschiedlichen Ergebnissen führen?, GRUR 1998, 857.

Grabowski, H., Vernon, J., Brand Loyalty, Entry, and Price Competition in Pharmaceuticals after the 1984 Drug Act, 35 Journal of Law and Economics 1992, 331.

-, Longer Patents For Increased Generic Competition: The Waxman-Hatch Act After One

Decade, vol. 10 (Supp. 2) PharmacoEconomics 1996, 110.

Green, J. G., Scotchmer, S., On the division of profit in sequential innovation, 26:1 RAND Journal of

Economics Spring 1995, 20.

Hansen, B., Neue Problem der zweite Indikation in der europäischen und nationalen Patentpraxis, GRUR Int. 1988, 379.

Helms, R., Preface, s. xxii, in Helms, R. B., (ed), Drugs and Health, Washington D.C. 1981.

Hieber, T., Die Zulässigkeit von Versuchen an patentierten Erfindungen nach § 11 Nr. 2 PatG 1981, GRUR 1996, 439.

Hofinger, S., Determinants of an Active Patent Policy - An Empirical Study, EPI Information 1996, 87.

Jacob, R., Some Recent Cases of Significance in the UK, IIC 1997, 880.

-, Scope of patents in the UK - is there a doctrine of equivalence?, NIR 1999, 475. 
Janicke, P. M., The Crisis in Patent Coverage: Defining Scope of an Invention by Function, 8 Harvard Journal of Law \& Technology 1994, 155.

Jayaraman, K. S., US patent office withdraws patent on Indian herb, 389 Nature 0409 1997, 6.

Jucker, E. M., Drug Innovation and Patents, American Patent Lawyers Association Quarterly Journal 1982, 81.

Karet, I., A Question of Epistemology, [1996] EIPR 97.

-, Over-broad Claims: An Inventive Step by the EPO, [1996] EIPR 561.

Kellner, H., Salz in der Suppe oder Sand im Getriebe? - Anmerkungen zu Schutzrechtszertifikaten, GRUR 1999, 805.

Kiley, T. D., Patents on Random Complementary DNA Fragments?, 257 Science 1992, 915.

Kitch, E. W., The Nature and Function of the Patent System, 20 Journal of Law \& Economics 1977, 265.

Kjeldgaard, R. H., Marsh, D. R., Recent Developments in the Patent Protection of Plant-based Technology in the United States, [1997] EIPR 16.

Klöpsch, F., a comment to the decision X ZB 4783 from Bundesgerichthof, GRUR 1983, 733.

Koenig, M. E. D., A Bibliometric Analysis of Pharmaceutical Research, 12 Research Policy 1983, 15.

Kolker, P. L., The Supplementary Protection Certificate: The European Solution to Patent Term Restoration, Intellectual Property Quarterly 1997, 249.

Krol, T.F., Coleman, J. C., Bryant, P. J., Scientific Competitive Intelligence in R\&D, 30 Drug information journal 1996, 250.

Kushan, J P., Protein patents and the doctrine of equivalents: Limits on the expansion of patent rights, 6:1 High Technology Law Journal 1991, 109.

Köhler, G., Milstein, C., Continuos Cultures of Fused Cells Secreting Antibodies of Predefined Specificity, 256 Nature 1975, 495.

Lederer, F., Equivalence of Chemical Product Patents, IIC 1999, 275.

Lerner, J., The importance of patent scope: an empirical analysis, 25:2 RAND Journal of Economics Summer 1994, 319.

-, Patenting in the shadow of competitors, 38:2 Journal of Law \& Economics 1995, 463.

Levin, M., Udviklingen i Norden, Allmän inledning, NIR 1996, 409.

Mackenzie, M., Cambrioso, A., Keating, P., The commercial application of a scientific discovery: The case of the hybridoma technique, 17 Research Policy 1988, 155.

Maebius, S. B., Novel DNA Sequences and the Utility Requirement, JPTOS September 1992, 651.

Marquis, H. L., An Economic Analysis of the Patentability of Chemical Compounds, 63:1 JPTOS 1981, 3.

Mcinerney, A., Biotechnology: Biogen v. Medeva in the House of Lords, [1998] EIPR 14.

von Meibom, W., och Pitz, J., Experimental Use and Compulsory Licence Under German Patent Law, Patent World June/July 1997, 27.

Merges, R. P., A Brief Note on Blocking Patents and Reverse Equivalents: Biotechnology as an Example”, JPTOS November 1991, 878.

-, Uncertainty and the Standard of Patentability, 7:1 High Technology Law Journal 1992, 1.

-, Intellectual Property Rights and Bargaining Breakdown: The Case of Blocking Patents, 62

Tennesse Law Review 1994, 75. Also published in Intellectual Property Law Review 1996, 69.

-, The end of friction? Property rights and contract in the "newtonian” world of on-line commerce, 12:1 Berkely Technology Law Journal 1997.

Merges, P., Nelson, R., On the Complex Economics of Patent Scope, 90 Columbia Law Review 1990, 839.

Miller, S. A., Should patenting of surgical procedures and other medical techniques by physicians be banned? 36:2 IDEA 1996, 255.

von Morzé, H., Hanna, P., Critical and Practical Observations regarding Pharmaceutical Patent Tem Restoration in the European Communities (Part 1) JPTOS June 1995, 479.

Moufang, R., Methods of medical treatment under patent law, IIC 1993, 18.

Multimear, J., The Challange to Merck v Stephar, [1996] EIPR 100.

Oosting, B., Second Medical Use Claims: Recent Developments, Patent World March 1999, 24.

Oser, A., Patenting (Partial) Gene Sequences Taking Particular Account of the EST Issue, IIC 1999, 1.

Pagenberg, J., The Inventive Step Under European Law, IIC 1978, 351.

-, (no titel) in Beier, F-K., Haertel, K. and Schricker, G., Europäisches Patentübereinkommen -

Münchener Gemeinschaftskommentar, Köln, Berlin, Bonn, München 1984.

-, More Refined Rules of Claim Interpretation in Germany - Are They Necessary?, IIC 1995, 228.

de Pastors, A., Supplementary Protection Certificates. Situation after Two Years of Operation of the EC 1786/92 SPC Regulation, 17:3 World Patent Information 1995, 189.

Paterson, G., Die Neuheit von Verwendungsansprüchen, GRUR 1996, 1093.

Pedrazzini, M. M., Zur patentrechtlichen Problematik von Versuchen, die ein fremdes Patentrecht benützen, GRUR Int. 1996, 373. 
Penan, H., R \& D strategy in a techno-economic network: Alzheimer’s disease therapeutic strategies, 25 Research Policy 1996, 337.

Raff, K., No protection for Fruits of Research under the Supplementary Protection Certificate Scheme, [1996] EIPR 508.

Rauh, P. A., Jaenichen, H-R., Novelty and Inventive Step in Inventions having Proteins or DNA Sequences as their Subject matter, JPTOS May 1988, 313. Also published in GRUR 1987, 753.

Reisman, J. M., Physicians and Surgeons as Inventors: Reconciling Medical Process Patents and Medical Etics, 10 High Technology Law Journal 1995, 355.

Roberts, T., Broad Claims for Biotechnological Inventions, [1994] EIPR 371.

Roberts, C., The Prospects of Success of the National Institute of Health's Human Genome Application, [1994] EIPR 30

Rogge, R., The Concept of Novelty and European Patent Law, IIC 1997, 443.

Scharen, U., Der Schutzbereich des Patents im Falle verschiedener Einwände des Beklagten eines

Verletzungsprozesses, GRUR 1999, 285.

Scotchmer, S., Standing on the Shoulders of Giants: Cumulative Research and the Patent Law, 5:1 Journal of Economic Perspective 1991 s. 29 ff.

Sheiness, D., Patenting Gene Sequences, JPTOS February 1996, 121.

Sherman, B., Patent Law in a Time of Change: Non-Obviousness and Biotechnology, 10 Oxford Journal of Legal Studies 1990, 278.

Sneader, W., Chronology of Drug Introduction s. 7 ff, in Hansch, C., Taylor, J. B., (ed), Comprehensive medicinal chemistry: The rational design, mechanistic study and therapeutic application of chemical compounds, Oxford 1990.

Spangenberg, R., The Novelty of ‘Selection’ Inventions, IIC 1997, 808.

de Stevens, G., Lead Structure Discovery and Development, s. 261 ff, in Hansch, C., Dayton, C. (ed), Comprehensive Medicinal Chemistry, Oxford 1990.

Straus, J., Neuheit, ältere Anmeldungen und unschädliche Offenbarungen im europäischen und deutschen Patentrecht, GRUR Int. 1994, 89.

-, Current Issues in Patenting Research Results Close to Industrial Application, 7 in European Research Structures - Changes and Challenges, Rosenheim 1994.

-, Patentrechtliche Probleme der Gentherapie, GRUR 1996, 10.

-, Abhängigkeit bei Patenten auf genetische Information - ein Sonderfall?, GRUR 1998, 314.

-, The Development of Equivalence In Legal Theory And Case Law In Germany Before and After EPC, NIR 1999, 459.

Suchy, H., Patent Protection for a Second Medical Use, IIC 1982, 471.

Szabo, G. S. A., The Problem and Solution Approach in the European Patent Office, IIC 1995, 457.

Takenaka, T., Doctrine of equivalents after Hilton Davis: a comparative law analysis, 22 Rutgers Computer \& Technology Law Journal 1996, 479.

Tauchner, P., Reflections on the German Terfenadine Prodrug Case, Patent World April 1999, 19.

Thums, D., Patent Protection for Medical Treatment - A Distinction Between Patent and Medical Law, IIC 1996, 423.

Törnroth, L., Andra medicinska indikationen särskilt i belysningen av förarbetena, NIR 1987, 147.

Valenzuela, P., et. al., Nucleotide sequence of the gene coding for the major protein of hepatitis B virus surface antigen, 280 Nature 197908 30, 815.

Vossius, C., et al., Der Terfenadine-Verletzungsstreit; zum Stand der Neuheitsprüfung, GRUR 1994, 473.

Wade, F., Inventor of Hybridoma Technology Failed to File for Patent, 208 Science 1980, 693.

Wells, N., Problem/Solution Approach in the EPO, [1999] EIPR 220.

Werner, R., Biobusiness in the Pharmaceutical Industry, 27:2 Drug Research 1996, 1091.

Wibbelmann, J., Protection of 'Products Directly Obtained by Processes' According to Article 64(2) EPC, [1996] EIPR 174.

-, Broad Claims: A Nuisance, [1997] EIPR 515.

Wiggins, S. N., The Pharmaceutical Research and Development Decision Process, 55, in Helms, R. B. (ed), Drugs and Health, Washington D. C. 1981.

Witte, D., Ensing, K., Franke, J-P., De Zeeuw, R., Development and registration of chiral drugs, 15 Pharmaceutical World Science 1993, 10.

Zucker, G. L., Darby, M. R., Present at the biotechnological revolution: transformation of technological identity for a large incumbent pharmaceutical firm, 26 Research Policy 1997, 429. 